\title{
International guidelines for groin hernia management
}

\author{
The HerniaSurge Group ${ }^{1}$
}

Received: 5 February 2017/Accepted: 13 September 2017/Published online: 12 January 2018

(C) The Author(s) 2018. This article is an open access publication

\begin{abstract}
Introduction Worldwide, more than 20 million patients undergo groin hernia repair annually. The many different approaches, treatment indications and a significant array of techniques for groin hernia repair warrant guidelines to standardize care, minimize complications, and improve results. The main goal of these guidelines is to improve patient outcomes, specifically to decrease recurrence rates and reduce chronic pain, the most frequent problems following groin hernia repair. They have been endorsed by all five continental hernia societies, the International Endo Hernia Society and the European Association for Endoscopic Surgery.
\end{abstract}

The guidelines have been endorsed by the following societies: European Hernia Society (EHS), Americas Hernia Society (AHS), Asia Pacific Hernia Society (APHS), Afro Middle East Hernia Society (AMEHS), Australasian Hernia Society, International Endo Hernia Society (IEHS), European Association for Endoscopic Surgery and Other Interventional Techniques (EAES).

The full list of collaborator names from the Steering Committee and HerniaSurge Group are listed in the Acknowledgements section at the end of the article.

The comments to this article are available at https://doi.org/10.1007/ s10029-017-1673-0, https://doi.org/10.1007/s10029-017-1674-z, https://doi.org/10.1007/s10029-017-1675-y, https://doi.org/10.1007/ s10029-017-1676-x, https://doi.org/10.1007/s10029-017-1677-9, https://doi.org/10.1007/s10029-017-1678-8, https://doi.org/10.1007/ s10029-017-1679-7.

The HerniaSurge Group

m.p.simons@olvg.nl

1 HerniaSurge Group, OLVG Hospital, Eerste Oosterparkstraat 9, 1091 AC Amsterdam, The Netherlands
Methods An expert group of international surgeons (the HerniaSurge Group) and one anesthesiologist pain expert was formed. The group consisted of members from all continents with specific experience in hernia-related research. Care was taken to include surgeons who perform different types of repair and had preferably performed research on groin hernia surgery. During the Group's first meeting, evidence-based medicine (EBM) training occurred and 166 key questions (KQ) were formulated. EBM rules were followed in complete literature searches (including a complete search by The Dutch Cochrane database) to January 1, 2015 and to July 1, 2015 for level 1 publications. The articles were scored by teams of two or three according to Oxford, SIGN and Grade methodologies. During five 2-day meetings, results were discussed with the working group members leading to 136 statements and 88 recommendations. Recommendations were graded as "strong" (recommendations) or "weak" (suggestions) and by consensus in some cases upgraded. In the Results and summary section below, the term "should" refers to a recommendation. The AGREE II instrument was used to validate the guidelines. An external review was performed by three international experts. They recommended the guidelines with high scores.

Results and summary The risk factors for inguinal hernia (IH) include: family history, previous contra-lateral hernia, male gender, age, abnormal collagen metabolism, prostatectomy, and low body mass index. Peri-operative risk factors for recurrence include poor surgical techniques, low surgical volumes, surgical inexperience and local anesthesia. These should be considered when treating IH patients. IH diagnosis can be confirmed by physical examination alone in the vast majority of patients with appropriate signs and symptoms. Rarely, ultrasound is necessary. Less commonly still, a dynamic MRI or CT scan 
or herniography may be needed. The EHS classification system is suggested to stratify IH patients for tailored treatment, research and audit. Symptomatic groin hernias should be treated surgically. Asymptomatic or minimally symptomatic male IH patients may be managed with "watchful waiting" since their risk of hernia-related emergencies is low. The majority of these individuals will eventually require surgery; therefore, surgical risks and the watchful waiting strategy should be discussed with patients. Surgical treatment should be tailored to the surgeon's expertise, patient- and hernia-related characteristics and local/national resources. Furthermore, patient healthrelated, life style and social factors should all influence the shared decision-making process leading up to hernia management. Mesh repair is recommended as first choice, either by an open procedure or a laparo-endoscopic repair technique. One standard repair technique for all groin hernias does not exist. It is recommended that surgeons/surgical services provide both anterior and posterior approach options. Lichtenstein and laparo-endoscopic repair are best evaluated. Many other techniques need further evaluation. Provided that resources and expertise are available, laparo-endoscopic techniques have faster recovery times, lower chronic pain risk and are cost effective. There is discussion concerning laparo-endoscopic management of potential bilateral hernias (occult hernia issue). After patient consent, during TAPP, the contra-lateral side should be inspected. This is not suggested during unilateral TEP repair. After appropriate discussions with patients concerning results tissue repair (first choice is the Shouldice technique) can be offered. Day surgery is recommended for the majority of groin hernia repair provided aftercare is organized. Surgeons should be aware of the intrinsic characteristics of the meshes they use. Use of so-called low-weight mesh may have slight short-term benefits like reduced postoperative pain and shorter convalescence, but are not associated with better longer-term outcomes like recurrence and chronic pain. Mesh selection on weight alone is not recommended. The incidence of erosion seems higher with plug versus flat mesh. It is suggested not to use plug repair techniques. The use of other implants to replace the standard flat mesh in the Lichtenstein technique is currently not recommended. In almost all cases, mesh fixation in TEP is unnecessary. In both TEP and TAPP it is recommended to fix mesh in M3 hernias (large medial) to reduce recurrence risk. Antibiotic prophylaxis in average-risk patients in low-risk environments is not recommended in open surgery. In laparo-endoscopic repair it is never recommended. Local anesthesia in open repair has many advantages, and its use is recommended provided the surgeon is experienced in this technique. General anesthesia is suggested over regional in patients aged 65 and older as it might be associated with fewer complications like myocardial infarction, pneumonia and thromboembolism. Perioperative field blocks and/or subfascial/subcutaneous infiltrations are recommended in all cases of open repair. Patients are recommended to resume normal activities without restrictions as soon as they feel comfortable. Provided expertise is available, it is suggested that women with groin hernias undergo laparoendoscopic repair in order to decrease the risk of chronic pain and avoid missing a femoral hernia. Watchful waiting is suggested in pregnant women as groin swelling most often consists of self-limited round ligament varicosities. Timely mesh repair by a laparo-endoscopic approach is suggested for femoral hernias provided expertise is available. All complications of groin hernia management are discussed in an extensive chapter on the topic. Overall, the incidence of clinically significant chronic pain is in the $10-12 \%$ range, decreasing over time. Debilitating chronic pain affecting normal daily activities or work ranges from 0.5 to $6 \%$. Chronic postoperative inguinal pain (CPIP) is defined as bothersome moderate pain impacting daily activities lasting at least 3 months postoperatively and decreasing over time. CPIP risk factors include: young age, female gender, high preoperative pain, early high postoperative pain, recurrent hernia and open repair. For CPIP the focus should be on nerve recognition in open surgery and, in selected cases, prophylactic pragmatic nerve resection (planned resection is not suggested). It is suggested that CPIP management be performed by multi-disciplinary teams. It is also suggested that CPIP be managed by a combination of pharmacological and interventional measures and, if this is unsuccessful, followed by, in selected cases (triple) neurectomy and (in selected cases) mesh removal. For recurrent hernia after anterior repair, posterior repair is recommended. If recurrence occurs after a posterior repair, an anterior repair is recommended. After a failed anterior and posterior approach, management by a specialist hernia surgeon is recommended. Risk factors for hernia incarceration/strangulation include: female gender, femoral hernia and a history of hospitalization related to groin hernia. It is suggested that treatment of emergencies be tailored according to patient- and hernia-related factors, local expertise and resources. Learning curves vary between different techniques. Probably about 100 supervised laparo-endoscopic repairs are needed to achieve the same results as open mesh surgery like Lichtenstein. It is suggested that case load per surgeon is more important than center volume. It is recommended that minimum requirements be developed to certify individuals as expert hernia surgeon. The same is true for the designation "Hernia 
Center". From a cost-effectiveness perspective, day-case laparoscopic IH repair with minimal use of disposables is recommended. The development and implementation of national groin hernia registries in every country (or region, in the case of small country populations) is suggested. They should include patient follow-up data and account for local healthcare structures. A dissemination and implementation plan of the guidelines will be developed by global (HerniaSurge), regional (international societies) and local (national chapters) initiatives through internet websites, social media and smartphone apps. An overarching plan to improve access to safe IH surgery in low-resource settings (LRSs) is needed. It is suggested that this plan contains simple guidelines and a sustainability strategy, independent of international aid. It is suggested that in LRSs the focus be on performing high-volume Lichtenstein repair under local anesthesia using low-cost mesh. Three chapters discuss future research, guidelines for general practitioners and guidelines for patients.

Conclusions The HerniaSurge Group has developed these extensive and inclusive guidelines for the management of adult groin hernia patients. It is hoped that they will lead to better outcomes for groin hernia patients wherever they live. More knowledge, better training, national audit and specialization in groin hernia management will standardize care for these patients, lead to more effective and efficient healthcare and provide direction for future research.

Keywords Hernia - Inguinal hernia · Groin hernia ·

Femoral hernia $\cdot$ Inguinal hernia treatment $\cdot$ Inguinal hernia repair - Open inguinal hernia - Laparoscopic inguinal hernia $\cdot$ Shouldice $\cdot$ Lichtenstein - TEP · TAPP · Standard of care $\cdot$ Guideline $\cdot$ Practice guideline

\section{Chapters}

PART 1

\section{Management of inguinal hernias in adults}

1. Introduction

2. Risk factors for the development of inguinal hernias in adults

3. Diagnostic modalities
4. Groin hernia classification

5. Indications: treatment options for symptomatic and asymptomatic patients

6. Surgical treatment of inguinal hernias

7. Individualization of treatment options

8. Occult hernias and bilateral repair

9. Day surgery

10. Meshes

11. Mesh fixation

12. Antibiotic prophylaxis

13. Anesthesia

14. Postoperative pain: prevention and management

15. Convalescence

\section{PART 2}

\section{Specific aspects of groin hernia management}

16. Groin hernias in women

17. Femoral hernia management

18. Complications: prevention and treatment

19. Pain: prevention and treatment

20. Recurrent inguinal hernias

21. Emergency treatment of groin hernia

\section{PART 3}

Quality, research and global management Quality aspects

22. Training and the learning curve

23. Specialized centers and hernia specialists

24. Costs

25. Registries

26. Outcomes and quality assessment

27. Dissemination and implementation

Global groin hernia management

28. Inguinal hernia surgery in low-resource settings

Research, general practitioner and patient perspectives

29. Questions for research

30. Summary for general practitioners

31. Management of groin hernias from patients' perspectives 

High W W are very confident that the estimate of effect lies close to the true effect for this outcome.
Moderate $\quad$ We are moderately confident that the estimate of effect lies close to the true effect for this outcome.
Low W W have limited confidence that the estimate of effect lies close to the true effect for this outcome.
Very Low $\quad$ We have no evidence, we are unable to estimate an effect, or we have no confidence in the estimate of effect for this outcome.
Statements and recommendations were made with the final grade according to the evidence and then after discussions within the HerniaSurge Group in some instances they were upgraded or downgraded. A recommendation was formulated as "suggestions" for weak and "recommendations" for strong evidence.

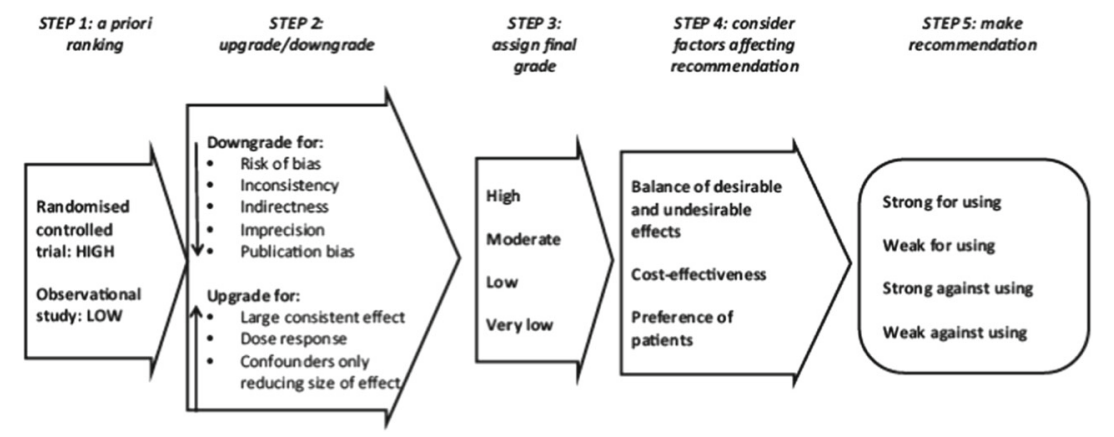

Figure 1. Quality of evidence according to Grade

\section{PART 1 \\ Management of inguinal hernias in adults}

\section{Chapter 1}

\section{HerniaSurge: international guidelines for groin hernia management}

\section{Introduction}

M. P. Simons, N. van Veenendaal, H. M. Tran, B. van den Heuvel and H. J. Bonjer

Lifetime occurrence of groin hernia-viscera or adipose tissue protrusions through the inguinal or femoral canal-is $27-43 \%$ in men and $3-6 \%$ in women. ${ }^{1}$ Inguinal hernias are almost always symptomatic; and the only cure is surgery. ${ }^{2}$ A minority of patients are asymptomatic but even a watchand-wait approach in this group results in surgery in approximately $70 \%$ within 5 years. ${ }^{2}$

Worldwide, inguinal hernia repair is one of the most common surgeries, performed on more than 20 million people annually. ${ }^{1}$ Surgical treatment is successful in the majority of cases, but recurrences necessitate reoperations in $10-15 \%$ and long-term disability due to chronic pain (pain lasting longer than 3 months) occurs in 10-12\% of patients. Approximately $1-3 \%$ of patients have severe chronic pain. This has a tremendous negative effect globally on health and healthcare costs.

However, better outcomes are definitely possible. Our objective is to improve groin hernia patient care worldwide by developing and globally distributing standards of care based on all available evidence and experience.

Currently, groin hernia treatment is not standardized. Three hernia societies have separately published guidelines aimed at both improving treatment and enhancing the education of surgeons involved in groin hernia treatment. In 2009, the European Hernia Society (EHS) published guidelines covering all aspects of inguinal hernia treatment in adult patients. ${ }^{3}$ The EHS guidelines were updated in 2014. ${ }^{4}$ The International Endo Hernia Society (IEHS) published guidelines in 2011 covering laparo-endoscopic groin hernia repair. ${ }^{5}$ In 2013, the European Association for Endoscopic Surgery (EAES) published a consensus document focused on aspects of laparo-endoscopic treatments. $^{5,6}$ These three societies began collaborating in 
2014, concluding it was both necessary and logical to develop a universal set of guidelines for groin hernia treatment. "Groin Hernia Guidelines" was selected as the name for the collaborative effort since information on femoral hernias was included for the first time. A movement was launched to develop a state-of-the-art series of guidelines spearheaded by passionate hernia experts for all aspects of abdominal wall hernia treatment. The European societies-EHS, IEHS and EAES-invited scientific societies worldwide with a focus on groin hernias to participate. The project was named "HerniaSurge" (http://www. herniasurge.com), forged from the combination of "hernia" and "surge" as a metaphor for waves crossing all continents.

\section{Evolution of groin hernia surgery}

The first groin hernia surgeries were done during the end of the sixteenth century. They involved hernia sac reduction and resection and posterior wall reinforcement of the inguinal canal by approximating its muscular and fascial components. Subsequently, many hernia repair variants were introduced. Prosthetic material utilization commenced in the 1960s, initially only in elderly patients with recurrent inguinal hernias. Favorable long-term results of these mesh repairs encouraged adoption of mesh repair in younger patients. Presently, the majority of surgeons in the world favor mesh repair of inguinal hernias. In Denmark, with its complete IH repair statistics in a national database, mesh use is currently close to $100 \% .^{7}$ In Sweden, mesh use is above $99 \%{ }^{8}$ In the early 1980 s, minimally invasive techniques for groin hernia repair were first performed and reported on in the scientific literature, adding another management modality. Laparoscopic Trans Abdominal Pre-Peritoneal (TAPP) and Totally Extra Peritoneal (TEP) endoscopic techniques, collectively, "laparo-endoscopic surgery", have been developed as well.

The fact that so many different repairs are now done strongly suggests that a "best repair method" does not exist. Additionally, large variations in treatments result from cultural differences amongst surgeons, different reimbursement systems and differences in resources and logistical capabilities.

Surgeons searching for "best" treatment strategies are challenged by a vast diverse scientific literature, much of which is difficult to interpret and apply to one's local practice environment. As noted, hernia repair techniques vary broadly, dependent upon setting. Mesh use probably varies from 0 to $5 \%$ in low-resource settings to $95 \%$ in settings with the highest resources. Currently, open mesh repair (mainly Lichtenstein repair) is still most frequently used. There are specialist hernia surgeons and specialized hospitals that promote non-mesh repair especially in patients with a low-risk profile for recurrence. Meshes used in gynecological operations have caused many lawsuits and the spin-off is a justified alertness by media and the public questioning its safety in inguinal hernia repair. There are concerns about influence of insurance companies and industry. There are patients that refuse the use of mesh.

Laparo-endoscopic surgery use varies from zero to a maximum of approximately 55\% in some high-resource countries. The average use in high-resource countries is largely unknown except for some examples like Australia $(55 \%),{ }^{9}$ Switzerland $(40 \%),{ }^{10}$ the Netherlands $(45 \%)$ and Sweden $(28 \%) .{ }^{8}$ Sweden has a national registry with complete coverage. Interesting are the following percentages for the year 2015: Lichtenstein 64\%, TEP 25\%, TAPP $3 \%$, open pre-peritoneal mesh $3.3 \%$, combined open and pre-peritoneal $2.7 \%$ and tissue repair in $0.8 \%$. The German Herniamed registry which contains data on about 200,000 patients (not complete national coverage, so possibly biased) contains interesting information confirming that a wide variety of techniques are in use. The percentages over the period 2009-2016 were: TAPP 39\%, TEP 25\%, Lichtenstein 24\%, Plug 3\%, Shouldice 2.6\%, Gilbert PHS $2.5 \%$ and Bassini $0.2 \%$. Other reliable data from Asia and America are lacking and often outdated once published. Table 1 indicates current hernia repair techniques.

Table 1. Current inguinal hernia repair techniques

\begin{tabular}{|l|l|}
\hline Non-mesh techniques & Shouldice \\
& Bassini (and many variations) \\
& Desarda \\
\hline Open mesh techniques* & Lichtenstein \\
& Trans inguinal pre-peritoneal (TIPP) \\
& Trans rectal pre-peritoneal (TREPP) \\
& Plug and patch \\
& PHS (bilayer) \\
& Variations \\
\hline Endoscopic techniques & Totally extra-peritoneal (TEP) \\
& Trans abdominal pre-peritoneal repair (TAPP) \\
& Single incision laparoscopic repair (SILS) \\
& Robotic repair \\
\hline *These can be modified; and different types of mesh are in use. &
\end{tabular}




\section{Future directions}

Standardizing groin hernia repairs and improving outcomes requires that many questions be answered. Best operative techniques should have the following attributes: low incidence of complications (pain and recurrence), relatively easy to learn, fast recovery, reproducible results, and cost effectiveness. Treatment of groin hernia patients will improve if we honor all stakeholders' interests (patients, hospitals, surgeons and society).

Worldwide, groin hernia surgery outcomes need improvement. Recurrence rates-as measured by the proxy of reoperations-still range from 10 to $15 \%$; although the increasing use of mesh has resulted in falling recurrence rates. ${ }^{11}$ There are great concerns about the complication of chronic pain which still occurs in $10-12 \%$ of patients.

\section{Our process}

The HerniaSurge guidelines that follow have been developed to address all questions concerning groin hernia repair in adults, worldwide. They contain recommendations for all groin hernia types, in all kinds of patients and in all parts of the world. It has been written by and endorsed by experts from every continent and from all the major hernia societies-European, Americas, Asia-Pacific, Afro-Middle-East and Australasian. Fifty expert surgeons from 19 countries crafted these state-of-the-art guidelines. We consider this work a "living document", open to interpretation, modification and improvement over time with increasing experience and knowledge.

The involved experts have extensive clinical and scientific experience and a combined scholarly output of hundreds of publications focused on various aspects of groin hernia management. They are experienced in open non-mesh, open mesh and both TEP and TAPP techniques. The HerniaSurge steering committee has done its best to include and honor all treatment approaches, without prejudice and self-interest. Although evidence in the scientific literature forms the foundation for the guidelines, we searched to incorporate patients' wishes and surgeon's expectations. Factors like financial resources and logistics were taken into account as well. Our aim was to offer unbiased guidance to all surgeons and patients wherever they reside.

\section{Guideline formulation}

The HerniaSurge guidelines are developed according to the AGREE instrument II (Appraisal of Guidelines for
Research and Evaluation). They are not a textbook, so extensive background information is not included. However, they represent the results of an extensive literature search spanning to 1 January 2015 for systematic reviews and to 1 July 2015 for randomized controlled trials and best evidence. During five 2-day meetings (Amsterdam April 2014, Edinburgh June 2014, Warsaw October 2014, Cologne February 2015 and Milano April/May 2015) and a 4-day meeting in Amsterdam in September 2015, a standard evidence-based process was rigorously used. Teams of two or three HerniaSurge members performed standard search strategies and scored greater than 3500 articles according to Oxford, SIGN and Grade methodology. ${ }^{12,} 13$ Level of evidence was first graded up or down by teams and later in all recommendations by the whole committee. Then, the statements and recommendations were developed and these were also graded during three consensus meetings. Statements are scored according to the levels very low, low, moderate or high. The recommendations contain the terms "recommend" when strong and "suggest" when weak. The grading consists of moving up or down in level after discussing the evidence in HerniaSurge meetings (Fig. 1). The first consensus was sought within the committee of 50 surgeons. The second consensus was sought via the internet and the final consensus during the EHS Rotterdam meeting of June 2016. The results of the consensus studies (including further consensus meetings during the APHS in October 2016 and AHS in March 2017 meetings) will be published separately. This strategy of combining evidence and expert opinion by consensus led to some very strong recommendations that not only reflect the evidence in literature, but also truly reflect the opinions of 50 international leaders in groin hernia surgery. Expert opinion in this case is the opinion of the entire committee. For some important recommendations, long and passionate discussions led to the consensus found in these guidelines. Our discussions transcended countries and cultures and withstood pressures from finance and/or industry-motivated opinions. Statements and recommendations sometimes strongly favor certain treatments but are not necessarily suited to use in all parts of the world depending on local tradition, training capabilities and/or resources. The adage applies that any technique, thoroughly taught and frequently performed with good results, is valid. Some techniques are easily learned and offer good results whilst others might be very difficult to master but offer great results. All these techniques are highly dependent on the surgeon's knowledge of anatomy, caseload and dedication to groin hernia surgery. 
HerniaSurge would like to stress the importance of shared decision-making with patients. Type of hernia, patient profile, surgeons' expertise, patient's wishes and expectations, logistical possibilities and local resources are all key factors that finally lead to a treatment advice.

All search strategies, tables with articles and background information will be published on HerniaSurge's website (https://www.herniasurge.com). All articles are filed per chapter in Mendeley ${ }^{\mathrm{R}}$ reference manager.

We would like to emphasize the fact that the "International Guidelines for Groin Hernia Management" is NOT a legal document, merely guidelines. If surgeons choose not to follow strong recommendations, they should do so in consultation with their patients and document this in the medical record.

HerniaSurge encourages the establishment of local and national registries because they are valuable for audit and research. HerniaSurge predicts an increase in training of hernia specialist surgeons and the formation of hernia centers, but acknowledges that training and educating general surgeons who work in general practice in the shortterm will have a greater impact on the results of groin hernia surgery. Furthermore, HerniaSurge is committed to develop E-learning modules and a "HerniaSurge App" to aid surgeons and patients around the world.

The HerniaSurge Group has formulated a large number of new research questions. The guidelines will be updated every 2 years as new evidence is published. The expiration date for this document is June 1, 2018.

The guidelines were externally reviewed by professors Jeekel (Europe), Ramshaw (USA) and Sharma (Asia). The Agree scores are published in the website of HerniaSurge (https://www.herniasurge.com).

\section{Chapter 2}

\section{Risk factors for the development of inguinal hernias in adults}

\author{
L. N. Jorgensen, W. W. Hope, and T. Bisgaard
}

\section{Introduction}

Numerous risk factors exist for the development of primary inguinal hernias (IH) and recurrent inguinal hernias (RIH) in adults, some better studied than others. These risk factors span a range, from acquired to genetic and modifiable to immutable. Some are under the surgeon's control, but many are not.

For the purposes of this chapter (unless stated otherwise), IH repair is considered synonymous with IH diagnosis. The studies referenced below do not distinguish between open and laparo-endoscopic repairs or between direct and indirect hernias. Femoral hernias are not considered in this review nor are IHs in children except for a brief mention.

\section{Key questions}

KQ02.a What are the risk factors for the development of primary inguinal hernias in adults?

KQ02.b What are the acquired, demographic and perioperative risk factors for recurrence after treatment of $\mathrm{IH}$ in adults?
Statements and Recommendation

Statement
Important intrinsic risk factors for the development of primary inguinal hernias include: inheritance, a previous contralateral hernia, male gender, age and abnormal collagen metabolism.
Statement
Important acquired risk factors for the development of primary inguinal hernias are previous prostatectomy and low body mass index.
Statement
Future studies on primary inguinal hernia formation should consider these inborn and acquired risk factors.

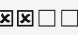
Several important intrinsic/demographic (anatomy, female gender, abnormal collagen metabolism), acquired (obesity), and Statement perioperative risk factors (poor surgical technique, low surgical volume, surgical inexperience, and local anesthesia) for recurrent IH development exist.
Acquired, surgical and perioperative risk factors are recommended to be strongly considered since they are potentially modifiable and can influence the type of repair performed.
冈खा
खखा
खणम
खण口
Strong* upgraded 


\section{Evidence in literature}

A medical literature search for primary IH risk factors identified 989 studies. Included are a discussion of one systematic review, two randomized controlled trials (RCTs), 24 cohort or registry studies, five case-control studies and five diagnostic studies in the material below.

A medical literature search for RIH risk factors identified 1191 studies. A discussion follows of two systematic reviews, two RCTs, 31 cohort or registry studies, one casecontrol study and four diagnostic studies.

\section{Primary inguinal hernia}

The lifelong cumulative incidence of $\mathrm{IH}$ repair in adults is $27-42.5 \%$ for men and $3-5.8 \%$ for women. ${ }^{14-17}$

Risk factors associated with IH formation (evidence level-high):

- Inheritance (first degree relatives diagnosed with IH elevates IH incidence, especially in females). ${ }^{18,} 19$

- Gender (IH repair is approximately 8-10 times more common in males).

- Age (peak prevalence at 5 years, primarily indirect and 70-80 years, primarily direct). ${ }^{16,20-22}$

- Collagen metabolism (a diminished collagen type I/III ratio).

- Prostatectomy history (especially open radical). ${ }^{23-35}$

- Obesity (inversely correlated with IH incidence). ${ }^{19,21,36-38}$

Risk factors associated with IH formation (evidence level-moderate):

- Primary hernia type (both indirect and direct subtypes are bilaterally associated). ${ }^{39}$

- Increased systemic levels of matrix metalloproteinase$2 .^{40-43}$

- Rare connective tissue disorders (e.g. Ehlers-Danlos syndrome). ${ }^{44}$

Risk factors associated with IH formation (evidence level-low):

- Race (IHs are significantly less common in black adults). ${ }^{21}$

- Chronic constipation. ${ }^{19,45}$

- Tobacco use (inversely correlated with IH incidence). ${ }^{37}$

- Socio-occupational factors.

There is contradictory evidence that social class, occupational factors and work load affect the risk of $\mathrm{IH}$ repair. $^{46,} 47$ Heavy lifting may predispose to $\mathrm{IH}$ formation. $^{48}$

Risk factors associated with $\mathrm{IH}$ formation (evidence level-very low):
- Pulmonary disease (COPD and chronic cough possibly increasing the risk of $\mathrm{IH}$ formation). ${ }^{48,49}$

Liver disease, renal disease and alcohol consumption have not been properly investigated to determine if they are risk factors for IH formation.

\section{Recurrent inguinal hernia}

Risk factors for RIH with a high level of evidence include female gender, ${ }^{49-59}$ direct versus indirect $\mathrm{IH},{ }^{58,59}$ annual $\mathrm{IH}$ repair volume of less than five cases ${ }^{60}$ and limited surgical experience. $^{56,61-68}$ However, this last risk factor may be modifiable by surgical coaching. ${ }^{69-72}$

Risk factors for RIH with a moderate level of evidence include: presence of a sliding hernia, ${ }^{73}$ a diminished collagen type I/III ratio, ${ }^{40,74,75}$ increased systemic matrix metalloproteinase levels, ${ }^{42,59,74,75}$ obesity $^{37,59}$ (although questioned in two very small studies ${ }^{57,76}$ ) and open hernia repair under local anesthesia by general surgeons. ${ }^{53,} 77 \mathrm{~A}$ recent meta-analysis examining features of 100,000-200,000 repairs demonstrated that size $(<3$ versus $\geq 3 \mathrm{~cm}$ ) and bilaterality did not affect the risk of recurrence. $^{59}$

Incorrect surgical technique is likely the most important reason for recurrence after primary $\mathrm{IH}$ repair. Within this broad category of poor surgical technique are included: lack of mesh overlap, improper mesh choice, lack of proper mesh fixation, amongst others.

Several other potential risk factors have not been well studied or have low or very low levels of evidence supporting an association. Early postoperative hematoma formation $^{78}$ and emergent surgery $50,52,58,59$ may be risk factors for hernia recurrence but the association is not conclusive. Low (1-7 drinks/week) versus no ethanol consumption may protect against hernia recurrence. The effect of high ethanol consumption is unclear. ${ }^{53}$ Increased age ${ }^{57,59,79,80} \mathrm{COPD},{ }^{57,59,76-82}$ prostatectomy, ${ }^{76}$ surgical site infection, ${ }^{78,}{ }^{83}$ cirrhosis, ${ }^{84}$ chronic constipation, ${ }^{76}$ a positive family history. ${ }^{80,85}$ and smoking. ${ }^{53,57,80,85}$ have not been consistently shown to be risk factors for RIH. Incompletely studied factors which may impact the risk of IH recurrence are chronic kidney disease, social class, occupation, work load, pregnancy, labor, race and postoperative seroma occurrence.

Conclusion: several demographic (anatomy, female gender, abnormal collagen metabolism), acquired (obesity), and perioperative risk factors (insufficient surgical technique, low surgical volume, surgical inexperience and local anesthesia) for RIH were identified. Risk factors for IH and $\mathrm{RIH}$ are not comparable. In daily surgical practice, attention should be paid to perioperative surgical factors as they are modifiable. Allocation arms in future outcome studies should be balanced according to these demographic and acquired risk factors. 


\section{Chapter 3}

\section{Diagnostic modalities}

H. Niebuhr, M. Pawlak and M. Śmietański

\section{Introduction}

History and clinical examination are usually all that are required to confirm the diagnosis of a clinically evident groin hernia. Imaging may be required if there is vague groin swelling and diagnostic uncertainty, poor localization of swelling, intermittent swelling not present at time of physical examination, and other groin complaints without swelling.
An apparent hernia with clear clinical features such as a reducible groin bulge with local discomfort usually requires no further investigation. However, when patients present with groin complaints and hernia is not clearly the diagnosis, the question arises about which imaging modality to use. Ultrasonography (US) is now widely available but rarely magnetic resonance imaging (MRI), computed tomography (CT) and herniography may play a role as well. Laparoscopy is not generally considered part of the diagnostic process for groin complaints and bulges and is not considered further in this chapter.

\section{Key questions}

KQ03.a Which diagnostic modality is the most suitable for diagnosing groin hernias?

Differential diagnosis of groin swellings

\begin{tabular}{|c|c|c|c|c|}
\hline Inguinal & Inguino-scrotal & Femoral & Inguino-femoral & Scrotal \\
\hline Inguinal hernia & Inguinal hernia & Femoral hernia & $\begin{array}{l}\text { Inguinal lymph } \\
\text { nodes }\end{array}$ & $\begin{array}{l}\text { Skin: boils, } \\
\text { sebaceous cysts, } \\
\text { papillomas, warts }\end{array}$ \\
\hline Lymph nodes & $\begin{array}{l}\text { Hydrocele: } \\
\text { encysted } \\
\text { hydrocele of the } \\
\text { cord, } \\
\text { infantile } \\
\text { hydrocele, } \\
\text { hydrocele of the } \\
\text { hernia sac }\end{array}$ & Lymph nodes & $\begin{array}{l}\text { Distended psoas } \\
\text { bursa }\end{array}$ & $\begin{array}{l}\text { Subcut. tissue: } \\
\text { lymph scrotum, } \\
\text { filariasis } \\
\text { Tunica vaginalis: } \\
\text { hydrocele, } \\
\text { pyocele, } \\
\text { hematocele, } \\
\text { chylocele }\end{array}$ \\
\hline $\begin{array}{l}\text { Encysted } \\
\text { hydrocele of the } \\
\text { cord }\end{array}$ & $\begin{array}{l}\text { Spermatic cord: } \\
\text { varicocele, } \\
\text { funiculitis, lymph } \\
\text { varix, diffuse } \\
\text { lipoma of the cord, } \\
\text { hematoma of the } \\
\text { cord }\end{array}$ & Saphena varix & $\begin{array}{l}\text { Effusion in the hip } \\
\text { joint }\end{array}$ & $\begin{array}{l}\text { Testis: Orchitis } \\
\text { (acute/chronic), } \\
\text { neoplasms }\end{array}$ \\
\hline $\begin{array}{l}\text { Undescended } \\
\text { testis }\end{array}$ & $\begin{array}{l}\text { Testis: } \\
\text { undescended, } \\
\text { ectopic testis }\end{array}$ & Ectopic testis & & $\begin{array}{l}\text { Epididymis: } \\
\text { cysts, acute or } \\
\text { chronic infections }\end{array}$ \\
\hline $\begin{array}{l}\text { In females or } \\
\text { pregnant women: } \\
\text { round ligament } \\
\text { varicoses }\end{array}$ & & & & $\begin{array}{l}\text { Spermatic cord: } \\
\text { varicocele, lymph } \\
\text { varix }\end{array}$ \\
\hline
\end{tabular}


KQ03.b Which diagnostic modality is the most suitable for diagnosing patients with obscure pain or doubtful swelling? KQ03.c Which diagnostic modality is the most suitable for diagnosing recurrent groin hernias?

KQ03.d Which diagnostic modality is the most suitable for diagnosing the course of chronic pain after groin hernia surgery?
The 1999 prospective cohort study showed that US had a specificity of 0.945 and a sensitivity of 0.815 for detecting groin hernias. ${ }^{86}$ MRI demonstrated a specificity of 0.963 and a sensitivity of $0.945{ }^{86}$ A 2013 meta-analysis revealed that groin US had a specificity of 0.86 and a sensitivity of $0.77 .{ }^{105}$

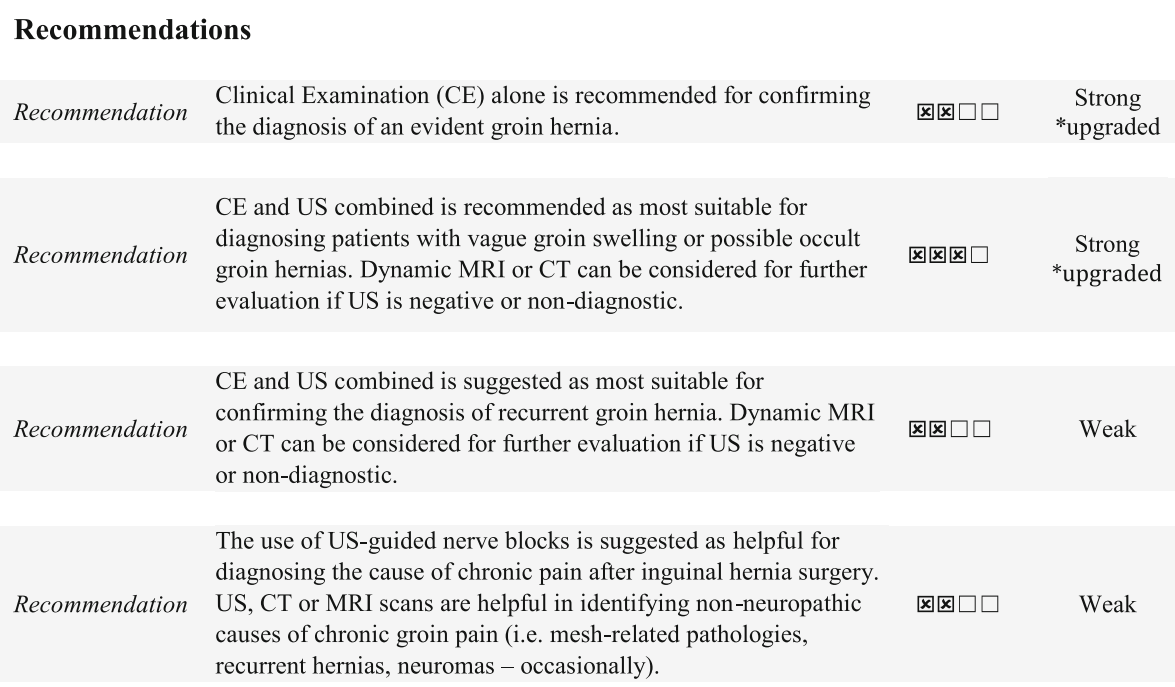

\section{Evidence in literature}

The gold standard for hernia diagnosis is clinical examination (CE) of the groin with a sensitivity of 0.745 and a specificity of 0.963 reported in a prospective cohort study from 1998. ${ }^{86}$ Three consensus guidelines have been published on groin hernia treatment. ${ }^{3,}$ 6, 87 All published statements on diagnostic workup are weak, mainly focusing on $\mathrm{CE}$ alone. Only groin pain that is obscure or groin swelling of unclear origin (possible occult hernia) are noted to require further diagnostic investigation. ${ }^{88,}{ }^{89}$ No consensus exists presently on the best imaging modality for these diagnostic dilemmas.

$\mathrm{CE}$ alone can miss hernias, especially those that are small (e.g. femoral hernias in obese women and men) and multiple hernias where only some of the hernias are apparent with physical examination. ${ }^{90}$ US, MRI, CT and herniography have all been studied in various settings in an attempt to close this "diagnostic gap". ${ }^{88,}$, $91-104$

Two studies with a total of 510 patients showed that US is highly sensitive and a useful way to identify hernias. ${ }^{88,96}$ Several other studies have echoed this finding. ${ }^{89,100,101,103}$
Two studies support the use of CE in combination with US to confirm the diagnosis of inguinal hernias. CE plus US was found to be superior to CE alone in both studies. ${ }^{89,96}$

Two prospective cohort studies-both of low qualityshowed that US performed poorly in the detection of occult groin hernias. ${ }^{106,107}$ Both studies did recommend the use of US for interval assessment of patients with equivocal findings since those with equivocal findings seem to have a high incidence of groin hernias.

In conclusion, challenging hernia diagnoses like femoral and clinically occult hernias can be evaluated with US since it is: routinely available, relatively specific, cost effective, repeatable, useful in diagnosing other conditions, delivers no ionizing radiation and well accepted by patients. $^{86,88-90,106-114}$

In pregnant women, colour-duplex US is useful for an entity presenting with an inguinal lump and pain, round ligament varicosity. ${ }^{109,115,116}$

When groin US is negative or non-diagnostic, dynamic MRI, dynamic CT and even herniography may be considered in an attempt to establish a diagnosis. ${ }^{117}$ Dynamic in this context refers to Valsalva manoeuvre during testing in an attempt to force a possibly occult or small hernia into its 
abnormal channel and more clearly demonstrate its presence. Herniography can only diagnose hernias, not other pathologies. MRI can diagnose adductor tendonitis, pubic osteitis, hip arthrosis, bursitis iliopectinea, and endometriosis amongst other conditions. If these ailments are part of the differential diagnosis, then MRI is the most suitable diagnostic tool. ${ }^{118,119} \mathrm{CT}$ can diagnose hernias as well and should be used when US is negative and MRI is not possible.

CE plus US is recommended as most suitable for the evaluation of patients suspected of having recurrent groin hernias. If diagnostic doubt exists after CE and US, MRI or CT should be considered. One prospective study and one retrospective case-control study, both of low quality, have addressed the issue of imaging for groin hernia recurrence. $^{120,121}$

US, CT or MRI scans are helpful in identifying nonneuropathic causes of chronic groin pain by identifying mesh-related pathologies, recurrent hernias and occasionally neuromas. ${ }^{122}$ A tailored, thoughtful approach to imaging is required since each of these imaging modalities possesses certain strengths and weaknesses and is not equally suited to diagnose all the listed conditions.

\section{Chapter 4}

\section{Groin hernia classification}

\author{
D. Cuccurullo and G. Campanelli
}

\section{Introduction}

In day-to-day surgical practice a classification system for groin hernias is seldom used other than to describe hernia types in general terms (lateral/indirect, medial/direct, recurrent, and femoral). However, a consensus classification system is needed in order to perform research, tailor treatments to hernia types, and perform quality audits. Presently it is uncertain which hernia classification system is most suited to achieving this purpose.

\section{Key questions}

KQ04.a Is a groin hernia classification system necessary, and if so, which classification system is most appropriate?

\section{Recommendation \\ Use of the EHS classification system for inguinal hernias is \\ Recommendation suggested for the purposes of performing research, tailoring treatments and performing quality audits.}

冈खण $\quad$ Weak
The use of US-guided nerve blocks is helpful in diagnosing the cause of chronic pain after surgery. A prospective cohort study described that the US-guided transversus abdominis plane block provided better the cause of pain and control than blind ilio-hypogastric nerve block after inguinal hernia repair. ${ }^{123}$ Considering the much higher number of patients $(n=273)$ compared to a randomized controlled trial with 24 included patients the quality rating of this Pain Catastrophizing Scale (PCS) could be determined as "moderate". ${ }^{124}$ In another publication, the authors renounced the use of imaging as a helpful way to diagnose the cause of postoperative inguinal pain. ${ }^{104}$ In short, it seems that US-guided nerve blocks are helpful in pinpointing the cause of chronic pain after groin hernia repair. Due to a lack of new studies and conflicting results in the available literature, the evidence supporting our recommendation on this KQ is considered "weak".

\section{Evidence in literature}

The 2009 EHS guidelines recommended that the EHS classification system be used. ${ }^{3}$ A 2015 literature review failed to reveal new proposed classification systems or new evidence on the value of the EHS system. ${ }^{125}$ However, it is the opinion of the HerniaSurge members that one uniform system be adopted.

For inguinal hernia repairs, it is increasingly clear that surgeons tailor techniques to suit various patients and different hernia types. It is also necessary to compare results across different techniques and perform medical audits. More hernia registries are recommended and will require that a consensus classification system be adopted. However, for now there is no consensus amongst general surgeons or hernia specialists on a preferred system.

The primary purpose of any disease classification system is to allow for severity stratification so that reasonable comparisons can be made between treatment strategies. ${ }^{125}$ Additionally, a classification system must be simple and easy to use. Given the large number of operative techniques and their variations for groin hernia repair, it 
appears that no one classification system can satisfy all presently. However, an expert panel analyzed the known systems to date (Nyhus, Gilbert, Rutkow, Schumpelick, Harkins, Casten Halverson, McVay, Lichtenstein, Bendavid, Stoppa, Alexandre and Zollinger) and developed the EHS system by consensus. ${ }^{125-132}$ HerniaSurge suggests this system be used since it fulfills most requirements and is relatively simple to use.

The EHS system was not developed to classify hernia types preoperatively. This is a disadvantage. It is suggested that complex cases be managed by hernia specialists. A classification to inform decision-making about these complex cases would be helpful. However, many complex cases are easy to describe and do not require further classification (e.g. multiple recurrences and chronic pain). for any $\mathrm{IH}$, including asymptomatic IHs because it was considered safe, effective, and associated with low morbidity. However, the natural history of untreated IHsespecially the incidence of complications-was unknown. Current literature suggests the possibility of surgical overtreatment of men with asymptomatic IHs. Also, the morbidity of inguinal herniorrhaphy has been re-evaluated over the last two decades and current evidence suggests that the incidence of chronic post-herniorrhaphy pain is much higher than previously realized. ${ }^{134}$

Inguinal herniorrhaphy is one of the most common operations performed by general surgeons. Therefore, considering the number of IH repairs performed worldwide annually, the consequences of overtreatment are significant. This has spurred recent studies to evaluate a watchful

\begin{tabular}{|l|l|l|l|l|l|}
\hline $\begin{array}{l}\text { EHS groin } \\
\text { classification system }\end{array}$ & \multicolumn{5}{|c|}{ Primary / Recurrent } \\
\hline & 0 & 1 & 2 & 3 & X \\
\hline Lateral (L) & & & & & \\
\hline Medial (M) & & & & & \\
\hline Femoral (F) & & & & & \\
\hline
\end{tabular}

For a detailed explanation please see the publication. ${ }^{125}$

For now, the classification system for groin hernias is mired in some controversy and disagreement. However, the best available evidence and expert opinion supports the adoption of the EHS system as classification system refinements evolve.

\section{Chapter 5}

\section{Indications: treatment options for symptomatic and asymptomatic patients}

B. van den Heuvel, A. R. Wijsmuller and R. J. Fitzgibbons

\section{Introduction}

Approximately one-third of inguinal hernia (IH) patients are asymptomatic. ${ }^{133}$ Until recently, IH management involved surgical repair regardless of the presence of symptoms, the rationale being that surgery for asymptomatic IHs prevents hernia complications (incarceration or strangulation). Surgical management was recommended waiting strategy in men with asymptomatic IHs. ${ }^{135,}{ }^{136} \mathrm{~A}$ critical appraisal of these studies and previous assumptions is presented.

Based on the current literature, it is not possible to determine if a watchful waiting management strategy is safe for symptomatic men with IHs. Similarly, it is impossible to determine the hernia complication rate (strangulation or bowel obstruction) in symptomatic patients. Additionally, watchful waiting raises ethical issues about observing symptomatic patients.

\section{Key questions}

KQ05.a Is a management strategy of watchful waiting safe for men with symptomatic inguinal hernias?

KQ05.b What is the risk of a hernia complication (strangulation or bowel obstruction) in this population?

KQ05.c Is a management strategy of watchful waiting safe for men with asymptomatic inguinal hernias?

KQ05.d What is the risk of a hernia complication (strangulation or bowel obstruction) in this population?

KQ05.e Are emergent inguinal herniorrhaphies associated with higher morbidity and mortality?

KQ05.f What is the crossover rate from watchful waiting to surgery? 


\begin{tabular}{|c|c|c|c|}
\hline \multicolumn{4}{|c|}{ Statements and Recommendations } \\
\hline Statement & $\begin{array}{l}\text { There is a low complication risk (incarceration or strangulation) } \\
\text { in asymptomatic or minimally symptomatic men with inguinal } \\
\text { hernias. }\end{array}$ & खण口 & \\
\hline Statement & $\begin{array}{l}\text { Emergent repair of incarcerated or strangulated inguinal hernias } \\
\text { in men is associated with higher morbidity and mortality } \\
\text { compared with elective repair in men with symptomatic } \\
\text { inguinal hernias. }\end{array}$ & 冈冈口 & \\
\hline Statement & $\begin{array}{l}\text { There is no evidence to support watchful waiting as a } \\
\text { management strategy in men with symptomatic inguinal } \\
\text { hernias. No data exist on the risk of incarceration or } \\
\text { strangulation in this population. }\end{array}$ & 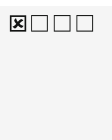 & \\
\hline Statement & $\begin{array}{l}\text { Most men with minimally symptomatic or asymptomatic } \\
\text { inguinal hernias will develop symptoms and require surgery. }\end{array}$ & ब目田 & \\
\hline Statement & $\begin{array}{l}\text { Although most patients will develop symptoms and need } \\
\text { surgery, watchful waiting for minimal or asymptomatic inguinal } \\
\text { hernias is safe since the risk of hernia complications is low. } \\
\text { Management decision is made between the surgeon and patient. }\end{array}$ & 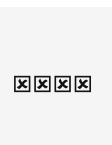 & \\
\hline Recommendation & $\begin{array}{l}\text { Discussions with patients about timing of hernia repair are } \\
\text { recommended to involve attention to social environment, } \\
\text { occupation and overall health. The lower morbidity of elective } \\
\text { surgery has to be weighed against the higher morbidity of } \\
\text { emergency surgery. }\end{array}$ & 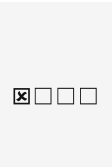 & $\begin{array}{l}\text { Strong } \\
\text { *upgraded }\end{array}$ \\
\hline
\end{tabular}

\section{Evidence in literature}

The literature search on this topic yielded six randomized controlled trials (RCTs), two systematic reviews and three cohort-controlled studies. Two study groups produced all six RCTs. ${ }^{135,136}$

A 2006 trial of 720 men with minimally symptomatic or asymptomatic IHs randomized subjects to either primary surgery or watchful waiting (WW). ${ }^{135}$ Primary outcomes were pain interfering with normal activities and change in physical function as measured by the physical component score of the SF-36 at 2 years. Secondary outcomes included complications, and patient-reported pain, functional status, activity levels and satisfaction. Pain interfering with daily activity occurred in $5.1 \%$ of the WW group and $2.2 \%$ in the primary surgery group at 2 years $(p=0.52)$. SF-36 improvement from baseline was seen in both groups. One hernia incarceration occurred within the 2-year minimum follow-up period and another occurred after 4.5 years (relative risk of 1.8 per 1000 patient years). The crossover rates were high for both groups. At 2 years, $17 \%$ crossed over from surgery to WW and $23 \%$ from WW to surgery. A WW strategy was deemed safe and acceptable since acute incarcerations rarely occurred. A secondary analysis found that those who developed symptoms had no greater risk of operative complications or recurrence than those undergoing elective hernia repairs.
A cost-effectiveness analysis was performed on the groups, calculating both costs and quality-adjusted lifeyears (QALYs). ${ }^{137}$ At 2 years, those in the surgery group had a \$1831 higher mean cost per patient when compared with WW group subjects. The cost per additional QALY in the surgery group was $\$ 59,065$. WW was judged to be a cost-effective management option for men with minimal or absent hernia symptoms.

These same groups were restudied 7 years later. $^{2}$ Crossover rates, crossover reasons and time to crossover were investigated. The crossover rate from WW to surgery was $50 \%$ at 7.3 years from randomization. Median crossover time was 3.7 years in men over 65 and 8.3 years in those 65 and younger $(p=0.001)$. The estimated crossover rate at 10 years was $68 \%$ using Kaplan-Meier analysis. The primary reason for crossover was pain. When patients over 65 at time of original study enrollment were analyzed, the estimated 10-year crossover rate was $79.4 \%$. This compares with a $62 \% 10$-year crossover estimate for those 65 or younger at enrollment. In the 10-year follow-up only three men $(2.4 \%)$ underwent surgery for a hernia accident. There was no mortality. The incidence of a hernia accident for the entire cohort was 0.2 per 100 person-years. These studies support the idea that men with IHs and minimal or absent symptoms should be counseled that although WW is 
safe, symptoms will likely progress and an operation may be needed. A follow-up cost analysis has yet to be reported.

Another 2006 study randomized 160 men over the age of 55 with asymptomatic IHs to either WW (80 patients) or surgery ( 80 patients). ${ }^{136}$ The primary outcome was pain at 1 year as measured by the SF-36. Cost was a secondary outcome. At 6 months, improvement-in most SF-36 dimensions-was observed in the surgery group compared with the WW group. This effect had dissipated at 12 months and there were no significant inter-group differences in visual analogue pain scores at rest or with activity. Analgesic use between groups did not differ. The only notable inter-group difference at 12 months was in a single SF-36 item indicating perceived change in health. The 1-year crossover rate from surgery to WW was 10 and $19 \%$ from WW to surgery. A single hernia incarceration occurred at 574 days. Primary surgical repair added 407.9 GBP in costs per patient (approximately \$591 US).

Long-term follow-up data were published in $2011 .^{138} \mathrm{At}$ 5 years, $54 \%$ had crossed over from WW to surgery and an estimated $72 \%$ crossed over at 7.5 years. The most common crossover reason was pain. The estimated median time between randomization and crossover was 4.6 years. In 7.5 years, two patients required emergent hernia repair. The study's authors concluded that a WW strategy is of little value since the majority of $\mathrm{WW}$ patients will require surgery in the near term.

Two systematic reviews have appraised primary repair versus WW for minimally symptomatic or asymptomatic IHs in men. ${ }^{139,} 140$ Both reviews included mostly observational studies and pooled data on morbidity and mortality. Morbidity and mortality after elective repair was 8 and $0.2-0.5 \%$, respectively, versus 32 and $4-5.5 \%$ following emergent repair (a 10- to 20 -fold increase in mortality). Risk factors for the observed increased morbidity and mortality include: age greater than 49 years, symptom duration, the presence of a femoral hernia, ASA class over two and nonviable bowel. Incarceration/strangulation risk factors are: symptom duration, age and hernia site (femoral). However, the reviews acknowledge that the incarceration/strangulation risk is low and that watchful waiting may be justified in selected patients.

Notably, both systematic reviews were published prior to the long-term RCTs cited above demonstrating symptom development over time in most men with minimally symptomatic or asymptomatic IHs. Symptom development (primarily pain) will prompt surgery. While it is true that incarcerations rarely occur in the WW group and are associated with defined risk factors, morbidity and mortality rates increase alarmingly when an IH strangulates.

A 2014 study reported on clinical consequences after the inception of a watchful waiting strategy. ${ }^{141}$ Regionally, a
WW policy was instituted in the United Kingdom for those with asymptomatic IHs. Outcomes of approximately 1000 patients before, and 1000 patients after, the policy's inception were compared retrospectively. The period following the policy change saw a $59 \%$ rise in the incidence of emergent hernia repair (3.6 vs 5.5\%). Emergent repair was also associated with significantly more adverse events (4.7 vs $18.5 \%$ ). Mortality spiked from 0.1 to $5.4 \%$. However, this was a retrospective study and did not report on the prior histories of those requiring emergent herniorrhaphies. Therefore, conclusions should be made with caution.

\section{Discussion, consensus and clarification of grading}

The initial results of a WW strategy in men with asymptomatic or minimally symptomatic IHs were promising. Complications occurred uncommonly and WW seemed cost effective in the short term. However, a longer-term view revealed high crossover rates due to symptom development, mostly pain. Whether WW is ultimately cost effective remains to be determined.

Observational studies have shown that emergent herniorrhaphy is associated with increased morbidity and mortality. Unfortunately, it is not possible currently to accurately predict which WW patients will develop symptoms or suffer a hernia complication. This foreknowledge would of course allow more tailored management.

Because of the increased morbidity and mortality associated with emergent herniorrhaphy, the expert group advises that each patient with an asymptomatic or minimally symptomatic inguinal hernia be informed about the expected natural history of their condition, the timing, and the risks of emergency hernia surgery. Although robust support for a WW strategy and timing of surgery is not to be found in the present medical literature the expert group has upgraded its recommendation on this subject. This is because patient health-related, life style and social factors should all influence the shared decision-making process leading up to hernia management.

\section{Chapter 6}

\section{Surgical treatment of inguinal hernias}

Th. J. Aufenacker, F. Berrevoet, R. Bittner, D. C. Chen, J. Conze, F. Kockerling, J. F. Kukleta, M. Miserez, M. C. Misra, M. P. Simons, H. M. Tran, S. Tumtavitikul 


\section{General introduction}

Choosing the best or most suitable groin hernia repair technique is a true challenge. The best operative technique should have the following attributes: low risk of complications (pain and recurrence), (relatively) easy to learn, fast recovery, reproducible results and cost effectiveness. The decision is also dependent upon many factors like: hernia characteristics, anesthesia type, the surgeon's preference, training, capabilities and logistics. The patient's wishes must be considered. There are cultural differences between surgeons, countries and regions. Emotions may play a role as well.

Accordingly, the HerniaSurge Group had some passionate discussions when developing this chapter. One single standard technique for all hernias does not exist (see also Chapter 7 on individualization).

In most situations a mesh repair is preferred. However, a minority of surgeons hold the opinion that mesh use should be avoided as much as possible. There is an ongoing discussion concerning the results of specialist centers like The Lichtenstein Hernia Clinic and The Shouldice Hospital. There are low-resource settings where mesh cannot be afforded. There are high-volume laparo-endoscopic surgeons who passionately advocate a TEP or TAPP in almost all cases. There are special mesh implants (often expensive) used by surgeons who have been successful with them for many years. How then can one reconcile these opinions and conflicts?

Although accurate and recent facts are not available, in most countries the Lichtenstein repair is probably the first choice in a majority of cases. It is a very good technique, but its outcomes may be bettered by a more difficult technique like the TEP when early postoperative recovery and the occurrence of chronic pain are considered. It is self-evident that a surgeon performing a technique and striving for optimal results should know the technique very well. Excellent training and a high caseload are the foundations of good surgery.

When comparing the best Lichtenstein outcomes with the best TEP/TAPP, it is noted that the differences are very small. It is challenging though when examining results reported in the literature because often the techniques being compared are not performed in a standardized manner by equally skilled and experienced surgeons. Therefore, this might not be true when comparing an average Lichtenstein to an average TEP/TAPP or Shouldice because of the former's lower complexity. Furthermore, applying research results to the approach for an individual patient is problematic as well. It is often far from clear whether the results of an RCT can be generalized to one's practice setting or patients within that setting.
In the 2009 European Guidelines, raw data were used to conclude that laparo-endoscopic and open repair were comparable in long-term follow-up of a minimum of 48 months. 3,142

When reading this chapter, we should realize that potential biases exist and these are caused by: lack of a clear chronic pain definition, variations in duration of chronic pain, age differences for the risk of chronic pain, lack of a generally agreed-upon classification system describing the type of hernias, differences in level of surgical expertise, differences in case load needed to maintain a certain technique, safety issues regarding training of the surgeons/residents in the world in difficult techniques like the TEP and TAPP, and costs of procedures, amongst others. In fact, all these factors must be considered when studying the evidence presented in the different chapters.

The chapters were researched and written by different teams, but the statements and recommendations were agreed upon by the whole HerniaSurge Group. Many lively discussions during the meetings and via email led to an internet consensus vote. There are recommendations that have been upgraded. The support for these decisions is at the end of each chapter.

\section{Key questions}

KQ06.a Which non-mesh technique is the preferred repair method for inguinal hernias?

KQ06.b Which is the preferred repair method for inguinal hernias: mesh or non-mesh?

KQ06.c Which is the preferred open mesh technique for inguinal hernias: Lichtenstein or other open flat mesh and implants via an anterior approach?

KQ06.d Which is preferred open mesh technique: Lichtenstein versus open pre-peritoneal?

KQ06.e Is TEP or TAPP the preferred laparo-endoscopic technique for inguinal hernias?

KQ06.f When considering recurrence, pain, learning curve, postoperative recovery and costs which is preferred technique for inguinal hernias: best open mesh (Lichtenstein) or a laparo-endoscopic (TEP and TAPP) technique? KQ06.g In males with unilateral primary inguinal hernias which is the preferred repair technique, laparo-endoscopic (TEP/TAPP) or open pre-peritoneal?

KQ06.h Which is the preferred technique in bilateral inguinal hernias? Open mesh or laparo-endoscopic approach?

\section{Key question}

KQ06.a Which non-mesh technique is the preferred repair method for inguinal hernias? 


\section{Introduction}

The 2009 European Guidelines opined that the Shouldice inguinal hernia repair was the best non-mesh technique. ${ }^{3}$ Since then, no studies have offered new evidence concerning a comparison between non-mesh techniques. Questions remain concerning the value of a non-mesh technique in certain cases like indirect hernias (EHS L1 and L2) in young male patients. There are questions concerning the results of Shouldice when performed in specialist centers or by specialist hernia surgeons. There are no RCTs performed in these centers. There are also regions (low-resource countries in particular) where mesh is not available and surgeons must use the best non-mesh technique. Also some patients refuse a mesh implant. Which non-mesh technique is best therefore remains an important question. discussion, the authors conclude that the review is flawed by: the inclusion of low-quality RCTs, non-blinded outcomes assessments, lack of external validity by patient selection (only healthy patients were included), high lostto-follow-up rates, no patient-oriented outcomes and the above-mentioned potential bias. Nevertheless, the large number of patients and consistent results do make the results useable in clinical practice. The level of the review with RCTs is downgraded to moderate. Since this systematic review was done, no new RCT comparing Shouldice with other non-mesh techniques has been published. ${ }^{142}$ The level of recommendation is strong.

Other non-mesh techniques

A 2012 RCT, in which 208 patients were randomized, described the Desarda technique compared with a Lichtenstein technique. ${ }^{144}$ Follow-up at 36 months found

\section{Recommendation \\ Statement The Shouldice technique has lower recurrence rates \\ than other suture repairs

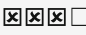

\section{Evidence in literature}

Systematic Review Cochrane 2012

A 2012 review covered all prior RCTs (until September 2011) concerning results of the Shouldice technique versus other open techniques (mesh and non-mesh). ${ }^{142}$ Eight RCTs with 2865 patients are contained, comparing mesh versus non-mesh IH repair. Most of these trials had inadequate randomization methods, did not mention dropouts and did not blind patients and surgeons to the technique used. Recurrence rate was a primary outcome in all and pain could only be analyzed in three trials. Pain definitions and measurements were not standardized. Studies were heterogeneous, with concerns that techniques were not standardized. The results show that in Shouldice versus other non-mesh (8 studies) the recurrence rate was lower in Shouldice (OR 0.62, 95\% 0.45-0.85 NNH 40). Six studies reported an OR in favor of the Shouldice technique. One included study reported the most data and its weight in the analysis was $59.56 \% .^{143}$ The results reflect different degrees of surgeon's familiarity with the techniques, making it impossible to eliminate the "handcraft" variable from surgical trials. Shouldice also results in less chronic pain (OR 0.3; 95\% CI 0.4-1.22) and lower rates of hematoma formation (OR $0.84 ; 95 \%$ CI $0.63-1.13$ ), but slightly higher infection rates (OR 1.34; 95\% CI 0.7-2.54). It is more time consuming and leads to a slightly increased hospital stay (WMD 0.25; 95\% CI 0.01-0.49). In their recurrence rates in each group of $1.9 \%$ and no significant differences in pain. As this is a new technique with some non-randomized studies showing promising results, it is worthy of mention in the guidelines. The level of the RCT is moderate and no recommendations can be formulated. The Desarda technique needs further investigation.

Large database studies

The large databases from Denmark and Sweden indicate results of non-mesh techniques, but cannot differentiate between different techniques so conclusions cannot be made concerning the quality of the Shouldice technique. ${ }^{145}$ In a 2004 questionnaire study, ${ }^{11,145,146}$ using results from the Danish database, chronic pain was more common after primary IH repair in young males, but there was no difference in pain when comparing Lichtenstein with nonmesh Marcy and Shouldice repairs. The databases conclude less recurrences after mesh repair, but not at the cost of more chronic pain.

\section{Guidelines}

The 2009 European Guidelines concluded that the Shouldice hernia repair technique is the best non-mesh repair method with a $1 \mathrm{~A}$ level of evidence. ${ }^{3}$

\section{Discussion, consensus and clarification of grading}

When considering the results from the systematic review, large databases and guideline conclusions, we conclude that Shouldice is superior to other non-mesh techniques 
especially when considering recurrence rates. In the systematic review the level of evidence was downgraded to moderate. But combining all the evidence, and after consensus by HerniaSurge, we concluded that a recommendation, upgraded to "strong" was supportable. In other words, in non-mesh repair, perform a Shouldice.

Although no studies exist on a comparison of the learning curves of the different non-mesh techniques, the HerniaSurge group agrees that the Shouldice technique is not easy to learn. In The Shouldice Hospital, surgeons are only considered qualified after 300 cases! It is well known that in many (mainly low resource) countries a (modified) Bassini is still performed.

Another matter is a discussion concerning the results of only high ligation and sac resection versus Shouldice in young adults with L1 and L2 IH. HerniaSurge is of the opinion that this issue needs further research. We are unable to formulate a statement on it at this time.

KQ06.b Which is the preferred repair method for inguinal hernias: mesh or non-mesh?

M. P. Simons, J. Conze and M. Miserez

\section{Introduction}

The 2009 European Guidelines concluded that all male adults over the age of 30 with a symptomatic IH should be operated on using a mesh-based technique (grade A). ${ }^{3}$ In most countries, the use of mesh has been accepted by the majority of surgeons as the best approach to decrease risk of recurrence. There are concerns about mesh causing more chronic pain. Other reasons not to use mesh include: higher cost or non-availability of meshes in low-resource settings, lack of surgical expertise with mesh, and patient refusal of a mesh repair. It remains to be seen whether a mesh-based technique is indicated in all cases (see also Chapter 7 on individualization).

\section{Evidence in literature}

Systematic Review Cochrane 2012

A 2012 systematic review covered all prior RCTs (until September 2011) concerning results of Shouldice versus other open techniques. ${ }^{142}$ The review contains 6 RCTs including 1565 patients and compared Shouldice versus open mesh (Lichtenstein in all studies except one with plug and patch) for IH repair. The overall RCT quality is low. Recurrence rates were the primary outcome. Pain definitions and measurements were not standardized. Studies were heterogeneous. There are concerns that techniques were not standardized and no classification was applied.

The results show, that in Shouldice versus mesh Lichtenstein, recurrence rate were higher in Shouldice (5 studies) (OR 3.65, 95\% 1.79-7.47, NNH 36). Although not the primary endpoint in most trials, there were no significant differences between Shouldice and Lichtenstein for postoperative stay, chronic pain, seroma/hematoma and wound infection, but operative time was shorter for mesh repair (WMD $9.64 \mathrm{~min}$; 95\% CI 6.96-12.32).

The authors concluded that the review is flawed by lowquality RCTs, non-blinded outcomes assessment, external validity concerns due to patient selection (generally healthy patients were studied), high lost-to-follow-up rates, lack of patient-oriented outcomes and the above-mentioned potential bias concerning surgical technique. Nevertheless, the large number of patients and consistent results do make the results useful.

Other RCTs since the systematic review

Since September 2011, three RCTs have been published describing a non-mesh versus mesh repair but they were excluded because they either did not include Shouldice repairs, ${ }^{147-149}$ Lichtenstein repairs, ${ }^{147,}{ }^{150}$ or had a very short follow-up. ${ }^{148-150}$

One 2012 RCT, in which 208 patients were randomized, compared the Desarda technique with a Lichtenstein technique. At 36-month follow-up, the recurrence rate in

\section{Statement and Recommendations}

$\begin{array}{lll} & \begin{array}{l}\text { The use of open non-mesh repair in specific } \\ \text { patients or types (e.g. young males with lateral } \\ \text { hernia L1 and L2) of inguinal hernia as an } \\ \text { acceptable alternative to a mesh technique has } \\ \text { not been adequately investigated so far. }\end{array} \\ \text { Recommendation } \quad \begin{array}{l}\text { A mesh-based repair technique is recommended } \\ \text { for patients with inguinal hernias. }\end{array} & \text { Strong } \\ \text { A non-mesh repair for inguinal hernia can be } \\ \text { suggested in cases where the patient refuses a } \\ \text { mesh and/or after a shared decision making. }\end{array}$


each group was $1.9 \%$ and no significant differences in pain were found. The Desarda technique is new and the subject of some non-randomized studies showing promising results, but the technique needs further investigation. The 2012 RCT is graded as moderate. No recommendations about its use can be made at this point.

\section{Large database studies}

Two publications from the Danish Hernia Database describe recurrence after 96 months following open nonmesh versus Lichtenstein. The recurrence rate after open non-mesh repair was 8 versus $3 \%$ for Lichtenstein. $^{11,151,152}$ These studies are flawed because the Shouldice group consisted of only $13 \%$ of all suture repairs and that reoperation rather than recurrence rates were used. However, they do offer insights though about outcomes in a general population being treated by general surgeons (see Chapter 25 concerning the value of database studies). A 2004 questionnaire study of the Danish database found that chronic pain occurred more commonly after primary $\mathrm{IH}$ repair in young males. But, no differences in pain occurred when comparing Lichtenstein with Marcy and Shouldice non-mesh repair techniques. The database studies also found fewer recurrences after mesh repair.

\section{Guidelines}

The European Guidelines concluded that all male adults over the age of 30 years with a symptomatic IH should be operated on using a mesh technique (grade A). ${ }^{3}$ They also recommend that a mesh technique be used for inguinal hernia correction in young men (18-30 years of age and irrespective of the type of inguinal hernia). The conclusion was based on a lack of evidence that the recurrence risk after L1-2 IH in younger men is acceptably lower than in men above 30. This question is not being researched probably due to the fact that almost all male patients are now treated with mesh techniques.

\section{Cohort studies}

There is lower level evidence that the Shouldice technique has a recurrence rate of less than $2 \%$ especially when performed in high-volume expert settings like the Shouldice Hospital. ${ }^{153}$ These data come primarily from expert centers. Often the studies suffer from inadequate follow-up and there is patient selection bias in some. This gives rise to a dispute between open non-mesh surgeons and surgeons advocating mesh repair on the true value of the Shouldice repair. Resolution is unlikely unless an RCT is performed with adequate methods truly comparing techniques by surgeons qualified and experienced in both approaches. This might be possible using large databases provided identification of Shouldice technique is done. It is clear from all high-level studies though that in general practice, mesh is superior to non-mesh especially when measuring recurrence rate. It is absolutely recommended that studies be performed into the value of Shouldice versus mesh in young male patients with lateral (L1) inguinal hernia. One important study with long-term follow-up after Shouldice indicated that hernia type (indirect versus direct) was not an independent risk factor. ${ }^{154}$ Recurrence after Shouldice after 2 and 5 years was, respectively, 4.3 and $6.7 \%$. Out of 21 recurrences 20 were direct. Out of 20 recurrences 7 were after an indirect hernia with an enlarged internal ring, 6 after indirect with a weakened posterior wall and 7 after a direct hernia (n.s.). There are cohort studies concerning Shouldice that indicate that classification matters and the risk of recurrence is higher after a direct non-mesh repair. ${ }^{80}$ An analysis of the location of the hernial gap revealed 83 lateral hernias $(48.5 \%)$ and 88 medial hernias (medial or combined, $51.5 \%$ ). The recurrence rate was $13.6 \%$ for medial or combined hernias and $8.4 \%$ for pure lateral hernias. This was not significant. Furthermore, it is unknown whether a high ligation and sac resection (herniotomy) has comparable results to Shouldice in these patient groups.

\section{Discussion, consensus and grading clarification}

Compared to non-mesh techniques mesh-based techniques have a lower recurrence rate and an equal risk of postoperative pain. Despite the mentioned limitations of the 2012 review, the large number of patients and consistent results make available evidence reliable and useable in practice. There is no conclusive evidence that mesh causes more chronic pain. It remains to be seen whether a mesh-based technique is indicated in all cases such as small lateral hernias (EHS L1 and L2) (see Chapter 7 on individualization).

It is unclear whether it is appropriate to compare the results of the Shouldice technique, usually performed by highly trained surgeons and/or in specialized centers, to the open mesh repair techniques which tend to be performed by generalists. Specialized centers have not published their results in a reliable manner. Many cohort studies contain bias and thus lack external validity. It is necessary to improve knowledge concerning this question concerning the value of non-mesh techniques especially for long-term recurrence rate and chronic pain. Although the level of evidence seems only moderate, by consensus in HerniaSurge the recommendation to use a mesh-based technique in inguinal hernia repair is upgraded to "strong".

KQ06.c Which is the preferred mesh for open inguinal hernia repair: anterior flat mesh, self-gripping mesh or three-dimensional implants (plug-and-patch and bilayer) via an anterior approach?

M. Miserez, J. Conze and M. Simons 


\section{Introduction}

The Lichtenstein technique with the onlay placement of a flat mesh is the criterion standard in open IH repair. ${ }^{155}$ Many alternatives to the original Lichtenstein technique have been described. The plug-and-patch (or mesh-plug) technique was the first, ${ }^{156}$ followed by the Trabucco technique. ${ }^{157}$ and the Prolene ${ }^{\circledR}$ Hernia System (PHS). ${ }^{158}$

In the Trabucco technique, a polypropylene plug is combined with a semi-rigid flat pre-shaped polypropylene mesh. Neither implant is fixed. The spermatic cord is placed subcutaneously. At the time of the first EHS guidelines on the treatment of $\mathrm{IH}$ in adults, no long-term comparative follow-up data were available on any of these techniques, ${ }^{3}$ but this changed at the time of the update with level 1 studies of the 2009 EHS guidelines. In addition, self-gripping meshes have been designed in an attempt to reduce or abandon the need for traumatic mesh fixation in Lichtenstein repair and decrease the risk for acute and chronic pain. assess recurrence rates and chronic pain after a median follow-up of 7.6 years $\left(n=180,81 \%\right.$ follow-up rate). ${ }^{161}$ Recurrence rates for Lichtenstein and plug-and-patch were 5.6 and $9.9 \%$, respectively $(p=0.770)$. Moderate or severe pain was reported in 5.6 and $5.5 \%$, respectively $(p=0.785)$. The second study-which also included recurrent hernias - evaluated patients by means of physical examination after a 6.5-year median follow-up and had similar findings ( $n=528,76 \%$ follow-up rate). ${ }^{162}$ Recurrence rates for Lichtenstein and plug-and-patch were 8.1 and $7.8 \%$, respectively (OR 0.92 n.s.) and chronic persistent pain (VAS $>3$ ). More reoperations occurred in the Lichtenstein group (OR 0.43, $p=0.016)$.

Prolene $^{\circledR}$ Hernia System (PHS)

At the time of the EHS update, two meta-analyses of six RCTs were published comparing PHS and Lichtenstein (follow-up ranging from 12 to 48 months). ${ }^{159,} 163$ In addition, one long-term follow-up study (5-year follow-up) was available. ${ }^{164}$ No differences in recurrence or chronic

\section{Statements and Recommendations}

$\begin{array}{cl}\text { Statement } & \begin{array}{l}\text { The recurrence rate and postoperative chronic pain are } \\ \text { comparable between plug-and-patch/ PHS and the } \\ \text { Lichtenstein technique. }\end{array} \\ & \begin{array}{l}\text { Self-gripping meshes do not provide any benefit in the } \\ \text { short- and medium-term versus the Lichtenstein } \\ \text { technique except a somewhat decreased operative } \\ \text { time. }\end{array} \\ \text { Statement } & \begin{array}{l}\text { Despite comparable results, three dimensional } \\ \text { implants (plug-and-patch and bilayer) are not } \\ \text { recommended because of the excessive use of foreign } \\ \text { material, the need to enter both the anterior and } \\ \text { posterior planes and the additional cost. }\end{array} \\ & \begin{array}{l}\text { The use of other implants to replace the standard flat } \\ \text { mesh in the Lichtenstein technique is currently not } \\ \text { recommended. }\end{array}\end{array}$

\section{Evidence in literature}

\section{Plug-and-patch}

The recent 2014 EHS guidelines update, ${ }^{4}$ with level 1 studies, included data on the comparison between plugand-patch versus Lichtenstein from two meta-analyses of seven RCTs. ${ }^{159,} 160$ These showed shorter operative times for the plug-and-patch (by 5-10 min), but otherwise comparable outcomes in the short- and long-term (follow-up ranging from 0.5 to 73 months).

Long-term follow-up data from two of the RCTs were published in 2014. The first study used a questionnaire to pain were found. The data on operative times and perioperative complications were contradictory in the metaanalyses, although no differences were seen for postoperative wound hematoma formation or infection in either.

A 2014 long-term outcome study (mean follow-up of 7.6 years) also include a PHS arm and these data are reported below, ${ }^{161}$ confirming earlier results. The recurrence rates for Lichtenstein and PHS were 5.6 and 3.3\%, respectively $(p=0.770)$. The incidence of chronic pain (moderate or severe) was 5.6 and $6.7 \%$, respectively $(p=0.785)$. 
A large-pore version of the PHS, the Ultrapro ${ }^{\circledR}$ Hernia System (UHS), was launched recently. One RCT compares Lichtenstein and the UHS. ${ }^{165}$ Another RCT compared the plug-and-patch technique with a 4D Dome ${ }^{\circledR}$ device in 95 patients. ${ }^{166}$ The "dome device" consists of a largely resorbable dome-shaped plug (90\% poly-L-lactic acid and $10 \%$ polypropylene) associated with a flat lightweight polypropylene mesh. Because of poor methodological quality (according to SIGN criteria), neither paper is further discussed here.

\section{Trabucco}

One RCT compared the Lichtenstein with the Trabucco technique in 108 patients under local anesthesia. ${ }^{167}$ The Trabucco technique was an average of $10 \mathrm{~min}$ faster vs. Lichtenstein $(p=0.04)$. There were no differences in postoperative pain (primary outcome) or groin discomfort at 6 months. At an average follow-up of 8 years (only telephone follow-up after 1 year), there were no recurrent hernias.

\section{Self-gripping mesh}

The first study on the use of the self-gripping Parietene Progrip@ mesh (large-pore polypropylene with resorbable polylactic acid micro-grips) found less pain on the first postoperative day when compared with the use of another large-pore non-gripping polypropylene mesh. ${ }^{168}$ Subsequently, four other RCTs comparing self-fixating largepore mesh vs suture fixation in Lichtenstein have been published up to 2013. ${ }^{169-172}$ These studies have been evaluated in five different meta-analyses, all published in 2013 and 2014 in different journals. ${ }^{173-177}$ All confirmed no difference in acute or chronic pain and recurrence rates.

Three additional RCTs were published in $2014,{ }^{178-180}$ and another two were published with long-term data from an RCT published earlier. ${ }^{181,182}$ All confirmed comparable recurrence rates and acute and chronic pain incidence in both groups. The self-fixation mesh is likely to be more expensive than standard fixation, but the operative time was shorter in the Progrip $\odot$ group (by a range of 1-12 min).

Since only data on medium-term follow-up are available (range 6-24 months), we advise the authors of the previously mentioned trial data to follow-up their patients at $3-5$ years and publish their updated results on chronic pain and recurrence rates.

\section{Discussion, consensus and grading clarification}

Plug-and-patch and PHS are acceptable treatments for primary IHs, but have no benefit vs. the Lichtenstein technique, except a somewhat shorter operative time for the plug-and-patch technique. However, both the anterior and posterior compartment are entered and scarred, making a subsequent repair for recurrence more difficult. Also, the amount of foreign material is higher than for a simple flat mesh. And-in the case of a combined hernia-the placement strategy for the device or plug is not standardized. The additional cost of the device needs to be taken into account as does the small chance of mesh migration/ erosion with the use of plugs. Therefore, the Lichtenstein technique with a flat mesh is considered to be superior. See also Chapter 10 on mesh in which the problems of meshplug erosion and migration are described.

Self-gripping mesh is an acceptable form of treatment for primary IHs, although only medium-term data are available and no specific information on the outcome in larger (direct) hernias. It has no benefits over the Lichtenstein technique other than a somewhat shorter operative time. Here also, the device's additional cost must be considered.

For these reasons, the recommendations to use the Lichtenstein technique with a standard flat mesh vs the use of self-gripping mesh or three-dimensional implants are upgraded to strong by the HerniaSurge Group.

KQ06.d Which is the preferred open mesh technique for inguinal hernias: Lichtenstein or any open pre-peritoneal technique?

F. Berrevoet, Th. Aufenacker and S. Tumtavitikul

\section{Introduction}

Open pre-peritoneal mesh techniques have gained more attention in the repair of IHs during the last two decades as a result of technical and commercial considerations. Surgeons should understand that "open pre-peritoneal techniques" as originally described by Nyhus, ${ }^{183}$ include several different approaches including the trans-inguinal pre-peritoneal repair described by Pélissier (TIPP), ${ }^{184}$ the posterior Kugel technique, ${ }^{185}$ transrectus pre-peritoneal approach (TREPP) ${ }^{186}$ Onstep approach, ${ }^{187}$ Ugahary technique, ${ }^{188}$ Wantz technique, ${ }^{189}$ and Rives' technique, ${ }^{190}$ for anterior pre-peritoneal repair. Note that TIPP, Onstep, and Rives' techniques approach the pre-peritoneal space through an anterior dissection opening the inguinal canal. Kugel, TREPP, Ugahary and Wantz use a posterior approach to open repair without entering the inguinal canal anteriorly.

Onstep is comparable with the PHS/UHS system, although there is only one mesh layer reinforcing the medial side pre-peritoneally, and the lateral side as in the Lichtenstein technique.

There are no data comparing the open pre-peritoneal techniques with each other, so no recommendation can be made about the preferred open pre-peritoneal technique. However, we are able to make the following statements based on limited data about pre-peritoneal techniques. The use of these techniques is suggested to be performed in research conditions. 


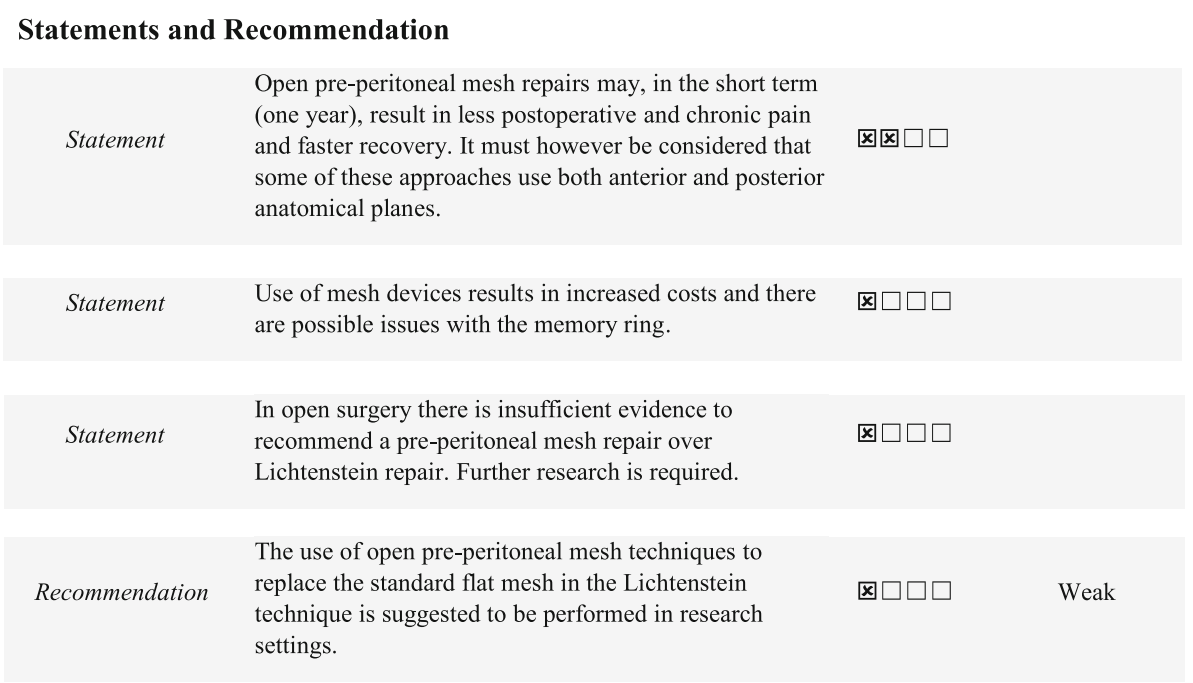

\section{Evidence in literature}

Two meta-analyses, one systematic review and three RCTs were identified out of 596 publications as suitable for inclusion and analysis below.

Cochrane Systematic Review 2009

A 2009 Cochrane Systematic Review included three eligible trials with 569 patients. ${ }^{191}$ Due to methodological limitations in the three trials considerable variations were found in acute pain (risk range 38.67-96.51\%) and chronic pain (risk range 7.83-40.47\%) across control groups. Two trials involving 322 patients found less chronic pain after pre-peritoneal repair (relative risk 0.18). These same two trials also found less acute pain (relative risk 0.17). One study of 247 patients found more chronic pain after preperitoneal repair (relative risk 1.17). This study reported that acute pain was nearly omnipresent and thus comparable in both intervention arms (relative risk 0.997, NNT 333). Early and late hernia recurrence rates were similar across the studies. Conflicting results were reported for other early outcomes like infection and hematoma formation.

Both pre-peritoneal and Lichtenstein repairs were seen as reasonable approaches since they resulted in similarly low hernia recurrence rates. There is some evidence that pre-peritoneal repairs cause less, or at least comparable, acute and chronic pain when compared with the Lichtenstein procedure. However, the Systematic Review authors emphasized the need for homogeneous high-quality randomized trials comparing elective pre-peritoneal IH repair techniques with the Lichtenstein repair to assess chronic pain incidence.

Meta-analysis 2013

A 2013 meta-analysis of 12 RCTs involving 1437 patients considered open trans-inguinal pre-peritoneal repair (TIPP) versus Lichtenstein in both primary and recurrent IHs. ${ }^{192}$ Unpublished data were used and data were extracted from a four-arm study using only two relevant arms. The "TIPP technique" was considered to be the Kugel approach, the actual TIPP technique and the Rives' technique. The metaanalysis concluded that the "TIPP repair" was associated with a reduced risk of chronic groin pain (RR $0.48 ; 95 \% \mathrm{CI}$ $0.26,0.89 ; z=2.33 ; p<0.02)$ without increasing the incidence of inguinal hernia recurrence (RR $0.18 ; 95 \% \mathrm{CI}$ $0.36,1.83 ; z=0.51 ; p=0.61)$. It was also concluded that-accounting for the significant heterogeneity amongst the different trials-the "TIPP technique" is comparable with the Lichtenstein repair in terms of hernia recurrence risk, postoperative complications, operation duration and postoperative pain intensity.

A second meta-analysis published in 2014, was judged to be of low methodological quality according to SIGN criteria and was withdrawn from analysis. ${ }^{193}$

RCT 2012

A 2012 study of TIPP versus Lichtenstein randomized 301 patients and used chronic postoperative pain at 1 year as the primary outcome measure. ${ }^{194}$ Patients and outcome assessors were blinded. Significantly fewer TIPP patients had continuous chronic pain, 3.5 versus $12.9 \%$ in the Lichtenstein group $(p=0.004)$. No significant intergroup differences were noted for other severe adverse events, including recurrence.

Another RCT, comparing Kugel versus Lichtenstein repair, was withdrawn from analysis due to low methodological quality by SIGN criteria. ${ }^{195}$ The same is true for another RCT comparing TIPP versus Lichtenstein repair. ${ }^{196}$ 


\section{Discussion, consensus and grading clarification}

From the available evidence, it can be concluded that open pre-peritoneal repairs seem as effective as the Lichtenstein repair in terms of recurrence and may possibly result in less postoperative pain and faster recovery. However, the caveat is that it is predominantly the anterior trans-inguinal pre-peritoneal technique (TIPP) and the posterior preperitoneal technique, as described by Kugel, which have been compared to the Lichtenstein repair. This caution is reinforced in the 2009 European Guidelines and the 2014 update. $^{3,} 4$ The various other open pre-peritoneal techniques have not been sufficiently studied to differentiate one from another.

Concerns about these surgical techniques may exist regarding both cost and long-term safety for some of these mesh devices. For the Kugel mesh there is an abundant amount of foreign material present. Problems with the initial recoil ring resulted in pain and even bowel perforation. ${ }^{197}$ The recent version of this mesh type now contains a resorbable memory ring. This being said, whether it is TIPP, Kugel, TREPP or others, the mesh choice is not strictly connected to the applied technique.

Mesh devices are more costly than flat meshes. However, a 2013 study found no differences in hospital costs between TIPP and Lichtenstein repairs. When productivity gains were included in the analysis, significant differences in cost favoring the TIPP modality were noted $(p=0.037) .{ }^{198}$ Individual surgeons and healthcare systems may wish to consider this point, depending on practice setting and reimbursement systems.

HerniaSurge acknowledges the potential value of open pre-peritoneal mesh techniques. The committee expressed concerns that some of these approaches use both anterior and posterior anatomical planes which has a theoretical disadvantage when a recurrence needs repair. There remains a need for learning curve studies, RCTs and registry studies, with long-term follow-up, to permit firmer conclusions.

KQ06.e Is TEP or TAPP the preferred laparo-endoscopic technique?

R. Bittner, F. Köckerling, J. Kukleta, S. Tumtavitikul and M. Misra

\section{Introduction}

Trans-abdominal pre-peritoneal (TAPP) and total extraperitoneal (TEP) differ although both techniques are in widespread use. In both, mesh is inserted in the pre-peritoneal plane but use a different access to that plane. In TEP, a totally pre-peritoneal approach is used with or without the help of a dissection balloon. In TAPP a laparoscopy is performed. The approach difference confers a theoretical advantage favoring TAPP. The anatomy is easier to identify when starting with a laparoscopy and the presence and type of hernia on the contralateral side can be identified before starting dissection. In TEP it is not necessary to open and close the peritoneum. Studies comparing TAPP and TEP show similar complication rates for seroma, scrotal edema, cord swelling, testicular atrophy, urinary bladder injury, inguinal nerve lesions, chronic pain and recurrence. Access-related complications can differ: there is increased risk of visceral injuries during trans-abdominal entry with TAPP while there is increased risk of vascular injuries during extra-peritoneal entry and dissection during TEP. 


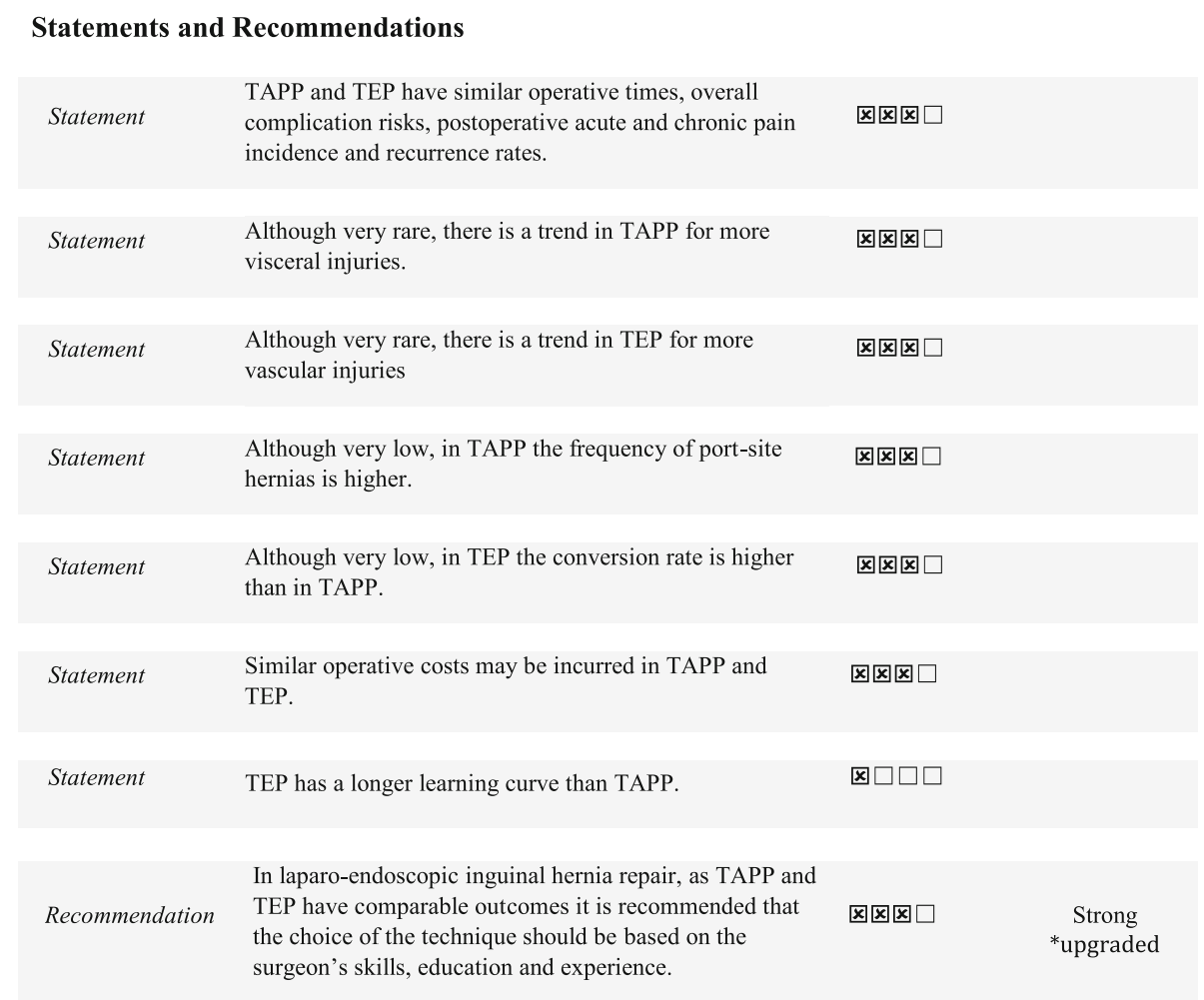

\section{Evidence in literature}

Out of hundreds of articles that were identified in the search 42 (including 2 abstracts) were analyzed. Out of these eight were systematic reviews. ${ }^{199-206}$ and three were large registry studies. ${ }^{207-209}$

Analyses of the RCTs and of the comparative nonrandomized studies showed many types of bias. A variety of confounding factors potentially impacting results were not mentioned or accounted for and were not identified by multivariate analyses. Most of the randomized studies lacked statistical power. ${ }^{210-215}$ The numbers of patients per intervention group were inadequate resulting in the risk of a type II error. ${ }^{210,} 211$, 215-217 Methods of patient allocation to one of the two techniques were not clearly stated. ${ }^{213,218,219}$ Surgeon's levels of experience with both techniques were not studied. In five of the studies, surgeons started laparoscopic hernia repair with TAPP, then, after gaining experience, switched to TEP. Thus the level of experience in laparoscopic surgery was not equivalent at the study's beginning. ${ }^{220-224}$ The cited high early recurrence rates $(>25 \%)$ and long operative times strongly suggest that the studied surgeons had not yet completed the learning curve. ${ }^{210}, 211,217,219,221,223-225$ Technical details such as mesh and fixation types, which could influence postoperative pain and/or recurrence, were omitted. ${ }^{67,210-213,216,218,226-234}$ Some of the studies employed overly small meshes $(<10 \times 15 \mathrm{~cm})$ or mesh of different size for TAPP and TEP. ${ }^{219}, 221,223-226,229$ Finally, follow-up duration differed for the TAPP and TEP groups (24-42.5 vs 9-28.8 months). ${ }^{210,} 211,215-218$, $226,227,233,235,236$

\section{Operation time, recurrence rate, pain, costs, access- related complications and conversion}

Due to the heterogeneity and weaknesses of the TAPP vs TEP studies, results varied greatly. The most recently published meta-analysis of ten RCTs failed to show any significant differences in operative times, total complication rates, hospital length of stay, recovery time, pain, recurrence rates or costs between TAPP and TEP. ${ }^{222}$

\section{Operation time}

In 22 comparisons, TAPP operative times varied from 34.5 to $104.5 \mathrm{~min}$ (median of $57 \mathrm{~min}$ ) and TEP operative times varied from 32.5 to $110 \mathrm{~min}$ (median of $62.3 \mathrm{~min}) .^{207,}$ 209-214, 216, 217, 219, 223-226, 229, 231, 232, 234-238

\section{Complication rates}

In 24 comparisons, TAPP complication rates ranged from 1.23 to $49 \%$ (median of $11.4 \%$ ) and TEP complication rates ranged from 1.3 to $50.3 \%$ (median $12.5 \%$ ). ${ }^{207}$, 209-214, $216-219,221,222,224-227,229,231-237,239$ One registry study reported a lower complication rate for TAPP ${ }^{207}$ while another for TEP. ${ }^{209}$ 


\section{Recurrence rates}

In 23 comparisons, TAPP recurrence rates varied between 0 and $25 \%$ (median $2.3 \%$ ) and TEP recurrence rates between 0 and $16.7 \%$ (median 0.6\%). ${ }^{67}$ 211-213, $216-219,222,223,225,227-229,232-238,240$ Interestingly, an analysis of the 1990-1998 literature (TAPP and TEP, 13 studies each) showed a TAPP recurrence rate of $1.33 \%$ and a TEP recurrence rate of $0.6 \%$. In the 1999-2008 period (seven TAPP and eight TEP studies), recurrence rates dropped to $0.77 \%$ for TAPP and $0.54 \%$ for TEP, possibly reflecting improved technical performance over time. ${ }^{5,241}$ Pain

A qualitative systematic review of 71 TAPP and TEP studies showed no difference in acute pain intensity or duration. $^{201}$ The same is generally true for chronic pain, with six studies showing no difference $^{212,214,219,229,232,234}$ and two ${ }^{213,230}$ slightly better outcomes after TAPP (1.15 vs. $3.03 \% ;^{230} 5$ vs. $\left.9 \% .^{213}\right)$. Costs

A large population-based study in German hospitals found no differences in TAPP and TEP costs. ${ }^{208}$

\section{Access-related complications and conversion}

The frequency of visceral access-related complications

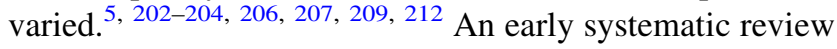
analyzing the results of 6 comparative studies and 3 case series showed that when using TAPP, visceral lesions occurred in $0.6 \%(54 / 9141)$ versus $0.2 \%(12 / 5803)$ in TEP. $^{206}$ On the other hand, after TEP vascular lesions occurred more often compared to TAPP (0.41 vs. $0.28 \%)$.

Port-site hernias were more common after TAPP ( 0.4 vs. $0.026 \%) .5,202-204,206,207,209,212$ The conversion rate in TEP was higher than in TAPP $(0.47$ vs. $0.26 \%)$. A recently published systematic review ${ }^{5}$ analyzed 8 comparative studies and 7 case series and found similar results: visceral injuries TAPP $0.21 \%$ vs. TEP $0.11 \%$; vascular injuries TAPP $0.25 \%$ vs. TEP $0.42 \%$; port-site hernias TAPP $0.6 \%$ vs. TEP $0.05 \%$ and conversion rate TAPP $0.16 \%$ vs. TEP $0.66 \%$. 5, 202-204, 206, 207, 209, 212

In a large German hernia registry (Herniamed) ${ }^{209}$ TAPPrelated visceral injuries (bowel, urinary bladder) were seen in $0.27 \%$ cases $(29 / 10,887)$ versus $0.1 \%$ in TEP $(7 / 6700)$, the difference was not statistically significant. Correspondingly to the literature, vascular complications were seen in $1.39 \%$ cases after TEP and in $1.13 \%$ after TAPP. This difference was significant $(p=0.03)$. Reoperation rates were not significantly different (TAPP $0.9 \%$ and TEP $0.2 \%$ ). Interestingly, the overall complication rate as reported by the Swiss hernia registry ${ }^{207}$ after TAPP was lower than after TEP (1.7 vs. $4.2 \%$ ), whereas the German registry ${ }^{209}$ showed more complications after TAPP (5.37 vs. $2.89 \%$ ).

\section{Length of hospital stay}

In the update of the IEHS guidelines TAPP had a longer hospital stay than TEP. ${ }^{241}$ A large population-based study in German hospitals found a significantly shorter hospital stay in TAPP compared to TEP. ${ }^{208}$ However, a similar study from Switzerland reported an advantage for TEP (2.3 vs. 2.9 days, $p=0.002) .{ }^{207}$ A randomized controlled study from India recently published could not demonstrate any significant difference between both techniques. ${ }^{234}$

\section{Guidelines}

The 2009 EHS Guidelines describe that no hard conclusions concerning the difference in results between TEP and TAPP can be offered. ${ }^{3}$ The only conclusion (level 2A) was that TAPP seemed to be associated with a higher risk of port site and visceral injuries and TEP a higher conversion rate. Both other guidelines (IEHS and EAES) reported similar results to those described above. $^{3,5,6}$

\section{Discussion, consensus and clarification of grading}

Only three of 29 RCTs and observational studies focused on primary, unilateral hernias in men. ${ }^{215,237,240}$ In spite of all variations and limitations of most of the comparative studies, all eight meta-analyses and systematic reviews inclusive of these studies concluded that insufficient evidence exists to recommend the use of one technique over the other. ${ }^{199-206}$ Each technique has different, very rare, but serious complications associated with it. One registry study reported a lower TAPP complication rate, ${ }^{207}$ while another reported a lower TEP complication rate. ${ }^{209}$ Operative team ease and experience are important factors in the decision to use one technique preferentially. ${ }^{236}$ HerniaSurge recommends that both techniques are suited for treatment of inguinal hernia(s).

KQ06.f When considering recurrence, pain, learning curve, postoperative recovery and costs which is preferred technique for primary unilateral inguinal hernias: best open mesh (Lichtenstein) or a laparo-endoscopic (TEP and TAPP) technique?

F. Köckerling, H. Tran and D. Chen

\section{Introduction}

In the EHS guidelines, open Lichtenstein and laparo-endoscopic IH techniques (TEP/TAPP) are recommended as the best evidence-based options for repair of primary unilateral hernias provided the surgeon is sufficiently experienced in the specific procedure. 3,4

The HerniaSurge committee thought it prudent to account for all important factors when considering recommendations on Lichtenstein and laparo-endoscopic techniques. It seems clear that when considering postoperative pain, recovery speed and chronic pain, the laparoendoscopic techniques are superior. In TEP and TAPP expert hands, especially when performing high-volume 
surgery, those techniques are probably also cost effective and very safe. However, many of the studies in this area suffer from weakness such as: lack of clear endpoints in pain assessment, definitions, quality of the surgeon's technique and caseload per surgeon. Additionally, there is a well-documented difference in learning curve and initial costs favoring Lichtenstein.

In order to properly address the key question, all metaanalyses and RCTs must be excluded that compared laparo-endoscopic techniques with either, open techniques other than Lichtenstein, and/or those that enrolled patients other than males with primary unilateral IHs. laparo-endoscopic procedures when compared with the Lichtenstein repair including: a lower incidence of wound infection (OR $0.39 ; 95 \%$ CI $0.26-0.61 ; p=0.00003$ ), a reduction in hematoma formation (OR $0.69 ; 95 \% \mathrm{CI}$ $0.54-0.90 ; p=0.005$ ), and nerve injury (OR $0.46 ; 95 \% \mathrm{CI}$ $0.35-0.61 ; p<0.00001)$, an earlier return to normal activities or work $(-1.35 ; 95 \%$ CI -1.72 to -0.97 ; $p<0.00001$ ), and fewer incidences of chronic pain syndrome (OR 0.56; 95\% CI 0.44-0.70; $p<0.00001) .{ }^{244}$ No difference was found in total morbidity or in the incidence of intestinal lesions, urinary bladder lesions, major vascular lesions, urinary retention and testicular problems. ${ }^{244} \mathrm{Sig}$ nificant advantages for the Lichtenstein repair included a

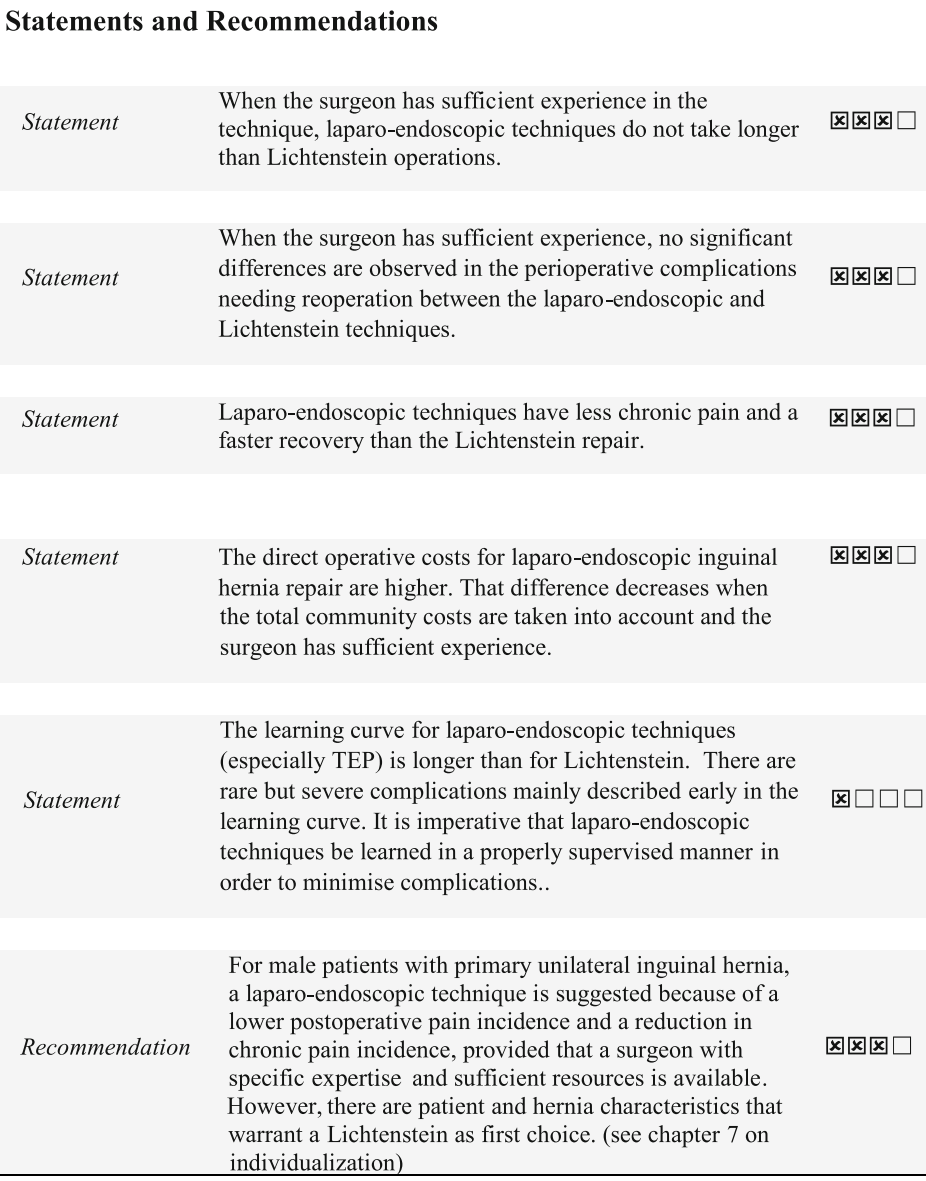

Statement

The direct operative costs for laparo-endoscopic inguinal hernia repair are higher. That difference decreases when the total community costs are taken into account and the surgeon has sufficient experience.

\section{冈खा}

The learning curve for laparo-endoscopic techniques (especially TEP) is longer than for Lichtenstein. There are rare but severe complications mainly described early in the learning curve. It is imperative that laparo-endoscopic techniques be learned in a properly supervised manner in order to minimise complications.

For male patients with primary unilateral inguinal hernia, a laparo-endoscopic technique is suggested because of a lower postoperative pain incidence and a reduction in

Recommendation chronic pain incidence, provided that a surgeon with specific expertise and sufficient resources is available. However, there are patient and hernia characteristics that warrant a Lichtenstein as first choice. (see chapter 7 on individualization)

\section{Evidence in literature}

\section{Systematic reviews and meta-analyses}

In meta-analyses from 1999, 2000, 2003 and 2012, TEP and TAPP are compared with all open procedures used for IH repair. ${ }^{199,202,242,243}$ Only in a 2005 meta-analysis subgroup analysis were the TAPP and TEP techniques jointly compared with the Lichtenstein operation. ${ }^{244}$ This subgroup analysis found significant advantages for the shorter operating time [TAPP/TEP $65.7 \mathrm{~min}(40-109)$ vs Lichtenstein $55.5 \min$ (34-99); $p=0.01$, a lower incidence of seroma formation (OR 1.42; 95\% CI 1.13-1.79; $p=0.003$ ), and fewer hernia recurrences (OR 2.00; 95\% CI $1.46-2.74 ; p=0.00001) .{ }^{244}$ The latter was strongly influenced by the Veterans Affairs Multicenter Trial, where the minimum mesh size in endoscopic surgery was $7.6 \times 150 \mathrm{~cm} .{ }^{245}$ When this study is excluded, there is no 
difference in the recurrence rates between open and laparoendoscopic surgery.

\section{RCTs}

For comparison of the laparo-endoscopic (TEP, TAPP) with the open Lichtenstein technique for male primary unilateral inguinal hernia many studies must be excluded. This is because they included female patients, bilateral hernias and/ or recurrent hernias or compared TEP and TAPP with other open procedures or used too small meshes or combined IH repair with laparoscopic cholecystectomy. ${ }^{212,213,216,225,}$ 228, 246-277 In the comparison of 1237 laparo-endoscopic (TEP, TAPP) operations with 1281 Lichtenstein operations from RCTs fulfilling the inclusion criteria, ${ }^{211,217,278-288}$ no differences have been observed in the intraoperative or postoperative complications following primary unilateral $\mathrm{IH}$ repair in males. Clear advantages have been observed for the laparo-endoscopic techniques in terms of early postoperative pain, analgesic consumption, and return to normal daily activities and to work. When the surgeon had sufficient experience in the respective technique (i.e. after completing the learning curve), no significant difference was detected in the recurrence rate (TEP vs Lichtenstein with median followup of 5.1 years 2.4 vs $1.2 \% ; p=0.109$ and TAPP vs Lichtenstein with median follow-up of 52 months 1.3 vs $1.2 \%$; ns) $)^{282,288}$ between the laparo-endoscopic and Lichtenstein techniques. Likewise, chronic pain occurred significantly less often after laparo-endoscopic than after Lichtenstein operation (TEP vs Lichtenstein with follow-up of 5 years 9.4 vs $18.8 \%$ and TAPP versus Lichtenstein with median follow-up of 52 months slight pain 14.8 vs $23.7 \%$, moderate pain 1.2 vs $5.3 \%$ and severe pain 0 vs $3.9 \%) .^{283,284,287}$ In the three RCTs. ${ }^{280,281,285}$ with at least 100 patients in each arm, the operative time for TEP was either similar to, or shorter than, the Lichtenstein operative time. The direct operative costs for laparo-endoscopic techniques are higher than for the Lichtenstein operation. $^{217,278,279,284}$ However, that difference decreases when all community costs are taken into account. ${ }^{278,284}$

\section{Large database studies}

A 2015 analysis of the Herniamed Registry compared the prospective data collected for males undergoing primary unilateral IH repair using either TEP or open Lichtenstein repair. $^{289}$ Inclusion criteria were: a minimum age of 16 years, male gender, primary unilateral $\mathrm{IH}$, elective operation and availability of data on 1-year follow-up by a questionnaire of the general practitioner and patient. In total, 17,388 patients were enrolled, 10,555 (60.7\%) had a Lichtenstein repair and $6833(39.3 \%)$ a TEP repair.

On multivariable analyses, surgical technique had no significant effect on the recurrence rate (estimated OR $0.77595 \%$ CI $0.549-1.093 ; p=0.146$ ) or on the chronic pain rate needing treatment (estimated OR $1.06695 \% \mathrm{CI}$ $0.860-1.321 ; p=0.560)$. Nor did the complication-related reoperation rates differ significantly between the two techniques (estimated OR 1.356 95\% CI $0.960-1.913$; $p=0.084$ ). TEP was found to have benefits on the postoperative complications rate (estimated OR $2.15295 \% \mathrm{CI}$ 1.734-2.672; $p<0.001$ ), pain-at-rest rate (estimated OR $1.23195 \%$ CI 1.049-1.444; $p=0.011$ ), and pain-on-exertion rate (OR $1.42095 \%$ CI 1.264-1.596; $p<0.001$ ).

\section{Guidelines}

The 2009 EHS guidelines concluded, ${ }^{3}$ mainly on the basis of the 2005 meta-analysis, ${ }^{244}$ that endoscopic IH techniques result in a lower incidence of wound infection, hematoma formation and an earlier return-to-normal activities or work than the Lichtenstein technique. Laparoendoscopic IH techniques have a longer operative time and a higher incidence of seroma formation than the Lichtenstein technique. Endoscopic repair results in a lower incidence of chronic pain/numbness than the Lichtenstein technique.

The learning curve for performing a laparo-endoscopic hernia repair, especially TEP, is longer than that for open Lichtenstein repair, and ranges between 50 and 100 procedures, with the first 30-50 being most critical. ${ }^{3}$

From a hospital perspective, an open mesh procedure is the most cost-effective operation. ${ }^{3}$ In cost-utility analyses including quality of life, endoscopic techniques may be preferable since they cause less numbness and chronic pain. $^{3}$

In the 2014 EHS guidelines update, ${ }^{4}$ a new meta-analysis was included. It contained studies with a follow-up of more than 48 months (including two new RCTs on TEP vs Lichtenstein). There was a non-significant difference in severe chronic pain $(p=0.12)$ and in recurrence when data from one surgeon in the Eklund trial. ${ }^{282}$ were excluded. This was because of unacceptable recurrence rates in the endoscopic group (32\%) due to technical failure.

\section{Discussion, consensus}

When the surgeon has sufficient experience in the respective techniques, laparo-endoscopic and Lichtenstein techniques have comparable operation times, perioperative complication rates needing reoperation and recurrence rates. Endoscopic techniques show advantages in terms of early and later postoperative pain and speed of recovery. In the EHS guidelines update, data were analyzed from studies with a follow-up of more than 48 months. This analysis yielded a non-significant difference in severe chronic pain and long-term recurrence. The direct operative costs for laparo-endoscopic IH repair are higher, but fall to levels comparable with the Lichtenstein repair when considering quality-of-life aspects and total community costs. Study quality heterogeneity-lack of clear pain endpoints, definitions, quality of surgeon's technique, 
caseload per surgeon, and lack of hernia classificationmake the evaluation of complication risks difficult. Furthermore, there is a well-documented difference in learning curve and initial costs favoring Lichtenstein.

Large RCTs with good external validity and large-scale database studies are urgently needed to compare endo- patients. Additionally, logistical and financial constraints may limit the availability of quality laparo-endoscopic repairs, especially in lower resource settings.

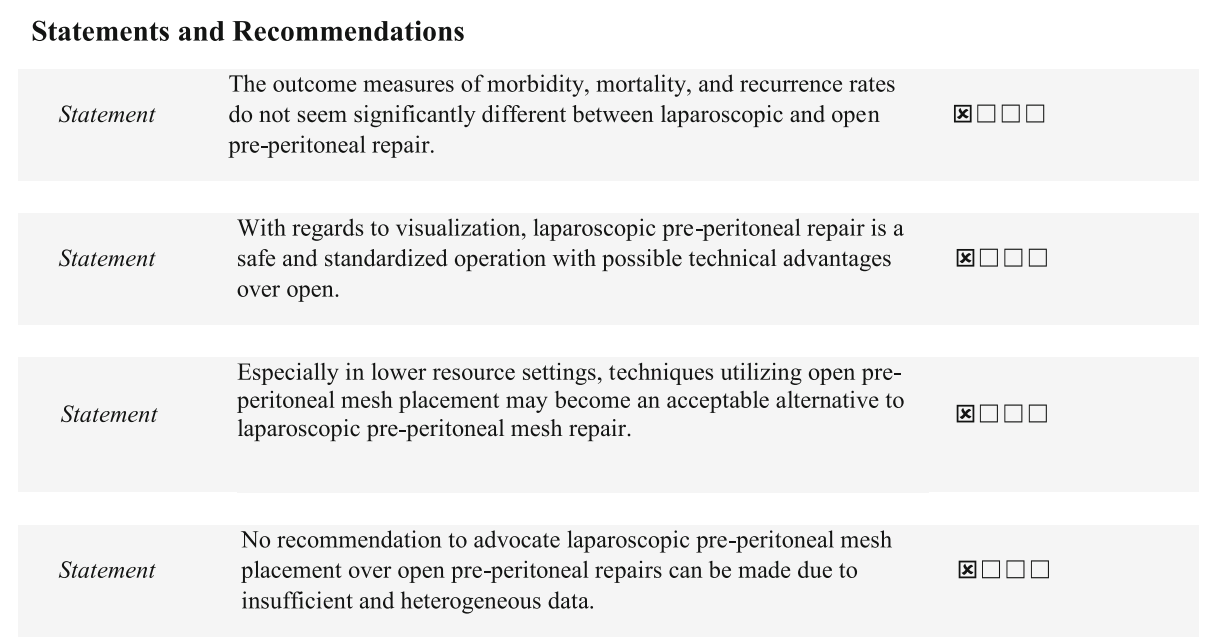

scopic with Lichtenstein operations for primary unilateral IHs in males. These studies must carefully select participating surgeons, to ensure that the learning curve has been completed for the respective surgical technique. A major investment is needed worldwide to make the learning curve for (laparo-endoscopic) hernia surgery as smooth as possible by ensuring optimal training facilities and circumstances.

HerniaSurge recommends a standardization of the laparo-endoscopic and Lichtenstein techniques, structured training programs and continuous supervision of trainees and surgeons within the learning curve.

KQ06.g In males with unilateral primary inguinal hernias which is the preferred repair technique, laparo-endoscopic (TEP/TAPP) or open pre-peritoneal?

F. Berrevoet, M. Misra and D. Chen

\section{Introduction}

Evidence suggests that pre-peritoneal mesh placement is preferred over anterior mesh placement because of the physiologic mesh location and placement of the mesh away from the groin nerves. There is clinical interest about whether the various surgical approaches to achieve preperitoneal mesh positioning leads to different patient outcomes. Laparo-endoscopic IH repair has been studied in detail with good results, but has a rather long learning curve, potentially higher procedure costs and potential risks associated with general anesthesia in certain types of

\section{Evidence in literature}

The literature comparing laparo-endoscopic techniques with open pre-peritoneal mesh placement for primary unilateral IHs is extremely limited and heterogeneous.

A 2002 meta-analysis compared laparo-endoscopic IH repair with open $\mathrm{IH}$ repair techniques. ${ }^{290}$ However, the early laparo-endoscopic trials control groups included in this meta-analysis were poorly standardized; and often included only suture repairs such as the Bassini, McVay, or Shouldice. In later studies, plug-and-patch repairs were the main cohort in the groups that considered open pre-peritoneal mesh techniques.

Although the authors concluded that open pre-peritoneal hernia repair provides equivalent outcomes at lower costs and has potentially less severe complications compared with laparoscopic techniques, the included studies and available literature do not address our key question adequately.

An RCT of 49 patients compared open pre-peritoneal repair and TAPP. ${ }^{291}$ This small study concluded that the open repairs were associated with fewer complications and recurrences and that laparoscopic TAPP was associated with higher costs but no advantage in median time to return-to-work.

The SCUR Hernia repair study, ${ }^{292}$ which compared 613 patients randomized to three groups (open suture repair, open pre-peritoneal repair with polypropylene mesh and TAPP) demonstrated that although TAPP resulted in both shorter time to full recovery and shorter time to return-to- 
work, it was more expensive and had a higher complication rate. There was no significant difference regarding recurrences at 1 year in the three groups (3\% overall). Another small four-arm randomized trial of 100 patients studied laparoscopic TAPP and TEP as well as open pre-peritoneal repair and Lichtenstein repair. ${ }^{211}$ The laparoscopic repair groups showed less postoperative pain and achieved significantly faster return-to-normal domestic activities and to-work compared to Lichtenstein repair patients. However, this study is of low methodological value according to SIGN criteria.

The currently available literature does not allow us to provide any recommendation about whether laparoscopic mesh placement in the pre-peritoneal plane is superior to open pre-peritoneal techniques. Further research is necessary. The learning curve of pre-peritoneal techniques needs to be evaluated and the theoretical advantage of a better visualization in laparo-endoscopic repair techniques (against potential higher cost and complications) must be researched.

KQ06.h What is the preferred technique in bilateral hernia?

A. C. de Beaux, M. P. Simons
There has been little new evidence on the preferred surgical approach for primary unilateral inguinal hernia. The outcomes for TEP and TAPP, when comparing unilateral versus bilateral, are similar, especially when taking into account the number of hernias repaired. ${ }^{297-299}$ One prospective non-randomized clinical study compared 53 patients undergoing bilateral Lichtenstein with 75 patients undergoing bilateral TEP repair. The authors reported that the TEP group had a shorter operation time, lower postoperative complication rate and shorter hospital stay. ${ }^{300}$

The 2009 EHS guidelines concluded with only moderate evidence that bilateral hernia is preferably treated by a laparo-endoscopic method provided expertise is available. ${ }^{3}$ This seems self-evident as the advantages of laparo-endoscopic repair (faster recovery, lower risk of chronic pain and cost effectiveness) are increased when performing two hernia repairs via the same three key hole incisions. No new high-level research was found, so the recommendation of the EHS guidelines have been used in the HerniaSurge guidelines. The EAES guidelines concluded that, especially in bilateral groin hernia, an endoscopic approach is an excellent choice (level 1B consensus 96\%). ${ }^{6}$ (see also Chapter 7 individualization). HerniaSurge by consensus decided to upgrade the level of recommendation.

\section{Recommendation

\section{Evidence in literature}

The 2009 EHS guidelines, recommended for bilateral primary inguinal hernia repair, either a bilateral Lichtenstein or endoscopic approach. ${ }^{3}$ The socio-economic benefits of the endoscopic approach over the Lichtenstein approach led to a suggestion, that the endoscopic repair was preferred, especially in younger patients. As for a primary unilateral hernia, the local/national hernia expertise in open versus endoscopic techniques will have a big influence in surgical approach chosen. ${ }^{293-296}$ In addition, the relative contra-indications to an endoscopic approach, such as fitness for general anesthesia, previous lower abdominal surgery and size of each hernia will influence individual surgeon choice of surgical approach (see Chapter 7). Another question in helping to decide the surgical approach is whether both hernias need to be repaired at the same time? A large symptomatic hernia on one side, and a small asymptomatic hernia on the other in an elderly man may only justify a unilateral repair under local anesthetic on the symptomatic side.

\section{Chapter 7}

\section{Individualization of treatment options}

B. van den Heuvel, M. P. Simons and U. Klinge

\section{Introduction}

Inguinal hernia treatment has changed markedly over the past seven decades. Prior to the 1950s, hernia surgery involved an anatomical reconstruction of the inguinal canal with sutures. $^{3}, 5,200,211,226,256,258,282,283,288,301-322$ When the tension-free mesh repair was introduced it resulted in a hernia repair revolution. Many new mesh applications and variations were developed including open, anterior and posterior approaches, and endoscopic techniques (Fig. 2). 3, 5, 200, 211, 226, 256, 258, 282, 283, 288, 301-322 
The adage applies that any technique, thoroughly taught and frequently performed with good results, is valid. Some techniques are easily learned and offer good results whilst others might be very difficult to master but offer great results. All these techniques are highly dependent on the surgeon's knowledge of anatomy, caseload and dedication to groin hernia surgery. Therefore, the question confronting result, questions arise as to which factors should properly guide surgical decision-making? Can IH treatment be standardized, or should it be individualized? If individualized, which determinants should influence surgeon's choices?

We have tried to answer the questions posed.

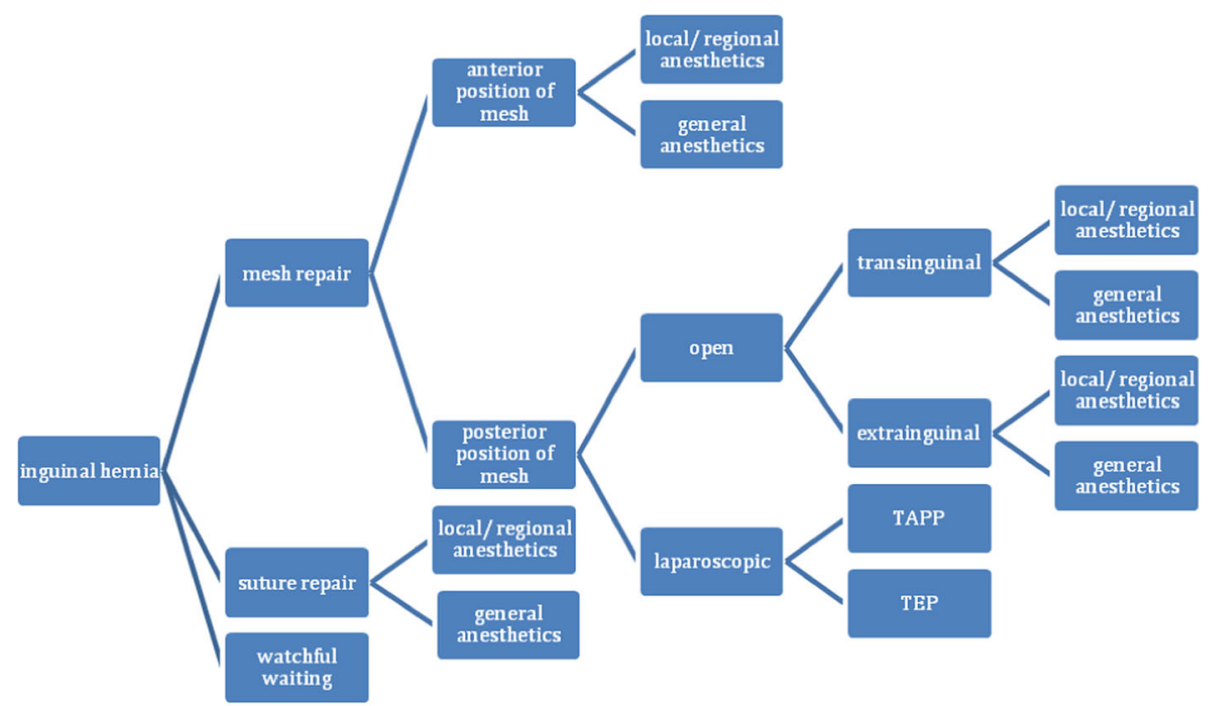

FIGURE 2. TREATMENT OPTIONS FOR INGUINAL HERNIA

hernia surgeons is: "Which technique should be used in which case?" Individual techniques have varying advantages and disadvantages such as the possibility of surgery under local anesthetic, simultaneous contralateral hernia repair, avoidance of scar tissue in recurrent hernias by choosing a different approach, amongst many others. As a

\section{Key question}

KQ07.a In inguinal hernia repair, when should treatment be individualized?

\begin{tabular}{|c|c|c|c|}
\hline \multicolumn{4}{|c|}{ Recommendations } \\
\hline Recommendation & $\begin{array}{l}\text { Laparo-endoscopic repair is recommended for the repair of } \\
\text { primary bilateral inguinal hernias provided that a surgeon } \\
\text { with specific and sufficient resources is available. }\end{array}$ & 冈खि & $\begin{array}{c}\text { Strong } \\
\text { *upgraded }\end{array}$ \\
\hline Recommendation & $\begin{array}{l}\text { In patients with pelvic pathology or scarring due to radiation } \\
\text { or pelvic surgery, or for those on peritoneal dialysis, consider } \\
\text { an anterior approach. }\end{array}$ & 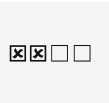 & $\begin{array}{c}\text { Strong } \\
\text { *upgraded }\end{array}$ \\
\hline Recommendation & $\begin{array}{l}\text { It is recommended that surgeons tailor treatments based on } \\
\text { expertise, local/national resources, and patient- and hernia- } \\
\text { related factors. }\end{array}$ & 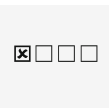 & $\begin{array}{c}\text { Strong } \\
\text { *upgraded }\end{array}$ \\
\hline Recommendation & $\begin{array}{l}\text { Since a generally accepted technique, suitable for all inguinal } \\
\text { hernias, does not exist, it is recommended that } \\
\text { surgeons/surgical services provide both an anterior and a } \\
\text { posterior approach option. }\end{array}$ & 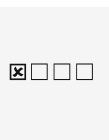 & $\begin{array}{c}\text { Strong } \\
\text { *upgraded }\end{array}$ \\
\hline
\end{tabular}




\section{Evidence in literature}

There are no reviews, RCTs or cohort studies comparing different techniques in specific situations. Since no mesh technique is proven to be superior, technique chosen often depends on surgeons' preferences.

One 2012 publication addresses surgical preferences in IH repair. ${ }^{294}$ A survey questionnaire was distributed to 100 endoscopic surgeons at the 2010 European Association of Endoscopic Surgery (EAES) annual meeting. The participating surgeons were asked to indicate preferred surgical technique in specific clinical scenarios, including patient age, gender, physical activity capabilities, physical characteristics, emergency situations, and hernia size and type. Surgeons were able to choose between open, TAPP or TEP repair in a variety of patient scenarios. Eighty-two percent of the surgeons chose a tailored approach and indicated that their choice of repair depended on the listed patient characteristics. Interestingly, only $6 \%$ of the surgeons were able to routinely offer patients all three techniques.

\section{Discussion, consensus and grading clarification}

The HerniaSurge Group has identified possible factors influencing the type of IH repair. These factors involve: patient characteristics, surgical expertise, local/national resources, and logistics (Table 2). Future research must address the issue of individualized treatment in specific cases. The HerniaSurge Group currently offers consensusbased examples of tailored surgical approaches in specific circumstances.

\section{Additional recommendations for individualization}

In the different chapters of these guidelines some recommendations are made with regard to indicated surgical technique. We have outlined these recommendations in this chapter, but refer to these specific chapters for detailed background information. In addition to these recommendations the consensus-based recommendations are outlined.

For recurrent IHs, use the opposite approach (e.g. for recurrence after anterior repair use a posterior technique, and vice versa) (Chapter 10).

In high-risk IH patients with extensive comorbidities consider an open mesh repair under local anesthesia (Chapter 16).

For IH patients with high preoperative pain, consider laparo-endoscopic repair (Chapter 18).

Consider a laparo-endoscopic approach in active young patients with IHs (Chapter 18).

In femoral hernia patients, a pre-peritoneal mesh repair is recommended (Chapter 25).

In female patients with IHs a laparo-endoscopic repair is recommended, providing expertise and resources are available (Chapter 26).

Use a laparo-endoscopic approach in patients with bilateral IHs (Chapter 6h).

In male patients with a large scrotal or irreducible hernia, an open mesh repair or a trans-abdominal laparoscopic repair (TAPP) is recommended (HerniaSurge consensus).

Table 2. Determinants of surgeons' preferences

\begin{tabular}{|ll|}
\hline SURGEON & $\begin{array}{l}\text { Surgeons expertise } \\
\text { LATIENT }\end{array}$ \\
CHARACTERISTICS & High preoperative pain \\
& Gender \\
& Comorbidity (smoking, collagen disease, obesity, ascites) \\
& Previous medical history (pelvic surgery, pelvic radiation, lower abdominal \\
& surgery) \\
& Previous hernia surgery \\
& Occupation \\
& Physical activity \\
& Age \\
& Contraindication for general anesthetics \\
\hline HERNIA & Size \\
CHARACTERISTICS & Type \\
& Primary or recurrent \\
& Reducibility \\
& Unilateral or bilateral \\
\hline EMERGENCY & Incarcerated hernia \\
SITUATION & Strangulated hernia \\
\hline
\end{tabular}




\section{Chapter 8}

\section{Occult hernias}

A. C. de Beaux, N. Schouten and J. F. Kukleta

\section{Introduction}

An occult hernia, as defined by the HerniaSurge Group, is an asymptomatic hernia not detectable by physical examination.

IH formation is considered a bilateral condition based on etiology, yet for many patients presentation with a unilateral symptomatic hernia is typical. Occasionally, a contralateral hernia will be evident on physical examination, but a number of patients will have a contralateral occult hernia at the time of initial presentation which may become symptomatic later. Another patient subset will develop a contralateral hernia de novo which may require repair at a later date.

\section{Key questions}

KQ08.a In those with unilateral overt primary IHs, what is the likelihood that they will also have a contralateral occult IH?

KQ08.b In those with unilateral overt primary IHs, what is the likelihood that they will develop contralateral overt hernias over time?

KQ08.c In patients who have undergone a unilateral TEP and negative contralateral exploration, what is the risk of developing an overt hernia on the disease-free side at a later date?

KQ08.d In those where an occult contralateral IH is seen during TAPP will it become symptomatic if not repaired? KQ08.e In those with overt unilateral primary inguinal hernias without contraindications to bilateral TEP or TAPP repair, should bilateral repair be performed?

\section{Statements and Recommendations}

\begin{tabular}{|c|c|c|c|}
\hline Statement & $\begin{array}{l}\text { In patients with unilateral overt primary inguinal hernias, an } \\
\text { occult contralateral inguinal hernia is sometimes seen at } \\
\text { time of laparoscopic inguinal hernia surgery. The true } \\
\text { incidence is unknown, and will vary depending on a number } \\
\text { of surgeon and patient factors. }\end{array}$ & 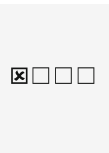 & \\
\hline Statement & $\begin{array}{l}\text { In patients who have undergone a unilateral inguinal hernia } \\
\text { repair, the chance of developing a contralateral inguinal } \\
\text { hernia increases with time; however, the true incidence is } \\
\text { unknown. }\end{array}$ & 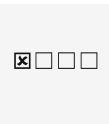 & \\
\hline Statement & $\begin{array}{l}\text { There is a low risk for the development of a contralateral } \\
\text { overt inguinal hernia following a previously negative TEP } \\
\text { exploration. }\end{array}$ & 冈ロ $\square$ & \\
\hline Statement & $\begin{array}{l}\text { The percentage of occult hernias noted at TAPP that become } \\
\text { symptomatic will increase over time; however, the true } \\
\text { incidence is unknown. }\end{array}$ & 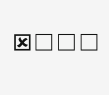 & \\
\hline Recommendation & $\begin{array}{l}\text { It is recommended that the contralateral groin be inspected at } \\
\text { the time of TAPP repair. If a contralateral inguinal hernia is } \\
\text { found at the time of surgery and prior informed consent was } \\
\text { obtained, repair is recommended. }\end{array}$ & 冈ᄆ $\square$ & $\begin{array}{c}\text { Strong } \\
\text { *upgraded }\end{array}$ \\
\hline Recommendation & $\begin{array}{l}\text { In those with overt unilateral primary inguinal hernias } \\
\text { without contralateral hernias, routine bilateral TAPP repair } \\
\text { is not suggested. }\end{array}$ & 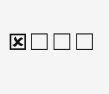 & Weak \\
\hline Recommendation & $\begin{array}{l}\text { Routine exploration by TEP of the contralateral groin in an } \\
\text { asymptomatic patient with no clinical hernia is not } \\
\text { suggested. }\end{array}$ & 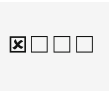 & Weak \\
\hline
\end{tabular}




\section{Evidence in literature}

Evidence for the recommendations and statements in this chapter is largely derived from retrospective case series involving relatively small numbers of patients. Some RCTs address certain aspects of the topics presented.

A number of studies have reported on the incidence of occult contralateral hernias at the time of bilateral TEP exploration for a clinically diagnosed unilateral hernia. These studies report incidence rates ranging from 5 to $58 \% .^{323-331}$ In TAPP exploration, clinically occult contralateral hernias are observed in $13-22 \%$ of patients. ${ }^{325,332,} 333$ However, the laparoscopic parameters for contralateral hernia presence or absence are not well defined in these studies, so it is difficult to know how such variation may account for the large variation in occult hernias reported. Additionally, the natural history of these small incidentally discovered defects is poorly understood and the clinical relevance of repair is unknown. ${ }^{323}$

In those with primary unilateral IHs, the lifetime risk of developing a contralateral IH is not known exactly. One study reported a $48 \%$ incidence of overt contralateral hernia development following TEP repair at 13 years followup. ${ }^{57}$ Others report the incidence of subsequent contralateral hernia repair after primary unilateral TEP repair as: $3.2 \%$ at 3 years, $3.5 \%$ at 5 years, and $3.8 \%$ at 10 years. $^{334-336}$

Several RCTs involving patients who have undergone repair of unilateral primary IHs have reported on contralateral hernia formation during various follow-up periods. One study reported a 5 -year $10 \%$ contralateral hernia incidence. ${ }^{138}$ An RCT with a nearly 11-year follow-up compared open-suture to open-mesh repair of unilateral primary IHs and found contralateral hernia formation in $21 \%$ of non-mesh patients and $25 \%$ of mesh patients. ${ }^{76}$ Another RCT of TEP vs open-mesh repair, reported that $10.7 \%$ of the TEP repair group and $7.3 \%$ of the open-repair group developed contralateral hernias at 5 years. ${ }^{337}$

Some surgeons perform contralateral exploration at the time of unilateral primary IH TEP repair. Two retrospective cohort studies address this subject. Notably, the laparoscopic features of a normal groin versus an occult hernia are not defined nor are the nature and completeness of follow-up. One study, with a 5.9-year median follow-up, reported that $8.1 \%$ of patients developed a contralateral $\mathrm{IH}$ after unilateral TEP repair with negative contralateral exploration. ${ }^{328}$ The annual calculated risk was $1.2 \%$ for contralateral hernia formation after a previously negative TEP exploration $(1.6 \%$ at 1 year, $5.9 \%$ at 5 years and $11.8 \%$ at 10 years). The median time to contralateral hernia development was 3.7 years (range $0-12$ years). However, almost $60 \%$ of the study population had already undergone bilateral repair. The remaining 40\% (409 patients) underwent unilateral repair and contralateral exploration and are therefore not representative of most hernia surgeon's practices. A second cohort study with 38-month median follow-up (range 10-82 months) reported a $1.1 \%$ incidence of contralateral overt hernia formation following unilateral TEP repair with contralateral exploration. ${ }^{326}$ Thirty percent of the study population had already undergone bilateral repair.

Two studies address the subject of contralateral (preperitoneal) exploration at the time of unilateral primary $\mathrm{IH}$ TAPP repair. In one, the presence of a so-called incipient hernia was identified during TAPP contralateral exploration in 5\% of patients. ${ }^{332}$ An incipient occult hernia was defined as a looming or beginning hernia with a defect too small to allow protrusion. After a mean follow-up of 112 months (range 16-218 months) 21\% of patients (13 patients) developed a symptomatic hernia. In the same study, a true contralateral occult hernia had been identified and repaired in $8 \%$ of patients during their initial surgery. Another study reported that with a 12-month median follow-up, six of 21 patients (29\%) with a contralateral "incidental hernia defect" seen on TAPP exploration developed an overt (i.e. symptomatic) $\mathrm{IH}^{338}$

Routine contralateral exploration or "preventive" mesh placement in a normal groin is controversial. Visualization of the contralateral side in TAPP repair for an overt unilateral hernia is easily done without additional dissection of the contralateral side. However, without dissection of the contralateral side, some cases of lipoma of the cord will be missed. Unlike the TAPP approach, the TEP repair requires additional dissection to diagnose a contralateral hernia. Bilateral repair proponents cite a number of advantages to their approach including: poor clinical accuracy in hernia diagnosis especially in obese patients, the benefits to the patient and the healthcare system of one operation, and possible prevention of a hernia-related complications during future contralateral side surgery. Opponents focus on the potential to do harm to a normal or near-normal groin and the associated risk of chronic pain following surgery on a normal groin. There is a lack of evidence to allow good decision-making on this issue. The decision to proceed with routine bilateral repair mandates appropriate informed consent and a high level of surgical skill.

A number of surgeons now perform "preventive" bilateral laparoscopic hernia repair in the majority of patients with symptomatic unilateral hernias. ${ }^{327,} 339$ Others advocate routine contralateral exploration with mesh repair in those in whom a "hernia defect" is found. ${ }^{325,332,333}$ The decision to explore a potentially normal groin may be influenced by the surgeon's mind-set, his operative expertise and his complication rate. However, the medical evidence to support this decision is either lacking or weak at present. 
Most studies comparing the outcomes of unilateral versus bilateral TAPP repair, report a longer operation time (in the region of $25 \mathrm{~min}$ ), but no differences in morbidity, time to recovery, reoperation and recurrence rate. ${ }^{333,340}$ One national cohort study reported a significant difference in the rate of postoperative surgical complications occurring within 30 days (such as hematoma, seroma and wound infection) between unilateral and bilateral $\mathrm{IH}$ repair by TAPP. The postoperative complications necessitated reoperation in $0.9 \%$ of patients after unilateral, and in $1.9 \%$ of patients after bilateral, IH repair. However, this study reported that these differences in intraoperative and postoperative complications between unilateral and bilateral repair decreased in experienced high-volume hernia centers. ${ }^{297}$ Furthermore, there is no evidence that exploration of a contralateral groin and mesh placement at TAPP when no hernia is present has the same risk as that of a true hernia repair.

In TEP repair, operation time is reported to be $7-10 \mathrm{~min}$ longer for a bilateral, compared to a unilateral, repair. No difference in recurrence rate, postoperative complications, conversion rate and time to recovery were reported by several studies. ${ }^{324,327,328,330,341-343}$ One study did report a slightly increased risk of intra-abdominal complications (specific complications were not described) and surgical postoperative complications (hematoma and wound infection) in the bilateral TEP group compared to the unilateral TEP group. ${ }^{299}$ Again, it is unknown if exploration of a normal groin carries the same risk as exploration of a groin with a hernia, although two studies have reported no significant morbidity from such a practice. ${ }^{326,328}$

Discussion

Almost all the studies cited in this chapter suffer from data heterogeneity and lack of a uniform definition of an "occult hernia". Therefore, the category "occult hernia" might include those with: actual protrusion of normally intraabdominal contents, a "beginning" hernia, or even just a patent processus vaginalis without herniation. A patent processus vaginalis is observed in $12 \%$ of patients, but only $12 \%$ of these develop an indirect hernia within 5 years. This compares with $3 \%$ of patients with an obliterated processus vaginalis. ${ }^{20,} 344$

Many of the important clinical questions on the subject of a proper approach to occult hernias cannot be definitively answered by the currently available evidence. However, it is likely that up to $50 \%$ of patients who develop an $\mathrm{IH}$, will either present with clinically evident bilateral IHs, or develop a contralateral IH in their lifetime. Risk factors to identify this group of patients and to inform the decision on bilateral repair should be areas of future research. HerniaSurge recommends a trial with long-term follow-up specifically addressing the question whether there is a need for bilateral repair in patients with a one- sided symptomatic IH, perhaps identifying high-risk groups of early contralateral hernia development. Until evidence is available to further inform this dilemma, it is recommended to discuss the possible surgical options with patients before surgery as part of individualized treatment.

\section{Chapter 9}

\section{Day surgery}

W.M.J. Reinpold, H. Niebuhr and D. Lomanto

\section{Introduction}

Day surgery for IH repair has become increasingly common over the past several decades. Synonyms for "day surgery" include: outpatient surgery, ambulatory surgery, same-day surgery, day case, and short-stay surgery and indicate that patient discharge occurs the day of operation. It is commonly known that day surgery is safe and feasible for many IH repairs. ${ }^{3}$ Several studies prove that day surgery is cost effective when compared with inpatient treatment. However, it is unclear which complex IHs should not be repaired as day cases. In these Guidelines, "complex cases" include:

1. Groin hernias with signs of incarceration, strangulation, infection, relevant preoperative chronic pain, difficult local findings in the groin such as large (irreducible) scrotal hernias, (multiple) recurrence(s), a relevant history of lower abdominal surgery, radiation, and comparable problems;

2. Groin hernias in patients with relevant comorbidities: cardiovascular, respiratory, endocrine, hepatic, renal and gastrointestinal pathologies, mental disorders, anxiety, immune deficiencies, post-transplantation status, coagulopathies and anti-thrombotic medications;

3. Difficult intraoperative findings (severe adhesions, abnormal anatomy, excessive bleeding) and intraoperative complications such as damage to viscera, blood vessels, nerves and genitals;

4. Symptoms and signs of postoperative local complications: bleeding, hematoma, thromboembolism, urinary retention, bowel obstruction, peritonitis, sepsis, infection, orchitis and/or general complications (cardiovascular, respiratory, renal, hepatic, gastrointestinal, cerebral organ failure, anxiety, psychic and mental distress).

The current evidence on ambulatory surgery for $\mathrm{IH}$ repair is presented. 


\section{Key questions}

KQ09.a Which inguinal hernias can be safely repaired in day surgery?

KQ09.b Can endoscopic and open herniorrhaphies be performed safely in day surgery?

KQ09.c Can patients with severe comorbidities (ASA III or higher) be safely treated in day surgery?

KQ09.d Can patients with complex inguinal hernias (e.g. scrotal hernias) be safely treated in day surgery? readmission following day surgery for IHs. ${ }^{353}$ According to a publication of outpatient surgery including groin hernia repair in more than 564,000 United States Medicare beneficiaries older than 65 years, the 7-day mortality rate was 37 per 100,000 cases. However, there are no reports in the medical literature of death or severe complications being directly related to day surgery

Although open tension-free repair under local anesthetic seems most suitable for day surgery, published series support the use of other surgical and anesthetic techniques

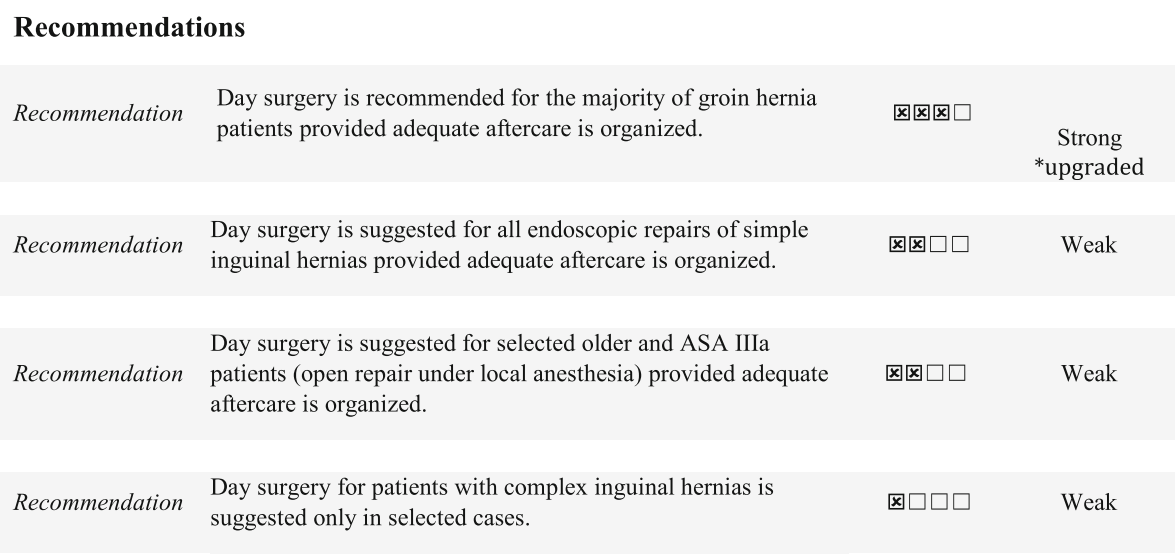

\section{Evidence in literature}

Day surgery for IH repair involves patient discharge the same day of surgery after a period of medically supervised recovery. ${ }^{345}$

The year 1955 marks the first publication on the advantages of day surgery repair of IH including: quicker mobilization, lower cost and a patient-friendly experience. ${ }^{346}$ Subsequently, several retrospective case series and three small randomized studies were published comparing inguinal herniorrhaphy day surgery with inpatient treatment. ${ }^{347-350}$ Another randomized study surveyed patient preference for site (inpatient or outpatient) of surgery. ${ }^{351}$ These studies all concluded that day surgery is cheaper than, and as safe and effective as, inpatient repair of selected IHs. Additionally, many cohort studies exist concerning various other aspects of day surgery for IHs. These studies span the outpatient surgery spectrum including: general, regional and local anesthesia; classical operative techniques; open tension-free repairs; and endoscopic techniques. All support the notion that day surgery is a safe option for many IH patients.

A 2006 Danish study of nearly 19,000 days surgery patients noted a $0.8 \%$ hospital readmission rate. ${ }^{352}$ A 2012 Danish multicenter study of over 57,700 days surgeries found a $1.1 \%$ complication rate leading to hospital in this setting. Day surgery should be considered for all simple inguinal herniorrhaphies (both open and endoscopic) provided adequate aftercare is organized. ${ }^{280,353,354}$ However, after laparoscopic repair (TAPP/TEP) and posterior open-mesh repair, severe pre-peritoneal or retroperitoneal bleeding, may occur in rare circumstances. In most cases, this infrequent complication occurs within the first $48 \mathrm{~h}$ postoperatively. Since the laparoscopic management of large hematomas is often only possible after immediate diagnosis, short-stay treatment of these patients can also be considered. There are no reports of Stoppa's open pre-peritoneal approach being performed on outpatients.

There are insufficient data to routinely recommend outpatient repair of complex IHs (see above). However, if adequate aftercare is arranged, some of these cases may be suitable for ambulatory surgery.

Operations on strangulated and acutely incarcerated hernias should not be performed as day cases.

Barring the exclusions cited above, IH day surgery can be considered for every patient with satisfactory care at home, including stable ASA III patients. ${ }^{355-359}$

Day surgery should also be considered for the elderly, including octogenarians. ${ }^{360-362}$ However, nonagenarians should be excluded since even elective IH repair in those 
over 90 has a tenfold higher mortality rate compared with younger patients. ${ }^{296}$

A recent publication based on data from 82,911 patients with IH operations documented in the German hernia registry "Herniamed" revealed that patients with prophylactic or therapeutic use of platelet aggregation inhibitors and oral anticoagulants had a significant higher risk of bleeding complications (3.9 vs $1.1 \% ; p<0.001)$ compared to those patients without such a medication. ${ }^{363}$ These data suggest that IH day surgery of patients on anticoagulants cannot generally be recommended.

A number of additional factors will either encourage or discourage day surgery. The anesthesiologist's preoperative assessment is extremely important, because he/she has primary responsibility for the perioperative- and immediately postoperative phase. ${ }^{357}$ Other hospital-, physicianand patient-related factors must be considered also. ${ }^{345}$ In a facility with considerable day surgery experience and a good infrastructure (i.e. easy availability of pre-assessment consultation and a smoothly functioning day surgery center), a large percentage of $\mathrm{IH}$ repairs may occur in day surgery. Surgical factors (quick operations and few complications) and anesthetic factors (effective pain and nausea control making rapid patient discharge possible) may influence the decision to proceed with day surgery.

Day surgery for IH repair is becoming increasingly more popular. $^{354,} 356$ In Spain in 2005, day surgery inguinal herniorrhaphies constituted $34 \%$ of the total. ${ }^{364}$ From 2000 to 2010 the rate of $\mathrm{IH}$ day surgeries in the Netherlands increased from 36 to $54 \% .^{265,296}$ Data from the Swedish National Registry indicate that $75 \%$ of IH repairs are performed in day surgery. From 2000 to 2009 the incidence of day surgery for IHs increased from 62 to $87 \%$ in the Northern Italian Veneto region. ${ }^{358}$ However, this considerable regional variation is not explained solely by the scientific evidence supporting the acceptability of day surgery IH repair. Healthcare financing and reimbursement almost certainly play a role. ${ }^{365}$

\section{Discussion}

Our present and future challenge is to provide ever more effective, less invasive, and safe ambulatory hernia surgery to a broadening array of complex, aged and sicker patients. More studies are needed on these high-risk groups to determine acceptable safety and outcome parameters. For now, the available evidence supports the idea that many patients are well served by day surgery repair of IHs.

\section{Chapter 10}

\section{Meshes}

D. Weyhe and U. Klinge

\section{General introduction}

Because of human anatomy and physiology, mesh must conform to a certain structure and stability profile. Requirements for mesh construction include: sufficient strength to reinforce the repair, the ability to stretch, elasticity, the ability to integrate into tissues without forming blocking scars, a low risk of precipitating chronic inflammation, and a low risk of bacterial adherence. Although postoperative complications may occur due to poor surgical technique or patient-specific risk factors, the risk of complications may be increased by the use of a poorly designed mesh. Mesh selection is therefore an important factor to consider if one wishes to optimize surgical outcomes.

The porosity, elasticity, strength and the polymer itself are mechanical properties, which all influence tissue reaction. A general classification, which is based on a specific property of the mesh and which is able to reflect all risks, currently is not available, and even hardly conceivable. Unfortunately, most of studies have only used the term lightweight (LWM) and heavyweight (HWM) as classification criteria; and no further details of the meshes were given in the published data. This must be considered in evaluation of results, statements and recommendations outlined below.

\section{Key question}

KQ10.a Is there a "best mesh"? What characteristics are important and can be used for classifying the mesh-related risks? 


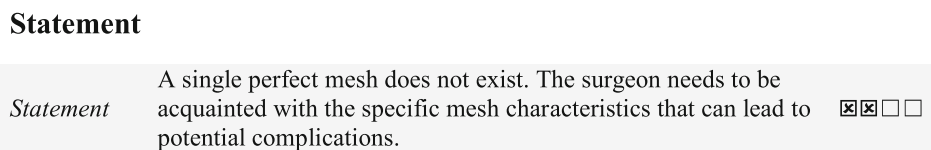

potential complications.

Pore size, effective porosity type of polymer filament structure,
and the weaving of meshes are known parameters for the
assessment of the biocompatibility of meshes.
Using the weight of the implant as a singular parameter for
mesh classification is not acceptable.

\section{Evidence in literature}

Various factors may impact mesh-related complications. ${ }^{366-379}$ These factors have been identified from human anatomy studies, studies of mesh-related failures, numerous preclinical tests in animal species, and in vitro tests.

- Material reduction can decrease mesh-related complication risk; larger pore flat meshes have a lower risk of mesh-related complications than do small-pore flat meshes.

- A tensile strength $>16 \mathrm{~N} / \mathrm{cm}^{2}$ is unnecessary for meshes used in groin hernia repair. ${ }^{373,377,380}$

- Shrinkage and stiffness of flexible meshes is affected by scar tissue. Smaller inter-filament distances and pores have an increased risk of bridging by scar tissue. $^{366,378}$

- For mechanical stress, mesh deformation lengthwise is linked to pore-size reduction. Therefore, prevention of pore collapse to avoid bridging scars requires high structural load stability of the textile construction. $^{381-386}$

- Plugs, when compared with flat meshes, have higher risks of extensive fibrosis and are more likely to stimulate an intense inflammatory reaction, thereby resulting in nonconforming biomechanical properties. $^{366,382}$

\section{Mechanical properties}

Characterization and classification of in vivo mesh materials must account for functional and biological outcomes. Modifications of polymers will result in substantially different biological responses. Any attempt to stratify meshes' impact on surgical outcomes has to consider the complex interplay between the polymer, the textile structure with fiber, the total amount of material, the porosity, the configuration of textile bindings, the implant location, and the mechanical strain placed upon the implant. None of these parameters in isolation are able to predict the inflammatory and fibrotic tissue response and classify meshes across all mesh-related complications. Due to manufacturing process, textile meshes often have considerable anisotropy with different mechanical properties when stressed vertically or horizontally. Therefore, any measurement of strength and elasticity is strongly affected by the setting of the test procedure (e.g., tensile strength tested on mesh strips or by puncture test, width of the mesh sample, or distinct directions of the mesh fibers in the test unit). As a result, the strength and elasticity of anisotropic meshes cannot be expressed as a single number. ${ }^{373,} 379$ Current data on physiological biomechanical requirements are flawed and only provide rough estimates for the mesh's mechanical characteristics. In groin hernia repair, the tensile strength of meshes does not need to be $>16 \mathrm{~N} / \mathrm{cm},{ }^{2}$ but it is unknown whether a minimum strength requirement exists. For construction of a mesh a monofilamental polypropylene composition is recommended, as multifilamental meshes tend to show a higher infection potential. ${ }^{387}$ Mesh shrinkage is seen as a consequence of the contracting scar tissue in the mesh area. Depending of the local inflammatory activity and the amount of scar, it is found in a range from $20 \%$ up to $90 \%$ in the so-called meshoma. ${ }^{388}$ Preclinical studies show that high structural stability may help to reduce mesh shrinkage. $^{381}$

Pore size and effective porosity

One mesh classification focuses on the risk for mesh infection and separates meshes with pores $<10 \mu \mathrm{m}$ (high risk for infection) from those with pores $>75 \mu \mathrm{m}$ (low risk). ${ }^{370}$ Another classification stratifies by risk for fibrotic bridging (defined as pores completely filled by scar), separating large-pore meshes $(>1 \mathrm{~mm}$, effective porosity $>0 \%)$ from small-pore meshes $(<1 \mathrm{~mm}$, effective porosity $=0 \%) .{ }^{366}$ Small-pore constructions have a higher risk for fibrotic bridging, whereas large-pore constructions have a lower risk. A pore size $>1.0 \mathrm{~mm}$ defines "largepore-size" but there is no consensus on this definition. Some guidelines use a definition for large-pore-size as $>1.5 \mathrm{~mm} .^{241}$ For the newer meshes, larger pore size is 
usually associated with reduced amount of material. Importantly, pore size measurement is not accurate if looking only at length or width in one dimension, ignoring the geometry of the pore. However, a technique does exist

\section{Key question}

KQ10.b Which mesh characteristics with impact on clinical outcome should be considered?
Statements and Recommendation
Statement
Evidence supports the contention that mesh characteristics influence clinical outcomes.
凶国要
The evidence for the effect of pore size alone on outcome in
Statement clinical trials is too low; therefore, no recommendation can be made.

to provide an accurate measurement of the critical pore sizes, which can avoid fibrotic bridging. ${ }^{371}$ Therefore, studies using only the designation "small pore" or "large pore" have inherent limitations unless they use the technique described by Mühl ${ }^{371}$ or an equivalent.

Amount of material

The weight of a mesh in $\mathrm{g} / \mathrm{m}^{2}$ has been used to classify the devices in groups of higher or lower inflammation, foreignbody reaction, risk for infection and fibrosis. ${ }^{366,370}$ Correspondingly sub-grouping of meshes by weight has been proposed. ${ }^{372,}{ }^{373}$ However, mesh weight is strongly affected by the specific density of the chosen polymer, e.g., polyvinylidene fluoride (PVDF) has a specific density of $1.77 \mathrm{~g} / \mathrm{cm}^{3}$ and is therefore considerably heavier than polypropylene $\left(0.91 \mathrm{~g} / \mathrm{cm}^{3}\right)$ or polyester $\left(1.38 \mathrm{~g} / \mathrm{cm}^{3}\right) .^{389}$ Therefore, the overall weight of meshes might vary considerably despite comparable mesh construction. ${ }^{390}$ Furthermore, the use just of weight without considering the porosity is inappropriate to be able to predict the tissue response. Meshes with very small pores induced remarkably increased inflammation despite reduced weight. ${ }^{391}$ Thus, weight alone is an inappropriate parameter for mesh classification in hernia surgery.

\section{Conclusion}

A single classification system that considers all relevant risk factors for all kind of complications, e.g. pain, infection, recurrence, or operative complications is difficult if not impossible to develop. Thus HerniaSurge recommends that surgeons be acquainted with the fact that every specific device has its specific risk pattern, which is strongly affected by the surgical procedure and the patient's biology.

\section{Evidence in literature}

Meshes in open and laparoscopic repair

Overall, 23 RCTs relate mesh material to some clinical outcomes. ${ }^{147,164,170,182,392-410}$ Eight of those 23 RCTs did not find significant differences. However, all the trials are small and are too underpowered to detect any differences of practical concern. Therefore, the lack of any significant difference does not automatically imply equality of the compared meshes with regard to the observed outcome, and thus provide no arguments against a possible impact of the mesh material for outcome. 15 RCTs confirmed an impact of the material on outcome. There is strong evidence that mesh selection can change clinical outcomes (e.g. foreign-body sensation, chronic pain, sperm motility ${ }^{411}$ and recurrence). The effect of mesh selection on risk/ benefit ratios for individual patients has yet to be defined. Large-pore size meshes

Currently, no distinction is made between large-pore-size and lightweight meshes. Research to date has focused mainly on mesh weight. Only one study compared a mesh with 3-4 $\mathrm{mm}$ to a 1-mm mesh in Lichtenstein technique. ${ }^{412}$ A significant difference with regard to chronic pain was not observed. However, preclinical studies suggest that meshes with larger pores $(>1 \mathrm{~mm})$ positively influence integration into adjacent tissue. ${ }^{381,} 413,414$

\section{Lightweight meshes}

The so-called lightweight meshes (LWM) are typically defined as mesh constructs with large-pore size and reduced weight. However, lightweight meshes with small pores are also available. ${ }^{390}$ Considering the major impact of pore size on tissue reaction, comparisons of meshes with different weight have to include only materials with similar pore sizes. There are only a small number of studies on this issue, which compare different outcomes of only large-pore 
meshes of different weight in Lichtenstein, TEP or TAPP surgery. These studies will be discussed in KQ10c.

\section{Discussion}

Though some of the clinical (RCT)studies have presented significantly different results between different treatment groups, many could not, and ended up in non-significant differences. This is often interpreted as equivalence, which is not justified.

Complications in mesh-based hernia surgery sometimes are mesh-related, but often result from failures during the surgical procedure, impaired wound healing, and/or by material-induced inflammation and scarring with subsequent functional damage. A mix of risk factors for complications is always at play. These limitations and confounders mean that statistically significant differences are achieved only in some studies focussing just on the comparison of materials. Whereas a significantly improved outcome in a comparative clinical study can serve as an all their possible confounders almost is impossible in clinical studies!

\section{Key question}

KQ10.c Are outcomes influenced by mesh weight? Do lightweight meshes have benefits in open or laparoscopic IH repair?

\section{Introduction}

There is an ongoing debate about the mesh type best suited for IH repair. So-called LWMs are supposedly associated with lower discomfort and less pain. However, they are feared to result in higher recurrence rates than so-called HWMs. ${ }^{393,}$ 415-417 The analysis presented below-with special attention to data from meta-analyses-is intended to clarify the issue.

\begin{tabular}{|c|c|c|c|}
\hline \multicolumn{4}{|c|}{ Statement and Recommendation } \\
\hline Statement & $\begin{array}{l}\text { There exists no clearly defined weight limit for LWMs and } \\
\text { HWMs. Therefore, the effect of weight differences alone on } \\
\text { surgical outcomes is unknown. }\end{array}$ & 曰曰凶冈 & \\
\hline Statement & $\begin{array}{l}\text { Use of so-called light weight meshes (LWM) compared to } \\
\text { heavy weight meshes (HWM) in open inguinal hernia surgery } \\
\text { may have some slight short-term benefit (shorter } \\
\text { convalescence). Meta analyses describe reduced pain after } 6 \\
\text { months in open surgery. For TEP/ TAPP there are no relevant } \\
\text { differences in the long term. }\end{array}$ & 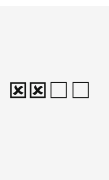 & \\
\hline Recommendation & $\begin{array}{l}\text { Before a clear definition of LWM and HWM is developed, } \\
\text { the selection of mesh based solely on the terms "lightweight" } \\
\text { or "heavyweight" is not recommended. }\end{array}$ & 冈खण口 & $\begin{array}{l}\text { Strong } \\
\text { *upgraded }\end{array}$ \\
\hline Recommendation & $\begin{array}{l}\text { HerniaSurge suggests large pore }(1-1,5 \mathrm{~mm}) \text { monofilament } \\
\text { synthetic flat meshes with a burst strength of } 16 \mathrm{~N} / \mathrm{m}^{2} \text { and } \\
\text { consisting of a minimum tensile strength in all directions } \\
\text { (including subsequent tearing force) of } 16 \mathrm{~N} / \mathrm{cm}^{2} \text {. }\end{array}$ & 冈囚 $\square$ & Weak \\
\hline Recommendation & $\begin{array}{l}\text { When considering postoperative pain after inguinal hernia } \\
\text { repair it is suggested to consider a so-called LWM although } \\
\text { probably these are only short term benefits. }\end{array}$ & 冈ख $\square \square$ & Weak \\
\hline
\end{tabular}

argument for a specific device, as obviously the impact exceeds the risk of an alpha-error; however, the non-finding of a significant difference may not serve as an argument due to the low statistical power in most studies. Cohorts of less than 1000 patients usually are related with an unacceptable risk for a beta-error (which means that the finding is not representing the truth). The absence of a significant finding therefore usually results from the limitations of the sample size rather than can be regarded as a real fact. Consecutively, to prove the similarity of two materials with

\section{Evidence in literature}

\section{Open surgery}

Some prospective randomized studies using Lichtenstein technique show advantages for LWM during the first postoperative weeks and months ( $<3$ months) with regard to pain. ${ }^{404,412,418,419}$ Two of those studies evaluated longterm chronic pain (60 months follow-up). Those studies do not find differences between LWM and HWM. Metaanalyses find fewer instances of chronic pain for LWM in the long term. ${ }^{415,} 419-421$ Those analyses are strongly 
influenced by one study with a follow-up of only 12 months. A difference between LWM and HWM in Lichtenstein technique with regard to recurrence rate was not found in those meta-analyses.

\section{TAPP surgery}

In TAPP there were no differences in perioperative or chronic pain with LWM. However, postoperative recovery following TAPP seems to improve with LWMs. ${ }^{422}$ One study with a 3-year follow-up did find a significant difference between light $\left(35 \mathrm{~g} / \mathrm{m}^{2}\right)$ and very-lightweight mesh $\left(15 \mathrm{~g} / \mathrm{m}^{2}\right)$ in TAPP repairs. A lower incidence of chronic pain with the use of extra-light mesh was shown in this study. ${ }^{423}$ However, results of this study should be viewed with caution since both meshes were LWM, and the weight difference of $20 \mathrm{~g} / \mathrm{m}^{2}$ only results in an overall difference in weight of $0.218 \mathrm{~g}$ for a $10 \mathrm{~cm} \times 15 \mathrm{~cm}$ mesh. ${ }^{390}$ This difference might be viewed as not clinically relevant.

Another study showed that, despite higher perioperative analgesia requirements with HWM, the incidence of chronic pain is similar to that seen with LWM. ${ }^{424}$ Recurrence rates following TAPP repairs are the same with LWM and HWM. $^{422}$ In conclusion, with regard to chronic pain and recurrence rates until now a relevant difference between LWM and HWM in TAPP technique has not been verified, whereas clinical convalescence seems to improve with LWM. TEP surgery

To date, 1650 patients have been studied over periods ranging from 3 to 12 months in prospective randomized trials. Some studies have found slight advantages concerning chronic groin pain and other symptoms like foreign-body feeling or

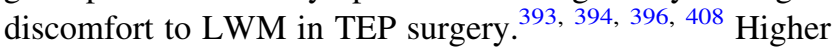
risk for recurrence rate when using LWM was not observed.

\section{Systematic reviews and meta-analysis}

Three meta-analyses reviewing various aspects of TEP or TAPP laparo-endoscopic surgery have been published. ${ }^{415-417}$ Review results varied slightly with regard to endpoints, recurrence rates, postoperative pain, chronic pain, return-to-work and seroma formation. One of the meta-analyses concluded that short- and long-term results following surgery with either LWM or HWM are comparable across all relevant endpoints. ${ }^{416}$ A second concluded that there were probably higher recurrence rates with LWM, but less groin pain and foreign-body sensation. ${ }^{417}$ The third also concluded that LWM was associated with less groin pain and foreign-body sensation, but found no increase in recurrence rate. ${ }^{415}$ All called for more studies on the topic; two suggested that studies with longer followup times be performed. The three meta-analyses differed broadly due to study selection for inclusion, heterogeneity of the selected studies, and quality assessment of the included studies. Additionally, the three meta-analyses only included RCTs published prior to 2012. Since then, two relevant RCTs have been published. A large 2015 study found no difference between LWM and HWM in the incidence of groin pain and foreign-body sensation. ${ }^{425} \mathrm{~A}$ 2012 study concluded that, compared with a HWM, an LWM provided no reduction in chronic groin pain and foreign-body sensation at 3 -year follow-up. ${ }^{391}$ There were no inter-group differences in recurrence rates.

\section{Discussion}

Regarding the many debates over different techniques and different implants, the quality of the meta-analyses on mesh is crucial for good decision-making and guidance of surgical practice. Unfortunately, most of the studies demonstrate a considerable heterogeneity of studies when defining inclusion criteria, comparing techniques and material, or outcome. As the final result often depends on the specific data of only some few studies, their inclusion or exclusion may lead to conflicting conclusions. Also, selection criteria remain quite unclear in some cases.

For example, a 2012 publication did not take into account some prospective randomized trials. ${ }^{405,422,424}$ and instead case control studies were included. ${ }^{426}$ A 2013 article included the aforementioned studies, but also included a surgeon's-choice randomized study that was mistakenly considered to be computer generated. Also, the three metaanalyses from 2012 to 2013 did not properly account for differences in fixation techniques and combined modified laparo-endoscopic techniques (TEP/TAPP) as well. Some of the included studies did not describe the mesh fixation technique used or compared different fixation methods. $396,406,408,424,427$

Given the bias in all studies HerniaSurge can only weakly suggest to use an LWM when considering postoperative pain as outcome.

\section{Mesh-foreign-body reaction}

\section{Introduction}

Mesh implantation induces a foreign-body reaction in the recipient's tissues leading to an encapsulation of the polymer fibers by a granuloma of inflammatory and fibrotic cells. Since inflammation is related to scar formation, any chronic inflammatory process results in permanent cell turnover which in turn leads to scar accumulation and constantly increasing collagen deposition. Considering the functional consequences of excessive scarring, the matter of chronic inflammation at mesh/tissue interfaces is important since it may represent a permanent risk for patients. A related issue is whether the foreign-body reaction attenuates over time. Both issues impact risk assessment for mesh implants. 
Key question

KQ10.d Does chronic inflammation occur at mesh/tissue interfaces?

\section{Erosion}

\section{Introduction}

Erosion of foreign bodies in human tissue is a well-known phenomenon. Mesh is placed in soft tissues with rapid

\section{Statement \\ Some form of chronic inflammation causing fibrosis is \\ Statement $\quad$ seen in all mesh explants and must be considered when performing mesh repair \\ खख़}

\section{Evidence in literature}

Tissue reaction to mesh has been studied in various animal models (e.g. mice, rats, rabbits, sheep and others) with a follow-up of up to 2 years in rodents and up to 3 years in sheep. All these studies confirm persistent chronic inflammation at mesh/tissue interfaces as a consequence of physiologic foreign-body reactions. Inflammatory intensity remodeling of adjacent tissues. When biomechanical strain occurs, mesh migration is often observed in the direction of the pulling forces. HerniaSurge only speaks of "erosion".

\section{Key question}

KQ10.e Is late-onset mesh erosion unavoidable?

\section{Statements and Recommendations \\ Statement There is a lifetime risk for mesh erosion which seems to be higher with plugs versus flat mesh. \\ 冈曰 $\square \square$ \\ There is a lifetime risk for mesh erosion. Mesh-related \\ $\begin{array}{ll}\text { Statement } & \text { complications - including erosion (or migration) - should be } \\ \text { considered in the differential diagnosis in patients with }\end{array}$ \\ relevant symptoms in the region of their mesh.}

varies with mesh location, animal species, mesh material, textile construction, time and individual host response.

Studies of human mesh explants were published in 2007 and 2012 with follow-ups of 3-15 years. ${ }^{366,} 428$ Most meshes were explanted due to complications, which may lead to increases in local inflammation, whereas some mesh/tissue specimens were taken as biopsies during revision procedures for other reasons. Although inflammatory intensity varied considerably, a foreign-body granuloma with macrophages and foreign-body giant cells (reflecting persistent inflammation) has always been confirmed. Since chronic inflammation stimulates local fibrosis and scar formation, long-term complications of this meshadjacent process must be considered. The risk/benefit ratio for patients is unknown presently.

\section{Evidence in literature}

Mesh erosion has been reported with all current polymers and following all hernia repair procedures. ${ }^{429-494}$ A major message of all relevant studies is the fact that 20 postoperative years may pass before symptoms of mesh migration occur.

Risk of mesh movement is reduced by the use of large flat mesh in a tension-free setting. Smaller mesh surface area and tensile forces on the mesh increases the risk. Correspondingly, for groin hernias specifically, most reports describe early (2-3 years) plug migration. Flat mesh erosion is uncommon.

There are several reports of mesh erosion after hiatal hernia and incisional hernia repair. ${ }^{495}$

Up to now, there is no polymer or no mesh construction known that is free from the risk of erosion if placed in a setting with tensile forces. 
A 2015 MRI-based study of mesh erosion at 3 months following TAPP did not detect any substantial change in mesh location. ${ }^{429}$

\section{Mesh rejection reactions}

\section{Introduction}

While it is true that hernia meshes induce immunological reactions, there is no strong evidence of adaptive immunological reaction, i.e. leading to allergic reactions. If so-called mesh "rejection" seems to be occurring, a bacterial infection should be suspected.

\section{Key question}

KQ10.f Do mesh polymers elicit rejection reactions? patients. However, this has not been reported as of this chapter's writing.

\section{Mesh degradation}

\section{Introduction}

Degradation here refers to complete or partial fragmentation (after placement in living tissue) of a non-absorbable polymer used for hernia mesh fiber construction (e.g. ePTFE, polyester, polypropylene, and polyvinylidene fluoride). Over time, most polymers do show alteration or degradation of their polymeric structure. These changes may become clinically relevant when mechanical loading occurs. It may be prudent to assume that hernia mesh implant instability can occur after several decades.

\section{Statement}

Statement There is no evidence of true immunologically-based rejection of $\quad$ 囚曰口 current synthetic mesh materials.

\section{Evidence in literature}

In the medical literature, there is no human study of the immunogenicity of hernia mesh polymers. Some animal studies do exist, only one demonstrating antibodies against

\section{Key question}

KQ10.g Does mesh degradation occur?

\section{Statement}

polyester textiles in rats. ${ }^{496}$ There are no reports of detectable B-cell or T-cell responses to mesh of any type. In light of current knowledge, there is no need to consider allergic reactions to mesh.

Notably, only a few polymers (e.g. PVDF) can be used without additives and these are supplemented with color particles. It may be that some of these adjuvants might stimulate an allergic or autoimmune syndrome in some

\section{Evidence in literature}

Under electron microscopy, human mesh explants (polyester, polypropylene or ePTFE) all show signs of degradation. ${ }^{197,}$ 428, 497-500 PVDF has the highest resistance to degradation. ${ }^{501}$ Local infection or exposure to bodily fluids and cells can accelerate mesh degradation. ${ }^{502}$

Several investigators have studied textile structure resistance during repetitive loading in vitro and have found 
rapid and irreversible deformation of the textile structure. $^{367-369}$ The clinical relevance of this finding is unknown.

\section{Mesh risk for carcinogenesis}

\section{Introduction}

If mesh implants confer an increased carcinogenic risk, this will severely affect the risk/benefit ratio of mesh-based surgery.

\section{Key question}

KQ10.h Is there a risk for carcinogenesis at meshes' interfaces?
There is inadequate evidence in humans for the carcinogenicity of non-metallic implants other than those made of silicone. ${ }^{511}$

In summary, there is no evidence that meshes meaningfully increase carcinogenesis risk. Thus, the risk for mesh-related carcinogenesis need not be considered in the risk/benefit evaluation of a mesh repair.

Patient age risks

\section{Introduction}

Patient age is often a critical consideration in many surgical procedures. Many IH surgery patients have years of productive life ahead of them. Therefore, it is important to know if patient age affects the complication risk profile.

\section{Statement}

\section{Evidence in literature}

It is clear that foreign bodies like textile mesh can induce malignancies in rodents, particularly in rats. ${ }^{503-508}$ Thankfully though, there is no evidence that hernia meshes

\section{Key question}

KQ10.i Is there an age-associated risk for mesh-related complications?

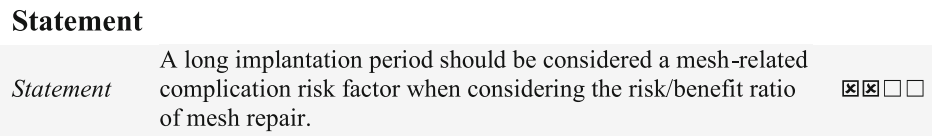

measurably increase the malignant transformation rate in humans.

There are, however, two reports worthy of mention. In one, abdominal wall fibromatosis developed in two patients after laparoscopic mesh placement. ${ }^{509}$ In another, aggressive squamous cell cancer occurred at the site of chronic mesh infection, and this may be regarded as mesh-induced cell proliferation/malignancy. 510

In 2000, The International Agency for Research on Cancer stated that "Polymeric implants prepared as thin smooth films (with the exception of poly-glycolic acid)) are possibly carcinogenic to humans (Group 2B)". 511

\section{Evidence in literature}

There are no adequately age-adjusted studies of complications following mesh-based $\mathrm{IH}$ repair. Also, no data exist on length of implantation period as an independent risk factor for complications.

There are, however, registry data indicating that increased patient age (especially $>65$ years) is a risk factor for complications. ${ }^{78}$

Several studies indicate that complications following mesh repair can occur after years. Mesh explantation, for example, usually occurs 2-3 years after implantation. $^{366,512}$ 
National registry data analyses usually show a nearly linear increase in reoperation rate, reflecting a permanent risk with an almost constant incidence over time. ${ }^{78,513,514}$ It is therefore, reasonable and logical to think that lifetime risk of complications will be increased for younger patients, though there are no data confirming this. A long implantation period should be considered a mesh-related complication risk factor when considering the risk/benefit ratio of mesh repair.

\section{Mesh shrinkage}

\section{Introduction}

Shrinkage of the mesh-caused mainly by collagen shortening-results in physiological wound contraction. This phenomenon, in turn, is related to scar tissue amount, influenced by surgery-induced local tissue trauma and patient-specific responses to tissue injury.

\section{Key question}

KQ10.j Does mesh shrinkage occur, and if so, to what extent?
Of note, mesh polymers themselves do not shrink, but the textile itself shortens, pulled together by the contracting scar. $^{366}$ Thick and stiff filaments in a rigid textile implant resist shrinkage more than large-pore meshes and offer flexibility adapted to surrounding tissues. ${ }^{381}$

Mesh shrinkage varies markedly. For plugs, a volume reduction due to shrinkage of up to $90 \%$ has been reported with the formation of a so-called meshoma. ${ }^{515}$ It is reasonable that mesh area shrinkage of greater than $50 \%$ increases postoperative risks and should be avoided if possible by minimizing surgical trauma and/or foreignbody reaction. $^{388,515-529}$

Studies of MRI-visible large-pore PVDF mesh report an up to $20 \%$ reduction in mesh surface area indicating a shortening across length and width of about $10 \% .{ }^{530}$ These studies confirm the results of a 2011 trial performed with digital computed radiographs and metal clips at a Lichtenstein mesh border. ${ }^{518}$

However, today these changes are small in relation to the accuracy of the CT/MRI measurements, and thus need to be confirmed by further studies.

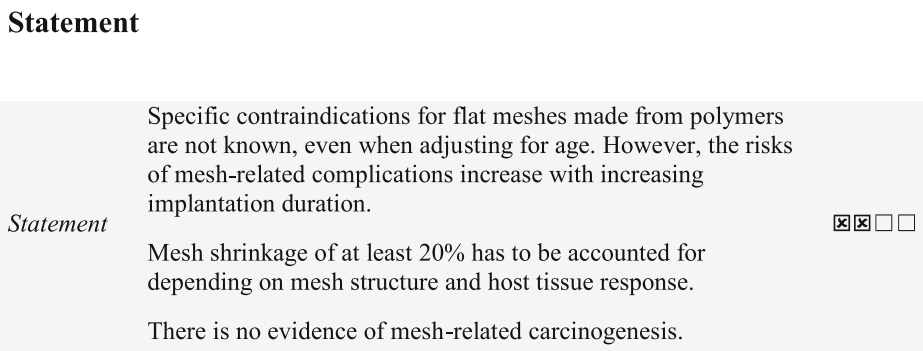

There is no evidence of mesh-related carcinogenesis.

\section{Evidence in literature}

It is known that certain patients develop enhanced scar formation and/or marked wound contraction, whereas others do not. It is also known that textile meshes induce a chronic foreign-body reaction with local inflammation and fibrosis (see KQ10e). In the case of small-pore meshes this reaction can bridge the entire inter-filament distance. ${ }^{366,382}$ Thus, small-pore meshes develop increased shrinkage in the area of surgical trauma. Mesh infection, with its resultant inflammation and increased fibrosis, exacerbates this process and results in even more shrinkage.

\section{Chapter 11}

\section{Mesh fixation}

R. H. Fortelny, D. L. Sanders and A. Montgomery

\section{Introduction}

Synthetic mesh fixation in both open and laparo-endoscopic hernia repair involves a consideration of the strength of fixation versus the risk of trauma to local tissues and 
nerve damage through entrapment. Mesh fixation complications include: mesh migration, adhesions, erosion and hernia recurrence, ${ }^{531-535}$ "meshoma" formation, ${ }^{536}$ tack hernias, ${ }^{537}$ chronic pain, ${ }^{538-543}$ and infection. ${ }^{544,}{ }^{545} \mathrm{~A}$ number of RCTs-also summarized in meta-analyseshave compared different mesh fixation methods in both open and laparo-endoscopic IH repair. Various mesh fixation methods exist including: tacks, staples, self-fixing, fibrin sealants (FSs), glues and sutures. However, consensus does not exist about a "best" fixation method, so methods used are based on surgeons' preferences. Evidence that a particular fixation method improves patientbased or surgical outcome measures may have a significant impact on clinical practice. Analyses below covers two topics: fixation in open hernia repair and fixation in laparoendoscopic hernia repair. Special patient-related circumstances are also highlighted.

\section{Open inguinal/femoral primary hernia repair}

\section{Key question}

\section{KQ11.a}

Which fixation methods are appropriate in primary open anterior mesh inguinal and femoral hernia repairs? fibrin sealant to sutures, one compared tacks to sutures, and one compared absorbable sutures to non-absorbable sutures. Per GRADE guidelines, none of the RCTs were rated as high quality. The most common reasons for low or very low study grading were: lack of power calculations, small subject numbers, short follow-up periods, and poorly matched groups (for age, hernia size and comorbidities).

\section{Recurrence}

Thirteen of 26 recurrences were reported in one study with a 5-year follow-up utilizing NB2C glue. ${ }^{552}$ There were no significantly different recurrence rates found between fixation methods in any of the RCTs, although long-term recurrence rates have not been determined and large hernias often have been excluded.

\section{Infection rates}

Surgical site infection (SSI) data were included in eight of the studies. No study distinguished between superficial and deep SSI. SSI diagnostic criteria were infrequently documented. Overall infection rates ranged from 0 to $3.5 \%$; and infection resulted in three mesh explantations. Choice of fixation method did not result in any significant difference in infection rates.

\section{Statement and Recommendation

In open anterior mesh groin hernia repairs there are no
differences in recurrence or surgical site infection rates
between different fixation methods. Fixation with glue (fibrin $\quad$ खtatement
sealant or cyanoacrylate) may reduce early postoperative and
chronic pain.

\section{Evidence in literature}

The search yielded eight systematic reviews on the subject of mesh fixation in primary open IH repair. $^{173,175,177,546-551}$ Seven of these reviews assessed IH repair using an anterior mesh repair while one assessed both open anterior and laparoscopic repairs.

\section{Systematic reviews on fixation methods}

Mesh fixation methods were assessed in one moderatequality systematic review of 12 RCTs involving 1992 primary IH repairs. ${ }^{551}$ Data heterogeneity precluded performance of a meta-analysis. Four studies compared $n$ butyl-2 cyanoacrylate (NB2C) glues to sutures, two compared self-fixing meshes to sutures, four compared

\section{Chronic pain}

All studies included chronic pain data. Most defined chronic pain as pain persisting beyond 3 months a range of definitions was though used (range 3-12 months). One study did not include a chronic pain definition. ${ }^{553}$ Five studies measured chronic pain incidence at 3 months, ${ }^{552,}{ }^{554-557}$ two only at 6 months, ${ }^{168,409}$ and three only at 1 year. ${ }^{171,558,559}$ One study used a composite endpoint of pain, numbness, and groin discomfort at 1 year (at 6 months if 1-year data were not available).

Overall, chronic pain rates ranged from 0 to $36.3 \%$. The combined chronic pain rates for mesh fixation of various types were: $14.7 \%$ for sutures, $7.6 \%$ for NB2C glue, $3.7 \%$ for FS, and $18.2 \%$ for self-fixing meshes. 
Nine studies reported no significant difference in chronic pain between fixation methods. Three identified a significant reduction with $\mathrm{NB} 2 \mathrm{C}$ glue ${ }^{556}$ or $\mathrm{FS}^{409}, 560$ compared with sutures. One RCT of moderate quality randomized 316 patients to either Tisseel $^{\circledR} /$ Tissucol $^{\circledR}$ or $2 / 0$ Prolene ${ }^{\circledR}$ sutures and reported a significant reduction in chronic pain at 6 months (defined as VAS > 3) with FS versus sutures $(8.1$ vs. $14.8 \%, p=0.035) .{ }^{560}$ A very lowquality RCT of 148 patients randomized to either Quixil ${ }^{\circledR}$ FS fixation of lightweight mesh or Vicryl ${ }^{\circledR}$ suture fixation of a heavyweight mesh found chronic pain at 6-month follow-up (determined by mean VAS scores) was lower in the FS/lightweight mesh group (0 vs. $7.8 \%, p<0.001){ }^{409}$ Two further RCTs, the first comparing suture fixation with self-gripping ProGrip mesh and the second comparing suture fixation with fibrin glue reported no difference in chronic pain. ${ }^{182,561}$

Pain within the first week postoperatively

Six RCTs reported on pain in the first postoperative week. Three studies noted significantly lower mean VAS scores at one or more assessment times within week one, with FS, ${ }^{409}$ NB2C glue, ${ }^{55}$ or self-fixing mesh ${ }^{168}$ compared with suture fixation. Two RCTs reported no significant difference in mean VAS scores between fixation methods. ${ }^{557,560} \mathrm{~A}$ significant reduction in postoperative pain within the first $24 \mathrm{~h}$ was observed with non-suture compared with suture fixation in three RCTs. The mean difference in VAS scores was $0.80(p<0.001)$ with $\mathrm{FS},{ }^{409} 1.44(p=0.031)$ with self-fixing mesh, ${ }^{168}$ and $0.90(p=0.003)$ with NB2C glue. ${ }^{55}$ Notably, all these RCTs were graded as very low quality because of small patient numbers or confounding variables. Furthermore, only one of these studies (FS versus suture fixation) showed a sustained difference in pain scores 1 week postoperatively. ${ }^{409}$ A subsequent moderatequality RCTs comparing fibrin glue fixation with suture fixation in 102 randomized patients, reported lower postoperative pain measured on a VAS of 1-10 with fibrin glue fixation at 1 week (VAS 0.28 lower than with suture fixation, $p<0.05$ ) and at 1 month (VAS 0.26 lower than with suture fixation, $p<0.05) .{ }^{561} \mathrm{~A}$ high-quality RCT including 557 men randomized to open anterior repair with suture fixation versus self-gripping ProGrip mesh reported that early postoperative pain scores were lower with self-gripping mesh than with sutured lightweight mesh: mean VAS $(0-150)$ pain score relative to baseline +1.3 and +8.6 , respectively, at discharge $(p=0.033)$, and mean surgical pain scale score relative to baseline +4.2 and +9.7 respectively on day $7(p=0.027)$. Although the results of these studies reveal statistically significant reduced pain after atraumatic fixation, the clinical significance of small changes in VAS scores in unclear. ${ }^{182}$

\section{Operative time}

Operative times were reported in 10 RCTs. Five reported significantly shorter operative times with non-suture mesh fixation. Two of these studies compared self-fixing meshes with suture fixation and reported $9-\min (p=0.01)^{171}$ and 12 -min $(p=0.008)^{168}$ reductions in mean operative times. Similarly, reduced mean operative times of $6 \mathrm{~min}$ were reported in two studies comparing NB2C glue with suture fixation. ${ }^{552,556}$ A reduced mean operative time of $18 \mathrm{~min}$ $(p<0.001)$ was reported in one study comparing FS with suture fixation. ${ }^{409}$ Three meta-analyses, all published in 2013 - two of moderate. ${ }^{546,547}$ and one of low quality 548 _have examined glue versus suture fixation in open anterior mesh $\mathrm{IH}$ repair. Despite methodological differences, all three meta-analyses reported an approximate 2-3 min shorter operative time with glue compared with sutures. The clinical significance of this small difference is debatable. One of the meta-analyses reported no difference in other outcomes including chronic pain (RR 1.60; $95 \% \mathrm{CI}$ $0.78,3.28 ; z=1.28 ; p=0.20)$, while the other two reported reduced postoperative pain (RR $0.46,95 \%$ CI $0.22-0.97 ; p=0.01$ ) and chronic pain (RR $0.51,95 \% \mathrm{CI}$ $0.31-0.87 ; p=0.01)$. These differences are remarkable, given that the articles were all published within the same year, and may reflect selection criteria for included studies and the meta-analysis methods used.

Three additional meta-analyses, all published in 2013/2014, and all of low quality, have examined selffixing meshes compared with suture fixation in open anterior mesh $\mathrm{IH}$ repair. ${ }^{173,175,177}$ All reviewed data from the same primary studies of 1353 patients. No inter-group differences in recurrence, chronic pain or SSI were found. However, shorter operative times (range of 1-9 min) were noted with self-fixing mesh.

Laparo-endoscopic inguinal/femoral primary hernia repair

\section{Key questions}

KQ11.b Is mesh fixation necessary in endoscopic TEP inguinal/femoral hernia repair in adults?

KQ11.c Are there specific indications for mesh fixation in endoscopic TEP inguinal/femoral hernia repair in adults?

KQ11.d Is mesh fixation ever recommended in laparoscopic TAPP inguinal/femoral hernia repair in adults?

KQ11.e If using mesh fixation, what types should be used in TEP and TAPP inguinal/femoral hernia repairs? 


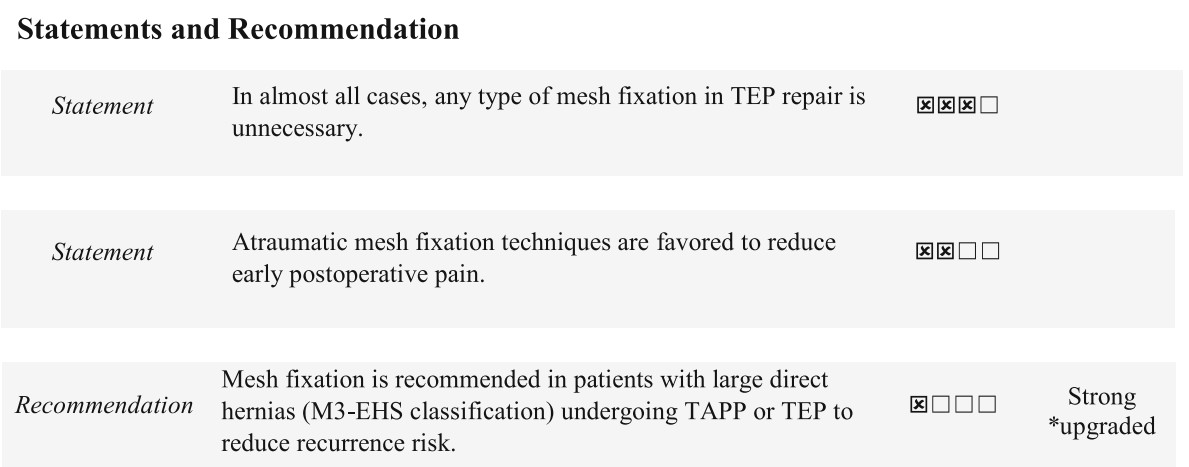

\section{Evidence in literature}

Pubmed and Cochrane databases were systematically searched, yielding a total of 67 papers of which 34 were included after applying strict inclusion (SIGN) criteria. Following the GRADE approach for Guidelines the reviews by Schäfer et al., ${ }^{562}$ Morales-Conde. ${ }^{563}$ and Fortelny. ${ }^{549}$ were excluded. Of the 34 included papers, five are systematic reviews/meta-analyses, ${ }^{550,}{ }^{564-567} 17$ are RCTs ${ }^{568-584}$ and 12 are case control studies (CCS). ${ }^{581-594}$

\section{Fixation versus non-fixation in TEP and TAPP}

The systematic review and meta-analyses ${ }^{565-567}$-all judged to be of moderate quality per GRADE guidelinesrevealed no significant differences in the rates of recurrence or postoperative pain between permanent tack fixation and non-fixation in either TEP or TAPP.

\section{Recurrence}

For TEP repair, the results of six RCTs, ${ }^{573,575,579,581,583,584}$ three case control studies, ${ }^{573,593,594}$ and two meta-analyses ${ }^{566,567}$ demonstrate no significant risk of recurrence following mesh non-fixation.

For TAPP repair, one RCT of moderate quality, comparing tack fixation with non-fixation demonstrated no significant difference in recurrence risk.

Notably, the RCTs cited above contain only limited information on hernia-defect size and type. This is especially true regarding the percentage of large direct hernias (type M3, EHS classification).

Based on the results of a multivariate analysis of 11,230 cases from a Herniamed registry study, ${ }^{595}$ a significant risk of recurrence is found not only in the group of non-fixation in case of direct hernias but also for combined hernias [combined versus medial: OR $1.137 \quad(95 \%$ CI 0.656-1.970); lateral versus medial: OR 0.463 (95\% CI $0.303-0.707) ; p<0.001]$

\section{Acute and chronic pain}

The three meta-analyses ${ }^{565-567}$ of eight RCTs revealed no significant differences in acute and chronic postoperative pain ${ }^{566,567,} 573$ Of the RCTs studying TEP repair $^{573,575,579,581,583}$ only one ${ }^{575}$ detected significantly less acute and chronic pain in the non-fixation group. The sole RCT on TAPP repair $^{582}$ showed no significant difference for chronic pain in the non-fixation group. Of three case control TEP repair studies, ${ }^{593,594,596}$ only one ${ }^{596}$ revealed a significantly lower rate of acute postoperative pain in the non-fixation group.

Reporting on preoperative pain is one of the greatest shortcomings of almost all studies. This information is essential to identify patients at high risk for postoperative chronic pain. Furthermore, the pain assessment within the different studies displays significant heterogeneity.

The Swedish Hernia Register study on the impact of mesh fixation on chronic pain in TEP in primary IH repair in men enrolled 1110 patients. It compared permanent fixation (PF) with no fixation (NF) or non-permanent fixation $(\mathrm{NPF})^{597}$ and revealed no difference regarding the primary endpoint of pain $(p<0.462)$ using Inguinal Pain Questionnaire and SF-36 subscales as well as no difference between PF- and NF-groups including subgroups of medial hernias during a 7.5-year follow-up.

\section{Operative time}

In several meta-analyses, including data from both TEPand TAPP-RCTs, no significant differences in operative times have been reported. ${ }^{297,}$ 565, 566, 573, 575, 579, 581, 583 A separate meta-analysis including three TEP$\mathrm{RCTs}^{579,581,593}$ revealed a significant reduction in operative time when mesh non-fixation was used.

Surgical site infection

Two RCTs ${ }^{581,582}$ and one CCS ${ }^{594}$ on SSI demonstrated no difference between fixation and non-fixation groups.

\section{Permanent versus non-permanent fixation (staple/tack vs glue) in TEP repair}

\section{Recurrence}

Two meta-analyses of moderate quality ${ }^{550,564}$ found no significant recurrence rate difference between staple and glue fixation methods. The results of three RCTs ${ }^{568,572,580}$ 
included in the meta-analyses, ${ }^{550}$ as well as another four $\mathrm{CCSs}^{585,591,592,598}$ confirmed these findings.

Acute and chronic pain

One systematic review ${ }^{550}$ analyzed only RCTs including TAPP repairs ${ }^{574,577,578}$ and one TEP repair ${ }^{580}$ Concerning acute pain, the review analysis detected no significant difference between staple and fibrin sealant groups. A significant difference was found, however, in the incidence of chronic pain favoring the fibrin sealant group. Another review ${ }^{564}$ included one $\mathrm{RCT}^{580}$ and three CCSs ${ }^{591,592,598}$ and reported on chronic pain incidence only. Both reviews 550,564 revealed significant advantages of glue fixation in lessening the incidence of chronic pain. However, as noted, only one $\mathrm{RCT}^{580}$ was included in these two systematic reviews. In total, three RCTs have been published ${ }^{568,572,580}$ and detected no significant difference in chronic pain when glue was compared to staple fixation. Three case control trials, ${ }^{591,592,598}$ however, found significantly less chronic pain in the glue fixation group.

Operative time

Two systematic reviews ${ }^{550,564}$ failed to demonstrate an operative time difference between groups undergoing different fixation methods. Similarly, one $\mathrm{RCT}^{580}$ and one case control trial ${ }^{598}$ also noted no significant difference although a different case control trial ${ }^{585}$ revealed longer operative times in the glue group.

\section{Surgical site infection}

SSI rates were not significantly impacted by different fixation methods across a systematic review, ${ }^{564}$ two $\mathrm{RCTs}^{568,580}$ and two case control trials ${ }^{591,592}$ that examined the subject.

\section{Permanent versus non-permanent fixation (staple/tack vs glue) in TAPP repair}

\section{Recurrence}

One meta-analysis of moderate quality that included only $\mathrm{RCTs}^{574,}$ 576-578 specifically addressed glue versus staple fixation in TAPP repair. ${ }^{550}$ and reported no significant intergroup difference. The results of six RCTs ${ }^{569,571,574,576-578}$ and three case control trials ${ }^{586,588,589}$ confirmed this finding. In addition to the meta-analyses and RCTs, a recently published study from the Danish Hernia Database included 1535 patients and detected no significant difference using Cox regression analysis [hazard ratio $0.8 ; 95 \% \mathrm{CI}(0.5-1.2)]^{599}$ in long-term reoperation rates and clinical recurrences (median follow-up time of 31 months) in patients undergoing TAPP IH repair with mesh fixation by fibrin sealant compared to tacks.

Acute and chronic pain

One systematic review $^{550}$ that included four $\mathrm{RCTs}^{574,}{ }^{576-578}$ found no significant difference in acute postoperative pain between glue- and staple-fixation

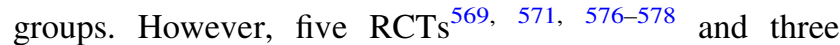
$\mathrm{CCSs}^{586,588,589}$ found significantly less acute pain after glue versus staple fixation.

One systematic review ${ }^{550}$ revealed a significantly higher incidence of chronic pain when the staple group was compared with the glue group. In contrast, three of six $\mathrm{RCTs}^{571,574,576}$ and two of three case control trials ${ }^{588,589}$ reported no significant difference. An important criticism of the systematic review ${ }^{550}$ was that it included 1-month followup data from one study as chronic pain data. Another study ${ }^{574}$ showing no difference was excluded for unknown reasons.

\section{Operative time}

No significant difference was seen between fixation methods in the systematic review. ${ }^{550}$

\section{Surgical site infection}

Two RCTs ${ }^{571,578}$ and two CCSs ${ }^{586,589}$ reported on surgical site infection and no significant difference in SSI risk was detected between fixation methods.

\section{Self-fixing mesh in TAPP}

One moderate-quality RCT compared self-fixing mesh to glue fixation in TAPP repair. ${ }^{570}$ Short-term follow-up at 3 months found no hernia recurrences and no significant differences in postoperative pain between groups. A CCS had similar results. ${ }^{587}$

\section{Discussion}

In open primary groin hernia repair beyond the use of sutures (non- or late-resorbable) for mesh fixation new atraumatic devices (e.g. fibrin glue, cyanoacrylate, self-fixating meshes) are safe in terms of recurrence (1 year) and reduce the risk of acute postoperative pain (weak suggestion). Self-gripping mesh is an acceptable form of treatment for primary IHs, although only medium-term data are available and no specific information on the outcome in larger (direct) hernias. It has no benefits over the Lichtenstein technique other than a somewhat shorter operative time. The device's additional cost must be considered (Chapter 6c). Glue fixation in the Lichtenstein technique can be performed in hernias limited to MII or LII types (EHS classification) according to HerniaSurge Group consensus.

In TEP and TAPP inguinal/femoral hernia repair nonfixation of mesh is recommended in almost all hernia types except large medial defects (M3 EHS classification) where mesh fixation is recommended. The fixation of large medial defects in TEP/TAPP is expert opinion and consensus within the HerniaSurge Group. A crucial precondition in large medial defects is the use of an adequate size and overlap of mesh and the reduction of the dead space caused by the dilated transverse facia. To minimize the risk of acute postoperative pain atraumatic fixation techniques (fibrin glue, cyanoacrylate) should be considered. 


\section{Chapter 12}

\section{Antibiotic prophylaxis}

\author{
A. Montgomery, Th. J. Aufenacker and J. Bingener
}

\section{Introduction}

Prophylactic antibiotics in inguinal herniorrhaphies are intended to prevent infections, which is particularly
KQ12.b Are prophylactic antibiotics indicated in open mesh repair in a high-risk patient in a low-risk environment?

KQ12.c Are prophylactic antibiotics indicated in open mesh repair in any patient in a high-risk environment?

KQ12.d Are prophylactic antibiotics indicated in laparoscopic repair in any patient in any risk environment?

$\begin{aligned} & \text { Statement and Recommendations } \\ & \text { There is an increased risk of wound infections in patients } \\ & \text { undergoing bilateral open hernia repairs and recurrent hernia } \\ & \text { repairs }\end{aligned}$
$\begin{array}{ll}\text { In open mesh repair, administration of antibiotic prophylaxis } \\ \text { in average-risk patients in a low-risk environment is not } \\ \text { recommended. }\end{array}$
$\begin{array}{ll}\text { Administration of antibiotic prophylaxis in open mesh repair } \\ \text { in high-risk patients in a low-risk environment is suggested. }\end{array}$

important when prosthetic material is used. However, unwarranted antibiotic use may create problems, notably patient allergies, $C$. difficile infection, bacterial resistance and increased costs, amongst others. Antibiotic use is widely accepted in patients with risk factors and in contaminated and infected conditions. However, prophylactic antibiotic use should be questioned under clean conditions in patients with limited risk factors for infection. Current evidence is presented.

\section{Key questions}

KQ12.a Are prophylactic antibiotics indicated in open mesh repair in an average-risk patient in a low-risk environment?

\section{Evidence in literature}

The latest Cochrane meta-analysis, encompassing 11 RCTs, was published in 2012. ${ }^{600}$ Additional relevant and crucial data were abstracted from papers published in 2013 and 2014. ${ }^{4,601,602}$ In total, 17 RCTs involving 5709 patients were included to formulate the recommendations. Eight of the articles included in this analysis are of high or moderate quality while the rest are of low or very low quality.

Difficulties in data interpretation stem from the fact that inclusion criteria vary broadly across the RCTs. This variation encompasses patient risk factors (e.g. immunosuppression, diabetes, heart failure), hernia characteristics (e.g. primary, bilateral, recurrent), and operative or postoperative interventions (e.g. wound infection incidence, hair shaving, drain use, seroma puncture). The current 
analysis accounts for this variation and defines averagerisk patients as those with primary hernias and minimal individual or operative risk factors. Of note, only elective operations are included in the 17 RCTs. High-risk patients-with comorbidities like diabetes-are only referenced in two of the 17 articles, representing $8.3 \%$ of all patients. $^{603,604}$

There is a potential risk of resistance to the prophylactic antibiotic given varying between countries and different settings. This problem is not highlighted in any study.

The wound infection rates in the placebo groups varied widely, from 0 to $18 \%$, likely reflecting the basal wound infection rates in the study population. High wound infection rates were noted in studies from Pakistan, Turkey, Japan and parts of India and Spain, and may reflect local differences in perioperative and operative practice.

Highly regarded guidelines and expert opinions hold that a less than $5 \%$ wound infection rate in the placebo group defines a low-risk environment. This cut-off has been used for this analysis. ${ }^{4,601}$ Accordingly, the 17 RCTs have been divided into those involving low- and high-risk environments and analyzed for potential benefit of antibiotic prophylaxis. A total of seven studies with 2838 patients comprise the low-risk environment group and ten studies with 2871 patients make up the high-risk environment group.

The overall meta-analysis results of the RCTs have to be corrected for a large clinical diversity (inclusion criteria variations regarding diabetes and recurrent hernia) and methodological diversity (surgical variations: drain use, average surgical time, seroma aspiration, timing of shaving) by using the random effect model.

Wound infections occurred in 2.3\% (33/1444) of the low-risk environment placebo group and 1.6\% (23/1394) of the prophylaxis group, confirming a lack of evidence for prophylactic antibiotic benefit in the low-risk environment group (OR 0.72; 95\% CI 0.42-1.24; NNT 158) (Fig. 3). Nine $(0.3 \%)$ surgical site infections occurred, with no difference between placebo and prophylaxis groups.

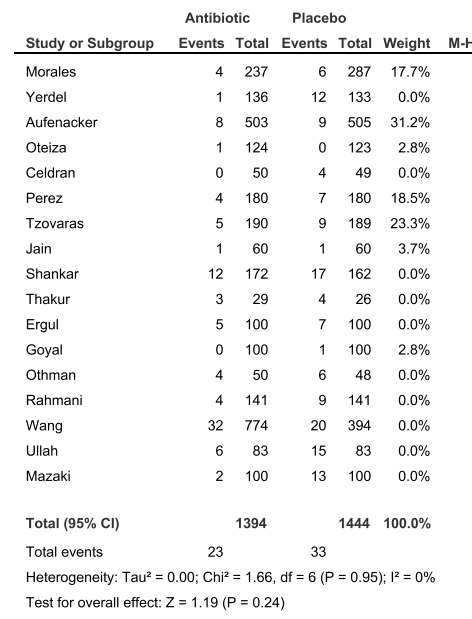

Odds Ratio

, Random, $95 \% \mathrm{Cl}$ Year $0.80[0.22,2.88] 2000$ $0.07[0.01,0.58] 2001$ $0.89[0.34,2.33] 2004$ $3.00[0.12,74.36] 2004$ $0.10[0.01,1.91] 2004$
$0.56[0.16)$ $0.10[0.01,1.91] 2004$
$0.56[0.16,1.95] 2005$ $0.56[0.16,1.95] 2005$ $1.50[0.18,1.04] 2007$ $0.64[0.30,1.38] 2010$ $0.63[0.13,3.15] 2010$ $0.70[0.21,2.28] 2011$ $0.33[0.01,8.20] 2011$ $0.61[0.16,2.31] 2011$ $0.43[0.13,1.42] 2012$ $0.81[0.46,1.43] 2013$ $0.35[0.13,0.96] 2013$ $0.14[0.03,0.62] 2014$ $0.72[0.42,1.24]$ Heterogeneity: $\mathrm{Tau}^{2}=0.00 ; \mathrm{Ch}^{2}=1.66, \mathrm{df}=6(\mathrm{~T})$
Test for overall effect: $Z=1.19(\mathrm{P}=0.24)$
Odds Ratio

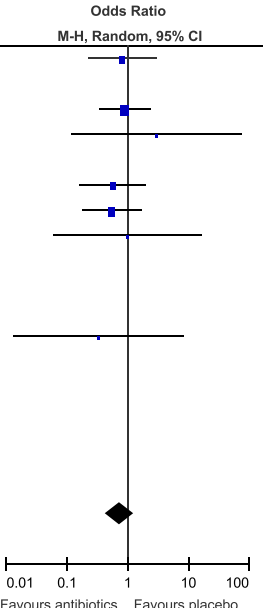

Figure 3. Pooled data of 7 studies in low-risk environments ( $<5 \%$ wound infection) on the use of antibiotic prophylaxis in the prevention of wound infection after mesh inguinal repair. 
Wound infection rates in the high-risk environment group were $8.7 \%(107 / 1236)$ in the placebo group and $4.2 \%(69 / 1635)$ in the prophylactic antibiotics group showing a clear benefit of antibiotic prophylaxis in this setting (OR 0.49; 95\% CI 0.33-0.74, NNT 24) (Fig. 4). Fourteen $(0.45 \%)$ patients developed deep surgical site infections with no difference between placebo and antibiotic prophylaxis. repair, use of drains, timing of shaving, seroma aspiration, long operative time and bilateral repairs. Due to the low incidence of infection, the number needed to treat was 323 to prevent one infection. Therefore, the clinical relevance of this conclusion can be argued.

There is only one small, low-quality RCT demonstrating no wound infections in any group in laparo-endoscopic IH repairs. Data from large patient cohorts in national registers

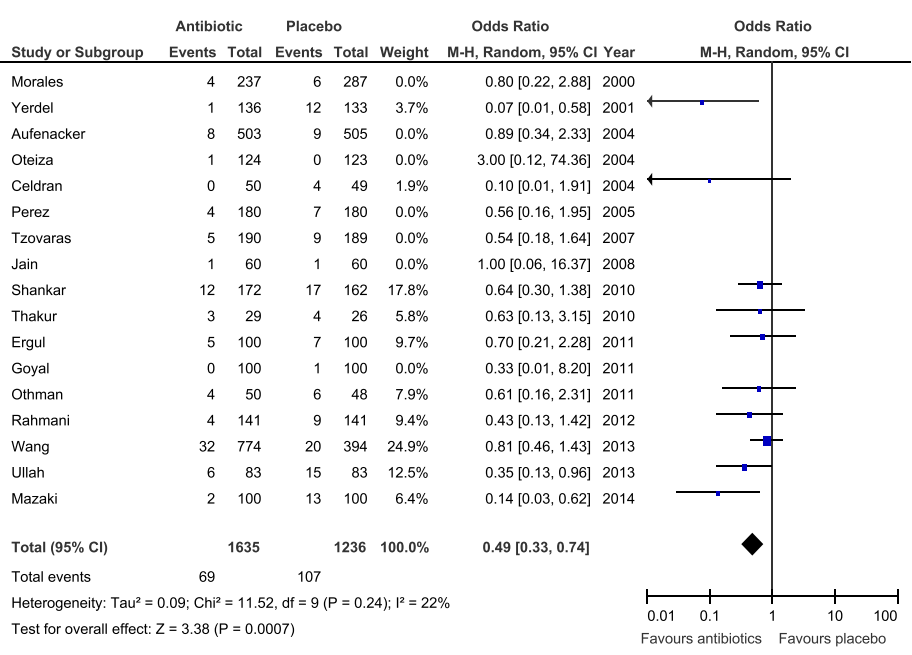

Figure 4. Pooled data of 10 studies on the use of antibiotic prophylaxis in the prevention of wound infection in centers with a high incidence $(>5 \%)$ of wound infection after mesh inguinal repair

The 2014 annual report of the Swedish Inguinal Hernia Register, which covers $95 \%$ of all hernia operations, revealed that $5.6 \%$ out of the 14,053 patients operated upon received antibiotic prophylaxis. Primarily high-risk patients as defined by national guidelines received antibiotics. Postoperative infection rates were reported as $1.2 \%$ in males and $1.5 \%$ in females. ${ }^{605}$

Germany's national register "HerniaMed" reported on the use of antibiotic prophylaxis. ${ }^{606}$ enrolling 85,000 patients (57\% laparo-endoscopic operations). Antibiotic prophylaxis was administered in $70 \%$ of patients and infection was seen in $0.2 \%$ in the laparo-endoscopic group and $0.6 \%$ in the open surgery group. In a multivariate analysis on wound healing the OR was 0.318 (CI 0.23-0.44) comparing laparo-endoscopic to open operation. It is concluded that endoscopic repair per se has such a high benefit in reducing wound infections, that the administration of antibiotic prophylaxis is not necessary. For open repair it was concluded that there was a benefit for antibiotic prophylaxis, but this summary statement did not account for factors like: reason for open or endoscopic do not support the use of antibiotic prophylaxis in these patients. ${ }^{606,607}$

\section{Special circumstances for antibiotic use}

There are very limited data on high-risk patients in a lowrisk environment. Two small studies address this issue but only include a few patients who might be considered to have any increased risk for postoperative surgical site infection. A consensus does not exist on what constitutes a high-risk patient in a low-risk environment for hernia surgery. However, common surgical practice includes antibiotic prophylaxis for increased-risk patients and these currently also include those undergoing IH repair. This is an area ripe for further studies.

Univariate and multivariate analysis of individual trials reveals an increased risk of wound infections in patients undergoing bilateral open hernia repairs and recurrent hernia repairs. This is likely due to increased operative time. There are insufficient data to draw conclusions on antibiotic prophylaxis for high-risk patients with diabetes or immunosuppression. 
In a high-risk environment (defined by a $>5 \%$ incidence of wound infection) there is a significant benefit of antibiotic prophylaxis. Therefore, in institutions with high wound infection rates, antibiotic prophylaxis is highly recommended. Furthermore, in these institutions the general risk factors influencing wound infections should be checked (like hygiene routines, shaving on the day before surgery and seroma aspiration, etc.). ${ }^{608}$

It is a fact that in some countries prophylactic antibiotics are a required indicator and considered a quality measure by Centers for Medicare and Medicaid services. HerniaSurge recommends these countries to reconsider this and adjust requirements to evidence-based guidelines. performed via epidural, spinal and paravertebral routes. However, a discussion of paravertebral anesthesia is not included in this section since limited data are available on this technique.

The ideal anesthetic technique: provides good perioperative and postoperative analgesia, produces optimal operating conditions by immobility, is associated with few complications, facilitates early patient discharge, and is cost effective. The EHS guidelines on IH treatment recommends that local anesthesia be considered for all adult patients with primary reducible unilateral IHs.

\begin{tabular}{|c|c|c|c|}
\hline \multicolumn{4}{|c|}{ Statements and Recommendations } \\
\hline Statement & $\begin{array}{l}\text { When compared with general anesthesia, local anesthesia is associated } \\
\text { with faster mobilization, earlier hospital discharge, lower hospital and } \\
\text { total healthcare costs, and fewer complications such as urinary } \\
\text { retention and early postoperative pain. However, when surgeons } \\
\text { inexperienced in its use administer local anesthesia, more hernia } \\
\text { recurrences might result. }\end{array}$ & 凶冈凶冈 & \\
\hline Statement & $\begin{array}{l}\text { When compared with regional anesthesia, local anesthesia is associated } \\
\text { with earlier hospital discharge, lower hospital and total healthcare } \\
\text { costs, and a lower incidence of urinary retention. However, when } \\
\text { surgeons inexperienced in its use administer local anesthesia, more } \\
\text { hernia recurrences might result. }\end{array}$ & అ曰⿴囗十 & \\
\hline Recommendation & $\begin{array}{l}\text { Local anesthesia is recommended for open repair of reducible inguinal } \\
\text { hernias, provided surgeons/teams are experienced in local anesthesia } \\
\text { use. }\end{array}$ & 曰国田 & $\begin{array}{c}\text { Strong } \\
\text { *upgraded }\end{array}$ \\
\hline Recommendation & $\begin{array}{l}\text { Correctly performed local anesthesia is suggested to be a good } \\
\text { alternative to general or regional anesthesia in patients with severe } \\
\text { systemic disease. }\end{array}$ & 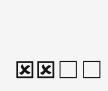 & weak \\
\hline
\end{tabular}

\section{Chapter 13}

\section{Anesthesia}

\section{A. R. Wijsmuller and P. Nordin}

\section{Key question}

KQ13.a Does local anesthesia influence outcomes after open repair of reducible inguinal hernia when compared with general or regional anesthesia?

\section{Introduction}

General, regional and local anesthetic techniques are used to facilitate open IH surgery. Regional anesthesia can be

\section{Evidence in literature}

We identified one meta-analysis and five reviews comparing local to general anesthesia. ${ }^{609-614}$ Of 17 randomized trials found, ${ }^{615-631}$ the most recent are included in the reviews. $^{616,620,629}$ SIGN analysis of the 2009 meta-analysis revealed methodological shortcomings. ${ }^{610}$ One shortcoming was the performance of a meta-analysis on urinary retention despite heterogeneity between studies. In addition, urinary retention data from the largest RCT comparing general to local anesthesia were omitted. These omitted figures demonstrate a lower incidence of urinary retention after local anesthesia when compared with general anesthesia. $^{629}$ A more recent 2012 review did not perform a meta-analysis because of included study design variation $^{611,614}$ and found a lower incidence of urinary retention following local anesthesia. ${ }^{614}$ 
When compared with general anesthesia, local anesthesia is more cost effective when hospital and total healthcare costs are considered ${ }^{632}$ and provides earlier patient mobilization and hospital discharge ${ }^{614}$ Although perioperative pain sensation is reported and can sometimes be a reason for conversion to general anesthesia, ${ }^{619}$ early postoperative pain seems less in the local anesthesia group. ${ }^{614}$ Some randomized studies report no inter-group difference in satisfaction or quality of life with respect to the operation and the first postoperative week. ${ }^{614,615,617}$ Others report higher patient satisfaction with the anesthetic technique for patients randomized to local anesthesia. $^{618,620}$

We identified five reviews ${ }^{609,611-614}$ and 11 randomized trials $^{618,620,624,625,629,633-638}$ comparing local to spinal anesthesia. The most recent meta-analysis, published in $2012,{ }^{614}$ did not include one randomized trial of spinal versus local anesthesia. ${ }^{634}$ The authors of this meta-analysis performed an analysis with respect to urinary retention and found a lower incidence of urinary retention in local anesthesia patients. ${ }^{614}$ The incidence of reported postoperative pain varies, ranging from no difference to less early postoperative pain after local anesthesia. ${ }^{614}$ Two randomized trials reported no differences in postoperative nausea. ${ }^{618,} 620$ However, the largest randomized trial (with more subjects than the other two trials combined) reported less postoperative nausea in the local anesthetic group. ${ }^{629}$ The majority of studies report faster hospital discharge after local anesthesia. ${ }^{614}$ Local is more cost effective than spinal anesthesia when hospital and total healthcare expenditures are compared. ${ }^{632}$ Crossover rates from local and regional anesthesia to general anesthesia strongly favor local anesthesia (1.9 versus $9.6 \%$, respectively). ${ }^{629}$

Hernia registries provide insights into IH recurrence risks with different anesthetic modalities. A Swedish Hernia Registry analysis of 59,823 patients found that local anesthesia is associated with an increased risk of reoperation for recurrence after primary IH repair. ${ }^{50}$ Using local anesthesia as a reference, they reported reoperation relative risks of 0.76 and 0.79 for regional and general anesthesia, respectively. A Danish Hernia Database analysis of 43,123 patients reported an increased reoperation rate after local anesthesia versus general or regional anesthesia after direct-but not indirect-hernia repair. $^{77}$ The same database analysis found lower reoperation rates following hernia repair by private hernia surgeons with uniform use of local anesthesia when compared with primary IH repair by general surgeons (possibly due to inexperience). They concluded that local anesthesia use in a general hospital might be a direct hernia recurrence risk factor, stressing the importance of experience in the administration of local anesthesia.

Cardiovascular disease accounts for most of the mortality associated with elective hernia repair (see Chapter 18). ${ }^{243}$ Therefore, correctly performed local anesthesia might be preferable to regional and general anesthesia in frail patients with severe systemic diseases (ASA class III). An RCT has demonstrated that local anesthesia is associated with a superior ventilation and oxygenation pattern when compared with general and regional anesthesia. ${ }^{635}$

\section{Discussion, consensus, clarification of grading}

Evidence strongly supports the idea that local anesthesia has several advantages over general or regional anesthesia in elective reducible $\mathrm{IH}$ repairs. As suggested by hernia database analysis, hernia recurrence may be more common following operation employing local anesthesia. Experience in local anesthetic administration might negate this downside risk.

ASA class III patients undergoing IH repairs may benefit by the administration of local anesthetic over regional or general anesthetic. However, the evidence for this potential benefit is weak.

\section{Key question}

KQ13.b Are outcomes different when open inguinal hernia repairs are performed with regional versus general or local anesthesia?

\section{Introduction}

The EHS Guidelines recommend against the use of spinal anesthesia in open anterior IH repairs in adults. ${ }^{3}$ They also cite general anesthesia with short-acting agents combined with local infiltration anesthesia as a valid alternative to local anesthesia alone. ${ }^{3}$ 


\section{Statements and Recommendations}

When compared with regional anesthesia, general anesthesia
offers no clear advantages regarding the incidence of
postoperative pain, postoperative nausea, cost, or patient
satisfaction. Its use allows for faster patient discharge, which is
of uncertain clinical significance. Some studies report a higher
incidence of urinary retention with regional anesthesia.

\section{Evidence in literature}

Five reviews ${ }^{611-614,} 639$ and nine RCTs ${ }^{620,} 624,629,631$, 635, 640-642 comparing general to regional anesthesia were identified. The majority of these RCTs compared general, regional and local anesthesia. ${ }^{620,624,629,635,642}$ Two of these five RCTs were excluded from this analysis since they mainly focused on pulmonary function assessment. ${ }^{624,}{ }^{635}$ A 2002 review, based mostly on cohort studies, and including 26,653 patients undergoing hernia repair with either general or spinal anesthesia, did not report a statistically significant inter-group difference (3 versus $2.4 \%$, respectively). ${ }^{611}$

A 2012 review of four randomized trials with 180 patients reported inconclusive results on early postoperative pain. ${ }^{614}$ The review indicated that there might be a reduction in analgesic need in the early postoperative period following spinal anesthesia. The effect on postoperative nausea was similarly inconclusive with one ${ }^{620}$ of two RCTs reporting a significant difference favoring spinal anesthesia while the other found no difference. ${ }^{620,641}$

The same 2012 review reported faster patient discharge after general anesthesia. No inter-group difference is reported in patient satisfaction scores. The incidence of urinary retention is not reported in the review.

The largest $\mathrm{RCT}^{629}$ comparing local, general and regional anesthesia was not included in the section of the 2012 review comparing general to regional anesthesia. The excluded RCT randomized 397 patients to either regional or general anesthesia. The majority of patients (62\%) in both groups received local anesthetic infiltration as well. Pain, nausea, early postoperative complications, hospital length of stay, patient satisfaction and costs were not significantly different between groups. ${ }^{615,} 629,632$ However, regional anesthesia patients were significantly more likely to require bladder catheterization for urinary retention.

Another recent systematic review ${ }^{639}$ excluded this $\mathrm{RCT}^{629}$ as well because many patients underwent two different anesthetic modalities. This systematic review also reported a lower incidence of urinary retention in the general anesthesia group. Less early postoperative pain was seen in the regional anesthesia group. There were no differences between groups in the incidence of other complications.

An analysis done on 29,033 elective groin hernia repairs from the Danish Hernia Database found a higher incidence of medical complications in patients aged 65 years and older after regional anesthesia (1.17\%) compared with general anesthesia $(0.59 \%){ }^{643}$ Complications included myocardial infarction, pneumonia and venous thromboembolisms.

\section{Discussion, consensus, clarification of grading}

Some high-quality medical evidence is available to address KQ13.b. Several RCTs support the statements and recommendations above. Barring the questionable value of a statistically significant but clinically negligible faster patient discharge, no clear benefits of general over spinal anesthesia have been reported except in those 65 and older. Urinary retention might be more frequent following regional anesthesia. A moderate level of evidence supports the recommendation above.

\section{Key question}

KQ13.c Can surgical residents/registrars safely perform open inguinal hernia repair using local anesthesia? 


\section{Introduction}

Local anesthesia has several advantages to regional and general anesthesia. However, data from hernia registries suggest that the hernia reoperation rate may be higher after local anesthesia when compared with general or regional anesthesia. ${ }^{50}$ Reoperation rates after hernia repair by private surgeons using local anesthesia are lower than those seen following primary $\mathrm{IH}$ repair in general hospitals. A higher level of expertise in local anesthesia administration seems to be associated with a lower reoperation risk. Does this also apply to physicians in the midst of learning curves like surgical residents/registrars? performing IH repair under local anesthesia. ${ }^{648}$ Beginners, defined as those who have repaired less than six hernias under local anesthesia, had a significantly higher recurrence rate. The study authors concluded that beginners should be closely supervised during their first six operations.

These few studies suggest that in the case of $\mathrm{IH}$ repairs done under local anesthesia, experience in local anesthesia administration influences recurrence/reoperation rates. Trainees can safely perform these operations, but supervision by a surgeon with the requisite experience is necessary to achieve optimal outcomes.

\section{Statements \\ Statement Open inguinal hernia repair under local anesthesia can be safely \\ performed by trainees under supervision of surgeons experienced $\quad$ $\quad \square \square$ \\ in the administration of local anesthesia.}

\section{Evidence in literature}

Five observational studies have examined complication rates after open $\mathrm{IH}$ repair under local anesthesia by trainees. ${ }^{644-648}$ We excluded one of these articles from analysis because it only investigated results in patients operated on by fully trained surgeons who wanted to learn local anesthetic administration. ${ }^{647}$ An Italian language article. $^{644}$ was also excluded from analysis, leaving three English-language publications for review. 645, 646, 648 Two studies reported no complication rate differences including no difference in recurrence rate after 10 years of follow-up after trainee-performed operations versus consultant-performed operations despite use of higher local anesthetic volumes by trainees. ${ }^{645,646}$ One study investigated the influence of experience on recurrence rates in 24 surgeons

\section{Chapter 14}

\section{Early postoperative pain prevention and management}

\author{
P. Nordin and A. R. Wijsmuller
}

\section{Introduction}

Several approaches to postoperative pain management have been studied including various medical treatments and interventions like the use of local anesthetics. This chapter reviews the literature on preoperative, perioperative, and postoperative interventions designed to treat pain after open groin hernia repair.

\section{Key questions}

KQ14.a Do preoperative or perioperative local anesthetic methods affect patients' pain experiences after open groin hernia repair?

KQ14.b Which is the most effective oral analgesic pain management regimen after groin hernia repair? 


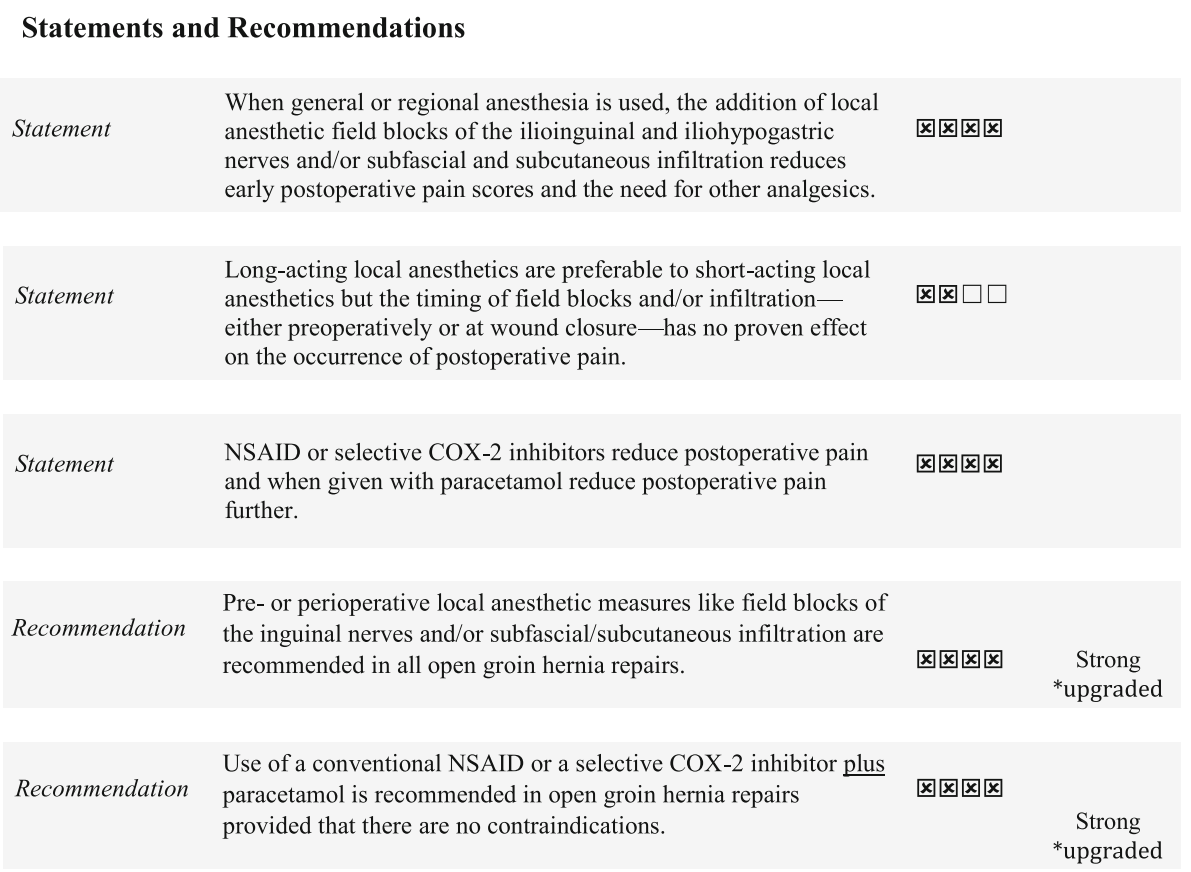

\section{Evidence in literature}

Herniorrhaphy postoperative pain prevention measures include the use of preoperative and intraoperative local anesthetic infiltration and/or preoperative or intraoperative field block and paravertebral block and conventional NSAIDs or selective COX-2 inhibitors.

The use of a preoperative or intraoperative field block (mostly of the ilio-inguinal and ilio-hypogastric nerves) with or without local wound infiltration is superior to placebo or no treatment for reducing early postoperative pain scores and the need for supplementary analgesics. ${ }^{649-653}$

Seven randomized trials reported that field block of the ilio-inguinal and ilio-hypogastric nerve with wound infiltration was superior to no treatment or placebo for reducing postoperative pain scores and supplementary analgesic requirements. ${ }^{654-660}$

A 2012 review $^{614}$ summarized four randomized trials comparing wound infiltration with local anesthetic to placebo. ${ }^{642}$, $661-663$ Wound infiltration was found to be superior to placebo for reducing early postoperative pain scores and the use of supplementary analgesics. Wound infiltration also lengthened the time-to-first-analgesic request.

A 2015 randomized trial of wound infiltration versus placebo found no difference in pain incidence 3 months postoperatively. ${ }^{664}$

A prospective, double-blind, randomized trial compared subfascial to subcutaneous local anesthetic infiltration and reported improved early postoperative pain scores after subfascial infiltration. ${ }^{665}$ Another randomized study compared combined subfascial and subcutaneous infiltration to subcutaneous or subfascial infiltration alone. Combination infiltration resulted in improved early postoperative pain scores, less supplementary analgesic need and longer timeto-first-analgesic request. ${ }^{666}$

Two studies compared local anesthetic infiltration to placebo or no treatment and found local infiltration superior with respect to early postoperative pain and supplemental analgesic use. ${ }^{667,668}$

Three studies investigated local anesthetic timing, comparing preoperative to at/near-wound-closure infiltration. ${ }^{669-671}$ Two of the three studies reported no differences in early postoperative pain and supplemental analgesic use after preoperative field block versus at-wound-closure field block during general anesthesia. ${ }^{669,}{ }^{670}$ The third study compared pre-incisional and before-wound-closure infiltration during general anesthesia concluded that pre-incisional infiltration with lidocaine was a more effective method of providing postoperative analgesia. ${ }^{671}$ The 2012 review referenced above concluded that preoperative and at-wound-closure local anesthetic regimens had equal benefit in reducing pain scores and supplemental analgesic use. $^{614}$

Two studies found that ultrasound-guided nerve blocks (involving the ilio-hypogastric/ilio-inguinal nerves) were superior to anatomic-landmark nerve blocks at providing effective analgesia. ${ }^{672,673}$

Paravertebral nerve blocks (PVBs) are established methods of providing analgesia to thoracic- and 
abdominal-surgery patients including those undergoing groin hernia repair. A PVB has the potential to offer sustained pain relief with minimal side effects. One systematic review $^{674}$ and three randomized studies ${ }^{675-677}$ found a tendency to less postoperative pain in PVB-patients when compared with general-anesthesia and spinal-anesthesia patients.

The transversus abdominis plane (TAP) block is a relatively new regional anesthetic technique developed in an attempt to reduce postoperative pain. It has evolved from a landmark technique to an ultrasound-guided one. Four randomized studies comparing TAP blocks with either placebo, local anesthetic infiltration, or no treatment reported conflicting results with respect to early postoperative pain and analgesic use. ${ }^{123}, 654,678,679$ A 2010 Cochrane Database Systematic Review found only limited evidence to suggest that the use of perioperative TAP blocks is opioid sparing or reduces pain scores after abdominal surgery. ${ }^{680}$

In addition to the preoperative and intraoperative pain prevention and treatment methods above, non-opioid and non-steroidal anti-inflammatory medications (acetaminophen, NSAIDs and selective COX-2 inhibitors) should be used for postoperative pain management. ${ }^{681-685}$ Paracetamol (acetaminophen) has insufficient effect as single-agent therapy for moderate-to-severe pain. However, the combination of paracetamol and a non-steroidal anti-inflammatory drug, given in a timely manner, seems to be optimal and provides sufficient analgesic during the early recovery phase provided that there is no contraindication. $^{614,686}$

Opioids may cause adverse effects such as nausea, vomiting, and constipation, amongst others which may delay postoperative recovery. Therefore, non-opioid analgesics should be used whenever possible. However, opioids can be used for moderate- or high-intensity pain, in addition to non-opioid analgesia or when the combination of an NSAID and paracetamol is not sufficient or is contraindicated. $^{687}$

Several small studies of varying quality seem to indicate that local anesthetic administration via intra-wound catheters by repeat bolus or continuous infusion is more efficacious than placebo at reducing postoperative pain. ${ }^{68-693}$ Potential benefits and risks of this technique need further study with RCTs and other means.

\section{Discussion and grading clarification}

Inguinal hernia repair results in pain postoperatively and the optimal method(s) to treat this pain remain(s) controversial. However, it is clear that local anesthetic field blocks and subfascial and/or subcutaneous local infiltration reduces early postoperative pain scores and the need for supplemental analgesics. Therefore, when general or regional anesthesia is used, local anesthetic field blocks and infiltration is recommended in all open groin hernia surgeries. Additionally, the combination of a conventional NSAID or a selective COX-2 inhibitor plus paracetamol reduces postoperative pain and is also recommended.

A weakness in the review presented in this chapter stems from the variation in quality of the available randomized trials. Although postoperative pain was our focus, it was not always the primary endpoint of the included studies.

There is strong evidence for preoperative and intraoperative inguinal field blocks and wound infiltration with seven randomized studies showing superiority to no treatment or to placebo. Four randomized trials found wound infiltration superior to placebo. Provided that there is no contraindication, the use of a conventional NSAID or a selective COX-2 inhibitor is also recommended with four randomized trials and one review showing reduced postoperative pain when compared to placebo. There is also strong evidence to support the use of paracetamol in combination with conventional NSAIDs/selective COX-2 inhibitors. Opioids are recommended in limited circumstances as described above.

\section{Chapter 15}

\section{Convalescence}

T. Bisgaard and L. N. Jorgensen

\section{Introduction}

Convalescence duration-defined as sick leave from work and time away from leisure-is an important feature of the recovery phase following $\mathrm{IH}$ surgery. However, most studies have not investigated the impact of recommendations on short duration convalescence. 


\section{Key question}

KQ15.a What is the recommended duration of convalescence following uncomplicated inguinal hernia repair? sports. $^{210,246,254,292,697,703}$ (low-to-moderate level of evidence).

The available medical evidence supports the idea that work and leisure activities can be resumed by most patients

Statement and Recommendation
$\begin{array}{ll}\text { Physical activity restrictions are unnecessary after } \\ \text { uncomplicated inguinal hernia repair and do not affect } \\ \text { recurrence rates. Patients should be encouraged to resume } \\ \text { normal activities as soon as possible. }\end{array}$
$\begin{aligned} & \text { Patients are recommended to resume normal activities without } \\ & \text { restrictions within three to five days or as soon as they feel } \\ & \text { comfortable. }\end{aligned}$

\section{Evidence in literature}

The literature search identified 327 studies of which we included one systematic review, 14 RCTs, three cohort studies and four case-control studies.

\section{Discussion}

Surgeons' recommendations for physical activity restrictions and/or sick leave duration are highly variable, rarely evidence-based, and greatly affect the duration of absence from normal activity. ${ }^{146,694-696}$ No study has demonstrated that early return to normal activities and work after IH repair increases hernia recurrence risk or complications.

In a nationwide multicentre prospective questionnaire study with controls from the Danish Hernia Database of 2365 patients with convalescence duration as the primary outcome found that a short duration of convalescence (even as short as 1 day) following open IH repair may be recommended without increasing hernia recurrence risk. ${ }^{146}$

Pain and wound-related problems are the most often cited reasons for not resuming work or leisure activities as recommended (evidence level—high). ${ }^{146}$ A 2012 study of 162 laparoscopic IH repair patients found that convalescence duration was a median of 5 days (range 1-40) from work and 3 days (range 1-49) from leisure activities when the recommendation was for 1 day. ${ }^{695}$ Patient expectation preoperatively for time off work was the only independent factor that predicted prolonged convalescence. Postoperatively, self-arranged planned sick leave, and complaints of pain and fatigue were the primary reasons for not resuming normal activities within the first 3 days after operation (evidence level-low). ${ }^{695}$

In studies where duration of convalescence was secondary outcome using non-restricted recommendations ( $\leq 2$ days) reported 1 week absence from domestic activities, ${ }^{210}, 223,245,246,260,697-699$ 1-2 weeks absence from work, ${ }^{210}, 223,246,250,254,257,260,281,292,697,699-704$ and 1-3 weeks after physical activities including within 3-5 days following elective laparoscopic or open $\mathrm{IH}$ repair without risk of hernia recurrence or other complications. The recommendations have been upgraded by HerniaSurge.

\section{PART 2}

\section{Specific aspects of Groin Hernia Management}

16. Groin hernias in women

17. Femoral hernias

18. Complications: prevention and treatment

19. Pain: prevention and treatment

20. Recurrent inguinal hernias

21. Emergency treatment of groin hernia

\section{Chapter 16}

\section{Groin hernias in women}

N. Schouten, H. Eker, K. Bury, and F. Muysoms

\section{Introduction}

Groin hernia repairs are 8-10 times more common in men compared with women. ${ }^{1,2}$ Inguinal hernias (IHs) occur 9-12 times more commonly in men, whereas femoral hernias occur approximately 4 times more commonly in women. ${ }^{3}$ These differences may be explained by the greater distance between the pubic tubercle and the internal ring and the wider rectus abdominis muscle in females. ${ }^{4}$

No systematic reviews or randomized controlled trials (RCTs) specifically address groin hernia repair in women. Data are collected mainly from subgroup analyses of epidemiological studies from national databases. Reoperation rates after anterior hernia repairs in women are higher 
when compared with men. In roughly $40 \%$ of reoperations in women, femoral hernia recurrences are found, suggesting that these "recurrences" might represent hernias overlooked during primary operations. ${ }^{5-11}$ Therefore, the European Hernia Society (EHS) guidelines on groin hernia treatment recommend laparoscopic repair in women covering both the inguinal and femoral orifices. ${ }^{12}$

\section{Key Questions}

KQ16.a In women with a groin lump, what is the best diagnostic modality and is a preoperative diagnosis necessary?

KQ16.b What is the optimal treatment for women with groin hernias?

KQ16.c What is the risk of incarceration/strangulation in women with groin hernias? What is the incidence of emergent inguinal/femoral hernia repair in women? What are the outcomes?
A meta-analysis of ultrasound as an initial diagnostic imaging modality for groin hernias showed a high sensitivity and positive predictive value in cases confounded by diagnostic uncertainty. The study cited low expense and minimal risk as advantages over other radiologic methods. ${ }^{13}$ However, ultrasound and clinical examination is "operator dependent" and may be unable to distinguish an inguinal from a femoral hernia. Both may also miss femoral hernias entirely. ${ }^{14-16}$

The literature confirms that femoral hernias are frequently found in women undergoing groin hernia repair, but that a correct preoperative diagnosis of these hernias is uncommon. ${ }^{14,15}$

In a few large epidemiological studies from national databases, reoperation rates after open anterior groin hernia repairs in females are higher when compared with reoperation rates in males. In approximately $40 \%$ of reoperations after anterior mesh or non-mesh repairs, a femoral "recurrence" is found, representing a nearly tenfold risk of this finding in women. ${ }^{5-9,} 17$ The explanation for this

$\begin{array}{ll}\begin{array}{l}\text { Statements and Recommendations } \\ \text { Statement }\end{array} & \begin{array}{l}\text { No clinical or diagnostic tests can reliably distinguish inguinal } \\ \text { from femoral hernias. A combination of Clinical examination } \\ \text { and ultrasound and a high suspicion of a possible femoral } \\ \text { hernia are best strategies. }\end{array} \\ \text { Statement } & \begin{array}{l}\text { Femoral hernias occur more often in women. } \\ \text { Statement }\end{array} \\ \text { Femoral hernias are more likely than inguinal hernias to } \\ \text { incarcerate and strangulate. The exact risks and outcome of } \\ \text { femoral hernia in women are unknown. }\end{array}$

\section{Evidence in literature}

When an overt groin hernia is present, the diagnosis can often be confirmed by physical examination. Textbooks state that a femoral hernia produces swelling inferolateral to the pubic tubercle and an inguinal hernia causes superomedial swelling; however, this subtle distinction is often difficult to discern, particularly in obese women. phenomenon may be that femoral hernias are overlooked during the initial operations, since an open anterior repair does not always involve opening of the transversalis fascia or preperitoneal space exploration. Subgroup analysis from the same studies supports this contention by noting that reoperation rates after laparoscopic approaches (TEP, TAPP) are lower when compared to Lichtenstein or other open anterior approaches. $5,7,8,10,11$ 
Two small cohort studies focused on hernia repair in women also suggest that an open anterior repair is associated with a relatively high postoperative complication rate and a higher incidence of postoperative pain compared with women undergoing TEP hernia repair. ${ }^{18,19}$

The high frequency of femoral hernias in women and the high risk of femoral recurrence mentioned above highlight the need for preperitoneal exploration and repair in all women with groin hernias. A laparoscopic preperitoneal repair offers a thorough view of the entire myopectineal orifice and creates easy access to, and coverage of, both inguinal and femoral defects. This also obviates the need for a correct preoperative diagnosis. ${ }^{7,15,16}$ Anatomically and with regard to mesh application, there is a little difference between the laparoscopic and open preperitoneal approach, but laparoscopic repair techniques have a long learning curve (see chapter 22 on learning curve). ${ }^{20}$ In experienced hands though, laparoscopic hernia repair seems to be associated with a slightly lower reoperation risk than open preperitoneal repair. $9,10,21$ watchful waiting strategy in women with groin hernias. Timely hernia repair is recommended.

\section{Discussion}

There are no systematic reviews or RCTs specifically addressing groin hernias in women, but several subgroup analyses from large epidemiological studies of national databases provide information about groin hernias and groin hernia repairs in women. It is abundantly clear that femoral hernias are more common in women. This argues for the use of a preperitoneal laparoscopic approach in women with groin hernias. In addition, since strangulation risk is unacceptably high, timely elective repair of groin hernias in women is strongly advised.

\section{Key question}

KQ16.d How is a groin lump in a pregnant female diagnosed and treated?

$\begin{aligned} & \text { Statements and Recommendation } \\ & \text { Statement } \\ & \text { Groin hernia formation is uncommon during pregnancy. } \\ & \text { The most likely diagnosis in a woman with the onset of a groin } \\ & \text { lump during pregnancy is round ligament varicosity. } \\ & \text { Statement } \\ & \text { Watchful waiting is suggested in pregnant females with groin } \\ & \text { Recommendation }\end{aligned} \quad$ Swelling

Femoral hernias carry a higher risk of strangulation when compared with IHs. In the Swedish and Danish hernia registries, 36-39\% of femoral hernias were emergently repaired versus $5 \%$ of IHs. ${ }^{10,22}$

About $17 \%$ of women with groin hernias require emergent repair versus about $5 \%$ of men. ${ }^{9,} 22$ Furthermore, patients with femoral hernias have a greater risk of undergoing bowel resection (23\% of patients undergoing emergent femoral hernia repair compared with approximately $5 \%$ of patients undergoing emergent $I H$ repair). Bowel resection occurred in $17 \%$ of women undergoing emergent groin hernia repair. There are no specific data about bowel resection after femoral hernia repair.

A woman's risk of death in the 30 days after elective femoral hernia repair is comparable to the risk of dying in an age- and gender-matched population $(<0.1 \%)$. However, $3.8 \%$ of women die within the 30 days following emergent femoral hernia repair., 22

The higher incidence of femoral hernias in women and the associated strangulation risk argues strongly against a

\section{Evidence in literature}

IH formation during pregnancy is rare with a prevalence estimated as $1 ; 2000 .^{23} \mathrm{~A}$ watchful waiting strategy is recommended for those who develop IHs during pregnancy. $^{24}$

Although mainly described in small case series and case reports, the onset of a groin lump in pregnancy is often a round ligament varicosity rather than a hernia. ${ }^{25} \mathrm{~A}$ round ligament varicocele arises from the veins draining the round ligament and the inguinal canal. It is a rare entity associated with pregnancy, presents with a groin lump typically early in the third trimester, progressively enlarges during pregnancy, and regresses soon after delivery. ${ }^{25}$ The diagnosis can be easily confirmed by color Doppler sonography. If, in fact, a groin lump consisting of varicose veins occurs, symptoms will spontaneously abate after delivery and expectant management can be used. Varicocele resection might be necessary only in cases with severe pain. 


\section{Discussion}

There are several case reports and case series, but only one small prospective cohort study describing the onset of a groin lump in pregnancy associated with varicose veins of the round ligament rather than a groin hernia. All conclude that a watchful waiting strategy is safe and preferred. It seems prudent to confirm the diagnosis with color Doppler sonography. The true prevalence of groin hernia formation during pregnancy is unknown, difficult to determine, and only mentioned-but not investigated-in two small cohort studies. The level of evidence supporting the statements in this section is low because of limited medical literature on the subject.

\section{Key question}

KQ16.e What is the best management of the round ligament in women who undergo groin hernia repair? is, therefore, advised to avoid division of the round ligament in open (anterior) hernia repair. If the ligament is divided nonetheless, care should be taken to properly address any incidentally ligated nerves.

Division of the round ligament in laparoscopic hernia repair is optional and might facilitate optimal mesh placement. The round ligament is enveloped by the peritoneum and may lead to lower folding of the mesh or peritoneum sliding beneath. For this reason, many surgeons choose to divide it in laparoscopic repair. There are fewer implications of division in the preperitoneal space as the nerves are not adherent to the ligament until it enters the internal ring. Division of the round ligament should, therefore, be performed proximal to the genital branch meeting, which is typically best performed at the fusion with the peritoneum where division has no functional implication.

\section{Statement and Recommendation

Concerning management of the round ligament in women the
division of the genital branch of the genitofemoral nerve carries
a small risk of deafferentation, hypersensitivity, and ipsilateral
labial numbness. \\ It is suggested to avoid division of the round ligament in open repair. Division of the round ligament is optional in laparo- endoscopic repair, but should preferentially be performed proximal to the genital branch meeting at the fusion with the peritoneum.}

\section{Evidence in literature}

One unaddressed issue in the management of groin hernia repair in women is whether the round ligament should be divided or spared during surgery. Although there is no evidence in the literature to support either, there are some anatomy-based considerations to take into account, mainly based on extrapolation from extended experience with anterior and retroperitoneal neurectomy.

The round ligament is attached to the uterus through the broad ligament of the uterus, enters the inguinal canal, and finally terminates in the digital process of fat of the labia majora. The genital branch of the genitofemoral nerve mostly meets the round ligament at the internal ring, but sometimes it may join earlier. Division of the round ligament in open repair inherently implies simultaneous division of the genital nerve and likely division of the ilioinguinal nerve. Sacrifice typically has minimal morbidity or consequence but carries a small risk of deafferentation hypersensitivity and ipsilateral labial numbness that may contribute to complaints of sexual dysfunction. It

\section{Discussion}

There is no literature that addresses the sparing or division of the round ligament in groin hernia repair in women. The statements are based on anatomical considerations and thorough discussion with experts in anterior and retroperitoneal neurectomy.

\section{Chapter 17}

\section{Femoral hernias}

H. Eker, N. Schouten, K. Bury, and F. Muysoms

\section{Introduction}

Elective and emergent femoral hernia repairs constitute roughly $2-4 \%$ of all groin hernia repairs. However, the true femoral hernia incidence is likely lower than $2-4 \%$, since 
this estimate is skewed by the high percentage of surgically treated femoral hernias compared to IHs. Medical literature focused on femoral hernias is scant and studies lack sufficient power to draw firm conclusions. However, large systematic reviews on IHs provide data that can inform decision-making about femoral hernia management. ${ }^{26,}{ }^{27}$

Some topics in this chapter (e.g., suture and mesh choice, prevention, and treatment of complications) were assumed to be comparable to IH repair and were not evaluated separately.

\section{Key questions}

KQ17.a Does tissue repair in femoral hernia have a higher recurrence rate than mesh repair?

KQ17.b Following femoral hernia repair are there differences in recurrence rates or the incidence of chronic pain between open anterior mesh repair and open posterior mesh repair?

KQ17.c Following open and endoscopic femoral hernia repairs are there differences in recurrence rates and or postoperative pain?

KQ17.d Should asymptomatic femoral hernias always be treated surgically? recommendation. Watchful waiting is discouraged, since the risks of serious and potentially lethal complications such as strangulation and bowel resection are unacceptably high. ${ }^{27-30}$ Several clinically significant differences were found in outcomes following elective and emergent femoral hernia repair. When compared to elective repair, emergent femoral hernia repair is associated with a greater risk of small bowel resection and a longer length of hospital stay. $22,31,32$

In contrast to IH repair, primary suture repair of femoral hernia is still an accepted technique in elective and emergency settings. A cohort study from a specialized hernia center concluded that there were no significant differences regarding recurrence rate between tissue-based and mesh repair of femoral hernias. ${ }^{29}$ However, studies from the Danish Hernia Database and the Swedish Hernia Registry all concluded that recurrence and reoperation rates after mesh repair were significantly lower. ${ }^{10,} 33$

Which mesh or plug should be used in open femoral hernia repair was investigated in two RCTs and a large national database study. ${ }^{3-35}$ Significantly better results concerning recurrence, postoperative pain, and foreignbody sensation were found in the RCT for preperitoneal mesh repair compared to plug repair. ${ }^{35}$ No differences in

\begin{tabular}{|c|c|c|c|}
\hline Statement & $\begin{array}{l}\text { In femoral hernia surgery tissue repair has a higher recurrence } \\
\text { rate than mesh repair. }\end{array}$ & 冈खि口 & \\
\hline Statement & $\begin{array}{l}\text { Preperitoneal open mesh repair for femoral hernia results in } \\
\text { significantly fewer recurrences and less postoperative pain and } \\
\text { foreign body sensation than open mesh repair. }\end{array}$ & 冈冈口 & \\
\hline Statement & $\begin{array}{l}\text { Laparo-endoscopic repair of femoral hernias results in } \\
\text { significantly fewer recurrences and less postoperative pain than } \\
\text { anterior repair. }\end{array}$ & 冈囚 $\square \square$ & \\
\hline Statement & $\begin{array}{l}\text { Laparo-endoscopic repair offers the opportunity to identify all } \\
\text { types of groin hernia. }\end{array}$ & 冈खि口 & \\
\hline Recommendation & $\begin{array}{l}\text { Mesh is recommended to be used in elective femoral hernia } \\
\text { repairs. }\end{array}$ & 冈खि & $\begin{array}{l}\text { Strong } \\
\text { *upgraded }\end{array}$ \\
\hline Recommendation & $\begin{array}{l}\text { Providing expertise is available, a laparo-endoscopic procedure } \\
\text { is recommended for elective femoral hernia repair. }\end{array}$ & 冈口 $\square$ & $\begin{array}{c}\text { Strong } \\
\text { *upgraded }\end{array}$ \\
\hline Recommendation & $\begin{array}{l}\text { Timely elective repair is recommended for all patients with } \\
\text { femoral hernias. }\end{array}$ & 冈囚 $\square \square$ & $\begin{array}{c}\text { Strong } \\
\text { *upgraded }\end{array}$ \\
\hline
\end{tabular}

Two systematic reviews (SRs) were identified that focused on IH repair but included data and recommendations on femoral hernias. ${ }^{26,}{ }^{27}$ KQ 17.d, concerning whether an asymptomatic femoral hernia should be electively repaired, received the strongest consensus hernia recurrence were found in a retrospective study. The large database study showed no differences in postoperative pain between different mesh types and anatomical locations for the mesh devices. 
The medical literature strongly supports the advantages of elective laparoscopic femoral hernia repair. ${ }^{10}$ Three cohort studies from specialized hernia centers found that endoscopic repairs provide the opportunity to unerringly diagnose conditions which may have been obscure preoperatively. ${ }^{7,14,15}$ Several other cohort studies of various sizes found significantly fewer recurrences after elective endoscopic femoral hernia repairs compared to open. $8,10,33$ The aforementioned arguments strongly support a laparoendoscopic approach for femoral hernia repair.

\section{Chapter 18}

\section{Complications: prevention and treatment}

S. Smedberg, W. Reinpold, A. Wijsmuller, and R. Fitzgibbons

\section{Introduction}

Common primary outcome measures in IH surgery studies include: recurrence, chronic postoperative pain, and wound infection. Usually reported as secondary outcomes are: urinary and sexual dysfunction, hematoma, seroma, infrequent visceral and vascular injuries, late postoperative complications, and mortality. These secondary measures are indispensable when considering the success and value of different types of $\mathrm{IH}$ repairs. Only these are reported in this chapter.

\section{Urinary retention}

\section{Key questions}

KQ18.a Is early postoperative pain associated with increased urinary retention risk?

KQ18.b Is there an age-associated postoperative urinary retention risk?

KQ18.c Does intraoperative parenteral fluid restriction reduce urinary retention risk?

KQ18.d Is there an increased risk of postoperative urinary retention with open anterior repair?

KQ18.e When is prophylactic urinary bladder catheterization indicated before hernia operation?

KQ18.f Is there effective prophylactic medication to decrease urinary retention?

\begin{tabular}{|c|c|c|c|}
\hline \multicolumn{4}{|l|}{ Statements } \\
\hline Statement & $\begin{array}{l}\text { There is only indirect evidence that pain results in increased } \\
\text { urinary retention rates based upon the experience with painful } \\
\text { tack fixation vs non-fixation techniques. LOE = weak }\end{array}$ & 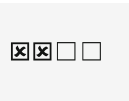 & \\
\hline Statement & Urinary retention after inguinal hernia repair increases with age. & 曰曰凶ख & \\
\hline Statement & $\begin{array}{l}\text { Minimizing the amount of parenteral fluids given to patients } \\
\text { undergoing inguinal herniorrhaphy may result in a lower incidence } \\
\text { of urinary retention. }\end{array}$ & $\otimes \otimes \square \square$ & \\
\hline Statement & $\begin{array}{l}\text { Open anterior repair performed under local anesthesia has a lower } \\
\text { incidence of urinary retention compared to endoscopic repair. }\end{array}$ & 凤凶冈囚 & \\
\hline Statement & $\begin{array}{l}\text { There is no difference in the incidence of urinary retention between } \\
\text { open repair and endoscopic repair when performed under general } \\
\text { anesthesia. }\end{array}$ & అ冈ख & \\
\hline Statement & $\begin{array}{l}\text { There is no evidence that routine use of a urinary catheter is } \\
\text { necessary for either conventional or laparoscopic inguinal } \\
\text { herniorrhaphy. A history of a prostatectomy or previous urinary } \\
\text { emptying problems is a relative indication for urinary } \\
\text { catheterization. }\end{array}$ & అखि口 & \\
\hline Statement & $\begin{array}{l}\text { Prazosin, phenoxybenzamine hydrochloride or tamsulosin may be } \\
\text { effective in preventing urinary retention. }\end{array}$ & 冈曰 $\square$ & \\
\hline Recommendation & $\begin{array}{l}\text { It is suggested to ask the patient to void the bladder prior to groin } \\
\text { hernia surgery Routine use of a urinary catheter is not } \\
\text { recommended. }\end{array}$ & 冈खि & Weak \\
\hline
\end{tabular}




\section{Evidence in literature}

The incidence of urinary retention following IH repair varies widely in published series ranging from less than $1 \%$ to greater than $20 \%{ }^{36,37}$ The most common predisposing factor for postoperative urinary retention (POUR) after an $\mathrm{IH}$ repair is the use of general or regional anesthesia. ${ }^{38-42}$ Most authorities feel that regional anesthesia (spinal or epidural) is worse than general. However, in a study pooling data from 70 non-randomized and two randomized studies, the incidence of urinary retention with local anesthesia was $0.37 \%$ (33 in 8991 patients), with regional anesthesia $2.42 \%$ (150 in 6191 patients), and with general anesthesia 3\% (344 in 11,471 patients). The need for general anesthesia for most laparoscopic IH repairs (LIH) almost certainly accounts for the higher incidence of POUR after LIH repair which has been reported to be as high as $22 \% .^{37}$ Other factors which have been inconsistently incriminated for POUR (i.e., some studies reporting statistically significant differences after LIH, while others do not) include: over-hydration with intravenous fluid during surgery, ${ }^{37}$ bilateral hernia repairs, ${ }^{43}$ increased BMI ${ }^{43}$ use of opioid analgesics, ${ }^{44}$ older age,${ }^{44,}{ }^{45}$ prostatic symptoms,${ }^{45}$ and prolonged operative time. ${ }^{43,}{ }^{45}$ In a metaanalysis performed by Tam et al., the incidence of POUR was found to be higher in patients with tack fixation presumably because of increased pain (3.1 vs $>1.0 \%)$, but this difference did not reach statistical significance. ${ }^{46}$ Other meta-analyses have not demonstrated a difference in urinary retention when comparing tack fixation with glue. ${ }^{47}$ Meta-analyses of RCTs comparing various hernia techniques have not shown a difference in urinary retention rates among the various laparoscopic and open techniques. ${ }^{48,} 49$ Many surgeons routinely place urinary catheters for inguinal herniorrhaphy especially when done laparoscopically. ${ }^{43}, 45$ This seems to be more a surgical tradition, not an evidence-based practice. A year study looked at patients in two time periods, an earlier one when urinary catheters were used routinely, and a later one when they were not. A marked improvement in urinary complications (cystitis, urinary retention and hematuria) occurred when the practice of routine urinary catheterization was abandoned. ${ }^{50}$ One of the largest laparoscopic TAPP series also showed that the procedure can be safely conducted without the use of a urinary catheter. ${ }^{51}$ Urinary retention can be treated by either intermittent catheterization or temporary placement of an indwelling urinary catheter. Prophylactic use of alpha-1 receptors' antagonists such as prazosin, phenoxybenzamine hydrochloride, or tamsulosin has been shown in some studies to be an effective strategy to prevent postoperative urinary retention. ${ }^{49-51}$ Overall urinary function as measured by the American Urological Association Symptom Score is improved by hernia repair providing that a Foley catheter was not used at the time of the index operation. ${ }^{52}$

Sexual dysfunction, testicular hormone function, and ischemic orchitis

Inguinal hernia surgery can cause damage to different nerves, disturbances of testicular circulation, and damage to the vas deferens which can lead to various long-lasting harms to the patient. Nerve damage can cause chronic pain that could interfere with sexual activity. Disturbances of testicular circulation can result in initially severe pain followed by atrophy of the testicle and thereby impaired hormone production. Division of the vas deferens will cause obstruction for the passage of sperm. In case of bilateral injuries of different types, both hormone changes and infertility could result in a disaster for the patient.

Sexual problems after hernia operations are probably under-reported, patients often being unwilling to discuss their sexual concerns. The heading "sexual dysfunction" includes several interacting factors, making the topic's bounds difficult to delineate. For that reason, it is also difficult to measure the incidence of sexual dysfunction.

\section{Key questions}

KQ18.g What defines "sexual dysfunction" after IH surgery?

KQ18.h What is the incidence of sexual dysfunction after IH surgery?

KQ18.i Are ischemic orchiditis causes known; and can this complication be prevented?

KQ18.j Does hernia repair with heavyweight mesh cause more testicular pain than hernia repair with lightweight mesh?

KQ18.k Are methods of repair or bilateral operation related to risks of impaired spermatogenesis and hormone production?

KQ18.I Can sexual dysfunction following hernia repair be treated surgically? 


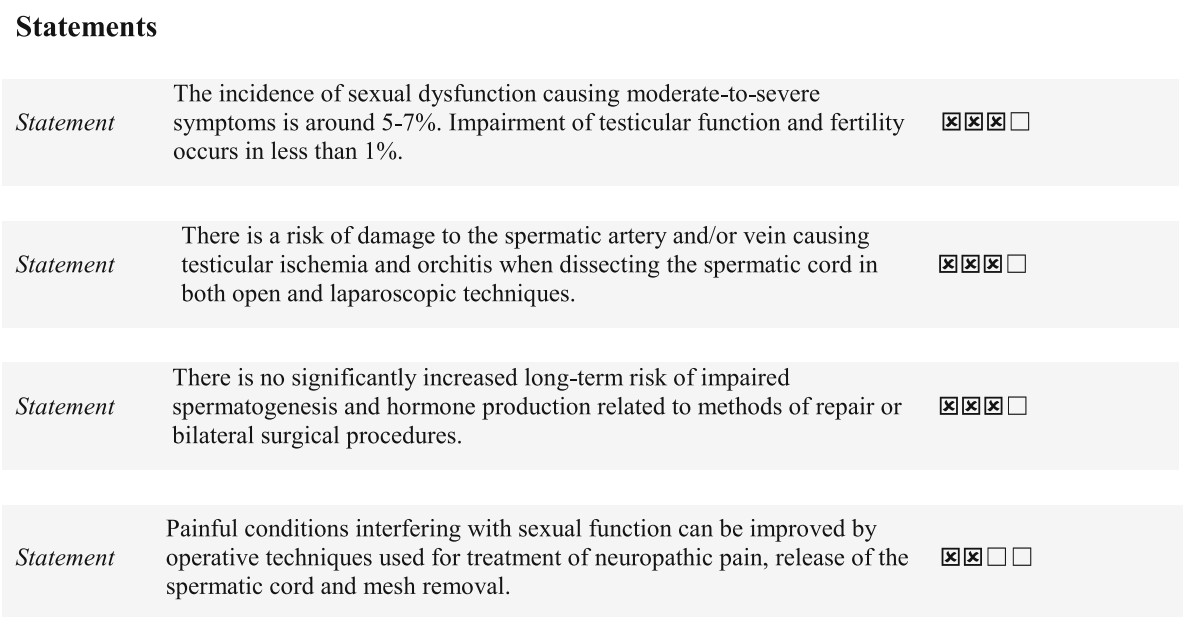

\section{Evidence in literature and discussion}

There is no generally accepted definition of sexual dysfunction after hernia operations. Publications can roughly be divided into those reporting on pain having a negative effect on sexual function such as neuropathic pain in the groin, pubalgia, and orchialgia (discussed in Chapter 19 on pain, briefly mentioned in this text), those focusing on negative effects of hernia operations on fertility and testicular gonadal function and those evaluating complications having an influence on both symptoms and function, i.e., dysejaculation and ischemic orchitis.

Groin or genital pain interfering with sexual activity was assessed in two follow-up questionnaire studies from the Danish Hernia Database. In the first, consisting mainly of open repairs, $28 \%$ admitted to some pain, while the second study of exclusively laparoscopic repairs reported $11 \%$ some pain ${ }^{53,54}$ with $2.8 \%$ of the mainly open and $2.4 \%$ of the laparoscopic group reporting that pain moderately to severely impaired their sexual activity. The incidence of dysejaculation $^{55}$ felt to be caused by spermatic duct trauma and/or a mesh-related inflammatory reaction along the duct typically causing pain at the superficial inguinal ring) was 7.6 and $3.1 \%$, respectively. ${ }^{56}$

Ischemic orchitis is caused by damage to the arterial and/or venous structures in the spermatic cord. An early clinical review suggested that the condition was related to venous thrombosis caused by the operative trauma. ${ }^{57}$ In a subsequent study, when distal indirect sacs were left in situ and recurrent hernias were operated upon with preperitoneal technique, the risk of ischemic orchitis was reduced from 0.65 to $0.03 \%$ and from 2.25 to $0.97 \%$, respectively. ${ }^{58}$ In most cases, the acute condition subsides postoperatively, but may result in testicular atrophy. ${ }^{59}$ In atrophic testicles after ischemic orchitis, seminiferous tubes are usually absent. Leydig cells producing testosterone and supporting Sertoli cells are, however, usually still present and have a normal appearance. ${ }^{57}$ In a meta-analysis of randomized studies of lightweight mesh vs heavyweight mesh, the incidence of testicular atrophy was reported as $0.8 \%$ irrespective of repair method. ${ }^{60}$

Testicular function is related to testicular perfusion. ${ }^{59}$ Animal studies have shown considerable structural changes in the cord and testicle after hernia repair, more pronounced after the use of mesh. ${ }^{59,61,62}$ In clinical studies, mesh repairs have negative effects on testicular perfusion and testicular volume, and reduce testosterone levels and sperm motility during the early postoperative period. ${ }^{59,63}$ However, long-term follow-up has not shown statistically significant differences in testicular perfusion or spermatogenesis compared to preoperative values. ${ }^{64,}{ }^{65}$ Further studies are in progress. ${ }^{66}$

Apart from above-mentioned complications, infertility may be caused by operative injury to the vas deferens by division, ligation, clipping, stapling, electrocauterization, and scarification. Damage to the vas is estimated to occur in $0.3 \%$ of adults and $0.8-2.0 \%$ of children. ${ }^{67}$ The inflammatory response to mesh can be so severe that vas obstruction results. ${ }^{68}$ One of the major arguments for the routine use of mesh in IH surgery, however, is to preserve fertility. The theory is that by decreasing the generally accepted recurrence rate in the general population from 10 to $15 \%$ seen with Bassini and its variants to less than $5 \%$ with the mesh tension-free approach, reoperative surgery, with its heavy toll of testicular loss, is avoided. ${ }^{69}$ The development of sperm antibodies as a result of extravasation of sperm from an injured duct is of particular concern, because the argument is challenged that the patient is protected by the opposite testicle when a unilateral inguinal herniorrhaphy is done. ${ }^{70,71}$

There was no increased risk of male infertility after bilateral hernia surgery with or without mesh when compared to the general population in a prospective 
questionnaire study within the Swedish Hernia Register. ${ }^{72}$ In a retrospective register study of bilateral hernia operations, a significantly higher incidence of infertility was found in a subgroup receiving mesh on both sides compared to sutured repairs. ${ }^{73}$ However, the risk was very low, and both studies concluded that there is no increased infertility risk after hernia repairs using mesh. ${ }^{73}$

A recent publication on surgical treatment of chronic inguinodynia with tailored neurectomy, funicular release, and/or mesh removal confirmed beneficial effects on dysejaculation with a significant reduction of VAS scores in 20 patients from 55 to 21 ( $p<0.001$ ). Sexual life normalized in two-thirds of the patients. ${ }^{74}$ Vasovagal anastomosis to correct infertility after hernia surgery is most often reported after hernia operations performed when the patient was a child. ${ }^{75}$ In adult patients with previous polypropylene, mesh repairs and infertility caused by vasal obstruction, reconstruction, and eventual fertility restoration are possible in only a minority of patients. ${ }^{68}$

\section{Hematoma and perioperative vascular incidents}

The EHS guidelines on IH treatment in adults report that a significantly lower incidence of hematoma formation occurs following endoscopic versus open repairs. ${ }^{12}$ The occurrence of a hematoma is more clinically obvious at inspection when performing an open than a laparoendoscoic repair. The definition of a hematoma that would be clinically relevant in both open and laparoendoscopic surgery is lacking, which makes results difficult to compare. A "moderate" preperitoneal bleeding in laparoendoscopic repairs might be of the same magnitude as a wound hematoma that would be easily diagnosed in open repair.

No trials include hematoma as a primary outcome. If studies are planned that include hematoma formation, it is HerniaSurge opinion that only symptomatic hematomas should be considered a postoperative complication.

\section{Key questions}

KQ18.m Is hematoma formation related to hernia repair method or mesh use?

KQ18.n Are intraoperative bleeding and postoperative hematoma formation related to a surgeon's level of experience?

\section{Evidence in literature}

Nine reviews and meta-analyses have compared open with endoscopic IH procedures, however, not all report on hematoma formation. ${ }^{76-84}$

Several of the studies that did investigate the incidence of hematoma formation report it to be lower in hematoma incidence after endoscopic versus open hernia repair but do not cite exact differences. ${ }^{76,77}$

A 2003 meta-analysis, which included the mean incidence of hematoma formation from 33 trials, reported a significantly lower incidence after endoscopic versus open techniques. $^{82}$ This difference was attributed mainly to the TEP procedure which is associated with significantly less hematoma formation than are open repairs. No significant difference in hematoma formation incidence was found when TAPP and open repairs were compared. When Shouldice versus endoscopic and Lichtenstein versus endoscopic repairs were compared, a significantly lower incidence of hematoma formation was seen after endoscopic repairs. ${ }^{80-83}$

Another meta-analysis which included 3410 patients, compared Shouldice repair to open mesh repairs and to other open non-mesh repairs and reported no significant difference in the incidence of hematoma formation between Shouldice repairs versus either open mesh repair or non-mesh repair. ${ }^{85}$

A different meta-analysis compared open preperitoneal mesh repair with Lichtenstein repair and found no difference in hematoma incidence. ${ }^{86}$

Three other meta-analyses reported no difference in hematoma incidence amongst different types of open mesh repairs. $^{87-89}$

One RCT and three cohort studies have examined the influence of the endoscopic IH repair learning curve on postoperative complications including hematoma formation. $^{20,90-92}$

The RCT compared endoscopically operated patients with open-repair patients and investigated the effect of surgical residents' postgraduate level. Besides a difference in the incidence of hernia recurrence, no difference was found in overall complication rate. ${ }^{90}$

A retrospective cohort study investigated the learning curve influence on morbidity in laparoscopic IH repair (TAPP). ${ }^{92}$ No inter-group morbidity differences were noted

\footnotetext{
Statements

Statement Hematoma incidence is reduced after endoscopic IH repair

Statement There is inadequate medical evidence to link hematoma formation risk to a surgeon's level of experience. 
between those operated upon by young trainees under supervision and those operated upon by experienced surgeons. ${ }^{92}$ However, it is possible that some hematomas were missed owing to the study's retrospective design.

A prospective cohort study also investigated the impact of surgeons' experience in TEP endoscopic hernia repair patients. Significantly more complications were noted in surgeons' first 100 cases compared with those of more experienced surgeons. $^{91}$

This finding is mirrored by another prospective cohort study that reported a significant decrease in postoperative complication rates with enhanced learning curve experience with the TEP procedure ${ }^{20}$ (see also chapter 22). However, a direct comparison of hematoma formation incidence was not made.

\section{Discussion}

The clinical relevance of hematoma formation following $\mathrm{IH}$ repair is unclear, since there is no hematoma severity classification and hematoma-related interventions are usually not reported. Two cohort studies do report significant decreases in overall complications associated with progress along the TEP repair learning curve. However, this information is too indirect to allow conclusions about hematoma formation and surgeons' level of experience. In addition, other outcome measures must be weighed when considering which repair type to undertake.

\section{Anticoagulants}

Anticoagulants and platelet aggregation inhibitors lower the incidence of thromboembolic events perioperatively and postoperatively, and may also affect the incidence of hematoma formation after open or endoscopic IH repair.

\section{Key question}

KQ18.0 Which patients undergoing anticoagulant or antiplatelet therapy are at risk of significant hematoma formation following hernia repair?

\section{Evidence in literature}

Eight studies have examined anticoagulated hernia repair patients, five RCTs and three retrospective cohort studies. $^{93-100}$ Two of the RCTs were excluded from our analysis, because their results did not directly answer the KQs posed above. $^{95,} 96$ One retrospective cohort study has investigated the influence of platelet aggregation inhibitors on the incidence of hematoma formation. ${ }^{101}$

A 1981 study randomized otherwise healthy male adults undergoing open hernia repair to either prophylactic heparin $(5000 \mathrm{U} 1 \mathrm{~h}$ before surgery and every $12 \mathrm{~h}$ thereafter for 4 days) or placebo. ${ }^{94}$ One hematoma occurred in the 30 study patients. There were no thromboembolic complications. The study authors concluded that there were no significant inter-group differences.

A 1986 study randomized unilateral hernia patients to either prophylactic heparin (5000 $\mathrm{U}$ heparin $1 \mathrm{~h}$ before surgery and every $12 \mathrm{~h}$ for 4 days) or placebo. ${ }^{93}$ All patients were discharged on postoperative day 5 and a variety of hernia repair techniques were employed. Significantly more hematomas were seen in the heparin group.

Another study involving the Bassini-Lotheisen repair randomized to heparin at $5000 \mathrm{U} 2 \mathrm{~h}$ preoperatively and every $8 \mathrm{~h}$ for 5 days and placebo. ${ }^{96}$ Significantly more hematomas occurred in the heparin group. No thromboembolic complications occurred. The study authors concluded that heparin should be administered only to those with an increased thromboembolic risk. Notably, the heparin dose used was higher than that used in other comparable studies.

A 2000 retrospective study reviewed 465 patients undergoing Shouldice repair. Healthy patients did not receive anticoagulation. Prophylactic heparin was given to those with risk factors for thromboembolism or for a longer operation. A higher incidence of hematoma formation and surgical reintervention was seen in the heparin group. ${ }^{100}$

A case-matched retrospective cohort study from 2008 examined patients who developed hematomas after open IH repair and reported warfarin use as the only significant predictor of postoperative bleeding. ${ }^{98}$

\footnotetext{
Statement and recommendation

Perioperative management of patients on anticoagulant or

Statement

antiplatelet therapy should be governed by local or institutional protocol.

A cautious approach should be taken when operating on patients undergoing anticoagulant or antiplatelet therapy even after bridging with LMWH. The development of local protocols is suggested to guide management of these patients.
} 
Another 2008 study, done retrospectively, compared patients on warfarin with INRs in the 2-3 range with those in the 3-4 range, and reported that an INR of 3-4 was associated with an increased risk of postoperative hematoma formation $(p=0.03)$. The study authors concluded that IH repair can safely be done in patients on warfarin with an INR $<39.9$

A 2014 retrospective review of 1839 patients, including 40 who continued warfarin perioperatively, reported no significant difference in hematoma formation between these patients and those who had discontinued warfarin or a case-matched control group. ${ }^{102}$

One 2011 study investigated clopidogrel effects in 46 patients undergoing open or endoscopic hernia repair. Patients were divided into those who had received clopidogrel $<7$ days before operation and those who had received clopidogrel $\geq 7$ days before operation. No significant differences in bleeding complications were reported. ${ }^{101}$

\section{Key questions}

KQ18.p What are the risk factors for postoperative seroma formation?

KQ18.q Is there an association between open anterior repair method and postoperative seroma formation?

KQ18.r Do certain endoscopic or open preperitoneal techniques increase the risk of postoperative seroma formation?

KQ18.s Can the risk of postoperative seroma formation be reduced surgically?

KQ18.t Does drain usage reduce the risk of postoperative seroma formation?

KQ18.u Is there an association between hernia sac treatment modality and seroma/hematoma formation?

KQ18.v Does the use of abdominal binders or comparable wound compression devices prevent seroma/hematoma formation?
Statements
$\begin{array}{ll}\text { The risk factors for postoperative seroma formation include: } \\ \text { statement } & \text { scrotal hernia, coagulopathy and congestive liver failure. }\end{array}$
冈खण
Statement
Inverting and fixing the lax fascia transversalis during
laparoscopic repair of large direct hernia sacs may reduce the risk $\quad$ 冈 $\mathbf{\nabla} \square$
of seroma and hematoma formation.
Since clinically significant seroma formation following IH repair
Recommendation
is uncommon routine drain insertion after primary uncomplicated
repair is not suggested.
冈ख $\square$
Weak

\section{Discussion}

Most anticoagulant-related studies on hernia patients are dated and were performed before day surgery was common and during an era when patients spent several days postoperatively in hospital. In addition, patient activity levels between those admitted and those discharged are unclear. Stasis is a known risk factor for thromboembolic complications and patient mobilization levels are poorly described in most studies. In addition, operative techniques have changed over time. Therefore, the available study results generally do not apply to the patient groups of interest in the modern era.

\section{Seroma}

Seroma assessment in IH repair studies is hampered by the lack of standardized definitions for this condition. ${ }^{103}$ No trials include seroma as a primary outcome. If studies are planned that include seroma formation, it is our groups' opinion that only symptomatic seromas should be considered a postoperative complication.

\section{Evidence in literature}

The reported incidence of seroma formation after IH repair varies between 0.5 and $12.2 \%$. Seroma formation risk factors are as follows: coagulopathy, congestive liver diseases, and cardiac insufficiency. ${ }^{103,104}$

Several meta-analyses report that seroma formation incidence is significantly higher following endoscopic and laparoscopic (TAPP/TEP) versus open hernia repair. ${ }^{82-84,} 105$ A 2013 RCT confirmed this finding, although its clinical relevance is uncertain.

Another meta-analysis of mesh versus non-mesh open techniques across 13 RCTs found no significant difference in seroma formation incidence. ${ }^{106}$ Neither did a metaanalysis of 8 RCTs with 2919 patients comparing Lichtenstein with mesh-plug repair. ${ }^{89}$ Another study found that seromas were the most frequent complication after TAPP repair of scrotal hernias. ${ }^{107}$ An RCT comparing TAPP repair with titanized lightweight mesh versus TAPP repair with heavyweight mesh found significantly fewer seromas in the lightweight group. ${ }^{108}$ Two recent RCTs reported 
significantly more seromas following TEP versus TAPP repair. $^{109,110}$

Two studies found that seroma and hematoma formation incidence is lessened after TAPP and TEP repair of large
KQ18.y Is mesh migration-with the attendant risk of pain and severe complications-related to: mesh type, mesh shape, repair method, wound infection, or hernia type?

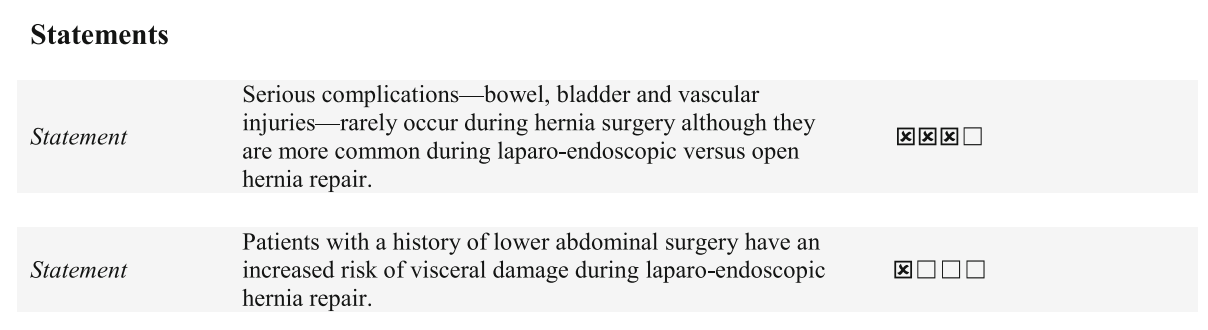

direct defects by inverting the lax fascia transversalis and closing the defect. ${ }^{111}{ }^{112}$ A recent meta-analysis of laparoscopic IH repair comparing mesh fixation with tissue glue versus tack fixation reported no difference in seroma formation. ${ }^{113}$ Another meta-analysis found a lower seroma incidence after glue mesh fixation compared with suture mesh fixation in open $\mathrm{IH}$ repair. ${ }^{47}$

Two studies found that drain insertion may prevent seroma formation especially in complicated and large scrotal hernias. ${ }^{114,115}$ Two other studies demonstrated that drain insertion after TEP repair may also reduce the incidence of seroma formation. ${ }^{116,117}$

Most seroma resolves spontaneously over 6-8 weeks. Since infections following seroma aspiration are regularly described, it is our groups' firm opinion that only symptomatic seromas be treated.

Studies of drain usage to prevent seroma formation provide conflicting results. One study of 100 patients found no benefit following open repair. However, another study involving 301 patients found fewer seromas after a 24-h drainage period. ${ }^{105,114}$

Since clinically significant seroma formation following $\mathrm{IH}$ repair is uncommon, our group recommends against routine drain insertion after primary uncomplicated repair.

There is no evidence that binders and other compression devices prevent hematoma and seroma formation.

\section{Infrequent complications}

\section{Key questions}

KQ18.w How common are serious complications during hernia surgery?

KQ18.x Are serious complications more common during endoscopic hernia surgery in patients with a history of previous abdominal surgery?

\section{Evidence in literature}

One meta-analysis comparing endoscopic to Lichtenstein and other open mesh techniques reported 13 incidents in 3640 operations $(0.4 \%)$ of potentially serious operative complications (defined as bowel, bladder, and vascular injuries). $^{83}$

Another meta-analysis comparing endoscopic to Shouldice and other non-mesh techniques reported 4 incidents in 5900 operations $(0.1 \%)$ of potentially serious operative complications. $^{84}$

A systematic review and meta-analysis of TEP versus Lichtenstein reported no inter-group differences in severe adverse events from the patient's perspective, although 1068 events - including chronic pain and recurrencesoccurred in 5397 patients. We calculated that, if chronic pain and recurrences had been excluded, 78 (1.4\%) severe events occurred. $^{76}$

In one study, infrequent serious intraoperative complications were reported to occur more frequently in endoscopic versus IH surgeries, although there was no overall morbidity difference. ${ }^{83}$

A Cochrane review of laparoscopic versus open techniques found that operative complications were uncommon, but more frequent, in the laparoscopic group. ${ }^{82}$ Another Cochrane review found an increased incidence of operative complications with the TAPP over the TEP approach. ${ }^{118}$ The study reported that serious events were bowel perforation, urinary bladder damage, and vascular injuries. $^{82-84,118}$

First trocar introduction during hernia operations has the potential to cause bowel and vascular injury. A variety of techniques including: use of blunt-tipped trocars, optical access trocars, and needlescopic 3-5-mm instruments have been studied, and are shown to reduce complications and improve safety. ${ }^{119-121}$ 
One study has shown that patients with a history of lower abdominal surgery are at increased risk for visceral injury during laparoscopic hernia operation. ${ }^{122}$

Vascular injuries at dissection and mesh fixation or suturing in the preperitoneal space typically involve the epigastric vessels or the aberrant obturator vessels crossing the Cooper ligament, the so-called corona mortis. ${ }^{123}$

Subcutaneous carbon dioxide emphysema can occur during TEP repair. This rare but serious condition affects the respiratory and/or cardiovascular system. ${ }^{124,125}$

Infrequent serious late complications related to mesh, mesh fixation, port-site hernia formation, and intra-abdominal adhesions have all been reported. ${ }^{12,118,126,127}$

Port-site hernias occur mostly after TAPP operations with a frequency of $0-3.7 \%$ according to a Cochrane review $^{118}$ and up to $8 \%$ after TAPP operations of recurrences after previous preperitoneal-mainly TAPP-repairs of primary hernias. ${ }^{126}$ Closure of port sites $\geq 1 \mathrm{~cm}$ is recommended. $^{12}$

The risk of intestinal obstruction after hernia surgery was calculated in a study based on data from the Swedish Hernia Register. Ninety patients-representing $0.3 \%$ of 33,275 operations on primary hernias-had intestinal obstruction considered to be related to the hernia operation. TAPP was the only operative technique associated with an increased relative risk of obstruction. ${ }^{127}$

Mesh complications (see chapter 10 on meshes)-excluding pain and problems related to the mesh itself or its fixation-are rare and can take years to develop. These generally are not mentioned in RCTs and only occasionally in meta-analyses. Clinical observations of these complications are most often published as case reports. Late mesh-related complications are associated most often with polypropylene meshes. Sometimes deep infections, including abscesses, develop around mesh. Erosion into hollow organs including the bowel or bladder and ingrowth and obstruction of the spermatic duct has been reported. ${ }^{128}$ Mesh migration into the abdominal cavity, the bowel or bladder or into the scrotum and mainly associated with mesh plugs in open techniques and preperitoneal meshes placed laparoscopically. ${ }^{129-131}$ Mesh exposed to the abdominal cavity through peritoneal defects (e.g., after hernia sac resections and peritoneal tears) may cause bowel adhesions resulting in bowel obstruction. ${ }^{132}$ Adhesions and obstruction caused by tacks have been reported. ${ }^{133}$

In a register study of postoperative complications and recurrence risks spanning 150,514 operations, those with complications such as hematoma and severe pain documented in the medical record at 30-day follow-up had a significantly increased relative risk (RR 1.23 and RR 1.84, respectively) of reoperation for recurrent hernia. ${ }^{134}$

\section{Discussion}

Serious complications related to hernia operations are rare. When they do occur, their details and descriptions are often published as secondary outcomes. Reviews of complications are often based on collections of individual cases in RCTs, retrospective follow-up cohorts, and case reports. Prospective registration of specific complications in a national registry is difficult, hampered by practical limitations on reporting of details and by compliance issues when reported. Secondary outcomes are frequently ill defined making comparisons difficult and potentially unreliable. These confounders should be considered when interpreting reviews on severe and/or rare complications.

\section{Mortality}

The mortality rate associated with elective hernia surgery is no higher than the mortality rate in the general population when compared to Cause-of-Death registers calculating expected deaths considering age and gender of the population, and often somewhat lower given patient selection criteria for operation, ${ }^{135}$ with high-risk patients being excluded from elective hernia repairs. This is not the case for emergent hernia repair. It is important to know the risk factors for incarceration and strangulation and patient characteristics such as medical history, age, and physical condition that place patients at increased risk of death.

\section{Key question}

KQ18.z What is the 30-day mortality rate following groin hernia repair? What are the causes of this mortality?

$\begin{array}{ll}\text { Statements } & \\ \text { Statement } & \begin{array}{l}\text { Death in the } 30 \text { days following inguinal hernia repair is very rare } \\ \text { and mainly associated with emergent repair. }\end{array} \\ \text { Statement } & \begin{array}{l}\text { Femoral hernias are associated with an increased likelihood of } \\ \text { emergent repair. }\end{array} \\ \text { Statement } & \begin{array}{l}\text { Mortality in the } 30 \text { days following elective hernia repair is mainly } \\ \text { related to medical comorbidities. }\end{array}\end{array}$ 


\section{Evidence in literature}

A 2011 systematic review summarized mortality rates following elective and emergent $\mathrm{IH}$ repairs. The review encompassed 85,585 operations reported in 14 publications over a 50 -year period. The average reported mortality was $0.5 \%$ (range $0-2 \%$ ) representing 470 patients. ${ }^{136}$

An average mortality rate of $5.8 \%$ was found for emergent IH repair. Of 7404 emergent hernia operations reported in 18 publications during the same study period, $22 \%$ were performed for femoral hernias and bowel resections were done in $14 \%$ of the emergent hernia operations. Factors associated with a statistically significant increase in morbidity and mortality included: age over 49 years, delayed presentation to hospital, presence of a femoral hernia, non-viable bowel, and ASA class above two. ${ }^{136}$

The INCA Trialists' Collaboration published a metaanalysis in 2011 on which approach-operation or observation-would be best for asymptomatic or mildly symptomatic male IH patients. A literature review and a Markov model of relevant parameters like mortality associated with elective or emergent hernia repair were incorporated into the meta-analysis. The mortality associated with elective IH was $0.2 \%$ (596 of 242,207 patients). The mortality associated with emergent $\mathrm{IH}$ repair (excluding femoral hernias) was $4 \%$ (715 of 18,092 patients). ${ }^{137}$

A 1996 study analyzed data from 30,675 IH operations performed on patients of all ages, during the period 1976-1986 in a geographically defined population. The study included data on the nine percent of patients who underwent an emergent operation. Of note, emergent operations were much more common in patients over 50 years of age. On average, emergent operation patients were older than elective operation patients and had a significantly greater postoperative mortality rate. No deaths were reported in day surgery patients. ${ }^{138}$

Another study on elective hernia repair in the elderly found a tenfold increase in mortality for non-agenarians versus octogenarians (3 versus $0.3 \%$, respectively). ${ }^{139}$

A Scottish audit of mortality following hernia surgery reported 91 fatalities (18 elective) in 28,760 inguinal and femoral hernia operations on a surgical ward or within 30 days of surgery during the period 1994-1997. Mortality was $0.8 \%$ following $\mathrm{IH}$ repair, and $3.1 \%$ following femoral hernia repair. Cardiopulmonary and neurological diseases were the principle comorbidities in the patient population. ${ }^{140}$

A cost-utility analysis of treatment options for IH done at Massachusetts General Hospital and Harvard Medical School analyzed data from over 1.5 million adult patients. Data from national databases and 51 RCTs were analyzed. A mathematical model derived from the data predicted that surgical mortality increased $8.7 \%$ per 1-year age increase. As an example, the calculated mortality risk for a 25 -yearold male operated on for primary IH was $0.0065 \%$ and was postulated to be 125 times higher $(0.81 \%)$ for a 65 -year-old male undergoing operation for a recurrent IH with obstruction. These calculations were found to be in agreement with outcomes seen in national hernia registries. ${ }^{141}$

From 1992 to 2005 , data on 107,838 groin hernias were entered in the Swedish Hernia Register. ${ }^{32}$ Five thousand two hundred and eighty of 104,911 (5.1\%) IHs were treated emergently compared with 1068 of 2927 (36.5\%) femoral hernias. Twenty-two percent of the operations in women were for femoral hernia versus $1 \%$ in men. In both men and women, operation for femoral hernia (including emergent operation) was associated with a sevenfold mortality increase. Mortality for elective hernia repair was not higher than the background mortality recorded in the national Cause-of-Death register, but increased sevenfold after emergent operations and 20-fold with bowel resection. Seventeen percent of women and $5.1 \%$ of men underwent emergent surgery. The overall standardized mortality ratio (observed/expected death in the population) within 30 days following hernia repair was 1.4 in men and 4.2 in women. In elective surgery, this ratio was lower, 0.67 and 0.85 , respectively. Cardiovascular disease accounted for $59 \%$ of the observed mortality in elective cases. $^{32}$

Another Swedish Hernia Register study looked at 3980 patients operated on for femoral hernia. Women were at increased risk for emergent operation, 40.6 versus $28.1 \%$ in men. Emergent femoral hernia operation was associated with a tenfold increase in mortality. The mortality risk of elective femoral hernia operation did not exceed that of the general population. ${ }^{22}$

A registry study of patients who died within 30 days of surgery examined causes of treatment delay and death. ${ }^{135}$ In $37 \%$ of patients with signs of bowel obstruction, documentation was missing on physical examination of the groin. These patients had an increased time-to-surgery when compared with patients with a palpable lump. Women and those with femoral hernias were significantly less likely to have a groin examination done.

A cohort study from the Danish Hernia Database of 29,033 elective groin hernia repairs analyzed: 30-day postoperative morbidity, prolonged length of stay, and death. Morbidity increased with age. Regional anesthesia was associated with an increased risk of complications. Thirty-day mortality following elective hernia repair was $0.12 \%{ }^{142}$ Another Danish multicenter study of 57,709 day surgery procedures reported no day-surgery-related deaths. ${ }^{143}$ 


\section{Discussion}

Mortality risk calculations and cause-of-death analyses require large data sets. Prospective registration of operations facilitates these calculations and reduces selection bias, thereby making the results more representative of the overall hernia surgery population. National registers are useful when calculating mortality risks, since the results can be verified and compared with national Cause-of-Death registers.

Emergent hernia surgery is associated with considerably increased mortality. Symptom duration before diagnosis patterns may differ depending on structures and organs involved and the type of repair performed. While certain predisposing neuroanatomic and technical factors can be avoided, chronic postoperative inguinal pain (CPIP) remains a complex challenge with several psychological, social, genetic, and behavioral influences.

\section{Key question}

KQ19P.a How is chronic pain defined? What is its prevalence after IH repair?

\section{Statement and Recommendation \\ Overall, the incidence of clinically significant chronic pain is \\ Statement in the 10 to $12 \%$ range, decreasing over time. Debilitating \\ chronic pain affecting normal daily activities or work ranges \\ from 0.5 to $6 \%$. \\ Recommendation \\ Chronic pain should be defined as $\geq$ bothersome moderate pain impacting daily activities lasting $\geq$ three months postoperatively.}

and treatment delays are factors that contribute to this increased mortality.

In elective hernia repair, medical comorbidities are the primary contributor to cause of death and must be considered when planning operations, especially in the elderly.

\section{Key question}

KQ19P.b What are the risk factors for CPIP?

\section{Statement}

CPIP risk factors include: young age, female gender, high preoperative pain, early high postoperative pain, recurrent hernia and open hernia repair.

\section{Chapter 19}

\section{Pain: prevention and treatment}

A. Wijsmuller, D. Chen, L. Liem, M. Loos, W. Reinpold, and S. Smedberg

\section{Pain prevention}

Definition of chronic postoperative inguinal pain (CPIP) Chronic pain is a frequent long-term complication following nearly all surgical procedures. However, there are no consensus definitions of exactly what constitutes chronic pain after specific operations. With IH repair, pain

\section{Evidence in literature}

\section{Definition of chronic pain}

In 1986, the International Association for the Study of Pain defined chronic pain as pain lasting more than 3 months. ${ }^{144}$ This definition was considered valid in epidemiological studies $^{145}$ and was used in randomized studies on hernia surgery, even if pain related to surgery was not part of the objectives when the definition was formulated.

However, with mesh hernia repairs, the inflammatory healing process may last longer than this defined 3-month interval due to tissue reaction to the mesh. Therefore, some systematic review authors have used pain lasting 6 months or more as a criterion for inclusion in their reviews. ${ }^{146}$ 
In spite of these varied interpretations of chronic pain, we recommend that the widely accepted $\geq 3$-month time period be used to define CPIP. We also recommend that the definition of CPIP includes a level of discomfort rated by the patient as $\geq$ moderate and impacting daily activities.

\section{Prevalence of chronic pain}

As noted in various guidelines, CPIP incidences vary from 0.7 to $>75 \% .^{104,147}$ The reported incidence of pain varies greatly due in part to the pain definition used in the guideline.

One RCT looking at Kugel versus Lichtenstein repair considered any visual analog score (VAS) of pain $>0$ at 3 months to be chronic pain, and reported incidences of 20.7 and $40.5 \%$, respectively. ${ }^{148}$ Another prospective follow-up study of hernia repairs performed during 1992-1993 (premesh) used different definitions. Mild pain was defined as occasional pain/discomfort, not limiting activity, with return-to-prehernia lifestyle. Moderate pain was defined as pain preventing return-to-preoperative activities. Severe pain was defined as pain incapacitating the patient frequently or interfering with daily activities. ${ }^{149}$

Follow-up data in this study and reporting on any groin/ inguinal pain within the last month found a $62.9 \%$ incidence at 1 year and a $53.6 \%$ incidence at 2 years. The incidence of moderate-to-severe pain was $11.9 \%$ at 1 year and $10.6 \%$ at 2 years.

A Danish Hernia Database 1-year questionnaire followup study found that $28.7 \%$ of patients reported hernia-area
Follow-up interval is an important metric, since pain tends to decrease over time. In the same Danish Hernia Database group, those with pain after 1 year were followed up after 6 years. After 6 years, less pain was reported by $75.8 \%$ of patients, the same pain by $16.7 \%$, and increased pain by $7.5 \% .^{53}$

In a meta-analysis of open preperitoneal versus Lichtenstein repair, chronic postoperative pain of greater than 6-month duration was found in 7.1 and $12.3 \%$, respectively. ${ }^{86}$ In another meta-analysis of TEP versus Lichtenstein, chronic postoperative pain of greater than 3 months duration was reported in 12.5 and $16.8 \%$, respectively. ${ }^{76}$

Meta-analyses and guidelines on surgical technique find that chronic pain occurs less frequently after endoscopic versus open procedures. ${ }^{83,84}$ Mesh use reduces the risk of chronic pain and surgical "fine tuning" on mesh choice and fixation method (or no fixation) may help to decrease its incidence (see chapters 10 and 11).

Overall, the incidence of clinically significant CPIP is in the $10-12 \%$ range, decreasing over time. ${ }^{53,147,151}$ Debilitating CPIP affecting normal daily activities or work ranges from 0.5 to $6 \%{ }^{147}$

Systematic reviews published within the last decade have generally found similar predictors for CPIP development. $^{104,146,151,152}$ The International Endohernia Society guidelines summarized from the literature: young age, female gender, preoperative pain level, and postoperative pain intensity as risk factors for chronic postoperative pain. ${ }^{104}$ One author has summarized risk factors for postherniorrhaphy inguinal pain (Table 3). ${ }^{152}$

Table 3. Risk factors for chronic post-herniorrhaphy inguinal pain

Preoperative:
Young age, female gender, high pain intensity level (inguinal/elsewhere), lower preoperative
optimism, impairment of everyday activities, operation for a recurrent hernia, genetic
predisposition (DQB ${ }^{*}$ 03:02 HLA haplotype).
Experimentally-induced pain:
High pain intensity to tonic heat stimulation can identify patients at risk for chronic pain.
Perioperative:
Less experienced surgeon/not dedicated hernia center, open repair technique, mesh type:
heavyweight, mesh fixation: suture, staple, ilio-inguinal nerve neurolysis in Lichtenstein repair.
Postoperative:
Postoperative complications (hematoma, infection), high early postoperative pain intensity,
lower perceived control over pain, sensory dysfunction in the groin

pain in the last month, $11 \%$ reported work- or leisure-activity impairment, and $4.5 \%$ had received medical treatment for pain. ${ }^{150}$
Population-based studies from the Swedish Hernia Register on long-term pain after hernia repair are in accord with the systematic reviews cited above. Pain interfering with daily activity occurred in 98 of 2421 (6\%) patients 
followed 1-6 years after the operation. Pain risk factors included: age below median, female gender, direct hernia, open technique, postoperative complications noted in patient files at 30-day review, recurrent hernia repair, and chronic pain of other origin in the 3 years prior to operation. ${ }^{153}$ Similar results were found in another register study of 2456 patients followed for 2-3 years. ${ }^{154}$

Another author, in a more holistic review, confirmed the same risk factors and added the significance of mental state, anxiety, and patient expectations. ${ }^{151}$

A prospective cohort study investigated psychological models for prediction of chronic postoperative pain after hernia surgery. These models are useful for predicting acute pain and in non-surgical contexts, for predicting transition from acute to chronic pain. A finding of higher pain intensity 1 -week postoperatively predicted higher pain intensity at 4 months. Lower preoperative optimism was an independent risk factor for chronic pain occurrence. ${ }^{155}$

A systematic review of predictive experimental pain studies of quantitative sensory testing investigated mechanical, thermal, and electrical stimuli. ${ }^{156}$ The review found that preoperative pain tests may predict $4-54 \%$ of the variance in postoperative pain occurrence. However, the review concluded that there is no simple reliable prognostic assessment method for postoperative pain. ${ }^{156}$

\section{Discussion}

A consensus on the definition of CPIP does not exist, although a time threshold has been suggested. Most IH literature uses a 3-month duration to define chronic pain. As discussed, for mesh-based repairs, a 6-month time frame may be more appropriate.

In a similar manner, discomfort severity that seems clinically meaningful has been set at a level of "bothersome moderate pain impacting daily activities." Using this cutoff, roughly $10-12 \%$ of patients experience this chronically after IH surgery.

Pain etiology does not enter into the definition of pain itself. However, etiology, treatment, prognosis, and, above all, prevention, is of utmost importance for the research into pain following IH surgery.

\section{Inguinal nerve anatomy}

\section{Introduction}

CPIP is thought to be primarily of a neuropathic origin. Therefore, knowledge of the most common inguinal nerve distribution patterns and variants is paramount in its prevention and treatment.

\section{Key question}

KQ19P.c What is the most common inguinal nerve distribution pattern and how common is it?

\section{Evidence in literature}

Several anatomic studies have been performed to elucidate the course of one or more of the inguinal nerves. ${ }^{157-170}$ Anesthesiology-based studies have been done to improve nerve block success. ${ }^{157-159}$ Surgical anatomical studies have been done in the hopes of preventing nerve injury during different approaches in this area. ${ }^{157,160-168}$ These anesthesiology-based and surgical anatomical studies report data on retroperitoneal and/or anterior nerve distribution patterns.

This section focuses on the most common course of the ilioinguinal (IIN), iliohypogastric (IHN), and the genitofemoral (GFN) nerves retroperitoneally over the quadratus lumborum and psoas muscles and anteriorly after they pierce the transverse abdominal muscle (TAM).

Pooled results of anatomical studies indicate that the IHN was present in 864 of 879 inguinal dissections $(98 \%$, range $60-100 \%) .{ }^{157,}{ }^{159-166,} 168$ The IIN was present in 1217 of 1443 dissections (84\%, range 56-100\%). ${ }^{157-169}$ The genital branch (GB) of the GFN was present in 256 of 258 dissections $(99 \%)^{160,165,166,170}$.

In $68 \%$ (578 of 844 ) of dissections, the IIN and IHN emerged separately lateral to the psoas muscle instead of emerging as a single trunk ${ }^{157-161,163,168}$. Thereafter, the IHN runs anteriorly over the quadratus lumborum muscle to pierce the TAM usually just cranial to the iliac crest, and runs shortly between the TAM and the internal oblique muscle (IOM) to pierce the IOM and run dorsally to the external oblique muscle (EOM). The IIN does the same, except that in most cases a part of its course is caudal to the iliac crest, anterior to the iliac muscle. ${ }^{160}$

The GFN emerges through the psoas muscle as a single branch in the majority of patients $(58 \%)$ and divides into femoral and genital branches anterior to the psoas muscle. ${ }^{165}$

In most patients, the IHN innervates the hypogastric region, after a course just cranial to the spermatic cord. Cutaneous innervation of the medial thigh, pubic, and scrotal/labial area and inguinal crease is provided jointly by the IIN and GB. The same applies to the motor innervation of the cremasteric muscle. When present, the IIN usually runs anteriorly and parallel to the spermatic cord. The course of the genital branch is usually laterocaudal at the level of the internal inguinal ring. ${ }^{166}$

The dorsal nerve branch of the pudendal nerve, which originates from $\mathrm{S} 2$ to $\mathrm{S} 4$, innervates the posterior scrotum/ 
labia. ${ }^{162}$ Data from a chronic pain patient series in which treatment consisted of triple neurectomy-that was extended to nerve tissue surrounding the vas deferens in some patients-suggest that the testicles are viscerally innervated by autonomic nerve fibers located with the lamina propria of the vas deferens originating from the deep pelvis plexus and referred to as paravasal nerves. ${ }^{171,172}$

Variations in the distribution pattern of inguinal nerves exist on several levels in the course of each nerve. Because of this logarithmic increase in different types of distribution patterns, a classic distribution pattern and its incidence cannot be determined.

\section{Key question}

KQ19P.d What are the most common variations in anterior inguinal nerve distribution patterns?

\section{Evidence in literature}

Common variations in inguinal nerve distribution patterns include a proximal common trunk for the IHN and IIN, an emergence of the GFN through the psoas muscle as two separate branches and variation in cutaneous innervation by the IIN and GB of the medial thigh, pubic, and scrotal/ quality anatomic study suggests that in these cases, the sensory component of the IIN follows the course of the GB after interconnections between the IIN and GB proximally at the height of the internal ring or at the lumbar level. ${ }^{165}$ Interconnections between all inguinal nerves have been described. Some studies note the absence of cutaneous innervation by the GB. ${ }^{165}$ One study found the GB in all dissections, but in 18 of 64 of those dissections (28\%), it did not contain sensory fibers for cutaneous innervation. ${ }^{165}$

Nerve management during open inguinal hernia repair

\section{Introduction}

Surgeons can either recognize or ignore the courses of the inguinal nerves during open IH repair. The IHN and IIN can be seen directly. The GB, running adjacent to the cremasteric vessels in the majority of cases, can be indirectly determined by the course of those vessels.

\section{Key question}

KQ19P.e Does a "nerve-recognition" approach reduce the incidence of acute and chronic pain following open inguinal hernia repair?

\section{Recommendation \\ Nerve anatomy awareness and recognition during chronic post-herniorrhaphy pain.

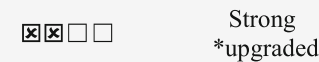

labial area and inguinal crease.

In 266 of 844 dissections (32\%, range 9-50\%), there is a single trunk for the IHN and IIN emerging laterally from behind the psoas muscle after which it usually divides somewhere after passing the quadratus lumborum muscle before piercing the IOM ${ }^{157-161,163,168}$. Notably in one study, in 44 of 256 dissections (17\%) with a common IHN/IIN trunk, the trunk divided beyond the anterior superior iliac spine (ASIS). In a subgroup, this trunk would divide after perforating through the aponeurosis of the EOM. ${ }^{168}$ This pattern may have been misinterpreted in other studies as an absent IIN, leading to an underestimation of the IINs true prevalence. One study describes that the GFN emerges through the psoas muscle as separate femoral and genital branches in 27 of 64 dissections $(42 \%){ }^{165}$

Several studies describe a variation with an absent IIN (range 7-44\%). ${ }^{158,165-167}$ In this case, sensory innervation of the medial thigh, pubic, and scrotal area and inguinal crease is mostly provided by the GB. ${ }^{158,}{ }^{165-167}$ A high-

\section{Evidence in literature}

A literature search was done for studies comparing only nerve-recognizing (N-R) with nerve-ignoring (N-I) approaches. Several studies that lacked a group in which nerves were ignored were excluded. ${ }^{173-176}$ One review was found $^{177}$ that included data from two cohort studies investigating the influence of an N-R versus and N-I approach. $^{178,179}$

A high-quality prospective multicentre cohort study compared the influence of preservation versus division of the IIN, IHN, and GB during open mesh herniorrhaphy. At 6 months postoperatively, the incidence of moderate-tosevere pain was $4.7 \%$ in $189 \mathrm{~N}-\mathrm{I}$ (no nerves identified) patients and $0 \%$ in 310 patients in whom all nerves were identified and preserved $(p=0.02){ }^{178}$

An older study compared chronic pain in N-I versus N-R McVay-repair patients. ${ }^{179} \mathrm{~A}$ four-point scale was used for symptom reporting $(1=$ no pain, $2=$ minor, $3=$ major, $4=$ persistent or disabling) with a follow-up of $>5$ years. Symptoms graded as 3 or 4 occurred in $3.7 \%$ of 297 N-I 
patients and $1.6 \%$ of 614 patients in whom all nerves were identified $(p<0.001)$.

The conclusion from both studies was that N-R (all three nerves) results in a significantly lower incidence of chronic pain.

\section{Discussion}

Both cited studies were observational and, therefore, "low" grade. In addition, in the McVay-repair study, only one surgeon performed all operations possibly resulting in a systematic bias and, therefore, a "very low" grade. The multicenter center study quality was "high." Overall, the evidence quality on the subject is "low." prophylactic inguinal neurectomy on chronic pain. A separate search was conducted for studies involving the IIN, the IHN, and the GB of the GFN.

\section{Key questions}

KQ19P.f Does prophylactic ilioinguinal nerve resection reduce pain incidence?

KQ19P.g Does prophylactic iliohypogastric nerve resection reduce pain incidence?

KQ19P.h Does prophylactic resection of the GB of the GFN reduce pain incidence?

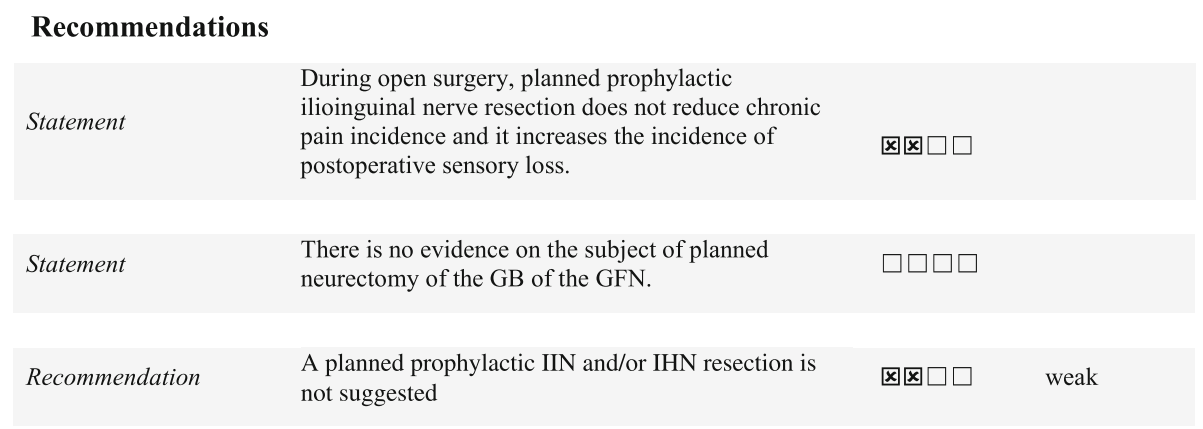

The GRADE system also assesses benefit-to-harm ratio. An N-R approach presumably improves operative outcomes by avoiding iatrogenic nerve injury, suture entrapment of nerves, and mesh-stimulated scarring with resultant nerve damage. Chronic pain leads to disability, repeated clinical encounters, consultations with anesthesiologists and other specialists, additional imaging studies, and extra costs in various ways. Although there is only a "low" level of medical evidence to support it, a strong recommendation for an $\mathrm{N}-\mathrm{R} /$ nerve-preservation approach seems justified, since this is associated with less chronic pain. To be clear, N-R/preservation in this context does NOT involve formal surgical dissection and identification, but rather simple recognition of nerves and their course as they are encountered.

\section{Prophylactic nerve resection}

\section{Introduction}

Medical literature describes different nerve handling techniques: nerve preservation, prophylactic neurectomy (resection, removal of a segment of the nerve along the inguinal canal), and pragmatic neurectomy (in cases of nerve injury or if mesh/nerve interference occurs). A search was conducted for studies investigating the influence of

\section{Ilioinguinal nerve}

\section{Evidence in literature}

Three meta-analyses ${ }^{177,} 180,181$ and seven RCTs ${ }^{182-188}$ have investigated the influence of IIN resection during open IH mesh repair. Studies investigating a pragmatic approach to perioperative inguinal nerve handling were excluded, since they did not compare a group in which the IIN was prophylactically neurectomized with a group in which it was preserved. ${ }^{175,178,189,190}$

A 2012 meta-analysis ${ }^{180}$ (MA-12) covers all prior RCTs $^{182-184,186,188}$ except two. ${ }^{185,187}$ One of these two was published after the 2012 meta-analysis. ${ }^{187}$ We have excluded it from our discussion due to a systematic bias resulting from inadequate study design. The other was also excluded from the meta-analysis and from our discussion because of selection bias. ${ }^{185}$ This meta-analysis reported no inter-group differences in chronic pain scores and numbness at 6- and 12-month postoperatively. ${ }^{180}$ However, increased sensory loss was reported at 6- and 12-month postoperatively following IIN resection.

These chronic pain outcomes were confirmed by another 2007 meta-analysis ${ }^{177}$ (MA-07). A 2011 meta-analysis (MA-11) of fewer studies than reference MA-12 reported a lower chronic pain incidence after IIN resection on the basis of fewer studies than were analyzed by reference 
MA-11. ${ }^{181}$ References MA-12 and MA-11 reported a moderate-to-high degree of heterogeneity with respect to chronic pain as an outcome measure and questioned the suitability of pooling results. This heterogeneity is present in several RCTs on the subject as well.

\section{Discussion}

The data from the meta-analyses were graded as moderate, since the included RCTs are moderately to highly heterogeneous. Evidence from several of the RCTs was also graded as moderate due to high loss-to-follow-up rates, ${ }^{182}$ small sample size, ${ }^{188}$ and possible selection bias. ${ }^{183}$

The GRADE system also assesses the benefit-to-harm ratio and treatment effect magnitude. Clear benefits of prophylactic IIN division/resection have not been reported. Possible harm might result from a higher rate of sensory loss but the clinical consequence of this loss is unclear. Therefore, the treatment effect magnitude is low, leaving us unable to make a strong recommendation. Finally, since study follow-up durations are 12-month maximum and delayed long-term painful conditions may occur following neurectomy (i.e., neuroma and deafferentation hypersensitivity), some of these adverse outcomes may have been missed in presently available series.

Iliohypogastric nerve

\section{Evidence in literature}

Two RCTs ${ }^{184,191}$ and three cohort studies ${ }^{174,192,193 \text { _but }}$ no reviews-were found comparing IHN neurectomy with IHN preservation.

One $\mathrm{RCT}^{191}$ and one cohort study ${ }^{193}$ were eliminated from this critical appraisal, the RCT, because of systematic bias due to comparison of two adjustments of the surgical technique. The cohort study was eliminated because of imprecision due to small sample size and possible selection bias.

Another RCT compared chronic pain incidence after tension-free self-gripping mesh repair with sutured Lichtenstein repair and recorded the type of nerve management. ${ }^{174}$ It too was omitted from our critical appraisal, since it did not report on the incidence of chronic pain after pure prophylactic IHN. Therefore, we deemed the evidence to justify prophylactic IHN neurectomy too indirect.
One $\mathrm{RCT}^{184}$ and one cohort study ${ }^{192}$ were critically appraised. Both reported no significant differences in the incidence of chronic pain or sensory loss 1-year postoperatively.

\section{Discussion}

Although the included RCT was well conducted, only one study of its type exists. Therefore, we considered the evidence quality to be moderate. The GRADE system assesses the benefit-to-harm ratio as well as the treatment effect magnitude and no benefit of prophylactic IHN resection has been reported. Furthermore, the development of a painful neuroma may have been missed as adverse outcome in the presently available series. In short, no positive treatment effect has been shown, making a strong recommendation for IHN resection unsupportable.

Genital branch of the genitofemoral nerve

\section{Evidence in literature}

No studies were found comparing prophylactic resection of the genital branch of the GFN with preservation of this nerve

\section{Pragmatic neurectomy}

\section{Introduction}

Pragmatic neurectomy refers to nerve resection or removal of a segment of a nerve that is "at risk." An "at-risk" nerve, in turn, is the one that has been damaged during surgery, is in danger of being traumatized due to interference with mesh position, or is likely to be included in the fibrotic process around mesh. Our search on this topic encompassed studies reporting on pain incidence following pragmatic resection of inguinal nerves.

\section{Key question}

KQ19P.i Does pragmatic (when nerve injury occurs or interferes with placement of the mesh) resection of inguinal nerves reduce pain incidence?

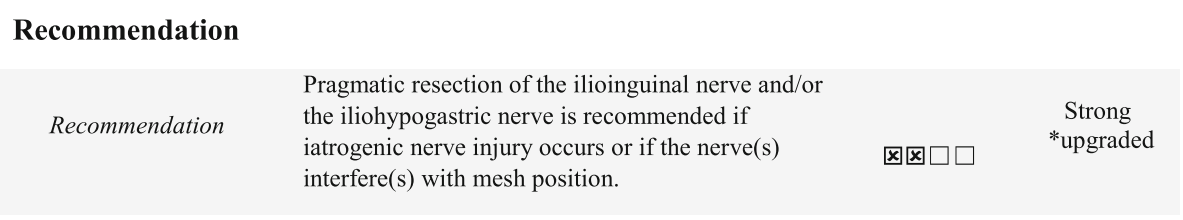




\section{Evidence in literature}

Our search found one cohort study comparing pragmatic neurectomy of "at-risk" nerves with a routine nervepreservation approach. ${ }^{173}$ Three other cohort studies involving only pragmatic neurectomy (without a nervepreservation control group) were identified, but excluded from analysis. ${ }^{178,189,194}$

The analyzed cohort study involved 364 Lichtenstein hernia repair patients who underwent neurectomies for "at-risk" nerves (traumatized, stretched, or interfered with by mesh). ${ }^{173}$ In a different group of 161 Lichtenstein patients, no recommendations were given for nerve handling and a nervepreservation routine was employed. There were no significant inter-group differences in the number of identified nerves. In the larger group, significantly more IHN resections were done (40 versus $12 \%$, respectively). No inter-group differences existed for IIN and GB resections. At 3-month postoperatively, there was significantly less pain in the nerves-at-risk resection group. It was concluded that, in Lichtenstein hernia repair patients, a pragmatic approach of at-risk neurectomy produced less chronic postoperative pain. ${ }^{173}$

Three other cohort studies were excluded from analysis. ${ }^{178,189,194}$ These studies investigated the influence of a pragmatic approach of "at-risk" nerves but did not compare this group to a group in which all nerves were preserved. For example, one study prospectively investigated a cohort of patients in which the influence of a pragmatic approach was investigated in all 172 patients: preservation of inguinal nerves unless it was felt that they would hinder the placement of the mesh or would become entrapped by the mesh or suture material, in which case, they were divided cleanly at the edge of the wound. ${ }^{194}$ They reported no differences in mean VAS score between the groups in which the IIN or IHN or GB was divided compared to the group in which all three nerves were preserved. Another study prospectively recorded type of nerve management in 973 primary IH patients undergoing elective Lichtenstein or Trabucco repair. ${ }^{178}$ No recommendations were made regarding identification or preservation of inguinal nerves. They reported no significant difference in moderate-tosevere pain at 6 months and 1 year between a group of 310 patients in which all nerves were identified and preserved and a group of 60 patients in which one or two nerves were divided and a group of 10 patients in which all nerves were divided. Furthermore, the third study prospectively recorded type of nerve management in 781 Lichtenstein and Shouldice repairs. ${ }^{189}$ They reported 12 patients with relevant chronic pain (VAS $>3$ ) at 5 years. In 11 of these 12 , IIN neurolysis had been performed during a Lichtenstein repair. The conclusion of this study was that mesh contact with a nerve removed from its natural bed should be avoided, supporting the notion that nerve resection is a better alternative to leaving an intact mobilized nerve in situ allowing mesh/nerve contact to occur.

\section{Discussion}

Overall, the strength of evidence on this topic is low. Only one cohort study exclusively compared a pragmatic resection of nerves 'at-risk' approach with a general routine of preservation, supporting pragmatic 'at-risk' neurectomy. ${ }^{173}$ One other cohort study indirectly supports the view that nerves should be pragmatically resected in case of iatrogenic damage or interference with the position of the mesh. ${ }^{178}$ Two other cohort studies report no downside to pragmatic neurectomy. The level of evidence is low, since only cohort studies were conducted.

When considering the benefit-to-harm ratio, IIN or IHN division does not influence the incidence of chronic pain. ${ }^{180}$ Sensory loss has been reported to increase following IIN neurectomy. ${ }^{180}$

Pragmatic resection of at-risk nerves seems justified and provides a better alternative to nerve preservation of an injured nerve or a nerve that interferes with mesh position.

\section{Hernia sac resection in indirect hernia}

\section{Introduction}

Hernia sac excision and ligation in indirect hernia management has been standard treatment for as long as radical hernia operations have been performed. The hernia sacpart of the parietal peritoneum-is well innervated. Early postoperative pain reduction might be achieved if the sac was resected or invaginated, but not ligated.

\section{Key question}

KQ19P.j Does hernia sac resection and ligation increase the incidence of acute and/or chronic pain?

\begin{abstract}
Statements
In indirect hernia management, sac invagination, without ligation, is associated with a reduction in the incidence of acute postoperative pain but an increased incidence of recurrence.

A possible increase in pain incidence should be weighed against a possible increase in recurrence incidence when considering indirect hernia sac ligation.

冈खि

冈凶田 


\section{Evidence in literature}

Until the 1970s, high ligation of the indirect hernia sac was generally considered a crucial part of the hernia repair in preventing recurrence. The 1978 edition of Hernia discussed this approach. ${ }^{195}$ A 1977 randomized study found no support for suturing of the peritoneum at abdominal wound closure. ${ }^{196}$

In the anterior abdominal wall, the peritoneum is innervated by parietal nerves also innervating the skin and muscles as opposed to the visceral and posterior peritoneum having a visceral innervation through the paravertebral ganglia and the vagal nerve. ${ }^{197}$ A prospective randomized study of hernia sac ligation of 110 indirect hernias in 105 male patients found that sac resection without ligation did not result in increased recurrences at 1-3-year follow-up. There was, however, a significant decrease in severe postoperative pain at 2-week and in moderate pain at 6-week follow-up in the non-ligated group. ${ }^{198}$ Increased pain after hernia sac ligation was attributed to peritoneal ischemia. A 2007 study randomized 477 Lichtenstein repair patients to ligated or non-ligated hernia sac groups. Significantly fewer non-ligated subjects had postoperative pain during a 30-day follow-up period

\section{Discussion}

Three RCTs have reached similar conclusions regarding postoperative pain. Indirect hernia sac invagination or resection without ligation results in less local pain in the immediate postoperative months and does not confer a long-term recurrence risk. There is a high level of evidence for this finding. However, a large register study with a 5-year follow-up term found that reoperation rates for recurrence were worse in invagination or resection without ligation patients. Therefore, our final recommendation received only a weak grade.

\section{Pubic pain and orchialgia}

\section{Introduction}

The periosteum is highly innervated, and if violated by fixation sutures or tacks, pain-intense and long-lastingis likely to occur. The resultant pain is somatic in nature, but, if intense, may be misinterpreted as neuropathic.

\section{Key question}

KQ19P.k Does mesh fixation to the pubic bone increase the incidence of acute and/or chronic pain?

\section{Recommendation}

and pain levels were significantly higher in the ligated group. ${ }^{199}$ Another study from 2014 randomized 167 Lichtenstein indirect hernia repair patients to one of three groups: sac dissected, not opened and inverted into the peritoneal cavity; sac excised without ligation; and sac resected and transfixed at the neck. ${ }^{200}$ Postoperative pain scores were $3.04,3.98$, and 4.06, respectively, significantly higher in the ligation group. Chronic pain and recurrence were not significantly different amongst the groups at 80-month follow-up.

A Swedish Hernia Registry study of 48,433 open anterior indirect IH repairs examined hernia sac management. $^{201}$ Hernia sac excision and ligation occurred in $49.5 \%$, invagination in $37.6 \%$, and division (the distal sac left in situ) in $12.9 \%$. Reoperations for recurrence occurred in $1.7,2.7$, and $1.7 \%$, respectively. However, within the subgroups of sutured repairs including a total of 6217 repairs, invagination of the sac did not increase the recurrence rate significantly.

\section{Evidence in literature}

While no studies focus specifically on periosteal pain, this entity is recognized as one of a number of causative factors in postherniorrhaphy pain syndromes. ${ }^{149,202-205}$

One study found at 2-year follow-up that non-neuropathic pain is the most common cause of chronic pain. Tenderness over the medial insertion of the inguinal ligament is the most common clinical finding. Therefore, authorities recommend against placing sutures in the area of the pubic bone. ${ }^{149}$ Another study mentions the issue of pain related to periosteal sutures but does not cite an incidence. $^{202}$

A third study found that 18 of 40 patients with nonneuropathic pain had periosteal pain probably caused by sutures. The 18 patients represented $12 \%$ of the total group of those with chronic postherniorrhaphy pain. ${ }^{203}$ Eight of these received an injection with lidocaine and corticosteroids resulting in pain reduction of more than $50 \%$ in their VAS scores. From a group of 237 Swedish Hernia Register patients operated on for persistent pain after groin 
hernia operations, 111 completed a questionnaire. Fourteen of these patients (13\%) had undergone pubic tubercle suture removal. ${ }^{205}$ Total or partial mesh removals were done at the same time and the results of suture removal alone were not calculated. The results within this subgroup did not significantly differ from the results in the whole study group of 111 patients answering the questionnaire, improvement being reported by $62 \%$ of the patients. In those with chronic postherniorrhaphy pain $(10-12 \%$ of IH repair patients), approximately $12-13 \%$ will have pain probably caused by tubercle mesh fixation, representing about $1 \%$ of chronic pain cases.

\section{Discussion}

Despite this being an uncommon problem and the subject of a limited number of publications, pain due to pubic bone periosteal interference is an important issue with a significant impact on patient wellbeing. Our recommendation on the subject is, therefore, ungraded to strong.

In open anterior mesh repairs, the mesh overlapping the medial pubic tubercle is, in fact, an "onlay" mesh part, meaning that local preparation for mesh placement is important. Overlap should compensate for mesh shrinkage. ${ }^{206}$ Deeply positioned sutures for medial mesh fixation should be avoided, and if local treatment with anesthetics and corticosteroids fail, surgical suture removal must be considered. ${ }^{203}$

The sensitive pubic bone area is also reachable via the preperitoneal route. The pectineal ligament along the pubic bone's superior ramus is commonly used for suture fixation. This ligament thins medially and sutures or tacks in that area may reach periosteum. However, no publications specifically address this issue.

\section{Orchialgia}

\section{Introduction}

Few CPIP patients develop testicular pain. If it develops, however, it can be debilitating and adversely affect sexual function and quality of life.

\section{Key question}

KQ19P.I What percentage of CPIP patients have orchialgia?

\section{Evidence in literature}

Orchialgia results from damage to the testicle's visceral innervation. These nerves run along the spermatic vessels and are derived from paravertebral ganglia mainly at the kidney level. It is known that ipsilateral orchialgia occurs in $6-44 \%$ of kidney donors. ${ }^{207,}{ }^{208}$ Laparoscopic standard procedure involves an en bloc dissection of the ureter and gonadal vein divided at the level of the iliac vessels' crossing. ${ }^{207}$ Orchialgia does not occur if a gonadal-veinsparing approach is used (so as not to disturb the vessel below the point of ligation at the renal vein). A study of genitofemoral neurectomy in 23 patients found that three of six patients with persistent neuralgia had significant orchialgia. None of the patients who had significant pain relief after neurectomy had preoperative testicular pain. ${ }^{209}$ Another study found that orchialgia was not affected by laparoscopic triple neurectomy in refractory neuropathic inguinodynia, because the testes have a separate innervation. ${ }^{210}$

Testicular pain occurs after open and laparoscopic hernia operations. Etiologies include: spermatic cord trauma, inflammation and fibrosis due to mesh ingrowth, and cord strangulation at the passage through a cut slit in the mesh.

Note that publications on testicular problems that do not cite patient numbers are excluded from our discussion.

A meta-analysis on endoscopic versus open mesh and non-mesh techniques found testicular atrophy or "problems" in 51 of 7622 operations $(0.7 \%)$ with no statistical significant inter-group differences in articles reporting on this issue. ${ }^{83,}{ }^{84}$ Chronic postoperative pain was reported in 598 of 8524 operations (7\%), endoscopic 5\%, and open $9 \%$. Orchialgia and testicular problems represented $10 \%$ of the chronic groin pain cases. ${ }^{83,84} \mathrm{~A}$ systematic review found the incidence of testicular pain to be 1-6\%. In another article reporting on those with severe or very severe groin pain, 22 of $120(18 \%)$ had testicular pain and 2.6-4.5\% had testicular atrophy. ${ }^{146} \mathrm{~A}$ meta-analysis of open preperitoneal versus Lichtenstein repair that included 2860 patients found testicular problems in 1.3 and $1.9 \%$, respectively, chronic pain in 7.1 and $12.3 \%$, respectively, for a total of $1.6 \%$ incidence of testicular problems and a $9.8 \%$ incidence of chronic pain. ${ }^{86}$ In four meta-analyses on lightweight versus heavyweight mesh in open hernia surgery published from 2011 to 2013, testicular problems

\section{Recommendation}


were all reported in the same four publications. There were no significant differences in testicular problems between groups. One of the meta-analyses reported testicular atrophy in $0.8 \%$ of patients and chronic pain in $11.2 \% .^{60}$ Irrespective of surgical technique, the incidence of orchialgia is about the same, roughly $10 \%$ of the incidence of CPIP.

\section{New directions in acute/chronic pain prevention.}

\section{Introduction}

Preoperative and intraoperative methods (various medications, psychological treatments and preparation, and educational programs) have been used in attempts to prevent chronic pain. Many of the techniques hinge on surveying psychological risk factors for chronic pain after hernia operations. A few psychologically based studies examining counseling or education have been published that related to hernia surgery.

\section{Key question}

KQ19P.m Can preoperative and perioperative topical and systemic medications reduce the incidence of chronic pain? KQ19P.n Can chronic postoperative pain be prevented or reduced by preoperative information and psychological preparation?
One systematic review on preemptive analgesia concluded that it can reduce acute postoperative pain. It also commented on the need for additional studies on gabapentin and pregabalin before recommending their use in chronic pain prophylaxis. ${ }^{214}$ However, the review summarized, the cause of chronic pain being multifactorial, that a combination of intraoperative and postoperative pain therapy is needed to minimize the risk of developing chronic pain. $^{214}$

Another systematic review found no evidence to support preemptive analgesia use in chronic postsurgical pain prevention. $^{215}$

A Cochrane review on high-dose topical capsaicin on intact skin for chronic neuropathic pain from postherpetic neuralgia and HIV neuropathy found a significant benefit during a 12 -week study period. ${ }^{216}$ One study of wound instillation of capsaicin showed superior analgesia versus placebo in the 3-4 days following IH repair. ${ }^{217}$ Follow-up at 2.5 years revealed that 5 of 20 patients in the capsaicin group had hyperalgesia compared to 1 of 16 in the placebo group. This trend did not rise to the level of statistical significance. ${ }^{218}$

Etanercept-a tumor necrosis factor-alpha inhibitorhas the potential to inhibit neuropathic pain. When this agent was used to treat postamputation pain, 5 of 6 soldiers in whom it was employed reported improvement. ${ }^{219}$ In a

\section{Statement}

\section{Perioperative prevention of chronic pain}

\section{Evidence in literature}

A review on persistent postsurgical pain concluded that nerve injury is the major factor causing chronic pain. It further surmised that preempting neuropathic pain requires a different approach from that needed for preventing inflammatory pain. ${ }^{211}$ Preemptive analgesia attempts to reduce sensory input from the surgical trauma causing sensitization and an increased risk of chronic postoperative pain. Analgesia timing is considered to be crucial. $^{212}$

A systematic review and meta-analysis including 11 clinical trials on perioperative administration of gabapentin or pregabalin found that they were effective in reducing the incidence of chronic postsurgical pain. ${ }^{213}$ Of eight gabapentin trials, four (one of which was a hernia repair study $^{211}$ ) reported lower incidence of pain and/or lower analgesic requirement $>2$ months after surgery. So did all three pregabalin trials. subsequent multicenter randomized study, etanercept was given 90 min before IH surgery and reduced postoperative pain by some measures. However, the effect was small, transient, and not statistically significant. ${ }^{220}$

\section{Preoperative education and psychological preparation}

\section{Evidence in literature}

In a systematic review of experimental pain studies investigating quantitative sensory testing, the variance in postoperative pain could be predicted in $4-54 \%$ of patients. Preoperative psychometric evaluations of vulnerability, anxiety, depression, and pain catastrophizing were less predictive. ${ }^{156}$ Numerous studies from a variety of disciplines have identified psychological risk factors for chronic pain. ${ }^{155}, 214,221-224$

Few studies exist on preoperative preparation and education of patients to reduce chronic postoperative pain. A prospective randomized study did evaluate the influence of 
a preoperative informative video before hernia surgery on postoperative quality of life. A benefit was noted at 3-month follow-up, but no difference was detectable at 6 months. ${ }^{225}$ The effect of music and music combined with therapeutic suggestions was studied in a prospective randomized manner in day case varicose vein and IH surgery. Either intervention demonstrated a modest effect on patients' pain in the immediate postoperative period. Longterm effects were not studied. ${ }^{226}$ The effect of postoperative education after hernia surgery was studied in a prospective randomized fashion. No difference in postoperative pain or return-to-work interval was found, but a small statistically significant benefit on pain-when-moving was found on postoperative day \#7. However, the study authors found no reason to recommend a change in standard practice regarding postoperative counseling. ${ }^{227}$

\section{Discussion}

The perioperative use of medications to prevent chronic pain has focused mainly on blocking central sensitization and the development of neuropathic pain. Gabapentin is the best studied and has a documented short-term benefit. In general, these benefits fade after 1-6 months. Pregabalin, though less well studied, has a similar effect. Capsaicin and etanercept have no proven long-term effect on chronic

\section{Pain treatment}

Chronic postoperative pain treatment after inguinal hernia repair

\section{Introduction}

Chronic pain is a significant complication after IH surgery leading to disability, dissatisfaction, and impaired productivity and quality of life. Despite a wide array of general pain treatment options, management of this burdensome condition remains challenging. Due to a paucity of evidence-based data of the subject and heterogeneous patient populations and pathologies, expert opinion plays a vital role in decision-making. For this reason, nearly all statements in the section below are only weakly supported but do represent a blend of an exhaustive literature review and expert opinion. The recommendations reflect current stateof-the-art and are important for clinicians and patients dealing with chronic pain.

\section{Key question}

KQ19T.a How should inguinal hernia repair patients with immediate, severe, postoperative pain be treated?

\section{Recommendation \\ Immediate severe/excruciating postoperative pain raises the possibility of vascular or nerve injury. Early re- operation on the same day is suggested to either exclude or manage these complications.}

pain. So-called "preventive analgesia" through multimodal approaches is predicated on the assumption that the only way to prevent central sensitization is to completely block any painful input from the surgical wound from time-ofincision to final wound healing. ${ }^{211,212}$

Preoperative psychological predispositions toward the development of chronic pain have been investigated in several surgical disciplines. This field remains poorly studied. The limited data available suggest only minor short-term effects on postoperative pain and no benefit in chronic pain prevention.

\section{Evidence in literature}

There is clear evidence that acute postoperative pain is a risk factor for chronic pain development. Early adequate pain management is vital to reduce the risk of conversion to chronic pain. ${ }^{228,} 229$ The existing guidelines of prevention and chronic pain management after IH repair address the issue of whether immediate reoperation is necessary. ${ }^{147}$

No studies exist on the appropriate treatment of acute severe pain after IH repair. In particular, no studies address whether early reoperation with neurectomy prevents chronic pain. From a purely pragmatic perspective; 
however, patients with immediate severe neuropathic pain postoperatively may be offered reoperation with neurectomy if appropriate.

\section{Key question}

KQ19T.b What should the initial approach be to IH repair patients with chronic postoperative pain (pain still present $>3$ months after surgery) (see treatment algorithm)? conservative modalities and pharmacological options (e.g., TCAs, SSRIs, gabapentin, and pregabalin). Then, nerve blocks and peripheral nerve stimulation were advocated as necessary. However, they acknowledged that these modalities are often ineffective and that surgical neurectomy provides the best results. Although the study group did not research the issue, the question then becomes which patients are suitable for neurectomy? They proposed a conservative treatment period of at least 6 months before neurectomy is done.

\section{Recommendation}

$\begin{array}{ll} & \text { A multidisciplinary team is suggested to manage } \\ \text { chronic pain patients. } & \text { Pharmacologic and interventional measures - including } \\ \text { Recommendation } & \begin{array}{l}\text { diagnostic and therapeutic nerve blocks - should } \\ \text { continue for a minimum of three months (minimum of } \\ \text { six months after hernia surgery). }\end{array}\end{array}$

\section{Evidence in literature}

Our search identified seven reviews describing different treatment options for chronic pain patients. $151,157,230-240$ A 1988 landmark paper presented a treatment algorithm for chronic pain after IH repair. ${ }^{230}$ The study authors concluded that pain persisting for 2-3 months required further investigation and treatment, starting with an IIN block at the ASIS. If this reduced pain to some extent, a repeat nerve block could be done, since repeated injections may interrupt the pain cycle. If an IIN block is ineffective, an anesthesiologist may be asked to perform a paravertebral block of the genitofemoral nerve. If transient pain reduction is achieved, a repeat block or neurectomy of the IIN or GB of the GFN is the next recommended step. If a block does not result in pain reduction, a course of pharmacological and adjunctive non-pharmacological therapy (i.e., psychotherapy, hypnosis, behavioral therapy, biofeedback, and acupuncture) is advocated.

A systematic review of CPIP management was done in $2005 .^{231}$ It concluded that neurectomy relieved chronic pain but also that studies demonstrating this finding suffered from methodological flaws. In particular, a clear pre, intra, and postoperative assessment was deemed necessary to provide a better understanding of therapeutic options.

Another study group emphasized that there is a broad differential diagnosis for chronic inguinal pain following IH repair. ${ }^{232}$ Examination with ultrasonography or magnetic resonance imaging may provide useful information and may detect recurrence, meshoma, or non-hernia-related causes of pain. This group suggested an initial trial of
In 2011, the international guidelines for the prevention and treatment of CPIP were published, providing consensus statements on best-available clinical recommendations. ${ }^{147}$ The guidelines advised a 1-year expectant period before remedial surgical treatment to allow the mesh/tissue interface inflammatory response to diminish. The guidelines further recommended a triple neurectomy if neurectomy was done. After endoscopic repair, a transabdominal or retroperitoneal approach was urged to remove the proximal parts of the nerves. They also concluded that the intramuscular part of the IHN should be resected during an open triple neurectomy.

More recently, others have proposed an algorithm for CPIP using the Delphian consensus method. ${ }^{233}$ They urge an expectant phase and-after recurrence has been excluded by imaging-referral to a multidisciplinary pain management team. If this approach fails, triple neurectomy and/or mesh explantation by an expert hernia surgeon is advocated.

In 2014, two CPIP reviews were published. One emphasized the complexity of, and the need for individualization in, treatment schemes making definitive broadly applicable treatment algorithms difficult to compose. ${ }^{152}$ The other concluded that while neurectomy provides the best results, improved studies with long-term outcome measures should be initiated. ${ }^{234}$

Most recently, data on 105 CPIP patients were published. On the basis of history, physical examination, and imaging, the 105 were partitioned into neuropathic (37) and non-neuropathic (68) pain groups. Twenty-eight underwent intervention with ultrasound-guided nerve blocks. Permanent pain reduction was achieved in 18 of the $28(62 \%)$. Implantable peripheral nerve stimulators were placed in 6 
of 28 who had temporary pain reduction following nerve block. Overall, this approach led to pain reduction in 24 of $28(83 \%)$ patients. $^{235}$

\section{Discussion}

A stepwise multidisciplinary approach starting with minimally invasive measures like analgesics and nerve blocks is advocated in all studies. Neurectomy seems reasonable after a minimum waiting period of 6 months without an adequate response to other therapy. Again, a paucity of evidence in this important subject area allows only a weak grade for the recommendation.

\section{Key question}

KQ19T.c Does non-pharmacological treatment work in CPIP? term pain reduction with gabapentin. ${ }^{237}$ However, the general pain literature on neuropathic pain (NeuPSIG guideline, http://www.neupsig.org/) does provide a stepwise treatment scheme. ${ }^{238}$

Limited evidence exists for the use of lidocaine and capsaicin patches in CPIP patients. One crossover trial of 21 patients found no benefit for lidocaine patches. ${ }^{239}$ Another study failed to show a statistically significant benefit of the capsaicin patch, although there was a trend toward less pain in the capsaicin group at 1 month. ${ }^{240}$

\section{Discussion}

There is little in the medical literature on non-pharmacological treatment options for CPIP. Lidocaine and capsaicin patches have not been proven to be effective for this condition.

\section{Statement}

Statement No benefit has been shown for lidocaine and capsaicin patch treatment of CPIP.

\section{Evidence in literature}

No studies exist on non-pharmacological treatments (e.g., physical therapy, acupuncture, and mind-body therapy) for CPIP, although there are some general articles on chronic pain modification and improvement by cognitive and emotional means. $^{236}$

\section{Key question}

KQ19T.d What is the effect of non-surgical interventional treatment on chronic pain after IH repair?

\section{Statements

There is insufficient evidence of the diagnostic and
therapeutic value of nerve blocks in chronic pain after
inguinal hernia repair. In clinical practice however, nerve $\quad$ Statement $\quad \begin{aligned} & \text { blocks can be useful in the diagnostic and therapeutic } \\ & \text { management of chronic pain after inguinal hernia repair. }\end{aligned}$ \\ Statement Pulsed radio frequency ablation may be an effective treatment for chronic pain after inguinal hernia repair. \\ ख๐० \\ $凶 \square \square \square$}

Remarkably, even pharmacological treatments (e.g. NSAIDs, acetaminophen/paracetamol, TCAs, SSRIs, gabapentin, pregabalin, and opioids) of CPIP have rarely been studied. Only one relevant case report describes long-

\section{Evidence in literature}

Limited evidence exists for the use of local nerve blocks for CPIP treatment. No studies have investigated whether nociceptive pain can be reliably distinguished from 
neuropathic pain. Only three studies have examined the effect of local anesthesia on chronic pain. ${ }^{241-243}$

The most notable of these is a small double-blind RCT demonstrating that ultrasound-guided IIN and IHN blocks did not produce pain relief. ${ }^{241}$ It is not clear from the study what percentage of patients had improperly placed nerve blocks despite ultrasound guidance.

A larger uncontrolled, retrospective study of 43 patients used local anesthetic and a corticosteroid injection with nerve stimulator or ultrasound guidance. Thirty-two percent of the patients were relieved of moderate-to-severe pain and nerve blocks, done as described, were considered to be an effective treatment modality. ${ }^{242}$

A case series describes CT-guided GFN block as an effective diagnostic and therapeutic option. ${ }^{243}$

Neuroablative techniques for chronic pain relief have been described. ${ }^{244-253}$ Cryoablation for chronic inguinal pain has been the subject of two case studies involving a total of ten patients. ${ }^{244,249}$ One of these studies reported a landmark) can be made. However, multiple authorities consider that nerve blocks serve an important diagnostic function and can be effective in the treatment of CPIP.

Cryoablation has been the subject of a few case reports involving few patients and limited follow-up. The initial positive results should be viewed accordingly.

All available studies on neuromodulation for CPIP cite sustained pain relief, quality-of-life improvement, and/or analgesic use reduction or cessation. However, these studies have significant limitations, such as retrospective design, case report or series design, lack of control group, short follow-up time, and no report of adverse events or complications. Presently, there is weak preliminary information in support of neuromodulation to treat CPIP.

\section{Key question}

KQ19T.e Is mesh removal without intentional neurectomy an effective treatment for chronic pain after IH repair?

\section{Statement \\ Statement There is insufficient evidence to support mesh removal alone without neurectomy in patients with CPIP}

$77.5 \%$ mean overall pain reduction with an average followup period of 8 months. ${ }^{244}$ Another successful cryoablation case involved a patient with chronic inguinal pain but no prior hernia surgery. ${ }^{249}$

The effects of peripheral nerve stimulation (PNS), dorsal root ganglion stimulation (DRGS), and spinal cord stimulation (SCS) on CPIP are new to the pain management field. Our literature search of PubMed and Embase yielded 117 articles using the terms "inguinal pain postherniorrhaphy." Excluding transcutaneous electrical nerve stimulation studies left 11 articles involving 64 patients undergoing PNS, SCS, or DRGS. The largest of these involved DRGS and pain relief of $76.8 \pm 8.2 \%(n=10)$ with a follow-up period of $17.4 \pm 5.7$ weeks. ${ }^{254}$ Another small study with a follow-up period of 12 months reported pain relief of $>75 \%$ and reduced pain medication intake with SCS. ${ }^{255}$ The other articles in this subject area are case reports or small case series.

\section{Discussion}

Few studies exist on nerve blocks for CPIP; therefore, no evidence-based recommendations for preferred technique (ultrasound-guided, neuro-stimulator directed, anatomic

\section{Evidence in literature}

Mesh removal can be considered if nociceptive pain due to mesh occurs. Compression of adjacent structures like the spermatic cord and surrounding inflammation is thought to be the mechanism of this pain. Often mesh is wrinkled and fibrotic causing pain in certain positions like sitting. However, some patients have pain that is both nociceptive and neuropathic. ${ }^{256}$ Therefore, the effect of mesh removal without neurectomy is difficult to interpret.

After a literature search, we identified 120 papers and considered nine to be suitable/eligible for inclusion in our review/discussion. ${ }^{205,}{ }^{257-264}$ In total, 278 operations for mesh removal were included. Five studies were retrospective case series. ${ }^{257-259,261,263}$ Efficacy rates in significantly reducing or eliminating pain ranged from 60 to $100 \%$. Data on the effect of mesh removal alone cannot easily be extracted, since the majority combined mesh removal with a tailored or triple neurectomy (82\%). Iatrogenic nerve injury during mesh removal was often an indication for simultaneous neurectomy. However, Campanelli et al. routinely performed a neurectomy. ${ }^{263}$ 


\section{Discussion}

Given the limitations of the literature on this subject, no conclusions can be reached regarding mesh removal sans neurectomy.

\section{Key question}

KQ19T.f What type of neurectomy should be performed in patients with chronic neuropathic pain (> 3 months) after IH repair?

\section{Discussion}

The literature quality on this subject area is poor, resulting in weakly supported recommendations. Given that mesh removal and neurectomy and the decisions around these procedures are complex, this will likely be the situation for some time. A high level of expertise and experience is required for positive outcomes. Neurectomy type is probably a secondary consideration relative to the selection of appropriate patients likely to benefit from nerve resection.

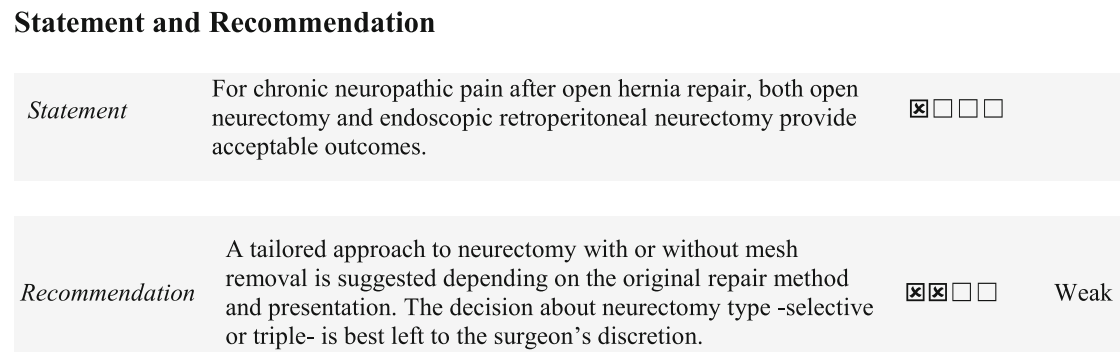

\section{Evidence in literature}

High level evidence is lacking. In total, 25 papers were identified. ${ }^{171,210,257-276}$ Most are retrospective case series. There are only two prospective studies. ${ }^{260,} 265$ The first one describes detailed preoperative and postoperative characteristics using mesh removal and a selective neurectomy. The second reports on 20 cases treated by endoscopic triple neurectomy (success rate 100\%). There are no studies comparing tailored and triple neurectomies. The results of endoscopic triple neurectomies were reported in seven case series/studies/trials. ${ }^{171,172,210,259,263,265,266}$ The remaining 18 studies reported on patients treated with a tailored neurectomy. ${ }^{257,258,260-262,264,267-277}$

The reported outcomes of triple neurectomy operations range from an $85-100 \%$ pain reduction. Selective single or double neurectomy studies generally report lower success rates.

Numerous confounding factors prohibit firm conclusions regarding a preferred neurectomy technique. First of all, most of the triple neurectomy data are derived from a single institute with reports including sequentially accumulated data. Furthermore, pain scores, follow-up, questionnaire, and neurologic examination techniques are inconsistent and mostly absent. Therefore, the heterogeneity in patient data prohibits firm conclusions.

\section{Chapter 20}

\section{Recurrent inguinal hernias}

H. Tran, D. Weyhe, and F. Berrevoet

\section{Introduction}

Recurrent inguinal hernia clearly still is a major health problem. It is estimated that, worldwide, approximately 20 million primary IH operations are performed annually. ${ }^{278}$ Recurrence rates in this same population can be as high as $15 \%{ }^{279}$ This figure is difficult to pinpoint, since recurrence rates vary with length of follow-up. ${ }^{280}$ Regardless, vast resources are committed to this problem.

Over 35 years after the introduction of mesh and 25 years after the first laparoendoscopic IH repair was performed, recurrence rates when compared to open-repair (tissue) techniques have not consistently decreased worldwide. $^{49,281,282}$ This situation needs improvement. Reorienting our thinking, such that recurrence is routinely considered a complication rather than an expectation is a necessary first step. 
Since recurrence rates are difficult to know, reoperation rates are used as a proxy, with the assumption that recurrences are up to twice as common as reoperations. ${ }^{11}$ In a 2014 long-term Danish observational study, the reoperation rate after primary Lichtenstein repair was 2.4 and 3.3\% after laparoscopic repair. ${ }^{281}$ A 2011 Swedish study found the cumulative 24 -month reoperation rate to be $1.7 \%$ for primary repair and $4.6 \%$ for recurrent repair. ${ }^{11}$ In Australia, the recurrence rate following $\mathrm{IH}$ repair is estimated at $7.9 \%$, and appears unchanged over 2 decades. ${ }^{283}$ This is, perhaps, disappointing, since, in Australia in 2014, 51\% of IH repairs were done laparoscopically, compared with $20 \%$ in 2000 . $^{284}$

Promisingly though, in highly specialized centers, $1 \%$ long-term recurrence rates have been achieved. ${ }^{285}$ These same investigators have found that the recurrence rate for laparoscopic recurrent IH repair after failed anterior repair approaches that of primary hernia repair. This strongly supports the notion that hernia surgery specialization may have a positive impact on outcomes, particularly recurrence rates. ${ }^{286}$

\section{Key question}

KQ20.a Are recurrence rates still too high despite innovations like endoscopic repair, anterior preperitoneal repair, and new mesh prosthetics? another. $^{288}$ These data suggest that virtually all $\mathrm{IH}$ patients-whether primary or recurrent-will require repair, usually because of pain or discomfort.

A 2014 cohort study of 1032 patients undergoing IH repair in the 16 months after the adoption of watchful waiting for asymptomatic or minimally symptomatic hernia compared with 978 in the 16 months before the adoption of watchful waiting showed a higher incidence of emergency repair (5.5 vs $3.6 \%, 95 \%$ confidence interval: $1.03-2.47)$, a higher adverse events rate (18.5 vs $4.7 \%$, adjusted OR: 3.68, 95\% CI 2.04-6.63), and higher mortality (5.4 vs $0.1 \%, p<0.001$, Fisher's exact test). ${ }^{289}$

Currently, there is no evidence on either watchful waiting or elective repair for those with recurrent IHs. Discussions about, and plans for repair, should be shared apace with recurrent IH patients.

\section{Open repair for recurrent inguinal hernia}

Details of prior hernia operations are important in planning for a recurrent $\mathrm{IH}$ repair. Regardless of the procedure chosen to repair a recurrent hernia, it is highly likely to be more difficult than a primary repair.

An anterior approach for recurrence after primary anterior repair means that scarred tissues with distorted tissue planes must be entered. In our experience/judgement, this increases the risk of testicular atrophy and nerve entrapment with consequent postherniorrhaphy chronic groin pain. If an endoscopic repair was previously per-

\section{Statement}

Statement Recurrence rates worldwide are still too high despite numerous innovations in inguinal hernia surgery.

\section{Key question}

KQ20.b Is surgery necessary for all recurrence patients? formed, then an anterior repair where tissue planes are undisturbed is recommended. ${ }^{290}$ At least one authority has stated, given that the extra-peritoneal space has already

\footnotetext{
Statement

There is no evidence to support or reject a watchful Statement waiting management strategy for those with recurrent inguinal hernias.
}

\section{Evidence in literature}

The current guidelines on a watchful waiting approach to patients with primary IHs remain unchanged from the 2009 EHS guidelines. ${ }^{12}$ However, our 2016 update states that while watchful waiting is relatively safe, there is a high likelihood of crossover to surgery (23\% at 2 years and $72 \%$ at 7.5 years in one study ${ }^{287}$ and $68 \%$ at 5 years in been dissected, an open preperitoneal approach including the PHS and Kugel should be avoided. ${ }^{291}$

\section{Key question}

KQ20.c Which management strategy is the best for recurrence after anterior repair? 


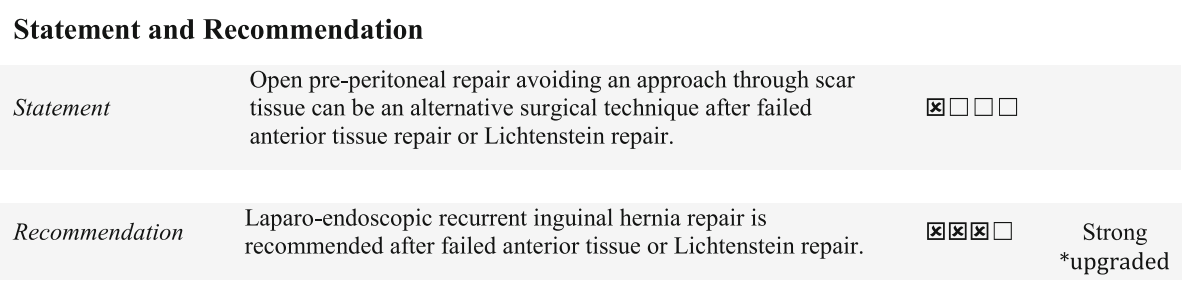

If the primary repair was a tissue repair, then either the anterior or posterior-either open or endoscopic-approach can be used for the recurrent hernia repair. If the primary repair was a mesh repair, then the entrance point should be via a space not previously entered.

For non-endoscopic surgeons, an anterior Lichtenstein approach has been recommended after a primary tissue repair. ${ }^{12}$ Care must be taken to prevent any potential (additional) damage to testicular vessels, since this may result in testicular atrophy. ${ }^{292}$ According to one study, incidental femoral hernias occur in up to $9 \%$ of recurrent IH patients. ${ }^{7}$ Therefore, groin exploration must include the femoral region.

When laparoendoscopic surgery is not an option, the open posterior approach represents an acceptable alternative. ${ }^{293,} 294$ This approach involves placing a large mesh piece posterior to the transversalis fascia via a trans-inguinal incision (Rives), or a muscle-splitting incision (Kugel and Wantz) or a lower midline incision (Stoppa). ${ }^{295}$ A report of 58 Stoppa operations for recurrence reported an overall rerecurrence rate of $12 \%{ }^{296}$ Nearly two-thirds of these occurred in the first few years after the technique was introduced at the study site. Further experience with the technique halved the rerecurrence rate in the same study.

The Prolene Hernia System ${ }^{\mathrm{R}}$ operation involves mesh leaflet placements anterior and posterior to the transversalis fascia. ${ }^{297,}{ }^{298}$ A connector holds the leaflets together. This technique requires a preperitoneal dissection via the anterior approach, difficult if the patient has already undergone a posterior approach either laparoscopic or open during primary repair. In addition, any prior anterior intervention would cause scarring, resulting in distorted tissue planes and increasing risk of testicular atrophy and nerve damage.

Since the potential for complications of open recurrent IH repair-including testicular atrophy and/or nerve entrapment and damage - is higher than for primary repair, we strongly suggest that this operation be performed by an expert hernia surgeon.

\section{Surgical options for patients with recurrence after anterior repair}

A 2016 study provided strong evidence that endoscopic recurrent hernia repair (TEP or TAPP) after anterior repair provides optimal outcomes while reducing the incidence of chronic pain and allowing earlier return-to-work or physical activity. ${ }^{299}$

Once an anterior repair has been done, an endoscopic repair will generally go through nearly undisturbed tissue planes, permitting relative ease of dissection. One study has demonstrated that the rate of recurrence of primary and recurrent IHs is similar. ${ }^{285}$ This likely depends on local expertise in part. It has been demonstrated that endoscopic repair after an anterior repair generally takes longer than a primary repair and increases the peritoneal tear incidence. $^{300}$

A 2014 meta-analysis summarized findings from six RCTs and 5 other studies comparing laparoscopic to open procedures in recurrent IH repair. The analysis of 1311 patients demonstrated that laparoscopy was associated with a lower incidence of wound infection and a shorter sick leave without an increase in operation time. ${ }^{301}$

While nationwide data on recurrence rates have remained disappointing high, there is evidence to suggest that in highly specialized hernia centers, rerecurrence rates of less than $2 \%$ can be achieved. For instance, a large institutional review reported a $2 \%$ rerecurrence rate after TAPP. ${ }^{83}$ Yet, another study of over 8000 patients found nearly identical rerecurrence rates of $1.1 \%$ for both primary and recurrent hernia repairs. ${ }^{302}$

A Swedish Hernia Register study of 850 recurrent IHs showed that posterior mesh repair (PMR)-either endoscopic of open preperitoneal-had a lower second recurrence rate versus anterior mesh repair (AMR) (5.6 vs $11 \%$, $p=0.025){ }^{303}$ An increased risk [3.21 (CI 1.33-7.44) $(p=0.009)]$ of a second recurrence was seen after anterior primary repair followed by AMR, and a decreased risk $[p=0.08$ (CI 0.01-0.94)] $(p=0.45)$ after PMR followed by AMR. ${ }^{303}$

Surgical options for patients with recurrence after TEP/ TAPP

\section{Key question}

KQ20.d What is the best operation for a recurrence after TEP/TAPP? 


\section{Recommendation}

Recommendation

Anterior repair is recommended after a failed posterior repair.

口回

Strong

*upgraded

After a failed TEP or TAPP repair, where the extraperitoneal space was entered, it is strongly recommended that an AMR (Lichtenstein)-which does not involve entering the posterior space-be performed. This recom-

\section{Key question}

KQ20.f What are the options for a recurrence in a patient with postherniorrhaphy chronic groin pain?

\section{Statement and Recommendation \\ Statement There are no studies to guide decision making for recurrent hernia patients with post-herniorrhaphy chronic groin pain.

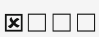 \\ खाम $\quad$ Weak

mendation remains the same as the EHS recommendation in $2009 .^{12}$

Surgical options for recurrence after anterior and posterior repairs

\section{Key question}

KQ20.e What is the optimal management strategy in a patient with recurrent hernia after failed anterior and posterior (laparoscopic or anterior preperitoneal) repairs?
Due to multiple prior interventions, anterior and/or laparoscopic, some patients with rerecurrence will develop postherniorrhaphy chronic groin pain. A tailored approach is urged, dependent upon the previous interventions and the significance of the recurrence (e.g., large incarcerated rerecurrence with small bowel obstruction risk). While an anterior approach may address the recurrence and potentially alleviate the chronic pain (if neurectomy and meshectomy are done), there are significant risks of testicular atrophy and even orchiectomy. In addition, subse-

Recommendation

The comparable recurrence rates after primary anterior and laparoscopic repair imply that patients are encountered presenting with rerecurrence after a laparoscopic or anterior preperitoneal repair and at least one anterior repair. Another anterior repair (e.g., Lichtenstein) would be expected to have a rerecurrence rate of three times that of a primary hernia repair. In addition, there would be significant risk of testicular atrophy and/or chronic groin pain. ${ }^{126}$ Relaparoscopy, either with a TAPP technique ${ }^{126}$ or a modified intraperitoneal onlay mesh technique, ${ }^{283}$ is feasible, but this decision, and the procedure itself, should be in the hands of an experienced laparoscopic hernia surgeon. ${ }^{286}$

Surgical options for recurrence with chronic groin pain quent recurrence is highly likely. All this must be explained to, and discussed with, the patient. Given the complexity of these cases and the high risk of complications, it is strongly suggested that patients in this category be managed by expert hernia experts.

\section{Conclusion}

Given the factors cited above, treatment of recurrent and serially recurrent IHs remains challenging. It may be possible to minimize rerecurrence and other complications using appropriate surgical technique, accounting for the previous approaches, and calling upon expert hernia surgeons to manage these cases. 


\section{Chapter 21}

\section{Emergency treatment of groin hernia}

\author{
M. López-Cano, S. Morales-Conde, and P. Chowbey
}

\section{Introduction}

Medical evidence is limited about the emergent treatment of groin hernias and the quality of that evidence is very low. Future research on the subject should focus on: incarceration and strangulation risk factors, diagnostic modalities, and optimal timing for emergency repair of incarcerated/strangulated groin hernias.

This chapter addresses key questions on best surgical approach (open anterior, posterior, or laparoscopic) and repair options (e.g., mesh versus non-mesh). It is important to consider the limitations imposed by the state of the currently available literature as we search for definitive evidence-based answers to key clinical questions.

The following definitions are used throughout this chapter.

Incarceration: Inability to reduce the hernia mass into the abdomen

Strangulation: The blood supply to the herniated tissues is compromised

\section{Key question}

KQ21.a Which groin hernias in adults are at increased risk for incarceration/strangulation? convalescence, type of anesthesia, and risk of complications, but description of results after emergency hernia is scarce". ${ }^{304}$ Randomized studies (RSs) addressing KQ21.a are not available. Several non-randomized studies (NRSs) with acceptable methodological quality have been published, however. Most are cohort studies although population-based studies exist also.

Patients who undergo emergent IH surgery had more hospitalizations than elective patients in the year preceding hernia repair. ${ }^{138}$ It has been noted that groin hernias in females have increased incarceration/strangulation risk. $^{22,}{ }^{305}$ Femoral hernias in particular have an increased risk of incarceration/strangulation ${ }^{22,30,305-307}$ and this risk seems to be increased if the hernia is right-sided ${ }^{306}$ (see also chapters 16 and 17).

In summary, an increased incarceration/strangulation risk is found for groin hernias in adults with the following features:

- Hernia-related hospitalizations in the year preceding hernia repair

- Female gender

- Femoral hernias, particularly those on the right side

\section{Discussion}

Understanding of incarceration/strangulation risks in adults with groin hernias will be improved by large-scale epidemiological studies, preferably based on national and international registries. However, the statement in KQ21.a has been upgraded to "strong", because the incarceration/ strangulation risk factors are consistent across the currently available studies. Even with the acknowledged low level of

\section{Statement}

\section{Evidence in literature}

It has been written that "most studies concerning hernia focus on recurrence rate, acute and chronic pain, evidence, the benefits of emergent or urgent surgery likely outweigh the risks. 


\section{Key question}

KQ21.b Which risk factors increase morbidity and mortality in adults with incarcerated/strangulated groin hernias?
- ASA class III and IV, BMI $>30$, and recurrent hernia ${ }^{30}$ and

- Anticoagulant use $e^{307}$

One study group has proposed a classification scheme to objectively reflect the degree of clinical and morphological

\section{Statement}

Statement

Increased morbidity and mortality are found amongst incarcerated/strangulated groin hernia patients with: age $>65$

冈ख $\square \square$

years, increased ASA classification, increased symptom duration,

increased weight, necessity for bowel resection, presence of a

recurrent hernia, femoral hernia presence, female gender, oral

anticoagulant use, and presence of bowel obstruction.

\section{Evidence in literature}

Morbidity and mortality are increased amongst incarcerated/strangulated adult groin hernia patients with the following risk factors.

- Age $>65$ years $^{30}, \quad 138, \quad 305, \quad 306,308$ especially octogenarians ${ }^{309}$

- Prolonged symptom duration ${ }^{305}$

- Delay to admission, diagnosis, and surgery ${ }^{22}$

- Prolonged time from admission to start of surgery ${ }^{308}$

- Incarceration for more than $24 \mathrm{~h}^{310}$

- Symptom duration of 3 or more days ${ }^{307}$

- Bowel obstruction 308

- Lack of health insurance 308

- Associated midline laparotomy for exploration after incarcerated/strangulated hernia reduction ${ }^{311}$

- Femoral hernia, ${ }^{30,}, 305-308$ especially right-sided ${ }^{306}$

- Female gender ${ }^{138,} 304,305$ changes in acutely incarcerated bowel. They found that acute incarceration time was the strongest predictor of bowel damage and subsequent infection. They also found that outcomes were worse in older patients, especially those with comorbidities and higher ASA scores. ${ }^{312}$

\section{Discussion}

The statement is graded as low evidence, because the available evidence leaves appreciable uncertainty about the magnitude of the effects of the various risks cited. More study in this area is clearly needed.

\section{Key question}

KQ 21.c Which diagnostic method is most suitable for the detection of incarcerated/strangulated groin hernias in adults?

\section{Statement and Recommendation}

$\begin{array}{ll}\text { Statement } & \begin{array}{l}\text { Clinical examination alone is sufficient for the diagnosis } \\ \text { of incarcerated/strangulated groin hernias in almost all } \\ \text { patients. Groin ultrasound and/or abdominopelvic CT can } \\ \text { provide additional diagnostic information and aid decision } \\ \text { making in selected cases. }\end{array} \\ \text { Recommendation } & \begin{array}{l}\text { Clinical examination of the groin is recommended in all } \\ \text { patients presenting with signs and symptoms of bowel } \\ \text { obstruction. }\end{array}\end{array}$




\section{Evidence in literature}

No RCTs, systematic reviews, or meta-analyses were found that address the KQ21.c. Two NRSs exist on ultrasound as a means to detect incarcerated/strangulated groin hernias. In the first of these, ultrasound was found to be helpful in diagnosing those with atypical presentations (e.g., obese patients). ${ }^{313}$ Hernia complications such as incarceration and strangulation are usually diagnosed or strongly suspected based on a constellation of signs and symptoms occasionally supported, or augmented, by laboratory data. However, sonography is required when the clinical picture is inconclusive or when the surgeon believes that he must

\section{Discussion}

Although the statement is only weakly supported by evidence, the recommendation was upgraded to strong, because potential benefits greatly outweigh risks. RCTs are needed on the value of imaging studies in the setting of suspected incarcerated/strangulated groin hernias.

\section{Key question}

KQ21.d Should adults with acutely incarcerated/strangulated IHs undergo repair emergently?

$\begin{array}{ll}\text { Statement } & \begin{array}{l}\text { Acutely incarcerated/strangulated groin hernias represent } \\ \text { surgical emergencies mandating timely surgery, taking } \\ \text { into account preoperative optimization and the capabilities } \\ \text { of local surgical facilities. }\end{array}\end{array}$

assess the contents of an incarcerated/strangulated groin hernia sac preoperatively. Sonographic findings associated with incarcerated hernias include: free fluid in the hernia sac (accuracy 96\%), bowel wall thickening within the hernia (accuracy 97\%), fluid within a herniated bowel loop (accuracy 94\%), or dilated intraabdominal bowel loops (accuracy 92\%). ${ }^{313}$ This ultrasound study did not comment on strangulated hernias due to a paucity of cases. ${ }^{313}$ In a second study, sonography was used as a guide to show the location and direction of the fascial defect when the inguinal mass was compressed with two hands. ${ }^{314}$ This ultrasound-guided maneuver may achieve incarcerated hernia reduction-reducing the number of emergent operation-and was found to be easier than blind manual reduction. Another advantage of sonography in this setting is its ability to diagnose other inguinal conditions presenting this groin pain or mass (e.g., varicocele, hydrocele, abscess, and lymphadenopathy). Notably, both studies included small patient numbers and their results must be interpreted cautiously.

No studies have been found regarding other imaging modalities in adults with incarcerated/strangulated groin hernias.

Regarding the importance of clinical examination, in a retrospective cohort study from the Swedish Hernia Register, ${ }^{135}$ the authors concluded that "In patients with bowel obstruction, physical examination of the groin may decrease the time-to-surgery and reduce imaging investigations in patients needing emergency surgery for groin hernia."

\section{Evidence in literature}

Although most would immediately and intuitively answer "yes!" to the question, there is no RCT supporting this opinion. However, results from NRSs consistently support the approach. A low-quality, comparative cohort study with small numbers of adult patients with incarcerated/ strangulated groin hernias analyzed time from symptom onset to surgery in two ways. ${ }^{315}$ The first comparison was between patients requiring bowel resection and those not requiring it. The second was between patients treated within $12 \mathrm{~h}$ of symptom onset with those treated outside that window. Both analyses found a significant increase in morbidity and mortality in those with delayed surgeries. Another similar study comparing patients operated upon within $24 \mathrm{~h}$ of symptom onset versus those operated on after that period found a statistically significant increase in bowel resection rates (29 vs $49 \%, p=0.047$ ).

In addition, data related to the question which risk factors increase morbidity and mortality impact this issue. In particular, presentation and treatment delays, incarceration duration, and prolonged symptom duration are related to increased morbidity and mortality in adults with incarcerated/strangulated groin hernias.

\section{Discussion}

Although all studies in this subject area are of low quality, the statement is graded as strong, since benefits to patients seem to clearly outweigh risks. RCTs would be difficult, if not frankly impossible, to perform; however, large-scale epidemiological studies based on national or international 
registries might further inform surgical decision-making on this crucial issue.

\section{Key questions}

KQ 21.e What is the optimal surgical approach for an acutely incarcerated/strangulated groin hernia?

\section{Key questions}

KQ21.f In patients with intestinal incarceration without signs of intestinal strangulation or concurrent bowel resection (i.e., a clean surgical field) is mesh-based repair recommended? Which mesh?

\section{Recommendation}

\section{Recommendation}

\section{Evidence in literature}

No randomized studies, systematic reviews, or comparative cohort studies address the question which approach is best. Only one low-quality randomized trial compares a meshbased open posterior approach with a mesh-based open anterior approach and found no differences except for an increased incidence of second incisions in the anterior approach group. A preperitoneal approach was judged to be as safe as an anterior Lichtenstein approach in incarcerated/ strangulated groin hernia repairs. ${ }^{316}$

A very low-quality comparative cohort study recommends a preperitoneal approach. ${ }^{317}$

No randomized studies or systematic reviews focus on TAPP or TEP repair techniques in patients with incarcerated/strangulated groin hernias. Only one low-quality comparative cohort study showed that TAPP repair is useful in elective and emergent cases such as those involving strangulated hernias in selected patients (those with scrotal hernias, previous pelvic or abdominal surgery, and severe cardiac or pulmonary problems were excluded). ${ }^{318}$

\section{Discussion}

The optimal technique for acutely incarcerated/strangulated IH repair remains an open issue, unknowable from the present literature. Well-conducted RCTs and other studies are needed to provide answers to the KQs posed above.

\section{Evidence in literature}

One low-quality cohort study compared open anterior polypropylene mesh repair with open anterior repair (anatomic repair, modified Bassini) without mesh. ${ }^{319}$ The majority of patients had intestinal incarceration without intestinal strangulation or concurrent bowel resection (i.e., a clean surgical field). No inter-group differences were found in wound infection rates or postoperative complications. No mesh-related problems were reported.

Another low-quality cohort study compared Lichtenstein repairs in incarcerated versus elective $\mathrm{IH}$ patients and found no inter-group differences. Most patients had clean surgical fields and were ASA class I and II. ${ }^{320}$

One low-quality randomized trial compared Lichtenstein with Bassini repairs in selected clean (no peritonitis, inflammatory hernias, or bowel resections) incarcerated groin hernia patients. In this study, the Lichtenstein repair was found to be safe and effective with an acceptable low rate of postoperative complications and no recurrences. ${ }^{321}$

All studies cited used polypropylene mesh. No data were found on absorbable mesh or biologic implants.

\section{Discussion}

In spite of low-quality medical evidence, the statement above received a strong grade, since benefits outweigh risks when mesh is used in emergent clean hernia operations. This is similar to the elective surgery experience. Definitive research is needed on this subject. 


\section{Key question}

KQ21.g In patients with intestinal strangulation and/or concurrent bowel resection (clean-contaminated surgical field) is mesh-based repair recommended. Which mesh? a wound protector was used at time of bowel resection and the area was lavaged with saline following bowel resection.

A low-quality systematic review with meta-analysis has been published and suffers from a number of flaws but represents the only SR that examines whether mesh repair

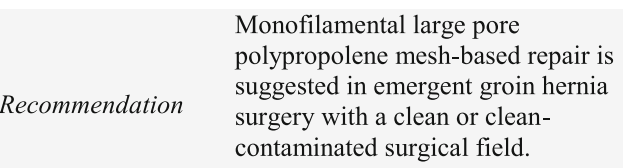

\section{Evidence in literature}

One well-done cohort study with a prospective analysis of Lichtenstein repairs in clean-contaminated fields (bowel resection vs no bowel resection) showed that acutely incarcerated groin hernias can be safely repaired with nonabsorbable mesh (monofilament polypropylene) with an acceptable wound infection and recurrence rate even when intestinal necrosis was present. ${ }^{322}$

Another small low-quality cohort study found no differences in morbidity, mortality, or wound- and mesh-related problems when comparing mesh repair (Lichtenstein) with Bassini in incarcerated/strangulated hernia repairs requiring bowel resection. ${ }^{323}$ Polypropylene mesh (type unspecified) was used after copious saline lavage of the surgical field.

Another cohort study compared mesh (PHS system) with non-mesh repairs in a mixed clean and clean-contaminated field population and found no differences. ${ }^{324}$ Contaminated-dirty field patients were excluded. The study supported the idea that use of prosthetic mesh in emergent hernia repairs is not contraindicated.

A well-done cohort retrospective study compared bowel resection with no bowel resection groups and concluded that mesh repair was safe in patients not requiring bowel resection. ${ }^{325}$ A further conclusion was that mesh use is not contraindicated in patients requiring bowel resection so long as the field is kept clean-contaminated during surgery. Gauze or is associated with a higher surgical site infection risk when compared with non-mesh techniques. ${ }^{326}$ The SRs authors recognize their review's weaknesses and conclude, "The mesh repair technique is a good option for the treatment of strangulated IHs in adults, giving an acceptable wound infection rate and fewer recurrences than non-mesh repair. Our study does not allow us to recommend the use of mesh in cases of bowel resection. We emphasize that, except for the two RCTs, the results are predicated on patient selection by careful surgeons."

Information to address the question is insufficiently available in the current medical literature.

\section{Discussion}

There is limited, low-quality evidence addressing the issues raised in this question. The statement is, therefore, only weakly supported. Appreciable uncertainty exists about the magnitude of benefits and risks. Definitive research is needed on this subject.

\section{Key question}

KQ21.h In stable patients with strangulated obstruction and peritonitis caused by a bowel perforation or an abscess due to necrosis of the omentum (contaminated-dirty surgical field) is mesh repair recommended. Which mesh?

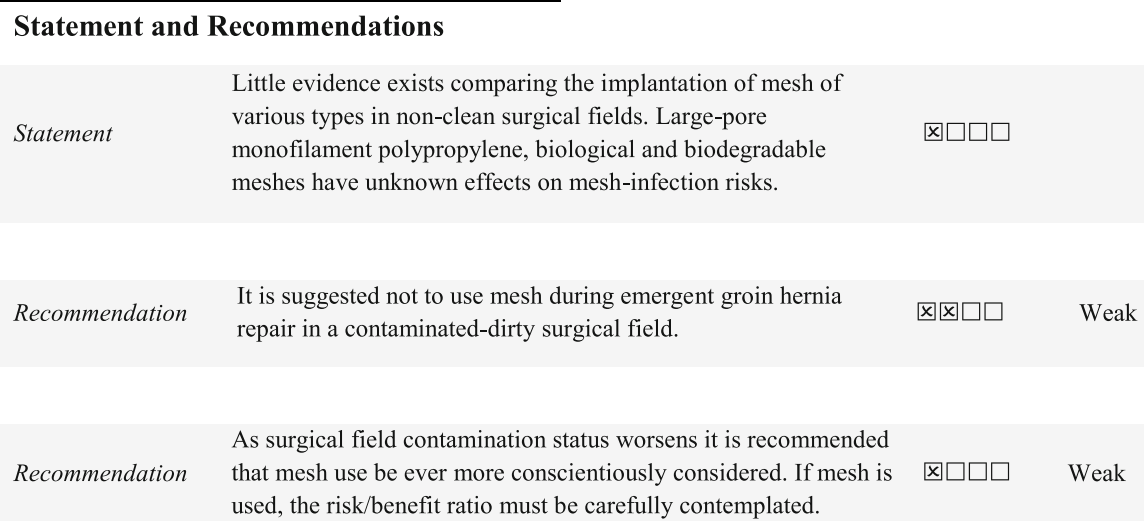




\section{Evidence in literature}

No medical literature addresses this question; therefore, the statements and recommendations are based on expert opinion.

\section{Key question}

KQ21.i Should adult patients with acutely incarcerated/ strangulated groin hernias receive antibiotic prophylaxis or treatment? risk patients (poorly defined, ASA class IV excluded), it may decrease major morbidity. However, this study suffers from the fact that is a preliminary report with risks of bias (no fully explained concealment allocation, no sample size calculation, major complications not fully defined, and small sample size).

A second publication is a review of "all published articles" about hernioscopy in adults and children. Data on 58 adults are included from one RCT, seven case reports, and two case series. The review concluded that in adults

Recommendation

Prophylactic intravenous antibiotics are suggested during and following emergent hernia surgery. They should be continued as

Recommendation

field.

Strong

upgraded

\section{Evidence in literature}

No medical literature directly addresses this question. However, most papers addressing other KQs related to emergent operations for incarcerated/strangulated groin hernias reference the use of intravenously antibiotics for 5 days postoperatively. Antibiotic choice varied across the studies.

\section{Key question}

KQ21.j In adults with acutely incarcerated/strangulated groin hernias, does hernia sac laparoscopy (hernioscopy) reduce morbidity and mortality in cases with spontaneous reduction of the hernia before viability assessment?

KQ21.k In adults with acutely incarcerated/strangulated groin hernias, is laparoscopy useful to check bowel viability even when an anterior approach is done? with incarcerated groin hernias, hernioscopy is useful to assess bowel viability after spontaneous hernia reduction. Furthermore, hernioscopy lessened the need for exploratory laparoscopy. However, the conclusion must be interpreted cautiously, because-in our opinion-the publication represents a narrative review based on the authors' subjective analysis.

The current medical literature does not address the question concerning the value of laparoscopic visualization.

\section{PART 3}

Quality * Research * Global Management

Quality Aspects

22. Training and the learning curve

23. Specialized centers and hernia specialists

\section{Statement}

\section{Evidence in literature}

One randomized study concludes that hernia sac laparoscopy seems to be an accurate and safe procedure with the potential to prevent unnecessary laparotomies after spontaneous incarcerated $\mathrm{IH}$ reduction. ${ }^{327}$ Particularly in high-
If a surgeon has any concern about bowel viability, bowel visualization is recommended. Depending on surgical approach, expertise, and facilities, bowel visualization may be undertaken by groin exploration, hernia sac laparoscopy,

formal laparoscopy or laparotomy.
24. Costs

25. Registries

26. Outcomes and quality assessment

27. Implementation: present and future aspects 
Groin Hernia Management Globally

28. Inguinal hernia surgery in low resource environments

Research, General Practitioners and Patient's Perspectives

29. Questions for research

30. Summary for general practitioners

31. Groin hernias: a patient's perspective

\section{Chapter 22}

\section{Training and the learning curve}

J. Bingener, R. Simmermacher, D. Lomanto and W. Reinpold

\section{General introduction}

This chapter's goal is to review evidence and provide guidance for two questions:

What are the learning curves of the different techniques?

What are the best methods to teach groin hernia repair?

Depending on circumstances, training to perform $\mathrm{IH}$ repair has different competency goals. In rural or low resource settings, training may be focused on the basic ability to perform any inguinal repair for patients with significant symptoms without causing mortality or serious morbidity (see also Chapter 28 ). ${ }^{1}$

The advent of laparoscopic techniques directed attention to the technical learning curve of surgical procedures. Learning curricula initially focused on the number of repetitions needed to achieve outcomes similar to experts. It soon became clear that skills proficiency or competence, rather than number of repetitions, correlated to improved patient outcomes. ${ }^{2}$ Technical competence is the ability to achieve a mastery level outcome in three consecutive attempts at a technical skill, where mastery level is calculated as the mean scores of several surgical experts (surgeons with excellent patient outcomes) in the same skill. ${ }^{3}$ The learning curve is defined as the time and effort necessary to achieve proficiency.

Assessing proficiency by objective standardized tools is possible but resource intense, ${ }^{4,5}$ thus the number of procedures performed, years spent in training, operative time and complication rates continue to be proxies for proficiency and metrics for learning curve progress.

Globally, options for training are diverse and evolving. Most surgical training programs include time for supervised performance of IH repairs. HerniaSurge considers "supervision" as the presence of a trained expert in the operating room. Training components are both cognitive and technical: groin area anatomy, procedural steps, intraoperative decision making and manual dexterity. These components should be safely acquired before independent practice occurs.

This chapter will not address the learning curve for pioneering a new technique or technology. Recommendations to safely guide these developments can be found in the IDEAL framework for surgical innovation. ${ }^{6}$ As a general rule, patient outcomes should be the same or better than for existing techniques once the novel approach is fully developed.

KQ22.a What is the learning curve for open inguinal hernia repair, anterior approach?

KQ22.b What is the learning curve for open inguinal hernia repair, posterior approach?

KQ22.c What is the learning curve for laparoscopic inguinal hernia repair, TEP?

KQ22.d What is the learning curve for laparoscopic inguinal hernia repair, TAPP?

KQ22.e What are the best methods to teach open hernia repair?

KQ22.f What are the best methods to teach laparoscopic inguinal hernia repair? 


\section{Statements and Recommendations}

Open anterior mesh repair by unsupervised trainees with less than 60

Statement cases or about 3 years' experience is on average associated with

higher recurrence rate and longer operative times.

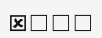

Complication rates do not differ between consultants and

supervised trainees regardless of training year. Involvement ब⿴囗口

$\begin{array}{ll}\text { Statement } & \text { supervised trainees regardless of training year. } \\ \text { of trainees may slightly prolong operative times. }\end{array}$

A goal-directed curriculum including review of anatomy, procedure steps, intraoperative decision making and

Statement technical skills training shortens the learning curve for laparoscopic hernia repair and improves patient outcomes.

The learning curve for TAPP and TEP seems to be similar. For novices, this includes significantly more procedures for laparo-endoscopic hernia repair than open mesh repair dependent on individual aptitude and the training

environment.

\begin{tabular}{|c|c|c|c|}
\hline Recommendation & $\begin{array}{l}\text { A goal-directed curriculum including review of anatomy, } \\
\text { procedure steps, intraoperative decision making and } \\
\text { proficiency based, simulation enhanced technical skills } \\
\text { training is recommended. }\end{array}$ & 凶खण & $\begin{array}{c}\text { Strong } \\
\text { *upgraded }\end{array}$ \\
\hline Recommendation & $\begin{array}{l}\text { Supervision of trainees should be provided until they have } \\
\text { reached safe proficiency levels. }\end{array}$ & 凤 & $\begin{array}{c}\text { Strong } \\
\text { *upgraded }\end{array}$ \\
\hline
\end{tabular}

\section{Evidence in literature}

Learning Curve: Tissue Repair

Although some may consider primary tissue repair of groin hernias to be easy and feasible, little is published about learning curves to independently perform a primary tissue repair in groin hernia patients. Therefore, no statement can be made regarding the learning curve for tissue repair. The mesh placement component of the open anterior approach is easier to learn for physicians already proficient in the anterior tissue repair ${ }^{7,8}$ than for novices without any experience in hernia repair. The following statements derive from published literature regarding surgical trainees in the mesh repair era.

\section{Learning Curve: Open Repair}

A registry study of 4406 patients ${ }^{9}$ demonstrated more recurrences if operating residents were unsupervised [unsupervised junior resident RR 21 (95\% CI 7.3-58.9), $p<0.001]$. Recurrence rates and operative times were also higher for junior residents $(<4$ years of postgraduate training) in a large randomized trial ${ }^{10}$ where residents were supervised. A 2012 study $^{11}$ found an average 16 min longer operative time for residents versus consultants on procedures for 28,000 patients captured in the American College of Surgeons National Surgical Quality Improvement Program (ACS-NSQIP), confirming earlier reports by others. ${ }^{12}$ In a recent study, 69 trainees were followed for 7 years with case log review and standardized technical competency assessments in UK NHS training programs. On average, the trainees achieved proficiency for independent IH repair after they had performed 64 repairs (range 12-73) which usually was reached in their fourth year of training. ${ }^{13}$

Learning Curve: Laparo-endoscopic Repair TEP Approach Irrespective of the definition, there seems to be consensus that laparo-endoscopic IH repair has a distinct learning curve. Evaluating learning curves in health technology is challenging. ${ }^{14}$

The figures below depict the reported complication rates (Fig. 5), operative times (Fig. 6) and recurrence rates (Fig. 7) in relation to procedure numbers from studies retrieved in literature search. The learning curve for laparo- 
endoscopic TEP shows some variation; however, it appears that, on average, more than 100 repairs are required to achieve outcomes comparable with open anterior mesh repair. Around 100 repairs represent about a 40\% higher case number to achieve proficiency in TEP compared to open IH mesh repair. There is limited evidence that the learning curve may flatten after about 400 procedures. ${ }^{15}$ More experience is likely required to achieve expert center outcomes.

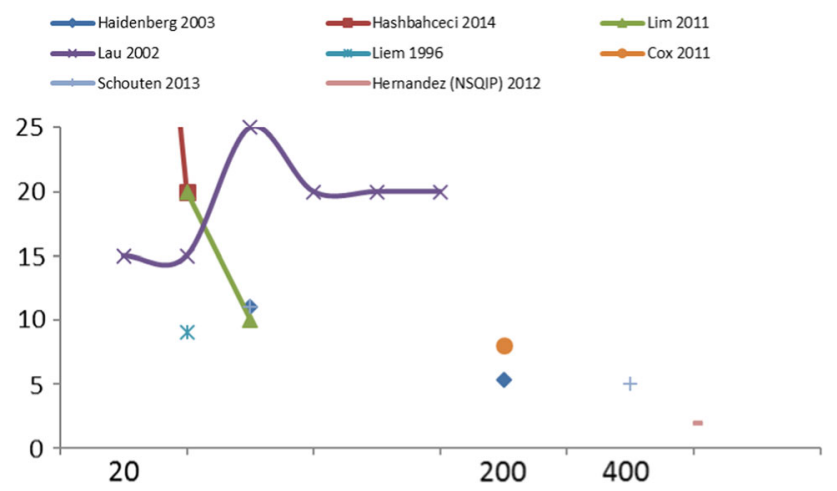

Figure 5: Published complications rates (\% in $y$ axis) correlated with case numbers. Complication rates for open IH repair captured for $>4000$ patients in the NSQIP data base are reported as $3 \% .^{15-22}$

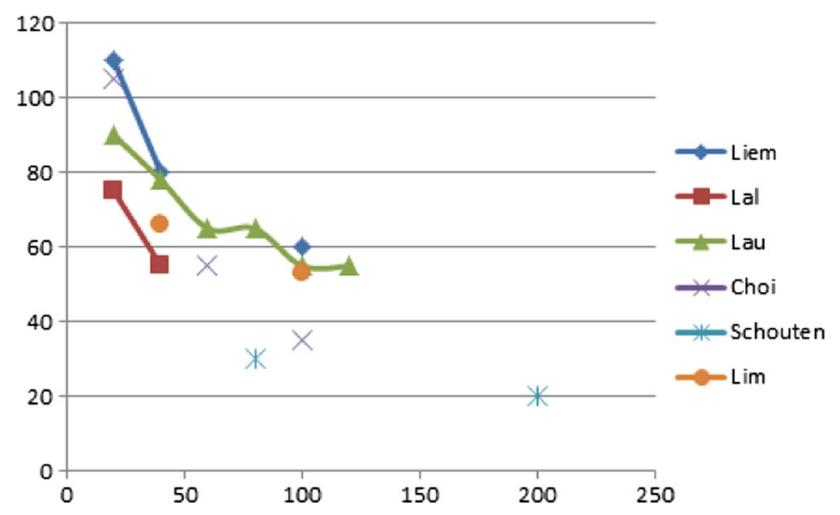

Figure 6: Reported operative time (minutes in $y$ axis) in six studies. ${ }^{15,17,19,21,23,24}$ correlated with case number. Note the mean time reported for general surgeons (not trainees) in NSQIP comprising $>4000$ cases is $45 \mathrm{~min} /$ case. $^{20}$

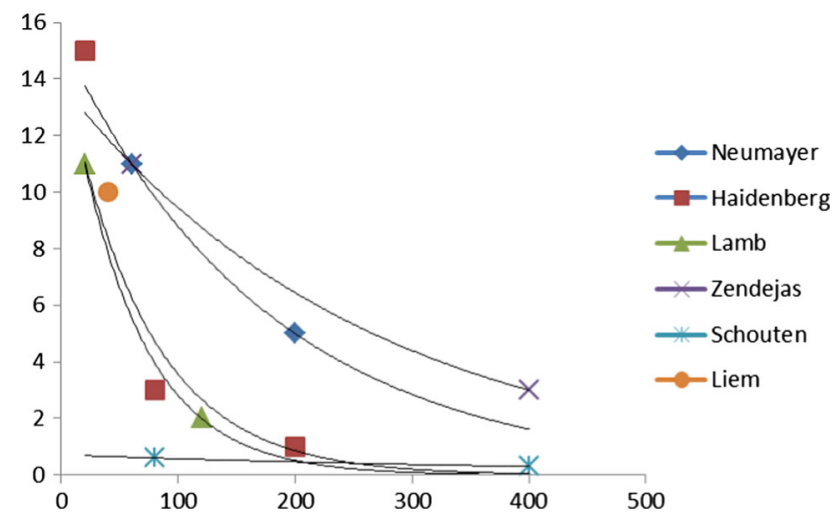

Figure 7: Reported recurrence rates (\% in $y$ axis) in six published studies correlated with case numbers. Note most studies reported using 10-15 cm polypropylene mesh for repair. $^{15,16,19,25-27}$

\section{Learning Curve: Laparo-endoscopic Repair, TAPP Approach}

Similar to TEP repair, TAPP repair appears to have a distinct learning curve compared to open anterior mesh repair. Five studies have addressed the topic, ${ }^{28-32}$ two of them $^{28,29}$ from the same center. The development and learning of the TAPP repairs appears to be included in study. ${ }^{28}$ After 300 repairs by the pioneers, complications and recurrences decreased significantly and these successes were passed on to subsequent trainees under well defined and rigorous training conditions. ${ }^{28,29}$ Notably, the program trainees experienced an operative time learning curve similar to the pioneers and were still considered trainees after they had performed $>200$ individual procedures. Another study ${ }^{31}$ also reported on all results including the initial learning curve and stated that recurrence rates improved after 200 cases, as the mesh size was changed to a larger mesh. A different study ${ }^{32}$ reported that there were significant improvements in conversions and admissions after 50 cases. Complication rates were halved, but were still $16 \%$ and did not reach statistically significant differences from the initial rates $(32 \%)$. We can thus extrapolate that the learning curve to get to outcomes comparable with open $\mathrm{IH}$ repair may have been longer.

Teaching: Open Hernia Repair

The literature search for teaching open hernia repair revealed two procedure-specific papers. One ${ }^{33}$ found that any simulation (high tech, low tech) improved performance over standard training with interactive simulation training showed the most improvement. Components of training are the understanding of anatomy, understanding of procedure steps and acquisition of technical skills; however, teaching 
anatomy on simulators does not necessarily lead to proficiency. ${ }^{34}$ Video assessment ${ }^{35}$ reliably reveals the proficiency level for open IH repair. One study ${ }^{5}$ noted that the mental workload for experts in open IH repair is lower than for novices, supporting the need for cognitive learning prior to technical performance.

\section{Teaching: Laparoscopic Hernia Repair}

A survey of more than 800 North American general surgeons and surgery residents found 59\% felt they lacked the requisite training for laparoscopic hernia repair and $26 \%$ were interested in learning the technique. They were most likely to seek education in a course followed by expert proctoring. ${ }^{36}$ The learning curve for the laparoscopic techniques may be significant enough to prevent some surgeons from offering the technique to their patients. This underscores the need for effective training methods to ensure that patients will benefit. ${ }^{36,37}$

The Cochrane Collaboration published a systematic review on laparoscopic surgical box model training for surgical trainees with limited prior laparoscopic experience. The review included a variety of procedures, including laparoscopic hernia repair. ${ }^{38}$ It found that laparoscopic box training improved patient outcome (e.g. length of stay), operative time and performance.

The review included a 2011 trial which demonstrated that by achieving a proficiency level in the simulation environment, residents performed better in the operating room than peers undergoing standard training and that their patients had fewer overnight admissions. The mastery training included cognitive learning (anatomy review and procedure steps learning) and technical skills training. ${ }^{3}$ The trainees required on average $69 \mathrm{~min}$ (range 13-193 min) and 16 attempts (range 7-27) to be able to perform the hernia repair in the low tech model ${ }^{39}$ in mastery time ( $2 \mathrm{~min}$ ). When this was translated to the operating room, the operative time was statistically significantly improved by $6 \mathrm{~min}$ for operations with residents who underwent training compared to operations with those who did not. The NSQIP data suggest that surgeons unaccompanied by residents perform laparoscopic hernia repair on average 20 min faster. In this RCT, undergoing purposeful proficiency training shortened the in-OR learning curve. Others have proposed similar simulators, checklists and curric$\mathrm{ula}^{4,}, 40-46$ with the same goal. In laparoscopic training in general, high tech or low tech environments may be less important than the fact that knowledge and skills are translated and measured. While some believe that intensive mentor presence and teaching of pitfalls is pivotal, ${ }^{40}$ other research disputes this. ${ }^{47,}{ }^{48}$ Residents do seem to be less frustrated with the low tech simulation. ${ }^{49}$ There are not enough data to prescribe the exact training modality in which the knowledge should be transferred. The available studies suggest that cognitive and technical components are essential for meaningful outcomes.

\section{Discussion, consensus and grading clarification}

The listed literature describes the current review of evidencebased knowledge to the best of our abilities. Several large registry-based studies and at least one large RCT have recently provided updated information on the learning curves for open and laparoscopic hernia repair. While none of our sources represent perfect data, many have similar results which led us to provide strong statements and recommendations for anterior mesh repair and laparoscopic TEP repairs.

We did not find enough published evidence on open tissue repair or an open posterior approach to reach firm statements or recommendations. It is known that in the Shouldice Hospital surgeons are supervised in their first 300 repairs, supporting our assumptions on learning curves to achieve expert performance.

In our review we postulated several benchmarks to delineate the progress of training to expert proficiency:

- Reaching minimum safety standards

- Reaching physician-reported outcomes similar to traditionally available procedures

- Reaching an institutional performance level at which the above standards and outcomes are met and patientreported outcomes exceed those of traditionally available procedures

Many surgeons have graciously described their experience with learning new procedures, especially the laparoendoscopic TEP approach. In evaluating these reports in the literature, several considerations apply:

- Given the overall small number of expected complications for hernia repair, large numbers of procedures are needed to identify a statistically significant change in an outcome (e.g., complication, recurrence rate). When a statistically significant increase in complication occurs in small patient cohorts (e.g., $n=20$ ), that may signal a large effect size in complication rates. 
- The development and learning of the techniques by early independent pioneers in the 1990s should be regarded separately from current structured surgical training programs.

Some surgeons find TAPP easier to learn than TEP. The data we reviewed on operative times and patient outcomes, however, do not strongly indicate that this is correct. It may be that entry into the preperitoneal space from the more familiar intra-abdominal environment decreases the disorientation in the preperitoneal space, or it may be that TAPP is indeed easier to learn.

Our statements and recommendations on how to teach laparoscopic skills were based on a systematic review of available studies that included a RCT on how to teach laparoscopic hernia repair. The mastery training used in the RCT did not close the gap to experts; it reduced it by a clinically relevant decrease in complications.

Other lower quality studies revealed largely similar results. There is, however, more available evidence on the learning curve than on the teaching methods. As more literature becomes available, the guidance on teaching methods may evolve as well.

In preparing these statements we have accessed new, good quality and relevant research. Thus, our statements and recommendations may update prior guidelines (e.g., EHS ${ }^{50}$ EAES $\left.^{51}\right)$. In addition, as stated above we set external benchmarks for the learning curve. For example, the fact that a complication rate decreased by $50 \%$ after 50 cases was important; however, if the patient outcomes were still lagged other options (e.g., open mesh repair as described in a large database) we did not describe the learning curve as complete.

We acknowledge that the statements and recommendations may represent challenges for training programs. Twenty-five years after the introduction of laparoscopic IH repair, surgeons and surgical trainees have, however, voiced concerns about being incompletely prepared. ${ }^{36,} 37$ Prior underestimation of the learning curve may have contributed to this unease.

\section{Chapter 23}

\section{Specialized centers and hernia specialists}

G. H. van Ramshorst, H. J. Bonjer, D. Cuccurullo, R. Bittner and H. M. Tran

\section{Introduction}

Terms like "specialization" or "specialized centers" are often undefined or poorly defined and rarely based on scientific standards of excellence. The term "hernia center" and terms like it are frequently used as marketing tools. ${ }^{52}$ Studies on IH repair-with good results-are often published by surgeons specialized in hernia surgery.

The definition of a hernia specialist requires objective parameters of expertise, annual case load, outcomes and contributions to education and science. It is recommended that surgeons complete their learning curves in multiple techniques, thus facilitating a patient-specific approach to each individual dependent upon comorbidities and surgical history. Most experienced hernia surgeons support the use of this patient-tailored approach (see Chapter 8). ${ }^{53}$

Recently, surgical procedures of various types have been qualified as "highly complex, low volume" and "low complex, high-volume." IH repair can reasonably be considered a high-volume procedure in the right setting. It has been shown that regular operating theater teams can shorten room turnover times, preparation times and procedure times and thereby increase daily patient volumes. ${ }^{54}$ The medical literature supports the notion that specialized centers with their high patient volumes achieve better results in laparoscopic and complex IH surgeries. The category "complex IH surgery" includes: multiple recurrences, chronic pain, and mesh infection. ${ }^{28,55,56}$

As in other types of surgery, the incidence of surgical complications is in large measure inversely related to a hernia surgeon's annual caseload. This is particularly true for laparoscopic hernia repair. The learning curve for open IH repair is shorter (see Chapter 22 on Learning Curve).

To improve $\mathrm{IH}$ repair outcomes, a continuous quality control and improvement cycle is recommended. Patient follow-up should be organized to detect and register longand short-term complications. Active involvement in training, education and science and a broad and deep clinical experience are essential for improving hernia surgery care. Regionalization of hernia care at specialized centers is vital as well.

The ability to discern a "true hernia center" of excellence from one with average experience and outcomes may lie in certification of hernia surgery centers. A seminal article from 2014 described the process and goals of hernia center certification in Germany. ${ }^{52}$ The article details that two certification processes exist in Germany. The nonprofit organization Surgical Review Corporation uses the designation, Certified Center of Excellence in Hernia Surgery (COEHS) while the German Hernia Society (GHS) and the German Society of General and Visceral Surgery use the term Certified Hernia Center. ${ }^{52}$ 
Certified Hernia Centers in Germany:

Level 1: Prerequisite to become a hernia center

Requires at least an annual hernia repair volume of 30 cases per surgeon registered in the Herniamed registry and follow-up. After 1 year at least $90 \%$ of all hernia patients must be entered into the Herniamed registry with information on comorbidities to allow for case mix variations. Quality outcome measures exist for infections, revisions, complications and follow-up rates.

\section{Level 2: Competence center}

It has higher annual volume stipulations and additional requirements for morbidity conferences, pain management and documentation (see Table 4).

\section{Level 3: Reference center}

It has still higher and more specific annual volume requirements and a variety of science- and education-based requirements. Level 3 (or reference) centers must be able to perform all laparoscopic and open techniques for hernia repair and must have formalized cooperative agreements with plastic surgeons (see Table 4).

All hernia surgeons in the healthcare system must be GHS and EHS members and subscribe to Hernia (http:// www.springer.com/medicine/surgery/journal/10029). ${ }^{52}$
A significant correlation between surgical volumes and better outcomes was demonstrated in a systematic review of 16 studies. ${ }^{57}$ However, this is not the case for all surgical interventions. The relationship between caseload and mortality held for pancreatic resections but not for colorectal surgery. ${ }^{58}$ It may be that as intervention complexity increases-requiring an interdisciplinary approach and advanced complication management-caseload becomes more important. However, an interdisciplinary approach or special complication management is rare in IH repair.

What constitute high- and low-volume centers for IH repair are unclear, making outcome comparisons difficult. Two large case series reported excellent results concerning recurrences in $\mathrm{TAPP}^{28}$ or TEP, ${ }^{56}$ but small case series have reached similar standards. ${ }^{59-62}$ One paper concluded that technique standardization and learning curve completion (of 50-100 cases) are the key parameters for performance quality. $^{29}$

Another study showed that in a high-volume center (defined as $>1000 \mathrm{IH}$ repairs/year) well-supervised trainees had longer operation times but similar complications rates and recurrences when compared with experienced surgeons. ${ }^{29}$ It seems that learning $\mathrm{IH}$ repair well in a short

Table 4 Overview of requirements for hernia centers in Germany

\begin{tabular}{|c|c|c|c|}
\hline Level & Level 1 & $\begin{array}{l}\text { Level } 2 \\
\text { Competence center }\end{array}$ & $\begin{array}{l}\text { Level } 3 \\
\text { Reference center }\end{array}$ \\
\hline $\begin{array}{l}\text { No of hernia patients } \\
\text { treated yearly }\end{array}$ & Min 30 & $\begin{array}{l}\text { Min } 200 \text { (min } 30 \text { incisional } \\
\text { hernia) }\end{array}$ & $\begin{array}{l}\text { Min } 250 \text { (min } 50 \text { incisional hernia, } 5 \\
\text { complex hernias, } 5 \text { hiatal hernias) }\end{array}$ \\
\hline Science & $\begin{array}{l}\text { Membership German and European Hernia } \\
\text { Society, subscription Hernia }{ }^{\mathrm{a}}\end{array}$ & $\begin{array}{l}\text { Yearly attendance at atleast } \\
\text { one hernia meeting }\end{array}$ & $\begin{array}{l}\text { At least two presentations at a hernia } \\
\text { congress or one publication }\end{array}$ \\
\hline Education & - & - & Education seminars, guest visits \\
\hline
\end{tabular}

${ }^{\text {a }}$ Obligatory for all levels

KQ23.a Does a center's volume affect IH surgery outcomes? time period requires a certain caseload. This experience may be easiest to obtain at a high-volume center.

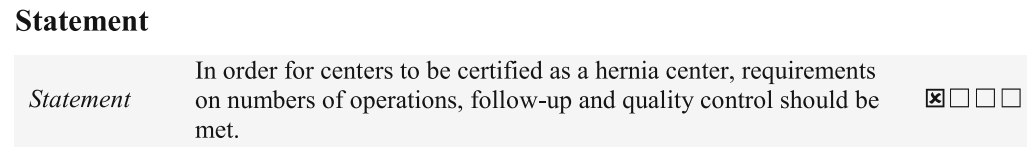


KQ23.b Do surgical volumes affect the outcomes of $\mathrm{IH}$ surgeries?
Few studies have compared high-volume surgeons' outcomes with low-volume surgeons' outcomes. Some

\section{Statements \\ Statement \\ The individual surgeon's case volume appears to be more important for IH surgery outcomes than a center's caseload. \\ Statement \\ A surgeon's case volume is inversely related to that surgeon's recurrence rate. \\ 冈■० \\ 冈खण}

A Swedish Hernia Registry study found that surgeons who performed one to five hernia repairs annually (any technique) had longer operation times and significantly higher reoperation rates than surgeons who did more repairs. ${ }^{63,} 64$ An NHS study found comparable results; surgeons' annual laparoscopic hernia repair caseload was inversely related to reoperation rates following laparoscopic repair of primary $\mathrm{IH}^{65}$ This was not the case for open repair. This study contained no information on surgeons' laparoscopic experience. The summed evidence suggests that higher case load correlates positively with fewer recurrences following primary laparoscopic IH repair.

A large RCT compared laparoscopic with open IH repair and found a $10.1 \%$ recurrence rate following laparoscopy. ${ }^{66}$ In the study, 69 surgeons performed 989 repairs. Prior to the study's commencement, only 20 of the surgeons self-reported an experience of more than 250 repairs. For this highly experienced group, the recurrence rate for laparoscopic repair of primary hernia dropped to $5.1 \%$ and was comparable to recurrence rate after open repair at $4.1 \%$. The authors concluded that an experience of $250 \mathrm{IH}$ repairs was necessary to achieve a significant reduction in recurrence rates. They defined a new category, "highly experienced surgeons" as those who had performed more than $250 \mathrm{IH}$ repairs.

A survey found that routine surgical practice varied with hernia surgery volume. Surgeons who performed more than 50 repairs annually were more likely to visualize and preserve inguinal nerves, ${ }^{67}$ a measure recommended for prevention of chronic pain. It is reasonable to assume that high-volume surgeons are more focused on chronic pain prevention. Notably though, this study did not document chronic pain incidence in relation to surgical volume.

A review article noted that recurrence rates after Shouldice repair by hernia specialists (term not defined in the article) were lower when compared with repairs by non-specialists. Wound infection rates were comparable between the groups. ${ }^{68}$ studies have compared open IH repairs by residents with repairs by full-trained surgeons. In one study, residents took more time to dissect and mobilize the sac and had significantly higher postoperative complication rates. Recurrence rates, however, were similar. More chronic pain occurred in the specialist-repair group. ${ }^{69}$

KQ23.c Does facility specialization affect IH surgery outcomes?

Emerging evidence suggests that high center volume is related to positive outcomes for a wide variety of surgical procedures and that reducing the number of centers undertaking complex surgical procedures is associated with better outcomes.

Complex IH repairs include those with re-recurrences, chronic pain or mesh infections. However, there are no studies comparing specialist with non-specialist center repairs of these cases.

Some have suggested that good outcomes in complex cases result from the aggregate effect of surgical expertise, high volumes, choice of more effective treatment modalities and other factors unrelated to surgical expertise. There may also be benefits of working with a highly skilled team that performs complex tasks repeatedly, has good knowledge of different techniques for abdominal wall repair and possesses extensive experience in the entire field of hernia surgery. There may be a need for hernia centers that offer "a complete hernia service" using a "tailored approach". 52

The National Outcomes Program established in 2009, evaluates healthcare outcomes in Italian hospitals and assesses the UK's National Health System (NHS). In addition to outcomes, the 2013 Program edition included a set of volume indicators for conditions with evidence of a volume-outcome association. However, due to a paucity of evidence, it is not possible to draw firm conclusions about hernia treatment from this data set. ${ }^{70}$

Another trial was also unable to establish a clear relationship between high-volume hernia centers and improved outcome. $^{71}$ 
However, another group reported marked differences in outcomes in relationship to individual surgeon's volume at three hospital types. So-called "occasional operators" dominated at university hospitals and had a significantly higher relative risk of recurrence compared with medium
In the some European countries and the United States, increase in surgical volume is often dependent on volume agreements with health insurance companies.

KQ23.d Does surgical specialization affect IH surgery outcomes?

\section{Statement \\ A Hernia specialist is a surgeon with mastery/expert level herni Statement surgery skills who actively train, educate and perform research in their field.}

and small hospitals. ${ }^{63}$ This finding supports the concept of regionalization to specialized settings with high case volumes and greater experience.

The impact of creating a surgical specialty referral center has been studied as well, specifically the financial and institutional volume impact. ${ }^{72}$ This study examined all hernia repairs in the period 2004-2011 comparing hernia repair type, volume and center financial performance. The ventral hernia repair (VHR) patient subset was further analyzed for previous repairs, comorbidities, referral patterns, and concomitant plastic surgery involvement. Prior to hernia center establishment, hernia procedures averaged 156 annually (years 1999-2003). Over the next 8-year period, 4927 hernia repairs were performed with an average of 616 hernia procedures per year. Annual billing increased yearly from 7 to $85 \%$ and averaged $37 \%$ per year. Comparing 2004 with 2011, procedural volume increased $234 \%$, and billing increased $713 \%$. During that period, there was a 2.5-fold increase in open VHRs, and plastic surgeon involvement increased almost eightfold, ( $p=0.004$ ). In $2005,51 \mathrm{VHR}$ patients had a previous repair, $27.0 \%$ with mesh, versus 114 previous VHR in $2011,58.3 \%$ with mesh $(p<0.0001)$. For VHR, in-state referrals from 2004 to 2011 increased $340 \%$ while out-ofstate referrals increased $580 \%$. In $2011,21 \%$ of all patients had more than four comorbidities, significantly increased from $2004(p=0.02)$. It was concluded that the establishment of a tertiary/regional referral hernia repair center led to a substantial increase in surgical volume, complexity, referral geography, and financial benefit to the institution.
It is difficult to separate surgeon caseload from specialization since they are highly correlated. The literature on surgeon caseload is described above. A publication from one expert group opines "... there is a need for hernia centers in which hernia surgery is practiced by specially accredited hernia surgeons who as far as possible master all hernia surgical techniques and play an active role in training and continuing education as well as in the field of science. ${ }^{52 "}$ This statement goes a long way toward defining a hernia specialist.

Specialized hernia centers outperform general surgical centers in laparoscopic and complex IH surgeries. ${ }^{28,55,56}$ Therefore, complex IH surgery should be performed by a hernia specialist.

In primary IH Lichtenstein repair, general surgeons' and supervised-residents' results were comparable with experts' results. ${ }^{73}, 74$ Similar findings were found for repairs with bilayer patches and plugs. ${ }^{75,76}$

Hernia surgery specialization can significantly impact the type of hernia surgery performed in a region. Prior to year 2000, less than $1 \%$ of inguinal repairs were performed laparoscopically in the Australian Capital Territory, population 400,000. Following the adoption and popularization of TEP repair in the state by a specialized hernia surgeon, laparoscopic repairs increased annually to 39\% in 2004. The value of hernia specialists developing and promulgating new techniques and offering continuing education to fellow surgeons and surgeons-in-training cannot be underestimated. 


\section{Chapter 24}

\section{Costs}

\section{G. H. van Ramshorst, R. Bittner, H. Eker and W. Hope}

\section{Introduction}

Factors influencing costs in inguinal hernia repair.

Cost calculations for IH repair are complex and difficult to perform. ${ }^{77}$ Overall costs, including pretreatment, treatment and posttreatment medical care, societal and employer costs are rarely completely reported in studies. Moreover, it should be considered that costs are not equal to charges. ${ }^{78}$ Charges are not necessarily related to costs, and are usually constructed using different formulas. Charges can vary greatly among hospitals and countries. Reimbursement of costs by insurance companies or patients varies widely between countries and hospitals, often depending on negotiations related to volume agreements. ${ }^{79}$ All of the aforementioned stages in the treatment process are associated with variable costs.

IH repair cost calculations are complex and difficult to perform. ${ }^{77}$ Total costs, including pretreatment, treatment and posttreatment medical care, societal and employer costs are rarely reported completely.

Data demonstrate clearly that hernia surgery cost calculations are determined by a large number of variables including:

Patient-specific characteristics, hernia pathology, anesthetic type, annual hernia case load, procedure type, surgeons' skills, fixation type (including no fixation), Complication frequency, operative setting, number of postoperative visits, sick leave duration, recurrence rate, salaries of personnel, equipment depreciation, share of costs from relevant support services.

As expected, published data on costs of an IH operation show huge variations, ranging from about 126 USD to more than 4116 USD $^{80,81}$ Even within one institution, there are huge variations in costs generated by individual providers. $^{81}$
Surgeons can only influence some of the factors above. Operating time, surgical intervention quality, and instrument and material choices are the surgeon's responsibility. ${ }^{82-86}$ An individual surgeon's experience and skill may significantly impact cost when factors such as operating time, complication rates and recurrences are considered. ${ }^{82,85,87}$

Studies report wide variations in quality of life and quality-adjusted life years (QALY) following IH repair. It is known for example, that patients receiving workers compensation take longer to return to work than those not receiving these compensations. ${ }^{79,88}$ Patient-related factors such as age, comorbidity, work type, employment history, local culture, and physicians' expectations influence recovery time but their contribution to costs are difficult to evaluate. ${ }^{89,90}$ Additional costs such as medication expenses, home care compensations and transportation-related expenditures add to the tally and are similarly difficult to capture fully. Rarely considered are patient loss of income, disability insurance costs, and costs associated with the patient's inability to care for others. Other relevant employer-related outlays include: insurance costs, productivity losses and worker replacement costs. ${ }^{79}$

Complicating comparisons between studies on costs is the fact that currency conversions over time are problematic and in some studies only percentages of cost differences were estimated. In other studies percentages of effectiveness differences were used to calculate incremental cost per recurrence avoided and incremental cost per added day of work or usual activity. ${ }^{80}$

Laparoscopic repair costs can change over time as new equipment is purchased, costs are spread over a higher volume of procedures, or the equipment is used for other procedures. $^{91}$

KQ24.a Is open or endoscopic inguinal hernia repair more cost effective?

KQ24.b What are the costs and cost differences between open and laparoscopic inguinal hernia repair?

\section{Statements \\ Statement Direct instituational costs are lower for open mesh repair than for laparo-endoscopic mesh repair.




\section{Statement and Recommendation}

$\begin{array}{ll}\text { Statement } & \begin{array}{l}\text { The higher institutional costs of laparo-endoscopic inguinal } \\ \text { hernia repair are mainly due to the use of expensive } \\ \text { disposable equipment. }\end{array} \\ \text { Recommendation } & \begin{array}{l}\text { From a cost-effectiveness perspective, day-case laparo- } \\ \text { endoscopic inguinal hernia repair with minimal use of } \\ \text { disposables is recommended. }\end{array}\end{array}$

\section{Evidence in literature}

Open tissue IH repair under local anesthetic is the least costly technique when materials alone are considered. However, due to longer return-to-work times and higher recurrence rates it may be less cost-effective when compared to mesh repair. ${ }^{92-94}$

Institutional costs were higher for laparoscopic repair (TAPP, TEP) when compared with open mesh techniques. ${ }^{62,82,83,}{ }^{95-121}$ In experienced centers with minimal disposables use, the cost of laparoscopic repair may be equivalent to, or lower than, the cost of open surgery.

However, some study data used to arrive at this conclusion may be flawed. Operating times in excess of $60 \mathrm{~min},{ }^{90,92,96,97,100,101,107,108,113,114,120-122}$ high recurrence rates for laparoscopic repair $(10 \%)^{66,92,123}$ and high conversion rates $(6-10 \%)^{82,115,118}$ may indicate lack of experience. Studies not mentioning instrument and material types are unsuitable for cost calculations.

Most papers state that higher laparoscopic surgery costs mainly reflect the use of expensive disposables and longer operating times. ${ }^{80,83,90,101,105-110,118,124-126}$ Multiple cost analyses demonstrate that if disposable trocars, graspers, preperitoneal balloons and stapling devices ("tackers") are included, ${ }^{127}$ direct costs are significantly higher for laparoscopic over open hernia repair. This was mainly true in the early laparoscopic hernia surgery era. ${ }^{82,89,96,97,100,108,117,119,124,128}$

Now, institutional costs for laparoscopic hernia repair may be comparable to, or lower than, open hernia repair costs. $^{79,90,91,117,129}$ One study shows that in large-volume laparoscopic surgery centers with minimal use of disposable instruments and no use of preperitoneal balloons and tackers for mesh fixation, the direct costs of laparoscopic repairs are comparable to open repairs. ${ }^{90}$ One recent study found lower TEP/TAPP costs when compared to open mesh repair. Data for this study were collected in 15 German hospitals and were used to analyze costs. The authors concluded that laparoscopic approaches are not necessarily associated with higher resource utilization when compared to open mesh repairs. ${ }^{85}$ A recent large English study had a similar finding. ${ }^{81}$ This study concluded that the mean cost of laparoscopic versus open hernia repair is comparable but laparoscopic repairs appear to offer higher costs per QALY versus open repairs. ${ }^{81}$

In contradiction to the results seen in studies of direct/ hospital costs, nearly all RCTs, systematic reviews and meta-analyses demonstrate that indirect/societal costs for laparoscopic IH repair are lower than open mesh repair. This finding is accounted for by more rapid recovery with less pain, $62,90,91,100,116,122,125,130$ shorter sick-leave time ${ }^{83}, 84,96,101,102,106,108,109,113,120,124-126,130-132$ better physio metric test results, ${ }^{79}, 95$ and decreased complications and recurrence rates as experience has grown. 79, 82, 90, 94, 101, 106, 108, 114, 116, 119, 126, 130-132

If both direct and indirect costs are tabulated, laparoscopic hernia repair appears to be more cost-effective than open hernia repair. ${ }^{81,102,105,119,126,131,133-136}$

KQ24.c Which surgeon-specific factors result in improved cost-effectiveness?

Cost-effectiveness may be enhanced by an increase in individual case load (more rapid depreciation of equipment costs, more experience), ${ }^{137}$ shortening of the learning curve (resulting in decreased operating times), proper supervision of residents and junior consultants, surgical technique improvements (resulting in lower complication and recurrence rates), technique standardization, systematic training including simulation-based training $46,83,87,97,100,132,138$ and use of non-disposable trocars and other instruments $^{83}, 90,91,107,136,139,140$ (see Chapter 22 material on learning curves). 
It has also been shown that, due to mesh technology improvements and a better understanding of the extent of inguinal floor dissection needed in hernias with defects of less than $3 \mathrm{~cm}$, expensive fixation devices are unnecessary. ${ }^{141,142}$

\section{Chapter 25}

\section{Groin Hernia Registries}

P. Nordin, A. Montgomery, L. N. Jorgensen, U. Klinge and T. Bisgaard

\section{Introduction}

Well-designed RCTs advance the scientific basis of our knowledge and promote evidence-based medicine because of their powerful internal validity. ${ }^{143,}{ }^{144}$ However, some aspects of medical care cannot be easily addressed by RCTs. Studies from well-validated registries can provide important information as well. Registry studies of large populations have the unique strength of reflecting clinical reality (e.g., outcomes in routine clinical practice) and thus provide the surgical community a high level of external validity.

KQ25.a When compared with RCTs, do well-validated IH quality registries, and the studies done on their databases, offer additional valuable evidence-based information to hernia surgeons? improvements at individual facilities. ${ }^{145,}{ }^{146}$ Registries can serve as the basis for observational studies, may detect and lead to the analysis of rare events, may provide data needed for RCTs, and facilitate questionnaire studies. Additionally, patients may be recruited from registers for clinical trials that address specific questions outside a registry's scope.

Registry data reflects effectiveness in routine care and possesses high external validity, provided their coverage is broadly inclusive of a national population. In contrast, RCTs and other trials are investigational and often report on efficacy obtained in expert hands when interventions are optimally applied to carefully selected subjects (Fig. 8) ${ }^{147}$ RCTs (Table 5) are widely recognized as the criterion standard in the evaluation of pharmacological interventions, but problems may arise if surgical techniques are compared. ${ }^{144}$

The optimal design for comparing surgical methods is a randomized study involving surgeons of equal skill levels who demonstrate equal levels of objectivity with the methods being compared. However, even in study settings, patient-related and surgeon-related factors, which cannot be controlled, influence outcomes. Technical skill variation will always exist and was demonstrated in an RCT measuring surgical skill. ${ }^{148}$ In this study, low surgical performance scores were highly correlated with 5-year hernia recurrence rates. The question naturally arises then, how to consider this issue when analyzing studies? Similarly, should RCTs be the only means we use to evaluate surgical methods?

Hernia surgery is usually considered within the purview of general surgery and is often performed by non-special-

$\begin{array}{ll}\text { Statements and recommendation } \\ \begin{array}{ll}\text { Hernia registries, with high coverage, allow monitoring of } \\ \text { clinical practice and provide high external validity whereas } \\ \text { RCTs define effects of a specific intervention with minimal } \\ \text { bias and high internal validity. }\end{array} \\ \begin{array}{ll}\text { Rare events can be detected early in hernia registries } \\ \text { highlighting potential problems soon after new techniques } \\ \text { and products are introduced into clinical practice. }\end{array} \\ \text { Statement } & \begin{array}{l}\text { Registry-based studies are important complements to RCTs, } \\ \text { in guideline development. }\end{array} \\ \text { Statement } & \begin{array}{l}\text { Countries or regions are suggested to develop and } \\ \text { implement registries with high coverage and long-term } \\ \text { follow-up for quality control in groin hernia patients. }\end{array}\end{array}$

\section{Evidence in literature}

Hernia registries provide long-term monitoring of surgical quality in unselected patients and facilitate surgical care ized surgeons or trainees. ${ }^{149}$ Factors like patient age, gender, comorbidities, hernia-specific conditions and surgeons' preferences and experience might influence surgical indications and the choice of operative technique. ${ }^{150-152}$ 
Table 5 Pros and cons with registry studies and randomized studies

\begin{tabular}{|c|c|c|}
\hline & Pros & Cons \\
\hline $\begin{array}{l}\text { Registry-based } \\
\text { studies }\end{array}$ & $\begin{array}{l}\text { High external validity } \\
\text { Includes all patients at aligned units } \\
\text { Involves many surgeons with varying level of skill and experience } \\
\text { Reflects routine clinical practice } \\
\text { Provides separate data from participating hospitals and aggregated } \\
\text { data for all participating units } \\
\text { Requires a limited contribution from all surgeons } \\
\text { Excellent tools for observing changes over time } \\
\text { Includes documentation and adjustment of several confounders } \\
\text { Permits post hoc subgrouping of patients at high risk } \\
\text { May investigate even rare events } \\
\text { Power increases over time }\end{array}$ & $\begin{array}{l}\text { Requires a limited contribution of a large number of } \\
\text { surgeons } \\
\text { Recurrence rate are replaced by re-operation for } \\
\text { recurrence } \\
\text { Generally lower rate of follow-up than in RCT } \\
\text { Low internal validity if not data are monitored }\end{array}$ \\
\hline RCT & $\begin{array}{l}\text { High internal validity } \\
\text { Allows for comparison of methods of repair under standardized } \\
\text { study conditions } \\
\text { Simple statistical analyses with comparative methods } \\
\text { Can prove the impact of a specific change in treatment on a } \\
\text { specific outcome in a specific setting } \\
\text { Short-term rates of recurrence and chronic pain can be determined }\end{array}$ & $\begin{array}{l}\text { Specified inclusion and exclusion criteria limit the } \\
\text { external validity } \\
\text { Inclusion of all consecutive patients is difficult } \\
\text { Results are mostly obtained by a limited number of } \\
\text { experts under optimal conditions } \\
\text { Extensive contribution from participating study } \\
\text { investigators is required } \\
\text { Follow-up for more than a few years is rarely possible } \\
\text { Focus on a single primary endpoint } \\
\text { All confounders are usual not considered } \\
\text { Post hoc subgrouping usually is not possible or justified } \\
\text { Usually insufficient power to detect rare events }\end{array}$ \\
\hline
\end{tabular}

For the reasons cited above, RCTs and registries should be considered alongside one another when evaluating various aspects of hernia repair. ${ }^{153}$

Ideally, a registry should follow patients from initial inclusion event to death. Provided consent is obtained to use personal identification numbers, patients can be tracked within a healthcare system and all subsequent encounters (e.g., reoperation) recorded. ${ }^{145,146,154}$ It is also possible to link registries of various types to detect and analyze risk factors contributing to unfavorable outcomes.

Coverage and data validity are crucial for registry studies. If a registry enrolls nearly all hernia patient encounters, the risk of skewed patient selection is minimized. Additionally, care must be taken when entering registry data since incorrect or missing data limit a registry's soundness. These factors influence the external validity or generalizability of conclusions reached in studies involving registry data and patients. In a perfect world, registry data and the conclusions about those data would exactly match the world outside the registry. This ideal is unlikely to be realized, but regional and national registries do include enormous data sets. Studies can be performed about time trends for repair methods, materials used, anesthetic type, patient gender and others topics. In contrast to studies performed at a single institution, registry studies can shed light on rarer events and conditions like: IHs in females, femoral hernias, serious complications and mortality. ${ }^{155,} 156$ It has been demonstrated the results abstracted from Danish and Swedish databases have changed clinical practice nationwide. ${ }^{157-159}$

Use of the checklists from the CONSORT statements, the STROBE curriculum and the RECORD statement are highly recommended to improve reporting quality for RCTs and observational studies. ${ }^{144,160,161}$

The 2012 EHS consensus meeting also spawned recommendations for reporting outcome results in abdominal wall repair across different study types. ${ }^{162}$

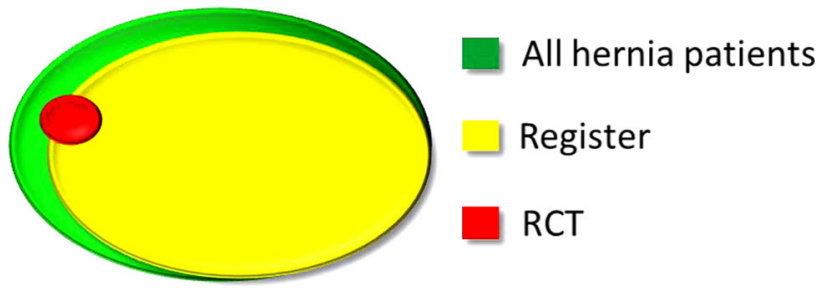

Figure 8. The potential coverage of patients operated on for an IH reported in a national or regional register compared to a randomized control trial. 


\section{Discussion}

There are several examples of RCTs with a major role in advancing the scientific basis of our knowledge and promote evidence-based groin hernia surgery. ${ }^{82,163,}{ }^{164}$ Hernia registers with high population based coverage, correct data and a great number of unselected patients have the unique possibility to study clinical reality and reflect outcomes in more routine clinical practice.

Registry-based studies are therefore important complements to RCTs. Currently, there are several examples of evidence-based national registry studies which are generalizable to the realm of groin hernia surgery. ${ }^{63}, 146,155-159$

\section{Chapter 26}

\section{Outcomes and Quality Assessment}

\section{D.L. Sanders, H. Eker and J. Bingener}

\section{Introduction}

Surgical outcome reporting is important in understanding the postoperative course of patients undergoing different types of groin hernia repair. It also serves to clarify how outcomes are affected by preoperative, surgical and postoperative variables (e.g., comorbidities, mesh type, mesh fixation method, and others).

KQ26.a What are the currently used methods for measuring surgeon-specific outcomes following groin hernia repair?

KQ26.b What are the currently used methods for measuring patient-based outcomes following groin hernia repair? recurrence, etc.) or patient-related (e.g., quality of life, patient satisfaction, etc.). Both are important in assessing quality and are interrelated. Devising a meaningful, intelligible and fair system for collecting data on quality is extremely complicated. Difficulties include:

\section{Questions with no answers raised for discussion:}

\section{What is measured?}

Which outcomes?

Should risk adjustment be performed?

How should risk adjustment be done (if it is done)?

Will outcome measurement lead to conservatism in surgical practice?

How are measurements made and are they accurate?

Are data collected accurately and reliably?

\section{Timing}

When does a valid outcome manifest after an operation? What follow-up time is required?

How is outcome information collected when routine follow-up is not done?

\section{Practicality}

Will outcome data collection significantly add to surgeons' workloads?

Will outcome data collection significantly add to other healthcare workers' workloads?

Will costs be increased?

Who will pay for these cost increases (if they occur)?

\section{How will the data be used and accessed?}

Data available to the lay public must be both understandable and detailed enough to provide valid decision making tools.

Will there be legal implications of outcome data collection and dissemination?

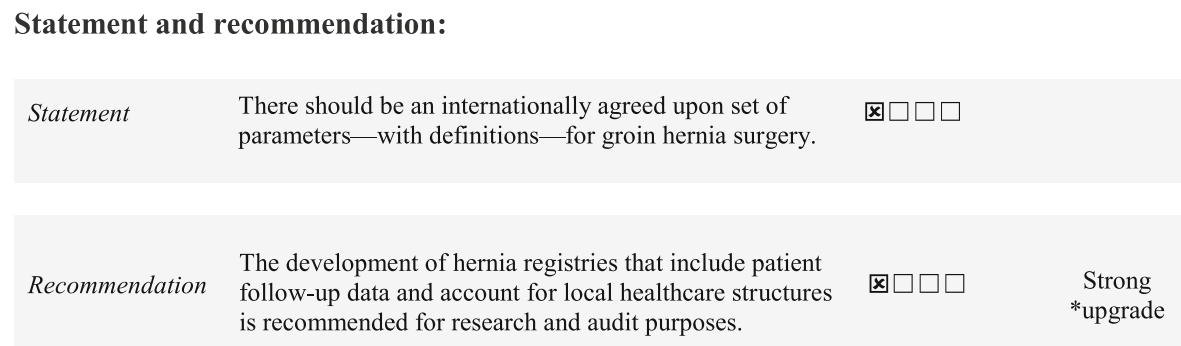

\section{Evidence and discussion}

A worldwide agenda now exists to ensure high standards in surgical practice by public dissemination of the outcomes of operations. ${ }^{165,166}$ Quality outcome indicators can be either surgeon-specific/clinical (e.g., length of stay,
Will there be regulatory or governmental implications?

Seven clinical outcomes assessment tools specific to hernia surgery are in use internationally, ${ }^{167-174}$ as is one patient outcomes assessment tool. ${ }^{175}$ Several hernia registries exist (Swedish Hernia Registry, Danish Hernia 
Registry, HerniaMed, Club Hernie, EuraHS, Dutch Hernia Registry, Evereg and AHSQC).

Large registries have the theoretical advantage of being able to capture information on rare adverse outcomes and disseminate that information to the surgical community at large. RCTs, which are often performed at expert centers, may lack this feature. Registries also can inform regulatory agencies and the public about important outcome differences between healthcare facilities.

Accurate, complete and valid data entry is crucial. A voluntary registry or a system lacking validity checks is at high risk for selection bias and input bias. Registry establishment and maintenance is costly and a stable funding source must be assured prior to registry development.

What constitutes a good registry?

Reasonable construct validity for a registry requires a robust system of data collection, follow-up and validation, agreed upon at the national level, and practical for the structure of the healthcare system in which it is imbedded. To deter risk-averse patient selection, predefined risk adjustment models are suggested.

Healthcare systems' structures vary broadly worldwide resulting in problems designing international registries. In many countries, routine follow-up is not done due to clinical and financial constraints. Additionally, patients experiencing adverse events may not present to their original healthcare provider, making adverse event data collection more difficult.

Time burdens, financial constraints, resource limitations and other factors place tremendous pressures on healthcare systems and their personnel worldwide. Quality data entry into registry databases may increase workload since many of these data points are already in the medical record. A method of minimizing data entry duplication would be to ensure that registry data entry occurs during the recording of clinical data entry. This, of course, would require local and national coordination. International registries could incorporate this feature as well.

\section{Patient Reported Outcomes}

Patient Reporting of Outcome Measures (PROMs) is another method of measuring outcomes. The United Kingdom's National Health Service (NHS) has used PROMs since April, 2009 to assess the quality of all NHSfunded care from patients' perspectives. ${ }^{176}$ PROMs measures patients' health status or health-related quality of life at a single point in time. Data are collected from short, selfcompleted questionnaires. For surgeries, health status information is gathered pre and postprocedure. Two generic measures are used to assess patients' self-reported outcomes following groin hernia surgery.

- The EQ-5D Index, a general measure of patients' quality of life
- The EQ-VAS, which provides a simple snapshot of patients' self-reported health.

The EQ-5D Index gives a general overview of patients' self-reported quality of life on five dimensions: health, anxiety and depression, ability to self-care, ability to carry out usual activities, and experience of pain or discomfort. Patients' scores on these questions are combined to give an index ranging from -0.594 to 1.0 (best possible score).

A problem with this approach is that IH patients generally do not have major problems with anxiety, depression or ability to self-care, dimensions included in the Index. What they do have is a specific local problem, that this generic health questionnaire will not identify or measure. At least two studies have shown clearly that generic instruments have poor discriminatory powers for distinguishing between satisfied and dissatisfied hernia repair patients. ${ }^{81,177}$ For unclear reasons, the NHS has failed to adopt a condition-specific IH questionnaire. Outcomespecific disease measures for hernia surgery such as the Carolinas comfort score exist and have been validated. ${ }^{81}$

Groin hernia repair outcome reporting is inconsistent and poorly defined, limiting meta-analyses, which themselves do not control for the differing definitions of assessed outcomes. A recent study published in the journal Hernia assessed type, frequency and definition of clinicianobserved and assessed outcomes and PROMs for instrument validity and frequency of domain reporting. ${ }^{178}$ Forty RCTs (10,810 patients) and seven meta-analyses (17,280 patients) were included in the review. ${ }^{178}$ No single PROM was reported by any study. There were 58 different clinician-observed outcomes, with recurrence ( $n=47,100 \%)$, wound infection $(n=33,70.2 \%)$, hematoma $(n=31$, $77.5 \%)$ and seroma formation $(n=22,46.8 \%)$ being most frequently reported. All studies measured patients' views, although only $12(30.0 \%)$ used validated instruments. The SF36 was the most commonly used multidimensional valid PROM $(n=7)$, and a visual analogue scale assessing pain ( $n=32$ ) was the most frequently used one-dimensional scale. Non-validated questionnaires assessed 25 other aspects of patients' health. Two meta-analyses defined recurrence, and three defined chronic pain; although neither ensured that included RCTs adhered to the definitions.

These results suggest that a standardized core outcome set is needed for hernia surgery to improve outcome reporting and evidence synthesis.

\section{Chapter 27}

\section{Dissemination and Implementation}

M. Pawlak, A. Wijsmuller and H. Eker 


\section{Introduction}

One of the goals of the HerniaSurge Group is the worldwide dissemination and implementation of our groin hernia management guidelines. They contain the most current evidence-based information and also show where scientific research is needed. They are important for guiding clinical practice and for the education of surgeons and for standardizing surgical training. However, without an ambitious implementation plan designed to reach targeted groups, the impact on hernia management could be disappointing. ${ }^{179,} 180$ Never before have any of the hernia surgery societies or inguinal guidelines focused on performing the difficult task of global recognition and awareness. ${ }^{50,51,181,182}$

HerniaSurge will create a guideline implementation trajectory and a transparent dissemination plan.

The following questions were identified:

- What are the target groups for the guidelines?

- What are the most important messages of the guidelines, both general and specific, for the targeted groups?

- Which channels can be used for guidelines distribution?

- How can the guidelines be supported by Internet tools, platforms, Apps and social media?

- What is the evaluation strategy for the implementation process? guideline. The barriers to implementing recommendations were sought and described independently in relevant chapters.

\section{Implementation and dissemination methods}

Branding-HerniaSurge. The aim is for the guidelines to be well-recognized, effective and disseminated worldwide.

Translation of the key statements and recommendations of the guidelines into languages that are most spoken: Mandarin, Spanish, French, Portuguese, Arabic, Russian, Japanese and German.

Website: http://www.HerniaSurge.com-a platform that consolidates the main aspects of the guidelines, gives insight into their development methodology, provides a database of the multimedia supplements and also includes resources for patients and medical professionals. ${ }^{184}$

1. Patient Resources

- Short videos explaining the pathology of IH and the most common surgical procedures.

- A brief explanation of the purpose for which the guidelines were created.

- Highlights of the most important issues that are of particular interest to IH patients (e.g., the prevention and treatment of chronic pain).

\section{Recommendation

\begin{tabular}{|c|c|c|}
\hline Recommendation & $\begin{array}{l}\text { HerniaSurge recommends that all countries or regions } \\
\text { develop a guidelines dissemination and implementation } \\
\text { strategy. }\end{array}$ & 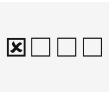 \\
\hline
\end{tabular}

\section{Target Groups}

The groups needing information about guidelines content include:

- Surgeons and physicians treating groin hernia patients

- Healthcare providers performing services for the treatment of hernias

- Groin hernia patients and their family members

\section{Message}

HerniaSurge was established as a joint effort of the EHS, EAES, IEHS, AHS, APHS, AMEHS and Australasian Hernia Society to develop guidelines for surgeons and healthcare providers who treat groin hernia patients. The guidelines include information on inguinal and femoral hernias in men and women and were developed according to the AGREE II instrument. ${ }^{183}$ A set of key recommendations of the guidelines will be identified on a global level by a vote during the international hernia congresses. The focus of the dissemination process will be placed on these key recommendations while providing access to the whole
2. Resources for medical professionals

- Full guidelines.

- Short explanations of main objectives, methods and key recommendations in several languages.

- Database with literature.

Social media: Facebook and LinkedIn pages including several selected topics (much more concise than the HerniaSurge website)

1. Resources for patients: three to four videos as mentioned above with simple explanations on the aim of the guidelines and the key recommendations that are of particular interest to patients, translated into different languages.

2. Resources for medical professionals: short explanations on main objectives, methods and key recommendations in several languages.

3. Direct links to http://www.HerniaSurge.com.

Publication of the key recommendations with reference to the full guidelines (on the HerniaSurge website) 
through every Hernia or National General Surgery Society after an inventory of these societies worldwide. Presentation of the key recommendations worldwide at hernia congresses (EHS/AHS/EAES/Annual congresses of Hernia Societies).

Mass media: several mass channels (for example, Euronews, BBC, CNBC) should be approached through media and communication departments to communicate the existence of the first worldwide surgical guidelines. Spokesmen will be chosen for this task.

The use of Modern Multimedia and Network tools.

The aim is not only to offer written guidelines, but also to offer assistance in their implementation.

- Video-tutorial videos for procedures. Videos of the most common hernia operations for the instruction of new learners (Lichtenstein, TEP, TAPP).

- Podcasts-recorded discussions on the guidelines conducted by recognized hernia experts and authorities. There will be a few selected essential topics like algorithms for groin hernia treatment pointing out the advantages and disadvantages of proposed procedures as well as defining the indications. Most importantly, this information will be submitted in an understandable and accessible fashion so that they are clear even for novice surgeons. The information should focus on the complexity of the treatment selection accordingly to defined factors such as gender, age, etc. These factors will be determined by the HerniaSurge Group and presented in the Podcast.

- Teaching-PowerPoint presentations will be developed and available on the website. Surgeons worldwide will be able to use these for teaching/learning in their own institutions.

- App-HerniaSurge will create an application for PC, smartphones and other devices which will help to analyze and select the best treatment option for individual patients according to the guidelines. Further it will contain critical information on the topic, an anatomical atlas of the groin region, answers on all frequently asked questions and a knowledge quiz to entertain and stimulate the curiosity of residents and experts.

\section{Evaluation and Revision}

The dissemination process will be monitored and audited by each of the national chapters. The level of implementation will be determined by a predefined set of criteria (guidelines awareness, target groups' attitudes, application of recommendations, and the effect on medical care).

During the dissemination, a study will be performed examining the quality and effectiveness of the process of implementation in two to three test countries that will be selected by the HerniaSurge Group. The most important key recommendations will be used for those test countries based on the up-to-date status of hernia surgery. The dissemination will be conducted and monitored by dedicated Ph.D. students. An evaluation after 5 years will be performed and analyzed so that improvements can be made.

The proposed dissemination plan is ambitious, however, looking through the prism of today's society that is oriented on fast collection and processing of information we need a clear, yet modern method for dissemination. It will be an innovative project that will determine the trend for the possibility and potential success of introducing future guidelines.

\section{Global Groin Hernia Management}

\section{Chapter 28}

\section{Inguinal Hernia Surgery in Low Resource Settings}

P. Nordin, D.L. Sanders, I. Konaté, R. Sani and M. P. Simons

\section{Introduction}

HerniaSurge (http://www.herniasurge.com) develops guidelines intended for use globally. This chapter contains guidelines on the performance of safe, cost-effective $\mathrm{IH}$ repair in low resource settings (LRSs). HerniaSurge believes that every patient with a groin hernia, wherever they may live, has the right to the best possible care. Nevertheless, it will take time to achieve a consistent high level of care throughout many areas in the world that lack the resources that are necessary and this is reflected in the recommendations made in this chapter.

There is a substantial burden of disease in countries where the majority of the world's groin hernia patients live. Although herniorrhaphy is one of the most commonly performed surgeries in LRSs, ${ }^{185,}{ }^{186}$ needs exceed capacity. This surgical "under-production" over time results in high hernia prevalence in populations. This in turn results in a high proportion of emergency surgery and significant morbidity and mortality. ${ }^{187-194}$ This, despite the fact that groin hernia repair is highly cost effective. ${ }^{195-197}$

\section{Key Questions:}

KQ28.a What is the epidemiology of inguinal hernia in LRSs?

KQ28.b Which types of inguinal hernia repairs are currently performed in LRSs?

KQ28.c What is the recommended operation for inguinal hernias in low resource environments?

KQ28.d What are the logistical challenges for safe groin hernia repair in low resource environments?

KQ28.e Should any special precautions be taken?

KQ28.f What is the most suitable mesh? 
KQ28.g What is the best way to educate surgeons in a sustainable manner in LRSs?

KQ28.h How can the internet and other technologies be used to teach physicians in LRSs?
Africa paint a very different clinical picture. A 1978 study of rural Ghanaian men estimated that $7.7 \%$ had an $\mathrm{IH}^{203}$ However, a 1969 study showed that the prevalence of $\mathrm{IH}$ was as high as $30 \%$ on Pemba Island in East Africa. ${ }^{204}$

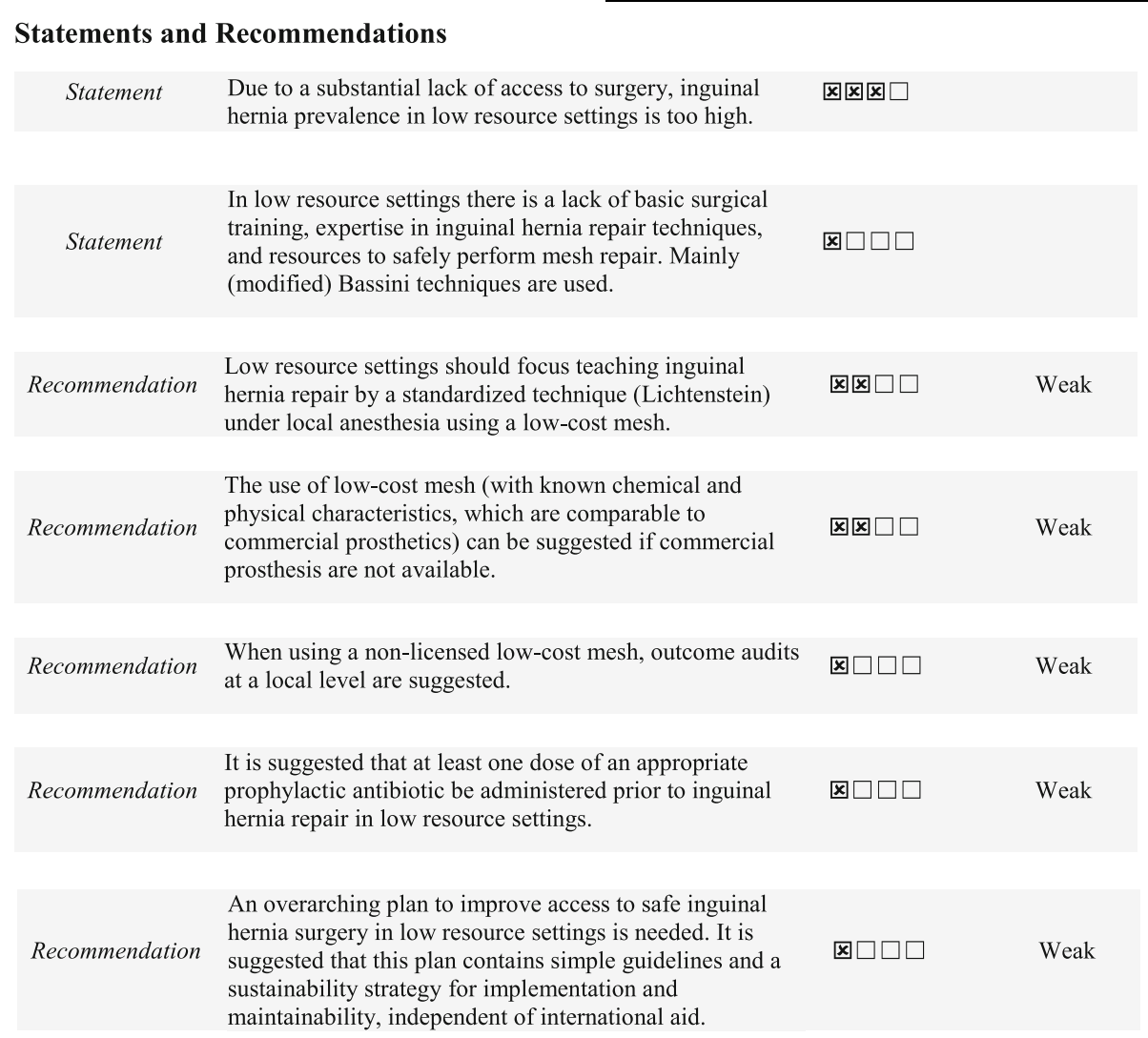

\section{Evidence in literature}

KQ28.a What is the epidemiology of inguinal hernia in LRSs?

IH epidemiology literature is limited, from both the developed world and particularly from LRSs. IH incidence-measure of probability of $\mathrm{IH}$ occurrence in a population within a specified time-is difficult to firmly establish although it seems unlikely that incidence varies much between countries. In contradistinction, IH prevalence-population proportion with IH at a given timeappears to be significantly higher in countries with poor healthcare access. ${ }^{194,}{ }^{198-202}$ The assumption is that most cases go untreated in resource-poor settings. The discrepancy in incidence versus repair rate results in high prevalence. This in turn has a huge economic impact on countries least able to shoulder that burden. ${ }^{197}$

A 1996 United Kingdom (UK) study found a lifetime risk of IH repair of $27 \%$ for men and $3 \%$ for women, an immense IH disease burden. ${ }^{186}$ Data from sub-Saharan
A prospective cohort study compared IHs in Ghana and the UK and revealed that two-thirds of Ghanaian hernias extended into the scrotum. This was the case in only $7 \%$ of UK IHs. ${ }^{205}$ The majority of these were longstanding rightsided indirect hernias. Ghanaian subjects had an average age of 34 versus 62 years in the UK cohort.

Inguinal hernias, occurring in the young, have a major impact on fragile economies. In the Ghanaian study, $64 \%$ of subjects experienced daily activity limitations and $16.3 \%$ of these individuals were unable to work.

A truly startling percentage of $\mathrm{IH}$ repairs are done on an emergent basis in sub-Saharan Africa- $65 \%$ in Ghana, $76 \%$ in Uganda, $33 \%$ in Sierra Leone and $25 \%$ in Nigeria. ${ }^{200,}{ }^{206-209}$ In contrast, $6 \%$ of IH repairs are performed as emergency in the EU. ${ }^{210}$ A 2007 Nigerian study reported that $20 \%$ of emergent IH repair patients died. ${ }^{211}$

In 2012, data from the National Health and Nutrition Examination Survey prospective cohort study of IHs were used to estimate IH disease burden in Ghana. ${ }^{199}$ Per this 
approach, the IH prevalence in the Ghanaian general population was $3.15 \%$ (range $2.79-3.5 \%$ ). The number of symptomatic hernias was estimated at 530,082 (range $469,501-588,980$ ). The annual incidence of symptomatic hernias was 210 per 100,000 individuals (range $186 / 100,000-233 / 100,000)$. It was concluded that at the estimated Ghanaian IH repair rate of 30 per 100,000 , a backlog of one million hernias needing repair develops each decade. The cost of repairing all symptomatic hernias in Ghana was estimated to be 53 million USD. Hernia elimination over a 10-year period would cost 106 million USD. Nearly five million disability-adjust life years (DALYs) would be saved by the repair of prevalent cases of symptomatic hernia in Ghana. These findings are supported by another study which estimated the unmet burden of IHs in sub-Saharan Africa. ${ }^{212}$ This study reported that the average district hospital performs 30 hernia repairs per 100,000 individuals per year (95\% CI 18-41), leaving an unmet need of 175 per 100,000 annually.

The same model was used to estimate Tanzanian IH prevalence. ${ }^{198}$ The prevalence of IH in Tanzanian adults was $5.36 \%$ while an estimated $12 \%$ of men had hernias. This equates to 683,904 Tanzanian adults with symptomatic IH. The annual incidence of IH in Tanzanian adults was 163 per 100,000 people. At Tanzania's current herniarepair rate, nearly one million hernia-in-need-of-repair backlog will develop over 10 years. Repair of the prevalent symptomatic hernias in Tanzania would save 4.4 million DALYs.

A 2012 study using data from the 2010 Global Burden of Disease (GBD) database quantified the burden of digestive diseases avertable by surgical care at first-level hospitals in low- and middle-income countries (LMICs). ${ }^{202}$ The study calculated the potential decrease in digestive disease burden if quality surgical services were universally available and accessible at first-level hospitals. It concluded that $74 \%$ of the burden of inguinal/femoral hernias in East Europe and Central Asia was avertable.

These disparities in surgical coverage highlight issues possibly amenable to rapid improvement. In East Europe and Central Asia, for example, the excess hernia burden can likely be addressed with few additional resources. Other regions may require a comprehensive reordering of priorities and resources to address their IH burden.

KQ28.b Which types of inguinal hernia repairs are currently performed in LRSs?

Groin hernia repair techniques have evolved over time. ${ }^{213}$ During the last 25 years, techniques with synthetic mesh have become the norm and are now the preferred technique in high-resource settings. They have demonstrated superiority over conventional non-mesh procedures, particularly because of their lower recurrence incidence. $^{50,214,215}$ Additional practice changes in high- resource environments are laparoscopy and day-case surgery. $^{216,} 217$

In LRSs, where out-of-pocket expenditures are significant and families often cope by borrowing money or selling assets to pay for surgery, mesh is often either unavailable or unaffordable. Most IHs in these settings are still repaired with the Bassini method (and many modifications) because of the high cost of mesh and the lack of training in mesh repair. $^{200,}$ 218-220

Occasional exceptions have been reported. A study from Nigeria found that mesh repair was well accepted with few complications at 1-year follow-up. ${ }^{221}$ Similarly, in rural Ghana and Uganda, mesh repair has been successfully used without significant complications. ${ }^{222,}{ }^{223}$ In India, mesh repair seems to be more common (or perhaps more commonly written about) than in other LRSs. ${ }^{224}$ Laparoscopy has been introduced in India as well. ${ }^{225}$ Nevertheless, mesh cost remains prohibitive in most LRSs.

KQ28.c What is the recommended operation for an inguinal hernia in low resource settings?

Most people with IHs live in LRSs. Many operative innovations such as laparo-endoscopic and mesh repair methods cannot be widely used in LRSs due to cost. Solutions that provide cheaper alternatives and do not compromise the safety and effectiveness of mesh repair are needed. One alternative to expensive synthetic mesh is sterilized low-cost "mosquito mesh". It too is a similar synthetic product originally intended for another purpose but is in use for hernia surgery in several locations. ${ }^{224,}{ }^{226-228}$ Several studies of "mosquito mesh" have shown promising results in terms of tissue reaction, outcomes, and cost-effectiveness. ${ }^{222,223,229,230}$

One animal study concluded that "mosquito mesh" might serve as a cheap substitute to other forms of mesh when the latter is unaffordable or unavailable. ${ }^{231}$ Two randomized trials have compared "mosquito mesh" with commercial mesh. One involved 40 patients from Burkina Faso and found no differences in outcomes at 30-day follow-up. ${ }^{232}$ One recent trial with 302 patients from eastern Uganda had a follow-up of 12-35 months. All patients included were operated on with the anterior mesh technique according to Lichtenstein, under local anesthesia, and the vast majority as day cases. Recurrence rate and postoperative complications did not differ significantly between low-cost mesh and those undergoing hernia repair with commercial mesh. ${ }^{223}$

Hernia repair with "mosquito mesh" has also been found to be highly cost effective in both Ghana and Ecuador. ${ }^{197,} 233$

KQ28.d What are the logistical challenges for safe groin hernia repair in low resource settings?

The challenge for hernia surgery in LRSs is to integrate the organizational structure of surgical care into the larger 
healthcare system. ${ }^{203}$ The healthcare systems in LRSs have variations in the range of services offered between hospitals in the same country. ${ }^{234}$ Studies have shown that properly functioning small hospitals and health centers in rural areas can deliver effective basic low-cost surgical services. ${ }^{187,}{ }^{235}$ However, many of them suffer from a lack of trained staff, equipment and integration of services delivery. ${ }^{236} \mathrm{~A}$ well-functioning hospital offering a narrow range of vital surgical services can be part of an integrated model of healthcare delivery. Integration aims to improve the service in relation to efficiency and quality, thereby maximizing use of resources and opportunities. ${ }^{237}$ The benefit of integration has been demonstrated in several settings. ${ }^{238}$

Health practitioners should have appropriate surgical and anesthetic equipment and supplies. It is important for hospitals to be able to administer appropriate anesthesia, whether local (LA), spinal, general (GA) or with tracheal intubation. $^{235}$

A meta-analysis demonstrated a striking disparity between anesthesia-related mortality in LRSs when compared with high income countries. ${ }^{239}$ Factors contributing to this disparity included: few qualified anesthetists, lack of appropriate training, limited supplies for safe patient monitoring, and limited supplies for the safe administration of anesthesia. ${ }^{240}$

Adequate surgical training of practitioners and the use of LA permit the vast majority of IH repairs to be done in LRSs. Studies have shown that IH repairs with LA allow return to normal activity a day earlier than GA, important in LRSs. ${ }^{241}$ Local anesthesia costs significantly less than spinal anesthesia and GA, another advantage in LRSs. ${ }^{234}$

Given these limitations and the inherently higher risk of GA, it is recommended that groin hernia repairs in LRSs be performed under LA.

Several strategies can be used to overcome the logistical challenge of cost. Surgical instrument packs and other materials can be bought at a discount from non-profit organizations. Healthcare facilities and manufacturers can donate these materials close to their expiration dates. ${ }^{242}$ If medical personnel and equipment are in short supply, short-term surgical missions by charitable organization can help reinforce the existing infrastructure. Sanitary mobile surgical platforms can be used in environments lacking modern sterile facilities. ${ }^{243}$ While short-term surgical missions have been promoted as a method of alleviating disease burden, the best way for charitable organizations to support surgical care in LRSs is through partnerships with local hernia societies and health practitioners. ${ }^{243}, 244$ Teaching and training local teams should be performed next to alleviate the waiting list. A partnership of this type is occurring presently in Ghana with Operation Hernia http://www.operationhernia.org.uk/. ${ }^{234}$ The effectiveness should particularly be evaluated in respect to the retention of surgical skills of the newly trained staff, to improvements in outcomes, and to the retention, in-country, of local healthcare providers. ${ }^{243,} 244$

A sustainable model to improve hernia surgery in LRS requires a national commitment to providing access to surgical services, especially in rural areas, and to adequately training practitioners. Safe, effective, accessible and cost-effective surgical services must be available to meet needs in LRSs. ${ }^{245}$

KQ28.e Should any special precautions be taken?

Only a few studies exist on interventions like antibiotics or nutritional supplementation pre- or postsurgery in LRSs. ${ }^{197,}$ 208, 246-248 One additional study analyzed IH patients in Ghana, Nigeria and the Ivory Coast operated on between 2005 and 2010. Mesh - either a standard brand polypropylene mesh or sterilized mosquito net-was used. Antibiotics were administered at the surgeon's discretion, with most patients receiving them. ${ }^{249}$

Summary statements from the studies cited above include:

- Antibiotics are recommended, particularly when mesh is implanted.

- For incarcerated hernias without bowel necrosis, a mesh repair with antibiotic coverage might be recommended.

- Antibiotics are recommended in all strangulated hernia repairs with or without bowel necrosis.

- Antibiotic administration was not standardized across the studies.

- No recommendations about nutritional supplementation were made.

Clearly, multi-centered RCTs in LRSs are needed to guide decision making about antibiotic use and nutritional supplementation.

KQ28.f Which mesh is most suitable for IH repair in LRSs?

In most resource-poor countries, sutured repair-with significantly inferior results compared with mesh-is common, since commercial mesh is either unavailable or unaffordable. ${ }^{184,} 250$

The hernia healthcare industry has developed over 200 mesh types with costs ranging from 40 to 6000 USD per piece. ${ }^{251}$ The most commonly used macro-porous polymers are polypropylene and polyester. Meshes differ marginally in their ultrastructure, filament type/construction, pore size, weight/density, tensile strength and elasticity. ${ }^{251} \mathrm{Com}$ mercial hernia meshes are class II medical devices and are required to undergo the Food and Drug Administration (FDA) pre-market notification process in the United States or the Medicines and Healthcare products Regulatory Agency (MHRA) or other authority approval in the UK and 
Europe prior to market release. ${ }^{252}$ Clearly these approved meshes are suitable for use in LRSs but are generally unaffordable there and therefore not used.

The use of mosquito net as an alternative to commercial prosthetics was pioneered in India by Dr. Tongaonkar. ${ }^{224}$ The first multicenter trial was performed there, using indigenous autoclaved and sterilized mosquito net mesh composed of polyethylene and polypropylene. The study reported a $6.9 \%$ incidence of complications, comparable to complications seen with Prolene mesh, with only one recurrence $(0.27 \%)$ and no adverse mesh reactions at up to 5 -year follow-up. More recently, a number of studies in developing countries have examined hernia repair with locally available mosquito net of various types. ${ }^{227,} 231,232,{ }^{253-257}$ Mosquito nets vary in construction, but most commonly consist of cotton, polyethylene, nylon and polyester polymers. ${ }^{258}$

Net pore size must be less than $1.2 \mathrm{~mm}$ to stop mosquitoes. However, many nets use a pore size of $0.6 \mathrm{~mm}$ to stop other biting insects. ${ }^{258}$ Several studies have demonstrated that mosquito net can be implanted with low complication rates, but not all mosquito nets are the same. In addition to pore size differences, some are constructed of unsuitable polymers, have coatings such as DEET, and have biomechanical properties that may produce intense inflammation, all of which may lead to mesh complications.

There are legitimate concerns about infection risk, foreign-body reaction, the effectiveness of sterilization procedures in LRS hospitals, and the safe use of locally sourced and prepared mosquito net for implantation.

A 2013 study compared the characteristics of mosquito net to other FDA- and MHRA-approved commercial meshes. ${ }^{230}$ The tested mosquito net was a low-density polyethylene homo-polymer (LDPE), knitted from monofilament fibers, the mean pore diameter was $1.9 \mathrm{~mm}$, with a $91.2 \%$ porosity, $53.7 \mathrm{~g} / \mathrm{m}^{2}$ mean mesh weight, and linear mass density of 152 denier, comparable to the "large pore" (class I) commercial meshes. The bursting force for polyethylene mosquito net was greater than that for UltraPro and Vypro (43.0 vs 35.5 and $27.2 \mathrm{~N} / \mathrm{cm}$, respectively). The mosquito net exhibited less anisotropy when compared with commercial meshes.

A randomized trial of nylon mosquito net versus commercial mesh in $40 \mathrm{IH}$ patients from Burkina Faso found no difference in short-term 30-day follow-up outcomes. ${ }^{232}$

A 10-year retrospective analysis was done of consecutive patients who underwent a total of 651 IH LDPE net repairs and were followed for 12-18 months. Thirty-two patients were lost to follow-up. Six superficial surgical site infections occurred $(0.9 \%)$, as did one seroma $(0.1 \%)$, and two hematomas $(0.3 \%)$. Two patients reported chronic pain $(0.3 \%)$. No recurrences or mesh rejections were reported.
The LDPE net was less than $0.03 \%$ the cost of commercial mesh. ${ }^{257}$

A recently published RCT comparing LDPE mesh with commercial mesh including 302 male patients concluded that there was no significant difference in recurrence or complication rates. ${ }^{223}$ The follow-up rate was $97.3 \%$ after 2 weeks and $95.6 \%$ after 1 year. Recurrence occurred in 1 patient $(0.7 \%)$ assigned to LDPE mesh and in no patients assigned to commercial mesh (absolute risk difference 0.7 percentage points; $95 \%$ confidence interval $[\mathrm{CI}]-1.2$ to 2.6; $p=1.0$ ). Postoperative complications occurred in 44 patients $(30.8 \%)$ assigned to the low-cost mesh and in 44 patients $(29.7 \%)$ assigned to the commercial mesh (absolute risk difference, 1.0 percentage point; $95 \% \mathrm{CI},-9.5$ to 11.6; $p=1.0$ ).

When mosquito net is used, tension-free IH repair is approximately one-third the cost of repair with a conventional alternative. ${ }^{227,229,} 233$ This finding is supported by a meta-analysis, which also found no increase in septic complications or recurrences. ${ }^{259}$

Mosquito net steam sterilization at $121{ }^{\circ} \mathrm{C}$ has been recommended but long-term follow-up data confirming sterility is lacking. Most of the currently used LDPE net is sterilized with ethylene oxide. ${ }^{222}$

Cost-effectiveness analyses have estimated the overall cost associated with mesh repair to be 12.88 USD per DALY averted (assuming 120.02 USD/hernia repair and 9.3 DALYs averted/person). ${ }^{197,}{ }^{233}$ Based on this figure, hernia repair using low-cost mesh is a more cost-effective intervention than oral dehydration or at-home HIV/AIDS treatment with antiretroviral therapy. ${ }^{202}$

Before universal acceptance of mosquito net for $\mathrm{IH}$ repair can be achieved; however, careful audit and followup studies are required, which may be difficult to do in LRSs.

KQ28.g What is the best way to sustainably educate surgeons in LRSs?

Groin hernia surgery is the most common surgery performed in LRSs even though access to surgical services is very limited. A lack of skilled healthcare personnel exacerbates this access problem. In sub-Saharan Africa, for example, most surgical and anesthesia services are provided by general physicians or non-physician clinicians rather than specialists. ${ }^{260,}{ }^{261}$ Hernia is a neglected condition in LRSs. Strategies to provide education, training, and resources and reorder priorities are necessary to change this situation.

Many surgical skill educational programs exist but are not especially focused on hernia surgery. It is known that continuing education improves patient safety. ${ }^{261} \mathrm{~A}$ conceptual hernia surgery education program could focus on three groups of LRS surgeons. 
- Surgeons needing focused training and skill development

- Hernia societies can create a hernia surgery certificate program whereby LRS surgeons receive a certificate of completion/competence after finishing a supervised course of study and demonstrate competent performance of a series of IH repair skills.

- Healthcare provider continuous education and skills training

- Open to surgeons and all others involved in IH patient care activities.

- May involve periodic visits from referral hospital personnel, telemedicine, review of educational materials.

- On-site support and training in hernia surgery by surgeon specialists from referral hospitals to outlying facilities.

- Operators/surgeons in outlying hospitals

- Can be visited on a rotating or as-needed basis by hernia specialists in a series of "surgical camps".

Few studies have evaluated the impact of short international training trips on the practice of local physicians following training trip participation. One study conducted in Ghana and Liberia reported on a 2-day surgical training course on tension-free mesh repair performed in a resource-limited setting. It also looked at the course's impact on local surgical practice. It concluded that a brief training course can significantly improve local practice. Operation Hernia is a UK-registered charity initiative involving the EHS and the Plymouth-Takoradi (Ghana) Hospital which trains, and teaches hernia surgery, in Africa. It sends volunteer teams to work alongside African surgeons, training them in local anesthetic administration and guiding/mentoring during hernia operations. Teams operate on a large volume of cases in a short time, often in two theatres simultaneously. $8,201,262$

When deciding which surgical services to offer facility capabilities and infrastructure must be considered. A wellequipped facility is necessary to support a strong education program in LRSs. ${ }^{261}$ Per the WHO Safe Surgery Initiate, operating theatres must be of adequate size, have appropriate lighting and have dependable electricity and water at a minimum. ${ }^{245}$

KQ28.h How can the internet and other technologies be used to teach physicians in LRSs?

Continuing education/training and data collection should be the focus of using new technologies to improve hernia surgery in LRSs. Internet use has already been highlighted by the cooperation between LMICs and high income countries (HIC) in the Global Surgery Project ${ }^{263}$ http://www.lancetglobalsurgery.org. Internet-based technologies are efficient ways of sharing surgical experience and may be a way to expand surgical education and strengthen local expertise in LRS. ${ }^{264,} 265$ One article describes that two surgeons from Paraguay and Brazil were trained by two international experts to perform a Lichtenstein IH repair using Google Glasses via an interactive online video stream. ${ }^{265}$ Multimedia are now used to disseminate medical content through archived and live video allowing physicians to stay current in a variety of settings. ${ }^{266}$

Most LRS physicians are connected to the World Wide Web. Advanced interactive technology allows experts to be virtually present, and assist through tele-mentoring, while other surgeons perform operations. ${ }^{265,} 266$

Internet-based data collection will facilitate the rapid development of hernia registries in LRSs as well as world hernia registries. The EHS now offers the global surgical community an online platform for registration and outcome measurements of abdominal wall hernia repairs (http:// www.eurahs.eu/HOME.php). ${ }^{168}$

The challenge in the short-term is the optimization of medical technology and clinical practice in order to deliver the best medical care and the highest patient satisfaction at the lowest cost. ${ }^{267}$ Research is needed on the impact of internet use and other technologies to achieve safe effective surgery globally.

\section{Research, General Practitioners and Patients perspectives}

\section{Chapter 29}

\section{Questions for research}

\section{B. van den Heuvel, M. P. Simons}

The members of Hernia Surge have attempted to evaluate all available literature on inguinal hernia surgery. Concerning many topics high quality designed studies have led to clear statements and conclusions. However, on many other topics high quality studies are lacking, resulting in great opportunities for future research. In this chapter we have attempted to sum up systematically research questions that we have encountered during the development of these guidelines. We hope that this chapter inspires hernia researchers to conduct new studies to answer these interesting questions. 


\section{Incidence and epidemiology}

Large epidemiologic studies or registry analysis could result in new insights in the incidence of groin hernia. The identification of modifiable life style and socio-occupational factors contributing to development of primary and recurrent inguinal hernia could help hernia surgeons in the future to further tailor surgical management.

\section{Pathophysiology}

It is becoming increasingly clear that the extracellular matrix and matrix metalloproteinases play a significant role in the pathogenesis of abdominal wall hernias. Further investigation into biomarkers which mirror its activities as well as strategies and methodologies to correct abnormalities could dramatically affect the incidence and treatment of abdominal wall pathologies. Interdisciplinary collaborative research with basic science will be necessary to properly investigate this complex environment.

\section{Classification}

Hernia classifications contribute to the possibility to compare and evaluate study outcomes and subsequent management strategy. Which classification system are the most suitable remains unknown. The EHS classification system is a simple system and easy to use. Future research should evaluate what the relevance of the EHS groin hernia classification is.

\section{Indications for surgery}

Watchful waiting (WW) has been a suggested management strategy in male patients with a minimal or asymptomatic inguinal hernia. Some aspects of WW need to be analyzed to fully establish its true value. Is a watchful waiting strategy ultimately cost-effective, considering high crossover rates due to symptom development? What are the risk factors for developing symptoms such as pain or incarceration in untreated male patients with a minimal symptomatic or asymptomatic inguinal hernia? What is the best timing for male patients with a minimal symptomatic or asymptomatic inguinal hernia to plan surgical repair in terms of cost-effectiveness? A large randomized controlled trial with long follow-up would be appropriate to answer this question.

\section{Surgical treatment of inguinal hernia}

There are many of studies performed on surgical techniques in inguinal hernia. However, not all techniques are equally well evaluated and there is still need for further research. Randomized controlled trials in centers where the various surgical techniques are mastered are ideal to address the following issues:
- What is the true recurrence rate and risk for chronic pain after Shouldice repair?

- Is only SAC resection in young patients with an L1 inguinal hernia a safe procedure in terms of recurrence rate?

- Is there a significant difference in results of tissue (Shouldice) repair between an indirect and a direct hernia?

- Are the outcomes after repair with a self-adhesive mesh comparable with a repair with a flat mesh for Lichtenstein?

- Do TEP and TAPP truly have equal results to each other?

- What are the advantages of the use of Prolene Hernia System (PHS) or UltraPro Hernia System (UHS) compared to Lichtenstein and TEP, TAPP?

- What are the long-term recurrence rates after inguinal hernia repair with PHS or UHS? Are these results significantly better to justify the use and subsequent scarring of both the anterior and posterior compartment?

- There is a need to design a large RCT comparing laparo-endoscopic and Lichtenstein repair in primary unilateral inguinal hernia repair in male patients by surgeons who are experts in both these respective techniques.

\section{Individualization in treatment options}

For many years now, the gold standard for inguinal hernia surgical treatment is a mesh repair. The mesh repair can be performed open or laparoscopically. Whether there is still an indication for non-mesh repair, or when a mesh repair needs to be done open or laparoscopically remains to be definitely determined. There is no uniform technique applicable to all patients. Hernia surgeons individualize, based mostly on their own experience. Scientific foundation is lacking. The essential question is: When do we individualize and does a tailored approach result in improved quality and outcomes? Scenarios where individualization might be in place are:

- Which surgical technique should be used in patients with an inguinal hernia with the following characteristics: high preoperative pain, smoking, collagen disease, obesity, ascites, physical active or elderly?

- Which surgical technique should be used in patients with an inguinal hernia with the following characteristics: small indirect, (large) medial or large lateral hernias, non-reducible hernias, incarcerated hernias or strangulated hernias? 
- What is the best management strategy in elderly patients with a minimal or asymptomatic inguinal hernia, watchful waiting or surgery?

- Is there an indication in certain cases (low risk for recurrence, high risk for pain) to perform non-mesh repair?

- Should open repair under local be promoted?

\section{Occult hernia and bilateral repair}

Some suggest that a prophylactic mesh repair on the contralateral side is indicated in older male patients with a medial inguinal hernia. The appropriateness of this strategy needs to be assessed. In which cases is prophylactic bilateral implementation of mesh indicated in unilateral inguinal hernia as a management strategy? Similarly, when an asymptomatic defect is found on the contralateral side during laparoscopic repair of a unilateral symptomatic inguinal hernia, is immediate treatment with mesh indicated? What is the natural course of such asymptomatic defects? There is a need for a prospective registry-based study of unilateral TEP and TAPP cases (with adequate follow-up) to investigate the true risk of lifetime bilateral (symptomatic and asymptomatic) IH.

\section{Day surgery}

We suggest to perform a registry study analyzing the safety of day surgery of the different types of inguinal hernia repair compared to short stay surgery with regards to severe bleeding, unnoticed visceral injury and thromboembolism.

\section{Meshes}

The gold standard for many types of hernia repair is the use of mesh. The long-run effect and interaction between mesh and bodily tissue still needs to be understood. The mesh has to fulfill many requirements and the ideal mesh has yet to be designed. The following research questions address these issues. As HerniaSurge we would like to emphasize that future in vivo research on mesh is of great importance to further improve quality after hernia surgery.

- How are the physiological requirements of mesh with focus on strength and elasticity to meet its functional needs defined?

- Which mesh material or design avoids scar entrapment or erosion?

- What is the value of bioactive meshes with drug release to avoid chronic pain, adhesions, or infection?

- What are the characteristics of the mesh surfaces to minimize the risk for bacterial adherence and for infection in contaminated wounds or surroundings?
- What are the molecular details of the wound healing process around a foreign body?

- Mesh related complications manifest with a considerable delay, the incidence rises with time and is higher for younger patients. Should there be a limitation by the patients' age to perform a mesh procedure? What is the impact of age on the risk-benefit ratio of meshes or mesh procedures?

- There is a need for a propensity score matching of large data from registries comparing the use of different kinds of meshes (e.g., large-pore versus small-pore) in primary unilateral inguinal hernia repair in male patients.

\section{Clinical outcome}

Clinical outcomes are influenced by the patients' biology, the surgical technique and surgical skills and the quality and characteristics of the mesh. It is unknown which of these factors dominates the clinical outcomes, or whether they all contribute equally. It is necessary to analyze the impact of these factors separately. A prediction model could be designed to optimize clinical outcomes in individual cases.

\section{Mesh fixation}

Mesh fixation remains subject of debate. Is mesh fixation necessary to minimize the risk of recurrence, or only in specific cases? And if fixation is needed, which fixation technique is to be used? And what are the disadvantages of fixation? The majority of the randomized controlled trials on mesh fixation include a follow-up of 1 or 2 years, which is the most severe drawback of these studies. Therefore, registry-based studies with a high number of patients and long-term follow-up are of additional value to the current randomized controlled trials on mesh fixations.

\section{Antibiotic prophylaxis}

The indication to use antibiotic prophylaxis is ruled by three factors; the varying standard of environment, patients risk factors and operative technique. A 5\% wound infection rate in patients not receiving antibiotic prophylaxis is defined as a low-risk environment. There is convincing evidence not to administer antibiotic prophylaxis in an average risk patients/low-risk environment and in any patient in any risk environment when using endoscopic/ laparoscopic repair.

There are very limited data on high-risk patients in a low-risk environment and no consensus exists on how to define these conditions. However, common surgical practice includes antibiotic prophylaxis for increased-risk patients and these currently also include those undergoing inguinal hernia repair. This is an area for further studies. 
This question is not adequately answered by a randomized controlled trial, since the potential number of factors, environmental and patient-related, are multifactorial and not well defined. Some of these questions might be answered from a well-designed register, including risk factors of importance and having a high coverage. More important than making more studies on fine-tuning indications for the use of antibiotic prophylaxis would be to implement the current guidelines on antibiotic prophylaxis and register the outcomes.

\section{Anesthesia}

Inguinal hernia repair can very well be performed under local anesthesia. Still very few surgeons offer this technique as an option. Optimization of local anesthesia technique is still to be determined. Should local anesthesia be achieved by a nerve block, local "en route" infiltration or a combination of the two? And in which dilution?

\section{Postoperative pain, prevention and management}

Postoperative pain is an adverse outcome of inguinal hernia repair. Even though the incidence is low, the impact on the patient's quality of life can be significant. A transversus abdominis plane (TAP) block placed by ultrasonography is a promising technique in the management of postoperative pain after inguinal hernia repair. Its value could be evaluated in a randomized controlled trial comparing the use of TAP block pre-, per- and postoperatively to prevent and manage postoperative pain.

Another treatment option is laparoscopic extraperitoneal neurectomy. Its value needs to be evaluated in both open and laparoscopic repairs. We suggest a randomized controlled trial comparing the effect of laparoscopic extraperitoneal neurectomy versus open peripheral neurectomy and total mesh removal for chronic pain after both open and laparoscopic mesh repair.

\section{Convalescence}

Postoperative instructions on when to resume specific activities vary largely depending on the preferences of the surgeon, surgical technique and cultural environment. Stimulation of activities leads to earlier return to normal activities and improved quality of life, without an increase in adverse events, such as recurrence or postoperative pain. However, which physical activity can be regained at what moment postoperatively after inguinal hernia repair (laparoscopic or open, mesh or non-mesh) remains unknown. And also, what is the socio-economic consequence of surgeons' recommendation for postoperative physical activity and sick leave duration?

\section{Groin hernia in women}

Inguinal hernia is less common in women, and subsequently little evidence supports one technique over another. Since there are so little women with an inguinal hernia, we suggest a large registry study with propensity score matching comparing laparo-endoscopic versus open groin hernia repair in women to answer this question: Which technique is preferred in women?

\section{Complications, prevention and treatment}

Adverse events after inguinal hernia repair include recurrence and chronic postoperative pain. Postoperative pain reduces quality of life significantly. With the use of different definitions of chronic postoperative inguinal pain (CPIP) it is hard to compare the outcomes of different studies and extract the preferred treatment. Therefore, worldwide consensus should be reached on a clear definition of CPIP and a uniform assessment of CPIP should be formulated. Subsequently, the impact of CPIP on daily activities can be analyzed, which is unknown until now. The long-term outcomes of treatment of CPIP remain unknown as well, and future registry analysis is recommended. Further on, a prediction model calculating the risk of developing CPIP would be helpful to a hernia surgeon to identify patients with an increased risk and tailor treatment. We recommend future researchers to develop such a model based on existing literature regarding this subject.

Severe complications after inguinal hernia surgery are rare. However, some hernia surgeons suggest that there might be an increased severe complication rate after laparo-endoscopic inguinal hernia, such as death. This severe complication rate might be underestimated due to insufficient number of included patients in trials and the associated surgical expertise in trial participating surgical centers. We therefore recommend to initiate a large registry study in countries where registration is obligatory to investigate the severe complication rate after laparoscopic inguinal hernia surgery.

\section{Emergency groin hernia treatment}

Medical evidence on treatment of acute groin hernias is limited and of poor quality. Future research should focus on identifying risk factors for developing incarceration and strangulation, diagnostic modalities, optimal timing and surgical approach. Nevertheless, in some of these aspects RCTs would be difficult, if not frankly impossible, to perform. Large-scale epidemiological studies based on national or international registries might further improve surgical decision making on this crucial issue. 


\section{Training and learning curve}

It is frequently stated that laparo-endoscopic inguinal hernia repair requires a longer learning curve when compared to open techniques, and endoscopic longer that laparoscopic. Learning curves are commonly expressed in numbers of surgeries performed. However, endoscopic competence of a resident prior to training is left out of the equation. Gradually it is believed that competence based training and learning is a much more viable model. Therefore, we recommend achieving consensus or guidelines on all aspects and surgical steps on groin hernia training. Subsequently, outcomes can be analyzed with regards to the surgeon's competences and secondly to the surgeon's caseload.

\section{Specialized centers and hernia specialists}

Hernia surgery is commonly performed by general surgeons in general hospitals. However, specialized hernia centers are emerging, focusing solely on hernia management. The additional value of these centers needs to be evaluated. Are the outcomes after hernia surgery in a specialized hernia center better compared to hernia surgery performed by a general surgeon in a general hospital? And if so, where should hernia surgery be performed? Which minimal conditions need to be fulfilled to perform hernia surgery in a non-specialized center? Additionally, which requirements are to be met, to call a center a Hernia center in terms of caseload, diagnostics, techniques performed, registry and scientific research participation? And equally, what would be the requirements to call oneself a hernia specialist?

\section{Costs}

The hernia literature needs standardized ways to report cost so that techniques may be equally compared. This would start with a review of the reported different cost models and then propose a standard model. Direct and indirect costs need to be taken into account, respecting international and cultural differences.

\section{Registries}

The use of registries has increased the last couple of years. Large sets of data have shown to be contributive in fields in which randomized controlled trials are lacking. It still needs to be determined whether national hernia registries improve outcomes of hernia treatment? And if so, should registration be internationally encouraged? What is the value of a registry compared to randomized controlled trials? And is data generated by registries valid? Can data from national registries be pooled to an international registry? Future research might find objective data to answer these questions.

A novel strategy is to use health quality registries as platforms for randomization, so-called Registry-based Randomised Clinical Trials (RRCT). By including a randomization module in a population based clinical registry with high coverage and unselected consecutive enrolment, the advantages of a RCT can be combined with the strengths of a large-scale registry. The advantages will be adequate power with unselected patients, facilitated followup, better control of confounding factors, and a powerful tool for conducting studies efficiently and cost-effectively.

\section{Outcomes and quality assessment}

A groin hernia operation is considered a success, not only in absence of complications, such as recurrence and chronic pain, but also if the patient is satisfied with all aspects of the repair. Patient Reported Outcome Measures (PROMs) assess how patients experience their illness and health after treatment. It is foreseen that in the future PROMs will have a growing significant meaning in the treatment of any condition or disease. The linkage of PROMs to national registries, yields opportunities to analyze numerous of variables in hernia surgery and their weight in quality outcomes. It is necessary to develop quality indicators that are well defined and feasible given the time and resources it needs to collect and analyze them.

\section{Implementation}

These current guidelines are an initiative of many surgical hernia societies. It would be interesting to conduct a survey in the future to evaluate surgeons' adherence to these guidelines. Guideline adherence is a tool to measure the value and implementation of the guidelines.

\section{Inguinal hernia surgery in low resource settings}

In low resource settings these current guidelines are less applicable. Mesh is not always available, and subsequent non-mesh techniques are the best surgical option. Question remains, whether it is feasible to implement a safe and cost-effective method of groin hernia mesh repair under local anesthesia in low income settings? Aspects of training, standardizing hernia care and financial aspects should be addressed.

\section{Proposed trials}

Apart from the trials mentioned in the previous text, we stimulate researchers to initiate the following specific trials:

- A randomized controlled trial including young male adults (18-25-years-old) with an inguinal hernia comparing SAC resection only with a Shouldice repair and 
a Lichtenstein/TEP. Follow-up should entail 5 years with primary outcomes recurrence and pain.

- A randomized controlled trial in a specialized environment comparing Shouldice with Lichtenstein and TEP/ TAPP.

- Propensity score matching analysis comparing Shouldice versus Lichtenstein versus TEP versus TAPP in large patient population from registries with an equal distribution of patient characteristics, risk factors and hernia findings.

- An RCT in which unilateral one sided symptomatic IH is compared to bilateral repair (laparo-endoscopically) stratified for medial and lateral hernias. Prospective analysis of the prognosis of an occult hernia should be performed.

- Large registry randomized controlled trials with longterm follow-up ( $>5$ years) comparing all surgical techniques (open non-mesh, open anterior mesh, open posterior mesh and laparo-endoscopic) in primary and recurrent hernia, unilateral and bilateral inguinal hernia repair in male and female patients. Patients should be operated by expert surgeons in the respective technique.

\section{Chapter 30}

\section{Summary for general practitioners}

N. van Veenendaal and M. P. Simons

\section{Background}

\section{Definition}

A groin hernia is defined as a protrusion of viscera or adipose tissue through the inguinal or femoral canal. This protrusion results in either an inguinal or femoral hernia.

In day-to-day practice a classification system for groin hernias is seldom used other than to describe hernia types in general terms such as: lateral/indirect, medial/direct, recurrent and femoral.

An occult hernia is an asymptomatic hernia not detectable by physical examination.

Epidemiology

The lifetime incidence of a groin hernia is $27-43 \%$ in men and $3-6 \%$ in women. Inguinal hernias (IHs) occur 9-12 times more commonly in men. Femoral hernias occur approximately 4 times more commonly in women.

Etiology/pathology

Numerous risk factors-mostly a combination of genetic and acquired features-exist for the development of primary IHs in adults. Risk factors associated with $\mathrm{IH}$ formation are inheritance, a previous contralateral hernia, male gender, elderly age, impaired collagen metabolism, low body mass index, obesity and a history of prostatectomy.

Symptoms

Groin hernias can be either asymptomatic or symptomatic. Approximately one-third of patients with IHs are asymptomatic. Roughly $70 \%$ of asymptomatic individuals with IHs will develop symptoms within 5 years, generally pain or discomfort.

\section{Diagnostics}

History, physical examination and diagnostic work-up

History and physical examination are usually all that are required to confirm the diagnosis of a clinically evident groin hernia. Approximately $95 \%$ of IHs can be diagnosed by physical examination. IHs produce swelling superomedial to the pubic tubercle and femoral hernias cause infero-lateral swelling. However, in practice this subtle distinction is often difficult to discern.

Imaging may be required if there is vague groin swelling and diagnostic uncertainty, poor localization of swelling, intermittent swelling not present at time of physical examination and other groin complaints without swelling. Physical examination and ultrasound combined are suitable for diagnosing patients with vague groin swelling or possible occult groin hernias. When groin ultrasound is negative or non-diagnostic, a dynamic MRI, dynamic CT or even herniography can be considered. Dynamic in this context refers to Valsalva maneuver during testing in an attempt to force a possibly occult or small hernia into its abnormal channel and more clearly demonstrate its presence.

In female patients, the existence of a femoral hernia should be excluded in all cases of a hernia in the groin. No clinical or diagnostic test can reliably distinguish inguinal from femoral hernias in women.

For the evaluation of patients suspected of having a recurrent groin hernia clinical examination and ultrasound are the most suitable. In case of diagnostic doubt after the ultrasound, MRI or CT can be considered.

\section{Management of groin hernia}

Treatment indications

Not all IHs require surgical treatment. There is a low risk of complications like incarceration or strangulation in asymptomatic or minimally symptomatic men with IHs. Therefore, in men, a watchful waiting management strategy is safe for minimally symptomatic or asymptomatic IHs. However, the crossover rate to surgery in men with minimally symptomatic or asymptomatic IHs is high due to the development of symptoms, mostly pain. Approximately 
$70 \%$ of men with these hernias will require surgery within 5 years.

Based on current literature it is not possible to determine if a watchful waiting management strategy is safe for symptomatic men with IHs. The risk of an $\mathrm{IH}$ becoming incarcerated is less than $3 \%$ per year. About $5 \%$ of men with groin hernias require emergent repair. In patients with symptomatic IHs surgical repair is recommended.

Femoral hernias carry a higher risk of incarceration and strangulation than IHs. Approximately $17 \%$ of women with groin hernias require emergent repair. Therefore, timely repair is recommended in women with groin hernias. In femoral hernia patients, even if symptoms are vague or absent, timely surgery is recommended.

At all times surgeons will tailor their treatment based on their expertise, patient- and hernia-related characteristics, local/national resources and logistics.

\section{Surgical treatment}

Worldwide, more than 20 million patients undergo groin hernia repair yearly. A generally accepted technique, suitable for all IHs, does not exist. There are many different techniques in routine use with varying advantages and disadvantages. Surgical repair of a groin hernia can be performed with or without mesh, using either an open approach or a laparo-endoscopic one. The surgeon will discuss the advantages and disadvantages of each technique with the patient. This is dependent upon the surgeon's expertise, local and regional resources and patient preferences.

Eighty-five percent of all IH repairs are performed using an open approach. In high-resource settings, 15-55\% are performed laparo-endoscopically. It is recommended that patients with symptomatic IHs be treated with a meshbased repair technique. The Lichtenstein technique with the onlay placement of a flat mesh is the criterion standard in open hernia repair and most frequently used. Trans-abdominal preperitoneal (TAPP) and total extraperitoneal (TEP) are laparo-endoscopic techniques in which a mesh is inserted in the preperitoneal plane. In TEP a totally preperitoneal approach is used with or without the help of a dissection balloon. In TAPP a laparoscopy is performed. TAPP and TEP have similar operative times, overall complication risks, postoperative acute and chronic pain incidence and recurrence rates. When a mesh is not available, the Shouldice technique is the first choice in nonmesh IH repair. The Shouldice technique has lower recurrence rates that other suture repairs.

A simple IH operation can be performed on a day surgery basis, unless the patient's comorbidities require clinical observation. Day surgery does require that adequate aftercare is organized. Day surgery of patients with complex IHs is suggested only in selected cases.
Women with groin hernias are advised to undergo laparoscopic repair with preperitoneal mesh placement.

Again, groin hernia management will be based on surgeon's expertise, patient- and hernia-related factors, available resources and logistics.

\section{Complications}

Surgical treatment of an $\mathrm{IH}$ is successful in the majority of cases. Complications of $\mathrm{IH}$ repair include: recurrences, chronic postoperative pain, wound infections, urinary and sexual dysfunction, hematoma, seroma, visceral and vascular injury (uncommon), late postoperative complications and mortality.

Risk factors for recurrent IHs are: incorrect surgical technique, female gender, direct IHs, a sliding hernia, collagen metabolism disorders and obesity. Recurrence necessitates reoperation in $5-15 \%$ of cases.

Ten to twelve percent of $\mathrm{IH}$ repair patients experience at least a bothersome level of moderate pain that impacts daily activities. Risk factors for chronic postoperative inguinal pain include: young age, female gender, high preoperative pain and, early high postoperative pain. Longterm disability due to chronic pain occurs in $10-12 \%$ of patients.

The incidence of urinary retention following $\mathrm{IH}$ repair varies from less than 1 to $20 \%$. The most common predisposing factor is the use of general or regional anesthesia. The incidence of sexual dysfunction causing symptoms of a moderate to severe degree is around 5-6\%. Impairment of testicular function and fertility occurs in less than $1 \%$.

Hematoma incidence is reduced after endoscopic $\mathrm{IH}$ repair compared with open repair. Most hematomas resolve spontaneously over 2-4 weeks and can be managed expectantly. Those with large, symptomatic or infected hematomas should be urgently referred back to their surgeons.

The reported incidence of seroma formation after $\mathrm{IH}$ repair varies between 0.5 and $12 \%$. Seroma formation risk factors are: coagulopathy, congestive liver diseases and cardiac insufficiency. There is no evidence that binders and other compression devices prevent hematoma and seroma formation. Most seroma resolve spontaneously over 6-8 weeks. Since infections following seroma aspiration are regularly described, only symptomatic seromas need to be treated.

Serious complications, such as bowel, bladder and vascular injuries, rarely occur during hernia surgery. They are more common (although still rare) during endoscopic versus open repair.

Death in the 30 days following IH repair is very rare and mainly associated with emergent repair or related to medical comorbidities. 


\section{Postoperative instructions}

Postoperative pain can be reduced by paracetamol, NSAIDs, or selective COX-2 inhibitors. The combination of paracetamol and an NSAID may be particularly effective.

A period of rest or a lifting restriction is not necessary after an IH operation. Patients can do what they feel capable of doing.

\section{Chapter 31}

\section{Groin hernias: a patient's perspective}

\author{
N. van Veenendaal, M. P. Simons, M. D. Burg
}

\section{Groin hernia: definition and some general comments}

Groin hernias occur due to muscular weakness in the lower abdominal wall in the general area of the crease between one's leg and abdomen. This weakness results in abdominal contents (abdominal organs, fat or bowels/intestines) bulging through the weak area.

Men and women can develop groin hernias but they are far more common in men.

A bulging or swelling in the groin region is often the first sign of a groin hernia. Pain or vague discomfort can occur but is not always present. Pain and bulging may worsen with coughing, sneezing, lifting, straining or prolonged standing. Occasionally, groin pain without bulging or swelling may indicate that a groin hernia is present. Sometimes, a groin hernia may be found by your healthcare provider on a routine physical examination.

Certain individuals are at increased risk for the development of a groin hernia. The list below contains certain features that may make groin hernia development more likely.

- Male gender

- Those with family members who have groin hernias

- So-called "impaired collagen metabolism" (collagen is a protein in many body tissues like muscle)

- Those with a previous hernia

- The elderly

- Those who've undergone removal of the prostate gland

- The obese

- Those who are extremely thin (so-called "low body mass index").

\section{Hernia "incarceration"}

Some groin hernias are "incarcerated" or trapped in their abnormal locations. The hernia contents then cannot return to their proper position in the body.
Hernia "strangulation"

Strangulated hernias are those that don't have proper blood supply. This means that whatever is in the hernia bulge may begin to die. Hernias that are suddenly far more painful than usual, red or larger than usual are likely to be strangulated. Vomiting or the inability to move one's bowels can also mean that a hernia is strangulated.

When to see a doctor

Immediately see a doctor if your groin hernia, that normally goes easily back into place, suddenly does not. Seek immediate medical attention if you have a groin hernia and develop any of the "strangulation" signs listed above. Do not eat or drink before leaving for hospital. Immediate surgery may be required.

\section{Groin hernia diagnosis}

Your medical history (what you tell the doctor) and the physical examination are usually all that are required to diagnose a groin hernia. An ultrasound or other testing can be done if the diagnosis is not obvious.

\section{Groin hernia treatment}

Groin hernias do not disappear without treatment; in fact, they often get larger and more painful with time. If you suspect that you have a groin hernia, see your general physician or surgeon. Your surgeon can discuss treatment options.

If you're a man with a groin hernia that causes few or no symptoms then a "watch-and-wait" approach may be reasonable. This is because the risk of serious complications-incarceration or strangulation-is low, making watchful waiting a safe strategy. Realize, however, that over time, many groin hernias without symptoms may start causing problems-mostly pain-and require surgery.

Women with groin hernias are often operated upon semi-urgently-even if they do not have symptoms-because their risk of hernia strangulation is higher than men.

Surgeons will tailor their treatment of your groin hernia based on a variety of factors including: their expertise, your medical history, the hernia itself, local (hospital and other) resources, and other considerations, including your wishes.

\section{Groin hernia operations: types and details}

Groin hernia surgeries are incredibly common. Worldwide, more than 20 million people have groin hernia repairs each year! It is important to realize that with groin hernia surgery, "one size does NOT fit all" and one repair technique, suitable for every situation, does NOT exist.

There are many different repair techniques in routine use with varying advantages and disadvantages. Your surgeon will discuss these and other issues with you and your family before proceeding. 
Groin hernia repair can be done with or without mesh. Mesh, if it is used, serves to reinforce and strengthen the area of the hernia.

Also, either an "open" or a "laparo-endoscopic" or socalled "key hole" approach can be used to repair a groin hernia. An "open" approach (which may also involve mesh) means that a surgical incision is made in the groin and the repair is done through this one incision. In a "laparo-endoscopic" approach, one or more small incisions are made and repair is done with the aid of tiny cameras and other small specialized surgical equipment. Many variations on these general themes are routinely used today.

Mesh is proven to be safe and is well tolerated by the human body.

Repair of your groin hernia may well be done in a day surgery (or "same day") surgery center. This means that you'll get to go home the day of your surgery once you're fully awake, recovered and ready.

Depending on your particular circumstances, groin hernia surgery can be done painlessly with local (area of the hernia only), regional (in a larger region of the body) or general (whole body) anesthesia. Again, your surgeon and the anesthesiologist will discuss these options with you.

\section{Complications}

No operation is risk free. Immediately after operation it could be difficult to urinate due to some pain in the operated region. Sometimes a catheter in the urinary bladder is needed to empty the bladder. In male patients it is not too uncommon that the catheter needs to be removed first after some days. Like every other operations, groin hernia surgery complications include: bleeding, infections, blood clot formation, pneumonia and others. Thankfully, these are all rare.

Specific groin hernia complications are described below.

\section{Hematoma}

A hematoma is a collection of blood in the body's tissues and can be recognized by a bluish discoloration and swelling in the area of the surgery, usually several days after surgery. The hematoma (blood) can spread to the base of the penis and scrotum in men, or into the labia majora (vaginal lips) in women. It usually goes away on its own after several days and should not concern you. A hematoma causing severe pain, marked swelling or black discoloration of the skin should prompt an urgent doctor visit.

\section{Seroma}

A seroma is a collection of blood plasma (not blood cells but the clear liquid in blood) that commonly collects after groin hernia surgery. Most go away without treatment in 6-8 weeks. Very large or infected seromas (both very uncommon) may require drainage. See your surgeon if you are concerned and he/she will advise you.

\section{Pain}

Pain-of some type-occurs after nearly all surgeries. Your surgeon will advise the proper treatment so that you are comfortable as you recover. The pain following groin hernia repair is usually mild and is commonly well treated with paracetamol and other non-narcotic pain relievers.

Some patients suffer from longer lasting or more moderate pain. Pain not controlled with recommended medications, or moderate, severe or long-lasting pain should prompt a visit to your surgeon. Severe chronic pain (thankfully very rare) can be debilitating and should prompt a visit to your surgeon who will advise other treatment options.

\section{Recurrence}

Groin hernias can recur in a small number of patients. See your surgeon if you start having the groin symptoms again that caused you to see a doctor originally. Treatments are available.

\section{Groin hernia operation: recovery}

Expect some pain or groin discomfort after surgical repair of your hernia. Depending on the surgical technique used and other factors (like the complications described above) almost all patients should be completely comfortable within 1-2 weeks. Use your pain relievers as recommended as you recover. Support the surgical area during coughing, sneezing or straining in the first few days after your operation. Do routine activities that you feel capable of doing. A period of rest or a lifting restriction is not necessary after a groin hernia operation.

Most surgeons will use dissolvable stitches, which do not need to be removed. If non-dissolvable stitches were used, they will be removed after a week.

If you have urgent questions or problems please consult your general practitioner, your surgeon or a hospital.

\section{Further reading}

The following website is endorsed by the HerniaSurge Guidelines Group: http://www.herniasurge.com

If information in this leaflet is missing or unclear, please inform the HerniaSurge Group via their website.

Acknowledgements The HerniaSurge Group gratefully acknowledges the able editing assistance of M.D. Burg, MD in the preparation of these chapters. Dr. Burg works as an Assistant Clinical Professor and Attending Physician in the UCSF/Fresno Emergency Medicine Residency Program.

The HerniaSurge Group gratefully acknowledges N.E. Simons (Medical Student) for managing the Mendeley reference manager.

The Guidelines development was sponsored by an educational and research grant from Johnson \& Johnson and Bard companies.

The HerniaSurge Group is very grateful for the financial support provided by The European Hernia Society. 
The final manuscript was reviewed externally by professors $\mathrm{J}$. Jeekel (Europe), B. Ramshaw (Americas) and A. Sharma (Asia).

Members of the HerniaSurge Group

The HerniaSurge Group acknowledges the following Steering Committee (SC) and other members:

\section{Steering Committee}

\begin{tabular}{|c|c|c|}
\hline M. P. Simons & (MSI) & The Netherlands (coordinator SC) \\
\hline M. Smietanski & $(\mathrm{MSM})$ & $\begin{array}{l}\text { Poland (European Hernia Society, } \\
\text { Treasurer, SC) }\end{array}$ \\
\hline H. J. Bonjer & (HJB) & $\begin{array}{l}\text { The Netherlands (European Association } \\
\text { for Endoscopic Surgery SC) }\end{array}$ \\
\hline R. Bittner & $(\mathrm{RB})$ & $\begin{array}{l}\text { Germany (International Endo Hernia } \\
\text { Society, SC) }\end{array}$ \\
\hline M. Miserez & (MMI) & Belgium (Editor Hernia, SC) \\
\hline $\begin{array}{l}\text { Th. } \\
\text { J. Aufenacker }\end{array}$ & $(\mathrm{TA})$ & The Netherlands (Statistical expert, SC) \\
\hline $\begin{array}{l}\text { R. } \\
\text { J. Fitzgibbons }\end{array}$ & $(\mathrm{RJF})$ & $\begin{array}{l}\text { USA (Americas Hernia Society, Editor } \\
\text { Hernia, SC) }\end{array}$ \\
\hline P. K. Chowbey & (PC) & India (Asia Pacific Hernia Society, SC) \\
\hline H. M. Tran & (HT) & $\begin{array}{l}\text { Australia (Australasian Hernia Society, } \\
\text { SC) }\end{array}$ \\
\hline R. Sani & (RSA) & $\begin{array}{l}\text { Niger (Afro Middle East Hernia Society, } \\
\text { SC) }\end{array}$ \\
\hline
\end{tabular}

\section{Members}

\begin{tabular}{|c|c|c|c|}
\hline F. Berrevoet & (FB) & Ghent & Belgium \\
\hline J. Bingener & $(\mathrm{JBC})$ & Rochester & USA \\
\hline T. Bisgaard & (TB) & Copenhagen & Denmark \\
\hline K. Bury & $(\mathrm{KB})$ & Gdansk & Poland \\
\hline G. Campanelli & (GC) & Milan & Italy \\
\hline D. C. Chen & $(\mathrm{DCH})$ & Los Angeles & USA \\
\hline J. Conze & (JC) & München & Germany \\
\hline D. Cuccurullo & (DCU) & Naples & Italy \\
\hline A. C. de Beaux & $(\mathrm{ADB})$ & Edinburgh & UK \\
\hline H. H. Eker & (HE) & Amsterdam & $\begin{array}{l}\text { The } \\
\text { Netherlands }\end{array}$ \\
\hline R. H. Fortelny & (RFO) & Vienna & Austria \\
\hline J. F. Gillion & (JG) & Antony & France \\
\hline B. J. van den Heuvel & $(\mathrm{BJH})$ & Amsterdam & $\begin{array}{l}\text { The } \\
\text { Netherlands }\end{array}$ \\
\hline W. W. Hope & (WWH) & Wilmington & USA \\
\hline L. N. Jorgensen & $(\mathrm{LNJ})$ & Copenhagen & Denmark \\
\hline U. Klinge & (UK) & Aachen & Germany \\
\hline F. Köckerling & $(\mathrm{FK})$ & Berlin & Germany \\
\hline J. F. Kukleta & $(\mathrm{JK})$ & Zurich & Switzerland \\
\hline I. Konate & (IK) & Saint Louis & Senegal \\
\hline A. L. Liem & (LL) & Utrecht & $\begin{array}{l}\text { The } \\
\text { Netherlands }\end{array}$ \\
\hline D. Lomanto & (DL) & Singapore & Singapore \\
\hline M. J. A. Loos & (MLO) & Veldhoven & $\begin{array}{l}\text { The } \\
\text { Netherlands }\end{array}$ \\
\hline
\end{tabular}

continued

\begin{tabular}{llll}
\hline M. Lopez-Cano & (MLC) & Barcelona & Spain \\
M. C. Misra & (MM) & New Delhi & India \\
A. Montgomery & (AM) & Malmö & Sweden \\
S. Morales-Conde & (SMC) & Sevilla & Spain \\
F. E. Muysoms & (FM) & Ghent & Belgium \\
H. Niebuhr & (HN) & Hamburg & Germany \\
P. Nordin & (PN) & Östersund & Sweden \\
M. Pawlak & (MP) & Gdansk & Poland \\
G. H. van Ramshorst & (GVR) & Amsterdam & The \\
& & & Netherlands \\
W. M. J. Reinpold & (WR) & Hamburg & Germany \\
D. L. Sanders & (DS) & Barnstaple & UK \\
N. Schouten & (NS) & Utrecht & The \\
& & & Netherlands \\
S. Smedberg & (SS) & Helsingborg & Sweden \\
R. K. J. Simmermacher & (RSI) & Utrecht & The \\
& & & Netherlands \\
S. Tumtavitikul & (ST) & Bangkok & Thailand \\
N. van Veenendaal & (NVV) & Amsterdam & The \\
& & & Netherlands \\
D. Weyhe & (DW) & Oldenburg & Germany \\
A. R. Wijsmuller & (AW) & Rotterdam & The \\
& & & Netherlands \\
& & &
\end{tabular}

External Reviewers

\begin{tabular}{lll}
\hline J. Jeekel & Rotterdam & Europe \\
A. Sharma & New Delhi & Asia \\
B. Ramshaw & Knoxville & Americas \\
\hline
\end{tabular}

\section{Disclaimer}

Since both Editors-in-Chief and one Associate Editor are coauthor of this manuscript, an additional independent review was conducted.

\section{Disclosures}

All HerniaSurge members are active in the scientific community. An additional course was given to all involved members to guarantee unbiased literature searches and review.

The guidelines are the property of HerniaSurge and they were financed through grants by Bard and Johnson \& Johnson. The sponsor had no direct or indirect influence on the methodology or the content of the guidelines.

TA, FB, TB, RB, KB, PC, ADB, HE, RFO, LNJ, IK, JK, LL, DL, MLO, AM, HN, PN, MP, MM, WR, DS, RSA, NS, RSI, MSI, SS, MSM, HT, ST, BJH, GVR, NVV, DW and AW report grants for meeting expenses related to the submitted work from Johnson \& Johnson and Bard, during the conduct of the study.

JBC declares conflict of interest not directly related to the submitted work by receiving grants from National Institutes of Health, Stryker Endoscopy and SAGES; and by receiving non-financial support from Nestle and Titan Medical and reports grants for meeting expenses related to the submitted work from Johnson \& Johnson and Bard, during the conduct of the study. 
HJB declares conflict of interest not directly related to the submitted work by receiving grants from Johnson \& Johnson, Medtronic, Olympus, Applied Medical and Stryker; and by receiving personal fees from Medtronic, Olympus and Cook and reports grants for meeting expenses related to the submitted work from Johnson \& Johnson and Bard, during the conduct of the study.

GC declares conflict of interest not directly related to the submitted work by receiving personal fees from Insightra, Insightra-Acelity and Johnson \& Johnson, and reports grants for meeting expenses related to the submitted work from Johnson \& Johnson and Bard, during the conduct of the study.

DCH declares conflict of interest not directly related to the submitted work by receiving support from Medtronic, and report grants for meeting expenses related to the submitted work from Johnson \& Johnson and Bard, during the conduct of the study.

JC declares conflict of interest not directly related to the submitted work by receiving personal fees from Johnson \& Johnson and Bard, and reports grants for meeting expenses related to the submitted work from Johnson \& Johnson and Bard, during the conduct of the study.

DCU declares conflict of interest not directly related to the submitted work by receiving personal fees from Gore, MSD and Bard, and reports grants for meeting expenses related to the submitted work from Johnson \& Johnson and Bard, during the conduct of the study.

RJF declares conflict of interest not directly related to the submitted work by receiving personal fees from Springer, and reports grants for meeting expenses related to the submitted work from Johnson \& Johnson and Bard, during the conduct of the study.

JG declares conflict of interest not directly related to the submitted work by receiving personal fees from Bard and Covidien Medtronic; and by receiving non-financial support from Peters, Cousin Biotech, Gore and organization of congresses and reports grants for meeting expenses related to the submitted work from Johnson \& Johnson and Bard, during the conduct of the study.

WWH declares conflict of interest not directly related to the submitted work by receiving personal fees from Bard and Gore and reports grants for meeting expenses related to the submitted work from Johnson \& Johnson and Bard, during the conduct of the study.

UK declares conflict of interest not directly related to the submitted work by receiving grants from Ethicon and FEG for research projects; and by receiving personal fees from Ethicon, FEG and Expert Testimony. In addition, Dr. Klinge has a patent PVDF mesh with royalties paid and reports grants for meeting expenses related to the submitted work from Johnson \& Johnson and Bard, during the conduct of the study.

FK declares conflict of interest not directly related to the submitted work by receiving grants from Johnson \& Johnson, Karl Storz, PFM Medical, Dahlhausen, B Braun, Menke Med and Bard; and by receiving personal fees from Tutogen and Bard and reports grants for meeting expenses related to the submitted work from Johnson \& Johnson and Bard, during the conduct of the study.

MLC declares conflict of interest not directly related to the submitted work by receiving personal fees from Johnson \& Johnson and Bard and reports grants for meeting expenses related to the submitted work from Johnson \& Johnson and Bard, during the conduct of the study.

MMI declares conflict of interest not directly related to the submitted work by receiving grants from Bard; and by receiving personal fees from Springer, Aspide, Bard, Braun, Ethicon and Gore and reports grants for meeting expenses related to the submitted work from Johnson \& Johnson and Bard, during the conduct of the study.

SMC declares conflict of interest not directly related to the submitted work by personal fees from Bard, Gore, Medtronic, Olympus and Teleflex and reports grants for meeting expenses related to the submitted work from Johnson \& Johnson and Bard, during the conduct of the study.
FM declares conflict of interest not directly related to the submitted work by receiving grants from Medtronic, Johnson \& Johnson, B. Braun, Dynamesh; and by receiving personal fees from Medtronic, Johnson \& Johnson, Dynamesh, Cousin Biotech and Bard; and by receiving non-financial support from Dynamesh and reports grants for meeting expenses related to the submitted work from Johnson \& Johnson and Bard, during the conduct of the study.

Open Access This article is distributed under the terms of the Creative Commons Attribution-NonCommercial 4.0 International License (http://creativecommons.org/licenses/by-nc/4.0/), which permits any noncommercial use, distribution, and reproduction in any medium, provided you give appropriate credit to the original author(s) and the source, provide a link to the Creative Commons license, and indicate if changes were made.

\section{References}

\section{PART 1}

1. Kingsnorth A, LeBlanc K (2003) Hernias: inguinal and incisional. Lancet 362:1561-1571. https://doi.org/10.1016/S01406736\%2803\%2914746-0

2. Fitzgibbons RJ, Ramanan B, Arya S et al (2013) Long-term results of a randomized controlled trial of a nonoperative strategy (watchful waiting) for men with minimally symptomatic inguinal hernias. Ann Surg 258(3):508-515. https://doi.org/10. 1097/SLA.0b013e3182a19725

3. Simons MP, Aufenacker T, Bay-Nielsen M et al (2009) European Hernia Society guidelines on the treatment of inguinal hernia in adult patients. Hernia 13:343-403. https://doi.org/10. 1007/s10029-009-0529-7

4. Miserez M, Peeters E, Aufenacker T et al (2014) Update with level 1 studies of the European Hernia Society guidelines on the treatment of inguinal hernia in adult patients. Hernia 18(2):151-163. https://doi.org/10.1007/s10029-014-1236-6

5. Bittner R, Arregui ME, Bisgaard T et al (2011) Guidelines for laparoscopic (TAPP) and endoscopic (TEP) treatment of inguinal hernia [International Endohernia Society (IEHS)]. Surg Endosc 25(9):2773-2843. https://doi.org/10.1007/s00464-0111799-6

6. Poelman MM, van den Heuvel B, Deelder JD et al (2013) EAES Consensus Development Conference on endoscopic repair of groin hernias. Surg Endosc 27(10):3505-3519. https://doi.org/ 10.1007/s00464-013-3001-9

7. Danish Hernia Database. https://www.herniedatabasen.dk/. Accessed 29 Oct 2017

8. Swedish Hernia Registry. http://www.svensktbrackregister.se/ images/stories/doc/verksamhetsberattelser/rapport16_170508. pdf. Accessed 29 Oct 2017

9. Tran H, Tran K, Turingan I, Zajkowska M, Lam V, Hawthorne W (2015) Single-incision laparoscopic inguinal herniorraphy with telescopic extraperitoneal dissection: technical aspects and potential benefits. Hernia 19:407-416. https://doi.org/10.1007/ s10029-015-1349-6

10. Tschuor C, Metzger J, Clavien PA, Vonlanthen R, Lehmann K (2015) Inguinal hernia repair in Switzerland. Hernia 19(5):741-745. https://doi.org/10.1007/s10029-015-1385-2

11. Bay-Nielsen M, Kehlet H, Strand L et al (2001) Quality assessment of 26,304 herniorrhaphies in Denmark: a prospective nationwide study. Lancet 358(9288):1124-1128. https://doi.org/ 10.1016/S0140-6736(01)06251-1 
12. Goldet G, Howick J (2013) Understanding GRADE: an introduction. J Evid Based Med 6(1):50-54. https://doi.org/10. 1111/jebm. 12018

13. Phillips B, Ball C, Sackett D, Badenoch D, Straus S, Haynes BDM (2001) Oxford centre for evidence-based medicine levels of evidence. Verfügbar unter http//www .... (March 2009):2001. https://doi.org/10.1111/j.1464-410X.2009.08556.x

14. Primatesta P, Goldacre MJ (1996) Inguinal hernia repair: incidence of elective and emergency surgery, readmission and mortality. Int J Epidemiol 25(4):835-839

15. Hernández-Irizarry R, Zendejas B, Ramirez T et al (2012) Trends in emergent inguinal hernia surgery in Olmsted County, MN: a population-based study. Hernia 16(4):397-403. https://doi.org/10.1007/s10029-012-0926-1

16. Burcharth J, Pedersen M, Bisgaard T, Pedersen C, Rosenberg J (2013) Nationwide prevalence of groin hernia repair. PLoS One 8(1):e54367. https://doi.org/10.1371/journal.pone.0054367

17. Ohene-Yeboah M, Abantanga F, Oppong J et al (2009) Some aspects of the epidemiology of external hernias in kumasi, Ghana. Hernia 13(5):529-532. https://doi.org/10.1007/s10029009-0491-4

18. Akin ML, Karakaya M, Batkin A, Nogay A (1997) Prevalence of inguinal hernia in otherwise healthy males of 20 to 22 years of age. J R Army Med Corps 143(2):101-102

19. Liem MS, van der Graaf Y, Zwart RC, Geurts I, van Vroonhoven TJ (1997) Risk factors for inguinal hernia in women: a case-control study. The Coala Trial Group. Am J Epidemiol 146(9):721-726

20. Van Wessem KJP, Simons MP, Plaisier PW (2003) The etiology of indirect inguinal hernias: congenital and/or acquired? Hernia 7(2):76-79. https://doi.org/10.1007/s10029-002-0108-7

21. Ruhl CE, Everhart JE (2007) Risk factors for inguinal hernia among adults in the US population. Am J Epidemiol 165(10):1154-1161. https://doi.org/10.1093/aje/kwm011

22. Quintas ML, Rodrigues CJ, Yoo JH, Rodrigues AJ Junior (2000) Age related changes in the elastic fiber system of the interfoveolar ligament. Rev Hosp Clin Fac Med Sao Paulo 55(3):83-86. https://doi.org/10.1590/S004187812000000300003

23. Nilsson H, Stranne J, Stattin P, Nordin P (2014) Incidence of groin hernia repair after radical prostatectomy: a populationbased nationwide study. Ann Surg 259(6):1223-1227. https://doi.org/10.1097/SLA.0b013e3182975c88

24. Stranne J, Johansson E, Nilsson A et al (2010) Inguinal hernia after radical prostatectomy for prostate cancer: results from a randomized setting and a nonrandomized setting. Eur Urol 58(5):719-726. https://doi.org/10.1016/j.eururo.2010.08.006

25. Lodding P, Bergdahl C, Nyberg M, Pileblad E, Stranne J, Hugosson J (2001) Inguinal hernia after radical retropubic prostatectomy for prostate cancer: a study of incidence and risk factors in comparison to no operation and lymphadenectomy. J Urol 166(3):964-967

26. Lin BM, Hyndman ME, Steele KE et al (2011) Incidence and risk factors for inguinal and incisional hernia after laparoscopic radical prostatectomy. Urology 77(4):957-962. https://doi.org/ 10.1016/j.urology.2010.12.011

27. Ku JH, Jeong CW, Park YH, Cho MC, Kwak C, Kim HH (2011) Nerve-sparing procedure in radical prostatectomy: a risk factor for hernia repair following open retropubic, pure laparoscopic and robot-assisted laparoscopic procedures. Scand J Urol Nephrol 45(3):164-170. https://doi.org/10.3109/00365599.2010. 544674

28. Lughezzani G, Sun M, Perrotte P et al (2010) Comparative study of inguinal hernia repair rates after radical prostatectomy or external beam radiotherapy. Int $\mathrm{J}$ Radiat Oncol Biol Phys 78(5):1307-1313. https://doi.org/10.1016/j.ijrobp.2009.09.065
29. Sun M, Lughezzani G, Alasker A et al (2010) Comparative study of inguinal hernia repair after radical prostatectomy, prostate biopsy, transurethral resection of the prostate or pelvic lymph node dissection. J Urol 183(3):970-975. https://doi.org/ 10.1016/j.juro.2009.11.036

30. Yoshimine S, Miyajima A, Nakagawa K, Ide H, Kikuchi E, Oya M (2009) Extraperitoneal approach induces postoperative inguinal hernia compared with transperitoneal approach after laparoscopic radical prostatectomy. Jpn J Clin Oncol 40(4):349-352. https://doi.org/10.1093/jjco/hyp172

31. Sekita N, Suzuki H, Kamijima $S$ et al (2009) Incidence of inguinal hernia after prostate surgery: open radical retropubic prostatectomy versus open simple prostatectomy versus transurethral resection of the prostate. Int J Urol 16(1):110-113. https://doi.org/10.1111/j.1442-2042.2008.02190.x

32. Koie $\mathrm{T}$, Yoneyama $\mathrm{T}$, Kamimura $\mathrm{N}$, Imai $\mathrm{A}$, Okamoto $\mathrm{A}$, Ohyama C (2008) Frequency of postoperative inguinal hernia after endoscope-assisted mini-laparotomy and conventional retropubic radical prostatectomies. Int J Urol 15(3):226-229. https://doi.org/10.1111/j.1442-2042.2007.01983.x

33. Abe T, Shinohara N, Harabayashi T et al (2007) Postoperative inguinal hernia after radical prostatectomy for prostate cancer. Urology 69(2):326-329. https://doi.org/10.1016/j.urology.2006. 09.043

34. Tsai P-J, Yu C-C, Lee Y-H, Huang J-K (2004) Inguinal hernia after radical retropubic prostatectomy-experience of Kaohsiung Veterans General Hospital. J Chin Med Assoc 67(3):141-144

35. Ichioka K, Yoshimura K, Utsunomiya N et al (2004) High incidence of inguinal hernia after radical retropubic prostatectomy. Urology 63(2):278-281. https://doi.org/10.1016/j.urology. 2003.09.038

36. Rosemar A, Angerås U, Rosengren A, Nordin P (2010) Effect of body mass index on groin hernia surgery. Ann Surg 252(2):397-401. https://doi.org/10.1097/SLA. 0b013e3181e985a1

37. Rosemar A, Angerås U, Rosengren A (2008) Body mass index and groin hernia: a 34-year follow-up study in Swedish men. Ann Surg 247(6):1064-1068. https://doi.org/10.1097/SLA. 0b013e31816b4399

38. Ichioka K, Kohei N, Yoshimura K, Arai Y, Terai A (2007) Impact of retraction of vas deferens in postradical prostatectomy inguinal hernia. Urology 70(3):511-514. https://doi.org/10. 1016/j.urology.2007.04.025

39. Burcharth J, Andresen K, Pommergaard H-C, Rosenberg J (2015) Groin hernia subtypes are associated in patients with bilateral hernias: a 14-year nationwide epidemiologic study. Surg Endosc 29(7):2019-2026. https://doi.org/10.1007/s00464014-3905-Z

40. Henriksen NA, Yadete DH, Sorensen LT, Ågren MS, Jorgensen LN (2011) Connective tissue alteration in abdominal wall hernia. Br J Surg 98(2):210-219. https://doi.org/10.1002/bjs.7339

41. Aren A, Gökçe AH, Gökçe FS, Dursun N (2011) Roles of matrix metalloproteinases in the etiology of inguinal hernia. Hernia 15(6):667-671. https://doi.org/10.1007/s10029-011-0846-5

42. Śmigielski J, Brocki M, Kuzdak K, Kołomecki K (2011) Serum MMP 2 and TIMP 2 in patients with inguinal hernias. Eur J Clin Investig 41(6):584-588. https://doi.org/10.1111/j.1365-2362. 2010.02445.x

43. Pascual G, Rodríguez M, Gómez-Gil V, Trejo C, Buján J, Bellón JM (2010) Active matrix metalloproteinase-2 upregulation in the abdominal skin of patients with direct inguinal hernia. Eur J Clin Investig 40(12):1113-1121. https://doi.org/10.1111/j. 1365-2362.2010.02364.x

44. Liem MSL, Van Der Graaf Y, Beemer FA, Van Vroonhoven TJMV (1997) Increased risk for inguinal hernia in patients with 
Ehlers-Danlos syndrome. Surgery 122(1):114-115. https://doi. org/10.1016/S0039-6060(97)90273-7

45. Sarosi GA, Wei Y, Gibbs JO et al (2011) A clinician's guide to patient selection for watchful waiting management of inguinal hernia. Ann Surg 253(3):605-610. https://doi.org/10.1097/SLA. 0b013e31820b04e9

46. Vad MV, Frost P, Bay-Nielsen M, Svendsen SW (2012) Impact of occupational mechanical exposures on risk of lateral and medial inguinal hernia requiring surgical repair. Occup Environ Med 69(11):802-809. https://doi.org/10.1136/oemed-2012100787

47. Svendsen SW, Frost P, Vad MV, Andersen JH (2013) Risk and prognosis of inguinal hernia in relation to occupational mechanical exposures - a systematic review of the epidemiologic evidence. Scand J Work Environ Health 39(1):5-26. https://doi.org/10.5271/sjweh.3305

48. Carbonell JF, Sanchez JL, Peris RT et al (1993) Risk factors associated with inguinal hernias: a case control study. Eur J Surg 159(9):481-486

49. Lau H, Fang C, Yuen WK, Patil NG (2007) Risk factors for inguinal hernia in adult males: a case-control study. Surgery 141(2):262-266. https://doi.org/10.1016/j.surg.2006.04.014

50. Nordin P, Haapaniemi S, van der Linden W, Nilsson E (2004) Choice of anesthesia and risk of reoperation for recurrence in groin hernia repair. Ann Surg 240(1):187-192. https://doi.org/ 10.1097/01.sla.0000130726.03886.93

51. van der Linden W, Warg A, Nordin P (2011) National register study of operating time and outcome in hernia repair. Arch Surg 146(10):1198-1203. https://doi.org/10.1001/archsurg.2011.268

52. Koch A, Edwards A, Haapaniemi S, Nordin P, Kald A (2005) Prospective evaluation of 6895 groin hernia repairs in women. Br J Surg 92(12):1553-1558. https://doi.org/10.1002/bjs.5156

53. Sorensen LT, Friis E, Jorgensen T et al (2002) Smoking is a risk factor for recurrence of groin hernia. World J Surg 26(4):397-400. https://doi.org/10.1007/s00268-001-0238-6

54. Bay-Nielsen MKH (2006) Inguinal herniorrhaphy in women. Hernia 10(1):30-33

55. Stylianidis G, Haapamäki MM, Sund M, Nilsson E, Nordin P (2010) Management of the hernial sac in inguinal hernia repair. Br J Surg 97(3):415-419. https://doi.org/10.1002/bjs.6890

56. El-Dhuwaib Y, Corless D, Emmett C, Deakin M, Slavin J (2013) Laparoscopic versus open repair of inguinal hernia: a longitudinal cohort study. Surg Endosc 27(3):936-945. https://doi.org/ 10.1007/s00464-012-2538-3

57. Brandt-Kerkhof A, Van Mierlo M, Schep N, Renken N, Stassen L (2011) Follow-up period of 13 years after endoscopic total extraperitoneal repair of inguinal hernias: a cohort study. Surg Endosc Other Interv Tech 25(5):1624-1629. https://doi.org/10. 1007/s00464-010-1462-7

58. Kald A, Nilsson E, Anderberg B et al (1998) Reoperation as surrogate endpoint in hernia surgery. A three year follow-up of 1565 herniorrhaphies. Eur J Surg 164(1):45-50. https://doi.org/ $10.1080 / 110241598750004940$

59. Burcharth J, Pommergaard HC, Bisgaard T, Rosenberg J (2015) Patient-related risk factors for recurrence after inguinal hernia repair: a systematic review and meta-analysis of observational studies. Surg Innov 22:303-317. https://doi.org/10.1177/ 1553350614552731

60. Nordin P, van der Linden W (2008) Volume of procedures and risk of recurrence after repair of groin hernia: national register study. BMJ 336(7650):934-937. https://doi.org/10.1136/bmj. 39525.514572.25

61. Lowham AS, Filipi CJ, Fitzgibbons RJ et al (1997) Mechanisms of hernia recurrence after preperitoneal mesh repair. Traditional and laparoscopic. Ann Surg 225(4):422-431. https://doi.org/10. 1097/00000658-199704000-00012
62. Neumayer LA, Gawande AA, Wang J et al (2005) Proficiency of surgeons in inguinal hernia repair: effect of experience and age. Ann Surg 242(3):344-348. https://doi.org/10.1097/01.sla. 0000179644.02187.ea

63. Feng B, He Z-R, Li J-W et al (2013) Feasibility of incremental laparoscopic inguinal hernia repair development in China: an 11-year experience. J Am Coll Surg 216(2):258-265. https://doi. org/10.1016/j.jamcollsurg.2012.10.020

64. Nixon SJ, Jawaid H (2009) Recurrence after inguinal hernia repair at ten years by open darn, open mesh and TEP-no advantage with mesh. Surgeon 7(2):71-74

65. Lamb ADG, Robson AJ, Nixon SJ (2006) Recurrence after totally extraperitoneal laparoscopic repair: implications for operative technique and surgical training. Surgeon 4(5):299-307. https://doi.org/10.1016/S1479-666X(06)80007-7

66. Edwards CC, Bailey RW (2000) Laparoscopic hernia repair: the learning curve. Surg Laparosc Endosc Percutan Tech 10(3):149-153

67. Felix E, Scott S, Crafton B et al (1998) Causes of recurrence after laparoscopic hernioplasty. A multicenter study. Surg Endosc 12(3):226-231. https://doi.org/10.1007/s004649900640

68. Champault GG, Rizk N, Catheline JM, Turner R, Boutelier P (1997) Inguinal hernia repair: totally preperitoneal laparoscopic approach versus Stoppa operation: randomized trial of 100 cases. Surg Laparosc Endosc 7(6):445-450. https://doi.org/10. 1097/00019509-199712000-00002

69. Bittner R, Schmedt CG, Schwarz J, Kraft K, Leibl BJ (2002) Laparoscopic transperitoneal procedure for routine repair of groin hernia. Br J Surg 89(8):1062-1066. https://doi.org/10. 1046/j.1365-2168.2002.02178.x

70. Bökeler U, Schwarz J, Bittner R, Zacheja S, Smaxwil C (2013) Teaching and training in laparoscopic inguinal hernia repair (TAPP): impact of the learning curve on patient outcome. Surg Endosc 27(8):2886-2893. https://doi.org/10.1007/s00464-0132849-z

71. Liem MS, van Steensel CJ, Boelhouwer RU et al (1996) The learning curve for totally extraperitoneal laparoscopic inguinal hernia repair. Am J Surg 171(2):281-285. https://doi.org/10. 1016/S0002-9610(97)89569-4

72. Frisén A, Starck J, Smeds S, Nyström PO, Kald A (2011) Analysis of outcome of Lichtenstein groin hernia repair by surgeons-in-training versus a specialized surgeon. Hernia 15(3):281-288. https://doi.org/10.1007/s10029-010-0780-y

73. Andresen K, Bisgaard T, Rosenberg J (2014) Sliding inguinal hernia is a risk factor for recurrence. Langenbeck's Arch Surg 400(1):101-106. https://doi.org/10.1007/s00423-014-1262-y

74. Rosch R, Junge K, Knops M, Lynen P, Klinge U, Schumpelick $\mathrm{V}$ (2003) Analysis of collagen-interacting proteins in patients with incisional hernias. Langenbeck's Arch Surg 387(11-12):427-432. https://doi.org/10.1007/s00423-002-03453

75. Zheng H, Si Z, Kasperk R et al (2002) Recurrent inguinal hernia: disease of the collagen matrix? World J Surg 26(4):401-408. https://doi.org/10.1007/s00268-001-0239-5

76. van Veen RN, Wijsmuller AR, Vrijland WW, Hop WC, Lange JF, Jeekel J (2007) Long-term follow-up of a randomized clinical trial of non-mesh versus mesh repair of primary inguinal hernia. Br J Surg 94(4):506-510. https://doi.org/10.1002/bjs. 5627

77. Kehlet H, Bay-Nielsen M (2008) Local anaesthesia as a risk factor for recurrence after groin hernia repair. Hernia 12(5):507-509. https://doi.org/10.1007/s10029-008-0371-3

78. Lundström K-J, Sandblom G, Smedberg S, Nordin P (2012) Risk factors for complications in groin hernia surgery. Ann Surg 255(4):784-788. $0 \mathrm{~b} 013 \mathrm{e} 31824 \mathrm{~b} 7 \mathrm{cb} 3$ 
79. Staarink M, Van Veen RN, Hop WC, Weidema WF (2008) A 10-year follow-up study on endoscopic total extraperitoneal repair of primary and recurrent inguinal hernia. Surg Endosc Other Interv Tech 22(8):1803-1806. https://doi.org/10.1007/ s00464-008-9917-9

80. Junge K, Rosch R, Klinge U et al (2006) Risk factors related to recurrence in inguinal hernia repair: a retrospective analysis. Hernia 10(4):309-315. https://doi.org/10.1007/s10029-0060096-0

81. Nilsson E, Kald A, Anderberg B et al (1997) Hernia surgery in a defined population: a prospective three year audit. Eur J Surg 163(11):823-829

82. Solorzano CC, Minter RM, Childers TC, Kilkenny JW, Vauthey JN (1999) Prospective evaluation of the giant prosthetic reinforcement of the visceral sac for recurrent and complex bilateral inguinal hernias. Am J Surg 177(1):19-22. https://doi.org/10. 1016/S0002-9610(98)00292-X

83. Gilbert AI, Felton LL (1993) Infection in inguinal hernia repair considering biomaterials and antibiotics. Surg Gynecol Obstet 177(2):126-130

84. Oh H-K, Kim H, Ryoo S, Choe EK, Park KJ (2011) Inguinal hernia repair in patients with cirrhosis is not associated with increased risk of complications and recurrence. World J Surg 35(6):1229-1233. https://doi.org/10.1007/s00268-011-1007-9

85. Jansen PL, Klinge U, Jansen M, Junge K (2009) Risk factors for early recurrence after inguinal hernia repair. BMC Surg 9(18):1471-2482. https://doi.org/10.1186/1471-2482-9-18

86. van den Berg JC, de Valois JC, Go PM, Rosenbusch G (1999) Detection of groin hernia with physical examination, ultrasound, and MRI compared with laparoscopic findings. Investig Radiol 34(12):739-743

87. Bittner R, Bingener-Casey J, Dietz U et al (2014) Guidelines for laparoscopic treatment of ventral and incisional abdominal wall hernias (International Endohernia Society (IEHS)—Part 1. Surg Endosc Other Interv Tech 28(1):2-29. https://doi.org/10.1007/ s00464-013-3170-6

88. Depasquale R, Landes C, Doyle G (2009) Audit of ultrasound and decision to operate in groin pain of unknown aetiology with ultrasound technique explained. Clin Radiol 64(6):608-614. https://doi.org/10.1016/j.crad.2008.11.006

89. Light D, Ratnasingham K, Banerjee A, Cadwallader R, Uzzaman MM, Gopinath B (2011) The role of ultrasound scan in the diagnosis of occult inguinal hernias. Int J Surg 9(2):169-172. https://doi.org/10.1016/j.ijsu.2010.10.014

90. Whalen HR, Gillian A, Kidd PJO (2011) Easily missed? Femoral hernias. BMJ 343:7668

91. Bradley M, Morgan D, Pentlow B, Roe A (2003) The groin hernia-an ultrasound diagnosis? Ann $\mathrm{R}$ Coll Surg Engl 85(3):178-180. https://doi.org/10.1308/003588403321661334

92. Cherian PT, Parnell AP (2008) The diagnosis and classification of inguinal and femoral hernia on multisection spiral CT. Clin Radiol 63(2):184-192. https://doi.org/10.1016/j.crad.2007.07. 018

93. Garner JP, Patel S, Glaves J, Ravi K (2006) Is herniography useful? Hernia 10(1):66-69. https://doi.org/10.1007/s10029005-0044-4

94. Geuens G, Bellinck P, Mulkens T et al (2011) Revisiting the role of herniography in the preoperative work-up of groin hernias? Acta Chir Belg 111(6):370-373

95. Hureibi KA, McLatchie GR, Kidambi AV (2011) Is herniography useful and safe? Eur J Radiol 80(2):e86-e90. https://doi.org/ 10.1016/j.ejrad.2010.08.017

96. Kim B, Robinson P, Modi H, Gupta H, Horgan K, Achuthan R (2015) Evaluation of the usage and influence of groin ultrasound in primary and secondary healthcare settings. Hernia 19(3):367-371. https://doi.org/10.1007/s10029-014-1212-1
97. Kitami M, Takase K, Tsuboi M et al (2009) Differentiation of femoral and inguinal hernias on the basis of anteroposterior relationship to the inguinal ligament on multidimensional computed tomography. J Comput Assist Tomogr 33(5):678-681. https://doi.org/10.1097/RCT.0b013e3181977a0a

98. Korenkov M, Paul A, Troidl H (1999) Color duplex sonography: diagnostic tool in the differentiation of inguinal hernias. J Ultrasound Med 18(8):565-568

99. Kraft BM, Kolb H, Kuckuk B et al (2003) Diagnosis and classification of inguinal hernias. Surg Endosc 17(12):2021-2024. https://doi.org/10.1007/s00464-002-9283-y

100. LeBlanc KE, LeBlanc LL, LeBlanc KA (2013) Inguinal hernias: diagnosis and management. Am Fam Phys 87(12):844-848

101. Lilly MC, Arregui ME (2002) Ultrasound of the inguinal floor for evaluation of hernias. Surg Endosc 16(4):659-662. https://doi.org/10.1007/s00464-001-8145-3

102. Miller J, Cho J, Michael MJ, Saouaf R, Towfigh S (2014) Role of imaging in the diagnosis of occult hernias. JAMA Surg 25141884. https://doi.org/10.1001/jamasurg.2014.484

103. Murphy KP, O'Connor OJ, Maher MM (2014) Adult abdominal hernias. AJR Am J Roentgenol 202(6):W506-W511. https://doi. org/10.2214/AJR.13.12071

104. Rosenberg J, Bisgaard T, Kehlet H et al (2011) Danish Hernia Database recommendations for the management of inguinal and femoral hernia in adults. Dan Med Bull 58(2):42-43

105. Robinson A, Light D, Kasim A, Nice C (2013) A systematic review and meta-analysis of the role of radiology in the diagnosis of occult inguinal hernia. Surg Endosc 27(1):11-18. https://doi.org/10.1007/s00464-012-2412-3

106. Alam A, Nice C, Uberoi R (2005) The accuracy of ultrasound in the diagnosis of clinically occult groin hernias in adults. Eur Radiol 15(12):2457-2461. https://doi.org/10.1007/s00330-0052825-7

107. Alabraba E, Psarelli E, Meakin K et al (2014) The role of ultrasound in the management of patients with occult groin hernias. Int J Surg 12(9):918-922. https://doi.org/10.1016/j.ijsu. 2014.07.266

108. Bradley M, Morgan J, Pentlow B, Roe A (2006) The positive predictive value of diagnostic ultrasound for occult herniae. Ann R Coll Surg Engl 88:165-167. https://doi.org/10.1308/ 003588406X95110

109. Grant T, Neuschler E, Hartz W 3rd (2011) Groin pain in women: use of sonography to detect occult hernias. J Ultrasound Med 30(12):1701-1707

110. Henriksen NA, Thorup JJL (2012) Unsuspected femoral hernia in patients with a preoperative diagnosis of recurrent inguinal hernia. Hernia 16(4):381-385. https://doi.org/10.1007/s10029012-0924-3

111. Lorenzini C, Sofia L, Pergolizzi FP, Trovato M (2008) The value of diagnostic ultrasound for detecting occult inguinal hernia in patients with groin pain. Chir Ital 60(6):813-817

112. Orchard JW, Read JW, Neophyton J, Garlick D (1998) Groin pain associated with ultrasound finding of inguinal canal posterior wall deficiency in Australian Rules footballers. Br J Sports Med 32(2):134-139

113. Pawlak M, Niebuhr H, Bury K (2015) Dynamic inguinal ultrasound: a diagnostic tool for hernia surgeons. Hernia 19(6):1033-1034. https://doi.org/10.1007/s10029-015-1356-7

114. Robinson P, Hensor E, Lansdown MJ, Ambrose NS, Chapman AH (2006) Inguinofemoral hernia: accuracy of sonography in patients with indeterminate clinical features. AJR Am J Roentgenol 187(5):1168-1178. https://doi.org/10.2214/AJR.05.1251

115. Lechner M, Fortelny R, Ofner D, Mayer F (2014) Suspected inguinal hernias in pregnancy-handle with care! Hernia 18(3):375-379. https://doi.org/10.1007/s10029-013-1082-y 
116. Pilkington SA, Rees M, Jones OGI (2004) Ultrasound diagnosis of round ligament varicosities mimicking inguinal hernias in pregnancy. Ann R Coll Surg Engl 86(5):400-401. https://doi. org/10.1308/1478708041782094

117. Garvey JFW (2012) Computed tomography scan diagnosis of occult groin hernia. Hernia 16(3):307-314. https://doi.org/10. 1007/s10029-011-0899-5

118. Barile A, Erriquez D, Cacchio A, De Paulis F, Di Cesare E, Masciocchi C (2000) Groin pain in athletes: role of magnetic resonance. Radiol Med 100(4):216-222

119. Drew MK, Osmotherly PG, Chiarelli PE (2014) Imaging and clinical tests for the diagnosis of long-standing groin pain in athletes. A systematic review. Phys Ther Sport 15(2):124-129. https://doi.org/10.1016/j.ptsp.2013.11.002

120. Shpitz B, Kuriansky J, Werener M et al (2004) Early postoperative evaluation of groins after laparoscopic total extraperitoneal repair of inguinal hernias. J Laparoendosc Adv Surg Tech A 14(6):353-357. https://doi.org/10.1089/lap.2004.14.353

121. Young J, Gilbert AI, Graham MF (2007) The use of ultrasound in the diagnosis of abdominal wall hernias. Hernia 11(4):347-351. https://doi.org/10.1007/s10029-007-0227-2

122. Hakeem A, Shanmugam V (2011) Current trends in the diagnosis and management of post-herniorraphy chronic groin pain. World J Gastrointest Surg 3(6):73-81. https://doi.org/10.4240/ wjgs.v3.i6.73

123. Aveline C, Le Hetet H, Le Roux A et al (2011) Comparison between ultrasound-guided transversus abdominis plane and conventional ilioinguinal/iliohypogastric nerve blocks for daycase open inguinal hernia repair. Br J Anaesth 106(3):380-386. https://doi.org/10.1093/bja/aeq363

124. Bischoff JM, Koscielniak-Nielsen ZJ, Kehlet H, Werner MU (2012) Ultrasound-guided ilioinguinal/iliohypogastric nerve blocks for persistent inguinal postherniorrhaphy pain: a randomized, double-blind, placebo-controlled, crossover trial. Anesth Analg 114(6):1323-1329. https://doi.org/10.1213/ANE. 0b013e31824d6168

125. Miserez M, Alexandre JH, Campanelli G et al (2007) The European hernia society groin hernia classification: simple and easy to remember. Hernia 11(2):113-116. https://doi.org/10. 1007/s10029-007-0198-3

126. Campanelli G, Pettinari D, Nicolosi FM, Cavalli M, Avesani EC (2006) Inguinal hernia recurrence: classification and approach. Hernia 10(2):159-161. https://doi.org/10.1007/s10029-0050053-3

127. Chowbey P, Khullar R, Mehrotra M, Sharma A, Soni V, Baijal M (2006) Sir Ganga Ram Hospital classification of groin and ventral abdominal wall hernias. J Minim Access Surg 2(3):106. https://doi.org/10.4103/0972-9941.27720

128. Holzheimer RG (2005) Inguinal hernia: classification, diagnosis and treatment-classic, traumatic and sportsman's hernia. Eur J Med Res 10(3):121-134

129. Zollinger RM (2004) An updated traditional classification of inguinal hernias. Hernia 8(4):318-322. https://doi.org/10.1007/ s10029-004-0245-2

130. Kingsnorth AN (2004) A clinical classification for patients with inguinal hernia. Hernia 8(3):283-284. https://doi.org/10.1007/ s10029-004-0228-3

131. Nyhus LM (2004) Classification of groin hernia: milestones. Hernia 8(2):87-88. https://doi.org/10.1007/s10029-003-0173-6

132. Zollinger RM (2003) Classification systems for groin hernias. Surg Clin North Am 83(5):1053-1063. https://doi.org/10.1016/ S0039-6109(03)00126-9

133. Hair A, Paterson C, Wright D, Baxter JN, O’Dwyer PJ (2001) What effect does the duration of an inguinal hernia have on patient symptoms? J Am Coll Surg 193(2):125-129. https://doi. org/10.1016/S1072-7515(01)00983-8
134. Nienhuijs S, Staal E, Strobbe L, Rosman C, Groenewoud H, Bleichrodt R (2007) Chronic pain after mesh repair of inguinal hernia: a systematic review. Am J Surg 194:394-400

135. Fitzgibbons RJ, Giobbie-Hurder A, Gibbs JO et al (2006) Watchful waiting vs repair of inguinal hernia in minimally symptomatic men: a randomized clinical trial. JAMA 295(3):285-292. https://doi.org/10.1001/jama.295.3.285

136. O’Dwyer PJ, Norrie J, Alani A, Walker A, Duffy F, Horgan P (2006) Observation or operation for patients with an asymptomatic inguinal hernia: a randomized clinical trial. Ann Surg 244(2):167-173. https://doi.org/10.1097/01.sla.0000217637. 69699.ef

137. Stroupe KT, Manheim LM, Luo P et al (2006) Tension-free repair versus watchful waiting for men with asymptomatic or minimally symptomatic inguinal hernias: a cost-effectiveness analysis. J Am Coll Surg 203:458-468. https://doi.org/10.1016/ j.jamcollsurg.2006.06.010

138. Chung L, Norrie J, O'Dwyer PJ (2011) Long-term follow-up of patients with a painless inguinal hernia from a randomized clinical trial. Br J Surg 98(4):596-599. https://doi.org/10.1002/ bjs. 7355

139. van den Heuvel B, Dwars BJ, Klassen DR, Bonjer HJ (2011) Is surgical repair of an asymptomatic groin hernia appropriate? A review. Hernia 15(3):251-259. https://doi.org/10.1007/s10029011-0796-y

140. Collaboration IT (2011) Operation compared with watchful waiting in elderly male inguinal hernia patients: a review and data analysis. J Am Coll Surg 212(2):251-259. https://doi.org/ 10.1016/j.jamcollsurg.2010.09.030

141. Hwang M, Bhangu A, Webster C, Bowley D, Gannon M, Karandikar S (2014) Unintended consequences of policy change to watchful waiting for asymptomatic inguinal hernias. Ann R Coll Surg Engl 96(5):343-347. https://doi.org/10.1308/ $003588414 X 13946184902000$

142. Amato B, Moja L, Panico S et al (2009) Shouldice technique versus other open techniques for inguinal hernia repair. Cochrane Database Syst Rev. CD001543. https://doi.org/10. 1002/14651858.CD001543.pub3

143. Hay J, Boudet M, Fingerhut A et al (1995) Shouldice inguinal hernia repair in the male adult: the gold standard? A multicenter controlled trial in 1578 patients. Ann Surg 222(6):719-727

144. Szopinski J, Dabrowiecki S, Pierscinski S, Jackowski M, Jaworski M, Szuflet Z (2012) Desarda versus Lichtenstein technique for primary inguinal hernia treatment: 3-year results of a randomized clinical trial. World J Surg 36(5):984-992. https://doi.org/10.1007/s00268-012-1508-1

145. Bay-Nielsen M, Nilsson E, Nordin P, Kehlet H (2004) Chronic pain after open mesh and sutured repair of indirect inguinal hernia in young males. Br J Surg 91(10):1372-1376. https://doi. org/10.1002/bjs.4502

146. Bay-Nielsen M, Thomsen H, Heidemann Andersen $F$ et al (2004) Convalescence after inguinal herniorrhaphy. Br J Surg 91(3):362-367. https://doi.org/10.1002/bjs.4437

147. Nakagawa M, Nagase T, Akatsu T et al (2013) A randomized prospective trial comparing clinical outcomes 3 years after surgery by Marcy repair and Prolene Hernia System repair for adult indirect inguinal hernia. Surg Today 43:1109-1115. https://doi.org/10.1007/s00595-012-0384-5

148. Naveen N, Srinath R (2014) A comparative study between modified Bassini's repair and Lichtenstein mesh repair of inguinal hernias in rural population. J Clin Diagn Res 8:88-91. https://doi.org/10.7860/JCDR/2014/7431.4016

149. Manyilirah W, Kijjambu S, Upoki A, Kiryabwire J (2012) Comparison of non-mesh (Desarda) and mesh (Lichtenstein) methods for inguinal hernia repair among black African 
patients: a short-term double-blind RCT. Hernia 16:133-144. https://doi.org/10.1007/s10029-011-0883-0

150. Lermite E, Arnaud JP (2012) Prospective randomized study comparing quality of life after Shouldice or mesh plug repair for inguinal hernia: short-term results. Surg Technol Int 22:101-106

151. Bay-Nielsen M, Kehlet H, Strand L et al (2004) The Danish Hernia Data Base-four years' results. Ugeskr Laeger 166:1894-1898

152. Bisgaard T, Bay-Nielsen M, Kehlet H (2008) Re-recurrence after operation for recurrent inguinal hernia. A nationwide 8-year follow-up study on the role of type of repair. Ann Surg 247(4):707-711. 0b013e31816b18e3

153. Malik A, Bell C, Stukel T, Urbach D (2015) Recurrence of inguinal hernias repaired in a large hernia surgical specialty hospital and general hospitals in Ontario, Canada. Can J Surg 1-7. https://doi.org/10.1503/cjs.003915

154. Arvidsson D, Berndsen FH, Larsson LG et al (2005) Randomized clinical trial comparing 5-year recurrence rate after laparoscopic versus Shouldice repair of primary inguinal hernia. Br J Surg 92(9):1085-1091. https://doi.org/10.1002/bjs.5137

155. Lichtenstein IL, Shulman AG, Amid PK, Montllor MM (1989) The tension-free hernioplasty. Am J Surg 157(2):188-193. https://doi.org/10.1016/0002-9610(89)90526-6

156. Robbins AW, Rutkow IM (1993) The mesh-plug hernioplasty. Surg Clin North Am 73(3):501-512

157. Trabucco E (1993) The office hernioplasty and the Trabucco repair. Ann Ital Chir 64(2):127-149

158. Murphy JW (2001) Use of the prolene hernia system for inguinal hernia repair: retrospective, comparative time analysis versus other inguinal hernia repair systems. Am Surg 67(10):919-923

159. Zhao G, Gao P, Ma B, Tian J, Yang K (2009) Open mesh techniques for inguinal hernia repair: a meta-analysis of randomized controlled trials. Ann Surg 250:35-42. https://doi.org/ 10.1097/SLA.0b013e3181ad63cc

160. Li J, Ji Z, Li Y (2012) Comparison of mesh-plug and Lichtenstein for inguinal hernia repair: a meta-analysis of randomized controlled trials. Hernia 16:541-548. https://doi.org/10.1007/ s10029-012-0974-6

161. Nienhuijs SW, Rosman C (2015) Long-term outcome after randomizing prolene hernia system, mesh plug repair and Lichtenstein for inguinal hernia repair. Hernia 19:77-81. https://doi.org/10.1007/s10029-014-1295-8

162. Droeser RA, Dell-Kuster S, Kurmann A et al (2014) Long-term follow-up of a randomized controlled trial of Lichtenstein's operation versus mesh plug repair for inguinal hernia. Ann Surg 259:966-972. https://doi.org/10.1097/SLA.0000000000000297

163. Sanjay P, Watt DG, Ogston SA, Alijani A, Windsor JA (2012) Meta-analysis of Prolene Hernia System mesh versus Lichtenstein mesh in open inguinal hernia repair. Surg J R Coll Surg Edinb Irel 10:283-289. https://doi.org/10.1016/j.surge.2012.06. 001

164. Pierides G, Vironen J (2011) A prospective randomized clinical trial comparing the prolene hernia system and the Lichtenstein patch technique for inguinal hernia repair in long term: 2- and 5-year results. Am J Surg 202:188-193. https://doi.org/10.1016/ j.amjsurg.2010.06.027

165. Karateke F, Ozyazici S, Menekse E et al (2014) ULTRAPRO hernia system versus Lichtenstein repair in treatment of primary inguinal hernias: a prospective randomized controlled study. Int Surg 99:391-397. https://doi.org/10.9738/INTSURG-D-1400064.1

166. Mutter D, Champault G, Binot D, Vix M, Leroy J, Marescaux J (2012) PerFix plug versus 4DDOME implants for inguinal hernia repair: prospective multicentric randomised controlled trial. Hernia 16(5):561-566. https://doi.org/10.1007/s10029012-0943-0

167. Ripetti V, La Vaccara V, Greco S, Bono F, Valeri S, Coppola R (2014) Randomised trial comparing Lichtenstein vs Trabucco vs Valenti techniques in inguinal hernia repair. Hernia 18:205-212. https://doi.org/10.1007/s10029-013-1089-4

168. Kapischke M, Schulze H, Caliebe A (2010) Self-fixating mesh for the Lichtenstein procedure-a prestudy. Langenbeck's Arch Surg 395:317-322. https://doi.org/10.1007/s00423-010-0597-2

169. Bruna Esteban M, Cantos Pallares M, Artigues Sanchez De Rojas E (2010) Use of adhesive mesh in hernioplasty compared to the conventional technique. Results of a randomised prospective study. [Spanish] TT-Utilizacion de mallas autoadhesivas en la hernioplastia frente a la tecnica convencional. Resultados de un estudio pr. Cir Esp 88:253-258

170. Kingsnorth A, Gingell-Littlejohn M, Nienhuijs S et al (2012) Randomized controlled multicenter international clinical trial of self-gripping Parietex ProGrip polyester mesh versus lightweight polypropylene mesh in open inguinal hernia repair: interim results at 3 months. Hernia 16:287-294. https://doi.org/ 10.1007/s10029-012-0900-y

171. Pierides G, Scheinin T, Remes V, Hermunen K, Vironen J (2012) Randomized comparison of self-fixating and sutured mesh in open inguinal hernia repair. Br J Surg 99:630-636. https://doi.org/10.1002/bjs.8705

172. Jorgensen LN, Sommer T, Assaadzadeh S et al (2013) Randomized clinical trial of self-gripping mesh versus sutured mesh for Lichtenstein hernia repair. $\mathrm{Br} \mathrm{J}$ Surg 100:474-481. https://doi.org/10.1002/bjs.9006

173. Zhang C, Li F, Zhang H, Zhong W, Shi D, Zhao Y (2013) Selfgripping versus sutured mesh for inguinal hernia repair: a systematic review and meta-analysis of current literature. J Surg Res 185(2):653-660. https://doi.org/10.1016/j.jss.2013.07.035

174. Sajid MS, Farag S, Singh KK, Miles WF (2014) Systematic review and meta-analysis of published randomized controlled trials comparing the role of self-gripping mesh against suture mesh fixation in patients undergoing open inguinal hernia repair. Updat Surg 66:189-196. https://doi.org/10.1007/s13304-0130237-9

175. Li J, Ji Z, Li Y (2014) The comparison of self-gripping mesh and sutured mesh in open inguinal hernia repair: the results of meta-analysis. Ann Surg 259(6):1080-1085. https://doi.org/10. 1097/SLA.0000000000000408

176. Pandanaboyana S, Mittapalli D, Rao A, Prasad R, Ahmad N (2014) Meta-analysis of self-gripping mesh (Progrip) versus sutured mesh in open inguinal hernia repair. Surg J R Coll Surg Edinb Irel 12:87-93. https://doi.org/10.1016/j.surge.2013.11. 024

177. Fang Z, Zhou J, Ren F, Liu D (2014) Self-gripping mesh versus sutured mesh in open inguinal hernia repair: system review and meta-analysis. Am J Surg 207(5):773-781. https://doi.org/10. 1016/j.amjsurg.2013.08.045

178. Chatzimavroudis G, Papaziogas B, Koutelidakis I et al (2014) Lichtenstein technique for inguinal hernia repair using polypropylene mesh fixed with sutures vs. self-fixating polypropylene mesh: a prospective randomized comparative study. Hernia 18:193-198. https://doi.org/10.1007/s10029-0131211-7

179. Nikkolo C, Vaasna T, Murruste M, Seepter H, Suumann J, Tein A, Kirsimagu U, Lepner U (2015) Single-center, single-blinded, randomized study of self-gripping versus sutured mesh in open inguinal hernia repair. J Surg Res 194(1):77-82. https://doi.org/ 10.1016/j.jss.2014.09.017

180. Porrero JL, Castillo MJ, Pérez-Zapata A et al (2015) Randomised clinical trial: conventional Lichtenstein vs. hernioplasty with self-adhesive mesh in bilateral inguinal hernia surgery. 
Hernia 19(5):765-770. https://doi.org/10.1007/s10029-0141316-7

181. Bruna Esteban M, Cantos Pallares M, Artigues Sanchez de Rojas E, Vila MJ (2014) Prospective randomized trial of longterm results of inguinal hernia repair using autoadhesive mesh compared to classic Lichtenstein technique with sutures and polypropylene mesh. Cir Esp 92:195-200. https://doi.org/10. 1016/j.ciresp.2013.03.009

182. Sanders DL, Nienhuijs S, Ziprin P, Miserez M, Gingell-Littlejohn M, Smeds S (2014) Randomized clinical trial comparing self-gripping mesh with suture fixation of lightweight polypropylene mesh in open inguinal hernia repair. Br J Surg 101:1373-1382. https://doi.org/10.1002/bjs.9598 (discussion 1382)

183. Nyhus LM, Stevenson JKHH (1959) Preperitoneal herniorrhaphy: a preliminary report in fifty patients. West J Surg Obstet 67:48-54

184. Pélissier EP (2006) Inguinal hernia: preperitoneal placement of a memory-ring patch by anterior approach. Preliminary experience. Hernia 10(3):248-252. https://doi.org/10.1007/s10029006-0079-1

185. Kugel RD (1999) Minimally invasive, nonlaparoscopic, preperitoneal, and sutureless, inguinal herniorrhaphy. Am J Surg 178(4):298-302. https://doi.org/10.1016/S0002-9610(99)001816

186. Koning GG, Andeweg CS, Keus F, van Tilburg MWA, van Laarhoven CJHM, Akkersdijk WL (2012) The transrectus sheath preperitoneal mesh repair for inguinal hernia: technique, rationale, and results of the first 50 cases. Hernia 16(3):295-299. https://doi.org/10.1007/s10029-011-0893-y

187. Lourenço A, Da Costa RS (2013) The ONSTEP inguinal hernia repair technique: initial clinical experience of 693 patients, in two institutions. Hernia 17(3):357-364. https://doi.org/10.1007/ s10029-013-1057-z

188. Ugahary F, Simmermacher R (1998) Groin hernia repair via a grid-iron incision: an alternative technique for preperitoneal mesh insertion. Hernia 2:123-125

189. Wantz G (1993) The technique of giant prosthetic reinforcement of the visceral sac performed through an anterior groin incision. Surg Gynecol Obstet 176(5):497-500

190. Rives J (1976) Surgical treatment of the inguinal hernia with dacron patch: principles, indications, technique and results. Int Surg 47:360-361

191. Willaert W, Berrevoet F, De Bacquer D, Rogiers X, Troisi R (2009) Open preperitoneal techniques versus Lichtenstein repair for inguinal hernia. Cochrane Database Syst Rev 4:1-28. https://doi.org/10.1002/14651858.CD008034

192. Sajid MS, Craciunas L, Singh KK, Sains P, Baig MK (2013) Open transinguinal preperitoneal mesh repair of inguinal hernia: a targeted systematic review and meta-analysis of published randomized controlled trials. Gastroenterol Rep 1:127-137. https://doi.org/10.1093/gastro/got002

193. Li J, Ji Z, Li Y (2014) Comparison of laparoscopic versus open procedure in the treatment of recurrent inguinal hernia: a metaanalysis of the results. Am J Surg 207:602-612. https://doi.org/ 10.1016/j.amjsurg.2013.05.008

194. Koning GG, Keus F, Koeslag L et al (2012) Randomized clinical trial of chronic pain after the transinguinal preperitoneal technique compared with Lichtenstein's method for inguinal hernia repair. Br J Surg 99:1365-1373. https://doi.org/10.1002/bjs. 8862

195. Arslan K, Erenoglu B, Turan E, Koksal H, Dogru O (2015) Minimally invasive preperitoneal single-layer mesh repair versus standard Lichtenstein hernia repair for inguinal hernia: a prospective randomized trial. Hernia 19(3):373-381. https://doi. org/10.1007/s10029-014-1306-9
196. Ray R, Kar M, Mukhopadhyay M (2014) Transinguinal preperitoneal technique of inguinal hernioplasty-a better alternative to Lichtenstein procedure. J Clin Diagn Res 8(5):NC01-NC03. https://doi.org/10.7860/JCDR/2014/7632. 4320

197. Wiegering A, Schlegel N, Isbert C et al (2013) Lessons and challenges during a 5-year follow-up of 21 Composix Kugel implantations. Hernia 17(4):435-443. https://doi.org/10.1007/ s10029-013-1096-5

198. Koning GG, Adang EMM, Stalmeier PFM, Keus F, Vriens PWHE, Van Laarhoven CJHM (2013) TIPP and Lichtenstein modalities for inguinal hernia repair: a cost minimisation analysis alongside a randomised trial. Eur $\mathbf{J}$ Health Econ 14:1027-1034. https://doi.org/10.1007/s10198-012-0453-0

199. Memon MA, Cooper NJ, Memon B, Memon MI, Abrams KR (2003) Meta-analysis of randomized clinical trials comparing open and laparoscopic inguinal hernia repair. Br J Surg 90:1479-1492

200. McCormack K, Wake BL, Fraser C, Vale L, Perez J, Grant A (2005) Transabdominal pre-peritoneal (TAPP) versus totally extraperitoneal (TEP) laparoscopic techniques for inguinal hernia repair: a systematic review. Hernia 9(2):109-114. https://doi. org/10.1007/s10029-004-0309-3

201. Tolver MA, Rosenberg J, Bisgaard T (2012) Early pain after laparoscopic inguinal hernia repair. A qualitative systematic review. Acta Anaesthesiol Scand 56:549-557. https://doi.org/10. 1111/j.1399-6576.2011.02633.x

202. O'Reilly EA, Burke JP, O'Connell PR (2012) A meta-analysis of surgical morbidity and recurrence after laparoscopic and open repair of primary unilateral inguinal hernia. Ann Surg 255(5):846-853. 0b013e31824e96cf

203. Bracale U, Melillo P, Pignata G et al (2012) Which is the best laparoscopic approach for inguinal hernia repair: TEP or TAPP? A systematic review of the literature with a network metaanalysis. Surg Endosc 26:3355-3366. https://doi.org/10.1007/ s00464-012-2382-5

204. Antoniou SA, Antoniou GA, Bartsch DK et al (2013) Transabdominal preperitoneal versus totally extraperitoneal repair of inguinal hernia: a meta-analysis of randomized studies. Am J Surg 206(245-252):e1. https://doi.org/10.1016/j.amjsurg.2012. 10.041

205. Wei FX, Zhang YC, Han W, Zhang YL, Shao Y, Ni R (2015) Transabdominal preperitoneal (TAPP) versus totally. Surg Laparosc Endosc Percutan Tech 25(5):375-383

206. McCormack K, Wake B, Perez J et al (2005) Laparoscopic surgery for inguinal hernia repair: systematic review of effectiveness and economic evaluation. Health Technol Assess 9(14):1-203 (iii-iv)

207. Gass M, Banz VM, Rosella L, Adamina M, Candinas D, Güller U (2012) TAPP or TEP? Population-based analysis of prospective data on 4,552 patients undergoing endoscopic inguinal hernia repair. World J Surg 36(12):2782-2786. https://doi.org/10.1007/s00268-012-1760-4

208. Wittenbecher F, Scheller-Kreinsen D, Röttger J, Busse R (2013) Comparison of hospital costs and length of stay associated with open-mesh, totally extraperitoneal inguinal hernia repair, and transabdominal preperitoneal inguinal hernia repair: an analysis of observational data using propensity score matching. Surg Endosc 27(4):1326-1333. https://doi.org/10.1007/s00464-0122608-6

209. Köckerling F, Bittner R, Jacob DA, Seidelmann L, Keller T, Adolf D, Kraft BKA (2015) TEP versus TAPP: comparison of the perioperative outcome in 17,587 patients with a primary unilateral inguinal hernia. Surg Endosc 29(12):3750-3760 
210. Schrenk P, Woisetschläger R, Rieger R, Wayand W (1996) Prospective randomized trial comparing postoperative pain and return to physical activity after transabdominal preperitoneal, total preperitoneal or Shouldice technique for inguinal hernia repair. Br J Surg 83(11):1563-1566. https://doi.org/10.1002/bjs. 1800831124

211. Hamza Y, Gabr E, Hammadi H, Khalil R (2010) Four-arm randomized trial comparing laparoscopic and open hernia repairs. Int J Surg 8(1):25-28. https://doi.org/10.1016/j.ijsu. 2009.09.010

212. Wang W, Chen J, Fang Q, Li J, Jin P-F, Li Z-T (2013) Comparison of the effects of laparoscopic hernia repair and Lichtenstein tension-free hernia repair. J Laparoendosc Adv Surg Tech A 23(4):301-305. https://doi.org/10.1089/lap.2012.0217

213. Pokorny H, Klingler A, Schmid T (2008) Recurrence and complications after laparoscopic versus open inguinal hernia repair: results of a prospective randomized multicenter trial. Hernia 12(4):385-389. https://doi.org/10.1007/s10029-0080357-1

214. Krishna A, Misra MC, Bansal VK, Kumar S, Rajeshwari S, Chabra A (2012) Laparoscopic inguinal hernia repair: transabdominal preperitoneal (TAPP) versus totally extraperitoneal (TEP) approach: a prospective randomized controlled trial. Surg Endosc 26(3):639-649. https://doi.org/10.1007/s00464-0111931-7

215. Zhu Q, Mao Z, Yu B, Jin J, Zheng M, Li J (2009) Effects of persistent $\mathrm{CO}_{2}$ insufflation during different laparoscopic inguinal hernioplasty: a prospective, randomized, controlled study. J Laparoendosc Adv Surg Tech 19:611-614. https://doi.org/10. 1089/lap.2009.0084

216. Dedemadi G, Sgourakis G, Christofides T, Kouraklis G, Karaliotas C (2006) Comparison of laparoscopic and open tensionfree repair of recurrent inguinal hernias: a prospective randomized study. Surg Endosc Other Interv Tech 20:1099-1104. https://doi.org/10.1007/s00464-005-0621-8

217. Butler RE, Burke R, Schneider JJ, Brar H, Lucha PA (2007) The economic impact of laparoscopic inguinal hernia repair: results of a double-blinded, prospective, randomized trial. Surg Endosc Other Interv Tech 21(3):387-390

218. Tetik C, Arregui ME, Dulucq JL et al (1994) Complications and recurrences associated with laparoscopic repair of groin hernias. A multi-institutional retrospective analysis. Surg Endosc 8(11):1313-1316

219. Zanghi A, Di Vita M, Lo Menzo E et al (2011) Multicentric evaluation by Verbal Rate Scale and euroqol-5D of early and late post-operative pain after TAPP and TEP procedures with mechanical fixation for bilateral inguinal hernias. Ann Ital Chir 82(6):437-442

220. Felix EL, Michas CA, Gonzalez MH Jr (1997) Laparoscopic hernioplasty: why does it work? Surg Endosc 11:36-41

221. Ramshaw B, Shuler FW, Jones HB et al (2001) Laparoscopic inguinal hernia repair: lessons learned after 1224 consecutive cases. Surg Endosc 15(1):50-54. https://doi.org/10.1007/ s004640001016

222. Weiser H, Klinge B (2000) Endoskopische operationstechniken. Erfahrungen und besonderheiten-leistenhernie. Viszeralchirurgie 35(3):316-320

223. Kald A, Anderberg B, Smedh K, Karlsson M (1997) Transperitoneal or totally extraperitoneal approach in laparoscopic hernia repair: results of 491 consecutive herniorrhaphies. Surg Laparosc Endosc 7(2):86-89. https://doi.org/10.1097/ 00019509-199704000-00002

224. Ramshaw BJ, Tucker JG, Conner T, Mason EM, Duncan TD, Lucas GW (1996) A comparison of the approaches to laparoscopic herniorraphy. Surg Endosc 10(1):29-32
225. Günal O, Ozer S, Gürleyik E, Bahçebaşi T (2007) Does the approach to the groin make a difference in hernia repair? Hernia 11(5):429-434. https://doi.org/10.1007/s10029-007-0252-1

226. Gong K, Zhang N, Lu Y et al (2011) Comparison of the open tension-free mesh-plug, transabdominal preperitoneal (TAPP), and totally extraperitoneal (TEP) laparoscopic techniques for primary unilateral inguinal hernia repair: a prospective randomized controlled trial. Surg Endosc 25(1):234-239. https://doi.org/10.1007/s00464-010-1165-0

227. Lepere M, Benchetrit S, Debaert M et al (2000) A multicentric comparison of transabdominal versus totally extraperitoneal laparoscopic hernia repair using PARIETEX meshes. J Soc Laparoendosc Surg 4(2):147-153

228. Belyansky I, Tsirline VB, Klima DA, Walters AL, Lincourt AE, Heniford TB (2011) Prospective, comparative study of postoperative quality of life in TEP, TAPP, and modified Lichtenstein repairs. Ann Surg 254(5):709-714. https://doi.org/10.1097/SLA. 0b013e3182359d07

229. Czechowski A, Schafmayer A (2003) TAPP versus TEP: a retrospective analysis 5 years after laparoscopic transperitoneal and total endoscopic extraperitoneal repair in inguinal and femoral hernia. [German] TT-TAPP versus TEPEine retrospektive Analyse funf Jahre postoperativ nach transper. Chirurg 74:1143-1149

230. Bright E, Reddy VM, Wallace D, Garcea G, Dennison AR (2010) The incidence and success of treatment for severe chronic groin pain after open, transabdominal preperitoneal, and totally extraperitoneal hernia repair. World J Surg 34(4):692-696. https://doi.org/10.1007/s00268-010-0410-y

231. Kald A, Anderberg B, Carlsson P, Park PO, Smedh K (1997) Surgical outcome and cost-minimisation-analyses of laparoscopic and open hernia repair: a randomised prospective trial with one year follow up. Eur J Surg 163:505-510

232. Shah NR, Mikami DJ, Cook C et al (2011) A comparison of outcomes between open and laparoscopic surgical repair of recurrent inguinal hernias. Surg Endosc 25:2330-2337. https://doi.org/10.1007/s00464-010-1564-2

233. Felix EL, Michas CA, Gonzalez MH (1995) Laparoscopic hernioplasty_TAPP vs TEP. Surg Endosc 9(9):984-989. https://doi.org/10.1007/BF00188456

234. Bansal VK, Misra MC, Babu D et al (2013) A prospective, randomized comparison of long-term outcomes: chronic groin pain and quality of life following totally extraperitoneal (TEP) and transabdominal preperitoneal (TAPP) laparoscopic inguinal hernia repair. Surg Endosc Other Interv Tech 27:2373-2382. https://doi.org/10.1007/s00464-013-2797-7

235. Cohen RV, Alvarez G, Roll S et al (1998) Transabdominal or totally extraperitoneal laparoscopic hernia repair? Surg Laparosc Endosc 8(4):264-268. https://doi.org/10.1097/00019509199808000-00004

236. Bobrzynski A, Budzynski A, Biesiada Z, Kowalczyk M, Lubikowski J, Sienko J (2001) Experience-the key factor in successful laparoscopic total extraperitoneal and transabdominal preperitoneal hernia repair. Hernia 5(2):80-83. https://doi.org/ 10.1007/s100290100004

237. Khoury N (1995) A comparative study of laparoscopic extraperitoneal and transabdominal preperitoneal herniorrhaphy. J Laparoendosc Surg 5(6):349-355

238. Papachristou E, Mitselou M, Finokaliotis N (2002) Surgical outcome and hospital cost analyses of laparoscopic and open tension-free hernia repair. Hernia 6(2):68-72. https://doi.org/10. 1007/s10029-002-0062-4

239. Wang J, Ji G, Yang Z et al (2013) Prospective randomized, double-blind, placebo controlled trial to evaluate infection prevention in adult patients after tension-free inguinal hernia repair. 
Int J Clin Pharmacol Ther 51:924-931. https://doi.org/10.5414/ CP201877e-pub

240. Van Hee R, Goverde P, Hendrickx L, Van der Schelling G, Totte E (1998) Laparoscopic transperitoneal versus extraperitoneal inguinal hernia repair: a prospective clinical trial. Acta Chir Belg 98:132-135

241. Bittner R, Montgomery MA, Arregui E et al (2015) Update of guidelines on laparoscopic (TAPP) and endoscopic (TEP) treatment of inguinal hernia (International Endohernia Society). Surg Endosc Other Interv Tech 29(2):289-321. https://doi.org/ 10.1007/s00464-014-3917-8

242. Chung RS, Rowland DY (1999) Meta-analyses of randomized controlled trials of laparoscopic vs conventional inguinal hernia repairs. Surg Endosc 13(7):689-694

243. Grant A (2000) Laparoscopic compared with open methods of groin hernia repair: systematic review of randomized controlled trials. Br J Surg 87:860-867. https://doi.org/10.1046/j.13652168.2000.01540.x

244. Schmedt CG, Sauerland S, Bittner R (2005) Comparison of endoscopic procedures vs Lichtenstein and other open mesh techniques for inguinal hernia repair: a meta-analysis of randomized controlled trials. Surg Endosc 19(2):188-199. https://doi.org/10.1007/s00464-004-9126-0

245. Neumayer L, Giobbie-Hurder A, Jonasson O et al (2004) Open mesh versus laparoscopic mesh repair of inguinal hernia. $\mathrm{N}$ Engl J Med 350:1819-1827 + 1922. https://doi.org/10.1056/ NEJMoa040093

246. Liem MS, van der Graaf Y, van Steensel CJ, Boelhouwer RU, Clevers GJMW et al (1997) Comparison of conventional anterior surgery and laparoscopic surgery for inguinal-hernia repair. N Engl J Med 336(22):1541-1547

247. Wright DM, Kennedy A, Baxter JN et al (1996) Early outcome after open versus extraperitoneal endoscopic tension-free hernioplasty: a randomized clinical trial. Surgery 119(5):552-557. https://doi.org/10.1016/S0039-6060(96)802662

248. Merello J, Guerra A, Madriz I, Guerra G (1997) Laparoscopic TEP versus open Lichtenstein hernia repair. Surg Endosc 11:545

249. Moreno-Egea A, Agauyo J (1999) Cirugia laparoscopica totalmente extraperitoneal frente a operacion de Lichtenstein en el tratamiento de la hernia inguinal. Cir Esp 66:53-57

250. Lal P, Kajla RK, Chander J, Saha R, Ramteke VK (2003) Randomized controlled study of laparoscopic total extraperitoneal versus open Lichtenstein inguinal hernia repair. Surg Endosc 17(6):850-856. https://doi.org/10.1007/s00464-002$8575-6$

251. Andersson B, Hallén M, Leveau P, Bergenfelz A, Westerdahl J (2003) Laparoscopic extraperitoneal inguinal hernia repair versus open mesh repair: a prospective randomized controlled trial. Surgery 133(5):464-472. https://doi.org/10.1067/msy.2003.98

252. Colak T, Akca T, Kanik A, Aydin S (2003) Randomized clinical trial comparing laparoscopic totally extraperitoneal approach with open mesh repair in inguinal hernia. Surg Laparosc Endosc Percutan Tech 13:191-195. https://doi.org/10.1097/00129689200306000-00010

253. Hildebrandt J, Levantin O (2003) Tension-free methods of surgery of primary inguinal hernias. Comparison of endoscopic, total extraperitoneal hernioplasty with the Lichtenstein operation. Chirurg 74(10):915-921. https://doi.org/10.1007/s00104003-0687-6

254. Bringman S, Ramel S, Heikkinen T-J, Englund T, Westman B, Anderberg B (2003) Tension-free inguinal hernia repair: TEP versus mesh-plug versus Lichtenstein: a prospective randomized controlled trial. Ann Surg 237(1):142-147. https://doi.org/10. 1097/00000658-200301000-00020
255. Zhiping T, Min T, Jingcheng Z (2007) Randomized comparative study on totally extra peritoneal prosthetic and tension free herniorrhaphy. J Laparoendosc Surg 12:391

256. Hallen M, Bergenfelz A, Westerdahl J (2008) Laparoscopic extraperitoneal inguinal hernia repair versus open mesh repair: long-term follow-up of a randomized controlled trial. Surgery 143(3):313-317

257. Langeveld HR, van't Riet M, Weidema WF et al (2010) Total extraperitoneal inguinal hernia repair compared with Lichtenstein (the LEVEL-Trial): a randomized controlled trial. Ann Surg 251(5):819-824. https://doi.org/10.1097/SLA. 0b013e3181d96c32

258. Eker HH, Langeveld HR, Klitsie PJ et al (2012) Randomized clinical trial of total extraperitoneal inguinal hernioplasty vs Lichtenstein repair: a long-term follow-up study. Arch Surg 147:256-260. https://doi.org/10.1001/archsurg.2011.2023

259. Payne JH, Grininger LM, Izawa MT, Podoll EF, Lindahl PJ, Balfour J (1994) Laparoscopic or open inguinal herniorrhaphy? A randomized prospective trial. Arch Surg 129(9):973-981

260. Stoker DL, Spiegelhalter DJ, Singh R, Wellwood JM (1994) Laparoscopic versus open inguinal hernia repair: randomised prospective trial. Lancet 343:1243-1245. https://doi.org/10. 1016/S0140-6736\%2894\%2992148-2

261. Maddern GJ, Rudkin G, Bessell JR, Devitt P, Ponte L (1994) A comparison of laparoscopic and open hernia repair as a day surgical procedure. Surg Endosc 8:1404-1408

262. Filipi CJ, Gaston-Johansson F, McBride PJ et al (1996) An assessment of pain and return to normal activity. Laparoscopic herniorrhaphy vs open tension-free Lichtenstein repair. Surg Endosc 10:983-986

263. Heikkinen T, Haukipuro K, Leppala J, Hulkko A (1997) Total costs of laparoscopic and Lichtenstein inguinal hernia repairs: a randomized prospective study. Surg Laparosc Endosc 7:1-5. https://doi.org/10.1097/00019509-199702000-00001

264. Sarli L, Pietra N, Choua O, Costi R, Thenasseril B, Giunta A (1997) Prospective randomized comparative study of laparoscopic hernioplasty and Lichtenstein tension-free hernioplasty. [Italian] TT-Confronto prospettico randomizzato tra ernioplastica laparoscopica ed ernioplastica tension-free secondo Lichtenstein. Acta Biomed Ateneo Parmense 68:5-10

265. Heikkinen TJ, Haukipuro K, Hulkko A (1998) A cost and outcome comparison between laparoscopic and Lichtenstein hernia operations in a day-case unit. A randomized prospective study. Surg Endosc 12(10):1199-1203. https://doi.org/10.1007/ s004649900820

266. Paganini AM, Lezoche E, Carle F et al (1998) A randomized, controlled, clinical study of laparoscopic vs open tension-free inguinal hernia repair. Surg Endosc 12:979-986

267. Wellwood J, Sculpher MJ, Stoker D et al (1998) Randomised controlled trial of laparoscopic versus open mesh repair for inguinal hernia: outcome and cost. Br Med J 317:103-110

268. Picchio M, Lombardi A, Zolovkins A, Mihelsons M, La Torre G (1999) Tension-free laparoscopic and open hernia repair: randomized controlled trial of early results. World J Surg 23:1004-1009

269. Jess P, Schultz K, Bendtzen K, Nielsen OH (2000) Systemic inflammatory responses during laparoscopic and open inguinal hernia repair: a randomised prospective study. Eur J Surg 166(7):540-544. https://doi.org/10.1080/110241500750008600

270. Douek M, Smith G, Oshowo A, Stoker DL, Wellwood JM (2003) Prospective randomised controlled trial of laparoscopic versus open inguinal hernia mesh repair: five year follow up. $\mathrm{Br}$ Med J 326:1012-1013

271. Mahon D, Decadt B, Rhodes M (2003) Prospective randomized trial of laparoscopic (transabdominal preperitoneal) vs open (mesh) repair for bilateral and recurrent inguinal hernia. Surg 
Endosc 17(9):1386-1390. https://doi.org/10.1007/s00464-0029223-x

272. Heikkinen T, Bringman S, Ohtonen P, Kunelius P, Haukipuro K, Hulkko A (2004) Five-year outcome of laparoscopic and Lichtenstein hernioplasties. Surg Endosc Other Interv Tech 18(3):518-522

273. Singh AN, Bansal VK, Misra MC et al (2012) Testicular functions, chronic groin pain, and quality of life after laparoscopic and open mesh repair of inguinal hernia: a prospective randomized controlled trial. Surg Endosc Other Interv Tech 26:1304-1317. https://doi.org/10.1007/s00464-011-2029-y

274. Abbas AE, Abd ME, Noaman N, Negm A, Amin M, Moatamed A (2012) Patient-perspective quality of life after laparoscopic and open hernia repair: a controlled randomized trial. Surg Endosc 26(9):2465-2470. https://doi.org/10.1007/s00464-0122212-9

275. Kargar S, Shiryazdi SM, Zare M, Mirshamsi MH, Ahmadi S, Neamatzadeh H (2015) Comparison of postoperative short-term complications after laparoscopic transabdominal preperitoneal (TAPP) versus Lichtenstein tension free inguinal hernia repair: a randomized trial study. Minerva Chir 70(2):83-89

276. Anadol ZA, Ersoy E, Taneri F, Tekin E (2004) Outcome and cost comparison of laparoscopic transabdominal preperitoneal hernia repair versus open Lichtenstein technique. J Laparoendosc Adv Surg Tech A 14(3):159-163

277. Sarli L, Villa F, Marchesi F (2001) Hernioplasty and simultaneous laparoscopic cholecystectomy: a prospective randomized study of open tension-free versus laparoscopic inguinal hernia repair. Surgery 129(5):530-536. https://doi.org/10.1067/msy. 2001.112962

278. Heikkinen TJ, Haukipuro K, Koivukangas P, Hulkko A (1998) A prospective randomized outcome and cost comparison of totally extraperitoneal endoscopic hernioplasty versus Lichtenstein hernia operation among employed patients. Surg Laparosc Endosc 8(5):338-344. https://doi.org/10.1097/00019509199810000-00003

279. Gokalp A, Inal M, Maralcan G, Baskonus I (2003) A prospective randomized study of Lichtenstein open tension-free versus laparoscopic totally extraperitoneal techniques for inguinal hernia repair. Acta Chir Belg 103(5):502-506

280. Lau H, Patil NG, Yuen WK (2006) Day-case endoscopic totally extraperitoneal inguinal hernioplasty versus open Lichtenstein hernioplasty for unilateral primary inguinal hernia in males: a randomized trial. Surg Endosc 20(1):76-81. https://doi.org/10. 1007/s00464-005-0203-9

281. Eklund A, Rudberg C, Smedberg S et al (2006) Short-term results of a randomized clinical trial comparing Lichtenstein open repair with totally extraperitoneal laparoscopic inguinal hernia repair. Br J Surg 93:1060-1068. https://doi.org/10.1002/ bjs. 5405

282. Eklund AS, Montgomery AK, Rasmussen IC, Sandbue RP, Bergkvist LA, Rudberg CR (2009) Low recurrence rate after laparoscopic (TEP) and open (Lichtenstein) inguinal hernia repair: a randomized, multicenter trial with 5-year follow-up. Ann Surg 249:33-38. https://doi.org/10.1097/SLA. 0b013e31819255d0

283. Eklund A, Montgomery A, Bergkvist L, Rudberg C (2010) Chronic pain 5 years after randomized comparison of laparoscopic and Lichtenstein inguinal hernia repair. $\mathrm{Br} \mathrm{J}$ Surg 97(4):600-608. https://doi.org/10.1002/bjs.6904

284. Eklund A, Carlsson P, Rosenblad A, Montgomery A, Bergkvist L, Rudberg C (2010) Long-term cost-minimization analysis comparing laparoscopic with open (Lichtenstein) inguinal hernia repair. Br J Surg 97(5):765-771. https://doi.org/10.1002/bjs. 6945
285. Dahlstrand U, Sandblom G, Ljungdahl M, Wollert S, Gunnarsson U (2013) TEP under general anesthesia is superior to Lichtenstein under local anesthesia in terms of pain 6 weeks after surgery: results from a randomized clinical trial. Surg Endosc Other Interv Tech 27:3632-3638. https://doi.org/10. 1007/s00464-013-2936-1

286. Dhankhar DS, Sharma N, Mishra T, Kaur N, Singh S, Gupta S (2014) Totally extraperitoneal repair under general anesthesia versus Lichtenstein repair under local anesthesia for unilateral inguinal hernia: a prospective randomized controlled trial. Surg Endosc 28(3):996-1002. https://doi.org/10.1007/s00464-0133269-9

287. Köninger J, Redecke J, Butters M (2004) Chronic pain after hernia repair: a randomized trial comparing Shouldice, Lichtenstein and TAPP. Langenbecks Arch Surg 389(5):361-365. https://doi.org/10.1007/s00423-004-0496-5

288. Butters M, Redecke J, Kninger J (2007) Long-term results of a randomized clinical trial of Shouldice, Lichtenstein and transabdominal preperitoneal hernia repairs. Br J Surg 94(5):562-565

289. Köckerling F, Stechemesser B, Hukauf M, Kuthe A, Schug-Pass C (2015) TEP versus Lichtenstein: which technique is better for the repair of primary unilateral inguinal hernias in men? Surg Endosc. https://doi.org/10.1007/s00464-015-4603-1

290. Voyles CR, Hamilton BJ, Johnson WD, Kano N (2002) Metaanalysis of laparoscopic inguinal hernia trials favors open hernia repair with preperitoneal mesh prosthesis. Am J Surg 184:6-10. https://doi.org/10.1016/S0002-9610\%2802\%2900878-4

291. Aitola P, Airo I, Matikainen M (1998) Laparoscopic versus open preperitoneal inguinal hernia repair: a prospective randomised trial. Ann Chir Gynaecol 87:22-25

292. Johansson B, Hallerbäck B, Glise H, Anesten B, Smedberg S, Román J (1999) Laparoscopic mesh versus open preperitoneal mesh versus conventional technique for inguinal hernia repair. Ann Surg 230(2):225

293. Trevisonno M, Kaneva P, Watanabe Y et al (2015) Current practices of laparoscopic inguinal hernia repair: a populationbased analysis. Hernia 19(5):725-733. https://doi.org/10.1007/ s10029-015-1358-5

294. Morales-Conde S, Socas M, Fingerhut A (2012) Endoscopic surgeons' preferences for inguinal hernia repair: TEP, TAPP, or OPEN. Surg Endosc 26(9):2639-2643. https://doi.org/10.1007/ s00464-012-2247-y

295. Stevenson AD, Nixon SJ, Paterson-Brown S (2010) Variation of laparoscopic hernia repair in Scotland: a postcode lottery? Surgeon 8(3):140-143. https://doi.org/10.1016/j.surge.2009.11. 001

296. de Lange DH, Kreeft M, van Ramshorst GH, Aufenacker TJ, Rauwerda JA, Simons MP (2010) Inguinal hernia surgery in the Netherlands: are patients treated according to the guidelines? Hernia 14(2):143-148. https://doi.org/10.1007/s10029-0090578-y

297. Jacob DA, Hackl JA, Bittner R, Kraft B, Köckerling F (2015) Perioperative outcome of unilateral versus bilateral inguinal hernia repairs in TAPP technique: analysis of 15,176 cases from the Herniamed Registry. Surg Endosc 29(12):3733-3740. https://doi.org/10.1007/s00464-015-4146-5

298. Köckerling F, Schug-Pass C, Adolf D, Keller T, Kuthe A (2015) Bilateral and unilateral total extraperitoneal inguinal hernia repair (TEP) have equivalent early outcomes: analysis of 9395 cases. World J Surg 39(8):1887-1894. https://doi.org/10.1007/ s00268-015-3055-Z

299. Gass M, Rosella L, Banz V, Candinas DGU (2012) Bilateral total extraperitoneal inguinal hernia repair (TEP) has outcomes similar to those for unilateral TEP: population-based analysis of prospective data of 6,505 patients. Surg Endosc 26:1364-1368 
300. Feliu X, Clavería R, Besora P et al (2011) Bilateral inguinal hernia repair: laparoscopic or open approach? Hernia 15(1):15-18. https://doi.org/10.1007/s10029-010-0736-2

301. Read RC (2009) Herniology: past, present, and future. Hernia 13(6):577-580. https://doi.org/10.1007/s10029-009-0582-2

302. Thomas AD, Rogers A (2004) Edoardo Bassini and the wound that inspires. World J Surg 28(10):1060-1062. https://doi.org/ 10.1007/s00268-004-7466-5

303. Bekker J, Keeman JN, Simons MP, Aufenacker TJ (2007) A brief history of the inguinal hernia operation in adults. Ned Tijdschr Geneeskd 151(16):924-931

304. Simons M (1996) Dissertation Shouldice in Amsterdam

305. Bendavid R (2003) Biography: Edward Earle Shouldice (1890-1965). Hernia 7(4):172-177. https://doi.org/10.1007/ s10029-003-0142-0

306. Read RC (2005) The contributions of Usher and others to the elimination of tension from groin herniorrhaphy. Hernia 9(3):208-211. https://doi.org/10.1007/s10029-005-0322-1

307. Usher F, Ochsner J, Tuttle JL (1958) Use of marlex mesh in the repair of incisional hernias. Am J Surg 24(12):969-974

308. Lichtenstein IL, Shulman AG (1986) Ambulatory outpatient hernia surgery. Including a new concept, introducing tensionfree repair. Int Surg 71(1):1-4

309. Lichtenstein IL, Shore JM (1976) Exploding the myths of hernia repair. Am J Surg 132(3):307-315. https://doi.org/10.1016/ 0002-9610(76)90381-0

310. Lichtenstein IL (1964) Local anesthesia for hernioplasty. Immediate ambulation and return to work: a preliminary report. Calif Med 100:106-109

311. Stoppa R, Petit J, Henry X (1975) Unsutured dacron prosthesis in groin hernias. Int Surg 60(8):411-412

312. Stoppa RE, Warlaumont CR, Verhaeghe PJ, Romero ER, M'Balla-N'Di CJ (1986) Prosthetic repair in the treatment of groin hernias. Int Surg 71:154-158

313. Ger R (1982) The management of certain abdominal herniae by intra-abdominal closure of the neck of the sac. Preliminary communication. Ann R Coll Surg Engl 64(5):342-344

314. Toy F, Smoot R (1991) Toy-Smoot laparoscopic hernioplasty. Surg Laparosc Endosc 1(3):151-155

315. Kingsley D, Vogt DM, Nelson MT, Curet MJ, Pitcher DE (1998) Laparoscopic intraperitoneal onlay inguinal herniorrhaphy. Am J Surg 176:548-553. https://doi.org/10.1016/S00029610\%2898\%2900274-8

316. Fitzgibbons RJ Jr, Camps J, Cornet DA et al (1995) Laparoscopic inguinal herniorrhaphy: results of a multicenter trial. Ann Surg 221:3-13

317. Sarli L, Pietra N, Choua O, Costi R, Cattaneo G (1997) Laparoscopic hernia repair: a prospective comparison of TAPP and IPOM techniques. Surg Laparosc Endosc 7:472-476. https://doi.org/10.1097/00019509-199712000-00008

318. Mesci A, Korkmaz B, Dinckan A, Colak T, Balci N, Ogunc G (2012) Digital evaluation of the muscle functions of the lower extremities among inguinal hernia patients treated using three different surgical techniques: a prospective randomized study. Surg Today 42(2):157-163. https://doi.org/10.1007/s00595-0110017-4

319. Choi YY, Han SW, Bae SH, Kim SY, Hur KY, Kang GH (2012) Comparison of the outcomes between laparoscopic totally extraperitoneal repair and prolene hernia system for inguinal hernia; review of one surgeon's experience. J Korean Surg Soc 82(1):40-44. https://doi.org/10.4174/jkss.2012.82.1.40

320. Myers E, Browne KM, Kavanagh DO, Hurley M (2010) Laparoscopic (TEP) versus Lichtenstein inguinal hernia repair: a comparison of quality-of-life outcomes. World J Surg 34(12):3059-3064
321. Bittner R, Schwarz J (2012) Inguinal hernia repair: current surgical techniques. Langenbecks Arch Surg 397(2):271-282. https://doi.org/10.1007/s00423-011-0875-7

322. Kuhry E, van Veen RN, Langeveld HR, Steyerberg EW, Jeekel J, Bonjer HJ (2007) Open or endoscopic total extraperitoneal inguinal hernia repair? A systematic review. Surg Endosc 21:161-166

323. Crawford DL, Hiatt JRPE (1998) Laparoscopy identifies unexpected groin hernias. Am J Surg 64:976-978

324. Sayad P, Abdo Z, Cacchione RFG (2000) Incidence of insipient contralateral hernia during laparoscopic hernia repair. Surg Endosc Other Interv Tech 14(6):543-545

325. Koehler RH (2002) Diagnosing the occult contralateral inguinal hernia: combined use of diagnostic laparoscopy and totally extraperitoneal laparoscopic repair. Surg Endosc Other Interv Tech 16(3):512-520. https://doi.org/10.1007/s00464-001-8166y

326. Saggar VR, Sarangi R (2007) Occult hernias and bilateral endoscopic total extraperitoneal inguinal hernia repair: is there a need for prophylactic repair?: results of endoscopic extraperitoneal repair over a period of 10 years. Hernia 11(1):47-49. https://doi.org/10.1007/s10029-006-0157-4

327. Pawanindra L, Philips P, Chander J, Ramteke VK (2010) Is unilateral laparoscopic TEP inguinal hernia repair a job half done? The case for bilateral repair. Surg Endosc. 24(7):1737-1745. https://doi.org/10.1007/s00464-009-0841-4

328. Zendejas B, Onkendi EO, Brahmbhatt RD, Greenlee SM, Lohse CMFD (2011) Contralateral metachronous inguinal hernia in adults: role for prophylaxis during the TEP repair. Hernia 15(4):403-408

329. O'Rourke A, Zell JA, Varkey-Zell TT, Barone JLBM (2002) Laparoscopic diagnosis and repair of asymptomatic bilateral inguinal hernias. Am J Surg 183:15-19

330. Bochkarev V, Ringley C, Vitamvas MOD (2007) Bilateral laparoscopic inguinal hernia repair in patients with occult contralateral inguinal defects. Surg Endosc 21(5):734-736

331. Tantia O, Jain M, Khanna SSB (2009) Laparoscopic repair of recurrent groin hernia: results of a prospective study. Surg Endosc 23:734-738

332. van den Heuvel B, Beudeker N, Van den Broek J, Bogte ADB (2013) The incidence and natural course of occult inguinal hernias during TAPP repair. Repair is beneficial. Surg Endosc 27:4142-4146

333. Griffin KJ, Harris S, Tang TY, Skelton N, Reed JB, Harris AM (2010) Incidence of contralateral occult inguinal hernia found at the time of laparoscopic trans-abdominal pre-peritoneal (TAPP) repair. Hernia 14(4):345-349. https://doi.org/10.1007/s10029010-0651-6

334. Thill V, Simoens C, Smets D, Ngongang C, da Costa PM (2008) Long-term results of a non-randomized prospective mono-centre study of 1000 laparoscopic totally extraperitoneal hernia repairs. Acta Chir Belg 108(4):405-408

335. Uchida H, Matsumoto T, Ijichi H, Endo YKT (2010) Contralateral occurrence after laparoscopic total extraperitoneal hernia repair for unilateral inguinal hernia. Hernia 14(5):481-484

336. Clark JJ, Limm W, Wong LL (2011) What is the likelihood of requiring contralateral inguinal hernia repair after unilateral repair? Am J Surg 202(6):754-7-8. https://doi.org/10.1016/j. amjsurg.2011.05.017

337. Wright D, Paterson C, Scott N, Hair A, O'Dwyer PJ (2002) Five-year follow-up of patients undergoing laparoscopic or open groin hernia repair: a randomized controlled trial. Ann Surg 235:333-337. https://doi.org/10.1097/00000658-20020300000004 
338. Thumbe VKED (2001) To repair or not to repair incidental defects found on laparoscopic repair of groin hernia. Surg Endosc 15:47-49

339. Chowbey PK, Pithawala M, Khullar R, Sharma A, Soni VBM (2006) Totally extraperitoneal repair of inguinal hernia: a case for bilateral repair. J Minim Access Surg 2(3):171-173

340. Wauschkuhn CA, Schwarz J, Boekeler UBR (2010) Laparoscopic inguinal hernia repair: gold standard in bilateral hernia repair? Results of more than 2800 patients in comparison to literature. Surg Endosc 24:3026-3030

341. Choi YY, Hur KY (2011) Simultaneous laparoscopic totally extraperitoneal repair of bilateral inguinal hernia: review of 1 surgeon experiences. Surg Laparosc Endosc Percutan Tech 21(4):264-266. https://doi.org/10.1097/SLE.0b013e31822177fd

342. Lau H, Patil NGYW (2003) A comparative outcome analysis of bilateral versus unilateral endoscopic extraperitoneal inguinal hernioplastics. J Laparoendosc Adv Surg Tech 13:153-157

343. Ismael M, Nair SGP (2010) Is prophylactic laparoscopic total extraperitoneal inguinal hernia repair on the contralateral side justified in less developed regions? A comparative study of bilateral to unilateral repair. J Laparoendosc Adv Surg Tech 20:533-536

344. Van Veen RN, Van Wessem KJP, Halm JA, Simons MP, Plaisier PW, Jeekel JLJ (2007) Patent processus vaginalis in the adult as a risk factor for the occurrence of indirect inguinal hernia. Surg Endosc 21:202-205

345. Go P, Rutten C, Grasveld-van Berkel M, van Montfort A (2002) Dagbehandeling in Nederland

346. Farquharson EL (1955) Early ambulation; with special reference to herniorrhaphy as an outpatient procedure. Lancet 269:517-519

347. Goulbourne IA, Ruckley CV (1979) Operations for hernia and varicose veins in a day-bed unit. Br Med J 2(6192):712-714

348. Michelsen MWF (1982) Comparison of outpatient and inpatient operations for inguinal hernia (1971 to 1978) (author's transl). [Article in German]. Zentralbl Chir 107(2):94-102

349. Pineault R, Contandriopoulos AP, Valois M, Bastian ML, Lance JM (1985) Randomized clinical trial of one-day surgery. Patient satisfaction, clinical outcomes, and costs. Med Care 23(2):171-182. https://doi.org/10.1097/00005650-19850200000008

350. Prescott RJ, Cutherbertson C, Fenwick N, Garraway WM, Ruckley CV (1978) Economic aspects of day care after operations for hernia or varicose veins. J Epidemiol Community Health 32(3):222-225

351. Ramyil VM, Ognonna BC, Iya D (1999) Patient acceptance of outpatient treatment for inguinal hernia in Jos, Nigeria. Cent Afr J Med 45(9):244-246

352. Engbaek J, Bartholdy J, Hjorts $\varnothing$ N-C (2006) Return hospital visits and morbidity within 60 days after day surgery: a retrospective study of 18,736 day surgical procedures. Acta Anaesthesiol Scand 50(8):911-919. https://doi.org/10.1111/j.13996576.2006.01090.x

353. Majholm B, Engbæk J, Bartholdy J et al (2012) Is day surgery safe? A Danish multicentre study of morbidity after 57,709 day surgery procedures. Acta Anaesthesiol Scand 56(3):323-331. https://doi.org/10.1111/j.1399-6576.2011.02631.x

354. McCormack K, Scott NW, Go PM, Ross S, Grant AM (2003) Laparoscopic techniques versus open techniques for inguinal hernia repair. Cochrane Database Syst Rev 1:CD001785. https://doi.org/10.1002/14651858.CD001785

355. Davies KE, Houghton K, Montgomery JE (2001) Obesity and day-case surgery. Anaesthesia 56(11):1112-1115. https://doi. org/10.1111/j.1365-2044.2001.1962-5.x
356. Jarrett PEM (2001) Day care surgery. Eur J Anaesthesiol 18(S23):32-35. https://doi.org/10.1046/j.1365-2346.2001. 018s23032.x

357. Prabhu A, Chung F (2001) Anaesthetic strategies towards developments in day care surgery. Eur J Anaesthesiol Suppl 23:36-42. https://doi.org/10.1046/j.1365-2346.2001.018s23036. $\mathrm{x}$

358. Sanjay P, Jones P, Woodward A (2006) Inguinal hernia repair: are ASA grades 3 and 4 patients suitable for day case hernia repair? Hernia 10(4):299-302. https://doi.org/10.1007/s10029005-0048-0

359. Mattila K, Vironen J, Eklund A, Kontinen VK, Hynynen M (2011) Randomized clinical trial comparing ambulatory and inpatient care after inguinal hernia repair in patients aged 65 years or older. Am J Surg 201:179-185. https://doi.org/10. 1016/j.amjsurg.2010.04.024

360. Huerta S, Pham T, Foster S, Livingston EH, Dineen S (2014) Outcomes of emergent inguinal hernia repair in veteran octogenarians. Am Surg 80(5):479-483

361. Palumbo P, Amatucci C, Perotti B et al (2014) Outpatient repair for inguinal hernia in elderly patients: still a challenge? Int $\mathrm{J}$ Surg 12(2):4-7. https://doi.org/10.1016/j.ijsu.2014.08.393

362. Pallati PK, Gupta PK, Bichala S, Gupta H, Fang X, Forse RA (2013) Short-term outcomes of inguinal hernia repair in octogenarians and nonagenarians. Hernia 17(6):723-727. https://doi. org/10.1007/s10029-012-1040-0

363. Köckerling F, Roessing C, Adolf D, Schug-Pass C, Jacob D (2016) Has endoscopic (TEP, TAPP) or open inguinal hernia repair a higher risk of bleeding in patients with coagulopathy or antithrombotic therapy? Data from the Herniamed Registry. Surg Endosc 30(5):2073-2081. https://doi.org/10.1007/s00464015-4456-7

364. Rodriguez-Cuellar E, Villeta R, Ruiz P et al (2005) National project for the management of clinical processes. Surgical treatment of inguinal hernia. Cir Esp 77(4):194-202

365. Weyhe D, Winnemöller C, Hellwig A et al (2006) (Section sign) $115 \mathrm{~b}$ SGB V threatens outpatient treatment for inguinal hernia. Analysis of outcome and economics. Chirurg 77(9):844-855. https://doi.org/10.1007/s00104-006-1208-1

366. Klinge U, Klosterhalfen B (2012) Modified classification of surgical meshes for hernia repair based on the analyses of 1,000 explanted meshes. Hernia 16(3):251-258. https://doi.org/10. 1007/s10029-012-0913-6

367. Velayudhan S, Martin D, Cooper-White J (2009) Evaluation of dynamic creep properties of surgical mesh prostheses-uniaxial fatigue. J Biomed Mater Res B Appl Biomater 91(1):287-296. https://doi.org/10.1002/jbm.b.31401

368. Li X, Kruger J, Jor J et al (2014) Characterizing the ex vivo mechanical properties of synthetic polypropylene surgical mesh. J Mech Behav Biomed Mater 37:48-55. https://doi.org/10.1016/ j.jmbbm.2014.05.005

369. Eliason BJ, Frisella MM, Matthews BD, Deeken CR (2011) Effect of repetitive loading on the mechanical properties of synthetic hernia repair materials. J Am Coll Surg 213(3):430-435. https://doi.org/10.1016/j.jamcollsurg.2011.05. 018

370. Amid P (1997) Classification of biomaterials and their related complications in abdominal wall hernia surgery. Hernia $1(1): 15-21$

371. Mühl T, Binnebösel M, Klinge U, Goedderz T (2008) New objective measurement to characterize the porosity of textile implants. J Biomed Mater Res Part B Appl Biomater 84(1):176-183. https://doi.org/10.1002/jbm.b.30859

372. Coda A, Lamberti R, Martorana S (2012) Classification of prosthetics used in hernia repair based on weight and 
biomaterial. Hernia 16(1):9-20. https://doi.org/10.1007/s10029011-0868-z

373. Deeken CR, Abdo MS, Frisella MM, Matthews BD (2011) Physicomechanical evaluation of polypropylene, polyester, and polytetrafluoroethylene meshes for inguinal hernia repair. J Am Coll Surg 212(1):68-79. https://doi.org/10.1016/j.jamcollsurg. 2010.09.012

374. Deeken CR, Thompson DM, Castile RM, Lake SP (2014) Biaxial analysis of synthetic scaffolds for hernia repair demonstrates variability in mechanical anisotropy, non-linearity and hysteresis. J Mech Behav Biomed Mater 38:6-16. https://doi.org/10.1016/j.jmbbm.2014.06.001

375. Maurer MM, Röhrnbaue B, Feola A, Deprest J, Mazza E (2014) Mechanical biocompatibility of prosthetic meshes: a comprehensive protocol for mechanical characterization. J Mech Behav Biomed Mater 40:42-58. https://doi.org/10.1016/j.jmbbm.2014. 08.005

376. Sahoo S, DeLozier KR, Erdemir A, Derwin KA (2015) Clinically relevant mechanical testing of hernia graft constructs. J Mech Behav Biomed Mater 41:117-118. https://doi.org/10. 1016/j.jmbbm.2014.10.011

377. Klinge U, Prescher A, Klosterhalfen B, Schumpelick V (1997) Entstehung und pathophysiologie der bauchwanddefekte. Chirurg 68(4):293-303

378. Klosterhalfen B, Junge K, Klinge U (2005) The lightweight and large porous mesh concept for hernia repair. Expert Rev Med Devices 2(1):103-117. https://doi.org/10.1586/17434440.2.1. 103

379. Saberski ER, Orenstein SB, Novitsky YW (2011) Anisotropic evaluation of synthetic surgical meshes. Hernia 15(1):47-52. https://doi.org/10.1007/s10029-010-0731-7

380. Klinge U, Klosterhalfen B, Conze J et al (1998) Modified mesh for hernia repair that is adapted to the physiology of the abdominal wall. Eur J Surg 164(12):951-960. https://doi.org/10. 1080/110241598750005138

381. Weyhe D, Cobb W, Lecuivre J et al (2015) Large pore size and controlled mesh elongation are relevant predictors for mesh integration quality and low shrinkage — systematic analysis of key parameters of meshes in a novel minipig hernia model. Int $\mathbf{J}$ Surg 22:46-53. https://doi.org/10.1016/j.ijsu.2015.07.717

382. Klosterhalfen B, Klinge U (2013) Retrieval study at 623 human mesh explants made of polypropylene-impact of mesh class and indication for mesh removal on tissue reaction. J Biomed Mater Res Part B Appl Biomater 101(8):1393-1399. https://doi. org/10.1002/jbm.b.32958

383. Barone WR, Amini R, Maiti S, Moalli PA, Abramowitch SD (2015) The impact of boundary conditions on surface curvature of polypropylene mesh in response to uniaxial loading. J Biomech 48(9):1566-1574. https://doi.org/10.1016/j.jbiomech. 2015.02.061

384. Feola A, Pal S, Moalli P, Maiti S, Abramowitch S (2014) Varying degrees of nonlinear mechanical behavior arising from geometric differences of urogynecological meshes. J Biomech 47(11):2584-2589. https://doi.org/10.1016/j.jbiomech.2014.05. 027

385. Feola A, Barone W, Moalli P, Abramowitch S (2013) Characterizing the ex vivo textile and structural properties of synthetic prolapse mesh products. Int Urogynecol J Pelvic Floor Dysfunct 24(4):559-564. https://doi.org/10.1007/s00192-012-1901-1

386. Shepherd JP, Feola AJ, Abramowitch SD, Moalli PA (2012) Uniaxial biomechanical properties of seven different vaginally implanted meshes for pelvic organ prolapse. Int Urogynecol J Pelvic Floor Dysfunct 23(5):613-620. https://doi.org/10.1007/ s00192-011-1616-8
387. Leber GE, Garb JL, Alexander AI et al (1960) Long-term complications associated with prosthetic repair of incisional hernias. Arch Surg 133:378-382

388. Kuehnert N, Kraemer NA, Otto J et al (2012) In vivo MRI visualization of mesh shrinkage using surgical implants loaded with superparamagnetic iron oxides. Surg Endosc Other Interv Tech 26(5):1468-1475. https://doi.org/10.1007/s00464-0112057-7

389. Weyhe D, Belyaev O, Müller C et al (2007) Improving outcomes in hernia repair by the use of light meshes - a comparison of different implant constructions based on a critical appraisal of the literature. World J Surg 31:234-244

390. Weyhe D, Schmitz I, Belyaev O et al (2006) Experimental comparison of monofile light and heavy polypropylene meshes: less weight does not mean less biological response. World J Surg 30(8):1586-1591. https://doi.org/10.1007/s00268-0050601-0

391. Nikkolo C, Murruste M, Vaasna T, Seepter H, Tikk T, Lepner U (2012) Three-year results of randomised clinical trial comparing lightweight mesh with heavyweight mesh for inguinal hernioplasty. Hernia 16(5):555-559. https://doi.org/10.1007/s10029012-0951-0

392. Sadowski B, Rodriguez J, Symmonds R et al (2011) Comparison of polypropylene versus polyester mesh in the Lichtenstein hernia repair with respect to chronic pain and discomfort. Hernia 15:643-654. https://doi.org/10.1007/s10029-011-0841-x

393. Chowbey PK, Garg N, Sharma A et al (2010) Prospective randomized clinical trial comparing lightweight mesh and heavyweight polypropylene mesh in endoscopic totally extraperitoneal groin hernia repair. Surg Endosc 24(12):3073-3079. https://doi. org/10.1007/s00464-010-1092-0

394. Chui LB, Ng WT, Sze YS, Yuen KS, Wong YT, Kong CK (2010) Prospective, randomized, controlled trial comparing lightweight versus heavyweight mesh in chronic pain incidence after TEP repair of bilateral inguinal hernia. Surg Endosc Other Interv Tech 24:2735-2738. https://doi.org/10.1007/s00464-0101036-8

395. Chen J, Lv Y, Shen Y, Liu S, Wang M (2010) A prospective comparison of preperitoneal tension-free open herniorrhaphy with mesh plug herniorrhaphy for the treatment of femoral hernias. Surgery 148(5):976-981. https://doi.org/10.1016/j.surg. 2010.02.006

396. Agarwal BB, Agarwal KA, Mahajan KC (2009) Prospective double-blind randomized controlled study comparing heavyand lightweight polypropylene mesh in totally extraperitoneal repair of inguinal hernia: early results. Surg Endosc Other Interv Tech 23:242-247. https://doi.org/10.1007/s00464-008-0188-2

397. Koch A, Bringman S, Myrelid P, Smeds S, Kald A (2008) Randomized clinical trial of groin hernia repair with titaniumcoated lightweight mesh compared with standard polypropylene mesh. Br J Surg 95:1226-1231. https://doi.org/10.1002/bjs.6325

398. Bringman S, Wollert S, Osterberg J, Smedberg S, Granlund H, Heikkinen T-J (2006) Three-year results of a randomized clinical trial of lightweight or standard polypropylene mesh in Lichtenstein repair of primary inguinal hernia. $\mathrm{Br} \mathrm{J}$ Surg 93(9):1056-1059. https://doi.org/10.1002/bjs.5403

399. Nienhuijs SW, van Oort I, Keemers-Gels ME, Strobbe LJ, Rosman C (2005) Randomized trial comparing the Prolene Hernia System, mesh plug repair and Lichtenstein method for open inguinal hernia repair. Br J Surg 92:33-38

400. Post S, Weiss B, Willer M, Neufang T, Lorenz D (2004) Randomized clinical trial of lightweight composite mesh for Lichtenstein inguinal hernia repair. $\mathrm{Br} \mathrm{J}$ Surg 91:44-48. https://doi.org/10.1002/bjs.4387

401. Sanjay P, Harris D, Jones P, Woodward A (2006) Randomized controlled trial comparing prolene hernia system and 
Lichtenstein method for inguinal hernia repair. ANZ J Surg 76:548-552. https://doi.org/10.1111/j.1445-2197.2006.03774.x

402. Paajanen H (2007) A single-surgeon randomized trial comparing three composite meshes on chronic pain after Lichtenstein hernia repair in local anesthesia. Hernia 11:335-339. https://doi. org/10.1007/s10029-007-0236-1

403. Moreno-Egea A, Carrillo-Alcaraz A, Soria-Aledo V (2013) Randomized clinical trial of laparoscopic hernia repair comparing titanium-coated lightweight mesh and medium-weight composite mesh. Surg Endosc Other Interv Tech 27:231-239. https://doi.org/10.1007/s00464-012-2425-y

404. Bury KSM (2012) Five-year results of a randomized clinical trial comparing a polypropylene mesh with a poliglecaprone and polypropylene composite mesh for inguinal hernioplasty. Hernia 16:549-553. https://doi.org/10.1007/s10029-012-0916-3

405. Peeters E, Spiessens C, Oyen R et al (2010) Laparoscopic inguinal hernia repair in men with lightweight meshes may significantly impair sperm motility: a randomized controlled trial. Ann Surg 252:240-246. https://doi.org/10.1097/SLA. 0b013e3181e8fac5

406. Langenbach MR, Schmidt J, Zirngibl H (2006) Comparison of biomaterials: three meshes and TAPP for inguinal hernia. Surg Endosc 20(10):1511-1517. https://doi.org/10.1007/s00464-0050078-9

407. Bringman S, Wollert S, Osterberg J et al (2005) One year results of a randomised controlled multi-centre study comparing Prolene and Vypro II-mesh in Lichtenstein hernioplasty. Hernia 9:223-227. https://doi.org/10.1007/s10029-005-0324-z

408. Heikkinen T, Wollert S, Osterberg J, Smedberg S, Bringman S (2006) Early results of a randomised trial comparing Prolene and Vypro II-mesh in endoscopic extraperitoneal inguinal hernia repair (TEP) of recurrent unilateral hernias. Hernia 10:34-40. https://doi.org/10.1007/s10029-005-0026-6

409. Lionetti R, Neola B, Dilillo S, Bruzzese D, Ferulano GP (2012) Sutureless hernioplasty with light-weight mesh and Wbrin glue versus Lichtenstein procedure: a comparison of outcomes focusing on chronic postoperative pain. Hernia 16:127-131. https://doi.org/10.1007/s10029-011-0869-y

410. Nikkolo C, Lepner U, Murruste M, Vaasna T, Seepter H, Tikk T (2010) Randomised clinical trial comparing lightweight mesh with heavyweight mesh for inguinal hernioplasty. Hernia 14:253-258. https://doi.org/10.1007/s10029-010-0630-y

411. Peeters E, Spiessens C, Oyen R et al (2014) Sperm motility after laparoscopic inguinal hernia repair with lightweight meshes: 3-year follow-up of a randomised clinical trial. Hernia 18:361-367. https://doi.org/10.1007/s10029-012-1028-9

412. Nikkolo C, Vaasna T, Murruste M, Seepter H, Kirsimägi U, Lepner U (2014) Randomized clinical study evaluating the impact of mesh pore size on chronic pain after Lichtenstein hernioplasty. J Surg Res 191(2):311-317. https://doi.org/10. 1016/j.jss.2014.04.022

413. Lake SP, Ray S, Zihni AM, Thompson DM, Gluckstein J, Deeken CR (2015) Pore size and pore shape-but not mesh density-alter the mechanical strength of tissue ingrowth and host tissue response to synthetic mesh materials in a porcine model of ventral hernia repair. J Mech Behav Biomed Mater 42:186-197. https://doi.org/10.1016/j.jmbbm.2014.11.011

414. Schug-Pass C, Tamme C, Tannapfel A, Kockerling F (2006) A lightweight polypropylene mesh (TiMesh) for laparoscopic intraperitoneal repair of abdominal wall hernias: comparison of biocompatibility with the DualMesh in an experimental study using the porcine model. Surg Endosc Other Interv Tech 20(3):402-409. https://doi.org/10.1007/s00464-004-8277-3

415. Sajid MS, Kalra L, Parampalli U, Sains PS, Baig MK (2013) A systematic review and meta-analysis evaluating the effectiveness of lightweight mesh against heavyweight mesh in influencing the incidence of chronic groin pain following laparoscopic inguinal hernia repair. Am J Surg 205:726-736. https://doi.org/10.1016/j.amjsurg.2012.07.046

416. Currie A, Andrew H, Tonsi A, Hurley PR, Taribagil S (2012) Lightweight versus heavyweight mesh in laparoscopic inguinal hernia repair: a meta-analysis. Surg Endosc 26:2126-2133. https://doi.org/10.1007/s00464-012-2179-6

417. Li J, Ji Z, Cheng T (2012) Lightweight versus heavyweight in inguinal hernia repair: a meta-analysis. Hernia 16:529-539. https://doi.org/10.1007/s10029-012-0928-z

418. Pielacinski K, Szczepanik AB, Wroblewski T (2013) Effect of mesh type, surgeon and selected patients' characteristics on the treatment of inguinal hernia with the Lichtenstein technique. Randomized trial. Wideochir Inne Tech Maloinwazyjne 8:99-106. https://doi.org/10.5114/wiitm.2011.32824

419. Śmietański M, Bury K, Śmietańska IA, Owczuk R, Paradowski $T$ (2011) Five-year results of a randomised controlled multicentre study comparing heavy-weight knitted versus low-weight, non-woven polypropylene implants in Lichtenstein hernioplasty. Hernia 15(5):495-501. https://doi.org/10.1007/s10029-0110808-y

420. Sajid M, Leaver C, Baig M et al (2012) Systematic review and meta-analysis of the use of lightweight versus heavyweight mesh in open inguinal hernia repair. Br J Surg 99:29-37

421. Uzzaman MM, Ratnasingham K, Ashraf N (2012) Meta-analysis of randomized controlled trials comparing lightweight and heavyweight mesh for Lichtenstein inguinal hernia repair. Hernia 16(5):505-518. https://doi.org/10.1007/s10029-012-0901-x

422. Bittner R, Leibl BJ, Kraft B, Schwarz J (2011) One-year results of a prospective, randomised clinical trial comparing four meshes in laparoscopic inguinal hernia repair (TAPP). Hernia 15:503-510. https://doi.org/10.1007/s10029-011-0810-4

423. Schopf S, von Ahnen T, von Ahnen M, Schardey H (2011) Chronic pain after laparoscopic transabdominal preperitoneal hernia repair: a randomized comparison of light and extralight titanized polypropylene mesh. World J Surg 35:302-310. https://doi.org/10.1007/s00268-010-0850-4

424. Bittner R, Schmedt CG, Leibl BJ, Schwarz J (2011) Early postoperative and one year results of a randomized controlled trial comparing the impact of extralight titanized polypropylene mesh and traditional heavyweight polypropylene mesh on pain and seroma production in laparoscopic hernia repair (TAPP). World J Surg 35:1791-1797

425. Burgmans JPJ, Voorbrood CEH, Schouten N et al (2015) Threemonth results of the effect of Ultrapro or Prolene mesh on postoperative pain and well-being following endoscopic totally extraperitoneal hernia repair (TULP trial). Surg Endosc Other Interv Tech 29(11):3171-3178. https://doi.org/10.1007/s00464014-4049-x

426. Horstmann R, Hellwig M, Classen C, Rottgermann S, Palmes D (2006) Impact of polypropylene amount on functional outcome and quality of life after inguinal hernia repair by the TAPP procedure using pure, mixed, and titanium-coated meshes. World J Surg 30:1742-1749. https://doi.org/10.1007/s00268005-0242-3

427. Champault G, Bernard C, Rizk N, Polliand C (2007) Inguinal hernia repair: the choice of prosthesis outweighs that of technique. Hernia 11(2):125-128

428. Costello CR, Bachman SL, Ramshaw BJ, Grant SA (2007) Materials characterization of explanted polypropylene hernia meshes. J Biomed Mater Res Part B Appl Biomater 83(1):44-49. https://doi.org/10.1002/jbm.b.30764

429. Hansen NL, Ciritsis A, Otto J, Busch D, Kuhl CK, Kraemer NA (2015) Utility of magnetic resonance imaging to monitor surgical meshes correlating imaging and clinical outcome of 
patients undergoing. Investig Radiol 50(7):436-442. https://doi. org/10.1097/RLI.0000000000000148

430. Acar T, Gomceli I, Tacyildiz R, Sozen S, Karakayali S, Aydin R (2002) Enterocutaneous fistula due to polypropylene mesh migration. Ir J Med Sci 171(3):172-174

431. Agrawal A, Avill R (2006) Mesh migration following repair of inguinal hernia: a case report and review of literature. Hernia 10(1):79-82. https://doi.org/10.1007/s10029-005-0024-8

432. Arroyo Q, Argüelles-Arias F, Jimenez-Saenz M, Herrerias-Gutierrez JM, Pellicer Bautista F (2011) Dysphagia caused by migrated mesh after paraesophageal hernia repair. Endoscopy 43(SUPPL. 2):257-258. https://doi.org/10.1055/s-0030-1256520

433. Aziz F, Zaeem M (2014) Chronic abdominal pain secondary to mesh erosion into ceacum following incisional hernia repair: a case report and literature review. J Clin Med Res 6(2):153-155. https://doi.org/10.14740/jocmr1730w

434. Baldassarre E, Valenti G, Porta IP, Vigano M (2006) Re: mesh migration into the bladder after TEP repair: a rare case report. Surg Laparosc Endosc Percutan Tech. 2006;16:52-53. Surg Laparosc Endosc Percutan Tech 16(5):377-378

435. Benedetti $M$, Albertario $S$, Niebel $T$ et al (2005) Intestinal perforation as a long-term complication of plug and mesh inguinal hernioplasty: case report. Hernia 9(1):93-95. https://doi.org/10.1007/s10029-004-0256-z

436. Bodenbach M, Bschleipfer T, Stoschek M, Beckert R, Sparwasser C (2002) Intravesical migration of a polypropylene mesh implant 3 years after laparoscopic transperitoneal hernioplasty. Urologe A 41(4):366-368

437. Borchert D, Kumar B, Dennis R, Alberts J (2008) Mesh migration following obturator hernia repair presenting as a bezoar inducing small intestinal obstruction. Hernia 12(1):83-85. https://doi.org/10.1007/s10029-007-0216-5

438. Carpelan-Holmström M, Kruuna O, Salo J, Kylänpää L, Scheinin T (2011) Late mesh migration through the stomach wall after laparoscopic refundoplication using a dual-sided PTFE/ePTFE mesh. Hernia 15(2):217-220. https://doi.org/10. 1007/s10029-010-0633-8

439. Carrozza V, Ivaldi L, Ferro A et al (2011) Inguinal hernia repair: an experimental study on mesh migration with the tension-free sutureless technique. 10 year follow-up. Minerva Chir 66(1):21-40

440. Celik A, Kutun S, Kockar C, Mengi N, Ulucanlar H, Cetin A (2005) Colonoscopic removal of inguinal hernia mesh: report of a case and literature review. J Laparoendosc Adv Surg Tech A 15(4):408-410. https://doi.org/10.1089/lap.2005.15.408

441. Chen MJ, Tian YF (2010) Intraperitoneal migration of a mesh plug with a small intestinal perforation: report of a case. Surg Today 40(6):566-568. https://doi.org/10.1007/s00595-0094107-5

442. Chowbey PK, Bagchi N, Goel A et al (2006) Mesh migration into the bladder after TEP repair: a rare case report. Surg Laparosc Endosc Percutan Tech 16(1):52-53. https://doi.org/10. 1097/01.sle.0000202185.34666.f1

443. Chuback JA, Singh RS, Sills C, Dick LS (2000) Small bowel obstruction resulting from mesh plug migration after open inguinal hernia repair. Surgery 127(4):475-476

444. D'Amore L, Gossetti F, Manto O, Negro P (2012) Mesh plug repair: can we reduce the risk of plug erosion into the sigmoid colon? Hernia 16(4):495-496. https://doi.org/10.1007/s10029012-0921-6

445. De Moor V, Zalcman M, Delhaye M, El Nakadi I (2012) Complications of mesh repair in hiatal surgery: about 3 cases and review of the literature. Surg Laparosc Endosc Percutan Tech 22(4):222-225. https://doi.org/10.1097/SLE. $0 \mathrm{~b} 013 \mathrm{e} 318253 \mathrm{e} 440$
446. Deligné E, Faucompret S, Louis C, Arigon JP, Bréda Y (2001) Intestinal obstruction by migration of wire mesh into the intestinal lumen following hernia repair. Ann Chir 126(6):590-593. https://doi.org/10.1016/S0003-3944(01)005569

447. Di Muria A, Formisano V, Di Carlo F, Aveta A, Giglio D (2007) Small bowel obstruction by mesh migration after umbilical hernia repair. Ann Ital Chir 78(1):59-60

448. Dieter R (1999) Mesh plug migration into scrotum: a new complication of hernia repair. Int J Surg 84(1):57-59

449. Ferrone R, Scarone PC, Natalini G (2003) Late complication of open inguinal hernia repair: small bowel obstruction caused by intraperitoneal mesh migration. Hernia 7(3):161-162. https://doi.org/10.1007/s10029-003-0129-x

450. Fukudome I, Dabanaka K, Okabayashi T et al (2014) A 58-yearold woman with mesh migration into the transverse colon. Am Surg 80(2):E40-E41

451. Gandhi D, Marcin S, Xin Z, Asha B, Kaswala D, Zamir B (2011) Chronic abdominal pain secondary to mesh erosion into cecum following incisional hernia repair: a case report and literature review. Ann Gastroenterol 24(4):321-324

452. Goswami R, Babor M, Ojo A (2007) Mesh erosion into caecum following laparoscopic repair of inguinal hernia (TAPP): a case report and literature review. J Laparoendosc Adv Surg Tech A 17(5):669-672. https://doi.org/10.1089/lap.2006.0135

453. Hamouda A, Kennedy J, Grant N, Nigam A, Karanjia N (2010) Mesh erosion into the urinary bladder following laparoscopic inguinal hernia repair; is this the tip of the iceberg? Hernia 14(3):317-319. https://doi.org/10.1007/s10029-009-0539-5

454. Hergueta-Delgado P, Marin-Moreno M, Morales-Conde S et al (2006) Transmural migration of a prosthetic mesh after surgery of a paraesophageal hiatal hernia. Gastrointest Endosc 64(1):120-121. https://doi.org/10.1016/j.gie.2006.01.034

455. Horzic M, Vergles D, Cupurdija K, Kopljar M, Zidak M, Lackovic Z (2011) Spontaneous mesh evacuation per rectum after incisional ventral hernia repair. Hernia 15(3):351-352. https://doi.org/10.1007/s10029-010-0655-2

456. Hume RH, Bour J (1996) Mesh migration following laparoscopic inguinal hernia repair. J Laparoendosc Surg 6(5):333-335

457. Jansen M, Otto J, Lynen Jansen P et al (2007) Mesh migration into the esophageal wall after mesh hiatoplasty: comparison of two alloplastic materials. Surg Endosc Other Interv Tech 21(12):2298-2303. https://doi.org/10.1007/s00464-007-9514-3

458. Jeans S, Williams GL, Stephenson BM (2007) Migration after open mesh plug inguinal hernioplasty: a review of the literature. Am Surg 73(3):207-209

459. Jensen JB, Jonler M, Lund L (2004) Recurrent urinary tract infection due to hernia mesh erosion into the bladder. Scand J Urol Nephrol 38(5):438-439. https://doi.org/10.1080/ 00365590410031689

460. Jha A, Nijhawan S, Pokharna R, Nepalia S, Suchismita A (2012) Colo-cutaneous fistula formation due to delayed mesh migration following lumbar hernia repair: colonoscopic diagnosis. Trop Gastroenterol 33(3):236-238

461. Kalish I, Bab̌bekov I, Ametov L, l'igitaliev SK (2014) Morphological characteristic of tissues, surrounding prosthesis in patients, suffering cutaneo-prosthetic fistulas and paraprosthetic hernias. [Article in Russian]. Klin Khir 4:34-46

462. Li W, Chen X, Zhan H, Yang F, Liu B, Zhou X (2014) Hernia mesh migration into bladder presenting as malignancy: potential source of diagnostic errors. Hernia 18(6):903-906

463. Liang X, Cai C, Yu H, Wang Y (2008) Strangulated bowel obstruction resulting from mesh plug migration after open inguinal hernioplasty: case report. Chin J Evid Med 121(2):183-184 
464. Libretti L, Ciriaco P, Carretta A et al (2006) Endobronchial migration of prosthetic patch after congenital diaphragmatic hernia repair. J Pediatr Surg 41(1):e65-e67. https://doi.org/10. 1016/j.jpedsurg.2005.10.075

465. Lo DJ, Bilimoria KY, Pugh CM (2008) Bowel complications after prolene hernia system (PHS) repair: a case report and review of the literature. Hernia 12(4):437-440. https://doi.org/ 10.1007/s10029-008-0338-4

466. Majeski J (1998) Migration of wire mesh into the intestinal lumen causing an intestinal obstruction 30 years after repair of a ventral hernia. South Med J 91(5):496-498

467. Millas SG, Mesar T, Patel RJ (2015) Chronic abdominal pain after ventral hernia due to mesh migration and erosion into the sigmoid colon from a distant site: a case report and review of literature. Hernia 19(5):849-852. https://doi.org/10.1007/ s10029-013-1182-8

468. Millikan KW, Cummings B, Doolas A (2003) The Millikan modified mesh-plug hernioplasty. Arch Surg 138(5):525-530. https://doi.org/10.1001/archsurg.138.5.525

469. Moorman M, Price P (2004) Migrating mesh plug complication of a well established hernia repair technique. Am Surg 70(4):298-299

470. Murphy JW, Misra DC, Silverglide B (2006) Sigmoid colonic fistula secondary to perfix-plug, left inguinal hernia repair. Hernia 10(5):436-438. https://doi.org/10.1007/s10029-0060113-3

471. Nelson EC, Vidoversuszky TJ (2011) Composite mesh migration into the sigmoid colon following ventral hernia repair. Hernia 15(1):101-103. https://doi.org/10.1007/s10029-0090623-x

472. Novaretti JPT, Silva RDP, Cotrim CAC, Souto LRM (2012) Migration mesh mimicking bladder malignancy after open inguinal hernia repair. Hernia 16(4):467-470. https://doi.org/10. 1007/s10029-010-0760-2

473. Ojo P, Abenthroth A, Fiedler P, Yavorek G (2006) Migrating mesh mimicking colonic malignancy. Am Surg 72(12):1210-1211

474. Olmi S, Uccelli M, Cesana GO et al (2013) Small bowel obstruction caused by mesh migration. Case report. G Chir 34(3):70-73

475. Panzironi G, De Vargas Macciucca M, Ricci F, Angelini P, De Cristofaro F (2000) A rare complication caused by prosthetic mesh in inguinal hernioplasty. A case report. Radiol Med 100(6):506-508

476. Pautrat K, Scotto B, Machet MC, Huten N, de Calan L (2004) Migration of a mesh prosthesis from hernia repair into the colonic lumen. J Chir (Paris) 141(6):378-380. https://doi.org/10. 1016/S0021-7697(04)95363-X

477. Porziella V, Cesario A, Lococo F et al (2012) Complete transmural gastric migration of PTFE mesh after surgery for a recurrent hiatal hernia. Eur Rev Med Pharmacol Sci 16(4):42-43

478. Pros I, Puyol M, Franco A et al (1990) Enterovesical fistula caused by a prosthesis made of synthetic material. Actas Urol Esp 14(4):282-285

479. Rasmussen M, Bisgaard $\mathrm{T}$ (2015) Late mesh migration into the colon after laparoscopic herniotomy. Ugeskr Laeger 136(4):1123-1125

480. Ratajczak A, Koscinski T, Banasiewicz T, Lange-Ratajczak L, Hermann JBA (2013) Migration of biomaterials used in gastroenterological surgery. Pol Przegl Chir 85(7):377-380

481. Riaz AA, Ismail M, Barsam A, Bunce CJ (2004) Mesh erosion into the bladder: a late complication of incisional hernia repair. A case report and review of the literature. Hernia 8(2):158-159. https://doi.org/10.1007/s10029-003-0187-0

482. Savoiz D, Ludwig C, Leissing C, Bolle J, Buhler L, Morel P (1997) Repeated macroscopic haematuria caused by intravesical migration of a preperitoneal prosthesis. Eur J Surg 163(8):631-632

483. Seker D, Kulacoglu H (2011) Long-term complications of mesh repairs for abdominal-wall hernias. J Long Term Eff Med Implants 21(3):205-218

484. Steinhagen E, Khaitov S, Steinhagen RM (2010) Intraluminal migration of mesh following incisional hernia repair. Hernia 14(6):659-662. https://doi.org/10.1007/s10029-010-0708-6

485. Stout C, Foret A, Christie D, Mullis E (2007) Small bowel volvulus caused by migrating mesh plug. Am Surg 73(8):796-797

486. Su Y-R, Chan P-H (2014) Mesh migration into urinary bladder after open ventral herniorrhaphy with mesh: a case report. Int Surg 99(4):410-413. https://doi.org/10.9738/INTSURG-D-1300037.1

487. Szitkaer B, Yzet T, Auwuier M et al (2010) Late complications from abdominal wall surgery: report of three cases of mesh migration into hollow viscus. J Radiol 91:59-64

488. Tiwari SK, Lal P (2014) A rare case of acute intestinal obstruction due to bowel entrapment in migrated onlay polypropylene mesh. Ann R Coll Surg Engl 96(7):e4-e5. https://doi.org/10.1308/003588414X13946184900200

489. Tokunaga Y, Tokuka A, Ohsumi K (2001) Sigmoid colon diverticulosis adherent to mesh plug migration after open inguinal hernia repair. Curr Surg 58(5):493-494. https://doi.org/ 10.1016/S0149-7944(01)00495-0

490. Ulukent SC, Kaya B, Bat O, Sahbaz NA, Akca O, Akyol A (2013) Prolene mesh migration into the rectum after sacral colpopexy presented with frozen pelvis. Int J Surg Case Rep 4(11):1004-1006. https://doi.org/10.1016/j.ijscr.2013.08.012

491. Voisard G, Feldman LS (2013) An unusual cause of chronic anemia and abdominal pain caused by transmural mesh migration in the small bowel after laparoscopic incisional hernia repair. Hernia 17(5):673-677. https://doi.org/10.1007/s10029013-1127-2

492. Yamamoto S, Kubota $\mathrm{T}$, Abe $\mathrm{T}$ (2015) A rare case of mechanical bowel obstruction caused by mesh plug migration. Hernia 19(6):983-985. https://doi.org/10.1007/s10029-0141247-3

493. Yilmaz I, Karakaş DO, Sucullu I, Ozdemir Y, Yucel E (2013) A rare cause of mechanical bowel obstruction: mesh migration. Hernia 17(2):267-269. https://doi.org/10.1007/s10029-0110867-0

494. You J, Onizuka N, Wong L (2014) Transgastric synthetic mesh migration, 9 years after liver resection. Case Rep Surg 2014:1-3. https://doi.org/10.1155/2014/412594

495. Stadlhuber RJ, El Sherif A, Mittal SK et al (2009) Mesh complications after prosthetic reinforcement of hiatal closure: a 28-case series. Surg Endosc Other Interv Tech 23(6):1219-1226. https://doi.org/10.1007/s00464-008-0205-5

496. Schlosser M, Wilhelm L, Urban G, Ziegler B, Ziegler M, Zippel $\mathrm{R}$ (2002) Immunogenicity of polymeric implants: long-term antibody response against polyester (Dacron) following the implantation of vascular prostheses into LEW.1A rats. J Biomed Mater Res 61(3):450-457. https://doi.org/10.1002/jbm.10096

497. Clave AA, Yahi H, Hammou JC, Montanari S, Gounon P, Clave $\mathrm{H}$ (2010) Polypropylene as a reinforcement in pelvic surgery is not inert: comparative analysis of 100 explants. Int Urogynecol J Pelvic Floor Dysfunct 21(3):261-270. https://doi.org/10.1007/ s00192-009-1021-8

498. Cozad MJ, Grant DA, Bachman SL, Grant DN, Ramshaw BJ, Grant SA (2010) Materials characterization of explanted polypropylene, polyethylene terephthalate, and expanded polytetrafluoroethylene composites: spectral and thermal analysis. J Biomed Mater Res Part B Appl Biomater 94(2):455-462. https://doi.org/10.1002/jbm.b.31675 
499. Riepe G, Heintz C, Kaiser E et al (2002) What can we learn from explanted endovascular devices? Eur J Vasc Endovasc Surg 24(2):117-122. https://doi.org/10.1053/ejversus2002.1677

500. Klink CD, Junge K, Binnebösel M et al (2011) Comparison of long-term biocompatibility of PVDF and PP meshes. J Investig Surg 24(6):292-299. https://doi.org/10.3109/08941939.2011. 589883

501. Mary C, Marois Y, King M et al (1998) Comparison of the in vivo behavior of polyvinylidene fluoride and polypropylene sutures used in vascular surgery. ASAIO J 44(3):199-206

502. Larena-Avellaneda Debus, Diener Dietz, Franke Thiede (2004) Species-dependent premature degradation of absorbable suture materials caused by infection-impact on the choice of thread in vascular surgery. Vasa 33:165-169

503. Bischoff F, Bryson G (1977) Intraperitoneal foreign body reaction in rodents. Res Commun Chem Pathol Pharmacol 18(2):201-214

504. Brand G, Brand I (1980) Investigations and review of literature relating to carcinogenesis. II. Communication: Cancer from foreign bodies (author's transl). Zentralbl Bakteriol Mikrobiol Hyg B 171(4-5):359-387

505. Brand G, Brand I (1980) Investigations and review of literature relating to carcinogenesis. I. Communication: Cancer from asbestos, schistosomiasis, and cicatrization (author's transl). Zentralbl Bakteriol Mikrobiol Hyg B 171(1):1-17

506. Ott G (1970) Fremdkorpersarkome (Foreign body induced sarcoma). Exp Med Pathol Klin 32:1-118

507. Paulini K, Beneke G, Korner B, Enders R (1975) The relationship between the latent period and animal age in the development of foreign body sarcomas. Beitr Pathol 154(2):161-169

508. Witherspoon P, Bryson G, Wright DM, Reid R, O'dwye P, Dwyer PJ (2004) Carcinogenic potential of commonly used hernia repair prostheses in an experimental model. Br J Surg 91(3):368-372. https://doi.org/10.1002/bjs.4462

509. Brown SBF, MacDuff E, O’Dwyer PJ (2013) Abdominal wall fibromatosis associated with previous laparoscopic hernia repair. Hernia 17(5):669-672. https://doi.org/10.1007/s10029-0131067-x

510. Birolini C, Minossi JG, Lima CF, Utiyama EM, Rasslan S (2014) Mesh cancer: long-term mesh infection leading to squamous-cell carcinoma of the abdominal wall. Hernia 18(6):897-901

511. No Authors Listed (1999) Surgical implants and other foreign bodies. IARC Monogr Eval Carcinog Risks Hum 74:1-409

512. Hawn MT, Snyder CW, Graham LA, Gray SH, Finan KR, Vick CC (2010) Long-term follow-up of technical outcomes for incisional hernia repair. J Am Coll Surg 210(5):648-655. https://doi.org/10.1016/j.jamcollsurg.2009.12.038

513. Nienhuijs SW, Rosman C (2015) Long-term outcome after randomizing prolene hernia system, mesh plug repair and Lichtenstein for inguinal hernia repair. Hernia 19(1):77-81

514. Flum DR, Horvath K, Koepsell T (2003) Have outcomes of incisional hernia repair improved with time? A population-based analysis. Ann Surg 237(1):129-135. https://doi.org/10.1097/ 00000658-200301000-00018

515. Amid PK (2003) The Lichtenstein repair in 2002: an overview of causes of recurrence after Lichtenstein tension-free hernioplasty. Hernia 7(1):13-16. https://doi.org/10.1007/s10029-0020088-7

516. Schoenmaeckers EJP, Van Der Valk SBA, Van Den Hout HW, Raymakers JFTJ, Rakic S (2009) Computed tomographic measurements of mesh shrinkage after laparoscopic ventral incisional hernia repair with an expanded polytetrafluoroethylene mesh. Surg Endosc Other Interv Tech 23(7):1620-1623. https://doi.org/10.1007/s00464-009-0500-9
517. Garcia-Urena M, Vega Ruiz V, Diaz Godoy A et al (2007) Differences in polypropylene shrinkage depending on mesh position in an experimental study. Am J Surg 193(4):538-542. https://doi.org/10.1016/j.amjsurg.2006.06.045

518. Silvestre AC, De Mathia GB, Fagundes DJ, Medeiros LR, Rosa MI (2011) Shrinkage evaluation of heavyweight and lightweight polypropylene meshes in inguinal hernia repair: a randomized controlled trial. Hernia 15(6):629-634. https://doi.org/10.1007/ s10029-011-0853-6

519. Khan RN, Jindal V, Bansal VK, Misra MC, Kumar S (2011) Does mesh shrinkage in any way depend upon the method of mesh fixation in laparoscopic incisional hernia repair? Surg Endosc Other Interv Tech 25(5):1690. https://doi.org/10.1007/ s00464-010-1363-9

520. Mamy L, Letouzey V, Lavigne JP et al (2011) Correlation between shrinkage and infection of implanted synthetic meshes using an animal model of mesh infection. Int Urogynecol $\mathrm{J}$ Pelvic Floor Dysfunct 22(1):47-52. https://doi.org/10.1007/ s00192-010-1245-7

521. Schug-Pass C, Sommerer F, Tannapfel A, Lippert H, Kockerling $F(2009)$ The use of composite meshes in laparoscopic repair of abdominal wall hernias: are there differences in biocompatibily?: Experimental results obtained in a laparoscopic porcine model. Surg Endosc Other Interv Tech 23(3):487-495. https://doi.org/10.1007/s00464-008-0085-8

522. Zinther NB, Wara P, Friis-Andersen H (2010) Shrinkage of intraperitoneal onlay mesh in sheep: coated polyester mesh versus covered polypropylene mesh. Hernia 14(6):611-615. https://doi.org/10.1007/s10029-010-0682-z

523. Langer C, Forster H, Konietschke F et al (2010) Mesh shrinkage in hernia surgery: data from a prospective randomized doubleblinded clinical study. Chirurg 81(8):735-742, 744-745. https://doi.org/10.1007/s00104-009-1853-2

524. Celik A, Altinli E, Koksal N et al (2009) The shrinking rates of different meshes placed intraperitoneally: a long-term comparison of the TiMesh, VYPRO II, Sepramesh, and DynaMesh. Surg Laparosc Endosc Percutan Tech 19(4):e130. https://doi. org/10.1097/SLE.0b013e3181aa598d

525. Zogbi L, Trindade EN, Trindade MRM (2013) Comparative study of shrinkage, inflammatory response and fibroplasia in heavyweight and lightweight meshes. Hernia 17(6):765-772. https://doi.org/10.1007/s10029-013-1046-2

526. Klein F, Ospina C, Rudolph B et al (2012) Formation of a chronic pain syndrome due to mesh shrinkage after laparoscopic intraperitoneal onlay mesh (IPOM). Surg Laparosc Endosc Percutan Tech 22(5):e288-e290. https://doi.org/10.1097/SLE. $0 \mathrm{~b} 013 \mathrm{e} 31825 \mathrm{efc} 3 \mathrm{c}$

527. Harrell A, Novitsky Y, Peindl R et al (2006) Prospective evaluation of adhesion formation and shrinkage of intra-abdominal prosthetics in a rabbit model. Am Surg 72(9):808-813

528. Jonas J (2009) The problem of mesh shrinkage in laparoscopic incisional hernia repair. Zentralbl Chir 134(3):209-213. https://doi.org/10.1055/s-0028-1098779

529. Beldi G, Wagner M, Bruegger LE, Kurmann A, Candinas D (2011) Mesh shrinkage and pain in laparoscopic ventral hernia repair: a randomized clinical trial comparing suture versus tack mesh fixation. Surg Endosc Other Interv Tech 25(3):749-755. https://doi.org/10.1007/s00464-010-1246-0

530. Ciritsis A, Hansen NL, Barabasch A et al (2014) Time-dependent changes of magnetic resonance imaging-visible mesh implants in patients. Invest Radiol 49(7):439-444. https://doi. org/10.1097/RLI.0000000000000051

531. Clarke T, Katkhouda N, Mason RJ et al (2011) Fibrin glue for intraperitoneal laparoscopic mesh fixation: a comparative study in a swine model. Surg Endosc Other Interv Tech 25(3):737-748. https://doi.org/10.1007/s00464-010-1244-2 
532. Hollinsky C, Kolbe T, Walter I et al (2010) Tensile strength and adhesion formation of mesh fixation systems used in laparoscopic incisional hernia repair. Surg Endosc Other Interv Tech 24(6):1318-1324. https://doi.org/10.1007/s00464-009-0767-x

533. Joels CS, Matthews BD, Kercher KW et al (2005) Evaluation of adhesion formation, mesh fixation strength, and hydroxyproline content after intraabdominal placement of polytetrafluoroethylene mesh secured using titanium spiral tacks, nitinol anchors, and polypropylene suture or polyglactin 910 suture. Surg Endosc Other Interv Tech 19(6):780-785. https://doi.org/ 10.1007/s00464-004-8927-5

534. Schwab R, Schumacher O, Junge K et al (2008) Biomechanical analyses of mesh fixation in TAPP and TEP hernia repair. Surg Endosc Other Interv Tech 22(3):731-738. https://doi.org/10. 1007/s00464-007-9476-5

535. Schwab R, Schumacher O, Junge K, Binnebosel M, Klinge U, Schumpelick V (2007) Fibrin sealant for mesh fixation in Lichtenstein repair: biomechanical analysis of different techniques. Hernia 11(2):139-145. https://doi.org/10.1007/s10029007-0195-6

536. Amid PK (2014) Radiologic images of meshoma. A new phenomenon causing chronic pain after prosthetic repair of abdominal wall hernias. Arch Surg 139:1297-1298

537. LeBlanc K (2003) Tack hernia: a new entity. J Soc Laparoendosc Surg 7(4):383-387

538. Stark E, Oestreich K, Wendl K, Rumstadt B, Hagmüller E (1999) Nerve irritation after laparoscopic hernia repair. Surg Endosc 13(9):878-881. https://doi.org/10.1007/s004649901124

539. Beattie G, Kumar S, Nixon S (2000) Laparoscopic total extraperitoneal hernia repair: mesh fixation is unnecessary. J Laparoendosc Adv Surg Tech 10(2):71-73. https://doi.org/10. 1089/lap.2000.10.71

540. Kumar S, Wilson RG, Nixon SJ, Macintyre IMC (2002) Chronic pain after laparoscopic and open mesh repair of groin hernia. $\mathrm{Br}$ J Surg 89(11):1476-1479. https://doi.org/10.1046/j.1365-2168. 2002.02260.x

541. Poobalan AS, Bruce J, Smith WC, King PM, Krukowski ZH, Chambers WA (2003) A review of chronic pain after inguinal herniorrhaphy. Clin J Pain 19:48-54

542. LeBlanc KA (2004) Laparoscopic incisional and ventral hernia repair: complications-how to avoid and handle. Hernia 8(4):323-331. https://doi.org/10.1007/s10029-004-0250-5

543. Wassenaar EB, Raymakers JTFJ, Rakic S (2007) Removal of transabdominal sutures for chronic pain after laparoscopic ventral and incisional hernia repair. Surg Laparosc Endosc Percutan Tech 17(6):514-516. https://doi.org/10.1097/SLE. 0b013e3181462b9e

544. Masini BD, Stinner DJ, Waterman SM, Wenke JC (2011) Bacterial adherence to suture materials. J Surg Educ 68(2):101-104. https://doi.org/10.1016/j.jsurg.2010.09.015

545. Edmiston CE, Seabrook GR, Goheen MP et al (2006) Bacterial adherence to surgical sutures: can antibacterial-coated sutures reduce the risk of microbial contamination? J Am Coll Surg 203(4):481-489. https://doi.org/10.1016/j.jamcollsurg.2006.06. 026

546. de Goede B, Klitsie PJ, van Kempen BJH et al (2013) Metaanalysis of glue versus sutured mesh fixation for Lichtenstein inguinal hernia repair. Br J Surg 100(6):735-742. https://doi.org/ $10.1002 /$ bjs. 9072

547. Ladwa N, Sajid MS, Sains P, Baig MK (2013) Suture mesh fixation versus glue mesh fixation in open inguinal hernia repair: a systematic review and meta-analysis. Int J Surg 11:128-135. https://doi.org/10.1016/j.ijsu.2012.12.013

548. Colvin HS, Rao A, Cavali M, Campanelli G, Amin AI (2013) Glue versus suture fixation of mesh during open repair of inguinal hernias: a systematic review and meta-analysis. World $\mathbf{J}$
Surg 37(10):2282-2292. https://doi.org/10.1007/s00268-0132140-4

549. Fortelny RH, Petter-Puchner AH, Glaser KS, Redl H (2012) Use of fibrin sealant (Tisseel/Tissucol) in hernia repair: a systematic review. Surg Endosc 26:1803-1812. https://doi.org/10.1007/ s00464-012-2156-0

550. Sajid MS, Ladwa N, Kalra L, McFall M, Baig MK, Sains P (2013) A meta-analysis examining the use of tacker mesh fixation versus glue mesh fixation in laparoscopic inguinal hernia repair. Am J Surg 206:103-111. https://doi.org/10.1016/j.amj surg.2012.09.003

551. Sanders DL, Waydia S (2014) A systematic review of randomised control trials assessing mesh fixation in open inguinal hernia repair. Hernia 18:165-176. https://doi.org/10.1007/ s10029-013-1093-8

552. Kim-Fuchs C, Angst E, Vorburger S, Helbling C, Candinas D, Schlumpf R (2012) Prospective randomized trial comparing sutured with sutureless mesh fixation for Lichtenstein hernia repair: long-term results. Hernia 16:21-27. https://doi.org/10. 1007/s10029-011-0856-3

553. Douglas J, Young W, Jones D (2002) Lichtenstein inguinal herniorrhaphy using sutures versus tacks. Hernia 6:99-101. https://doi.org/10.1007/s10029-002-0052-6

554. Hidalgo M, Castillo MJ, Eymar JL, Hidalgo A (2005) Lichtenstein inguinal hernioplasty: sutures versus glue. Hernia 9(3):242-244. https://doi.org/10.1007/s10029-005-0334-x

555. Nowobilski W, Dobosz M, Wojciechowicz T, Mionskowska L (2004) Lichtenstein inguinal hernioplasty using butyl-2cyanoacrylate versus sutures: preliminary experience of a prospective randomized trial. Eur Surg Res 36:367-370. https://doi.org/10.1159/000081646

556. Shen YM, Sun WB, Chen J, Liu SJ, Wang MG (2012) NBCA medical adhesive ( $n$-butyl-2-cyanoacrylate) versus suture for patch fixation in Lichtenstein inguinal herniorrhaphy: a randomized controlled trial. Surgery 151:550-555. https://doi.org/ 10.1016/j.surg.2011.09.031

557. Wong JU, Leung TH, Huang CC, Huang CS (2011) Comparing chronic pain between fibrin sealant and suture fixation for bilayer polypropylene mesh inguinal hernioplasty: a randomized clinical trial. Am J Surg 202:34-38. https://doi.org/10.1016/j. amjsurg.2010.05.004

558. Paajanen H, Kossi J, Silvasti S, Hulmi T, Hakala T (2011) Randomized clinical trial of tissue glue versus absorbable sutures for mesh fixation in local anaesthetic Lichtenstein hernia repair. Br J Surg 98:1245-1251. https://doi.org/10.1002/bjs. 7598

559. Paajanen H (2002) Do absorbable mesh sutures cause less chronic pain than nonabsorbable sutures after Lichtenstein inguinal herniorraphy? Hernia 6:26-28. https://doi.org/10.1007/ s10029-002-0048-2

560. Campanelli G, Pascual MH, Hoeferlin A et al (2012) Randomized, controlled, blinded trial of Tisseel/Tissucol for mesh fixation in patients undergoing Lichtenstein technique for primary inguinal hernia repair: results of the TIMELI trial. Ann Surg 255:650-657. https://doi.org/10.1097/SLA.0b013e31824b32bf

561. Bracale U, Rovani M, Picardo A et al (2014) Beneficial effects of fibrin glue (Quixil) versus Lichtenstein conventional technique in inguinal hernia repair: a randomized clinical trial. Hernia 18:185-192. https://doi.org/10.1007/s10029-012-1020-4

562. Schafer M, Vuilleumier H, Di Mare L, Demartines N (2010) Fibrin sealant for mesh fixation in endoscopic inguinal hernia repair: is there enough evidence for its routine use? Surg Laparosc Endosc Percutan Tech 20:205-212. https://doi.org/10. 1097/SLE.0b013e3181ed85b3

563. Morales-Conde S, Barranco A, Socas M, Alarcon I, Grau M, Casado MA (2011) Systematic review of the use of fibrin sealant 
in abdominal-wall repair surgery. Hernia 15:361-369. https://doi.org/10.1007/s10029-011-0809-x

564. Kaul A, Hutfless S, Le H et al (2012) Staple versus fibrin glue fixation in laparoscopic total extraperitoneal repair of inguinal hernia: a systematic review and meta-analysis. Surg Endosc Other Interv Tech 26:1269-1278. https://doi.org/10.1007/ s00464-011-2025-2

565. Sajid MS, Ladwa N, Kalra L, Hutson K, Sains P, Baig MK (2012) A meta-analysis examining the use of tacker fixation versus no-fixation of mesh in laparoscopic inguinal hernia repair. Int J Surg 10(5):224-231. https://doi.org/10.1016/j.ijsu. 2012.03.001

566. Teng YJ, Pan SM, Liu YL et al (2011) A meta-analysis of randomized controlled trials of fixation versus nonfixation of mesh in laparoscopic total extraperitoneal inguinal hernia repair. Surg Endosc Other Interv Tech 25:2849-2858. https://doi.org/ 10.1007/s00464-011-1668-3

567. Tam KW, Liang HH, Chai CY (2010) Outcomes of staple fixation of mesh versus nonfixation in laparoscopic total extraperitoneal inguinal repair: a meta-analysis of randomized controlled trials. World J Surg 34:3065-3074

568. Melissa CS, Bun TAY, Wing CK, Chung TY, Wai NEK, Tat LH (2014) Randomized double-blinded prospective trial of fibrin sealant spray versus mechanical stapling in laparoscopic total extraperitoneal hernioplasty. Ann Surg 259:432-437. https://doi. org/10.1097/SLA.0b013e3182a6c513

569. Tolver MA, Rosenberg J, Juul P, Bisgaard T (2013) Randomized clinical trial of fibrin glue versus tacked fixation in laparoscopic groin hernia repair. Surg Endosc Other Interv Tech 27:2727-2733. https://doi.org/10.1007/s00464-012-2766-6

570. Cambal M, Zonca P, Hrbaty B (2012) Comparison of selfgripping mesh with mesh fixation with fibrin-glue in laparoscopic hernia repair (TAPP). Bratisl Lek Listy 113:103-107

571. Brugger L, Bloesch M, Ipaktchi R, Kurmann A, Candinas D, Beldi G (2012) Objective hypoesthesia and pain after transabdominal preperitoneal hernioplasty: a prospective, randomized study comparing tissue adhesive versus spiral tacks. Surg Endosc Other Interv Tech 26:1079-1085. https://doi.org/10. 1007/s00464-011-2003-8

572. Subwongcharoen S, Ruksakul K (2013) A randomized controlled trial of staple fixation versus $\mathrm{N}$-butyl-2-cyanoacrylate fixation in laparoscopic inguinal hernia repair. J Med Assoc Thail 96:8-13

573. Garg P, Nair S, Shereef M et al (2011) Mesh fixation compared to nonfixation in total extraperitoneal inguinal hernia repair: a randomized controlled trial in a rural center in India. Surg Endosc 25(10):3300-3306. https://doi.org/10.1007/s00464-011$1708-\mathrm{z}$

574. Fortelny RH, Petter-Puchner AH, May C et al (2012) The impact of atraumatic fibrin sealant vs. staple mesh fixation in TAPP hernia repair on chronic pain and quality of life: results of a randomized controlled study. Surg Endosc Other Interv Tech 26:249-254. https://doi.org/10.1007/s00464-011-1862-3

575. Taylor C, Layani L, Liew V, Ghusn M, Crampton N, White S (2008) Laparoscopic inguinal hernia repair without mesh fixation, early results of a large randomised clinical trial. Surg Endosc 22(3):757-762. https://doi.org/10.1007/s00464-0079510-7

576. Boldo E, Armelles A, Perez de Lucia G et al (2008) Pain after laparoscopic bilateral hernioplasty: early results of a prospective randomized double-blind study comparing fibrin versus staples. [Erratum appears in Surg Endosc. 2008 May; 22(5):1210. Note: Armelles, Andres [added]; Perez de Lucia, Guillermo]. Surg Endosc 22:1206-1209

577. Olmi S, Scaini A, Erba L, Guaglio M, Croce E (2007) Quantification of pain in laparoscopic transabdominal preperitoneal
(TAPP) inguinal hernioplasty identifies marked differences between prosthesis fixation systems. Surgery 142:40-46. https://doi.org/10.1016/j.surg.2007.02.013

578. Lovisetto F, Zonta S, Rota E et al (2007) Use of human fibrin glue (Tissucol) versus staples for mesh fixation in laparoscopic transabdominal preperitoneal hernioplasty: a prospective, randomized study. Ann Surg 245:222-231. https://doi.org/10.1097/ 01.sla.0000245832.59478.c6

579. Koch CA, Greenlee SM, Larson DR, Harrington JR, Farley DR (2006) Randomized prospective study of totally extraperitoneal inguinal hernia repair: fixation versus no fixation of mesh. JSLS $10: 457-460$

580. Lau H (2005) Fibrin sealant versus mechanical stapling for mesh fixation during endoscopic extraperitoneal inguinal hernioplasty: a randomized prospective trial. Ann Surg 242:670-675. https://doi.org/10.1097/01.sla.0000186440.02977.de

581. Moreno-Egea A, Torralba Martinez JA, Morales Cuenca G, Aguayo Albasini JL (2004) Randomized clinical trial of fixation vs nonfixation of mesh in total extraperitoneal inguinal hernioplasty. Arch Surg 139:1376-1379. https://doi.org/10.1001/arch surg.139.12.1376

582. Smith AI, Royston CMS, Sedman PC (1999) Stapled and nonstapled laparoscopic transabdominal preperitoneal (TAPP) inguinal hernia repair: a prospective randomized trial. Surg Endosc 13:804-806. https://doi.org/10.1007/s004649901104

583. Ferzli GS, Frezza EE, Pecoraro AM, Ahern KD (1999) Prospective randomized study of stapled versus unstapled mesh in a laparoscopic preperitoneal inguinal hernia repair. J Am Coll Surg 188(5):461-465

584. Parshad R, Kumar R, Hazrah P, Bal S (2005) A randomized comparison of the early outcome of stapled and unstapled techniques of laparoscopic total extraperitoneal inguinal hernia repair. J Soc Laparoendosc Surg 9(4):403-407

585. Horisberger K, Jung MK, Zingg U, Schöb O (2013) Influence of type of mesh fixation in endoscopic totally extraperitoneal hernia repair (TEP) on long-term quality of life. World J Surg 37(6):1249-1257. https://doi.org/10.1007/s00268-013-1974-0

586. Wang MG, Tian ML, Zhao XF, Nie YS, Chen J, Shen YM (2013) Effectiveness and safety of $n$-butyl-2-cyanoacrylate medical adhesive for noninvasive patch fixation in laparoscopic inguinal hernia repair. Surg Endosc Other Interv Tech 27(10):3792-3798. https://doi.org/10.1007/s00464-013-2970-z

587. Fumagalli Romario U, Puccetti F, Elmore U, Massaron S, Rosati R (2013) Self-gripping mesh versus staple fixation in laparoscopic inguinal hernia repair: a prospective comparison. Surg Endosc Other Interv Tech 27:1798-1802. https://doi.org/10. 1007/s00464-012-2683-8

588. Bittner R, Gmahle E, Gmahle B, Schwarz J, Aasvang E, Kehlet $\mathrm{H}$ (2010) Lightweight mesh and noninvasive fixation: an effective concept for prevention of chronic pain with laparoscopic hernia repair (TAPP). Surg Endosc Other Interv Tech 24:2958-2964. https://doi.org/10.1007/s00464-010-1140-9

589. Ceccarelli G, Casciola L, Pisanelli MC et al (2008) Comparing fibrin sealant with staples for mesh fixation in laparoscopic transabdominal hernia repair: a case control-study. Surg Endosc Other Interv Tech 22(3):668-673. https://doi.org/10.1007/ s00464-007-9458-7

590. Santoro E, Agresta F, Buscaglia F et al (2007) Preliminary experience using fibrin glue for mesh fixation in 250 patients undergoing minilaparoscopic transabdominal preperitoneal hernia repair. J Laparoendosc Adv Surg Tech A 17(1):12-15. https://doi.org/10.1089/lap.2006.0107

591. Schwab R, Willms A, Kröger A, Becker HP (2006) Less chronic pain following mesh fixation using a fibrin sealant in TEP inguinal hernia repair. Hernia 10(3):272-277. https://doi.org/10. 1007/s10029-006-0080-8 
592. Topart P, Vandenbroucke F, Lozac'h P (2005) Tisseel vs tack staples as mesh fixation in totally extraperitoneal laparoscopic repair of groin hernias: a retrospective analysis. Surg Endosc Other Interv Tech 19:724-727. https://doi.org/10.1007/s00464004-8812-2

593. Lau H, Patil NG (2003) Selective non-stapling of mesh during unilateral endoscopic total extraperitoneal inguinal hernioplasty: a case-control study. Arch Surg 138(12):1352-1355. https://doi. org/10.1001/archsurg.138.12.1352

594. Khajanchee YS, Urbach DR, Swanstrom LL, Hansen PD (2001) Outcomes of laparoscopic herniorrhaphy without fixation of mesh to the abdominal wall. Surg Endosc 15(10):1102-1107. https://doi.org/10.1007/s004640080088

595. Mayer F, Niebuhr H, Lechner M et al (2016) When is mesh fixation in TAPP-repair of primary inguinal hernia repair necessary? The register-based analysis of 11,230 cases. Surg Endosc 30(10):4363-4371. https://doi.org/10.1007/s00464-0164754-8

596. Garg P, Rajagopal M, Varghese V, Ismail M (2009) Laparoscopic total extraperitoneal inguinal hernia repair with nonfixation of the mesh for 1,692 hernias. Surg Endosc 23(6):1241-1245. https://doi.org/10.1007/s00464-008-0137-0

597. Gutlic N, Rogmark P, Nordin P, Petersson U, Montgomery A (2015) Impact of mesh fixation on chronic pain in total extraperitoneal inguinal hernia repair (TEP): a nationwide register-based study. Ann Surg 263(6):1199-1206. https://doi.org/ 10.1097/SLA.0000000000001306

598. Novik B, Hagedorn S, Mörk UB, Dahlin K, Skullman S, Dalenbäck J (2006) Fibrin glue for securing the mesh in laparoscopic totally extraperitoneal inguinal hernia repair: a study with a 40-month prospective follow-up period. Surg Endosc Other Interv Tech 20(3):462-467. https://doi.org/10. 1007/s00464-005-0391-3

599. Fenger AQ, Helvind NM, Pommergaard H-C, Burcharth J, Rosenberg J (2015) Fibrin sealant for mesh fixation in laparoscopic groin hernia repair does not increase long-term recurrence. Surg Endosc 30(3):986-992. https://doi.org/10.1007/ s00464-015-4280-0

600. Sanchez-Manuel FJ, Lozano-García J, Seco-Gil JL (2012) Antibiotic prophylaxis for hernia repair. Cochrane Database Syst Rev 15(2):CD003769. https://doi.org/10.1002/14651858. CD003769.pub3

601. Aufenacker TJ (2013) The danger of performing meta-analysis and the impact of guidelines. Hernia 17(5):665-668. https://doi. org/10.1007/s10029-013-1141-4

602. Mazaki T, Mado K, Masuda H, Shiono M (2013) Antibiotic prophylaxis for the prevention of surgical site infection after tension-free hernia repair: a Bayesian and frequentist metaanalysis. J Am Coll Surg 217:784-788. https://doi.org/10.1016/ j.jamcollsurg.2013.07.386

603. Celdran A, Frieyro O, de la Pinta JC et al (2004) The role of antibiotic prophylaxis on wound infection after mesh hernia repair under local anesthesia on an ambulatory basis. Hernia 8:20-22. https://doi.org/10.1007/s10029-003-0164-7

604. Tzovaras G, Delikoukos S, Christodoulides G et al (2007) The role of antibiotic prophylaxis in elective tension-free mesh inguinal hernia repair: results of a single-centre prospective randomised trial. Int J Clin Pract 61(2):236-239. https://doi.org/ 10.1111/j.1742-1241.2006.00977.x

605. Swedish registry. http://www.svensktbrackregister.se/rapporter71/klinikrapporter/2014. Published 2014

606. Köckerling F, Bittner R, Jacob D et al (2015) Do we need antibiotic prophylaxis in endoscopic inguinal hernia repair? Results of the Herniamed Registry. Surg Endosc Other Interv Tech 29(12):3741-3749. https://doi.org/10.1007/s00464-0154149-2
607. Schwetling R, Barlehner E (1998) Is there an indication for general perioperative antibiotic prophylaxis in laparoscopic hernioplasty with implantation of alloplastic meshes?. [German] TT-Besteht eine indikation zur generellen perioperativen antibiotikaprophylaxe bei laparoskopischer. Zentralbl Chir 123:193-195

608. Mangram AJ, Horan TC, Pearson ML, Silver LC, Jarvis WR (1999) Guideline for prevention of surgical site infection, 1999. Am J Infect Control 27(2):97-134. https://doi.org/10.1016/ S0196-6553(99)70088-X

609. Cheek CM, Black NA, Devlin HB, Kingsnorth AN, Taylor RS, Watkin DF (1998) Groin hernia surgery: a systematic review. Ann R Coll Surg Engl 80(1):S1-S80

610. Reece-Smith AM, Maggio AQ, Tang TY, Walsh SR (2009) Local anaesthetic vs. general anaesthetic for inguinal hernia repair: systematic review and meta-analysis. Int $\mathbf{J}$ Clin Pract 63(12):1739-1742. https://doi.org/10.1111/j.1742-1241.2009. 02131.x

611. Jensen P, Mikkelsen T, Kehlet H (2002) Postherniorrhaphy urinary retention-effect of local, regional, and general anesthesia: a review. Reg Anesth Pain Med 27(6):612-617. https://doi.org/10.1053/rapm.2002.37122

612. Kehlet H, Aasvang E (2005) Groin hernia repair: anesthesia. World J Surg 29(8):1058-1061. https://doi.org/10.1007/s00268005-7969-8

613. Callesen T (2003) Inguinal hernia repair: anaesthesia, pain and convalescence. Dan Med Bull 50(3):203-218

614. Joshi GP, Rawal N, Kehlet H et al (2012) Evidence-based management of postoperative pain in adults undergoing open inguinal hernia surgery. Br J Surg 99(2):168-185. https://doi. org/10.1002/bjs.7660

615. Nordin P, Hernell H, Unosson M, Gunnarsson U, Nilsson E (2004) Type of anaesthesia and patient acceptance in groin hernia repair: a multicentre randomised trial. Hernia 8(3):220-225. https://doi.org/10.1007/s10029-004-0234-5

616. O'Dwyer PJ, Serpell MG, Millar K et al (2003) Local or general anesthesia for open hernia repair: a randomized trial. Ann Surg 237(4):574-579. https://doi.org/10.1097/01.SLA.0000059992. 76731.64

617. Gönüllü N, Çubukçu A, Alponat A (2002) Comparison of local and general anesthesia in tension-free (Lichtenstein) hernioplasty: a prospective randomized trial. Hernia 6(1):29-32. https://doi.org/10.1007/s10029-002-0040-x

618. Song D, Greilich NB, White PF, Watcha MF, Tongier WK (2000) Recovery profiles and costs of anesthesia for outpatient unilateral inguinal herniorrhaphy. Anesth Analg 91(4):876-881

619. Teasdale C, McCrum AM, Williams NB, Horton RE (1982) A randomised controlled trial to compare local with general anaesthesia for short-stay inguinal hernia repair. Ann R Coll Surg Engl 64(4):238-242

620. Ozgun H (2002) Comparison of local, spinal, and general anesthesia for inguinal herniorrhaphy. Eur J Surg 168:455-459. https://doi.org/10.1097/00132586-198804000-00042

621. Friemert B, Faoual J, Becker HP, Lampl L (2000) Eine prospektiv randomisierte Studie zur Leistenhernienreparation nach Shouldice Vorteile für die Lokalanaesthesie. Chirurg 71(1):52-57

622. Schmitz R, Shah S, Treckmann J, Schneider K (1997) Extraperitoneal, "tension free" inguinal hernia repair with local anesthesia-a contribution to effectiveness and economy. In: [German] TT-Der extraperitoneale, "Tension Free"-Leistenhernien Repair in Lokalanasthesie-Ein Beitrag fur Effizienz und Okonomie. Langenbecks Archiv fur Chirurgie. Supplement, pp 1135-1138

623. Milone F, Salvatore G, Leongito M, Milone M (2010) [Hernia repair and local anesthesia. Results of a controlled randomized 
clinical trial]. [Italian] TT-Ernioplastica inguinale e anestesia locale. Risultati di uno studio randomizzato e controllato. G Chir 31:552-555

624. Godfrey PJ, Greenan J, Ranasinghe DD (1981) Ventilatory capacity after three methods of anaesthesia for inguinal hernia repair: a randomized controlled trial. Br J Surg 68:587-589

625. Knapp RW, Mullen JT (1976) Clinical evaluation of the use of local anesthesia for repair of inguinal hernia. Am Surg 42:908-910

626. Peiper C, Töns C, Schippers E, Busch F, Schumpelick VCN-C00110104 (1994) Local versus general anesthesia for Shouldice repair of the inguinal hernia. World J Surg 18:912-916

627. Rau HM, Arlt G, Peiper C, Schumpelick V (1998) Surgery of inguinal hernia with local anesthesia-technique and results of a minimal invasive procedure. In: [German] TT-Leistenhernienchirurgie in Lokalanaesthesie-Technik und Ergebnisse eines "minimal invasiven" Verfahrens. Langenbecks Archiv fur Chirurgie. Supplement, pp 1024-1026

628. Aasbo V, Thuen A, Raeder J (2002) Improved long-lasting postoperative analgesia, recovery function and patient satisfaction after inguinal hernia repair with inguinal field block compared with general anesthesia. Acta Anaesthesiol Scand 46:674-678. https://doi.org/10.1034/j.1399-6576.2002.460607.x

629. Nordin P, Zetterstrom H, Gunnarsson U, Nilsson E (2003) Local, regional, or general anaesthesia in groin hernia repair: multicentre randomised trial. Lancet 362:853-858. https://doi. org/10.1016/S0140-6736\%2803\%2914339-5

630. Behnia R, Hashemi F, Stryker SJ, Ujiki GT, Poticha SM (1992) A comparison of general versus local anesthesia during inguinal herniorrhaphy. Surg Gynecol Obstet 174(4):277-280

631. Srivastava U, Kumar A, Saxena S, Neeraj C, Sehgal DR (2007) Comparison of local, spinal and general anaesthesia for inguinal hernia repair. J Anaesthesiol Clin Pharmacol 23:151-154

632. Nordin P, Zetterström H, Carlsson P et al (2007) Cost-effectiveness analysis of local, regional and general anaesthesia for inguinal hernia repair using data from a randomized clinical trial. Br J Surg 94(4):500-505. https://doi.org/10.1002/bjs.5543

633. Gultekin FA, Kuruahvecioglu O, Karamercan A, Ege B, Ersoy E, Tatlicioglu E (2007) A prospective comparison of local and spinal anesthesia for inguinal hernia repair. Hernia 11:153-156. https://doi.org/10.1007/s10029-006-0166-3

634. van Veen RN, Mahabier C, Dawson I et al (2008) Spinal or local anesthesia in Lichtenstein hernia repair: a randomized controlled trial. Ann Surg 247(3):428-433. https://doi.org/10.1097/SLA. 0b013e318165b0ff

635. Merhav H, Rothstein H, Eliraz A, Hana R, Pfeffermann R (1993) A comparison of pulmonary functions and oxygenation following local, spinal or general anaesthesia in patients undergoing inguinal hernia repair. Int Surg 78:257-261

636. Yang B, Liang MJ, Zhang YC (2008) Use of local or epidural anesthesia in inguinal hernia repair: a randomized trial [Chinese]. Zhonghua Wai Ke Za Zhi 46:1234-1236

637. Yilmazlar A, Bilgel H, Donmez C, Guney A, Yilmazlar T, Tokat O (2006) Comparison of ilioinguinal-iliohypogastric nerve block versus spinal anesthesia for inguinal herniorrhaphy. South Med J 99:48-51. https://doi.org/10.1097/01.smj.0000197298. 48311.80

638. Shafique N, Ur Rashid H, Ijaz Raja M, Saeed M (2015) Comparison of efficacy of spinal anaesthesia and subfascial local anaesthetic inguinal field block for open inguinal hernia repaira single institutional experience. J Ayub Med Coll Abbottabad 27(1):197-200

639. Bakota B, Kopljar M, Baranovic S, Miletic M, Marinovic M, Vidovic D (2015) Should we abandon regional anesthesia in open inguinal hernia repair in adults? Eur J Med Res 20(1):76. https://doi.org/10.1186/s40001-015-0170-0
640. Urbach K, Lee W, Sheely L, Lang F, Sharp R (1964) Spinal or general anesthesia for inguinal hernia repair? A comparison of certain complications in a controlled series. JAMA 190:25-29

641. Burney RE, Prabhu MA, Greenfield MLVH, Shanks A, O'Reilly M (2004) Comparison of spinal vs general anesthesia via laryngeal mask airway in inguinal hernia repair. Arch Surg 139:183-187. https://doi.org/10.1001/archsurg.139.2.183

642. Tverskoy M, Cozacov C, Ayache M, Bradley EL Jr, Kissin I (1990) Postoperative pain after inguinal herniorrhaphy with different types of anesthesia. Anesth Analg 70:29-35

643. Bay-Nielsen M, Kehlet H (2008) Anaesthesia and post-operative morbidity after elective groin hernia repair: a nation-wide study. Acta Anaesthesiol Scand 52(2):169-174. https://doi.org/10. 1111/j.1399-6576.2007.01514.x

644. Trombetta F, Moscato R, Mussa B et al (2003) Day surgery. An opportunity for postgraduate surgeons' training. Minerva Chir 58(2): $143-147$

645. Paajanen H (2003) Groin hernia repair under local anaesthesia: effect of surgeon's training level on long-term results. Ambul Surg. https://doi.org/10.1016/j.ambsur.2003.06.001

646. Sanjay P, Woodward A (2009) Local anaesthetic inguinal hernia repair performed under supervision: early and long-term outcomes. Ann R Coll Surg Engl 91(8):677-680. https://doi.org/10. 1308/003588409X12486167521073

647. Devlin B, Peter A (1994) Recurrence rates following local anaesthetic day case inguinal hernia repair by junior surgeons in a district general hospital. Ann R Coll Surg Engl 12:7

648. Kingsnorth AN, Britton BJ, Morris PJ (1981) Recurrent inguinal hernia after local anaesthetic repair. Br J Surg 68(4):273-275

649. Andersen FH, Nielsen K, Kehlet H (2005) Combined ilioinguinal blockade and local infiltration anaesthesia for groin hernia repair-a double-blind randomized study. $\mathrm{Br} \mathrm{J}$ Anaesth 94:520-523. https://doi.org/10.1093/bja/aei083

650. Ding Y, White PF (1995) Post-herniorrhaphy pain in outpatients after pre-incision ilioinguinal-hypogastric nerve block during monitored anaesthesia care. Can J Anaesth 42:12-15

651. Toivonen J, Permi J, Rosenberg PH (2001) Effect of preincisional ilioinguinal and iliohypogastric nerve block on postoperative analgesic requirement in day-surgery patients undergoing herniorrhaphy under spinal anaesthesia. Acta Anaesthesiol Scand 45:603-607. https://doi.org/10.1034/j.13996576.2001.045005603.x

652. Bugedo GJ, Carcamo CR, Mertens RA, Dagnino JA, Munoz HRCN-C-00296066 (1990) Preoperative percutaneous ilioinguinal and iliohypogastric nerve block with $0.5 \%$ bupivacaine for post-herniorrhaphy pain management in adults. Reg Anesth 15:130-133

653. O'Hanlon JJ, McCleane G, Muldoon T (1996) Preoperative application of piroxicam gel compared to a local anaesthetic field block for postoperative analgesia. Acta Anaesthesiol Scand 40:715-718

654. Petersen PL, Mathiesen O, Stjernholm P et al (2013) The effect of transversus abdominis plane block or local anaesthetic infiltration in inguinal hernia repair: a randomised clinical trial. Eur J Anaesthesiol 30:415-421. https://doi.org/10.1097/EJA. 0b013e32835fc86f

655. Santos GC, Braga GM, Queiroz FL, Navarro TP, Gomez RS (2011) Assessment of postoperative pain and hospital discharge after inguinal and iliohypogastric nerve block for inguinal hernia repair under spinal anesthesia: a prospective study. Rev Assoc Med Bras 57:545-549

656. Harrison CA, Morris S, Harvey JS (1994) Effect of ilioinguinal and iliohypogastric nerve block and wound infiltration with $0.5 \%$ bupivacaine on postoperative pain after hernia repair. $\mathrm{Br} \mathrm{J}$ Anaesth 72:691-693 
657. Nehra D, Gemmell L, Pye JK (1995) Pain relief after inguinal hernia repair: a randomized double-blind study. Br J Surg 82:1245-1247. https://doi.org/10.1002/bjs.1800820929

658. Johansson B, Hallerback B, Stubberod A et al (1997) Preoperative local infiltration with ropivacaine for postoperative pain relief after inguinal hernia repair: a randomised controlled trial. Eur J Surg 163:371-378

659. Fischer S, Troidl H, MacLean AA, Koehler L, Paul A (2000) Prospective double-blind randomised study of a new regimen of pre- emptive analgesia for inguinal hernia repair: evaluation of postoperative pain course. Eur J Surg 166:545-551

660. Narchi P, Carry PY, Catoire P et al (1998) Postoperative pain relief and recovery with ropivacaine infiltration after inguinal hernia repair. Ambul Surg 6:221-226. https://doi.org/10.1016/ S0966-6532\%2898\%2900029-8

661. Mulroy MF, Burgess FW (1999) Emanuelsson BMCN-C00307997. Ropivacaine $0.25 \%$ and $0.5 \%$, but not $0.125 \%$, provide effective wound infiltration analgesia after outpatient hernia repair, but with sustained plasma drug levels. Reg Anesth Pain Med 24:136-141

662. Dierking GW, Ostergaard E, Ostergard HT, Dahl JB (1994) The effects of wound infiltration with bupivacaine versus saline on postoperative pain and opioid requirements after herniorrhaphy. Acta Anaesthesiol Scand 38:289-292

663. Ausems ME, Hulsewe KW, Hooymans PM, Hoofwijk AG (2007) Postoperative analgesia requirements at home after inguinal hernia repair: effects of wound infiltration on postoperative pain. Anaesthesia 62:325-331. https://doi.org/10.1111/j. 1365-2044.2007.04991.x

664. Kurmann A, Fischer H, Dell-Kuster S et al (2015) Effect of intraoperative infiltration with local anesthesia on the development of chronic pain after inguinal hernia repair: a randomized, triple-blinded, placebo-controlled trial. Surgery 157:144-154. https://doi.org/10.1016/j.surg.2014.07.008

665. Yndgaard S, Holst P, Bjerre-Jepsen K, Thomsen CB, Struckmann J, Mogensen T (1994) Subcutaneously versus subfascially administered lidocaine in pain treatment after inguinal herniotomy. Anesth Analg 79:324-327

666. El-Radaideh KM, Al-Ghazo MA, Bani-Hani KE (2006) Combined subfascial and subcutaneous bupivacaine instillation for inguinal hernia wounds. Asian J Surg 29:242-246

667. Bays RA, Barry L, Vasilenko P (1991) The use of bupivacaine in elective inguinal herniorrhaphy as a fast and safe technique for relief of postoperative pain. Surg Gynecol Obstet 173:433-437

668. Sinclair R, Cassuto J, Hogstrom S et al (1988) Topical anesthesia with lidocaine aerosol in the control of postoperative pain. Anesthesiology 68:895-901

669. Dierking GW, Dahl JB, Kanstrup J, Dahl A, Kehlet H (1992) Effect of pre- vs postoperative inguinal field block on postoperative pain after herniorrhaphy. Br J Anaesth 68:344-348

670. Gill P, Kiani S, Victoria BA, Atcheson R (2001) Pre-emptive analgesia with local anaesthetic for herniorrhaphy. Anaesthesia 56:414-417

671. Ejlersen E, Andersen HB, Eliasen K, Mogensen T (1992) A comparison between preincisional and postincisional lidocaine infiltration and postoperative pain. Anesth Analg 74(4):495-498

672. Demirci A, Efe EM, Turker G et al (2014) Iliohypogastric/ilioinguinal nerve block in the inguinal hernia repair for postoperative pain management: comparison of the anatomical landmark and ultrasound guided techniques. Rev Bras Anestesiol 64(5):350-356. https://doi.org/10.1016/j.bjane.2014.01.001

673. Baerentzen F, Maschmann C, Jensen K, Belhage B, Hensler M, Borglum J (2012) Ultrasound-guided nerve block for inguinal hernia repair: a randomized, controlled, double-blind study. Reg
Anesth Pain Med 37:502-507. https://doi.org/10.1097/AAP. $0 \mathrm{~b} 013 \mathrm{e} 31825 \mathrm{a} 3 \mathrm{c} 8 \mathrm{a}$

674. Thavaneswaran P, Rudkin GE, Cooter RD, Moyes DG, Perera CL, Maddern GJ (2010) Brief reports: paravertebral block for anesthesia: a systematic review. Anesth Analg 110(6):1740-1744. https://doi.org/10.1213/ANE. 0b013e3181da82c8

675. Akcaboy EY, Akcaboy ZN, Gogus N (2009) Ambulatory inguinal herniorrhaphy: paravertebral block versus spinal anesthesia. Minerva Anestesiol 75:684-691

676. Klein SM, Pietrobon R, Nielsen KC et al (2002) Paravertebral somatic nerve block compared with peripheral nerve blocks for outpatient inguinal herniorrhaphy. Reg Anesth Pain Med 27:476-480. https://doi.org/10.1053/rapm.2002.35147

677. Hadzic A, Kerimoglu B, Loreio D et al (2006) Paravertebral blocks provide superior same-day recovery over general anesthesia for patients undergoing inguinal hernia repair. Anesth Analg 102:1076-1081. https://doi.org/10.1213/01.ane. 0000196532.56221.f2

678. Milone M, Di Minno MND, Musella M et al (2013) Outpatient inguinal hernia repair under local anaesthesia: feasibility and efficacy of ultrasound-guided transversus abdominis plane block. Hernia 17:749-755. https://doi.org/10.1007/s10029-0121022-2

679. Ebru Salman A, Yetisir F, Yurekli B, Aksoy M, Yildirim M, Kilic M (2013) The efficacy of the semi-blind approach of transversus abdominis plane block on postoperative analgesia in patients undergoing inguinal hernia repair: a prospective randomized double-blind study. Local Reg Anesth 6(1):1-7

680. Charlton S, Cyna AM, Middleton P (2009) Perioperative transversus abdominis plane (TAP) blocks for analgesia after abdominal surgery. Cochrane Database Syst Rev (2). https://doi. org/10.1002/14651858.CD007705

681. Schurr MJ, Faucher LD (2009) A prospective, randomized, comparative trial of a COX-2 selective nonsteroidal anti-inflammatory drug versus placebo in inguinal herniorrhaphy patients. Hernia 13:491-497. https://doi.org/10.1007/s10029009-0489-y

682. Turaga K, Wright A, Lee $\mathrm{R}$ et al (2008) A randomized trial of the peri-operative use of COX-2 inhibitors in Lichtenstein herniorrhaphy. Hernia 12:515-519. https://doi.org/10.1007/ s10029-008-0379-8

683. Beaussier M, Weickmans H, Paugam C et al (2005) A randomized, double-blind comparison between parecoxib sodium and propacetamol for parenteral postoperative analgesia after inguinal hernia repair in adult patients. Anesth Analg 100:1309-1315. https://doi.org/10.1213/01.ANE.0000150972. 88708.13

684. Dueholm S, Forrest M, Hjortso E, Lemvigh E (1989) Pain relief following herniotomy: a double-blind randomized comparison between naproxen and placebo. Acta Anaesthesiol Scand 33:391-394

685. Chen L-C, Elliott RA, Ashcroft DM (2004) Systematic review of the analgesic efficacy and tolerability of COX-2 inhibitors in post-operative pain control. J Clin Pharm Ther 29(3):215-229. https://doi.org/10.1111/j.1365-2710.2004.00558.x

686. Ong CKS, Seymour RA, Lirk P, Merry AF (2010) Combining paracetamol (acetaminophen) with nonsteroidal antiinflammatory drugs: a qualitative systematic review of analgesic efficacy for acute postoperative pain. Anesth Analg 110(4):1170-1179. https://doi.org/10.1213/ANE.0b013e3181cf9281

687. Wheeler M, Oderda GM, Ashburn MA, Lipman AG (2002) Adverse events associated with postoperative opioid analgesia: a systematic review. J Pain 3(3):159-180. https://doi.org/10.1054/ jpai.2002.123652 
688. Lau H, Patil NG, Lee F (2003) Randomized clinical trial of postoperative subfascial infusion with bupivacaine following ambulatory open mesh repair of inguinal hernia. Dig Surg 20:285-289. https://doi.org/10.1159/000071187

689. Sanchez B, Waxman K, Tatevossian R, Gamberdella M, Read B (2004) Local anesthetic infusion pumps improve postoperative pain after inguinal hernia repair: a randomized trial. Am Surg 70:1002-1006

690. Oakley MJ, Smith JS, Anderson JR, Fenton-Lee D (1998) Randomized placebo-controlled trial of local anaesthetic infusion in day-case inguinal hernia repair. Br J Surg 85:797-799. https://doi.org/10.1046/j.1365-2168.1998.00651.x

691. LeBlanc KA, Bellanger D, Rhynes VK, Hausmann M (2005) Evaluation of continuous infusion of $0.5 \%$ bupivacaine by elastomeric pump for postoperative pain management after open inguinal hernia repair. J Am Coll Surg 200:198-202. https://doi. org/10.1016/j.jamcollsurg.2004.10.011

692. Schurr MJ, Gordon DB, Pellino TA, Scanlon TA (2004) Continuous local anesthetic infusion for pain management after outpatient inguinal herniorrhaphy. Surgery 136:761-769. https://doi.org/10.1016/j.surg.2004.06.016

693. Wu CC, Bai CH, Huang MT, Wu CH, Tam KW (2014) Local anesthetic infusion pump for pain management following open inguinal hernia repair: a meta-analysis. Int J Surg 12:245-250. https://doi.org/10.1016/j.ijsu.2014.01.006

694. Kehlet H, Wilmore DW (2002) Multimodal strategies to improve surgical outcome. Am J Surg 183(6):630-641

695. Tolver MA, Strandfelt P, Forsberg G, Hjørne FP, Rosenberg J, Bisgaard T (2012) Determinants of a short convalescence after laparoscopic transabdominal preperitoneal inguinal hernia repair. Surgery 151(4):556-563. https://doi.org/10.1016/j.surg. 2011.08.020

696. Callesen T, Klarskov B, Bech K, Kehlet H (1999) Short convalescence after inguinal herniorrhaphy with standardised recommendations: duration and reasons for delayed return to work. Eur J Surg 165(3):236-241. https://doi.org/10.1080/ 110241599750007108

697. Fujita F, Lahmann B, Otsuka K, Lyass S, Hiatt JR, Phillips EH (2004) Quantification of pain and satisfaction following laparoscopic and open hernia repair. Arch Surg 139(6):596-600-602. https://doi.org/10.1001/archsurg.139.6. 596

698. Kapiris SA, Brough WA, Royston CM, O’Boyle C, Sedman PC (2001) Laparoscopic transabdominal preperitoneal (TAPP) hernia repair. A 7-year two-center experience in 3017 patients. Surg Endosc 15(9):972-975. https://doi.org/10.1007/ s004640080090

699. Wilson MS, Deans GT, Brough WA (1995) Prospective trial comparing Lichtenstein with laparoscopic tension-free mesh repair of inguinal hernia. Br J Surg 82(2):274-277

700. Eklund A, Rudberg C, Leijonmarck CE et al (2007) Recurrent inguinal hernia: Randomized multicenter trial comparing laparoscopic and Lichtenstein repair. Surg Endosc Other Interv Tech 21(4):634-640. https://doi.org/10.1007/s00464-006-9163y

701. Berndsen F, Arvidsson D, Enander LK et al (2002) Postoperative convalescence after inguinal hernia surgery: prospective randomized multicenter study of laparoscopic versus Shouldice inguinal hernia repair in 1042 patients. Hernia 6(2):56-61. https://doi.org/10.1007/s10029-002-0055-3

702. Fleming WR, Elliott TB, Jones RM, Hardy KJ (2001) Randomized clinical trial comparing totally extraperitoneal inguinal hernia repair with the Shouldice technique. $\mathrm{Br}$ J Surg 88(9):1183-1188. https://doi.org/10.1046/j.0007-1323.2001. 01865.x
703. Dirksen CD, Beets GL, Go PMNYH, Geisler FEA, Baeten CGMI, Kootstra G (1998) Bassini repair compared with laparoscopic repair for primary inguinal hernia: a randomised controlled trial. Eur J Surg 164:439-447. https://doi.org/10. 1080/110241598750004256

704. Lawrence K, Mcwhinnie D, Goodwin A et al (1995) Randomised controlled trial of laparoscopic versus open repair of inguinal hernia: early results. Br Med J 311(7011):981-985

\section{PART 2}

1. Zendejas B, Ramirez T, Jones $\mathrm{T}$ et al (2013) Incidence of inguinal hernia repairs in Olmsted County, MN. Ann Surg 257(3):520-526. 0b013e31826d41c6

2. Burcharth J, Pedersen M, Bisgaard T, Pedersen C, Rosenberg J (2013) Nationwide prevalence of groin hernia repair. PLoS One 8(1):e54367. https://doi.org/10.1371/journal.pone.0054367

3. Nilsson E, Kald A, Anderberg B et al (1997) Hernia surgery in a defined population: a prospective three year audit. Eur J Surg 163(11):823-829

4. Rosen A, Nathan H, Luciansky EOR (1989) The inguinal region: anatomic differences in men and women with reference to hernia formation. Acta Anat 136(4):306-310

5. Bay-Nielsen MKH (2006) Inguinal herniorrhaphy in women. Hernia 10(1):30-33

6. Mikkelsen T, Bay-Nielsen M, Kehlet H (2002) Risk of femoral hernia after inguinal herniorrhaphy. Br J Surg 89(4):486-488. https://doi.org/10.1046/j.0007-1323.2002.02058.x

7. Henriksen NA, Thorup JJL (2012) Unsuspected femoral hernia in patients with a preoperative diagnosis of recurrent inguinal hernia. Hernia 16(4):381-385. https://doi.org/10.1007/s10029012-0924-3

8. Burcharth J, Andresen K, Pommergaard HC, Bisgaard T, Rosenberg J (2014) Direct inguinal hernias and anterior surgical approach are risk factors for female inguinal hernia recurrences. Langenbeck's Arch Surg 399:71-76. https://doi.org/10.1007/ s00423-013-1124-z

9. Koch A, Edwards A, Haapaniemi S, Nordin P, Kald A (2005) Prospective evaluation of 6895 groin hernia repairs in women. Br J Surg 92(12):1553-1558. https://doi.org/10.1002/bjs.5156

10. Andresen K, Bisgaard T, Kehlet H, Wara P, Rosenberg J (2014) Reoperation rates for laparoscopic vs open repair of femoral hernias in Denmark: a nationwide analysis. JAMA Surg 149(8):853-857. https://doi.org/10.1001/jamasurg.2014.177

11. Sevonius D, Gunnarsson U, Nordin P, Nilsson E, Sandblom G (2011) Recurrent groin hernia surgery. Br J Surg 98(10):1489-1494. https://doi.org/10.1002/bjs.7559

12. Simons MP, Aufenacker T, Bay-Nielsen M et al (2009) European Hernia Society guidelines on the treatment of inguinal hernia in adult patients. Hernia 13:343-403. https://doi.org/10. 1007/s10029-009-0529-7

13. Robinson A, Light D, Nice C (2013) Meta-analysis of sonography in the diagnosis of inguinal hernias. J Ultrasound Med 32:339-346

14. Schouten N, Burgmans JPJ, van Dalen T et al (2012) Female "groin" hernia: totally extraperitoneal (TEP) endoscopic repair seems the most appropriate treatment modality. Hernia 16(4):387-392. https://doi.org/10.1007/s10029-012-0904-7

15. Putnis S, Wong A, Berney C (2011) Synchronous femoral hernias diagnosed during endoscopic inguinal hernia repair. Surg 
Endosc 25(12):3752-3754. https://doi.org/10.1007/s00464-0111781-3

16. Renzulli P, Frei E, Schafer M, Werlen S, Wegmuller HKL (1997) Preoperative Nyhus classification of inguinal hernias and type-related individual hernia repair. Surg Laparosc Endosc 7:373-377

17. Burcharth J, Pommergaard HC, Bisgaard T, Rosenberg J (2015) Patient-related risk factors for recurrence after inguinal hernia repair: a systematic review and meta-analysis of observational studies. Surg Innov 22:303-317. https://doi.org/10.1177/ 1553350614552731

18. Kark AEKM (2008) Groin hernias in women. Hernia 12(3):267-270

19. Lau H, Patil NGYW (2005) Is endoscopic totally extraperitoneal hernioplasty justified for the repair of groin hernia in female patients? Surg Endosc 19(12):1544-1548

20. Schouten N, Simmermacher RKJ, van Dalen T et al (2013) Is there an end of the "learning curve" of endoscopic totally extraperitoneal (TEP) hernia repair? Surg Endosc 27(3):789-794. https://doi.org/10.1007/s00464-012-2512-0

21. Tantia O, Jain M, Khanna SSB (2009) Laparoscopic repair of recurrent groin hernia: results of a prospective study. Surg Endosc 23:734-738

22. Dahlstrand U, Wollert S, Nordin P, Sandblom G, Gunnarsson U (2009) Emergency femoral hernia repair: a study based on a national register. Ann Surg 249(4):672-676. https://doi.org/10. 1097/SLA.0b013e31819ed943

23. Ochsenbein-Kölble N, Demartines N, Ochsenbein-Imhof N, Zimmermann R (2004) Cesarean section and simultaneous hernia repair. Arch Surg 139:893-895. https://doi.org/10.1001/ archsurg.139.8.893

24. Buch K, Tabrizian P, Divino C (2008) Managment of hernias in pregnancy. J Am Coll Surg 207(4):539-542

25. Lechner M, Fortelny R, Ofner D, Mayer F (2014) Suspected inguinal hernias in pregnancy-handle with care! Hernia 18(3):375-379. https://doi.org/10.1007/s10029-013-1082-y

26. Scott N, Go P, Graham P, McCormack K, Ross S, Grant A (2001) Open mesh versus non-mesh for groin hernia repair (Review). Cochrane Libr (3). https://doi.org/10.1002/14651858. CD002197.Copyright

27. McIntosh A, Hutchinson A, Roberts A, Withers H (2000) Evidence-based management of groin hernia in primary care-a systematic review. Fam Pract 17:442-447

28. Dahlstrand U, Sandblom G, Nordin P, Wollert S, Gunnarsson U (2011) Chronic pain after femoral hernia repair: a cross-sectional study. Ann Surg 254(6):1017-1021. https://doi.org/10. 1097/SLA.0b013e31822ba9b6

29. Chan G, Chan C-K (2008) Longterm results of a prospective study of 225 femoral hernia repairs: indications for tissue and mesh repair. J Am Coll Surg 207(3):360-367. https://doi.org/10. 1016/j.jamcollsurg.2008.04.018

30. Hernández-Irizarry R, Zendejas B, Ramirez T et al (2012) Trends in emergent inguinal hernia surgery in Olmsted County, MN: a population-based study. Hernia 16(4):397-403. https://doi.org/10.1007/s10029-012-0926-1

31. Humes DJ, Radcliffe RS, Camm C, West J (2013) Populationbased study of presentation and adverse outcomes after femoral hernia surgery. Br J Surg 100:1827-1832. https://doi.org/10. 1002/bjs.9336

32. Nilsson H, Stylianidis G, Haapamäki M, Nilsson E, Nordin P (2007) Mortality after groin hernia surgery. Ann Surg 245:656-660. https://doi.org/10.1097/01.sla.0000251364.32698. $4 \mathrm{~b}$

33. Bay-Nielsen M, Kehlet H, Strand L et al (2001) Quality assessment of 26,304 herniorrhaphies in Denmark: a prospective nationwide study. Lancet 358(9288):1124-1128. https://doi.org/ 10.1016/S0140-6736(01)06251-1

34. Ceriani V, Faleschini E, Sarli D et al (2006) Femoral hernia repair. Kugel retroparietal approach versus plug alloplasty: a prospective study. Hernia 10(2):169-174. https://doi.org/10. 1007/s10029-005-0059-X

35. Chen J, Lv Y, Shen Y, Liu S, Wang M (2010) A prospective comparison of preperitoneal tension-free open herniorrhaphy with mesh plug herniorrhaphy for the treatment of femoral hernias. Surgery 148(5):976-981. https://doi.org/10.1016/j.surg. 2010.02.006

36. Antonescu I, Baldini G, Watson D et al (2013) Impact of a bladder scan protocol on discharge efficiency within a care pathway for ambulatory inguinal herniorraphy. Surg Endosc 27(12):4711-4720. https://doi.org/10.1007/s00464-013-3119-9

37. Koch CA, Grinberg GG, Farley DR (2006) Incidence and risk factors for urinary retention after endoscopic hernia repair. Am J Surg 191(3):381-385. https://doi.org/10.1016/j.amjsurg.2005. 10.042

38. van Veen RN, Mahabier C, Dawson I et al (2008) Spinal or local anesthesia in Lichtenstein hernia repair: a randomized controlled trial. Ann Surg 247(3):428-433. https://doi.org/10.1097/SLA. 0b013e318165b0ff

39. Kehlet H, Aasvang E (2005) Groin hernia repair: anesthesia. World J Surg 29(8):1058-1061. https://doi.org/10.1007/s00268005-7969-8

40. Bakota B, Kopljar M, Baranovic S, Miletic M, Marinovic M, Vidovic D (2015) Should we abandon regional anesthesia in open inguinal hernia repair in adults? Eur J Med Res 20(1):76. https://doi.org/10.1186/s40001-015-0170-0

41. Symeonidis D, Baloyiannis I, Koukoulis G et al (2014) Prospective non-randomized comparison of open versus laparoscopic transabdominal preperitoneal (TAPP) inguinal hernia repair under different anesthetic methods. Surg Today 44:906-913. https://doi.org/10.1007/s00595-013-0805-0

42. Joshi GP, Rawal N, Kehlet H et al (2012) Evidence-based management of postoperative pain in adults undergoing open inguinal hernia surgery. Br J Surg 99(2):168-185. https://doi. org/10.1002/bjs.7660

43. Hudak KE, Frelich MJ, Rettenmaier CR et al (2015) Surgery duration predicts urinary retention after inguinal herniorrhaphy: a single institution review. Surg Endosc 29(11):3246-3250. https://doi.org/10.1007/s00464-015-4068-2

44. Patel JA, Kaufman AS, Howard RS, Rodriguez CJ, Jessie EM (2015) Risk factors for urinary retention after laparoscopic inguinal hernia repairs. Surg Endosc 29(11):3140-3145 https://doi.org/10.1007/s00464-014-4039-z

45. Sivasankaran MV, Pham T, Divino CM (2014) Incidence and risk factors for urinary retention following laparoscopic inguinal hernia repair. Am J Surg 207(2):288-292. https://doi.org/10. 1016/j.amjsurg.2013.06.005

46. Tam KW, Liang HH, Chai CY (2010) Outcomes of staple fixation of mesh versus nonfixation in laparoscopic total extraperitoneal inguinal repair: a meta-analysis of randomized controlled trials. World J Surg 34:3065-3074

47. Liu H, Zheng X, Gu Y, Guo S (2015) A meta-analysis examining the use of fibrin glue mesh fixation versus suture mesh fixation in open inguinal hernia repair. Dig Surg 31(6):444-451. https://doi.org/10.1159/000370249

48. Jensen P, Mikkelsen T, Kehlet H (2002) Postherniorrhaphy urinary retention-effect of local, regional, and general anesthesia: a review. Reg Anesth Pain Med 27(6):612-617. https://doi.org/10.1053/rapm.2002.37122

49. Treadwell J, Tipton K, Oyesanmi O, Sun F, Schoelles K (2012) Surgical options for inguinal hernia: comparative effectiveness review. Agency Healthc Res Qual Comp (70):1-1219 
50. Oehlenschlager J, Hjorne FP, Albers M et al (2010) Fewer urological complications after laparoscopic inguinal hernia repair without indwelling catheter. Dan Med Bull 57(9):A4176

51. Bittner R, Schmedt CG, Schwarz J, Kraft K, Leibl BJ (2002) Laparoscopic transperitoneal procedure for routine repair of groin hernia. Br J Surg 89(8):1062-1066. https://doi.org/10. 1046/j.1365-2168.2002.02178.x

52. Reed RD, Poston TL, Kerby JD, Richman JS, Colli JL, Hawn MT (2014) Effect of elective inguinal hernia repair on urinary symptom burden in men. Am J Surg 208(2):180-186. https://doi. org/10.1016/j.amjsurg.2014.02.004

53. Aasvang EK, Bay-Nielsen M, Kehlet H (2006) Pain and functional impairment 6 years after inguinal herniorrhaphy. Hernia 10(4):316-321. https://doi.org/10.1007/s10029-006-0098-y

54. Bischoff JM, Linderoth G, Aasvang EK, Werner MU, Kehlet H (2012) Dysejaculation after laparoscopic inguinal herniorrhaphy: a nationwide questionnaire study. Surg Endosc 26(4):979-983. https://doi.org/10.1007/s00464-011-1980-y

55. Bendavid R (1992) "Dysejaculation": an unusual complication of inguinal herniorrhaphy. Postgrad Gen Surg. 4:139-141

56. Aasvang EK, Mohl B, Kehlet H (2007) Ejaculatory pain: a specific postherniotomy pain syndrome? Anesthesiology 107(2):298-304. https://doi.org/10.1097/01.anes.0000270736. 28324.61

57. Wantz GE (1984) Complications of inguinal hernial repair. Surg Clin N Am 64(2):287-298

58. Fong Y, Wantz GE (1992) Prevention of ischemic orchitis during inguinal hernioplasty. Surg Gynecol Obstet 174(5):399-402

59. Tekatli H, Schouten N, van Dalen T, Burgmans I, Smakman N (2012) Mechanism, assessment, and incidence of male infertility after inguinal hernia surgery: a review of the preclinical and clinical literature. Am J Surg 204(4):503-509. https://doi.org/10. 1016/j.amjsurg.2012.03.002

60. Li J, Ji Z, Cheng T (2012) Lightweight versus heavyweight in inguinal hernia repair: a meta-analysis. Hernia 16:529-539. https://doi.org/10.1007/s10029-012-0928-z

61. Junge K, Binnebösel M, Kauffmann C et al (2011) Damage to the spermatic cord by the Lichtenstein and TAPP procedures in a pig model. Surg Endosc Other Interv Tech 25(1):146-152. https://doi.org/10.1007/s00464-010-1148-1

62. Peiper C, Junge K, Klinge U, Strehlau E, Ottinger A, Schumpelick V (2006) Is there a risk of infertility after inguinal mesh repair? Experimental studies in the pig and the rabbit. Hernia 10(1):7-12. https://doi.org/10.1007/s10029-005-0055-1

63. Peeters E, Spiessens C, Oyen R et al (2010) Laparoscopic inguinal hernia repair in men with lightweight meshes may significantly impair sperm motility: a randomized controlled trial. Ann Surg 252:240-246. https://doi.org/10.1097/SLA. 0b013e3181e8fac5

64. Peeters E, Spiessens C, Oyen R et al (2014) Sperm motility after laparoscopic inguinal hernia repair with lightweight meshes: 3-year follow-up of a randomised clinical trial. Hernia 18:361-367. https://doi.org/10.1007/s10029-012-1028-9

65. Aydede H, Erhan Y, Sakarya A, Kara E, Ilkgul O, Can M (2003) Effect of mesh and its localisation on testicular flow and spermatogenesis in patients with groin hernia. Acta Chir Belg 103(6):607-610

66. Schouten N, van Dalen T, Smakman N et al (2012) Male infertility after endoscopic totally extraperitoneal (Tep) hernia repair (Main): rationale and design of a prospective observational cohort study. BMC Surg 12(1):7. https://doi.org/10.1186/ 1471-2482-12-7

67. Sheynkin YR, Hendin BN, Schlegel PN, Goldstein M (1998) Microsurgical repair of iatrogenic injury to the vas deferens. J Urol 159:139-141
68. Shin D, Lipshultz LI, Goldstein M et al (2005) Herniorrhaphy with polypropylene mesh causing inguinal vasal obstruction. Ann Surg 241(4):553-558. https://doi.org/10.1097/01.sla. $0000157318.13975 .2 \mathrm{a}$

69. Fitzgibbons RJ Jr (2005) Can we be sure polypropylene mesh causes infertility? Ann Surg 241(4):559-561

70. Štula I, Družijanić N, Sapunar A, Perko Z, Bošnjak N, Kraljević D (2014) Antisperm antibodies and testicular blood flow after inguinal hernia mesh repair. Surg Endosc 28(12):3413-3420. https://doi.org/10.1007/s00464-014-3614-7

71. Chehval MJ, Doshi R, Kidd CF, Winkelmann T, Chehval V (2002) Antisperm autoantibody response after unilateral vas deferens ligation in rats: when does it develop? J Androl 23(5):669-673

72. Hallen M, Sandblom G, Nordin P, Gunnarsson U, Kvist U, Westerdahl J (2011) Male infertility after mesh hernia repair: a prospective study. Surgery 149(2):179-184. https://doi.org/10. 1016/j.surg.2010.04.027

73. Hallén M, Westerdahl J, Nordin P, Gunnarsson U, Sandblom G (2012) Mesh hernia repair and male infertility: a retrospective register study. Surgery 151(1):94-98. https://doi.org/10.1016/ j.surg.2011.06.028

74. Verhagen T, Loos MJA, Scheltinga MRM, Roumen RMH (2016) Surgery for chronic inguinodynia following routine herniorrhaphy: beneficial effects on dysejaculation. Hernia 20(1):63-68. https://doi.org/10.1007/s10029-015-1410-5

75. Matsuda T (2000) Diagnosis and treatment of post-herniorrhaphy vas deferens obstruction. Int J Urol 7(1):35-38. https://doi. org/10.1046/j.1442-2042.2000.00171.x

76. Koning GG, Wetterslev J, van Laarhoven CJ, Keus F (2013) The totally extraperitoneal method versus Lichtenstein's technique for inguinal hernia repair: a systematic review with meta-analyses and trial sequential analyses of randomized clinical trials. PLoS One 8(1):e52599. https://doi.org/10.1371/journal.pone. 0052599 [Electronic Resour]

77. O'Reilly EA, Burke JP, O'Connell PR (2012) A meta-analysis of surgical morbidity and recurrence after laparoscopic and open repair of primary unilateral inguinal hernia. Ann Surg 255(5):846-853. $0 \mathrm{~b} 013 \mathrm{e} 31824 \mathrm{e} 96 \mathrm{cf}$

78. Kuhry E, van Veen RN, Langeveld HR, Steyerberg EW, Jeekel J, Bonjer HJ (2007) Open or endoscopic total extraperitoneal inguinal hernia repair? A systematic review. Surg Endosc 21:161-166

79. Cavazzola LT, Rosen MJ (2013) Laparoscopic versus open inguinal hernia repair. Surg Clin N Am 93(5):1269-1279. https://doi.org/10.1016/j.suc.2013.06.013

80. Bittner R, Schwarz J (2012) Inguinal hernia repair: current surgical techniques. Langenbecks Arch Surg 397(2):271-282. https://doi.org/10.1007/s00423-011-0875-7

81. Grant A (2000) Laparoscopic compared with open methods of groin hernia repair: systematic review of randomized controlled trials. Br J Surg 87:860-867. https://doi.org/10.1046/j.13652168.2000.01540.x

82. McCormack K, Scott NW, Go PM, Ross S, Grant AM (2003) Laparoscopic techniques versus open techniques for inguinal hernia repair. Cochrane Database Syst Rev (1):CD001785. https://doi.org/10.1002/14651858.CD001785

83. Schmedt CG, Sauerland S, Bittner R (2005) Comparison of endoscopic procedures vs Lichtenstein and other open mesh techniques for inguinal hernia repair: a meta-analysis of randomized controlled trials. Surg Endosc 19(2):188-199. https://doi.org/10.1007/s00464-004-9126-0

84. Bittner R, Sauerland S, Schmedt C-G (2005) Comparison of endoscopic techniques vs Shouldice and other open nonmesh techniques for inguinal hernia repair: a meta-analysis of 
randomized controlled trials. Surg Endosc 19(5):605-615. https://doi.org/10.1007/s00464-004-9049-9

85. Amato B, Moja L, Panico S et al (2009) Shouldice technique versus other open techniques for inguinal hernia repair. Cochrane Database Syst Rev CD001543. https://doi.org/10. 1002/14651858.CD001543.pub3

86. Li J, Ji Z, Cheng T (2012) Comparison of open preperitoneal and Lichtenstein repair for inguinal hernia repair: a meta-analysis of randomized controlled trials. Am J Surg 204:769-778. https://doi.org/10.1016/j.amjsurg.2012.02.010

87. Sanjay P, Watt DG, Ogston SA, Alijani A, Windsor JA (2012) Meta-analysis of Prolene Hernia System mesh versus Lichtenstein mesh in open inguinal hernia repair. Surg J R Coll Surg Edinb Irel 10:283-289. https://doi.org/10.1016/j.surge.2012.06. 001

88. Zhao G, Gao P, Ma B, Tian J, Yang K (2009) Open mesh techniques for inguinal hernia repair: a meta-analysis of randomized controlled trials. Ann Surg 250:35-42. https://doi.org/ 10.1097/SLA.0b013e3181ad63cc

89. Li J, Ji Z, Li Y (2012) Comparison of mesh-plug and Lichtenstein for inguinal hernia repair: a meta-analysis of randomized controlled trials. Hernia 16:541-548. https://doi.org/10.1007/ s10029-012-0974-6

90. Wilkiemeyer M, Pappas TN, Giobbie-Hurder A, Itani KMF, Jonasson O, Neumayer LA (2005) Does resident post graduate year influence the outcomes of inguinal hernia repair? Ann Surg 241(6):879-884. https://doi.org/10.1097/01.sla.0000164076. 82559.72

91. Feliu-Palà X, Martín-Gómez M, Morales-Conde S, FernándezSallent E (2001) The impact of the surgeon's experience on the results of laparoscopic hernia repair. Surg Endosc 15(12):1467-1470. https://doi.org/10.1007/s00464-001-9017-6

92. Bökeler U, Schwarz J, Bittner R, Zacheja S, Smaxwil C (2013) Teaching and training in laparoscopic inguinal hernia repair (TAPP): impact of the learning curve on patient outcome. Surg Endosc 27(8):2886-2893. https://doi.org/10.1007/s00464-0132849-z

93. Van Ooijen B (1986) Subcutaneous heparin and postoperative wound hematomas: a prospective, double-blind, randomized study. Arch Surg 121:937-940

94. Goris RJ, Van Dongen LM, Deleu HW, Doesburg DW (1981) Low dose subcutaneous heparin and wound hematoma formation in inguinal hernia operations - a double-blind randomized clinical trial. Neth J Surg 33:19-22

95. Dommering M (1981) The site of injection for low dose heparin in inguinal herniorrhaphy; a double-blind trial. Neth J Surg 33:23-24

96. Dommering M (1979) Low-dose heparin prophylaxis in herniorrhaphy? A prospective trial in bleeding complications. Arch Chir Neerl 31:57-61

97. Schmitz R, Kansy M, Moser KH, Treckmann J, Byrne JG, Rice CL (1995) Effect of low-dose subcutaneous heparin on postoperative wound hematomas: randomized clinical trial on hospitalized inguinal hernia patients in Germany. World J Surg 19:416-419. https://doi.org/10.1007/BF00299176

98. Smoot RL, Oderich GS, Taner CB et al (2008) Postoperative hematoma following inguinal herniorrhaphy: patient characteristics leading to increased risk. Hernia 12(3):261-265. https://doi.org/10.1007/s10029-007-0313-5

99. Sanders DL, Shahid MK, Ahlijah B, Raitt JE, Kingsnorth AN (2008) Inguinal hernia repair in the anticoagulated patient: a retrospective analysis. Hernia 12(6):589-592. https://doi.org/10. 1007/s10029-008-0405-x

100. Mumme T, Peiper CLN (2000) Does anticoagulation increase the risk of wound hematoma in inguinal hernia surgery? Hernia 4(2):121-128
101. Chu EW, Telem DA, Chernoguz A, Divino CM (2011) Assessing the risk of clopidogrel-related bleeding complications in patients undergoing inguinal herniorrhaphy. Hernia 15(1):31-35. https://doi.org/10.1007/s10029-010-0732-6

102. Stucky C-CH, Garvey EM, Johnson DJ et al (2014) Challenging a surgical dictum: results from a 10-year experience on the safety of open inguinal herniorrhaphy in patients on chronic warfarin therapy. Hernia 19(1):1-5. https://doi.org/10.1007/ s10029-014-1257-1

103. Bhangu A, Singh P, Pinkney T, Blazeby JM (2015) A detailed analysis of outcome reporting from randomised controlled trials and meta-analyses of inguinal hernia repair. Hernia 19(1):65-75. https://doi.org/10.1007/s10029-014-1299-4

104. Bittner R, Arregui ME, Bisgaard T et al (2011) Guidelines for laparoscopic (TAPP) and endoscopic (TEP) treatment of inguinal hernia [International Endohernia Society (IEHS)]. Surg Endosc 25(9):2773-2843. https://doi.org/10.1007/s00464-0111799-6

105. Schmedt CG, Leibl BJ, Bittner R (2002) Endoscopic inguinal hernia repair in comparison with Shouldice and Lichtenstein repair. A systematic review of randomized trials. Dig Surg 19:511-517

106. Grant AM (2002) Open mesh versus non-mesh repair of groin hernia meta-analysis of randomized trials leased on individual patient data: the EU Hernia Trialists Collaboration. Hernia 6(3):130-136. https://doi.org/10.1007/s10029-002-0073-1

107. Leibl BJ, Schmedt CG, Kraft K, Ulrich M, Bittner R (2000) Scrotal hernias: a contraindication for an endoscopic procedure? Results of a single-institution experience in transabdominal preperitoneal repair. Surg Endosc 14(3):289-292. https://doi. org/10.1007/s004640000045

108. Bittner R, Schmedt CG, Leibl BJ, Schwarz J (2011) Early postoperative and one year results of a randomized controlled trial comparing the impact of extralight titanized polypropylene mesh and traditional heavyweight polypropylene mesh on pain and seroma production in laparoscopic hernia repair (TAPP). World J Surg 35:1791-1797

109. Bansal VK, Misra MC, Babu D et al (2013) A prospective, randomized comparison of long-term outcomes: chronic groin pain and quality of life following totally extraperitoneal (TEP) and transabdominal preperitoneal (TAPP) laparoscopic inguinal hernia repair. Surg Endosc Other Interv Tech 27:2373-2382. https://doi.org/10.1007/s00464-013-2797-7

110. Krishna A, Misra MC, Bansal VK, Kumar S, Rajeshwari S, Chabra A (2012) Laparoscopic inguinal hernia repair: transabdominal preperitoneal (TAPP) versus totally extraperitoneal (TEP) approach: a prospective randomized controlled trial. Surg Endosc 26(3):639-649. https://doi.org/10.1007/s00464-0111931-7

111. Berney CR (2012) The Endoloop technique for the primary closure of direct inguinal hernia defect during the endoscopic totally extraperitoneal approach. Hernia 16(3):301-305. https://doi.org/10.1007/s10029-011-0892-z

112. Reddy VM, Sutton CD, Bloxham L, Garcea G, Ubhi SS, Robertson GS (2007) Laparoscopic repair of direct inguinal hernia: a new technique that reduces the development of postoperative seroma. Hernia 11:393-396. https://doi.org/10.1007/ s10029-007-0233-4

113. Shah NS, Fullwood C, Siriwardena AK, Sheen AJ (2014) Mesh fixation at laparoscopic inguinal hernia repair: a meta-analysis comparing tissue glue and tack fixation. World J Surg 38:2558-2570. https://doi.org/10.1007/s00268-014-2547-6

114. Beacon J, Hoile RW, Ellis H (1980) A trial of suction drainage in inguinal hernia repair. Br J Surg 67:554-555. https://doi.org/ 10.1002/bjs. 1800670808 
115. Peiper C, Conze J, Ponschek N, Schumpelick V (1997) Value of subcutaneous drainage in repair of primary inguinal hernia. A prospective randomized study of 100 cases. [German] TT Stellenwert der subcutanen Drainage bei der Reparation primarer Leistenhernien. Eine prospektive randomisierte Studie an 100 F. Chirurg 68:63-67

116. Ismail M, Garg M, Rajagopal M (2009) Garg PCN-C-00721892. Impact of closed-suction drain in preperitoneal space on the incidence of seroma formation after laparoscopic total extraperitoneal inguinal hernia repair. Surg Laparosc Endosc Percutaneous Tech 19:263-266. https://doi.org/10.1097/SLE. 0b013e3181a4d0e1

117. Gao D, Wei S, Zhai C et al (2015) Clinical research of preperitoneal drainage after endoscopic totally extraperitoneal inguinal hernia repair. Hernia 19(5):789-794. https://doi.org/10. 1007/s10029-014-1310-0

118. Wake BL, Mccormack K, Fraser C, Vale L, Perez J, Grant A (2005) Transabdominal pre-peritoneal [TAPP] vs totally extraperitoneal [TEP] laparoscopic techniques for inguinal hernia repair. Cochrane Database Syst Rev 25(1):CD004703

119. String A, Berber E, Foroutani A, Macho JR, Pearl JM, Siperstein AE (2001) Use of the optical access trocar for safe and rapid entry in various laparoscopic procedures. Surg Endosc 15(6):570-573. https://doi.org/10.1007/s004640080056

120. Tinelli A, Malvasi A, Guido M et al (2011) Laparoscopy entry in patients with previous abdominal and pelvic surgery. Surg Innov 18(3):201-205. https://doi.org/10.1177/ 1553350610393989

121. Wada H, Kimura T, Kawabe A et al (2012) Laparoscopic transabdominal preperitoneal inguinal hernia repair using needlescopic instruments: a 15-year, single-center experience in 317 patients. Surg Endosc Other Interv Tech 26:1898-1902. https://doi.org/10.1007/s00464-011-2122-2

122. Ramshaw B, Shuler FW, Jones HB et al (2001) Laparoscopic inguinal hernia repair: lessons learned after 1224 consecutive cases. Surg Endosc 15(1):50-54. https://doi.org/10.1007/ s004640001016

123. Lau H, Lee F (2003) A prospective endoscopic study of retropubic vascular anatomy in 121 patients undergoing endoscopic extraperitoneal inguinal hernioplasty. Surg Endosc Other Interv Tech 17(9):1376-1379. https://doi.org/10.1007/s00464003-8800-y

124. Lo CH, Trotter D, Grossberg P (2005) Unusual complications of laparoscopic totally extraperitoneal inguinal hernia repair. ANZ J Surg 75:917-919

125. Saggar VR, Sarangi R (2007) Occult hernias and bilateral endoscopic total extraperitoneal inguinal hernia repair: is there a need for prophylactic repair? Results of endoscopic extraperitoneal repair over a period of 10 years. Hernia 11(1):47-49. https://doi.org/10.1007/s10029-006-0157-4

126. van den Heuvel B, Dwars BJ (2013) Repeated laparoscopic treatment of recurrent inguinal hernias after previous posterior repair. Surg Endosc 27(3):795-800. https://doi.org/10.1007/ s00464-012-2514-y

127. Bringman S, Blomqvist $P$ (2005) Intestinal obstruction after inguinal and femoral hernia repair: a study of 33,275 operations during 1992-2000 in Sweden. Hernia 9(2):178-183. https://doi. org/10.1007/s10029-004-0305-7

128. Goswami R, Babor M, Ojo A (2007) Mesh erosion into caecum following laparoscopic repair of inguinal hernia (TAPP): a case report and literature review. J Laparoendosc Adv Surg Tech A 17(5):669-672. https://doi.org/10.1089/lap.2006.0135

129. Ojo P, Abenthroth A, Fiedler P, Yavorek G (2006) Migrating mesh mimicking colonic malignancy. Am Surg 72(12):1210-1211
130. D’Amore L, Gossetti F, Manto O, Negro P (2012) Mesh plug repair: can we reduce the risk of plug erosion into the sigmoid colon? Hernia 16(4):495-496. https://doi.org/10.1007/s10029012-0921-6

131. Novaretti JPT, Silva RDP, Cotrim CAC, Souto LRM (2012) Migration mesh mimicking bladder malignancy after open inguinal hernia repair. Hernia 16(4):467-470. https://doi.org/10. 1007/s10029-010-0760-2

132. Eugene JR, Gashti M, Curras EB, Schwartz K, Edwards J (1998) Small bowel obstruction as a complication of laparoscopic extraperitoneal inguinal hernia repair. J Am Osteopath Assoc 98(9):510-511. https://doi.org/10.1089/lap.1998.8.61

133. Withers L, Rogers A (2006) A spiral tack as a lead point for volvulus. J Soc Laparoendosc Surg 10(2):247-249

134. Lundström K-J, Sandblom G, Smedberg S, Nordin P (2012) Risk factors for complications in groin hernia surgery. Ann Surg 255(4):784-788. https://doi.org/10.1097/SLA. 0b013e31824b7cb3

135. Nilsson H, Nilsson E, Angerås U, Nordin P (2011) Mortality after groin hernia surgery: delay of treatment and cause of death. Hernia 15(3):301-307. https://doi.org/10.1007/s10029-0110782-4

136. van den Heuvel B, Dwars BJ, Klassen DR, Bonjer HJ (2011) Is surgical repair of an asymptomatic groin hernia appropriate? A review. Hernia 15(3):251-259. https://doi.org/10.1007/s10029011-0796-y

137. Collaboration IT (2011) Operation compared with watchful waiting in elderly male inguinal hernia patients: a review and data analysis. J Am Coll Surg 212(2):251-259. https://doi.org/ 10.1016/j.jamcollsurg.2010.09.030

138. Primatesta P, Goldacre MJ (1996) Inguinal hernia repair: incidence of elective and emergency surgery, readmission and mortality. Int J Epidemiol 25(4):835-839

139. Pallati PK, Gupta PK, Bichala S, Gupta H, Fang X, Forse RA (2013) Short-term outcomes of inguinal hernia repair in octogenarians and nonagenarians. Hernia 17(6):723-727. https://doi. org/10.1007/s10029-012-1040-0

140. McGugan E, Burton H, Nixon SJ, Thompson AM (2000) Deaths following hernia surgery: room for improvement. J R Coll Surg Edinb 45(3):183-186. https://doi.org/10.1038/sj.cdd.4401724

141. Stylopoulos N, Gazelle GS, Rattner DW (2003) A cost-utility analysis of treatment options for inguinal hernia in 1,513,008 adult patients. Surg Endosc 17(2):180-189. https://doi.org/10. 1007/s00464-002-8849-z

142. Bay-Nielsen M, Kehlet H (2008) Anaesthesia and post-operative morbidity after elective groin hernia repair: a nation-wide study. Acta Anaesthesiol Scand 52(2):169-174. https://doi.org/10. 1111/j.1399-6576.2007.01514.x

143. Majholm B, Engbæk J, Bartholdy J et al (2012) Is day surgery safe? A Danish multicentre study of morbidity after 57,709 day surgery procedures. Acta Anaesthesiol Scand 56(3):323-331. https://doi.org/10.1111/j.1399-6576.2011.02631.x

144. Merskey H, Bogduk N (1986) Classification of chronic pain. Description of pain terms. Prepared by the International Association for the Study of Pain. Subcommittee on Taxonomy. Pain Suppl 3:1-226

145. VanDenKerkhof EG, Peters ML, Bruce J (2013) Chronic pain after surgery : time for standardization? A framework to establish core risk factor and outcome domains for epidemiological studies. Clin J Pain 29(1):2-8. https://doi.org/10.1097/ AJP.0b013e31824730c2

146. Aasvang E, Kehlet H (2005) Chronic postoperative pain: the case of inguinal herniorrhaphy. Br $\mathrm{J}$ Anaesth 95(1):69-76. https://doi.org/10.1093/bja/aei019

147. Alfieri S, Amid PK, Campanelli G et al (2011) International guidelines for prevention and management of post-operative 
chronic pain following inguinal hernia surgery. Hernia 15(3):239-249. https://doi.org/10.1007/s10029-011-0798-9

148. Nienhuijs S, Staal E, Keemers-Gels M, Rosman C, Strobbe L (2007) Pain after open preperitoneal repair versus Lichtenstein repair: a randomized trial. World J Surg 31(9):1751-1759. https://doi.org/10.1007/s00268-007-9090-7

149. Cunningham J, Temple WJ, Mitchell P, Nixon JA, Preshaw RM, Hagen NA (1996) Cooperative hernia study. Pain in the postrepair patient. Ann Surg 224:598-602

150. Bay-Nielsen M, Perkins FM, Kehlet H (2001) Pain and functional impairment 1 year after inguinal herniorrhaphy: a nationwide questionnaire study. Ann Surg 233(1):1-7. https://doi.org/10.1097/00000658-200101000-00001

151. Nienhuijs SW, Rosman C, Strobbe LJA, Wolff A, Bleichrodt RP (2008) An overview of the features influencing pain after inguinal hernia repair. Int J Surg 6(4):351-356. https://doi.org/ 10.1016/j.ijsu.2008.02.005

152. Bjurstrom MF, Nicol AL, Amid PK, Chen DC (2014) Pain control following inguinal herniorrhaphy: current perspectives. J Pain Res 7:277-290. https://doi.org/10.2147/JPR.S47005

153. Kalliomäki ML, Meyerson J, Gunnarsson U, Gordh T, Sandblom G (2008) Long-term pain after inguinal hernia repair in a population-based cohort; risk factors and interference with daily activities. Eur J Pain 12(2):214-225. https://doi.org/10.1016/j. ejpain.2007.05.006

154. Fränneby U, Sandblom G, Nordin P, Nyrén O, Gunnarsson U (2006) Risk factors for long-term pain after hernia surgery. Ann Surg 244(2):212-219. https://doi.org/10.1097/01.sla. 0000218081.53940 .01

155. Powell R, Johnston M, Smith WC et al (2012) Psychological risk factors for chronic post-surgical pain after inguinal hernia repair surgery: a prospective cohort study. 16(1999):600-610. https://doi.org/10.1016/j.ejpain.2011.08.010

156. Werner MU, Mjöbo HN, Nielsen PR, Rudin A (2010) Prediction of postoperative pain: a systematic review of predictive experimental pain studies. Anesthesiology 112(6):1494-1502. https://doi.org/10.1097/ALN.0b013e3181ded5a0

157. Klaassen Z, Marshall E, Tubbs RS, Louis RG, Wartmann CT, Loukas M (2011) Anatomy of the ilioinguinal and iliohypogastric nerves with observations of their spinal nerve contributions. Clin Anat 24(4):454-461. https://doi.org/10.1002/ca. 21098

158. Ndiaye A, Diop M, Ndoye JM et al (2010) Emergence and distribution of the ilioinguinal nerve in the inguinal region: applications to the ilioinguinal anaesthetic block (about 100 dissections). Surg Radiol Anat 32(1):55-62. https://doi.org/10. 1007/s00276-009-0549-0

159. Peschaud F, Malafosse R, Le Floch-Prigent P, Coste-See C, Nordlinger B, Delmas V (2006) Anatomical bases of prolonged ilio-inguinal-hypogastric regional anesthesia. Surg Radiol Anat 28(5):511-517. https://doi.org/10.1007/s00276-006-0132-x

160. Reinpold W, Schroeder AD, Schroeder M, Berger C, Rohr M, Wehrenberg U (2015) Retroperitoneal anatomy of the iliohypogastric, ilioinguinal, genitofemoral, and lateral femoral cutaneous nerve: consequences for prevention and treatment of chronic inguinodynia. Hernia 19(4):539-548. https://doi.org/10. 1007/s10029-015-1396-z

161. Mandelkow H (1988) The iliohypogastric and ilioinguinal nerves. Surg Radiol Anat 10(2):145-149

162. Whiteside JL, Barber MD, Walters MD, Falcone T (2003) Anatomy of ilioinguinal and iliohypogastric nerves in relation to trocar placement and low transverse incisions. Am J Obstet Gynecol 189(6):1574-1578. https://doi.org/10.1016/S00029378(03)00934-7

163. Rahn DD, Phelan JN, Roshanravan SM, White AB, Corton MM (2010) Anterior abdominal wall nerve and vessel anatomy: clinical implications for gynecologic surgery. Am J Obstet Gynecol 202(3):234.e1-234.e5. https://doi.org/10.1016/j.ajog. 2009.10.878

164. Avsar FM, Sahin M, Arikan BU, Avsar AF, Demirci S, Elhan A (2002) The possibility of nervus ilioinguinalis and nervus iliohypogastricus injury in lower abdominal incisions and effects on hernia formation. J Surg Res 107(2):179-185

165. Rab M, Ebmer JA, Dellon A (2001) Anatomic variability of the ilioinguinal and genitofemoral nerve: implications for the treatment of groin pain. Plast Reconstr Surg 108(6):1618-1623

166. Wijsmuller AR, Lange JFM, Kleinrensink GJ et al (2007) Nerve-identifying inguinal hernia repair: a surgical anatomical study. World J Surg 31(2):414-420. https://doi.org/10.1007/ s00268-006-0376-y

167. Moosman DA, Oelrich TM (1977) Prevention of accidental trauma to the iloinguinal nerve during inguinal hernoirrhaphy. Am J Surg 133(2):146-148

168. Papadopoulos NJ, Katritsis ED (1981) Some observations on the course and relations of the iliohypogastric and ilioinguinal nerves (based on 348 specimens). Anat Anz 149(4):357-364

169. Diop M, Dia A, Ndiaye A, Lo EA, Sow ML, Ndiaye PD (2000) Emergence and course of the ilioinguinal nerve of the groin. Morphologie 84(266):29-32

170. Liu W-C, Chen T-H, Shyu J-F et al (2002) Applied anatomy of the genital branch of the genitofemoral nerve in open inguinal herniorrhaphy. Eur J Surg 168(3):145-149. https://doi.org/10. 1080/110241502320127748

171. Amid PK, Hiatt JR (2007) New understanding of the causes and surgical treatment of postherniorrhaphy inguinodynia and orchalgia. J Am Coll Surg 205(2):381-385. https://doi.org/10. 1016/j.jamcollsurg.2007.04.001

172. Amid PK, Chen DC (2011) Surgical treatment of chronic groin and testicular pain after laparoscopic and open preperitoneal inguinal hernia repair. J Am Coll Surg 213:531-536. https://doi. org/10.1016/j.jamcollsurg.2011.06.424

173. Smeds S, Löfström L, Eriksson O (2010) Influence of nerve identification and the resection of nerves "at risk" on postoperative pain in open inguinal hernia repair. Hernia 14(3):265-270. https://doi.org/10.1007/s10029-010-0632-9

174. Smeds S, Nienhuijs S, Kullman E et al (2016) Identification and management of the ilio-inguinal and ilio-hypogastric nerves in open inguinal hernia repair: benefits of self-gripping mesh. Hernia 20(1):33-41. https://doi.org/10.1007/s10029-015-1372-7

175. Eker HH, Langeveld HR, Klitsie PJ et al (2012) Randomized clinical trial of total extraperitoneal inguinal hernioplasty vs Lichtenstein repair: a long-term follow-up study. Arch Surg 147:256-260. https://doi.org/10.1001/archsurg.2011.2023

176. Bischoff JM, Aasvang EK, Kehlet H, Werner MU (2012) Does nerve identification during open inguinal herniorrhaphy reduce the risk of nerve damage and persistent pain? Hernia 16(5):573-577. https://doi.org/10.1007/s10029-012-0946-x

177. Wijsmuller AR, Van Veen RN, Bosch JL et al (2007) Nerve management during open hernia repair. Br J Surg 94(1):17-22. https://doi.org/10.1002/bjs.5651

178. Alfieri S, Rotondi F, Di Giorgio A et al (2006) Influence of preservation versus division of ilioinguinal, iliohypogastric, and genital nerves during open mesh herniorrhaphy: prospective multicentric study of chronic pain. Ann Surg 243(4):553-558. https://doi.org/10.1097/01.sla.0000208435.40970.00

179. Izard G (1996) Traitement des hernies de l'aine par la technique de McVay.pdf. Ann Chir 50(9):755-766

180. Hsu W, Chen C-S, Lee H-C et al (2012) Preservation versus division of ilioinguinal nerve on open mesh repair of inguinal hernia: a meta-analysis of randomized controlled trials. World J Surg 36(10):2311-2319. https://doi.org/10.1007/s00268-012$1657-2$ 
181. Johner A, Faulds J, Wiseman SM (2011) Planned ilioinguinal nerve excision for prevention of chronic pain after inguinal hernia repair: a meta-analysis. Surgery 150(3):534-541. https://doi.org/10.1016/j.surg.2011.02.024

182. Picchio M, Palimento D, Attanasio U, Matarazzo PF, Bambini C, Caliendo A (2004) Randomized controlled trial of preservation or elective division of ilioinguinal nerve on open inguinal hernia repair with polypropylene mesh. Arch Surg 139(7):755-759. https://doi.org/10.1001/archsurg.139.7.755

183. Malekpour F, Mirhashemi SH, Hajinasrolah E, Salehi N, Khoshkar A, Kolahi AA (2008) Ilioinguinal nerve excision in open mesh repair of inguinal hernia-results of a randomized clinical trial: simple solution for a difficult problem? Am J Surg 195(6):735-740. https://doi.org/10.1016/j.amjsurg.2007.09.037

184. Karakayali F, Oksuz E, Turk E et al (2010) Effectiveness of multiple neurectomies to prevent chronic groin pain after tension-free hernia repair. Int Surg 95(1):40-48

185. Crea N, Pata G (2010) Effects of prophylactic ilioinguinal nerve excision in mesh groin hernia repair: short- and long-term follow-up of a randomized clinical trial. Am Surg 76:1275-1281

186. Mui WL-M, Ng CSH, Fung TM-K et al (2006) Prophylactic ilioinguinal neurectomy in open inguinal hernia repair: a doubleblind randomized controlled trial. Ann Surg 244(1):27-33. https://doi.org/10.1097/01.sla.0000217691.81562.7e

187. Khoshmohabat H, Panahi F, Alvandi AA, Mehrvarz S, Mohebi HA, Shams Koushki E (2012) Effect of ilioinguinal neurectomy on chronic pain following herniorrhaphy. Trauma Mon 17(3):323-328. https://doi.org/10.5812/traumamon.6581

188. Ravichandran D, Kalambe BG, Pain JA (2000) Pilot randomized controlled study of preservation or division of ilioinguinal nerve in open mesh repair of inguinal hernia. $\mathrm{Br} \mathrm{J}$ Surg 87(9):1166-1167. https://doi.org/10.1046/j.1365-2168.2000. 01529.x

189. Reinpold WMJ, Nehls J, Eggert A (2011) Nerve management and chronic pain after open inguinal hernia repair: a prospective two phase study. Ann Surg 254(1):163-168. https://doi.org/10. 1097/SLA.0b013e31821d4a2d

190. Sanders DL, Nienhuijs S, Ziprin P, Miserez M, Gingell-Littlejohn M, Smeds S (2014) Randomized clinical trial comparing self-gripping mesh with suture fixation of lightweight polypropylene mesh in open inguinal hernia repair. Br J Surg 101:1373-1382. https://doi.org/10.1002/bjs.9598 (discussion 1382)

191. Caliskan K, Nursal TZ, Caliskan E, Parlakgumus A, Yildirim S, Noyan T (2010) A method for the reduction of chronic pain after tension-free repair of inguinal hernia: iliohypogastric neurectomy and subcutaneous transposition of the spermatic cord. Hernia 14(1):51-55. https://doi.org/10.1007/s10029-009-0571-5

192. Pappalardo G, Frattaroli FM, Mongardini M et al (2007) Neurectomy to prevent persistent pain after inguinal herniorraphy: a prospective study using objective criteria to assess pain. World J Surg 31(5):1081-1086. https://doi.org/10.1007/s00268006-7627-9

193. Zanghì G, Stefano GDI, Arena M, Dio DDI, Basile F (2012) Iliohypogastric neurectomy in the prevention of postoperative pain following inguinal hernioplasty. G Chir 33(5):172-174

194. Bartlett DC, Porter C, Kingsnorth AN (2007) A pragmatic approach to cutaneous nerve division during open inguinal hernia repair. Hernia 11(3):243-246. https://doi.org/10.1007/ s10029-007-0209-4

195. Ferguson D (1978) Closure of the hernial sac-pro and con. In: Nyhus LM, Conde RE (eds) Hernia, 2nd edn. JB Lippincott, Philadelphia, pp 152-153

196. Ellis H, Heddle R (1977) Does the peritoneum need to be closed at laparotomy? Br J Surg 64(10):733-736
197. Smedberg SG, Broome AE, Gullmo A (1984) Ligation of the hernial sac? Surg Clin N Am 64:299-306

198. Tanaka K, Matsugami T, Chiba T (2002) The origin of sensory innervation of the peritoneum in the rat. Anat Embryol (Berl) 205(4):307-313. https://doi.org/10.1007/s00429-002-0254-9

199. Delikoukos S, Lavant L, Hlias G, Palogos K, Gikas D (2007) The role of hernia sac ligation in postoperative pain in patients with elective tension-free indirect inguinal hernia repair: a prospective randomized study. Hernia 11:425-428. https://doi. org/10.1007/s10029-007-0249-9

200. Othman I, Hady HA (2014) Hernia sac of indirect inguinal hernia: invagination, excision, or ligation? Hernia 18:199-204. https://doi.org/10.1007/s10029-013-1081-z

201. Stylianidis G, Haapamäki MM, Sund M, Nilsson E, Nordin P (2010) Management of the hernial sac in inguinal hernia repair. Br J Surg 97(3):415-419. https://doi.org/10.1002/bjs.6890

202. Poobalan AS, Bruce J, Smith WC, King PM, Krukowski ZH, Chambers WA (2003) A review of chronic pain after inguinal herniorrhaphy. Clin J Pain 19:48-54

203. Loos MJA, Roumen RMH, Scheltinga MRM (2007) Classifying post-herniorrhaphy pain syndromes following elective inguinal hernia repair. World J Surg 31(9):1760-1767. https://doi.org/10. 1007/s00268-007-9121-4

204. Hakeem A, Shanmugam V (2011) Current trends in the diagnosis and management of post-herniorraphy chronic groin pain. World J Gastrointest Surg 3(6):73-81. https://doi.org/10.4240/ wjgs.v3.i6.73

205. Magnusson N, Gunnarsson U, Nordin P, Smedberg S, Hedberg M, Sandblom G (2015) Reoperation for persistent pain after groin hernia surgery: a population-based study. Hernia 19(1):45-51. https://doi.org/10.1007/s10029-014-1340-7

206. Amid PK (2003) The Lichtenstein repair in 2002: an overview of causes of recurrence after Lichtenstein tension-free hernioplasty. Hernia 7(1):13-16. https://doi.org/10.1007/s10029-0020088-7

207. Shirodkar SP, Gorin MA, Sageshima J et al (2011) Technical modification for laparoscopic donor nephrectomy to minimize testicular pain: a complication with significant morbidity. Am J Transplant 11(5):1031-1034. https://doi.org/10.1111/j.16006143.2011.03495.x

208. Jalali M, Rahmani S, Joyce AD, Cartledge JJ, Lewis MH, Ahmad N (2012) Laparoscopic donor nephrectomy: an increasingly common cause for testicular pain and swelling. Ann R Coll Surg Engl 94(6):407-410. https://doi.org/10.1308/ $003588412 X 13171221592177$

209. Kennedy EM, Harms BA, Starling JR (1994) Absence of maladaptive neuronal plasticity after genitofemoral-ilioinguinal neurectomy. Surgery 116(4):665-671

210. Chen DC, Hiatt JR, Amid PK (2013) Operative management of refractory neuropathic inguinodynia by a laparoscopic retroperitoneal approach. JAMA Surg 148(10):962-967. https://doi.org/10.1001/jamasurg.2013.3189

211. Kehlet H, Jensen TS, Woolf CJ (2006) Persistent postsurgical pain: risk factors and prevention. Lancet 367(9522):1618-1625. https://doi.org/10.1016/S0140-6736(06)68700-X

212. Dahl JB, Kehlet H (2011) Preventive analgesia. Curr Opin Anaesthesiol 24(3):331-338. https://doi.org/10.1213/ANE. 0b013e31824011e 8

213. Clarke H, Bonin RP, Orser BA, Englesakis M, Wijeysundera DN, Katz J (2012) The prevention of chronic postsurgical pain using gabapentin and pregabalin: a combined systematic review and meta-analysis. Anesth Analg 115(2):428-442. https://doi. org/10.1213/ANE.0b013e318249d36e

214. Sittl R, Irnich D, Lang PM (2013) Update zur präemptiven Analgesie. Anaesthesist 62(10):789-796. https://doi.org/10. 1007/s00101-013-2225-3 
215. Kong VKF, Irwin MG (2007) Gabapentin: a multimodal perioperative drug? Br J Anaesth 99(6):775-786. https://doi.org/10. 1093/bja/aem316

216. Derry S, Asc R, Cole P, Tan T, Ra M (2013) Topical capsaicin (high concentration) for chronic neuropathic pain in adults (Review). Cochrane Database Syst Rev 28(2):CD007393. https://doi.org/10.1002/14651858.CD007393.pub3

217. Aasvang EK, Hansen JB, Malmstrom J et al (2008) The effect of wound instillation of a novel purified capsaicin formulation on postherniotomy pain: a double-blind, randomized, placebocontrolled study. Anesth Analg 107:282-291. https://doi.org/10. 1213/ane.0b013e31816b94c9

218. Aasvang EK, Hansen JB, Kehlet H (2010) Late sensory function after intraoperative capsaicin wound instillation. Acta Anaesthesiol Scand 54(2):224-231. https://doi.org/10.1111/j.13996576.2009.02068.x

219. Dahl E, Cohen SP (2008) Perineural injection of etanercept as a treatment for postamputation pain. Clin J Pain 24(2):172-175. https://doi.org/10.1097/AJP.0b013e31815b32c8

220. Cohen SP, Galvagno SM, Plunkett A et al (2013) A multicenter, randomized, controlled study evaluating preventive etanercept on postoperative pain after inguinal hernia repair. Anesth Analg 116:455-462. https://doi.org/10.1213/ANE.0b013e318273f71c

221. Peters ML, Sommer M, de Rijke JM et al (2007) Somatic and psychologic predictors of long-term unfavorable outcome after surgical intervention. Ann Surg 245(3):487-494. https://doi.org/ 10.1097/01.sla.0000245495.79781.65

222. Powell R, Johnston M, Smith WC et al (2013) Rehabilitation following surgery: clinical and psychological predictors of activity limitations. Rehabil Psychol 58(4):350-360. https://doi. org/10.1037/a0034660

223. Bruce J, Thornton AJ, Powell R et al (2014) Psychological, surgical, and sociodemographic predictors of pain outcomes after breast cancer surgery: a population-based cohort study. Pain 155(2):232-243. https://doi.org/10.1016/j.pain.2013.09.028

224. Azari L, Santoso JT, Osborne SE (2013) Optimal pain management in total abdominal hysterectomy. Obstet Gynecol Surv 68(3):215-227. 0b013e31827f5119

225. Zieren J, Menenakos C, Mueller JM (2007) Does an informative video before inguinal hernia surgical repair influence postoperative quality of life? Results of a prospective randomized study. Qual Life Res 16(5):725-729. https://doi.org/10.1007/s11136007-9171-y

226. Nilsson U, Rawal N, Enqvist B, Unosson M (2003) Analgesia following music and therapeutic suggestions in the PACU in ambulatory surgery; a randomized controlled trial. Acta Anaesthesiol Scand 47:278-283. https://doi.org/10.1034/j.13996576.2003.00064.x

227. Glindvad J, Jorgensen M (2007) Postoperative education and pain in patients with inguinal hernia. J Adv Nurs 57:649-657. https://doi.org/10.1111/j.1365-2648.2006.04157.x

228. Werner MU, Bischoff JM (2014) Persistent postsurgical pain: evidence from breast cancer surgery, groin hernia repair, and lung cancer surgery. Curr Top Behav Neurosci 20:3-29. https://doi.org/10.1007/7854_2014_285

229. Werner MU, Kongsgaard UE (2014) Defining persistent postsurgical pain: is an update required? Br J Anaesth 113(1):1-4. https://doi.org/10.1093/bja/aeu012

230. Lichtenstein IL, Shulman AG, Amid PK, Montllor MM (1988) Cause and prevention of postherniorrhaphy neuralgia: a proposed protocol for treatment. Am J Surg 155(6):786-790

231. Aasvang E, Kehlet H (2005) Surgical management of chronic pain after inguinal hernia repair. Br J Surg 92:795-801
232. Ferzli GS, Edwards E, Al-Khoury G, Hardin R (2008) Postherniorrhaphy groin pain and how to avoid it. Surg Clin N Am 88(1):203-216. https://doi.org/10.1016/j.suc.2007.10.006

233. Lange JFM, Kaufmann R, Wijsmuller AR et al (2014) An international consensus algorithm for management of chronic postoperative inguinal pain. Hernia. https://doi.org/10.1007/ s10029-014-1292-y

234. Werner MU (2014) Management of persistent postsurgical inguinal pain. Langenbecks Arch Surg 399(5):559-569. https://doi.org/10.1007/s00423-014-1211-9

235. Voorbrood CEH, Burgmans JPJ, van Dalen T et al (2015) An algorithm for assessment and treatment of postherniorrhaphy pain. Hernia 19(4):571-577. https://doi.org/10.1007/s10029015-1387-0

236. Bushnell MC, Čeko M, Low LA (2013) Cognitive and emotional control of pain and its disruption in chronic pain. Nat Rev Neurosci 14(7):502-511. https://doi.org/10.1038/nrn3516. Cognitive

237. Benito-León J, Picardo A, Garrido A, Cuberes R (2001) Gabapentin therapy for genitofemoral and ilioinguinal neuralgia. J Neurol 248(10):907-908. https://doi.org/10.1007/ s004150170080

238. Finnerup NB, Jensen TS, Lund K et al (2015) Pharmacotherapy for neuropathic pain in adults: a systematic review and metaanalysis. http://www.thelancet.com/neurologyArtic. Lancet Neurol 14:162-173. https://doi.org/10.1016/S14744422(14)70251-0

239. Bischoff JM, Petersen M, Uceyler N, Sommer C, Kehlet H, Werner MU (2013) Lidocaine patch $(5 \%)$ in treatment of persistent inguinal postherniorrhaphy pain: a randomized, doubleblind, placebo-controlled, crossover trial. Anesthesiology 119:1444-1452

240. Bischoff JM, Ringsted TK, Petersen M, Sommer C, Uceyler N, Werner MU (2014) A capsaicin (8\%) patch in the treatment of severe persistent inguinal postherniorrhaphy pain: a randomized, double-blind, placebo-controlled trial. PLoS One 9(10):e109144. https://doi.org/10.1371/journal.pone.0109144 (Electronic Resour)

241. Bischoff JM, Koscielniak-Nielsen ZJ, Kehlet H, Werner MU (2012) Ultrasound-guided ilioinguinal/iliohypogastric nerve blocks for persistent inguinal postherniorrhaphy pain: a randomized, double-blind, placebo-controlled, crossover trial. Anesth Analg 114(6):1323-1329. https://doi.org/10.1213/ANE. 0b013e31824d6168

242. Thomassen I, van Suijlekom JA, van de Gaag A, Ponten JEH, Nienhuijs SW (2013) Ultrasound-guided ilioinguinal/iliohypogastric nerve blocks for chronic pain after inguinal hernia repair. Hernia 17(3):329-332. https://doi.org/10.1007/s10029012-0998-y

243. Parris D, Fischbein N, Mackey S, Carroll I (2010) A novel CTguided transpsoas approach to diagnostic genitofemoral nerve block and ablation. Pain Med 11(5):785-789. https://doi.org/10. 1111/j.1526-4637.2010.00835.x

244. Fanelli RD, DiSiena MR, Lui FY, Gersin KS (2003) Cryoanalgesic ablation for the treatment of chronic postherniorrhaphy neuropathic pain. Surg Endosc Other Interv Tech 17(2):196-200. https://doi.org/10.1007/s00464-002-8840-8

245. Cohen SP, Foster A (2003) Pulsed radiofrequency as a treatment for groin pain and orchialgia. Urology 61:645

246. Rozen D, Ahn J (2006) Pulsed radiofrequency for the treatment of ilioinguinal neuralgia after inguinal herniorrhaphy. Mt Sinai J Med 73(4):716-718

247. Rozen D, Parvez U (2006) Pulsed radiofrequency of lumbar nerve roots for treatment of chronic inguinal herniorraphy pain. Pain Physician 9(2):153-156 
248. Mitra R, Zeighami A, Mackey S (2007) Pulsed radiofrequency for the treatment of chronic ilioinguinal neuropathy. Hernia 11(4):369-371. https://doi.org/10.1007/s10029-007-0191-x

249. Campos NA, Chiles JH, Plunkett AR (2009) Ultrasound-guided cryoablation of genitofemoral nerve for chronic inguinal pain. Pain Physician 12(6):997-1000

250. Kastler A, Aubry S, Piccand V, Hadjidekov G, Tiberghien F, Kastler B (2012) Radiofrequency neurolysis versus local nerve infiltration in 42 patients with refractory chronic inguinal neuralgia. Pain Physician 15(3):237-244

251. Kastler A, Aubry S, Barbier-Brion B, Jehl J, Kastler B (2012) Radiofrequency neurolysis in the management of inguinal neuralgia: preliminary study. Radiology 262(2):701-707. https://doi.org/10.1148/radiol.11110727

252. Werner MU, Bischoff JM, Rathmell JP, Kehlet H (2012) Pulsed radiofrequency in the treatment of persistent pain after inguinal herniotomy: a systematic review. Reg Anesth Pain Med 37:340-343. https://doi.org/10.1097/AAP.0b013e31824bea4e

253. Rosendal F, Moir L, De Pennington N, Green AL, Aziz TZ (2013) Successful treatment of testicular pain with peripheral nerve stimulation of the cutaneous branch of the ilioinguinal and genital branch of the genitofemoral nerves. Neuromodulation 16(2):121-123. https://doi.org/10.1111/j.1525-1403.2011. 00421.x

254. Schu S, Gulve A, Eldabe S et al (2014) Spinal cord stimulation of the dorsal root ganglion for groin pain-a retrospective review. Pain Pract 15(4):293-299. https://doi.org/10.1111/papr. 12194

255. Yakovlev AE, Al Tamimi M, Barolat G et al (2010) Spinal cord stimulation as alternative treatment for chronic post-herniorrhaphy pain. Neuromodulation 13(4):288-290. https://doi.org/ 10.1111/j.1525-1403.2010.00276.x

256. Amid PK (2014) Radiologic images of meshoma. A new phenomenon causing chronic pain after prosthetic repair of abdominal wall hernias. Arch Surg 139:1297-1298

257. Heise CP, Starling JR (1998) Mesh inguinodynia: a new clinical syndrome after inguinal herniorrhaphy? J Am Coll Surg 187(5):514-518

258. Rosen MJ, Novitsky YW, Cobb WS, Kercher KW, Heniford BT (2006) Combined open and laparoscopic approach to chronic pain following open inguinal hernia repair. Hernia 10(1):20-24. https://doi.org/10.1007/s10029-005-0032-8

259. Keller JE, Stefanidis D, Dolce CJ, Iannitti DA, Kercher KW, Heniford BT (2008) Combined open and laparoscopic approach to chronic pain after inguinal hernia repair. Am Surg 74(8):691-695

260. Aasvang EK, Kehlet H (2009) The effect of mesh removal and selective neurectomy on persistent postherniotomy pain. Ann Surg 249(2):327-334. https://doi.org/10.1097/SLA. 0b013e31818eec49

261. Koopmann MC, Yamane BH, Starling JR (2011) Long-term follow-up after meshectomy with acellular human dermis repair for postherniorrhaphy inguinodynia. Arch Surg (Chicago Ill 1960) 146(4):427-431. https://doi.org/10.1001/archsurg.2011.49

262. Bischoff JM, Enghuus C, Werner MU, Kehlet H (2013) Longterm follow-up after mesh removal and selective neurectomy for persistent inguinal postherniorrhaphy pain. Hernia 17(3):339-345. https://doi.org/10.1007/s10029-013-1073-z

263. Campanelli G, Bertocchi V, Cavalli M et al (2013) Surgical treatment of chronic pain after inguinal hernia repair. Hernia 17(3):347-353. https://doi.org/10.1007/s10029-013-1059-x

264. Valvekens E, Nijs Y, Miserez M (2015) Long-term outcome of surgical treatment of chronic postoperative groin pain: a word of caution. Hernia 19(4):587-594. https://doi.org/10.1007/s10029013-1125-4
265. Amid PK (2002) A 1-stage surgical treatment for postherniorrhaphy neuropathic pain. Arch Surg 137:100-104. https://doi. org/10.1001/archsurg.137.1.100

266. Amid PK (2004) Causes, prevention, and surgical treatment of postherniorrhaphy neuropathic inguinodynia: triple neurectomy with proximal end implantation. Hernia 8(4):343-349. https://doi.org/10.1007/s10029-004-0247-0

267. Bower S, Moore BB, Weiss SM (1996) Neuralgia after inguinal hernia repair. Am Surg 62(8):664-667

268. Deysine M, Deysine G, Reed W (2002) Groin pain in the absence of hernia: a new syndrome. Hernia 6(2):64-67. https://doi.org/10.1007/s10029-002-0057-1

269. Ducic I, West J, Maxted W (2008) Management of chronic postoperative groin pain. Ann Plast Surg 60:294-298. https://doi.org/10.1097/SAP.0b013e3180de600e

270. Giger U, Wente MN, Büchler MW, Krähenbühl S, Lerut J, Krähenbühl L (2009) Endoscopic retroperitoneal neurectomy for chronic pain after groin surgery. Br J Surg 96(9):1076-1081. https://doi.org/10.1002/bjs.6623

271. Lee CH, Dellon AL (2000) Surgical management of groin pain of neural origin. J Am Coll Surg 191(2):137-142. https://doi. org/10.1016/S1072-7515(00)00319-7

272. Loos MJ, Scheltinga MR, Roumen RM (2010) Tailored neurectomy for treatment of postherniorrhaphy inguinal neuralgia. Surgery 147(2):275-281. https://doi.org/10.1016/j.surg. 2009.08.008

273. Madura JA, Madura JA 2nd, Copper CM, Worth RM (2005) Inguinal neurectomy for inguinal nerve entrapment: an experience with 100 patients. Am J Surg 189(3):283-287. https://doi. org/10.1016/j.amjsurg.2004.11.015

274. Starling JR, Harms BA, Schroeder ME, Eichman PL (1987) Diagnosis and treatment of genitofemoral and ilioinguinal entrapment neuralgia. Surgery 102(4):581-586

275. Vuilleumier H, Hübner M, Demartines N (2009) Neuropathy after herniorrhaphy: indication for surgical treatment and outcome. World J Surg 33(4):841-845. https://doi.org/10.1007/ s00268-008-9869-1

276. Zacest AC, Magill ST, Anderson VC, Burchiel KJ (2010) Longterm outcome following ilioinguinal neurectomy for chronic pain. J Neurosurg 112(4):784-789. https://doi.org/10.3171/2009. 8.JNS09533

277. Kim D, Murovic J, Tiel R, Kline D (2005) Surgical management of 33 ilioingional and iliohypogastric neuralgias at Louisiana State University Health Sciences Center. Neurosurgery 56:1013-1020

278. Kingsnorth A, LeBlanc K (2003) Hernias: inguinal and incisional. Lancet 362:1561-1571. https://doi.org/10.1016/S0140$6736 \% 2803 \% 2914746-0$

279. Neumayer L, Giobbie-Hurder A, Jonasson O et al (2004) Open mesh versus laparoscopic mesh repair of inguinal hernia. N Engl J Med 350(18):1819-1827. https://doi.org/10.1056/ NEJMoa040093

280. Staarink M, Van Veen RN, Hop WC, Weidema WF (2008) A 10-year follow-up study on endoscopic total extraperitoneal repair of primary and recurrent inguinal hernia. Surg Endosc Other Interv Tech 22(8):1803-1806. https://doi.org/10.1007/ s00464-008-9917-9

281. Burcharth J, Andresen K, Pommergaard H-C, Bisgaard T, Rosenberg J (2014) Recurrence patterns of direct and indirect inguinal hernias in a nationwide population in Denmark. Surgery 155(1):173-177. https://doi.org/10.1016/j.surg.2013.06.006

282. Bisgaard T, Bay-Nielsen M, Kehlet H (2008) Re-recurrence after operation for recurrent inguinal hernia. A nationwide 8-year follow-up study on the role of type of repair. Ann Surg 247(4):707-711. 0b013e31816b18e3
https://doi.org/10.1097/SLA. 
283. Tran H, Tran K, Turingan I, Zajkowska M, Lam V, Hawthorne W (2015) Single-incision laparoscopic inguinal herniorraphy with telescopic extraperitoneal dissection: technical aspects and potential benefits. Hernia 19:407-416. https://doi.org/10.1007/ s10029-015-1349-6

284. Tran H (2013) Australian statistics. https://medicareaus tralia.gov.au/statistics/mbs_itme.shtml

285. Scheuerlein H, Schiller A, Schneider C, Scheidbach H, Tamme C, Köckerling F (2003) Totally extraperitoneal repair of recurrent inguinal hernia: results from 179 consecutive patients. Surg Endosc Other Interv Tech 17(7):1072-1076. https://doi.org/10. 1007/s00464-002-8957-9

286. Köckerling F, Jacob D, Wiegank W et al (2016) Endoscopic repair of primary versus recurrent male unilateral inguinal hernias: are there differences in the outcome? Surg Endosc 30(3):1146-1155. https://doi.org/10.1007/s00464-015-4318-3

287. Fitzgibbons RJ, Ramanan B, Arya S et al (2013) Long-term results of a randomized controlled trial of a nonoperative strategy (watchful waiting) for men with minimally symptomatic inguinal hernias. Ann Surg 258(3):508-515. https://doi.org/10. 1097/SLA.0b013e3182a19725

288. Chung L, Norrie J, O'Dwyer PJ (2011) Long-term follow-up of patients with a painless inguinal hernia from a randomized clinical trial. Br J Surg 98(4):596-599. https://doi.org/10.1002/ bjs. 7355

289. Hwang M, Bhangu A, Webster C, Bowley D, Gannon M, Karandikar S (2014) Unintended consequences of policy change to watchful waiting for asymptomatic inguinal hernias. Ann R Coll Surg Engl 96(5):343-347. https://doi.org/10.1308/ $003588414 X 13946184902000$

290. Rosenberg J, Bisgaard T, Kehlet H et al (2011) Danish Hernia Database recommendations for the management of inguinal and femoral hernia in adults. Dan Med Bull 58(2):42-43

291. Schroder DM, Lloyd LR, Boccaccio JE, Wesen CA (2004) Inguinal hernia recurrence following preperitoneal Kugel patch repair. Am Surg 70:132-136

292. Bingener J, Dorman JP, Valdes G (2003) Recurrence rate after laparoscopic repair of recurrent inguinal hernias: have we improved? Surg Endosc Other Interv Tech 17(11):1781-1783. https://doi.org/10.1007/s00464-002-8556-9

293. Katri KM (2009) Open preperitoneal mesh repair of recurrent inguinal hernia. Hernia 13(6):585-589. https://doi.org/10.1007/ s10029-009-0520-3

294. Karatepe O, Acet E, Altiok M, Adas G, Cakir A, Karahan S (2010) Preperitoneal repair (open posterior approach) for recurrent inguinal hernias previously treated with Lichtenstein tension-free hernioplasty. Hippokratia 14(2):119-121

295. Beitler JC, Gomes SM, Coelho ACJ, Manso JEF (2009) Complex inguinal hernia repairs. Hernia 13(1):61-66. https://doi.org/ 10.1007/s10029-008-0432-7

296. Langer I, Herzog U, Schuppisser JP, Ackermann C, Tondelli P (1996) Preperitoneal prosthesis implantation in surgical management of recurrent inguinal hernia. Retrospective evaluation of our results 1989-1994. Chirurg 67(4):394-402

297. Gilbert A, Berende C, Ruurda J, Olsman J, Hazenberg C, van Geffen H (2007) Inguinal hernia treatment with Prolene hernia system in a Dutch regional training hospital. Hernia 11(4):297. https://doi.org/10.1007/s10029-007-0224-5

298. Nienhuijs SW, Rosman C (2015) Long-term outcome after randomizing prolene hernia system, mesh plug repair and Lichtenstein for inguinal hernia repair. Hernia 19:77-81. https://doi.org/10.1007/s10029-014-1295-8

299. Sevonius D, Montgomery A, Smedberg S, Sandblom G (2015) Chronic groin pain, discomfort and physical disability after recurrent groin hernia repair: impact of anterior and posterior mesh repair. Hernia 20(1):1-11. https://doi.org/10.1007/s10029015-1439-5

300. Lau H (2004) Endoscopic totally extraperitoneal inguinal hernioplasty for recurrence after open repair. ANZ J Surg 74(10):877-880. https://doi.org/10.1111/j.1445-1433.2004. 03193.x

301. Li J, Ji Z, Li Y (2014) Comparison of laparoscopic versus open procedure in the treatment of recurrent inguinal hernia: a metaanalysis of the results. Am J Surg 207:602-612. https://doi.org/ 10.1016/j.amjsurg.2013.05.008

302. Muschaweck U et al (2007) How to treat recurrent inguinal hernia. In: Schumpelick V, Fitzgibbons RJ (eds) Recurrent hernia. Springer, Berlin, Heidelberg, pp 289-307. https://doi. org/10.1007/978-3-540-68988-1_26

303. Sevonius D, Sandblom G, Agger E, Smedberg S, Montgomery A (2015) The impact of type of mesh repair on 2nd recurrence after recurrent groin hernia surgery. World J Surg 39(2):315-322. https://doi.org/10.1007/s00268-014-2921-4

304. Kjaergaard J, Bay-Nielsen M, Kehlet H (2010) Mortality following emergency groin hernia surgery in Denmark. Hernia 14(4):351-355. https://doi.org/10.1007/s10029-010-0657-0

305. Kurt N, Oncel M, Ozkan Z, Bingul S (2003) Risk and outcome of bowel resection in patients with incarcerated groin hernias: retrospective study. World J Surg 27(6):741-743. https://doi. org/10.1007/s00268-003-6826-x

306. Suppiah A, Gatt M, Barandiaran J, Heng MS, Perry EP (2007) Outcomes of emergency and elective femoral hernia surgery in four district general hospitals: a 4-year study. Hernia 11(6):509-512. https://doi.org/10.1007/s10029-007-0262-z

307. de Guzmán CA-R, Picazo-Yeste J, Tenías-Burillo JM, MorenoSanz C (2013) Improved outcomes of incarcerated femoral hernia: a multivariate analysis of predictive factors of bowel ischemia and potential impact on postoperative complications. Am J Surg 205(2):188-193. https://doi.org/10.1016/j.amjsurg. 2012.03.011

308. Ge B-J, Huang Q, Liu L-M, Bian H-P, Fan Y-Z (2010) Risk factors for bowel resection and outcome in patients with incarcerated groin hernias. Hernia 14(3):259-264. https://doi.org/10. 1007/s10029-009-0602-2

309. Azari Y, Perry Z, Kirshtein B (2015) Strangulated groin hernia in octogenarians. Hernia 19(3):443-447. https://doi.org/10.1007/ s10029-013-1205-5

310. Pesic I, Karanikolic A, Djordjevic N et al (2012) Incarcerated inguinal hernias surgical treatment specifics in elderly patients. Vojnosanit Pregl 69(9):778-782. https://doi.org/10.2298/ VSP1209778P

311. Romain B, Chemaly R, Meyer N, Brigand C, Steinmetz JP, Rohr S (2012) Prognostic factors of postoperative morbidity and mortality in strangulated groin hernia. Hernia 16(4):405-410. https://doi.org/10.1007/s10029-012-0937-y

312. Duan S, Liu H, Niu J, Wang C, Chen S, Wang M (2014) Classifications of clinical and bowel morphological changes and their relationship with characteristics of patients with incarcerated groin hernias. Med Sci Monit 20:214-218. https://doi.org/ 10.12659/MSM.889786

313. Rettenbacher T, Hollerweger A, Macheiner P et al (2001) Abdominal wall hernias: cross-sectional imaging signs of incarceration determined with sonography. AJR Am J Roentgenol 177(5):1061-1066. https://doi.org/10.2214/ajr.177.5. 1771061

314. Chen S-C, Lee C-C, Liu Y-P et al (2005) Ultrasound may decrease the emergency surgery rate of incarcerated inguinal hernia. Scand J Gastroenterol 40(6):721-724. https://doi.org/10. 1080/00365520510015485

315. Tanaka N, Uchida N, Ogihara H, Sasamoto H, Kato H, Kuwano $\mathrm{H}$ (2010) Clinical study of inguinal and femoral incarcerated 
hernias. Surg Today 40(12):1144-1147. https://doi.org/10.1007/ s00595-009-4193-4

316. Karatepe O, Adas G, Battal M et al (2008) The comparison of preperitoneal and Lichtenstein repair for incarcerated groin hernias: a randomised controlled trial. Int J Surg 6:189-192. https://doi.org/10.1016/j.ijsu.2008.02.007

317. George SM, Mangiante EC, Voeller GR, Britt LG (1991) Preperitoneal herniorrhaphy for the acutely incarcerated groin hernia. Am Surg 57:139-141

318. Rebuffat C, Galli A, Scalambra MS, Balsamo FCN (2006) Laparoscopic repair of strangulated hernias. Surg Endosc Other Interv Tech 20:131-134. https://doi.org/10.1007/s00464-0050171-0

319. Papaziogas B, Lazaridis C, Makris J et al (2005) Tension-free repair versus modified Bassini technique (Andrews technique) for strangulated inguinal hernia: a comparative study. Hernia 9(2):156-159. https://doi.org/10.1007/s10029-004-0311-9

320. Bessa SS, Katri KM, Abdel-Salam WN, Abdel-Baki NA (2007) Early results from the use of the Lichtenstein repair in the management of strangulated groin hernia. Hernia 11(3):239-242. https://doi.org/10.1007/s10029-007-0207-6

321. Elsebae MMA, Nasr M, Said M (2008) Tension-free repair versus Bassini technique for strangulated inguinal hernia: a controlled randomized study. Int J Surg 6(4):302-305. https://doi.org/10.1016/j.ijsu.2008.04.006

322. Atila K, Guler S, Inal A, Sokmen S, Karademir S, Bora S (2010) Prosthetic repair of acutely incarcerated groin hernias: a prospective clinical observational cohort study. Langenbeck's Arch Surg 395(5):563-568. https://doi.org/10.1007/s00423-0080414-3

323. Ueda J, Nomura T, Sasaki J et al (2012) Prosthetic repair of an incarcerated groin hernia with small intestinal resection. Surg Today 42(4):359-362. https://doi.org/10.1007/s00595-0110019-2

324. Oida T, Kawasaki A, Mimatsu K et al (2012) Mesh vs. nonmesh repair for inguinal hernias in emergency operations. Hepatogastroenterology 59(119):2112-2114. https://doi.org/10. 5754/hge10309

325. Sawayama H, Kanemitsu K, Okuma T, Inoue K, Yamamoto K, Baba H (2014) Safety of polypropylene mesh for incarcerated groin and obturator hernias: a retrospective study of 110 patients. Hernia 18(3):399-406. https://doi.org/10.1007/s10029013-1058-y

326. Hentati H, Dougaz W, Dziri C (2014) Mesh repair versus nonmesh repair for strangulated inguinal hernia: systematic review with meta-analysis. World J Surg 38:2784-2790. https://doi.org/ 10.1007/s00268-014-2710-0

327. Sgourakis G, Radtke A, Sotiropoulos GC, Dedemadi G, Fouzas I, Karaliotas C (2009) Assessment of strangulated content of the spontaneously reduced inguinal hernia via hernia sac laparoscopy: preliminary results of a prospective randomized study. Surg Laparosc Endosc Percutaneous Tech 19:133-137. https://doi.org/10.1097/SLE.0b013e31819d8b8b

\section{PART 3}

1. Sani R, Sanoussi S, Didier JL, Salifou GM, Abarchi H (2013) Rural surgery in Niger: a multicentric study in 21 district hospitals. Indian J Surg 77(December):1-5. https://doi.org/10.1007/ s12262-013-1015-0

2. Zendejas B, Onkendi EO, Brahmbhatt RD, Greenlee SM, Lohse CMFD (2011) Contralateral metachronous inguinal hernia in adults: role for prophylaxis during the TEP repair. Hernia 15(4):403-408

3. Zendejas B, Cook DA, Hernández-Irizarry R, Huebner M, Farley DR (2012) Mastery learning simulation-based curriculum for laparoscopic TEP inguinal hernia repair. J Surg Educ 69(2):208-214. https://doi.org/10.1016/j.jsurg.2011.08.008

4. Kurashima Y, Feldman LS, Al-Sabah S, Kaneva PA, Fried GM, Vassiliou MC (2011) A tool for training and evaluation of laparoscopic inguinal hernia repair: the Global Operative Assessment Of Laparoscopic Skills-Groin Hernia (GOALSGH). Am J Surg 201(1):54-61. https://doi.org/10.1016/j.amj surg.2010.09.006

5. Tien T, Pucher PH, Sodergren MH, Sriskandarajah K, Yang GZ, Darzi A (2014) Differences in gaze behaviour of expert and junior surgeons performing open inguinal hernia repair. Surg Endosc Other Interv Tech 29(2):405-413. https://doi.org/10. 1007/s00464-014-3683-7

6. McCulloch P, Cook JA, Altman DG, Heneghan C, Diener MK (2013) IDEAL framework for surgical innovation 1: the idea and development stages. BMJ 346:f3012

7. Horeyseck G, Roland F, Rolfes N (1996) “Tension-free" repair of inguinal hernia: laparoscopic (TAPP) versus open (Lichtenstein) repair. [German] TT - Die "spannungsfreie" Reparation der Leistenhernie: laparoskopisch (TAPP) versus offen (Lichtenstein). Chirurg 67:1036-1040

8. Wang YT, Meheš MM, Naseem H-R et al (2014) Assessing the impact of short-term surgical education on practice: a retrospective study of the introduction of mesh for inguinal hernia repair in sub-Saharan Africa. Hernia 18(4):549-556. https://doi. org/10.1007/s10029-014-1255-3

9. Robson AJ, Wallace CG, Sharma AK, Nixon SJ, PatersonBrown S (2004) Effects of training and supervision on recurrence rate after inguinal hernia repair. Br J Surg 91(6):774-777. https://doi.org/10.1002/bjs.4540

10. Wilkiemeyer M, Pappas TN, Giobbie-Hurder A, Itani KMF, Jonasson O, Neumayer LA (2005) Does resident post graduate year influence the outcomes of inguinal hernia repair? Ann Surg 241(6):879-884. https://doi.org/10.1097/01.sla.0000164076. 82559.72

11. Papandria D, Rhee D, Ortega G et al (2012) Assessing trainee impact on operative time for common general surgical procedures in ACS-NSQIP. J Surg Educ 69(2):149-155. https://doi. org/10.1016/j.jsurg.2011.08.003

12. Paajanen H (2003) Groin hernia repair under local anaesthesia: effect of surgeon's training level on long-term results. Ambul Surg. https://doi.org/10.1016/j.ambsur.2003.06.001

13. Abdelrahman T, Long J, Egan R, Lewis WG (2016) Operative experience vs. competence: a curriculum concordance and learning curve analysis. J Surg Educ 73(4):694-698. https://doi. org/10.1016/j.jsurg.2016.01.011

14. Ramsay CR, Grant AM, Wallace SA, Garthwaite PH, Monk AF, Russell IT (2001) Statistical assessment of the learning curves of health technologies. Health Technol Assess 5:1-79

15. Schouten N, Simmermacher RKJ, van Dalen T et al (2013) Is there an end of the "learning curve" of endoscopic totally extraperitoneal (TEP) hernia repair? Surg Endosc 27(3):789-794. https://doi.org/10.1007/s00464-012-2512-0

16. Haidenberg J, Kendrick ML, Meile T, Farley DR (2003) Totally extraperitoneal (TEP) approach for inguinal hernia: the favorable learning curve for trainees. Curr Surg 60(1):65-68. https://doi.org/10.1016/S0149-7944(02)00657-8

17. Lau H, Patil NG, Yuen WK, Lee F (2002) Learning curve for unilateral endoscopic totally extraperitoneal (TEP) inguinal hernioplasty. Surg Endosc Other Interv Tech 16(12):1724-1728. https://doi.org/10.1007/s00464-001-8298-0 
18. Hasbahceci M, Basak F, Acar A, Alimoglu O (2014) A new proposal for learning curve of TEP inguinal hernia repair: ability to complete operation endoscopically as a first phase of learning curve. Minim Invasive Surg 2014:528517. https://doi.org/10. $1155 / 2014 / 528517$

19. Liem MS, van Steensel CJ, Boelhouwer RU et al (1996) The learning curve for totally extraperitoneal laparoscopic inguinal hernia repair. Am J Surg 171(2):281-285. https://doi.org/10. 1016/S0002-9610(97)89569-4

20. Hernández-Irizarry R, Zendejas B, Ali SM, Lohse CM, Farley DR (2012) Impact of resident participation on laparoscopic inguinal hernia repairs: are residents slowing us down? J Surg Educ 69(6):746-752. https://doi.org/10.1016/j.jsurg.2012.08. 013

21. Lim JW, Lee JY, Lee SE et al (2012) The learning curve for laparoscopic totally extraperitoneal herniorrhaphy by moving average. J Korean Surg Soc 83(2):92-96. https://doi.org/10. 4174/jkss.2012.83.2.92

22. Cox TC, Pearl JP, Parreno D, Moore RRE (2011) Fellowship training eliminates the learning curve for laparoscopy inguinal hernia repair. Surg Endosc 25:S216

23. Choi YY, Kim Z, Hur KY (2012) Learning curve for laparoscopic totally extraperitoneal repair of inguinal hernia. Can $\mathrm{J}$ Surg 55(1):33-36. https://doi.org/10.1503/cjs.019610

24. Lal K, Laghari ZH, Laghari A (2011) Laparoscopic total extra peritoneal mesh repair and open Lichtenstein mesh repair for the treatment of inguinal hernia. Med Channel 17:13-17

25. Neumayer LA, Gawande AA, Wang J et al (2005) Proficiency of surgeons in inguinal hernia repair: effect of experience and age. Ann Surg 242(3):344-348. https://doi.org/10.1097/01.sla. 0000179644.02187.ea

26. Lamb ADG, Robson AJ, Nixon SJ (2006) Recurrence after totally extraperitoneal laparoscopic repair: implications for operative technique and surgical training. Surgeon 4(5):299-307. https://doi.org/10.1016/S1479-666X(06)80007-7

27. Zendejas B, Onkendi EO, Brahmbhatt RD, Lohse CM, Greenlee SM, Farley DR (2011) Long-term outcomes of laparoscopic totally extraperitoneal inguinal hernia repairs performed by supervised surgical trainees. Am J Surg 201(3):379-384. https://doi.org/10.1016/j.amjsurg.2010.08.019

28. Bittner R, Schmedt CG, Schwarz J, Kraft K, Leibl BJ (2002) Laparoscopic transperitoneal procedure for routine repair of groin hernia. Br J Surg 89(8):1062-1066. https://doi.org/10. 1046/j.1365-2168.2002.02178.x

29. Bökeler U, Schwarz J, Bittner R, Zacheja S, Smaxwil C (2013) Teaching and training in laparoscopic inguinal hernia repair (TAPP): impact of the learning curve on patient outcome. Surg Endosc 27(8):2886-2893. https://doi.org/10.1007/s00464-0132849-z

30. Edwards CC, Bailey RW (2000) Laparoscopic hernia repair: the learning curve. Surg Laparosc Endosc Percutan Tech 10(3):149-153

31. Ridings P, Evans DS (2000) The transabdominal pre-peritoneal (TAPP) inguinal hernia repair: a trip along the learning curve. J R Coll Surg Edinb 45(1):29-32

32. Voitk AJ (1998) The learning curve in laparoscopic inguinal hernia repair for the community general surgeon. Can J Surg 41(6):446-450

33. Khatib M, Hald N, Brenton H et al (2014) Validation of open inguinal hernia repair simulation model: a randomized controlled educational trial. Am J Surg 208(2):295-301. https://doi. org/10.1016/j.amjsurg.2013.12.007

34. Rowse PG, Ruparel RK, Abdelsattar JM, AlJamal YN, Dy BM, Farley DR (2016) TEP and Lichtenstein anatomy: does simulation accelerate acquisition among interns? Hernia 20(3):411-416. https://doi.org/10.1007/s10029-015-1409-y
35. Driscoll PJ, Paisley AM, Paterson-Brown S (2008) Video assessment of basic surgical trainees' operative skills. Am J Surg 196(2):265-272. https://doi.org/10.1016/j.amjsurg.2007. 09.044

36. Trevisonno M, Kaneva P, Watanabe Y et al (2015) A survey of general surgeons regarding laparoscopic inguinal hernia repair: practice patterns, barriers, and educational needs. Hernia 19(5):719-724. https://doi.org/10.1007/s10029-014-1287-8

37. Zahiri HR, Park AE, Pugh CM, Vassiliou M, Voeller G (2015) "See one, do one, teach one": inadequacies of current methods to train surgeons in hernia repair. Surg Endosc Other Interv Tech 29(10):2867-2872. https://doi.org/10.1007/s00464-015-4411-7

38. Gurusamy K, Nagendran M, Toon C, Davidson B (2014) Laparoscopic surgical box model training for surgical trainees with limited prior laparoscopic experience (review). Cochrane Database Syst Rev 3(3):CD010478

39. Zendejas B, Cook DA, Bingener J et al (2011) Simulation-based mastery learning improves patient outcomes in laparoscopic inguinal hernia repair: a randomized controlled trial. Ann Surg 254(3):502-511. 0b013e31822c6994

40. Miserez M, Arregui M, Bisgaard T et al (2009) A standardized resident training program in endoscopic surgery in general and in laparoscopic totally extraperitoneal (TEP) inguinal hernia repair in particular. Surg Laparosc Endosc Percutan Tech 19(4):125-129. https://doi.org/10.1097/SLE.0b013e3181a9ce06

41. Hamilton EC, Scott DJ, Kapoor A et al (2001) Improving operative performance using a laparoscopic hernia simulator. Am J Surg 182(6):725-728

42. Shapiro SJ, Gordon LA, Daykhovsky L, Senter N (1994) The laparoscopic hernia trainer. The role of a life-like trainer in laparoendoscopic education. Endosc Surg Allied Technol 2(1):66-68

43. Poudel S, Kurashima Y, Kawarada Y et al (2015) Development and validation of a checklist for assessing recorded performance of laparoscopic inguinal hernia repair. Am J Surg. https://doi. org/10.1016/j.amjsurg.2015.09.014

44. Yamazaki M, Nomura E, Uchida K et al (2015) A prospective, single-arm, single-center, case series to determine the feasibility of safe skill transfer for transabdominal preperitoneal (TAPP) repair utilizing a hands-on mentorship model. Tokai J Exp Clin Med 40(4):161-164

45. Kurashima Y, Feldman L, Al-Sabah S, Kaneva P, Fried G, Vassiliou M (2011) A novel low-cost simulator for laparoscopic inguinal hernia repair. Surg Innov 18(2):171-175. https://doi. org/10.1177/1553350610395949

46. Kurashima Y, Feldman LS, Kaneva PA et al (2014) Simulationbased training improves the operative performance of totally extraperitoneal (TEP) laparoscopic inguinal hernia repair: a prospective randomized controlled trial. Surg Endosc 28(3):783-788. https://doi.org/10.1007/s00464-013-3241-8

47. Stefanidis D, Korndorffer JR, Heniford BT, Scott DJ (2007) Limited feedback and video tutorials optimize learning and resource utilization during laparoscopic simulator training. Surgery 142(2):202-206. https://doi.org/10.1016/j.surg.2007.03. 009

48. Bingener J, Boyd T, Van Sickle K et al (2008) Randomized double-blinded trial investigating the impact of a curriculum focused on error recognition on laparoscopic suturing training. Am J Surg 195(2):179-182. https://doi.org/10.1016/j.amjsurg. 2007.11.001

49. Brydges R, Hatala R, Zendejas B, Erwin PJ, Cook DA (2015) Linking simulation-based educational assessments and patientrelated outcomes: a systematic review and meta-analysis. Acad Med 90(2):246-256. https://doi.org/10.1097/ACM. 0000000000000549 
50. Simons MP, Aufenacker T, Bay-Nielsen M et al (2009) European Hernia Society guidelines on the treatment of inguinal hernia in adult patients. Hernia 13:343-403. https://doi.org/10. 1007/s10029-009-0529-7

51. Poelman MM, van den Heuvel B, Deelder JD et al (2013) EAES Consensus Development Conference on endoscopic repair of groin hernias. Surg Endosc 27(10):3505-3519. https://doi.org/ 10.1007/s00464-013-3001-9

52. Köckerling F, Berger D, Jost JO (2014) What is a certified hernia center? The example of the German Hernia Society and German Society of General and Visceral Surgery. Front Surg 1(July):1-4. https://doi.org/10.3389/fsurg.2014.00026

53. Morales-Conde S, Socas M, Fingerhut A (2012) Endoscopic surgeons' preferences for inguinal hernia repair: TEP, TAPP, or OPEN. Surg Endosc 26(9):2639-2643. https://doi.org/10.1007/ s00464-012-2247-y

54. Stepaniak PS, Vrijland WW, de Quelerij M, de Vries G, Heij C (2010) Working with a fixed operating room team on consecutive similar cases and the effect on case duration and turnover time. Arch Surg 145(12):1165-1170. https://doi.org/10.1001/ archsurg.2010.255

55. O'Dwyer PJ (2004) Current status of the debate on laparoscopic hernia repair. Br Med Bull 70:105-118. https://doi.org/10.1093/ bmb/ldh027

56. Tamme C, Scheidbach H, Hampe C, Schneider C, Köckerling F (2003) Totally extraperitoneal endoscopic inguinal hernia repair (TEP). Surg Endosc Other Interv Tech 17(2):190-195. https://doi.org/10.1007/s00464-002-8905-8

57. Killeen SD, O'Sullivan MJ, Coffey JC, Kirwan WO, Redmond HP (2005) Provider volume and outcomes for oncological procedures. Br J Surg 92(4):389-402. https://doi.org/10.1002/bjs. 4954

58. Finlayson EVA, Birkmeyer JD (2003) Effects of hospital volume on life expectancy after selected cancer operations in older adults: a decision analysis. J Am Coll Surg 196(3):410-417. https://doi.org/10.1016/S1072-7515(02)01753-2

59. Belyansky I, Tsirline VB, Klima DA, Walters AL, Lincourt AE, Heniford TB (2011) Prospective, comparative study of postoperative quality of life in TEP, TAPP, and modified Lichtenstein repairs. Ann Surg 254(5):709-714. https://doi.org/10.1097/SLA. 0b013e3182359d07

60. Zanghi A, Di Vita M, Lo Menzo E et al (2011) Multicentric evaluation by Verbal Rate Scale and EuroQoL-5D of early and late post-operative pain after TAPP and TEP procedures with mechanical fixation for bilateral inguinal hernias. Ann Ital Chir 82(6):437-442

61. Bansal VK, Misra MC, Babu D et al (2013) A prospective, randomized comparison of long-term outcomes: chronic groin pain and quality of life following totally extraperitoneal (TEP) and transabdominal preperitoneal (TAPP) laparoscopic inguinal hernia repair. Surg Endosc Other Interv Tech 27:2373-2382. https://doi.org/10.1007/s00464-013-2797-7

62. Wang W, Chen J, Fang Q, Li J, Jin P-F, Li Z-T (2013) Comparison of the effects of laparoscopic hernia repair and Lichtenstein tension-free hernia repair. J Laparoendosc Adv Surg Tech A 23(4):301-305. https://doi.org/10.1089/lap.2012.0217

63. Nordin P, van der Linden W (2008) Volume of procedures and risk of recurrence after repair of groin hernia: national register study. BMJ 336(7650):934-937. https://doi.org/10.1136/bmj. 39525.514572.25

64. van der Linden W, Warg A, Nordin P (2011) National register study of operating time and outcome in hernia repair. Arch Surg 146(10):1198-1203. https://doi.org/10.1001/archsurg.2011.268

65. El-Dhuwaib Y, Corless D, Emmett C, Deakin M, Slavin J (2013) Laparoscopic versus open repair of inguinal hernia: a longitudinal cohort study. Surg Endosc 27(3):936-945. https://doi.org/10.1007/s00464-012-2538-3

66. Neumayer L, Giobbie-Hurder A, Jonasson O et al (2004) Open mesh versus laparoscopic mesh repair of inguinal hernia. N Engl J Med 350:1819-1827 + 1922. http://dx.doi.org/10.1056/ NEJMoa040093

67. Ravindran R, Bruce J, Debnath D, Poobalan A, King PM (2006) A United Kingdom survey of surgical technique and handling practice of inguinal canal structures during hernia surgery. Surgery 139(4):523-526. https://doi.org/10.1016/j.surg.2005.09. 008

68. Stephenson BM (2003) Complications of open groin hernia repairs. Surg Clin N Am 83(5):1255-1278. https://doi.org/10. 1016/S0039-6109(03)00128-2

69. Frisén A, Starck J, Smeds S, Nyström PO, Kald A (2011) Analysis of outcome of Lichtenstein groin hernia repair by surgeons-in-training versus a specialized surgeon. Hernia 15(3):281-288. https://doi.org/10.1007/s10029-010-0780-y

70. Amato L, Colais P, Davoli M et al (2013) Volume and health outcomes: evidence from systematic reviews and from evaluation of Italian hospital data. Epidemiol Prev 37:1-100

71. Biau DJ, Halm JA, Ahmadieh H et al (2008) Provider and center effect in multicenter randomized controlled trials of surgical specialties: an analysis on patient-level data. Ann Surg 247(5):892-898. 0b013e31816ffa99

72. Williams KB, Belyansky I, Dacey KT et al (2014) Impact of the establishment of a specialty hernia referral center. Surg Innov 21(6):572-579. https://doi.org/10.1177/1553350614528579

73. Cueto Rozon R, De Baerdemacker Y, Polliand C, Champault G (2006) Surgical training and inguinal hernia repair. Ann Chir 131(5):311-315. https://doi.org/10.1016/j.anchir.2006.02.003

74. Shulman AG, Amid PK, Lichtenstein IL (1995) A survey of non-expert surgeons using the open tension-free mesh patch repair for primary inguinal hernias. Int Surg 80(1):35-36

75. Gilbert AI, Young J, Graham MF, Divilio LT, Patel B (2004) Combined anterior and posterior inguinal hernia repair: intermediate recurrence rates with three groups of surgeons. Hernia 8(3):203-207. https://doi.org/10.1007/s10029-004-0238-1

76. Gilbert AI, Graham MF, Young J, Patel BG, Shaw K (2006) Closer to an ideal solution for inguinal hernia repair: comparison between general surgeons and hernia specialists. Hernia 10(2):162-168. https://doi.org/10.1007/s10029-005-0054-2

77. Hahn S, Whitehead A (2003) An illustration of the modelling of cost and efficacy data from a clinical trial. Stat Med 22(6):1009-1024. https://doi.org/10.1002/sim.1459

78. Schurz J, Arregui M, Hammond J (1995) Open vs laparoscopic hernia repair. Analysis of costs, charges, and outcomes. Surg Endosc 9(3):1310-1317

79. Millikan KW, Deziel DJ (1996) The management of hernia. Considerations in cost effectiveness. Surg Clin North Am 76(1):105-116

80. Vale L, Grant A, McCormack K, Scott NW (2004) Cost-effectiveness of alternative methods of surgical repair of inguinal hernia. Int $\mathbf{J}$ Technol Assess Health Care 20:192-200. https://doi.org/10.1017/S0266462304000972

81. Coronini-Cronberg S, Appleby J, Thompson J (2013) Application of patient-reported outcome measures (PROMs) data to estimate cost-effectiveness of hernia surgery in England. J R Soc Med 106(7):278-287. https://doi.org/10.1177/ 0141076813489679

82. Langeveld HR, van't Riet M, Weidema WF et al (2010) Total extraperitoneal inguinal hernia repair compared with Lichtenstein (the LEVEL-Trial): a randomized controlled trial. Ann Surg 251(5):819-824. https://doi.org/10.1097/SLA. 0b013e3181d96c32 
83. Eklund A, Carlsson P, Rosenblad A, Montgomery A, Bergkvist L, Rudberg C (2010) Long-term cost-minimization analysis comparing laparoscopic with open (Lichtenstein) inguinal hernia repair. Br J Surg 97(5):765-771. https://doi.org/10.1002/bjs. 6945

84. Aly O, Green A, Joy M et al (2011) Is laparoscopic inguinal hernia repair more effective than open repair? J Coll Physicians Surg Pak 21:291-296

85. Wittenbecher F, Scheller-Kreinsen D, Röttger J, Busse R (2013) Comparison of hospital costs and length of stay associated with open-mesh, totally extraperitoneal inguinal hernia repair, and transabdominal preperitoneal inguinal hernia repair: an analysis of observational data using propensity score matching. Surg Endosc 27(4):1326-1333. https://doi.org/10.1007/s00464-0122608-6

86. Sgourakis G, Dedemadi G, Gockel I et al (2013) Laparoscopic totally extraperitoneal versus open preperitoneal mesh repair for inguinal hernia recurrence: a decision analysis based on net health benefits. Surg Endosc Other Interv Tech 27(7):2526-2541. https://doi.org/10.1007/s00464-012-2776-4

87. Koperna T (2004) How long do we need teaching in the operating room? The true costs of achieving surgical routine. Langenbecks Arch Surg 389(3):204-208

88. Salcedo-Wasicek MC, Thirlby RC (1995) Postoperative course after inguinal herniorrhaphy. A case-controlled comparison of patients receiving workers' compensation vs patients with commercial insurance. Arch Surg 130(1):29-32. https://doi.org/ 10.1001/archsurg.1995.01430010031006

89. McCormack K, Scott NW, Go PM, Ross S, Grant AM (2003) Laparoscopic techniques versus open techniques for inguinal hernia repair. Cochrane Database Syst Rev (1):CD001785. https://doi.org/10.1002/14651858.CD001785

90. Khajanchee YS, Kenyon TAG, Hansen PD, Swanström LL (2004) Economic evaluation of laparoscopic and open inguinal herniorrhaphies: the effect of cost-containment measures and internal hospital policy decisions on costs and charges. Hernia 8(3):196-202. https://doi.org/10.1007/s10029-004-0212-y

91. McCormack K, Wake B, Perez J et al (2005) Laparoscopic surgery for inguinal hernia repair: systematic review of effectiveness and economic evaluation. Health Technol Assess 9(14):1-203, iii-iv

92. Dirksen CD, Ament AJ, Adang EM et al (1998) Cost-effectiveness of open versus laparoscopic repair for primary inguinal hernia. Int J Technol Assess Health Care 14(3):472-483

93. Fleming WR, Elliott TB, Jones RM, Hardy KJ (2001) Randomized clinical trial comparing totally extraperitoneal inguinal hernia repair with the Shouldice technique. Br J Surg 88(9):1183-1188. https://doi.org/10.1046/j.0007-1323.2001. 01865. $\mathrm{x}$

94. Amato B, Moja L, Panico S et al (2009) Shouldice technique versus other open techniques for inguinal hernia repair. Cochrane Database Syst Rev. https://doi.org/10.1002/14651858. CD001543.pub3

95. Payne JH, Grininger LM, Izawa MT, Podoll EF, Lindahl PJ, Balfour J (1994) Laparoscopic or open inguinal herniorrhaphy? A randomized prospective trial. Arch Surg 129(9):973-981

96. Brooks DC (1994) A prospective comparison of laparoscopic and tension-free open herniorrhaphy. Arch Surg 129(4):361-366. $\quad$ https://doi.org/10.1001/archsurg.1994. 01420280031004

97. Lawrence K, Mcwhinnie D, Goodwin A et al (1995) Randomised controlled trial of laparoscopic versus open repair of inguinal hernia: early results. Br Med J 311(7011):981-985

98. Barkun JS, Wexler MJ, Hinchey EJ et al (1995) Laparoscopic versus open inguinal herniorrhaphy: preliminary results of a randomized controlled trial. Surgery 118:703-710. https://doi. org $110.1016 / \mathrm{S} 0039-6060 \% 2805 \% 2980038-8$

99. van den Oever R, Debbaut B (1996) Cost analysis of inguinal hernia surgery in ambulatory and inpatient management. Zentralbl Chir 121(10):836-840

100. Lawrence K, McWhinnie D, Goodwin A et al (1996) An economic evaluation of laparoscopic versus open inguinal hernia repair. J Public Health Med 18(1):41-48

101. Liem MS, Halsema JA, van der Graaf Y, Schrijvers AJ, van Vroonhoven TJ (1997) Cost-effectiveness of extraperitoneal laparoscopic inguinal hernia repair: a randomized comparison with conventional herniorrhaphy. Coala Trial Group. Ann Surg 226(6):668-676

102. Kald A, Anderberg B, Carlsson P, Park PO, Smedh K (1997) Surgical outcome and cost-minimisation-analyses of laparoscopic and open hernia repair: a randomised prospective trial with one year follow up. Eur J Surg 163:505-510

103. Damamme A, Samama G, D'Alche-Gautier MJ, Chanavel N, Brefort JL, Le Roux Y (1998) Medico-economic evaluation of treatment of inguinal hernia: Shouldice vs. laparoscopy. Ann Chir 52:11-16

104. Zieren J, Zieren HU, Jacobi CA, Wenger FA, Muller JM (1998) Prospective randomized study comparing laparoscopic and open tension-free inguinal hernia repair with Shouldice's operation. Am J Surg 175:330-333. https://doi.org/10.1016/S0002-9610\% 2898\%2900004-X

105. Heikkinen TJ, Haukipuro K, Hulkko A (1998) A cost and outcome comparison between laparoscopic and Lichtenstein hernia operations in a day-case unit. A randomized prospective study. Surg Endosc 12(10):1199-1203

106. Wellwood J, Sculpher MJ, Stoker D et al (1998) Randomised controlled trial of laparoscopic versus open mesh repair for inguinal hernia: outcome and cost. Br Med J 317:103-110

107. Paganini AM, Lezoche E, Carle F et al (1998) A randomized, controlled, clinical study of laparoscopic vs open tension-free inguinal hernia repair. Surg Endosc 12:979-986

108. Johansson B, Hallerbäck B, Glise H, Anesten B, Smedberg S, Román J (1999) Laparoscopic mesh versus open preperitoneal mesh versus conventional technique for inguinal hernia repair. Ann Surg 230(2):225

109. Jonsson B, Zethraeus N (2000) Costs and benefits of laparoscopic surgery-a review of the literature. Eur J Surg Acta Chir Suppl 585:48-56

110. Medical Research Council Laparoscopic Groin Hernia Trial (2001) Cost-utility analysis of open versus laparoscopic groin hernia repair: results from a multicentre randomized clinical trial. Br J Surg 88(5):653-661. https://doi.org/10.1046/j.13652168.2001.01768.x

111. Papachristou E, Mitselou M, Finokaliotis N (2002) Surgical outcome and hospital cost analyses of laparoscopic and open tension-free hernia repair. Hernia 6(2):68-72. https://doi.org/10. 1007/s10029-002-0062-4

112. Bataille N (2002) Clinical and economic evaluation of laparoscopic surgery for inguinal hernia. Return of a difficult clinical choice. [French] TT - Evaluation clinique et economique de la coeliochirurgie de la hernie de l'aine. Retour sur un difficile choix clinique. J Chir (Paris) 139:130-134

113. Schneider BE, Castillo JM, Villegas L, Scott DJ, Jones DB (2003) Laparoscopic totally extraperitoneal versus Lichtenstein herniorrhaphy: cost comparison at teaching hospitals. Surg Laparosc Endosc Percutan Tech 13(4):261-267

114. Andersson B, Hallén M, Leveau P, Bergenfelz A, Westerdahl J (2003) Laparoscopic extraperitoneal inguinal hernia repair versus open mesh repair: a prospective randomized controlled trial. Surgery 133(5):464-472. https://doi.org/10.1067/msy.2003.98 
115. Hildebrandt J, Levantin O (2003) Tension-free methods of surgery of primary inguinal hernias. Comparison of endoscopic, total extraperitoneal hernioplasty with the Lichtenstein operation. Chirurg 74(10):915-921. https://doi.org/10.1007/s00104003-0687-6

116. Anadol ZA, Ersoy E, Taneri F, Tekin E (2004) Outcome and cost comparison of laparoscopic transabdominal preperitoneal hernia repair versus Open Lichtenstein technique. J Laparoendosc Adv Surg Tech A 14(3):159-163

117. Hynes DM, Stroupe KT, Luo P et al (2006) Cost effectiveness of laparoscopic versus open mesh hernia operation: results of a Department of Veterans Affairs randomized clinical trial. J Am Coll Surg 203(4):447-457

118. Butler RE, Burke R, Schneider JJ, Brar H, Lucha PA (2007) The economic impact of laparoscopic inguinal hernia repair: results of a double-blinded, prospective, randomized trial. Surg Endosc Other Interv Tech 21(3):387-390

119. Kuhry E, van Veen RN, Langeveld HR, Steyerberg EW, Jeekel J, Bonjer HJ (2007) Open or endoscopic total extraperitoneal inguinal hernia repair? A systematic review. Surg Endosc 21:161-166

120. Gong K, Zhang N, Lu Y et al (2011) Comparison of the open tension-free mesh-plug, transabdominal preperitoneal (TAPP), and totally extraperitoneal (TEP) laparoscopic techniques for primary unilateral inguinal hernia repair: a prospective randomized controlled trial. Surg Endosc 25(1):234-239. https://doi.org/10.1007/s00464-010-1165-0

121. Smart P, Castles L (2012) Quantifying the cost of laparoscopic inguinal hernia repair. ANZ J Surg 82(11):809-812. https://doi. org/10.1111/j.1445-2197.2012.06189.x

122. Khan N, Babar TS, Ahmad M, Ahmad Z, Shah LA (2013) Outcome and cost comparison of laparoscopic transabdominal preperitoneal hernia repair versus open Lichtenstein technique. J Postgrad Med Inst 27:310-316

123. Beets GL, Dirksen CD, Go PMNYH, Geisler FEA, Baeten CGMI, Kootstra G (1999) Open or laparoscopic preperitoneal mesh repair for recurrent inguinal hernia? A randomized controlled trial. Surg Endosc 13:323-327. https://doi.org/10.1007/ s004649900981

124. Gholghesaei M, Langeveld HR, Veldkamp R, Bonjer HJ (2005) Costs and quality of life after endoscopic repair of inguinal hernia vs open tension-free repair: a review. Surg Endosc Other Interv Tech 19(6):816-821

125. Heikkinen T, Haukipuro K, Leppala J, Hulkko A (1997) Total costs of laparoscopic and Lichtenstein inguinal hernia repairs: a randomized prospective study. Surg Laparosc Endosc 7:1-5. https://doi.org/10.1097/00019509-199702000-00001

126. Heikkinen TJ, Haukipuro K, Koivukangas P, Hulkko A (1998) A prospective randomized outcome and cost comparison of totally extraperitoneal endoscopic hernioplasty versus Lichtenstein hernia operation among employed patients. Surg Laparosc Endosc 8(5):338-344. https://doi.org/10.1097/00019509199810000-00003

127. Ferzli GS, Frezza EE, Pecoraro AM, Ahern KD (1999) Prospective randomized study of stapled versus unstapled mesh in a laparoscopic preperitoneal inguinal hernia repair. J Am Coll Surg 188(5):461-465

128. Voyles CR, Hamilton BJ, Johnson WD, Kano N (2002) Metaanalysis of laparoscopic inguinal hernia trials favors open hernia repair with preperitoneal mesh prosthesis. Am J Surg 184:6-10. https://doi.org/10.1016/S0002-9610\%2802\%2900878-4

129. Jacobs VR, Morrison JE (2008) Comparison of institutional costs for laparoscopic preperitoneal inguinal hernia versus open repair and its reimbursement in an ambulatory surgery center. Surg Laparosc Endosc Percutan Tech 18(1):70-74. https://doi. org/10.1097/SLE.0b013e31815a58d7
130. Chung RS, Rowland DY (1999) Meta-analyses of randomized controlled trials of laparoscopic vs conventional inguinal hernia repairs. Surg Endosc 13(7):689-694

131. Stylopoulos N, Gazelle GS, Rattner DW (2003) A cost-utility analysis of treatment options for inguinal hernia in 1,513,008 adult patients. Surg Endosc 17(2):180-189. https://doi.org/10. 1007/s00464-002-8849-z

132. Winslow ER, Quasebarth M, Brunt LM (2004) Perioperative outcomes and complications of open vs laparoscopic extraperitoneal inguinal hernia repair in a mature surgical practice. Surg Endosc 18(2):221-227. https://doi.org/10.1007/s00464-0038934-y

133. Go PM (1998) Overview of randomized trials in laparoscopic inguinal hernia repair. Semin Laparosc Surg 5:238-241

134. Nyhus L (1995) Hernia moderators overview. Surg Endosc 9(3):1306-1310

135. Greenberg D, Peiser JG (2001) Costs and benefits of laparoscopic inguinal hernia repair-is there an economic justification? Harefuah 140(7):580-585, 680, 679

136. Basu S, Chandran S, Somers SS, Toh SKC (2005) Cost-effective laparoscopic TEP inguinal hernia repair: the Portsmouth technique. Hernia 9(4):363-367. https://doi.org/10.1007/s10029005-0006-x

137. Chatterjee S, Laxminarayan R (2013) Costs of surgical procedures in Indian hospitals. BMJ Open 3(6):e002844. https://doi. org/10.1136/bmjopen-2013-002844

138. Perniceni T, Danes M, Boudet MJ, Levard H, Gayet B (1998) Laparoscopy versus the Shouldice intervention in the treatment of unilateral inguinal hernia: can the operative surcosts be minimized? Gastroenterol Clin Biol 22:1061-1064

139. Farinas LP, Griffen FD (2000) Cost containment and totally extraperitoneal laparoscopic herniorrhaphy. Surg Endosc 14:37-40. https://doi.org/10.1007/s004649900007

140. Lau H, Lee F, Patil NG, Yuen WK (2002) Two hundred endoscopic extraperitoneal inguinal hernioplasties: cost containment by reusable instruments. Chin Med J (Engl) 115(6):888-891

141. Taylor C, Layani L, Liew V, Ghusn M, Crampton N, White S (2008) Laparoscopic inguinal hernia repair without mesh fixation, early results of a large randomised clinical trial. Surg Endosc 22(3):757-762. https://doi.org/10.1007/s00464-0079510-7

142. Bittner R, Montgomery MA, Arregui E et al (2015) Update of guidelines on laparoscopic (TAPP) and endoscopic (TEP) treatment of inguinal hernia (International Endohernia Society). Surg Endosc Other Interv Tech 29(2):289-321. https://doi.org/ 10.1007/s00464-014-3917-8

143. Begg C, Cho M, Eastwood S, Horton R, Moher D, Olkin I, Pitkin RRD, Schulz KF, Simel DSD (1996) Improving the quality of reporting of randomized controlled trials: the consort statement. JAMA 276(8):637-639. https://doi.org/10.1001/jama. 1996.03540080059030

144. Lepage L, Altman DG, Schulz KF et al (2001) The revised CONSORT statement for reporting randomized trials: explanation and elaboration. Ann Intern Med 134(8):663-694. https://doi.org/10.7326/0003-4819-134-8-200104170-00012

145. Nilsson E, Haapaniemi S (2000) The Swedish hernia register: an eight year experience. Hernia 4(2):286-289

146. Bay-Nielsen M, Kehlet H, Strand L et al (2001) Quality assessment of 26,304 herniorrhaphies in Denmark: a prospective nationwide study. Lancet 358(9288):1124-1128. https://doi.org/ 10.1016/S0140-6736(01)06251-1

147. Flay BR (1986) Efficacy and effectiveness trials (and other phases of research) in the development of health promotion programs. Prev Med (Baltim) 15(5):451-474. https://doi.org/10. 1016/0091-7435(86)90024-1 
148. Arvidsson D, Berndsen FH, Larsson LG et al (2005) Randomized clinical trial comparing 5-year recurrence rate after laparoscopic versus Shouldice repair of primary inguinal hernia. Br J Surg 92(9):1085-1091. https://doi.org/10.1002/bjs.5137

149. Deysine M, Soroff HS (1990) Must we specialize herniorrhaphy for better results? Am J Surg 160(3):239-240. https://doi.org/10. 1016/S0002-9610(06)80014-0

150. Devereaux P, Bhandari M, Clarke $M$ et al (2005) Need for expertise based randomised controlled trials: expertise based design has shortfalls. BMJ 330:88-91. https://doi.org/10.1136/ bmj.330.7494.791-b

151. Bell PR (1997) Surgical research and randomized trials. Br J Surg 84(6):737-738

152. McCulloch P, Taylor I, Sasako M, Lovett B, Griffin D (2002) Randomised trials in surgery: problems and possible solutions. BMJ 324(7351):1448-1451

153. Lilford R, Braunholtz D, Harris J, Gill T (2004) Trials in surgery. Br J Surg 91(1):6-16. https://doi.org/10.1002/bjs.4418

154. Ludvigsson JF, Otterblad-Olausson P, Pettersson BU, Ekbom A (2009) The Swedish personal identity number: possibilities and pitfalls in healthcare and medical research. Eur J Epidemiol 24(11):659-667. https://doi.org/10.1007/s10654-009-9350-y

155. Nilsson H, Nilsson E, Angerås U, Nordin P (2011) Mortality after groin hernia surgery: delay of treatment and cause of death. Hernia 15(3):301-307. https://doi.org/10.1007/s10029-0110782-4

156. Koch A, Edwards A, Haapaniemi S, Nordin P, Kald A (2005) Prospective evaluation of 6895 groin hernia repairs in women. Br J Surg 92(12):1553-1558. https://doi.org/10.1002/bjs.5156

157. Bay-Nielsen M, Kehlet H (2008) Anaesthesia and post-operative morbidity after elective groin hernia repair: a nation-wide study. Acta Anaesthesiol Scand 52(2):169-174. https://doi.org/10. 1111/j.1399-6576.2007.01514.x

158. Bisgaard T, Bay-Nielsen M, Kehlet H (2008) Re-recurrence after operation for recurrent inguinal hernia. A nationwide 8-year follow-up study on the role of type of repair. Ann Surg 247(4):707-711. 0b013e31816b18e3

https://doi.org/10.1097/SLA.

159. Nilsson H, Stranne J, Stattin P, Nordin P (2014) Incidence of groin hernia repair after radical prostatectomy: a populationbased nationwide study. Ann Surg 259(6):1223-1227. https://doi.org/10.1097/SLA.0b013e3182975c88

160. Bolignano D, Mattace-Raso F, Torino C et al (2013) The quality of reporting in clinical research: the CONSORT and STROBE initiatives. Aging Clin Exp Res 25(1):9-15. https://doi.org/10. 1007/s40520-013-0007-z

161. Benchimol EI, Smeeth L, Guttmann A et al (2015) The REporting of studies Conducted using Observational Routinelycollected health Data (RECORD) statement. PLoS Med. https://doi.org/10.1371/journal.pmed.1001885

162. Muysoms FE, Deerenberg EB, Peeters E et al (2013) Recommendations for reporting outcome results in abdominal wall repair: results of a Consensus meeting in Palermo, Italy, 28-30 June 2012. Hernia 17(4):423-433. https://doi.org/10.1007/ s10029-013-1108-5

163. Fitzgibbons RJ, Giobbie-Hurder A, Gibbs JO et al (2006) Watchful waiting vs repair of inguinal hernia in minimally symptomatic men: a randomized clinical trial. JAMA 295(3):285-292. https://doi.org/10.1001/jama.295.3.285

164. Campanelli G, Pascual MH, Hoeferlin A et al (2012) Randomized, controlled, blinded trial of Tisseel/Tissucol for mesh fixation in patients undergoing Lichtenstein technique for primary inguinal hernia repair: results of the TIMELI trial. Ann Surg 255:650-657. https://doi.org/10.1097/SLA.0b013e31824b32bf

165. Dahlke AR, Chung JW, Holl JL et al (2014) Evaluation of initial participation in public reporting of American College of surgeons NSQIP surgical outcomes on Medicare's hospital compare website. J Am Coll Surg. https://doi.org/10.1016/ j.jamcollsurg.2013.11.022

166. Chung L, Norrie J, O'Dwyer PJ (2011) Long-term follow-up of patients with a painless inguinal hernia from a randomized clinical trial. Br J Surg 98(4):596-599. https://doi.org/10.1002/ bjs. 7355

167. Etzioni DA (2015) Potential problems with the public reporting of risk-adjusted surgical outcomes. Dis Colon Rectum 58(5):540-542. https://doi.org/10.1097/DCR. 0000000000000368

168. Muysoms F, Campanelli G, Champault GG et al (2012) EuraHS: the development of an international online platform for registration and outcome measurement of ventral abdominal wall hernia repair. Hernia 16(3):239-250. https://doi.org/10.1007/ s10029-012-0912-7

169. Nilsson E, Haapaniemi S, Gruber G, Sandblom G (1998) Methods of repair and risk for reoperation in Swedish hernia surgery from 1992 to 1996. Br J Surg 85(12):1686-1691. https://doi.org/10.1046/j.1365-2168.1998.00886.x

170. Stechemesser B, Jacob DA, Schug-Pass C, Kockerling F (2012) Herniamed: an internet-based registry for outcome research in hernia surgery. Hernia 16(3):269-276. https://doi.org/10.1007/ s10029-012-0908-3

171. Jorgensen LN, Friis-Andersen H, Bay-Nielsen M, Kehlet H (2012) 13809 Danish Hernia database. Ugeskr Laeger 174(42): 2522

172. www.ethicon.com/healthcare. Accessed 28 Oct 2017

173. Cohen ME, Bilimoria KY, Ko CY, Hall BL (2009) Development of an American College of Surgeons National Surgery Quality Improvement Program: morbidity and mortality risk calculator for colorectal surgery. J Am Coll Surg 208(6):1009-1016. https://doi.org/10.1016/j.jamcollsurg.2009. 01.043

174. Velanovich V, Shadduck P, Khaitan L, Morton J, Maupin G, Traverso LW (2006) Analysis of the SAGES Outcomes Initiative groin hernia database. Surg Endosc Other Interv Tech 20(2):191-198. https://doi.org/10.1007/s00464-005-0436-7

175. www.alsgbi.org/resources/sword. Accessed 28 Oct 2017

176. Sanders. (2014). http://www.hscic.gov.uk//proms-background. Accessed 31 Oct 2017

177. Bailey J, Roland M, Roberts C (1999) Is follow up by specialists routinely needed after elective surgery? A controlled trial. J Epidemiol Community Health 53:118-124

178. Heniford BT, Walters AL, Lincourt AE, Novitsky YW, Hope WW, Kercher KW (2008) Comparison of generic versus specific quality-of-life scales for mesh hernia repairs. J Am Coll Surg 206(4):638-644. https://doi.org/10.1016/j.jamcollsurg.2007.11. 025

179. de Lange DH, Kreeft M, van Ramshorst GH, Aufenacker TJ, Rauwerda JA, Simons MP (2010) Inguinal hernia surgery in The Netherlands: are patients treated according to the guidelines? Hernia 14(2):143-148. https://doi.org/10.1007/s10029-0090578-y

180. Gagliardi AR, Brouwers MC, Palda VA, Lemieux-Charles L, Grimshaw JM (2009) An exploration of how guideline developer capacity and guideline implementability influence implementation and adoption: study protocol. Implement Sci 4:36. https://doi.org/10.1186/1748-5908-4-36

181. O'Connor JF (1940) Treatment of inguinal hernia in adults. Br Med J 2(4151):113-115. https://doi.org/10.1136/bmj.2.4153. 205-b

182. Bittner R, Arregui ME, Bisgaard T et al (2011) Guidelines for laparoscopic (TAPP) and endoscopic (TEP) treatment of inguinal hernia [International Endohernia Society (IEHS)]. Surg 
Endosc 25(9):2773-2843. https://doi.org/10.1007/s00464-0111799-6

183. www.agreetrust.org/agree-ii. Accessed 28 Oct 2017

184. van Barneveld TA, van den Broek L, Burgers JS, Schouten LMT, van de Steeg HC (2006) Implementeren van richtlijnen, een leidraad voor adviseurs. Version 1.0: 1-45. https:// www.zorginzicht.nl/documents. Accessed 31 Oct 2017

185. Primatesta P, Goldacre MJ (1996) Inguinal hernia repair: incidence of elective and emergency surgery, readmission and mortality. Int J Epidemiol 25(4):835-839

186. Laxminarayan R, Mills AJ, Breman JG et al (2006) Advancement of global health: key messages from the Disease Control Priorities Project. Lancet 367(9517):1193-1208. https://doi.org/ 10.1016/S0140-6736(06)68440-7

187. http://www.dcp-3.org/dcp2/chapters. Accessed 28 oct 2017

188. Galukande M, von Schreeb J, Wladis A et al (2010) Essential surgery at the district hospital: a retrospective descriptive analysis in three African countries. PLoS Med 7(3):1-10. https://doi. org/10.1371/journal.pmed.1000243

189. Nordberg EM (1984) Incidence and estimated need of caesarean section, inguinal hernia repair, and operation for strangulated hernia in rural Africa. Br Med J (Clin Res Ed) 289(6437):92-93. https://doi.org/10.1136/bmj.289.6437.92

190. Nabembezi J, Nordberg E (2001) Surgical output in Kibaale district, Uganda. East Afr Med J 78(7):379-381

191. Fente B, Ukoima H (2013) Incarcerated external anterior abdominal wall hernias. A 5 year experience in Niger Delta University Teaching Hospital, Okolobiri, Bayelsa State of Nigeria. Afr J Med Surg 1(1):001-005

192. Alvarez JA, Baldonedo RF, Bear IG, Solís JAS, Alvarez P, Jorge JI (2004) Incarcerated groin hernias in adults: presentation and outcome. Hernia 8(2):121-126. https://doi.org/10.1007/s10029003-0186-1

193. Gul M, Aliosmanoglu I, Kapan M et al (2012) Factors affecting morbidity and mortality in patients who underwent emergency operation for incarcerated abdominal wall hernia. Int Surg 97(4):305-309. https://doi.org/10.9738/CC114.1

194. Nilsson H, Stylianidis G, Haapamäki M, Nilsson E, Nordin P (2007) Mortality after groin hernia surgery. Ann Surg 245:656-660. https://doi.org/10.1097/01.sla.0000251364.32698. $4 \mathrm{~b}$

195. Lofgren J, Makumbi F, Galiwango E et al (2014) Prevalence of treated and untreated groin hernia in eastern Uganda. Br J Surg 101:728-734. https://doi.org/10.1002/bjs.9457

196. Grimes CE, Henry JA, Maraka J, Mkandawire NC, Cotton M (2014) Cost-effectiveness of surgery in low- and middle-income countries: a systematic review. World J Surg 38:252-263. https://doi.org/10.1007/s00268-013-2243-y

197. Chao TE, Sharma K, Mandigo M et al (2014) Cost-effectiveness of surgery and its policy implications for global health: a systematic review and analysis. Lancet Glob Health 2(6):334-345. https://doi.org/10.1016/S2214-109X(14)70213-X

198. Shillcutt SD, Clarke MG, Kingsnorth AN (2010) Cost-effectiveness of groin hernia surgery in the Western Region of Ghana. Arch Surg 145(10):954-961. https://doi.org/10.1001/ archsurg.2010.208

199. Beard JH, Oresanya LB, Akoko L, Mwanga A, Dicker RA, Harris HW (2014) An estimation of inguinal hernia epidemiology adjusted for population age structure in Tanzania. Hernia 18(2):289-295. https://doi.org/10.1007/s10029-013-1177-5

200. Beard JH, Oresanya LB, Ohene-Yeboah M, Dicker RA, Harris HW (2013) Characterizing the global burden of surgical disease: a method to estimate inguinal hernia epidemiology in Ghana. World J Surg 37(3):498-503. https://doi.org/10.1007/s00268012-1864-x
201. Ohene-Yeboah M, Abantanga FA (2011) Inguinal hernia disease in Africa: a common but neglected surgical condition. West Afr J Med 30(2):77-83. https://doi.org/10.4314/wajm.v30i2

202. Kingsnorth AN, Clarke MG, Shillcutt SD (2009) Public health and policy issues of hernia surgery in Africa. World J Surg 33(6):1188-1193. https://doi.org/10.1007/s00268-009-9964-y

203. Higashi H, Barendregt JJ, Kassebaum NJ, Weiser TG, Bickler SW, Vos T (2015) Surgically avertable burden of obstetric conditions in low- and middle-income regions: a modelled analysis. BJOG Int $\mathbf{J}$ Obstet Gynaecol 122(2):228-236. https://doi.org/10.1111/1471-0528.13198

204. Belcher DW, Nyame PK, Wurapa FK (1978) The prevalence of inguinal hernia in adult Ghanaian males. Trop Geogr Med 30(1):39-43

205. Yordanov Y, Stoyanov S (1969) The incidence of hernia on the island of Pemba. East Afr Med J 46(12):687-691

206. Sanders DL, Porter CS, Mitchell KCD, Kingsnorth AN (2008) A prospective cohort study comparing the African and European hernia. Hernia 12(5):527-529. https://doi.org/10.1007/s10029008-0369-x

207. Ohene-Yeboah M, Abantanga F, Oppong J et al (2009) Some aspects of the epidemiology of external hernias in Kumasi, Ghana. Hernia 13(5):529-532. https://doi.org/10.1007/s10029009-0491-4

208. Ohene-Yeboah M (2003) Strangulated external hernias in Kumasi. West Afr J Med 22(4):310-313

209. Harouna Y, Yaya H, Abdou I, Bazira L (2000) Prognosis of strangulated inguinal hernia in the adult: influence of intestinal necrosis. Apropos of 34 cases. Bull Soc Pathol Exot 93(5):317-320

210. McConkey SJ (2002) Case series of acute abdominal surgery in rural Sierra Leone. World J Surg 26(4):509-513. https://doi.org/ 10.1007/s00268-001-0258-2

211. Swedish registry (2014) http://www.svensktbrackregister.se/rap porter-71/klinikrapporter/2014. Published 2014

212. Mbah N (2007) Morbidity and mortality associated with inguinal hernia in northwestern Nigeria. West Afr $J$ Med 26(4):288-292. https://doi.org/10.4314/wajm.v26i4.28329

213. Grimes CE, Law RSL, Borgstein ES, Mkandawire NC, Lavy CBD (2012) Systematic review of met and unmet need of surgical disease in rural sub-saharan Africa. World J Surg 36(1):8-23. https://doi.org/10.1007/s00268-011-1330-1

214. Sachs M, Damm M, Encke A (1997) Historical evolution of inguinal hernia repair. World J Surg 21(2):218-223. https://doi. org/10.1007/s002689900220

215. Sakorafas GH, Halikias I, Nissotakis C et al (2001) Open tension free repair of inguinal hernias; the Lichtenstein technique. BMC Surg 1:3. https://doi.org/10.1186/1471-2482-1-3

216. Zendejas B, Ramirez T, Jones T et al (2012) Trends in the utilization of inguinal hernia repair techniques: a populationbased study. Am J Surg 203(3):313-317. https://doi.org/10. 1016/j.amjsurg.2011.10.005

217. Atkinson HDE, Nicol SG, Purkayastha S, Paterson-Brown S (2004) Surgical management of inguinal hernia: retrospective cohort study in southeastern Scotland, 1985-2001. BMJ 329(7478):1315-1316. https://doi.org/10.1136/bmj.38282. 675556.F7

218. Saia M, Mantoan D, Buja A et al (2013) Increased rate of day surgery use for inguinal and femoral hernia repair in a decade of hospital admissions in the Veneto Region (north-east Italy): a record linkage study. BMC Health Serv Res 13(1):349. https://doi.org/10.1186/1472-6963-13-349

219. Odula P, Kakande I (2009) Groin hernia at Mulago HospitalKampala-Uganda. East Afr J Surg 9(1):48-52

220. Mabula JB, Chalya PL (2012) Surgical management of inguinal hernias at Bugando Medical Centre in northwestern Tanzania: 
our experiences in a resource-limited setting. BMC Res Notes 5(1):585. https://doi.org/10.1186/1756-0500-5-585

221. Leive A, Xu K (2008) Coping with out-of-pocket health payments: empirical evidence from 15 African countries. Bull World Health Organ 86(11):849-856. https://doi.org/10.2471/ BLT.07.049403

222. Arowolo OA, Agbakwuru EA, Adisa AO, Lawal OO, Ibrahim $\mathrm{MH}$, Afolabi AI (2011) Evaluation of tension-free mesh inguinal hernia repair in Nigeria: a preliminary report. West Afr J Med 30(2):110-113

223. Stephenson BM, Kingsnorth AN (2011) Safety and sterilization of mosquito net mesh for humanitarian inguinal hernioplasty. World J Surg 35(9):1957-1960. https://doi.org/10.1007/s00268011-1176-6

224. Lofgren J, Nordin P, Ibingira C, Matovu A, Galiwango E, Wladis A (2016) A randomized trial of low-cost mesh in groin hernia repair. N Engl J Med 374:146-153

225. Tongaonkar R, Reddy B, Mehta V, Singh N, Shivade S (2003) Preliminary multicentric trial of cheap indigenous mosquito-net cloth for tension-free hernia repair. Indian J Surg 65:89-95

226. Swadia ND (2011) Laparoscopic totally extra-peritoneal inguinal hernia repair: 9 year's experience. Hernia 15(3):273-279. https://doi.org/10.1007/s10029-010-0781-x

227. Sorensen CG, Rosenberg J (2012) The use of sterilized mosquito nets for hernioplasty: a systematic review. Hernia 16:621-625. https://doi.org/10.1007/s10029-012-0973-7

228. Clarke MG, Oppong C, Simmermacher R et al (2009) The use of sterilised polyester mosquito net mesh for inguinal hernia repair in Ghana. Hernia 13(2):155-159. https://doi.org/10.1007/ s10029-008-0460-3

229. Gundre NP, Iyer SP, Subramaniyan P (2012) Prospective randomized controlled study using polyethylene mesh for inguinal hernia meshplasty as a safe and cost-effective alternative to polypropylene mesh. Updates Surg 64(1):37-42. https://doi.org/ 10.1007/s13304-011-0103-6

230. Stephenson BM, Kingsnorth AN (2011) Inguinal hernioplasty using mosquito net mesh in low income countries: an alternative and cost effective prosthesis. BMJ 343(December): $\mathrm{d} 7448$. https://doi.org/10.1136/bmj.d7448

231. Sanders DL, Kingsnorth AN, Stephenson BM (2013) Mosquito net mesh for abdominal wall hernioplasty: a comparison of material characteristics with commercial prosthetics. World J Surg 37(4):737-745. https://doi.org/10.1007/s00268-012-1900-x

232. Wilhelm TJ, Freudenberg S, Jonas E, Grobholz R, Post S, Kyamanywa P (2007) Sterilized mosquito net versus commercial mesh for hernia repair: an experimental study in goats in Mbarara/Uganda. Eur Surg Res 39(5):312-317. https://doi.org/ $10.1159 / 000104402$

233. Freudenberg S, Sano D, Ouangré E, Weiss C, Wilhelm TJ (2006) Commercial mesh versus Nylon mosquito net for hernia repair. A randomized double-blind study in Burkina Faso. World J Surg 30(10):1784-1789. https://doi.org/10.1007/s00268-0060108-3 (discussion 1790)

234. Shillcutt SD, Sanders DL, Teresa Butrón-Vila M, Kingsnorth AN (2013) Cost-effectiveness of inguinal hernia surgery in northwestern Ecuador. World J Surg 37(1):32-41. https://doi. org/10.1007/s00268-012-1808-5

235. Disease Control Priorities (2015) 3rd edition. World Bank Publications, Washington, DC, pp 151-172

236. Disease Control Priorities (2015) 3rd edition. World Bank Publications, Washington, DC, pp 213-230

237. Ozgediz D, Jamison D, Cherian M, McQueen K (2008) The burden of surgical conditions and access to surgical care in lowand middle-income countries. Bull World Health Organ 86(8):646-647. https://doi.org/10.1038/450494a
238. Dudley L, Garner P (2011) Strategies for integrating primary health services in low- and middle-income countries at the point of delivery. Cochrane Database Syst Rev (7):CD003318. https://doi.org/10.1002/14651858.CD003318.pub3

239. Lê G, Morgan R, Bestall J, Featherstone I, Veale T, Ensor T (2016) Can service integration work for universal health coverage? Evidence from Around the Globe, Health Policy. Health Policy, New York

240. Bainbridge D, Martin J, Arango M, Cheng D (2012) Perioperative and anaesthetic-related mortality in developed and developing countries: a systematic review and meta-analysis. Lancet 380(9847):1075-1081. https://doi.org/10.1016/S01406736(12)60990-8

241. Walker IA, Wilson IH (2008) Anaesthesia in developing countries—a risk for patients. Lancet 371(9617):968-969. https://doi. org/10.1016/S0140-6736(08)60432-8

242. Reece-Smith AM, Maggio AQ, Tang TY, Walsh SR (2009) Local anaesthetic vs. general anaesthetic for inguinal hernia repair: systematic review and meta-analysis. Int J Clin Pract 63(12):1739-1742. https://doi.org/10.1111/j.1742-1241.2009. 02131.x

243. Cavallo JA, Ousley J, Barrett CD et al (2014) A material costminimization analysis for hernia repairs and minor procedures during a surgical mission in the Dominican Republic. Surg Endosc 28(3):747-766. https://doi.org/10.1007/s00464-0133253-4

244. Disease Control Priorities (2015) 3rd edition. World Bank Publications, Washington, DC, pp 231-244

245. Shrime MG, Sleemi A, Ravilla TD (2014) Charitable platforms in global surgery: a systematic review of their effectiveness, cost-effectiveness, sustainability, and role training. World J Surg 39:10-20

246. World Alliance for Patient Safety (2008) Second global patient safety challenge: safe surgery saves lives. WHO Press, Geneva

247. Choua O, Djonga O, Sarrah I, Amour M, Kaboro M, Ngowe N (2014) Plasties herniaires la technique "tension free" de Lichtenstein. Notre experience a N'djamena (Tchad). Afr J Integr Health 3(1):01-04

248. Sani R, McGee J, Illo A et al (2004) The open tension free repair of inguinal hernia by the Lichtenstein procedure: our experience about 47 cases at the National Hospital of Niamey. J Afr Chir Dig 4(2):359-366

249. Brian Ostrow MD, FRCS(C). Groin Hernias in Africa. What is the most appropriate repair for Groin Hernias in Africa?. http:// www.ptolemy.ca/members/archives/2005/Groin_Hernias_Au gust_2005.pdf. Accessed 31 Oct 2017

250. Warwick A, Oppong C, Boateng Doah B, Kingsnorth A (2013) Inguinal hernia repair is safe in Africa. East Cent Afr J Surg 18(2):14-17

251. Rutkow IM (2003) Demographic and socioeconomic aspects of hernia repair in the United States in 2003. Surg Clin N Am 83(5):1045-1051, v-vi. https://doi.org/10.1016/S00396109(03)00132-4

252. Sanders DL, Kingsnorth AN (2012) Prosthetic mesh materials used in hernia surgery. Expert Rev Med Devices 9(2):159-179. https://doi.org/10.1586/erd.11.65

253. Ashar BS, Dang JM, Krause D, Luke MC (2011) Performing clinical studies involving hernia mesh devices: what every investigator should know about the FDA investigational device exemption (IDE) process. Hernia 15(6):603-605. https://doi.org/ 10.1007/s10029-011-0872-3

254. Oribabor FO, Amao OA, Akanni SOFS (2015) The use of nontreated mosquito-net mesh cloth for a tension free inguinal hernia repair: our experience. Niger J Surg Off Publ Niger Surg Res Soc 21(1):48-51. https://doi.org/10.4103/1117-6806. 152726 
255. Sanders DL, Kingsnorth AN, Moate R, Steer JA (2013) An in vitro study assessing the infection risk of low-cost polyethylene mosquito net compared with commercial hernia prosthetics. J Surg Res. https://doi.org/10.1016/j.jss.2013.01.047

256. Farmer D (2010) Surgeon, do you know where your DALYs are?: (Can you fix a hernia with a mosquito net?): comment on "Cost-effectiveness of groin hernia surgery in the Western Region of Ghana". Arch Surg 145(10):961

257. Udwadia TE (2007) Commercial mesh versus nylon mosquito net for hernia repair. A randomized double-blind study in Burkina Faso. World J Surg 31:858

258. Tongaonkar RR, Sanders DL, Kingsnorth AN (2013) Ten-year personal experience of using low density polyethylene (LDPE) mesh for inguinal hernia repair. Trop Med Surg 1(5):5-7. https://doi.org/10.4172/2329-9088.10001

259. Fox C (2009) Mosquito net: a story of the pioneers of tropical medicine. Rev Inst Med Trop Sao Paulo 51(2):72. https://doi. org/10.1590/S0036-46652009000200014

260. Yang J, Papandria D, Rhee D, Perry H, Abdullah F (2011) Lowcost mesh for inguinal hernia repair in resource-limited settings. Hernia 15(5):485-489. https://doi.org/10.1007/s10029-0110827-8

261. Bergström S (2005) Who will do the caesareans when there is no doctor? Finding creative solutions to the human resource crisis. BJOG Int J Obstet Gynaecol 112(9):1168-1169. https://doi.org/ 10.1111/j.1471-0528.2005.00719.x
262. www.essentialsurgery.ucsf.edu/bellagio/docs/bellagio_report. pdf. Accessed 28 Oct 2017

263. Grimes C, Lavy C (2010) Role of UK hospitals in supporting surgical training in Africa. Bull R Coll Surg Engl 92(10):1-4. https://doi.org/10.1308/147363510X533676

264. Davies JI, Meara JG (2015) Global surgery-going beyond the Lancet Commission. Lancet 386(9993):507-509. https://doi.org/ 10.1016/S0140-6736(15)60465-2

265. Rothenberg S, Holcomb G, Georgeson K, Irish M, Lucas E, Blinman T (2007) Web-based live telesurgery for minimally invasive procedures in children as an educational tool. $\mathrm{J} \mathrm{La}-$ paroendosc Adv Surg Tech 17(2):226-229. https://doi.org/10. 1089/lap.2006.0011

266. Datta N, MacQueen IT, Schroeder AD et al (2015) Wearable technology for global surgical teleproctoring. J Surg Educ 72(6):1290-1295

267. Ponsky TA, Rothenberg SS (2015) Modern, multi-media, advances in surgical information. Semin Pediatr Surg 24(3):124-129. https://doi.org/10.1053/j.sempedsurg.2015.02. 010

268. Geiger JD, Hirschl RB (2015) Innovation in surgical technology and techniques: challenges and ethical issues. Semin Pediatr Surg 24(3):115-121. https://doi.org/10.1053/j.sempedsurg.2015. 02.008 